\title{
Digital Tabulation of Geologic and Hydrologic Data from Water Wells in the Northern San Francisco Bay Region, Northern California
}

By D.S. Sweetkind and E.M. Taylor

Geology and Ground Water in Napa and Sonoma Valleys

Napa and Sonoma Counties California

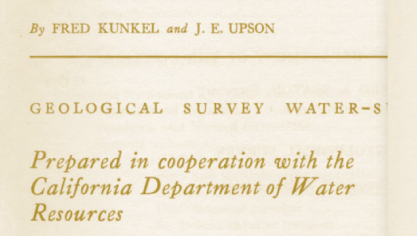

Resources

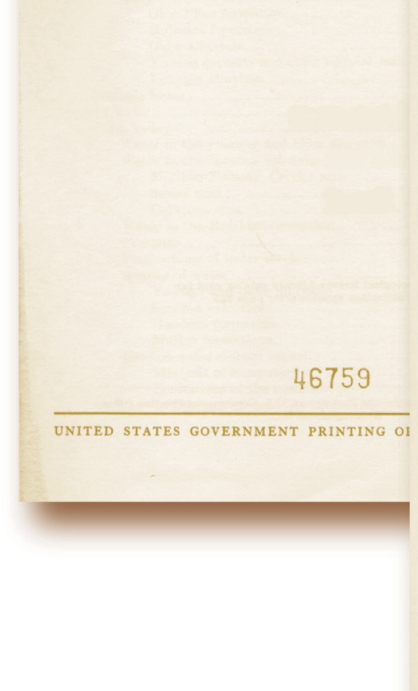

Geology and Ground Water in

Russian River Valley Areas and in Round, Laytonville and Little Lake Valleys Sonoma and Mendocino Counties, California By G. T. CARDWELL

Prepared in cooperation with the California Department of Water Resources

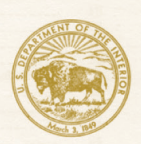

Water in the Santa Rosa and Petaluma Valley Areas

Sonoma County

\section{California}

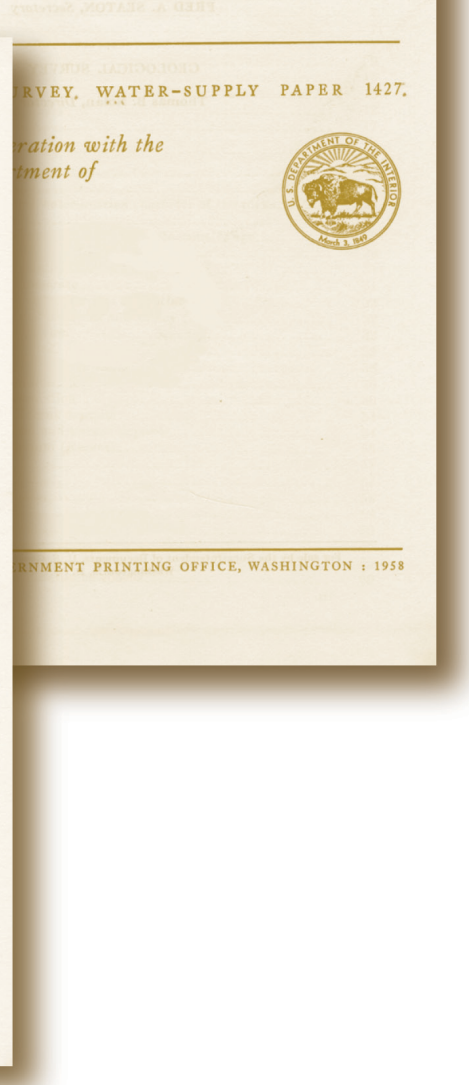

Open-File Report 2010-1063

U.S. Department of the Interior

U.S. Geological Survey 


\section{U.S. Department of the Interior \\ KEN SALAZAR, Secretary}

\section{U.S. Geological Survey \\ Marcia K. McNutt, Director}

U.S. Geological Survey, Reston, Virginia: 2010

For product and ordering information:

World Wide Web: http://www.usgs.gov/pubprod

Telephone: 1-888-ASK-USGS

For more information on the USGS—-the Federal source for science about the Earth, its natural and living resources, natural hazards, and the environment:

World Wide Web: http://www.usgs.gov

Telephone: 1-888-ASK-USGS

Suggested citation:

Sweetkind, D.S., and Taylor, E.M., 2010, Digital tabulation of geologic and hydrologic data from water wells in the northern San Francisco Bay region, northern California: U.S. Geological Survey Open-File Report 2010-1063, 125 p.

Any use of trade, product, or firm names is for descriptive purposes only and does not imply endorsement by the U.S. Government.

Although this report is in the public domain, permission must be secured from the individual copyright owners to reproduce any copyrighted material contained within this report. 


\section{Contents}

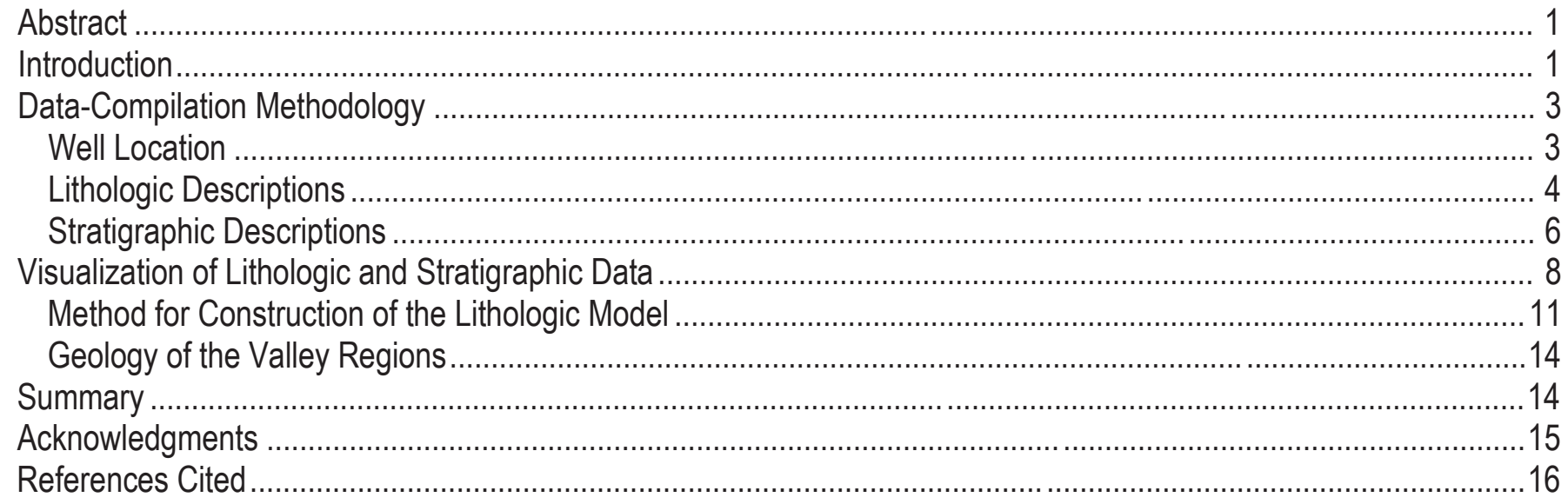

\section{Figures}

1. Location of well data described in three Water-Supply papers...................................................................... 2

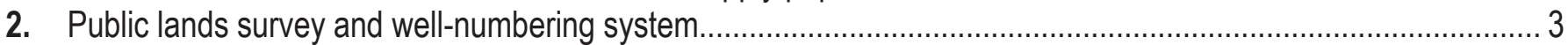

3. Topographic and simplified geologic maps of the Santa Rosa, Sonoma, and Napa Valleys showing well and vertical section locations...............................................................................................................

4. Frequency of (A) the number of wells drilled between 1918 and 1960 and $(B)$ the total depth of the wells

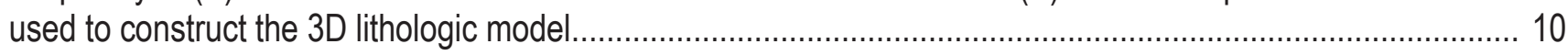

5. Three-dimensional portrayals of (A) well lithologic data and (B) well data with vertical sections in Santa Rosa, Sonoma, and Napa Valleys....................................................................................... 12

6. Detail of well data and vertical sections in (A) Santa Rosa Valley, (B), Sonoma Valley, and (C) the southern

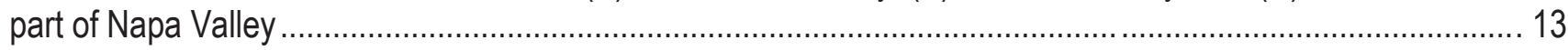

\section{Tables}

1. Generalized lithologic classes based on driller's descriptions. ..................................................................

2. Description of simplified stratigraphic units.......................................................................................

\section{Appendixes}

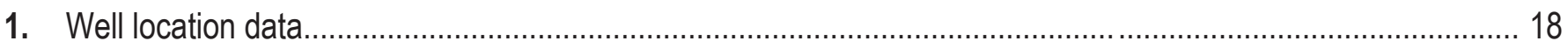

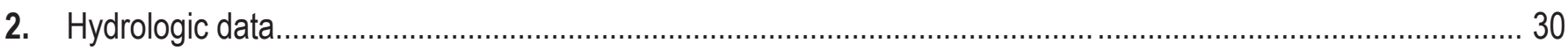

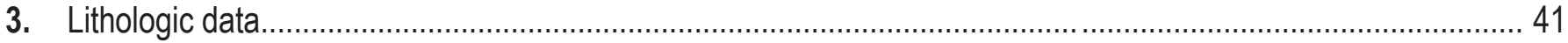




\section{Conversion Factors}

SI to Inch/Pound

\begin{tabular}{lcl}
\hline \multicolumn{1}{c}{ Multiply } & By & To obtain \\
\hline centimeter $(\mathrm{cm})$ & 0.3937 & inch (in) \\
millimeter $(\mathrm{mm})$ & 0.03937 & inch (in) \\
meter $(\mathrm{m})$ & 3.281 & foot (ft) \\
kilometer $(\mathrm{km})$ & 0.6214 & mile (mi) \\
\hline \multicolumn{3}{c}{ Area } \\
\hline square meter $\left(\mathrm{m}^{2}\right)$ & 0.0002471 & acre \\
square kilometer $\left(\mathrm{km}^{2}\right)$ & 247.1 & acre \\
\hline & Flow rate & \\
\hline liter per second $(\mathrm{L} / \mathrm{s})$ & 15.85 & gallon per minute $(\mathrm{gal} / \mathrm{min})$ \\
cubic meter per day $\left(\mathrm{m}^{3} / \mathrm{d}\right)$ & 264.2 & gallon per day (gal $/ \mathrm{d})$ \\
\hline
\end{tabular}

Temperature in degrees Fahrenheit $\left({ }^{\circ} \mathrm{F}\right)$ may be converted to degrees Celsius $\left({ }^{\circ} \mathrm{C}\right)$ as follows:

${ }^{\circ} \mathrm{C}=\left({ }^{\circ} \mathrm{F}-32\right) / 1.8$

Vertical coordinate information is referenced to the the North American Vertical Datum of 1988 (NAVD 88).

Horizontal coordinate information is referenced to the North American Datum of 1927 (NAD 27).

Elevation, as used in this report, refers to distance above the vertical datum. 


\title{
Digital Tabulation of Geologic and Hydrologic Data from Water Wells in the Northern San Francisco Bay Region, Northern California
}

\author{
By D.S. Sweetkind and E.M. Taylor
}

\begin{abstract}
Downhole lithologic information and aquifer pumping test data are reported from 464 wells from a broad area of the northern part of the Coast Ranges in California. These data were originally published in paper form as numerous tables within three USGS Water-Supply Papers describing geology and groundwater conditions in Napa and Sonoma Valleys, the Santa Rosa and Petaluma Valley areas, and in the Russian River Valley and areas in Sonoma and Mendocino Counties, Calif. The well data are compiled in this report in digital form suitable for use in a digital mapping environment. These data, although mostly from relatively shallow water wells, provide important subsurface information that displays the disposition and facies transition of lithologic units throughout this broad area. Well lithologic data themselves and simple three-dimensional interpolation of those data show distinct spatial patterns that are linked to subsurface stratigraphy and structure and can be used to aid in the assessment of the groundwater resources.
\end{abstract}

\section{Introduction}

In this Open-File report we compile in digital form geologic and hydrologic data from water wells in a broad area of the northern part of the Coast Ranges in California. These data were originally published as numerous tables within three USGS Water-Supply Papers describing geology and groundwater conditions in Napa and Sonoma Valleys (Kunkel and Upson, 1960), the Santa Rosa and Petaluma Valley areas (Cardwell, 1958), and in the Russian River Valley and areas in Sonoma and Mendocino Counties, Calif. (Cardwell, 1965) (fig. 1).

The northern part of the San Francisco Bay area has experienced rapid population growth and urbanization in recent decades. Resulting changes in land use within Sonoma County and the northern Coast Ranges require an understanding of subsurface geologic conditions and aquifer properties to aid in the assessment of the groundwater resources (California Department of Water Resources, 1982). The digital data compiled here are suitable for incorporation within a geographic information system (GIS) and for use in construction of three-dimensional (3D) geologic framework models that can be used to address these issues. 


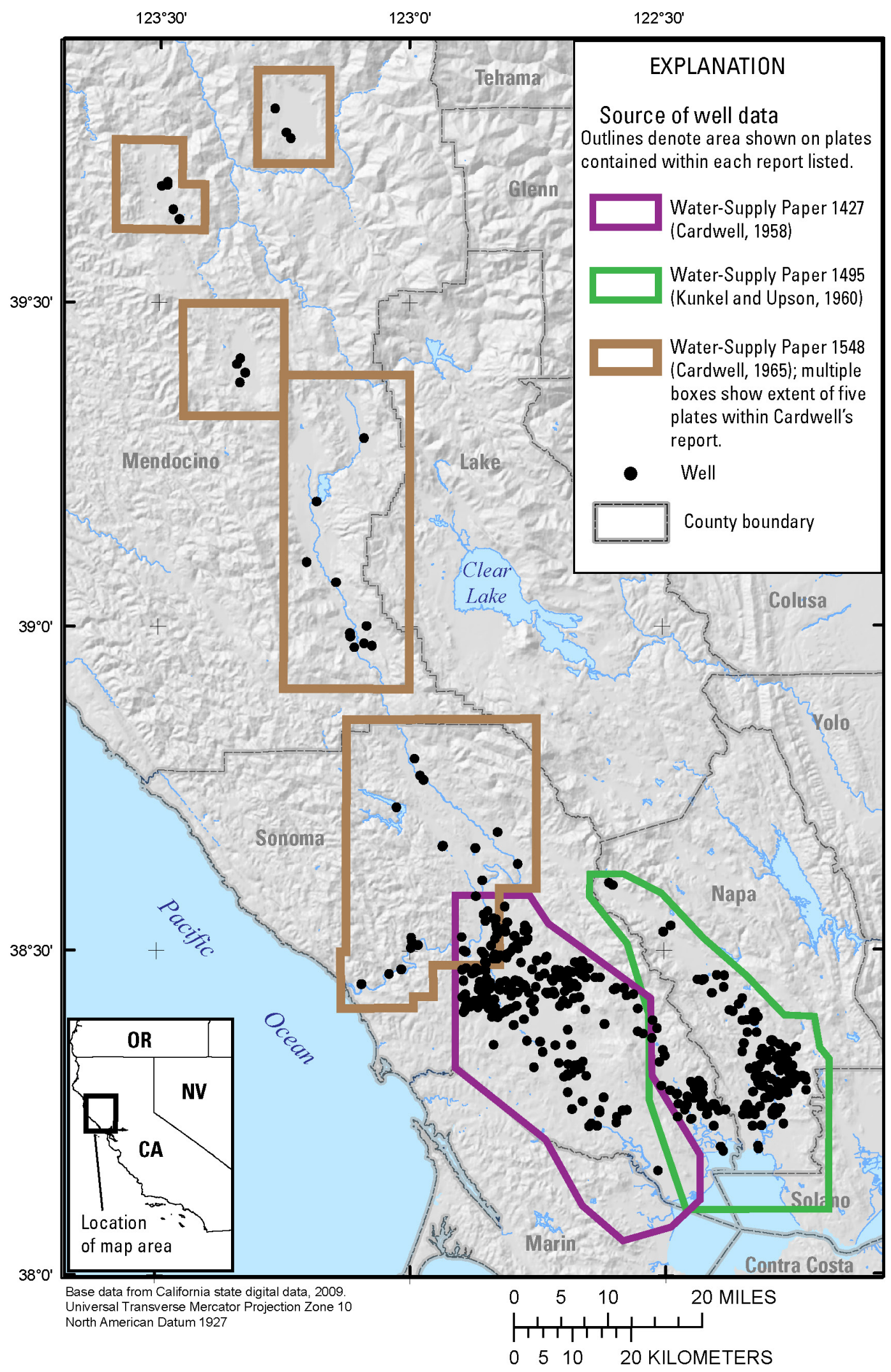

Figure 1. Location of well data described in three Water-Supply papers. 
This data tabulation includes all downhole lithologic information and aquifer pumping test data from 464 wells (plate 1; appendixes 1-3) described in the three USGS Water-Supply Papers (Cardwell, 1958; Kunkel and Upson, 1960; Cardwell, 1965) (fig. 1). A subset of these data, tabulating the lithology present at the water table for wells only in the Santa Rosa Valley area, was previously released by Valin and McLaughlin (2005).

\section{Data-Compilation Methodology}

In the three USGS Water-Supply Papers (Cardwell, 1958; Kunkel and Upson, 1960; Cardwell, 1965 ) and in this report, a well is defined as subsurface excavation or boring intended to be used to locate, sample, or develop groundwater or other material. Many of the wells have multiple zones of perforations, but for the purposes of this report these are considered to be all in the same well.

The focus of our data transcription was on wells that had downhole lithologic data and that had lithologic or stratigraphic information reported from the water-bearing interval (appendixes 1-3). There remain a number of data sets and tables from the three USGS Water-Supply Papers (Cardwell, 1958; Kunkel and Upson, 1960; Cardwell, 1965) that we did not transcribe, including water levels on wells for which no geologic data were reported and tables of water chemistry data. Our emphasis was on compiling subsurface geologic information and not necessarily in creating an exhaustive and complete transcription of these three published reports.

\section{Well Location}

The well-numbering system used in the three USGS Water-Supply Papers (appendix 1) locates the water wells according to their location in the rectangular system for the subdivision of public lands. Identification consists of the township number, north or south; the range number, east or west; and the section number (fig. 2). Each section is divided into sixteen 40 -acre tracts lettered consecutively (except I and $\mathrm{O}$ ), beginning with "A" in the northeast corner of the section and progressing in a boustrophedonic manner to "R" in the southeast corner. Within the 40 -acre tract, wells are numbered sequentially in the order they are inventoried. Many valley areas were privately acquired through land grants made by the Spanish or Mexican Governments and have never been formally surveyed into sections (plate 1). In order to locate wells in these areas, we subdivided these areas for reference purposes only by extending survey lines from adjacent areas or by superimposing an arbitrary land grid.

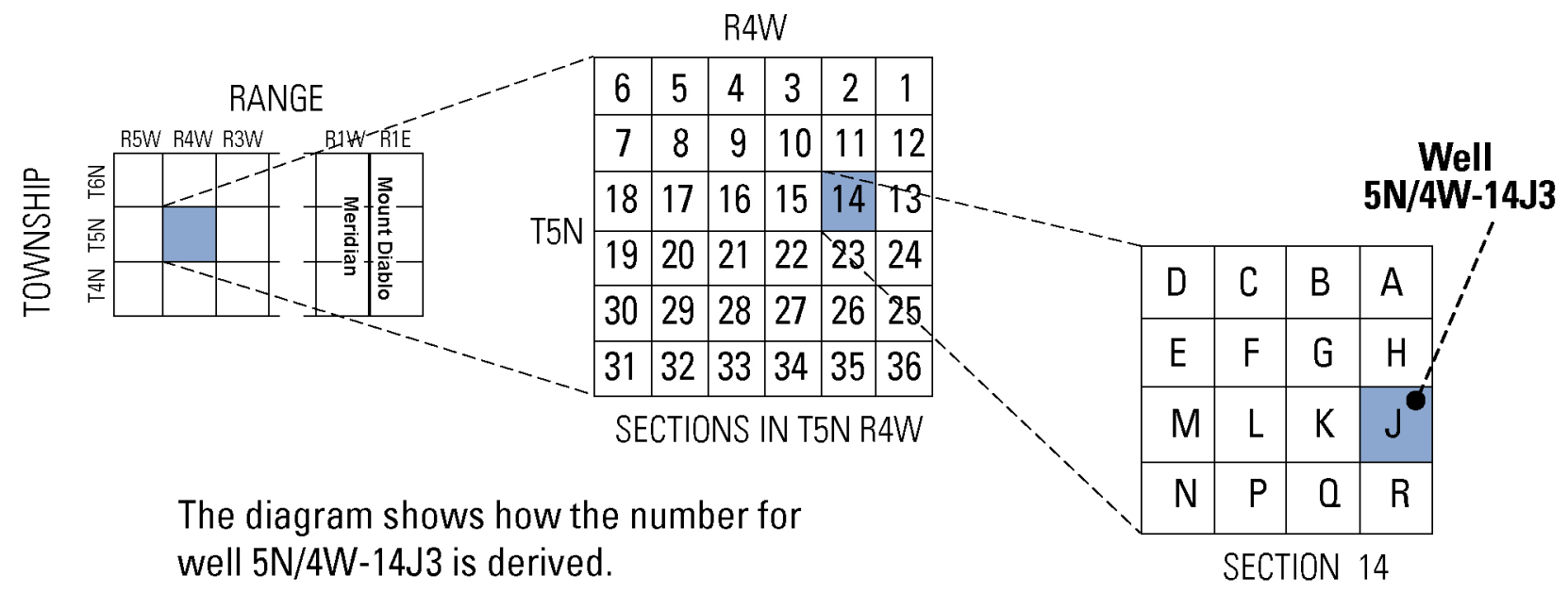

Figure 2. Public lands survey and well-numbering system. 
However, most of the well locations in the original studies were field-checked so that the wells were located with greater accuracy than the township-range-section designation. It was these fieldchecked locations that were plotted on the plates that accompany each Water-Supply paper and on this report's plate 1. Longitude and latitude coordinates for the wells were not published in the original reports. These well locations were determined by digitizing the well locations plotted on each map plate (appendix 1). Paper copies of plates showing well locations were scanned to a digital raster image and spatially referenced in a GIS using a digital topographic base. Well locations were digitized from the spatially referenced images. Accuracy of the digitized well locations is estimated in most cases to be within 50 meters $(\mathrm{m})$ of the plotted location. Well elevation and total drilled depth are transcribed directly from the source reports (appendix 1).

\section{Lithologic Descriptions}

Two types of lithologic data were presented in the three USGS Water-Supply Papers (Cardwell, 1958; Kunkel and Upson, 1960; Cardwell, 1965). Subsurface lithologic descriptions were presented in all three reports for each drilled downhole interval for a certain number of wells. These data are tabulated in appendix 3. In addition, in the Santa Rosa and Petaluma Valley areas (Cardwell, 1958) lithologic descriptions were reported for the water-bearing interval for those wells that had hydrologic data. These data are compiled in appendix 2 as part of the hydrologic data. For all of these data, the lithologic descriptions are those provided by the well driller and comprise a vast array of descriptive terms. Drillers' descriptions are generally short phrases that accompany a significant lithologic change. Typically, descriptions range from one to ten words that describe a change recognized by the driller as they penetrate a different unit; for example, descriptions may include information on grain size, presence or absence of gravel or large rocks, degree of consolidation, and rock type or abrupt color changes. Although the driller's descriptions can vary in length, detail, and accuracy, they are the fundamental geologic data set that was collected at the drill site. We preserve these descriptions exactly as they were originally reported by the drillers, as tabulated in the three USGS Water-Supply Papers (Cardwell, 1958; Kunkel and Upson, 1960; Cardwell, 1965). In appendixes 2 and 3 these basic descriptions are listed as "reported lithologic unit" or "reported geologic unit" and are clearly separated from our interpretations.

Drillers' descriptions were simplified to a small number of internally consistent lithologic classes (table 1) for all 464 wells. We assigned each described interval to a lithologic class based on our knowledge of the range of sediment types gained from observation of surface outcrops, lithologic descriptions from published reports (Allen, 2003; Davies, 1986; Powell and others, 2004; Wagner and others, 2005), similarity to more detailed subsurface descriptions (Powell and others, 2006), and on comparisons of downhole intervals in adjacent wells. Sediments were subdivided on the basis of grain size or mixtures of size classes. We attempted to recognize the degree of cementation or induration from driller's descriptions. This was possible in the sandy part of the section, where sand could be reliably separated from sandstone, but proved impossible for the coarse-grained fraction, where large clast size sometimes interfered with a driller's assessment of induration. Stratigraphic order and character of overlying and underlying intervals were considered in classifying the lithologic descriptions. Volcanic rocks were divided into two categories: (1) basaltic lava flows, and (2) ash or tuff. Basalts were assumed to be readily identified by the drillers, so these were split from the rest of the volcanic intervals. 
Table 1. Generalized lithologic classes based on driller's descriptions.

\begin{tabular}{|c|c|}
\hline $\begin{array}{l}\text { Lithology } \\
\text { class }\end{array}$ & Typical drillers' description \\
\hline Gravel & $\begin{array}{l}\text { Boulders; cemented gravel; gravel; loose rock; rock (if surrounded by clay and gravel and is less than } \\
5 \mathrm{ft} \text { thick); rubble. }\end{array}$ \\
\hline $\begin{array}{l}\text { Sand (or } \\
\text { sandstone) } \\
\text { and gravel }\end{array}$ & $\begin{array}{l}\text { Alluvial deposits; clayey sand and gravel; clayey sand with minor gravel or streaks of gravel; gravel } \\
\text { and (or with) streaks of clay; gravel with sand and clay; sand and gravel; sand and gravel with clay; } \\
\text { sand and rock; silty gravel; surface and boulders; cemented sand and gravel; sandstone and gravel. }\end{array}$ \\
\hline Sand & Blue, gray, red, or yellow sand; sand; shells (as only descriptor of interval). \\
\hline Sandstone & $\begin{array}{l}\text { Blue rock; graywacke; sand rock; sandstone; cemented sand; rock (when surrounded by shelly or } \\
\text { sandstone layers); sand and ledges; sandy rock. }\end{array}$ \\
\hline $\begin{array}{l}\text { Sand and } \\
\text { clay }\end{array}$ & $\begin{array}{l}\text { Adobe; clay sandstone; loam; quicksand; sand and clay; sand and shale; soil; silt; sticky sand; surface; } \\
\text { topsoil. }\end{array}$ \\
\hline $\begin{array}{l}\text { Clay, sand, } \\
\text { and gravel }^{2}\end{array}$ & $\begin{array}{l}\text { Clay, sand and gravel; conglomerate and agglomerate; gravel, sand and shale; sandy clay and gravel; } \\
\text { shale and gravel; shale and rock; topsoil and gravel or rock. }\end{array}$ \\
\hline $\begin{array}{l}\text { Clay, sand, } \\
\text { and trace } \\
\text { gravel }\end{array}$ & $\begin{array}{l}\text { Sandy clay and streaks of gravel; shale and boulders; shale and gravel; silty clay and some gravel; } \\
\text { clay and little gravel; clay and some boulders; clay and some gravel; clay and streaks gravel; clay } \\
\text { with gravel stringers. }\end{array}$ \\
\hline $\begin{array}{l}\text { Clay and } \\
\text { gravel }^{3}\end{array}$ & $\begin{array}{l}\text { Boulders in clay; cemented gravel and clay; clay and boulders; clay, gravel; clay with conglomerate; } \\
\text { clay and rock; clayey sand and gravel; embedded clay or gravel; gravel and clay; gravelly clay; } \\
\text { hardpan. }\end{array}$ \\
\hline $\begin{array}{l}\text { Clay and } \\
\text { sand (or } \\
\text { sandstone) }\end{array}$ & $\begin{array}{l}\text { Basin deposits; clay and sand; clay and shale; clay and silt; mud; diatomite; mudstone; sand clay } \\
\text { (brown, blue, green, orange, and yellow); sandy clay; silt; silty clay; shale; shale and sand; clay and } \\
\text { sandstone; gray rock when surrounded by shale; sandy claystone; siltstone. }\end{array}$ \\
\hline Clay & Clay, typically described as black, blue, brown, gray, green, tan, or yellow. \\
\hline Basalt & $\begin{array}{l}\text { Andesite; basalt; basalt and cinders; basalt and lava; basalt and sand; basalt boulders; black ash; black } \\
\text { volcanic rock; cinders; diorite; lava rock; porous volcanic rock. }\end{array}$ \\
\hline $\begin{array}{l}\text { Ash and(or) } \\
\text { tuff }\end{array}$ & $\begin{array}{l}\text { Altered ash; ash (with color descriptors blue, brown, gray, red, white, or yellow); ash flow tuff; } \\
\text { broken rock; clay and ash; lava ash; conglomerate and volcanic rock; multicolored rock; pumice } \\
\text { rock; rock (where described as red or black, or when surrounded by rocks that are described as } \\
\text { volcanic). }\end{array}$ \\
\hline $\begin{array}{l}\text { Undifferen- } \\
\text { tiated } \\
\text { basement }^{4}\end{array}$ & $\begin{array}{l}\text { Serpentine; basalt and serpentine; basalt and shale; black rock with quartz; blue and green rock or } \\
\text { clay when surrounded by graywacke, shale or serpentine; oily rock; rock with quartz and blue or } \\
\text { green rock, shale or clay; all units below any interval identified as serpentine, especially where } \\
\text { described as green and blue. }\end{array}$ \\
\hline No data & No data, rock (when not surrounded by volcanic units and greater than $5 \mathrm{ft}$ thick). \\
\hline \multicolumn{2}{|r|}{$\begin{array}{l}{ }^{2} \text { Conglomerate and agglomerate in some cases may refer to volcanic rocks. Rocks with these descriptors are included with } \\
\text { rocks where surrounding intervals also appear to be volcanic rocks. }\end{array}$} \\
\hline${ }^{3} \mathrm{Cla}$ & $\begin{array}{l}\text { and gravel, in some cases, may be weathered ash and volcanic rocks, but the distinction cannot be made without additional } \\
\text { tratigraphic context. }\end{array}$ \\
\hline${ }^{4} \mathrm{Or}$ & $\begin{array}{l}\text { units at depth are considered. When an interval at shallow depth is described by any of these terms other than serpentine } \\
\text { g intervals are not described as basement, this zone is not described as undifferentiated basement. Although } \\
\text { oasement is only used at depth in a drill hole, on rare occasion the Franciscan Complex is thrust over the Glen Ellen } \\
\text { as along the Trenton fault near the Russian River. }\end{array}$ \\
\hline
\end{tabular}




\section{Stratigraphic Descriptions}

The three USGS Water-Supply Papers (Cardwell, 1958; Kunkel and Upson, 1960; Cardwell, 1965) reported interpreted subsurface stratigraphy and name-specific geologic formations with the downhole lithologic interval data and with the geologic description of the water-bearing interval. We tabulated these formation picks as they appeared in the original reports and compiled the data in appendix 2. In addition, we have chosen to create a series of generalized stratigraphic unit classes which generally correspond to recognized stratigraphic units but do not require us to make specific formational assignments in the absence of subsurface control or detailed stratigraphic studies (table 2). In order to display well data in a simple fashion, rocks intercepted in the subsurface were classified into the following generalized stratigraphic unit classes: Mesozoic rocks, undifferentiated; Paleogene marine sedimentary rocks; Neogene continental sedimentary rocks; Neogene volcanic rocks; Neogene marine sedimentary rocks; Neogene to Quaternary continental deposits; and Quaternary continental deposits (table 2, appendix 2, plate 1).

In the northern California Coast Ranges, Mesozoic rocks of the Franciscan Complex, the Great Valley sequence, and the Coast Range ophiolite make up the basement. In the eastern part of the study area, the Mesozoic basement is overlain by Paleogene marine formations (plate 1). In the southern Napa Valley and to the east of Sonoma Valley, Neogene marine strata of the San Pablo Group overlie the basement. Because these rocks locally underlie the Sonoma Volcanics, we have included them with the older Paleogene marine sedimentary rocks (plate 1). 
Table 2. Description of simplified stratigraphic units.

\begin{tabular}{|c|c|c|c|c|c|}
\hline $\begin{array}{c}\text { Generalized } \\
\text { stratigraphic unit } \\
\text { class }\end{array}$ & $\begin{array}{l}\text { General age } \\
\text { range }^{1}\end{array}$ & $\begin{array}{l}\text { Examples of mapped geologic } \\
\text { units }^{2}\end{array}$ & $\begin{array}{l}\text { Estimated } \\
\text { thickness, } \\
\text { in meters }\end{array}$ & $\begin{array}{l}\text { Typical depositional } \\
\text { environment }{ }^{4}\end{array}$ & Typical lithologic description \\
\hline $\begin{array}{l}\text { Quaternary } \\
\text { continental deposits } \\
\text { (Qal) }\end{array}$ & Quaternary & $\begin{array}{l}\text { Includes units mapped as } \\
\text { younger and older alluvium and } \\
\text { alluvial fan and terrace deposits }\end{array}$ & $0-60$ & $\begin{array}{l}\text { Stream-channel, flood-plain } \\
\text { deposits, older alluvium and } \\
\text { terrace deposits }\end{array}$ & $\begin{array}{l}\text { Gravel; sand and gravel; sand, } \\
\text { clay, and gravel; sand and clay }\end{array}$ \\
\hline $\begin{array}{l}\text { Neogene to } \\
\text { Quaternary } \\
\text { continental deposits } \\
\text { (QTc) }\end{array}$ & $\begin{array}{l}\text { Early } \\
\text { Pleistocene(?) } \\
\text { and Pliocene }\end{array}$ & $\begin{array}{l}\text { Includes the Glen Ellen } \\
\text { Formation, the Huichica } \\
\text { Formation, and other unnamed } \\
\text { Tertiary continental deposits }\end{array}$ & $0-900$ & $\begin{array}{l}\text { Continental, piedmont, and } \\
\text { valley alluvial fans, local } \\
\text { lacustrine deposits }\end{array}$ & $\begin{array}{l}\text { Clay and sand; clay and gravel; } \\
\text { sand; sand and gravel; tuff; } \\
\text { conglomerate }\end{array}$ \\
\hline $\begin{array}{l}\text { Neogene marine } \\
\text { sedimentary rocks } \\
\text { (Twg) }\end{array}$ & $\begin{array}{l}\text { Late Miocene } \\
\text { to late } \\
\text { Pliocene }\end{array}$ & $\begin{array}{l}\text { Includes the Ohlson Ranch and } \\
\text { Wilson Grove Formations, and } \\
\text { rocks formerly assigned to the } \\
\text { Merced Formation }\end{array}$ & $0-1,800$ & $\begin{array}{l}\text { Deep to shallow marine, } \\
\text { locally transitional to } \\
\text { continental environments. }\end{array}$ & $\begin{array}{l}\text { Sand, sandstone, blue } \\
\text { sandstone; clay, sand or gravel } \\
\text { and shells; clay and sand }\end{array}$ \\
\hline $\begin{array}{l}\text { Neogene }^{5} \text { volcanic } \\
\text { rocks }(\mathrm{Tv})\end{array}$ & $\begin{array}{l}\text { Pliocene and } \\
\text { Miocene }\end{array}$ & $\begin{array}{l}\text { Includes Sonoma and } \\
\text { TolayVolcanics, and volcanics } \\
\text { of Burdell Mountain }\end{array}$ & $0-600$ & --- & Basalt; volcanic breccia; tuff \\
\hline $\begin{array}{l}\text { Neogene continental } \\
\text { sedimentary rocks } \\
\text { (Tp) }\end{array}$ & $\begin{array}{l}\text { Pliocene to } \\
\text { late Miocene }\end{array}$ & $\begin{array}{l}\text { Includes the Petaluma } \\
\text { Formation }\end{array}$ & $0-1,200$ & $\begin{array}{l}\text { Fluvial and lacustrine, with } \\
\text { estuarine and transitional } \\
\text { marine environment }\end{array}$ & $\begin{array}{l}\text { Clay; clay and sand; shale; } \\
\text { sand or sandstone }\end{array}$ \\
\hline $\begin{array}{l}\text { Paleogene marine } \\
\text { sedimentary rocks } \\
(\mathrm{Tu})\end{array}$ & $\begin{array}{l}\text { Eocene to } \\
\text { Miocene }\end{array}$ & $\begin{array}{l}\text { Includes units such as the } \\
\text { Neroly Formation and the } \\
\text { Markley Sandstone }^{3}\end{array}$ & $0->1,200$ & --- & Sandstone, shale \\
\hline $\begin{array}{l}\text { Mesozoic rocks, } \\
\text { undifferentiated } \\
(\mathrm{KJu})\end{array}$ & $\begin{array}{l}\text { Pre-Miocene; } \\
\text { predominantly } \\
\text { Jurassic and } \\
\text { Cretaceous }\end{array}$ & $\begin{array}{l}\text { Includes the Franciscan } \\
\text { Complex, the Great Valley } \\
\text { sequence, including the Coast } \\
\text { Range ophiolite }\end{array}$ & $>2,000$ & --- & $\begin{array}{l}\text { Sandstone, graywacke, chert, } \\
\text { serpentine }\end{array}$ \\
\hline
\end{tabular}

${ }^{1}$ General age ranges from Wagner and Bortungo (1982); Blake and others (2002); Graymer and others (2002).

${ }^{2}$ Mapped geologic units from Wagner and Bortungo (1982); Blake and others (2002); Graymer and others (2002); Graymer and others (2007).

${ }^{3}$ Wagner and Bortungo (1982) and Blake and others (2002) describe Eocene and Miocene sedimentary rocks in the region.

${ }^{4}$ Depositional environment listed only for Miocene and younger sedimentary rocks.

${ }^{5}$ May locally include Quaternary volcanic rocks from the Clear Lake volcanic field. 
Cenozoic basins developed above these older rocks as a response to tectonic processes at the plate margin (Fox, 1983; McLaughlin and others, 1996). The Neogene volcanic rocks of the northern San Francisco Bay region are part of a linear belt of volcanic fields that are progressively younger to the northwest (Fox, 1983) and are roughly coeval with basin formation and fault motion in the region. Neogene sedimentary rocks include the Petaluma Formation, mostly continental rocks with some transitional marine deposits, and the marine sandstones such as the Wilson Grove Formation. Rocks of the Petaluma Formation interfinger with both Neogene volcanic rocks and with the marine sandstones such as the Wilson Grove Formation. Overlying these rocks are younger continental gravels such as the Glen Ellen Formation and similar deposits.

\section{Visualization of Lithologic and Stratigraphic Data}

The relatively dense clustering of 358 wells in the Santa Rosa, Sonoma and Napa Valleys (fig. 3) was used to construct a 3D lithologic model of the region in order to help visualize the spatial distribution of subsurface lithologic data. The wells used in the visualization were drilled between 1918 and 1960, with the majority drilled in the 1950s (fig. 4A). Most of the wells were drilled for residential use, which at the time was predominantly in the valleys rather than the surrounding mountains. The total drilled depth of these wells varied from 6 to $633 \mathrm{~m}$; the average total depth was about $100 \mathrm{~m}$ (fig. $4 B$ ). Wells were located by latitude and longitude, as described above, and the elevation of the well was derived from a 30-m digital elevation model (DEM). 


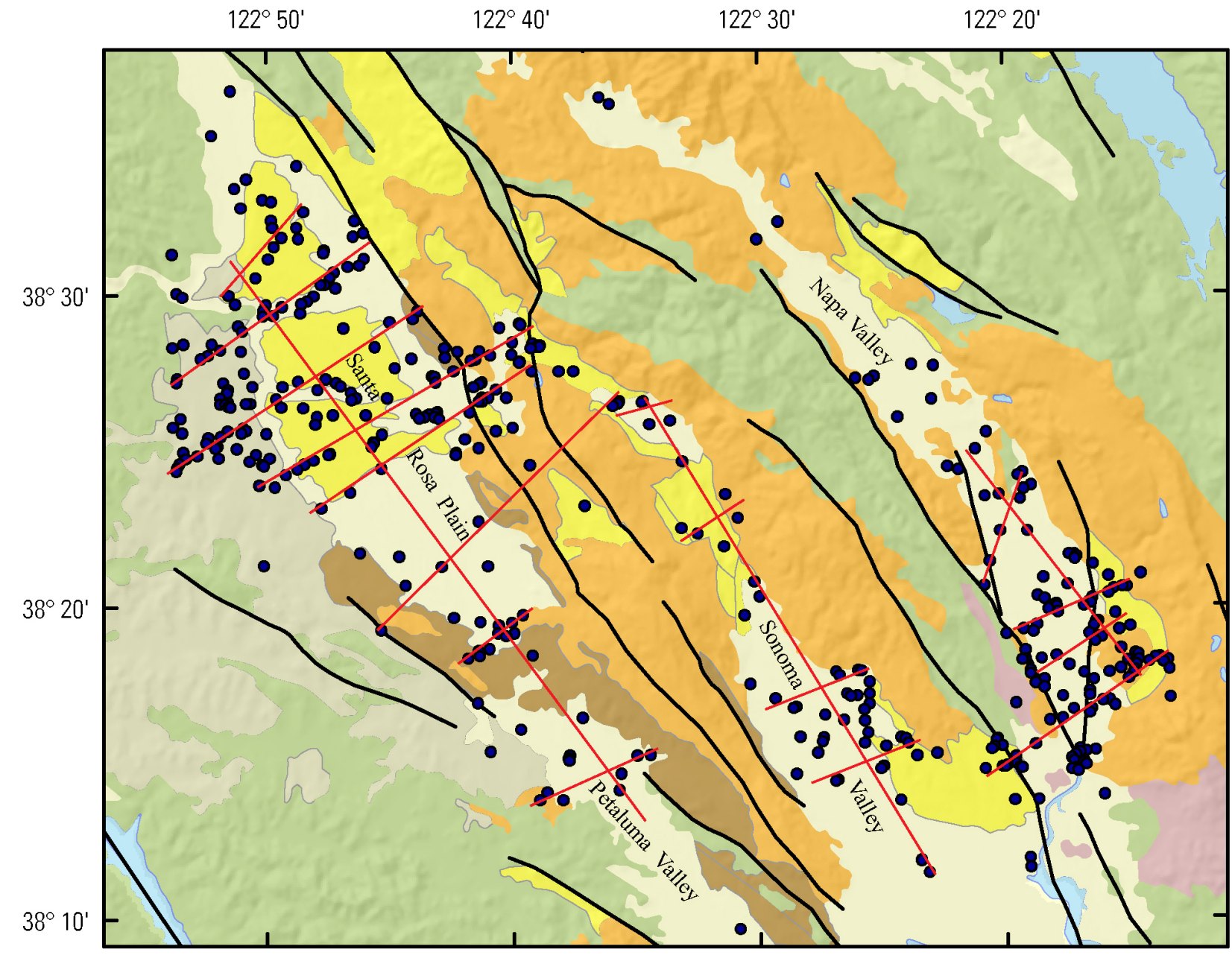

See plate 1 for base credit and sources of geologic mapping Universal Transverse Mercator Projection Zone 10

North American Datum 1927

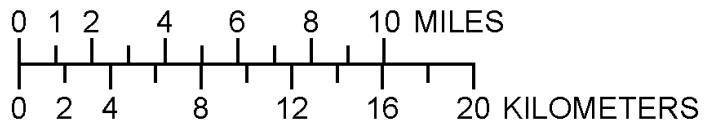

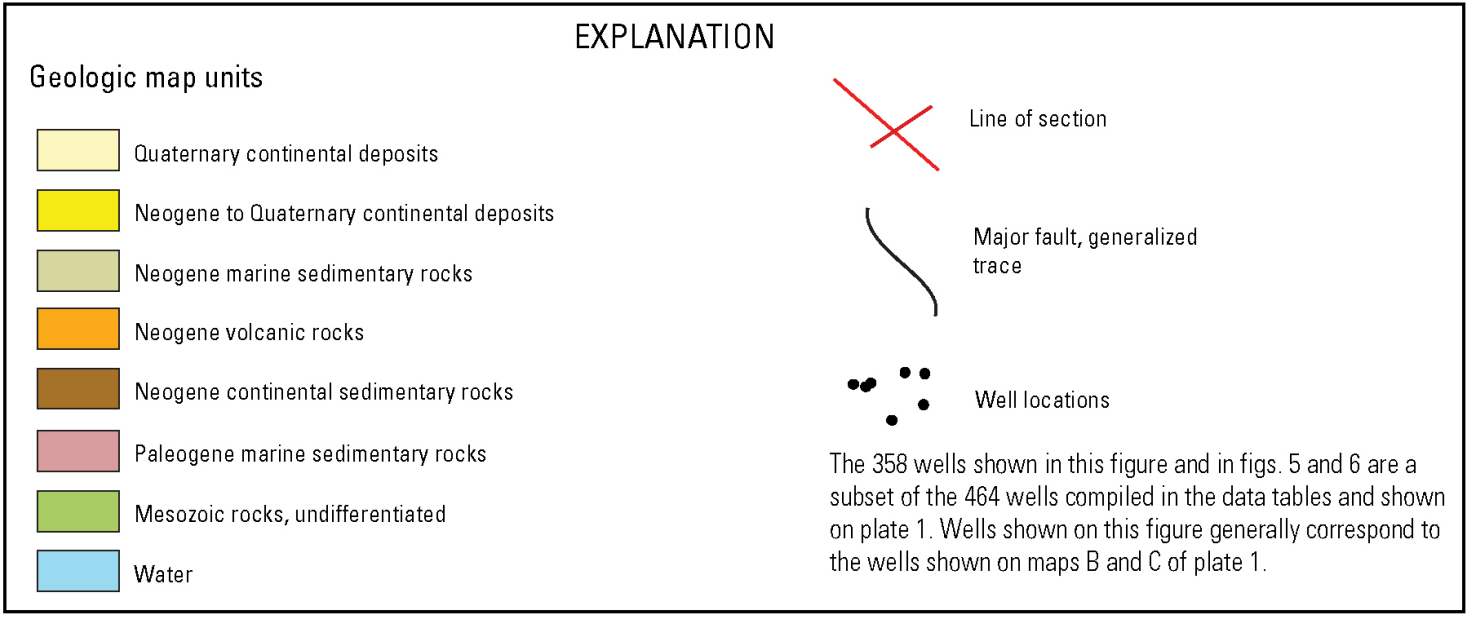

Figure 3. Topographic and simplified geologic maps of the Santa Rosa, Sonoma, and Napa Valleys showing well and vertical section locations. 


\section{A. Frequency of wells drilled between 1918 and 1959}

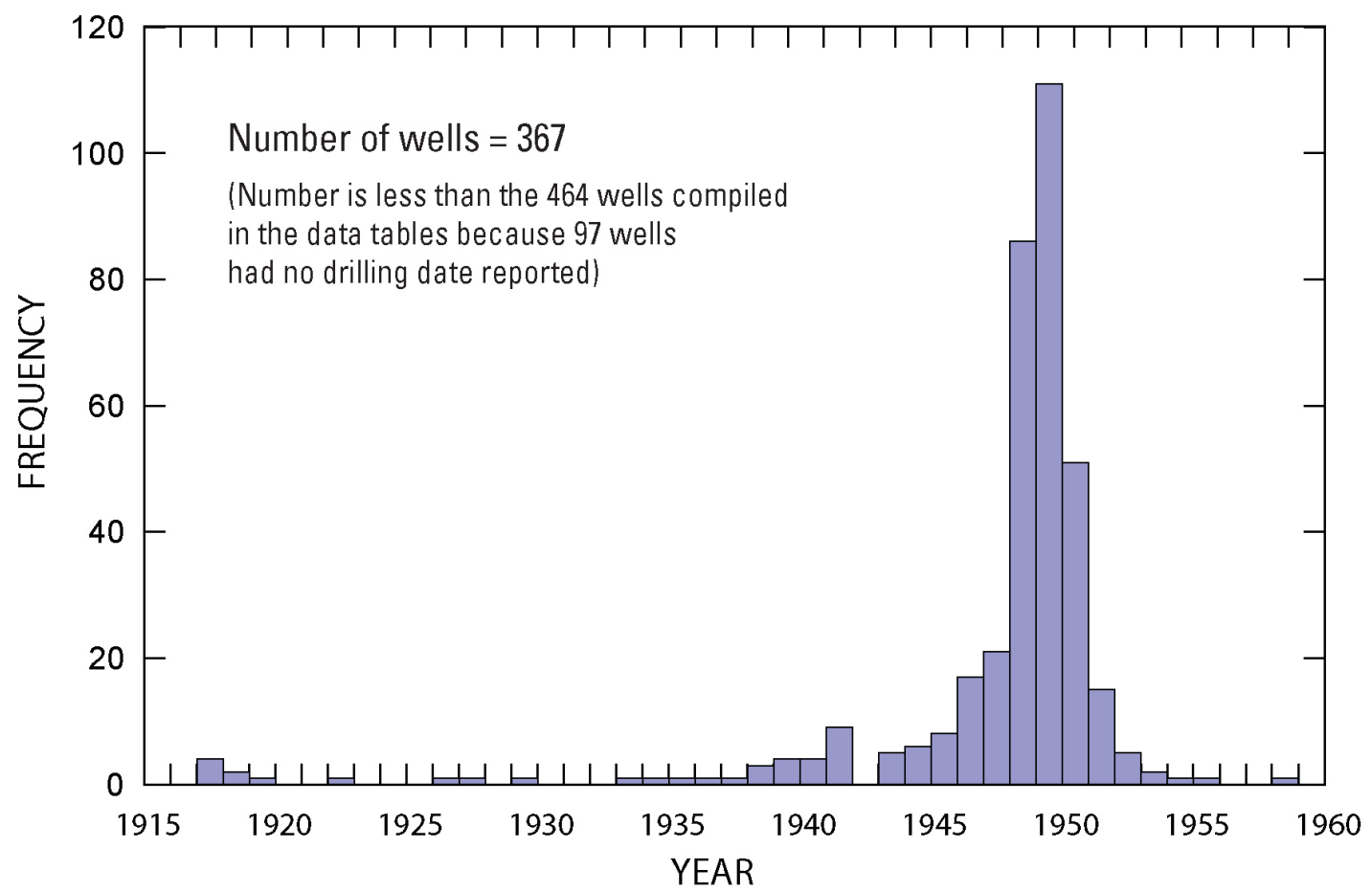

B. Total drilled depth of wells

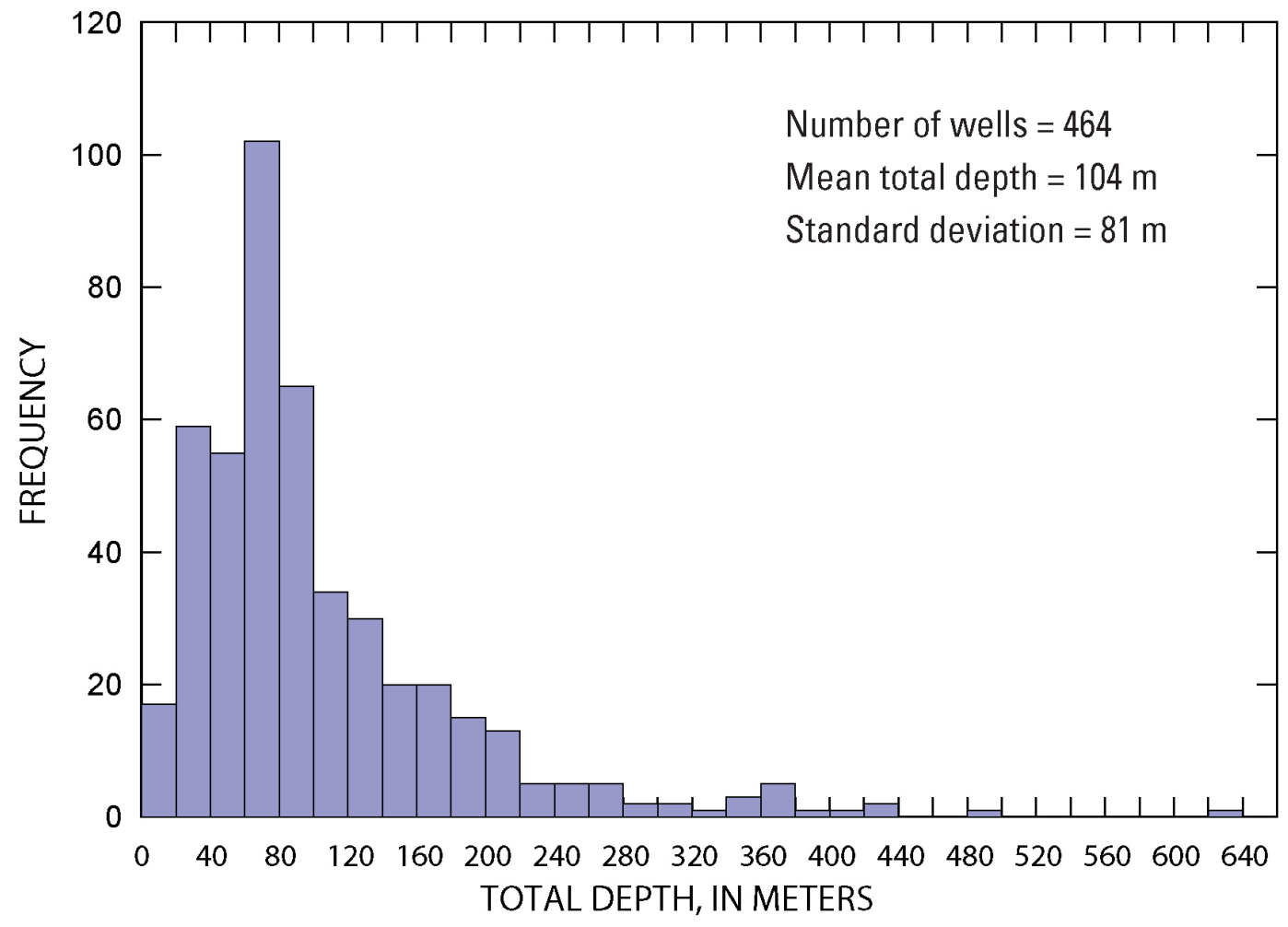

Figure 4. Frequency of (A) the number of wells drilled between 1918 and 1960 and (B) the total depth of the wells used to construct the $3 \mathrm{D}$ lithologic model. 


\section{Method for Construction of the Lithologic Model}

The 3D distribution of subsurface lithologic data was illustrated by constructing a $\mathrm{D}$ lithologic solid model of the area. Drillers' descriptions were simplified to a small number of internally consistent lithologic classes (table 1) for all 358 wells. Interpreted well lithologic data were numerically interpolated between wells by using a cell-based, 3D gridding process using the RockWorks 3D modeling software package (http://www.rockware.com/ accessed October, 2010). In this method, a solid modeling algorithm is used to extrapolate numeric codes that represent a lithologic class onto grid nodes throughout the 3D-model domain. Grid nodes between wells are assigned lithologic class values based on the relative horizontal proximity of each grid node to surrounding wells. Cell dimensions for the 3D interpolation were $500 \mathrm{~m}$ in the horizontal dimensions and $5 \mathrm{~m}$ in the vertical dimension. The solid model was constructed to $620 \mathrm{~m}$ below the surface, but trimmed at depth to $250 \mathrm{~m}$ to eliminate the modeling artifacts resulting from a few deep wells controlling the model results at greater depths. The upper surface of the solid model was trimmed with the DEM. The 3D distribution of lithologic classes within the solid model may be visualized by displaying a number of vertical cross sections sliced through the solid model (figs. 5 and 6). 


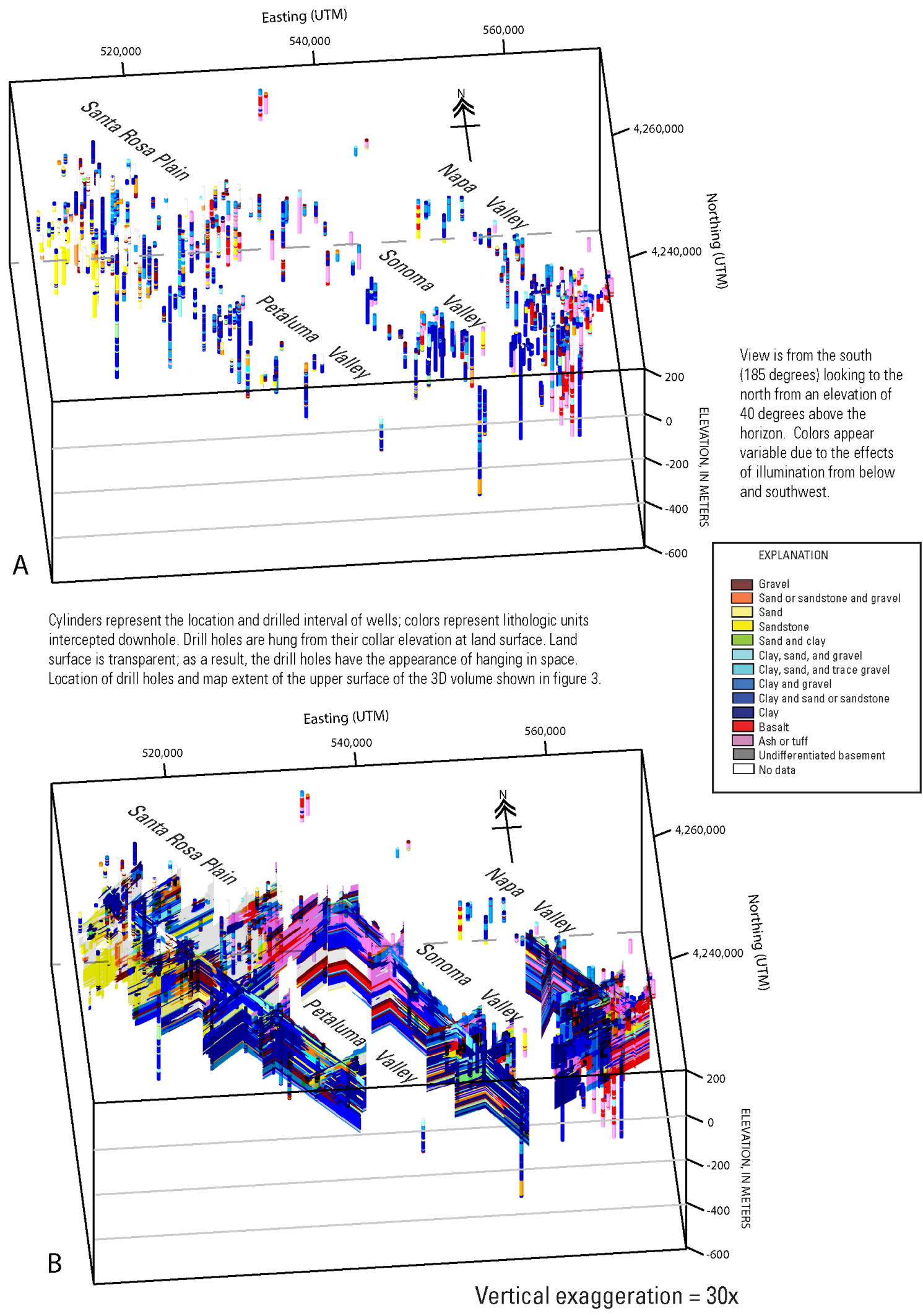

Figure 5. Three-dimensional portrayals of (A) well lithologic data and (B) well data with vertical sections in Santa Rosa, Sonoma, and Napa Valleys. 
A. Detail of Santa Rosa Valley

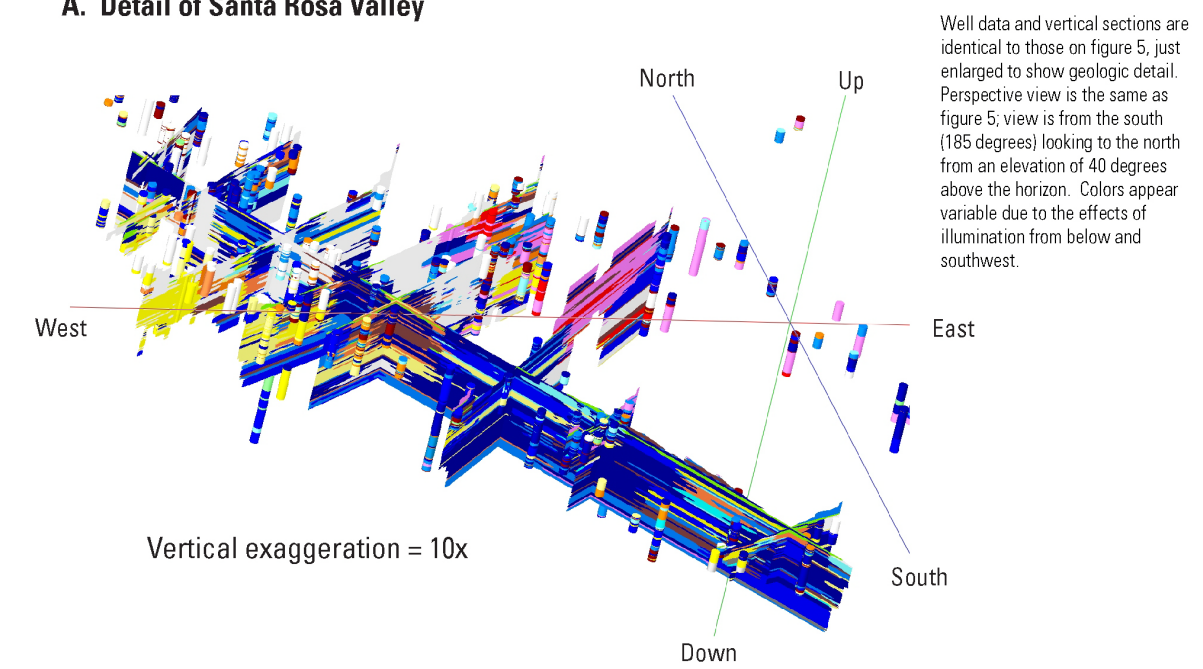

B. Detail of Sonoma Valley

Horizontal and vertical scales are not shown. Coordinate axes that portray north-south, east-west, and portray north-south, east-west, and
up-down direction are shown for

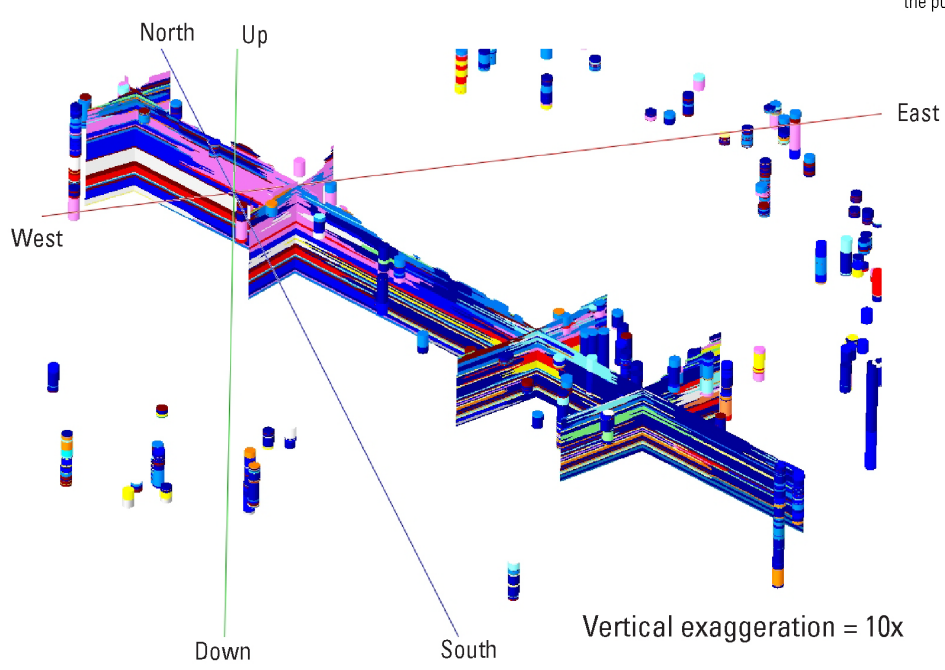

C. Detail of the southern part of Napa Valley

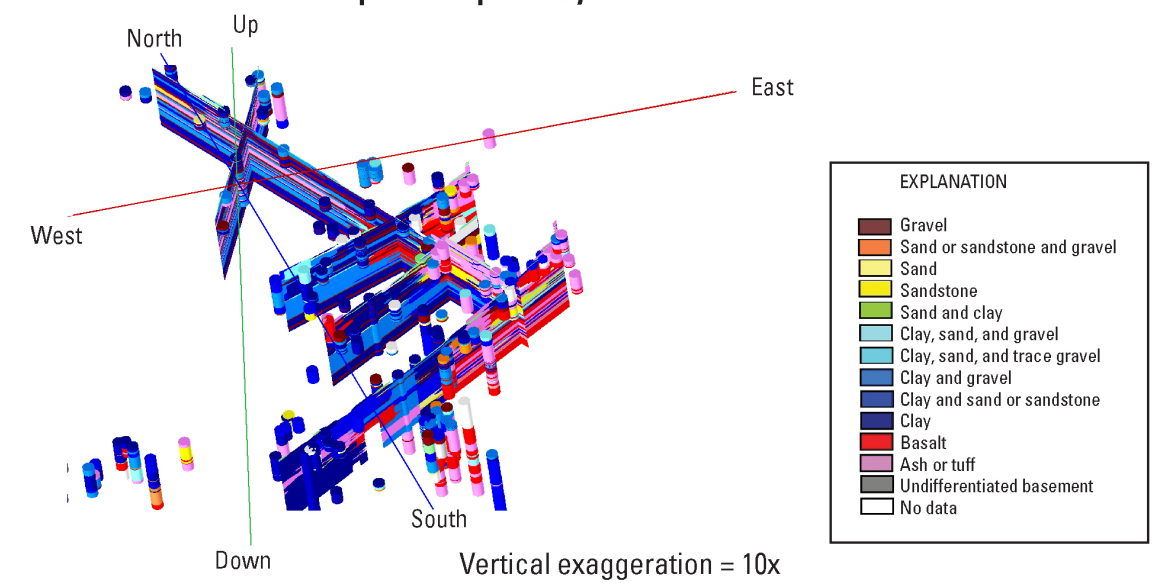

Figure 6. Detail of well data and vertical sections in (A) Santa Rosa Valley, (B), Sonoma Valley, and (C) the southern part of Napa Valley. 


\section{Geology of the Valley Regions}

Santa Rosa, Sonoma, and Napa Valleys are divided by ranges composed of Tertiary and Mesozoic rocks that isolate the areas from one another (fig. 3). The valleys are filled with Tertiary marine and continental sediments that may be interfingered with Tertiary volcanic rocks. The well lithologic data may be viewed in 3D space, with the location and drilled depth of wells portrayed as vertical cylinders of varying colors that correspond to separate lithologic classes (fig. 5). When viewed in 3D space, the lithologic classes derived from the drillers' descriptions fall into distinct spatial groupings (fig. 5A). Three fence diagrams were constructed from the 3D lithologic model by intersecting the solid model with multiple vertical sections (figs. 5b and 6). These fence diagrams were located where the highest density of well data occurs.

The hills northwest of the Santa Rosa Plain are dominated by sandy units that represent younger Tertiary marine sedimentary rocks deposited in shelf environments (figs. 5 and 6) (Powell and others, 2004, 2006). These sandy units are expressed as yellow intervals in the well cylinders and on the vertical sections. Beneath the Santa Rosa Valley, marine facies rocks transition to nonmarine deposits across a higher-energy dune and littoral environment (Powell and others, 2004, 2006) represented by gravel or sand and gravel deposits, expressed as brown and orange intervals, respectively, in the well cylinders and on the vertical sections (figs. 5 and 6). Beneath most of the central part of the Santa Rosa Valley and the Petaluma Valley to the southeast, clay-rich units interbedded with coarser material are characteristic of both the Quaternary and Tertiary continental sediments (Allen, 2003; Powell and others, 2004), and are observed in both the well-cylinders and the fence diagram as variable colors of dark blue with thin beds of yellow and orange (figs. 5 and 6). Santa Rosa Valley is flanked on the east primarily by tuff and basalt units that represent Tertiary volcanic rocks (plate 1), which are expressed as pink and red intervals, respectively, in the well-cylinders and on the vertical sections (figs. 5 and 6).

The north end of Sonoma Valley is characterized by tuff and basalt units (plate 1) that represent Tertiary volcanic rocks interbedded with clays and gravels of both Quaternary and Tertiary continental sediments (figs. 5 and 6). To the south of the town of Sonoma, the volcanic units dip southward beneath surficial sedimentary cover, and again the subsurface is predominantly clay interbedded with gravel and sand, typical of Tertiary continental sediments (figs. 5 and 6).

The southern part of Napa Valley is dominated by ash, tuff, and basalt units that represent Tertiary volcanic rocks (plate 1), expressed as pink and red intervals in the well cylinders and on the vertical sections (figs. 4 and 5). In the area southwest of the town of Napa and southeast of Sonoma Valley, the volcanic rocks interfinger with clay-rich units interbedded with coarser material that are characteristic of both the Quaternary and Tertiary continental sediments. Some of the abrupt lithologic transitions visible in the vertical sections result from the complex faulting along the western side of Napa Valley (plate 1).

\section{Summary}

This data tabulation includes all downhole lithologic information and aquifer pumping test data from 464 of the wells described in the three USGS Water-Supply Papers (Cardwell, 1958; Kunkel and Upson, 1960; Cardwell, 1965) that describe the northern part of the Coast Ranges in California (fig. 1). This report makes available in digital form a large subsurface well dataset so that subsurface lithologic and hydrologic information is available for incorporation within a GIS and use in construction of threedimensional geologic framework models that can be used to address water-resource issues.

The well data here, although mostly from relatively shallow water wells, provide important subsurface information that displays the disposition and facies transition of lithologic units between the 
four principal valleys at the southern end of the study area. Well lithologic data themselves and simple three-dimensional interpolation of those data show distinct spatial patterns (plate 1) that are linked to subsurface stratigraphy and structure and can be used in more sophisticated three-dimensional analysis of these basins.

\section{Acknowledgments}

We thank Alexandra Anderson (U.S. Geological Survey, retired) for transcribing the well data from the original reports, Daniel Burnham (former U.S. Geological Survey student intern) for spatially referencing the plates and digitizing well locations, and Meghan Patterson (U.S. Geological Survey, student) for digital data compilation. 


\section{References Cited}

Allen, J.R., 2003, Stratigraphy and tectonics of Neogene strata, northern San Francisco Bay Area: San Jose, Calif., San Jose State University, Master's thesis, 183 p.

Blake, M.C., Jr., Graymer, R.W., and Stamski, R.E., 2002, Geologic map and map database of western Sonoma, northernmost Marin, and southernmost Mendocino Counties, California: U.S. Geological Survey Miscellaneous Field Studies Map MF-2402, 42 p., scale 1:100,000, accessed November 2009 at http://pubs.usgs.gov/mf/2002/2402/.

Bryant, W.A. (compiler), 2005, Digital database of Quaternary and younger faults from the Fault Activity Map of California, version 2.0: Sacramento, Calif., California Geological Survey Accessed November 2009 at http://www.consrv.ca.gov/CGS/information/publications/QuaternaryFaults_ver2.htm/.

California Department of Water Resources, 1982, Santa Rosa Plain, evaluation of ground water resources-Sonoma County, v. 2: Sacramento, Calif., Bulletin 118-4, 107 p.

Cardwell, G.T., 1958, Geology and ground water in the Santa Rosa and Petaluma Valley areas, Sonoma County, California: U.S. Geological Survey Water-Supply Paper 1427, 273 p.

Cardwell, G.T., 1965, Geology and ground water in Russian River Valley areas and in Round, Laytonville, and Little Lake Valleys, Sonoma and Mendocino Counties, California: U.S. Geological Survey Water-Supply Paper 1548, 154 p.

Davies, E.A., 1986, Stratigraphic and structural relationships of the Miocene and Pliocene formations of the Petaluma Valley area of California: Berkeley, Calif., University of California, Berkeley, unpublished Master's thesis, $96 \mathrm{p}$.

Fox, K.F., Jr., 1983, Tectonic setting of late Miocene, Pliocene, and Pleistocene rocks in part of the Coast Ranges north of San Francisco, California: U.S. Geological Survey Professional Paper 1239, 33 p.

Graymer, R.W., Brabb, E.E., Jones, D.J., Barnes, J., Nicholson, R.S., and Stamski, R.E., 2007, Geologic map and map database of eastern Sonoma and western Napa counties, California: U.S. Geological Survey Scientific Investigations Map 2956, scale 1:100,000, accessed November 2009 at http://pubs.usgs.gov/sim/2007/2956/.

Graymer, R.W., Bryant, William, McCabe, C.A., Hecker, Suzanne, and Prentice, C.S., 2006, Map of Quaternary-active faults in the San Francisco Bay region: U.S. Geological Survey Scientific Investigations Map 2919, scale 1:275,000, accessed November 2009 at http://pubs.usgs.gov/sim/2006/2919/.

Graymer, R.W., Jones, D.L., and Brabb, E.E., 2002, Geologic map and map database of northeastern San Francisco Bay region, California-Most of Solano County and parts of Napa, Marin, Contra Costa, San Joaquin, Sacramento, Yolo, and Sonoma Counties: U.S Geological Survey Miscellaneous Field Studies Map MF-2403, 1 sheet, 1:100,000 scale, 28 p., accessed November 2009 at http://pubs.usgs.gov/mf/2002/2403/.

Graymer, R.W., Moring, B.C., Saucedo, G.J., Wentworth, C.M., Brabb, E.E., and Knudsen, K.L., 2006, Geologic map of the San Francisco Bay region: U.S. Geological Survey Scientific Investigations Map 2918, scale 1:275,000, accessed November 2009 at http://pubs.usgs.gov/sim/2006/2918/.

Kunkel, Fred, and Upson, J.E., 1960, Geology and ground water in Napa and Sonoma Valleys, Napa and Sonoma Counties, California: U.S. Geological Water-Supply Paper 1495, 252 p.

McLaughlin, R.J. Sliter, W.V., Sorg, D.H., Russell. P.C., and Sarna-Wojcicki, A.M., 1996, Large-scale right-slip displacement on the East San Francisco Bay Region fault system- Implications for the location of late Miocene to Pliocene plate boundary: Tectonics, v. 15, p. 1-18. 
Powell, C.L., II, Allen, J.R., and Holland, P.J., 2004, Invertebrate paleontology of the Wilson Grove Formation (late Miocene to late Pliocene), Sonoma and Marin Counties, California, with some observations on its stratigraphy, thickness, and structure: U.S. Geological Survey Open-File Report 2004-1017, 106 p., accessed November 2009 at http://pubs.usgs.gov/of/2004/1017/.

Powell, C.L., II, McLaughlin, R.J., and Wan, Elmira, 2006, Biostratigraphic and lithologic correlations of two Sonoma County Water Agency pilot wells with the type Wilson Grove Formation, Sonoma County, central California: U.S. Geological Survey Open-File Report 2006-1196, 37 p., accessed November 2009 at http://pubs.usgs.gov/of/2006/1196/.

Saucedo, G.J., Bedford, D.R., Raines, G.L., Miller, R.J., and Wentworth, C.M., 2000, GIS Data for the Geologic Map of California: Sacramento, Calif., California Department of Conservation, CD 200007.

Valin, Z.C., and McLaughlin, R.J., 2005, Locations and data for water wells of the Santa Rosa Valley, Sonoma County, California: U.S. Geological Survey Open-File Report 2005-,1318, 16 p. accessed November 2009 at http://pubs.usgs.gov/of/2005/1318/.

Wagner, D.L. and Bortugno, E.J., 1982, Geologic map of the Santa Rosa Quadrangle: California Division of Mines and Geology Regional Geologic Map Series, Map 2A, scale 1:250,000.

Wagner, D.L. Fleck, R.J., Sarna-Wojcicki, A.M., and Deino, Alan, 2005, Golden Gate to southern Sonoma County, Rodgers Creek Fault, Burdell Mountain, Donnell Ranch, and southern Sonoma Volcanics in Stevens, Calvin and Cooper, John, eds., Late Neogene transition from transform to subduction margin east of the San Andreas Fault in the wine country of the northern San Francisco Bay Area, California-Field trip 10 Guidebook and Volume Prepared for the Joint Meeting of the Cordilleran Section - Geological Society of America and Pacific Section-American Association of Petroleum Geologists, San Jose, California, April 29-May 1, 2005: Pacific Section-Society for Sedimentary Geology, p. 1-28.

\section{Appendixes}

Appendix 1. Well location data.

Appendix 2. Hydrologic data.

Appendix 3. Lithologic data. 
Appendix 1. Well Location Data

[Site IDs corrspond to locations shown on Plate 1; Well number and area correspond to descriptions in the Water-Supply Paper listed; latitude and longitude geographic coordinates have a horizontal datum of North American Datum 1927; Easting and Northing coordinates are in Universa Transverse Mercator projection zone 10, North American Datum 1927; system of quarter-quarter section designation is explained in the Water-Supply Papers; leaders (--), well number not assigned]

\begin{tabular}{|c|c|c|c|c|c|c|c|c|c|c|c|c|c|c|c|}
\hline Site ID & Well number & Area & Water-Supply Paper & Latitude & Longitude & Easting & Northing & $\begin{array}{l}\text { Township } \\
\text { (N) }\end{array}$ & $\begin{array}{l}\text { Range } \\
\text { (W) }\end{array}$ & Section & $\begin{array}{c}\text { Quarter- } \\
\text { quarter } \\
\text { section } \\
\text { designation }\end{array}$ & $\begin{array}{c}\text { Well } \\
\text { number } \\
\text { within } \\
\text { quarter- } \\
\text { quarter } \\
\text { section }\end{array}$ & $\begin{array}{l}\text { Land } \\
\text { surface } \\
\text { elevation, in } \\
\text { meters }\end{array}$ & $\begin{array}{l}\text { Total } \\
\text { depth of } \\
\text { well, in } \\
\text { meters }\end{array}$ & Geologic units intersected \\
\hline 1 & $22 / 13-2 A 3$ & Mendocino County; Round Valley & Cardwell (1965) & 39.7994 & -123.2698 & 476899 & 4405313 & 22 & 13 & 2 & A & 3 & 432.8 & 44.5 & Sedimentary rocks and pre-Cenozoic rocks \\
\hline 2 & $22 / 12-18 \mathrm{~N} 1$ & Mendocino County; Round Valley & Cardwell (1965) & 39.7623 & -123.2475 & 478799 & 4401199 & 22 & 12 & 18 & $\mathrm{~N}$ & 1 & 426.7 & 137.8 & Sedimentary rocks only \\
\hline 3 & 22/12-19G2 & Mendocino County; Round Valley & Cardwell (1965) & 39.7533 & -123.2382 & 479592 & 4400194 & 22 & 12 & 19 & G & 2 & 422.1 & 154.8 & Sedimentary rocks only \\
\hline 4 & 21/15-12K2 & $\begin{array}{l}\text { Mendocino County; Laytonville } \\
\text { Valley }\end{array}$ & Cardwell (1965) & 39.6859 & -123.4839 & 458507 & 4392802 & 21 & 15 & 12 & $\mathrm{k}$ & 2 & 495.3 & 76.2 & Sedimentary rocks only \\
\hline 5 & 21/15-13B1 & $\begin{array}{l}\text { Mendocino County; Laytonville } \\
\text { Valley }\end{array}$ & Cardwell (1965) & 39.6809 & -123.4844 & 458462 & 4392246 & 21 & 15 & 13 & B & 1 & 490.7 & 160.9 & Sedimentary rocks and pre-Cenozoic rocks \\
\hline 6 & 21/15-14A1 & $\begin{array}{l}\text { Mendocino County; Laytonville } \\
\text { Valley }\end{array}$ & Cardwell (1965) & 39.6791 & -123.4959 & 457474 & 4392052 & 21 & 15 & 14 & A & 1 & 509.0 & 14.9 & Sedimentary rocks only \\
\hline 7 & 21/14-30M1 & $\begin{array}{l}\text { Mendocino County; Laytonville } \\
\text { Valley }\end{array}$ & Cardwell (1965) & 39.6434 & -123.4725 & 459460 & 4388081 & 21 & 14 & 30 & M & 1 & 514.5 & 7.0 & Sedimentary rocks only \\
\hline 8 & 21/14-31J1 & $\begin{array}{l}\text { Mendocino County; Laytonville } \\
\text { Valley }\end{array}$ & Cardwell (1965) & 39.6284 & -123.4606 & 460473 & 4386401 & 21 & 14 & 30 & $\mathrm{~J}$ & 1 & 512.1 & 123.4 & Sedimentary rocks and pre-Cenozoic rocks \\
\hline 9 & $18 / 13-18 \mathrm{H} 1$ & $\begin{array}{l}\text { Mendocino County; Little Lake } \\
\text { Valley }\end{array}$ & Cardwell (1965) & 39.4144 & -123.3371 & 470978 & 4362605 & 18 & 13 & 18 & $\mathrm{H}$ & 1 & 408.4 & 107.9 & Sedimentary rocks only \\
\hline 10 & 18/13-19B1 & $\begin{array}{l}\text { Mendocino County; Little Lake } \\
\text { Valley }\end{array}$ & Cardwell (1965) & 39.4048 & -123.3447 & 470324 & 4361544 & 18 & 13 & 19 & B & 1 & 414.5 & 138.4 & Sedimentary rocks only \\
\hline 11 & 18/13-20P2 & $\begin{array}{l}\text { Mendocino County; Little Lake } \\
\text { Valley }\end{array}$ & Cardwell (1965) & 39.3920 & -123.3277 & 471782 & 4360124 & 18 & 13 & 20 & $\mathrm{P}$ & 2 & 428.2 & 21.9 & Sedimentary rocks only \\
\hline 12 & 18/13-31A1 & $\begin{array}{l}\text { Mendocino County; Little Lake } \\
\text { Valley }\end{array}$ & Cardwell (1965) & 39.3767 & -123.3383 & 470864 & 4358424 & 18 & 13 & 31 & A & 1 & 442.0 & 88.1 & Sedimentary rocks only \\
\hline 13 & 17/11-33D1 & $\begin{array}{l}\text { Russian River Valley area; Potter, } \\
\text { Ukiah, and Sanel Valleys }\end{array}$ & Cardwell (1965) & 39.2914 & -123.0908 & 492167 & 4348915 & 17 & 11 & 33 & D & 1 & 280.4 & 200.6 & Sedimentary rocks and pre-Cenozoic rocks \\
\hline 14 & $16 / 12 \mathrm{~W}-34 \mathrm{~N} 2$ & $\begin{array}{l}\text { Russian River Valley area; Potter, } \\
\text { Ukiah, and Sanel Valleys }\end{array}$ & Cardwell (1965) & 39.1931 & -123.1855 & 483978 & 4338019 & 16 & 12 & 34 & $\mathrm{~N}$ & 2 & 250.3 & 64.2 & Sedimentary rocks only \\
\hline 15 & $14 / 12-5 K 1$ & $\begin{array}{l}\text { Russian River Valley area; Potter, } \\
\text { Ukiah, and Sanel Valleys }\end{array}$ & Cardwell (1965) & 39.1001 & -123.2046 & 482306 & 4327695 & 14 & 12 & 5 & $\mathrm{~K}$ & 1 & 185.9 & 28.7 & Sedimentary rocks and pre-Cenozoic rocks \\
\hline 16 & $14 / 12-26 \mathrm{H}$ & $\begin{array}{l}\text { Russian River Valley area; Potter, } \\
\text { Ukiah, and Sanel Valleys }\end{array}$ & Cardwell (1965) & 39.0689 & -123.1461 & 487364 & 4324225 & 14 & 12 & 26 & $\mathrm{H}$ & -- & 167.6 & 19.8 & Sedimentary rocks only \\
\hline 17 & $13 / 11-8 \mathrm{H} 1$ & $\begin{array}{l}\text { Russian River Valley area; Potter, } \\
\text { Ukiah, and Sanel Valleys }\end{array}$ & Cardwell (1965) & 39.0014 & -123.0855 & 492599 & 4316724 & 13 & 11 & 8 & $\mathrm{H}$ & 1 & 251.5 & 57.0 & Sedimentary rocks only \\
\hline 18 & 13/11-18D1 & $\begin{array}{l}\text { Russian River Valley area; Potter, } \\
\text { Ukiah, and Sanel Valleys }\end{array}$ & Cardwell (1965) & 38.9907 & -123.1189 & 489707 & 4315543 & 13 & 11 & 18 & D & 1 & 149.4 & 18.6 & Sedimentary rocks only \\
\hline 19 & 13/11-18M1 & $\begin{array}{l}\text { Russian River Valley area; Potter, } \\
\text { Ukiah, and Sanel Valleys }\end{array}$ & Cardwell (1965) & 38.9846 & -123.1177 & 489805 & 4314865 & 13 & 11 & 18 & M & 1 & 149.4 & 19.2 & Sedimentary rocks only \\
\hline 20 & $13 / 11-22 G$ & $\begin{array}{l}\text { Russian River Valley area; Potter, } \\
\text { Ukiah, and Sanel Valleys }\end{array}$ & Cardwell (1965) & 38.9748 & -123.0904 & 492172 & 4313779 & 13 & 11 & 22 & G & -- & 236.2 & 62.5 & Sedimentary rocks only \\
\hline 21 & 13/11-21L2 & $\begin{array}{l}\text { Mendocino County; Laytonville } \\
\text { Valley }\end{array}$ & Cardwell (1965) & 38.9710 & -123.0750 & 493505 & 4313356 & 13 & 11 & 21 & $\mathrm{~L}$ & 2 & 167.6 & 67.1 & Sedimentary rocks only \\
\hline
\end{tabular}


Appendix 1. Well Location Data. _Continued

[Site IDs corrspond to locations shown on Plate 1; Well number and area correspond to descriptions in the Water-Supply Paper listed; latitude and longitude geographic coordinates have a horizontal datum of North American Datum 1927; Easting and Northing coordinates are in Universa Transverse Mercator projection zone 10, North American Datum 1927; system of quarter-quarter section designation is explained in the Water-Supply Papers; leaders (--), well number not assigned]

\begin{tabular}{|c|c|c|c|c|c|c|c|c|c|c|c|c|c|c|c|}
\hline Site ID & Well number & Area & Water-Supply Paper & Latitude & Longitude & Easting & Northing & $\begin{array}{l}\text { Township } \\
\text { (N) }\end{array}$ & $\begin{array}{l}\text { Range } \\
\text { (W) }\end{array}$ & Section & $\begin{array}{l}\text { Quarter- } \\
\text { quarter } \\
\text { section } \\
\text { designation }\end{array}$ & $\begin{array}{c}\text { Well } \\
\text { number } \\
\text { within } \\
\text { quarter- } \\
\text { quarter } \\
\text { section }\end{array}$ & $\begin{array}{l}\text { Land } \\
\text { surface } \\
\text { elevation, in } \\
\text { meters }\end{array}$ & $\begin{array}{l}\text { Total } \\
\text { depth of } \\
\text { well, in } \\
\text { meters }\end{array}$ & Geologic units intersected \\
\hline 22 & 13/11-19Q1 & $\begin{array}{l}\text { Russian River Valley area; Potter, } \\
\text { Ukiah, and Sanel Valleys }\end{array}$ & Cardwell (1965) & 38.9686 & -123.1097 & 490500 & 4313087 & 13 & 11 & 19 & Q & 1 & 144.8 & 34.7 & Sedimentary rocks only \\
\hline 23 & 11/10-17R1 & $\begin{array}{l}\text { Russian River Valley area; } \\
\text { Alexander Valley and Healdsburg } \\
\text { area }\end{array}$ & Cardwell (1965) & 38.7968 & -122.9908 & 500795 & 4294017 & 11 & 10 & 17 & $\mathrm{R}$ & 1 & 91.4 & 12.5 & Sedimentary rocks and pre-Cenozoic rocks \\
\hline 24 & 11/10-28L1 & $\begin{array}{l}\text { Russian River Valley area; } \\
\text { Alexander Valley and Healdsburg } \\
\text { area }\end{array}$ & Cardwell (1965) & 38.7708 & -122.9798 & 501757 & 4291137 & 11 & 10 & 28 & $\mathrm{~L}$ & 1 & 77.7 & 6.1 & Sedimentary rocks only \\
\hline 25 & 11/10-33A & $\begin{array}{l}\text { Russian River Valley area; } \\
\text { Alexander Valley and Healdsburg } \\
\text { area }\end{array}$ & Cardwell (1965) & 38.7644 & -122.9729 & 502355 & 4290429 & 11 & 10 & 33 & A & -- & 77.7 & 12.2 & Sedimentary rocks only \\
\hline 26 & 10/11-12Q1 & $\begin{array}{l}\text { Russian River Valley area; } \\
\text { Alexander Valley and Healdsburg } \\
\text { area }\end{array}$ & Cardwell (1965) & 38.7222 & -123.0257 & 497762 & 4285746 & 10 & 11 & 12 & Q & 1 & 67.1 & 16.8 & Sedimentary rocks only \\
\hline 27 & 10/9-26ட1 & $\begin{array}{l}\text { Russian River Valley area; } \\
\text { Alexander Valley and Healdsburg } \\
\text { area }\end{array}$ & Cardwell (1965) & 38.6836 & -122.8271 & 515035 & 4281476 & 9 & 8 & 26 & $\mathrm{~L}$ & 1 & 62.5 & 74.4 & Sedimentary rocks only \\
\hline 28 & 9/10-2B3 & $\begin{array}{l}\text { Russian River Valley area; } \\
\text { Alexander Valley and Healdsburg } \\
\text { area }\end{array}$ & Cardwell (1965) & 38.6631 & -122.9349 & 505665 & 4279187 & 9 & 10 & 2 & B & 3 & 43.3 & 86.9 & Sedimentary rocks only \\
\hline 29 & 9/10-2B1 & $\begin{array}{l}\text { Russian River Valley area; } \\
\text { Alexander Valley and Healdsburg } \\
\text { area }\end{array}$ & Cardwell (1965) & 38.6623 & -122.9357 & 505593 & 4279099 & 9 & 10 & 2 & B & 1 & 42.7 & 32.6 & Sedimentary rocks and pre-Cenozoic rocks \\
\hline 30 & 9/9-4E1 & $\begin{array}{l}\text { Russian River Valley area; } \\
\text { Alexander Valley and Healdsburg } \\
\text { area }\end{array}$ & Cardwell (1965) & 38.6590 & -122.8711 & 511217 & 4278735 & 9 & 9 & 4 & $\mathrm{E}$ & 1 & 54.9 & 35.7 & Sedimentary rocks only \\
\hline 31 & 9/8-7Q1 & $\begin{array}{l}\text { Russian River Valley area; } \\
\text { Alexander Valley and Healdsburg } \\
\text { area }\end{array}$ & Cardwell (1965) & 38.6346 & -122.7871 & 518534 & 4276044 & 9 & 8 & 7 & $\mathrm{Q}$ & 1 & 48.8 & 149.4 & Sedimentary rocks only \\
\hline 32 & 9/9-21R1 & $\begin{array}{l}\text { Russian River Valley area; } \\
\text { Alexander Valley and Healdsburg } \\
\text { area }\end{array}$ & Cardwell (1965) & 38.6090 & -122.8575 & 512405 & 4273192 & 9 & 9 & 21 & $\mathrm{R}$ & 1 & 25.9 & 54.1 & Sedimentary rocks only \\
\hline 33 & 9/9-33Е2 & $\begin{array}{l}\text { Russian River Valley area; } \\
\text { Alexander Valley and Healdsburg } \\
\text { area }\end{array}$ & Cardwell (1965) & 38.5850 & -122.8702 & 511305 & 4270525 & 9 & 9 & 33 & $\mathrm{E}$ & 2 & 27.4 & 20.1 & Sedimentary rocks only \\
\hline 34 & 8/9-19K1 & $\begin{array}{l}\text { Russian River Valley area; Lower } \\
\text { Russian River }\end{array}$ & Cardwell (1965) & 38.5218 & -122.8973 & 508953 & 4263513 & 8 & 9 & 19 & $\mathrm{k}$ & 1 & 24.4 & 19.5 & Sedimentary rocks and pre-Cenozoic rocks \\
\hline 35 & 8/10-20м3 & $\begin{array}{l}\text { Russian River Valley area; Lower } \\
\text { Russian River }\end{array}$ & Cardwell (1965) & 38.5211 & -122.9969 & 500267 & 4263432 & 8 & 10 & 20 & M & 3 & 24.4 & 37.5 & Sedimentary rocks only \\
\hline 36 & 8/10-29D1 & $\begin{array}{l}\text { Russian River Valley area; Lower } \\
\text { Russian River }\end{array}$ & Cardwell (1965) & 38.5155 & -122.9963 & 500327 & 4262809 & 8 & 10 & 29 & $\mathrm{D}$ & 1 & 18.3 & 55.8 & Sedimentary rocks and pre-Cenozoic rocks \\
\hline 37 & $8 / 10-29 \mathrm{H} 2$ & $\begin{array}{l}\text { Russian River Valley area; Lower } \\
\text { Russian River }\end{array}$ & Cardwell (1965) & 38.5096 & -122.9842 & 501380 & 4262146 & 8 & 10 & 29 & $\mathrm{H}$ & 2 & 9.1 & 38.1 & Sedimentary rocks and pre-Cenozoic rocks \\
\hline 38 & $8 / 10-29 \mathrm{~N} 1$ & $\begin{array}{l}\text { Russian River Valley area; Lower } \\
\text { Russian River }\end{array}$ & Cardwell (1965) & 38.5052 & -122.9981 & 500165 & 4261667 & 8 & 10 & 29 & N & 1 & 13.7 & 27.4 & Sedimentary rocks only \\
\hline 39 & 8/9-31A1 & $\begin{array}{l}\text { Russian River Valley area; } \\
\text { Alexander Valley and Healdsburg } \\
\text { area }\end{array}$ & Cardwell (1965) & 38.5007 & -122.8940 & 509246 & 4261175 & 8 & 9 & 31 & A & 1 & 19.8 & 99.1 & Sedimentary rocks only \\
\hline 40 & 8/9-32E3 & $\begin{array}{l}\text { Russian River Valley area; } \\
\text { Alexander Valley and Healdsburg } \\
\text { area }\end{array}$ & Cardwell (1965) & 38.4986 & -122.8904 & 509560 & 4260941 & 8 & 9 & 32 & $\mathrm{E}$ & 3 & 18.3 & 25.3 & Sedimentary rocks only \\
\hline
\end{tabular}


Appendix 1. Well Location Data. _Continued

[Site IDs corrspond to locations shown on Plate 1; Well number and area correspond to descriptions in the Water-Supply Paper listed; latitude and longitude geographic coordinates have a horizontal datum of North American Datum 1927; Easting and Northing coordinates are in Universa Transverse Mercator projection zone 10, North American Datum 1927; system of quarter-quarter section designation is explained in the Water-Supply Papers; leaders (--), well number not assigned]

\begin{tabular}{|c|c|c|c|c|c|c|c|c|c|c|c|c|c|c|c|}
\hline Site ID & Well number & Area & Water-Supply Paper & Latitude & Longitude & Easting & Northing & $\begin{array}{l}\text { Township } \\
\text { (N) }\end{array}$ & $\begin{array}{l}\text { Range } \\
\text { (W) }\end{array}$ & Section & $\begin{array}{c}\text { Quarter- } \\
\text { quarter } \\
\text { section } \\
\text { designation }\end{array}$ & $\begin{array}{c}\text { Well } \\
\text { number } \\
\text { within } \\
\text { quarter- } \\
\text { quarter } \\
\text { section }\end{array}$ & $\begin{array}{l}\text { Land } \\
\text { surface } \\
\text { elevation, in } \\
\text { meters }\end{array}$ & $\begin{array}{l}\text { Total } \\
\text { depth of } \\
\text { well, in } \\
\text { meters }\end{array}$ & Geologic units intersected \\
\hline 41 & 7/10-7D1 & $\begin{array}{l}\text { Russian River Valley area; Lower } \\
\text { Russian River }\end{array}$ & Cardwell (1965) & 38.4722 & -123.0168 & 498537 & 4258003 & 7 & 10 & 7 & D & 1 & 7.6 & 36.6 & Sedimentary rocks only \\
\hline 42 & 7/11-11J1 & $\begin{array}{l}\text { Russian River Valley area; Lower } \\
\text { Russian River }\end{array}$ & Cardwell (1965) & 38.4651 & -123.0406 & 496455 & 4257219 & 7 & 11 & 11 & $\mathrm{~J}$ & 1 & 12.2 & 41.5 & Sedimentary rocks only \\
\hline 43 & 7/111-17J1 & $\begin{array}{l}\text { Russian River Valley area; Lower } \\
\text { Russian River }\end{array}$ & Cardwell (1965) & 38.4492 & -123.0947 & 491736 & 4255458 & 7 & 11 & 17 & $\mathrm{~J}$ & 1 & 4.6 & 45.1 & Sedimentary rocks and pre-Cenozoic rocks \\
\hline 44 & 8/9-1M1 & Santa Rosa Valley & Cardwell (1958) & 38.5690 & -122.8126 & 516328 & 4268755 & 8 & 9 & 1 & M & 1 & 56.4 & 96.0 & Sedimentary rocks only \\
\hline 45 & 8/9-3P1 & Santa Rosa Valley & Cardwell (1958) & 38.5619 & -122.8467 & 513359 & 4267969 & 8 & 9 & 3 & $\mathrm{P}$ & 1 & 25.0 & 36.0 & Sedimentary rocks only \\
\hline 46 & 8/9-9H1 & Santa Rosa Valley & Cardwell (1958) & 38.5567 & -122.8547 & 512659 & 4267390 & 8 & 9 & 9 & $\mathrm{H}$ & 1 & 14.1 & 12.2 & Sedimentary rocks only \\
\hline 47 & 8/9-10R1 & Santa Rosa Valley & Cardwell (1958) & 38.5508 & -122.8357 & 514318 & 4266733 & 8 & 9 & 10 & $\mathrm{R}$ & 1 & 50.3 & 121.9 & Hydrology data only; no geologic information \\
\hline 48 & 8/9-11P1 & Santa Rosa Valley & Cardwell (1958) & 38.5498 & -122.8299 & 514826 & 4266626 & 8 & 9 & 11 & $\mathrm{P}$ & 1 & 40.0 & 45.7 & Sedimentary rocks only \\
\hline 49 & 8/9-15D1 & Santa Rosa Valley & Cardwell (1958) & 38.5463 & -122.8507 & 513014 & 4266237 & 8 & 9 & 15 & D & 1 & 20.0 & 40.8 & Sedimentary rocks only \\
\hline 50 & $8 / 9-13 C 1$ & Santa Rosa Valley & Cardwell (1958) & 38.5442 & -122.8080 & 516728 & 4266010 & 8 & 9 & 13 & c & 1 & 32.0 & 61.6 & Sedimentary rocks only \\
\hline 51 & 8/9-14L1 & Santa Rosa Valley & Cardwell (1958) & 38.5398 & -122.8299 & 514828 & 4265516 & 8 & 9 & 14 & $\mathrm{~L}$ & 1 & 29.0 & 80.8 & Sedimentary rocks only \\
\hline 52 & 8/8-17L1 & Santa Rosa Valley & Cardwell (1958) & 38.5396 & -122.7732 & 519769 & 4265506 & 8 & 8 & 17 & $\mathrm{~L}$ & 1 & 56.4 & 84.7 & Sedimentary rocks only \\
\hline 53 & 8/9-14P1 & Santa Rosa Valley & Cardwell (1958) & 38.5358 & -122.8292 & 514883 & 4265076 & 8 & 9 & 14 & $\mathrm{P}$ & 1 & 29.0 & 80.5 & Sedimentary rocks only \\
\hline 54 & $8 / 9-13 \mathrm{~N} 1$ & Santa Rosa Valley & Cardwell (1958) & 38.5358 & -122.8127 & 516322 & 4265070 & 8 & 9 & 13 & $\mathrm{~N}$ & 1 & 27.4 & 63.4 & Sedimentary rocks only \\
\hline 55 & 8/8-20B2 & Santa Rosa Valley & Cardwell (1958) & 38.5331 & -122.7670 & 520311 & 4264783 & 8 & 8 & 20 & B & 2 & 56.4 & 70.4 & Sedimentary rocks only \\
\hline 56 & 8/8-20D1 & Santa Rosa Valley & Cardwell (1958) & 38.5312 & -122.7744 & 519667 & 4264576 & 8 & 8 & 20 & D & 1 & 44.2 & 79.2 & Hydrology data only; no geologic information \\
\hline 57 & 8/9-23G1 & Santa Rosa Valley & Cardwell (1958) & 38.5307 & -122.8227 & 515451 & 4264511 & 8 & 9 & 23 & G & 1 & 22.9 & 134.1 & Sedimentary rocks only \\
\hline 58 & 8/9-24E1 & Santa Rosa Valley & Cardwell (1958) & 38.5300 & -122.8115 & 516432 & 4264427 & 8 & 9 & 24 & $\mathrm{E}$ & 1 & 24.4 & 100.6 & Hydrology data only; no geologic information \\
\hline 59 & 8/9-23L1 & Santa Rosa Valley & Cardwell (1958) & 38.5257 & -122.8279 & 515006 & 4263950 & 8 & 9 & 23 & $\mathrm{~L}$ & 1 & 22.9 & 130.8 & Sedimentary rocks only \\
\hline 60 & 8/8-19M1 & Santa Rosa Valley & Cardwell (1958) & 38.5237 & -122.7939 & 517964 & 4263740 & 8 & 8 & 19 & M & 1 & 32.0 & 79.2 & Sedimentary rocks only \\
\hline 61 & 8/8-19N1 & Santa Rosa Valley & Cardwell (1958) & 38.5226 & -122.7944 & 517927 & 4263617 & 8 & 8 & 19 & $\mathrm{~N}$ & 1 & 32.0 & 75.9 & Sedimentary rocks only \\
\hline 62 & 8/8-20Q1 & Santa Rosa Valley & Cardwell (1958) & 38.5193 & -122.7670 & 520315 & 4263254 & 8 & 8 & 20 & Q & 1 & 42.7 & 95.1 & Sedimentary rocks only \\
\hline 63 & $8 / 9-26 \mathrm{D} 1$ & Santa Rosa Valley & Cardwell (1958) & 38.5191 & -122.8321 & 514641 & 4263216 & 8 & 9 & 26 & D & 1 & 22.9 & 141.4 & Sedimentary rocks only \\
\hline 64 & $8 / 8-29 \mathrm{C} 1$ & Santa Rosa Valley & Cardwell (1958) & 38.5158 & -122.7703 & 520026 & 4262864 & 8 & 8 & 29 & c & 1 & 41.0 & 20.7 & Sedimentary rocks only \\
\hline 65 & 8/8-29D1 & Santa Rosa Valley & Cardwell (1958) & 38.5151 & -122.7778 & 519371 & 4262783 & 8 & 8 & 29 & D & 1 & 39.0 & 17.4 & Sedimentary rocks only \\
\hline 66 & 8/8-30G1 & Santa Rosa Valley & Cardwell (1958) & 38.5121 & -122.7873 & 518546 & 4262447 & 8 & 8 & 30 & G & 1 & 36.0 & 42.7 & Sedimentary rocks only \\
\hline 67 & 8/8-30L1 & Santa Rosa Valley & Cardwell (1958) & 38.5093 & -122.7899 & 518320 & 4262144 & 8 & 8 & 30 & $\mathrm{~L}$ & 1 & 13.1 & 141.1 & Sedimentary rocks only \\
\hline 68 & $8 / 9-27 \mathrm{~K} 1$ & Santa Rosa Valley & Cardwell (1958) & 38.5089 & -122.8401 & 513943 & 4262086 & 8 & 9 & 27 & $\mathrm{k}$ & 1 & 18.3 & 101.5 & Sedimentary rocks only \\
\hline 69 & 8/8-30N1 & Santa Rosa Valley & Cardwell (1958) & 38.5054 & -122.7936 & 518001 & 4261709 & 8 & 8 & 30 & N & 1 & 13.1 & 138.7 & Sedimentary rocks only \\
\hline 70 & 8/8-30N2 & Santa Rosa Valley & Cardwell (1958) & 38.5054 & -122.7962 & 517771 & 4261709 & 8 & 8 & 30 & $\mathrm{~N}$ & 2 & 12.5 & 182.9 & Sedimentary rocks only \\
\hline 71 & 8/8-31B1 & Santa Rosa Valley & Cardwell (1958) & 38.5036 & -122.7861 & 518649 & 4261504 & 8 & 8 & 31 & B & 1 & 35.1 & 64.0 & Sedimentary rocks only \\
\hline 72 & $8 / 9-33 \mathrm{G} 1$ & Santa Rosa Valley & Cardwell (1958) & 38.4997 & -122.8587 & 512323 & 4261058 & 8 & 9 & 33 & G & 1 & 30.5 & 109.1 & Hydrology data only; no geologic information \\
\hline 73 & $8 / 9-36 \mathrm{G} 1$ & Santa Rosa Valley & Cardwell (1958) & 38.4992 & -122.8010 & 517350 & 4261013 & 8 & 9 & 36 & G & 1 & 30.5 & 195.7 & Hydrology data only; no geologic information \\
\hline 74 & 8/9-36K1 & Santa Rosa Valley & Cardwell (1958) & 38.4967 & -122.8052 & 516992 & 4260733 & 8 & 9 & 36 & $\mathrm{k}$ & 1 & 29.0 & 403.9 & Hydrology data only; no geologic information \\
\hline 75 & 8/9-36L1 & Santa Rosa Valley & Cardwell (1958) & 38.4953 & -122.8095 & 516610 & 4260577 & 8 & 9 & 36 & L & 1 & 29.0 & 172.2 & Sedimentary rocks only \\
\hline 76 & 8/9-33J1 & Santa Rosa Valley & Cardwell (1958) & 38.4952 & -122.8541 & 512722 & 4260568 & 8 & 9 & 33 & $\mathrm{~J}$ & 1 & 13.7 & 99.1 & Sedimentary and volcanic rocks \\
\hline 77 & 8/9-35M1 & Santa Rosa Valley & Cardwell (1958) & 38.4948 & -122.8333 & 514541 & 4260517 & 8 & 9 & 35 & M & 1 & 19.8 & 144.8 & Hydrology data only; no geologic information \\
\hline 78 & $8 / 9-35 \mathrm{~K} 1$ & Santa Rosa Valley & Cardwell (1958) & 38.4937 & -122.8226 & 515469 & 4260400 & 8 & 9 & 35 & $\mathrm{k}$ & 1 & 24.4 & 151.8 & Sedimentary rocks only \\
\hline 79 & 8/9-34R1 & Santa Rosa Valley & Cardwell (1958) & 38.4917 & -122.8351 & 514384 & 4260180 & 8 & 9 & 34 & $\mathrm{R}$ & 1 & 19.8 & 109.7 & Sedimentary rocks only \\
\hline 80 & 8/8-34Q1 & Santa Rosa Valley & Cardwell (1958) & 38.4912 & -122.7307 & 523488 & 4260148 & 8 & 8 & 34 & $\mathrm{Q}$ & 1 & 105.2 & 86.9 & Hydrology data only; no geologic information \\
\hline 81 & 8/9-36P1 & Santa Rosa Valley & Cardwell (1958) & 38.4901 & -122.8103 & 516542 & 4260001 & 8 & 9 & 36 & $\mathrm{P}$ & 1 & 27.4 & 320.0 & Sedimentary and volcanic rocks \\
\hline
\end{tabular}


Appendix 1. Well Location Data. _Continued

[Site IDs corrspond to locations shown on Plate 1; Well number and area correspond to descriptions in the Water-Supply Paper listed; latitude and longitude geographic coordinates have a horizontal datum of North American Datum 1927; Easting and Northing coordinates are in Universa Transverse Mercator projection zone 10, North American Datum 1927; system of quarter-quarter section designation is explained in the Water-Supply Papers; leaders (--), well number not assigned]

\begin{tabular}{|c|c|c|c|c|c|c|c|c|c|c|c|c|c|c|c|}
\hline Site ID & Well number & Area & Water-Supply Paper & Latitude & Longitude & Easting & Northing & $\begin{array}{l}\text { Township } \\
\text { (N) }\end{array}$ & $\begin{array}{l}\text { Range } \\
\text { (W) }\end{array}$ & Section & $\begin{array}{l}\text { Quarter- } \\
\text { quarter } \\
\text { section } \\
\text { designation }\end{array}$ & $\begin{array}{c}\text { Well } \\
\text { number } \\
\text { within } \\
\text { quarter- } \\
\text { quarter } \\
\text { section }\end{array}$ & $\begin{array}{l}\text { Land } \\
\text { surface } \\
\text { elevation, in } \\
\text { meters }\end{array}$ & $\begin{array}{l}\text { Total } \\
\text { depth of } \\
\text { well, in } \\
\text { meters }\end{array}$ & Geologic units intersected \\
\hline 82 & 7/9-2C1 & Santa Rosa Valley & Cardwell (1958) & 38.4887 & -122.8286 & 514951 & 4259851 & 7 & 9 & 2 & c & 1 & 21.3 & 185.9 & Hydrology data only; no geologic information \\
\hline 83 & 8/9-34R2 & Santa Rosa Valley & Cardwell (1958) & 38.4886 & -122.8352 & 514374 & 4259838 & 8 & 9 & 34 & $\mathrm{R}$ & 2 & 19.8 & 149.7 & Sedimentary rocks only \\
\hline 84 & $7 / 8-3 \mathrm{Cl}$ & Santa Rosa Valley & Cardwell (1958) & 38.4872 & -122.7340 & 523201 & 4259700 & 7 & 8 & 3 & c & 1 & 53.3 & 71.6 & Sedimentary rocks only \\
\hline 85 & 7/8-4B3 & Santa Rosa Valley & Cardwell (1958) & 38.4855 & -122.7497 & 521831 & 4259509 & 7 & 8 & 4 & B & 3 & 45.0 & 46.3 & Sedimentary rocks only \\
\hline 86 & $7 / 7-5 F 1$ & Santa Rosa Valley & Cardwell (1958) & 38.4846 & -122.6619 & 529489 & 4259430 & 7 & 7 & 5 & $\mathrm{~F}$ & 1 & 107.0 & 50.0 & Sedimentary rocks only \\
\hline 87 & $7 / 7-5 F 2$ & Santa Rosa Valley & Cardwell (1958) & 38.4839 & -122.6608 & 529584 & 4259356 & 7 & 7 & 5 & $\mathrm{~F}$ & 2 & 99.1 & 83.8 & Sedimentary rocks only \\
\hline 88 & 7/9-3E1 & Santa Rosa Valley & Cardwell (1958) & 38.4835 & -122.8523 & 512879 & 4259265 & 7 & 9 & 3 & E & 1 & 45.7 & 39.6 & Sedimentary rocks only \\
\hline 89 & $7 / 7-6 \mathrm{G} 1$ & Santa Rosa Valley & Cardwell (1958) & 38.4823 & -122.6750 & 528348 & 4259167 & 7 & 7 & 6 & G & 1 & 89.9 & 103.0 & Sedimentary rocks only \\
\hline 90 & $7 / 8-6 \mathrm{H} 2$ & Santa Rosa Valley & Cardwell (1958) & 38.4824 & -122.7809 & 519109 & 4259152 & 7 & 8 & 6 & $\mathrm{H}$ & 2 & 41.1 & 121.0 & Sedimentary rocks only \\
\hline 91 & 7/9-3M1 & Santa Rosa Valley & Cardwell (1958) & 38.4806 & -122.8495 & 513124 & 4258939 & 7 & 9 & 3 & M & 1 & 30.5 & 39.9 & Sedimentary rocks only \\
\hline 92 & $7 / 7-5 \mathrm{~N} 1$ & Santa Rosa Valley & Cardwell (1958) & 38.4746 & -122.6664 & 529097 & 4258323 & 7 & 7 & 5 & $\mathrm{~N}$ & 1 & 82.0 & 26.8 & Sedimentary rocks only \\
\hline 93 & 7/7-8A1 & Santa Rosa Valley & Cardwell (1958) & 38.4738 & -122.6526 & 530308 & 4258241 & 7 & 7 & 8 & A & 1 & 96.0 & 117.3 & Hydrology data only; no geologic information \\
\hline 94 & 7/9-4N1 & Santa Rosa Valley & Cardwell (1958) & 38.4736 & -122.8699 & 511347 & 4258167 & 7 & 9 & 4 & $\mathrm{~N}$ & 1 & 85.3 & 36.6 & Sedimentary rocks only \\
\hline 95 & $7 / 9-5 \mathrm{~N} 1$ & Santa Rosa Valley & Cardwell (1958) & 38.4736 & -122.8893 & 509656 & 4258160 & 7 & 9 & 5 & $\mathrm{~N}$ & 1 & 51.8 & 89.0 & Sedimentary rocks and pre-Cenozoic rocks \\
\hline 96 & 7/7-9D3 & Santa Rosa Valley & Cardwell (1958) & 38.4731 & -122.6479 & 530718 & 4258156 & 7 & 7 & 9 & D & 3 & 121.9 & 97.2 & Sedimentary rocks only \\
\hline 97 & 7/7-9D2 & Santa Rosa Valley & Cardwell (1958) & 38.4729 & -122.6480 & 530709 & 4258133 & 7 & 7 & 9 & D & 2 & 114.3 & 98.5 & Sedimentary rocks only \\
\hline 98 & $7 / 7-8 \mathrm{~A} 2$ & Santa Rosa Valley & Cardwell (1958) & 38.4724 & -122.6492 & 530600 & 4258082 & 7 & 7 & 8 & A & 2 & 109.7 & 70.1 & Sedimentary rocks only \\
\hline 99 & 7/7-9D1 & Santa Rosa Valley & Cardwell (1958) & 38.4724 & -122.6480 & 530703 & 4258082 & 7 & 7 & 9 & D & 1 & 115.8 & 74.7 & Sedimentary rocks only \\
\hline 100 & 7/8-9D1 & Santa Rosa Valley & Cardwell (1958) & 38.4724 & -122.7593 & 520995 & 4258048 & 7 & 8 & 9 & D & 1 & 39.6 & 61.0 & Hydrology data only; no geologic information \\
\hline 101 & $7 / 9-7 \mathrm{G} 1$ & Santa Rosa Valley & Cardwell (1958) & 38.4719 & -122.8967 & 509009 & 4257973 & 7 & 9 & 7 & G & 1 & 18.9 & 100.9 & Hydrology data only; no geologic information \\
\hline 102 & 7/8-11G1 & Santa Rosa Valley & Cardwell (1958) & 38.4714 & -122.7125 & 525078 & 4257956 & 7 & 8 & 11 & G & 1 & 88.4 & 72.2 & Hydrology data only; no geologic information \\
\hline 103 & 7/7-8G2 & Santa Rosa Valley & Cardwell (1958) & 38.4712 & -122.6540 & 530183 & 4257952 & 7 & 7 & 8 & G & 2 & 88.4 & 129.5 & Hydrology data only; no geologic information \\
\hline 104 & 7/9-9F1 & Santa Rosa Valley & Cardwell (1958) & 38.4705 & -122.8642 & 511848 & 4257817 & 7 & 9 & 9 & $\mathrm{~F}$ & 1 & 20.1 & 68.9 & Sedimentary rocks only \\
\hline 105 & $7 / 8-12 \mathrm{H} 1$ & Santa Rosa Valley & Cardwell (1958) & 38.4697 & -122.6886 & 527164 & 4257773 & 7 & 8 & 12 & $\mathrm{H}$ & 1 & 140.2 & 77.7 & Hydrology data only; no geologic information \\
\hline 106 & $7 / 8-12 E 2$ & Santa Rosa Valley & Cardwell (1958) & 38.4696 & -122.7035 & 525870 & 4257753 & 7 & 8 & 12 & E & 2 & 74.7 & 91.4 & Hydrology data only; no geologic information \\
\hline 107 & 7/9-10E1 & Santa Rosa Valley & Cardwell (1958) & 38.4699 & -122.8501 & 513073 & 4257752 & 7 & 9 & 10 & E & 1 & 61.0 & 30.5 & Sedimentary rocks only \\
\hline 108 & 7/7-8M2 & Santa Rosa Valley & Cardwell (1958) & 38.4679 & -122.6666 & 529087 & 4257573 & 7 & 7 & 8 & M & 2 & 76.2 & 64.3 & Hydrology data only; no geologic information \\
\hline 109 & $7 / 7-7 F 2$ & Santa Rosa Valley & Cardwell (1958) & 38.4675 & -122.6812 & 527810 & 4257531 & 7 & 7 & 7 & $\mathrm{~F}$ & 2 & 74.7 & 97.5 & Sedimentary and volcanic rocks \\
\hline 110 & 7/9-8J1 & Santa Rosa Valley & Cardwell (1958) & 38.4676 & -122.8730 & 511076 & 4257495 & 7 & 9 & 8 & $\mathrm{~J}$ & 1 & 17.6 & 76.2 & Sedimentary rocks only \\
\hline 111 & $7 / 8-11 \mathrm{~K} 1$ & Santa Rosa Valley & Cardwell (1958) & 38.4666 & -122.7121 & 525114 & 4257424 & 7 & 8 & 11 & $\mathrm{k}$ & 1 & 91.4 & 77.4 & Sedimentary rocks only \\
\hline 112 & 7/8-10L1 & Santa Rosa Valley & Cardwell (1958) & 38.4659 & -122.7349 & 523124 & 4257332 & 7 & 8 & 10 & L & 1 & 41.1 & 64.6 & Sedimentary rocks only \\
\hline 113 & 7/9-8K1 & Santa Rosa Valley & Cardwell (1958) & 38.4661 & -122.8773 & 510704 & 4257328 & 7 & 9 & 8 & $\mathrm{k}$ & 1 & 19.0 & 106.7 & Sedimentary rocks only \\
\hline 114 & 7/8-12K1 & Santa Rosa Valley & Cardwell (1958) & 38.4654 & -122.6917 & 526898 & 4257296 & 7 & 8 & 12 & $\mathrm{k}$ & 1 & 146.3 & 105.8 & Sedimentary rocks only \\
\hline 115 & 7/8-12Q2 & Santa Rosa Valley & Cardwell (1958) & 38.4648 & -122.6944 & 526661 & 4257229 & 7 & 8 & 12 & Q & 2 & 131.1 & 29.0 & Sedimentary rocks only \\
\hline 116 & 7/7-8P2 & Santa Rosa Valley & Cardwell (1958) & 38.4643 & -122.6613 & 529548 & 4257183 & 7 & 7 & 8 & $P$ & 2 & 86.9 & 76.2 & Volcanic rocks only \\
\hline 117 & 717-8L1 & Santa Rosa Valley & Cardwell (1958) & 38.4640 & -122.6620 & 529491 & 4257149 & 7 & 7 & 8 & L & 1 & 76.2 & 84.7 & Sedimentary and volcanic rocks \\
\hline 118 & $7 / 8-9 \mathrm{R} 1$ & Santa Rosa Valley & Cardwell (1958) & 38.4609 & -122.7458 & 522181 & 4256776 & 7 & 8 & 9 & $\mathrm{R}$ & 1 & 36.6 & 57.0 & Sedimentary rocks only \\
\hline 119 & 7/8-14A1 & Santa Rosa Valley & Cardwell (1958) & 38.4593 & -122.7066 & 525602 & 4256610 & 7 & 8 & 14 & A & 1 & 53.3 & 274.6 & Sedimentary and volcanic rocks \\
\hline 120 & 7/7-16A1 & Santa Rosa Valley & Cardwell (1958) & 38.4590 & -122.6352 & 531831 & 4256600 & 7 & 7 & 16 & A & 1 & 99.1 & 80.2 & Sedimentary rocks only \\
\hline 121 & $7 / 7-15 \mathrm{C} 1$ & Santa Rosa Valley & Cardwell (1958) & 38.4589 & -122.6251 & 532710 & 4256590 & 7 & 7 & 15 & c & 1 & 114.3 & 121.0 & Sedimentary rocks only \\
\hline 122 & 7/7-17A1 & Santa Rosa Valley & Cardwell (1958) & 38.4589 & -122.6532 & 530254 & 4256581 & 7 & 7 & 17 & A & 1 & 88.4 & 257.9 & Sedimentary and volcanic rocks \\
\hline 123 & 7/9-15E2 & Santa Rosa Valley & Cardwell (1958) & 38.4582 & -122.8482 & 513244 & 4256461 & 7 & 9 & 15 & $\mathrm{E}$ & 2 & 38.1 & 67.1 & Hydrology data only; no geologic information \\
\hline 124 & 7/8-14D1 & Santa Rosa Valley & Cardwell (1958) & 38.4567 & -122.7209 & 524348 & 4256315 & 7 & 8 & 14 & D & 1 & 47.2 & 91.4 & Sedimentary rocks only \\
\hline 125 & 7/8-14E1 & Santa Rosa Valley & Cardwell (1958) & 38.4564 & -122.7192 & 524501 & 4256283 & 7 & 8 & 14 & $\mathrm{E}$ & 1 & 47.2 & 232.0 & Hydrology data only; no geologic information \\
\hline
\end{tabular}


Appendix 1. Well Location Data. _Continued

[Site IDs corrspond to locations shown on Plate 1; Well number and area correspond to descriptions in the Water-Supply Paper listed; latitude and longitude geographic coordinates have a horizontal datum of North American Datum 1927; Easting and Northing coordinates are in Universa Transverse Mercator projection zone 10, North American Datum 1927; system of quarter-quarter section designation is explained in the Water-Supply Papers; leaders (--), well number not assigned]

\begin{tabular}{|c|c|c|c|c|c|c|c|c|c|c|c|c|c|c|c|}
\hline Site ID & Well number & Area & Water-Supply Paper & Latitude & Longitude & Easting & Northing & $\begin{array}{l}\text { Township } \\
\text { (N) }\end{array}$ & $\begin{array}{l}\text { Range } \\
\text { (W) }\end{array}$ & Section & $\begin{array}{c}\text { Quarter- } \\
\text { quarter } \\
\text { section } \\
\text { designation }\end{array}$ & $\begin{array}{c}\text { Well } \\
\text { number } \\
\text { within } \\
\text { quarter- } \\
\text { quarter } \\
\text { section }\end{array}$ & $\begin{array}{l}\text { Land } \\
\text { surface } \\
\text { elevation, in } \\
\text { meters }\end{array}$ & $\begin{array}{l}\text { Total } \\
\text { depth of } \\
\text { well, in } \\
\text { meters }\end{array}$ & Geologic units intersected \\
\hline 126 & 7/9-18J1 & Santa Rosa Valley & Cardwell (1958) & 38.4552 & -122.8936 & 509288 & 4256124 & 7 & 9 & 18 & $\mathrm{~J}$ & 1 & 35.1 & 198.1 & Hydrology data only; no geologic information \\
\hline 127 & 7/8-18M1 & Santa Rosa Valley & Cardwell (1958) & 38.4550 & -122.7929 & 518075 & 4256114 & 7 & 8 & 18 & M & 1 & 24.4 & 195.1 & Hydrology data only; no geologic information \\
\hline 128 & 7/9-13M1 & Santa Rosa Valley & Cardwell (1958) & 38.4537 & -122.8116 & 516436 & 4255966 & 7 & 9 & 13 & M & 1 & 6.9 & 96.3 & Sedimentary rocks only \\
\hline 129 & 7/8-14M1 & Santa Rosa Valley & Cardwell (1958) & 38.4533 & -122.7182 & 524593 & 4255945 & 7 & 8 & 14 & M & 1 & 47.2 & 69.4 & Hydrology data only; no geologic information \\
\hline 130 & 7/8-13J1B & Santa Rosa Valley & Cardwell (1958) & 38.4532 & -122.6871 & 527303 & 4255939 & 7 & 8 & 13 & $\mathrm{~J}$ & 1 & 6.1 & 77.1 & Hydrology data only; no geologic information \\
\hline 131 & 7/8-18K1 & Santa Rosa Valley & Cardwell (1958) & 38.4533 & -122.7858 & 518691 & 4255923 & 7 & 8 & 18 & $\mathrm{k}$ & 1 & 25.9 & 128.0 & Sedimentary rocks only \\
\hline 132 & $7 / 9-18 \mathrm{~K} 1$ & Santa Rosa Valley & Cardwell (1958) & 38.4534 & -122.8939 & 509260 & 4255919 & 7 & 9 & 18 & $\mathrm{~K}$ & 1 & 35.1 & 81.1 & Hydrology data only; no geologic information \\
\hline 133 & 7/9-16L1 & Santa Rosa Valley & Cardwell (1958) & 38.4528 & -122.8621 & 512032 & 4255858 & 7 & 9 & 16 & $\mathrm{~L}$ & 1 & 67.1 & 77.7 & Hydrology data only; no geologic information \\
\hline 134 & $7 / 8-13 \mathrm{~J} 2$ & Santa Rosa Valley & Cardwell (1958) & 38.4522 & -122.6886 & 527174 & 4255823 & 7 & 8 & 13 & $\mathrm{~J}$ & 2 & 64.0 & 64.0 & Hydrology data only; no geologic information \\
\hline 135 & 7/8-18Q1 & Santa Rosa Valley & Cardwell (1958) & 38.4511 & -122.7828 & 518950 & 4255685 & 7 & 8 & 18 & Q & 1 & 24.4 & 268.5 & Sedimentary rocks only \\
\hline 136 & $7 / 8-13 \mathrm{~K} 1$ & Santa Rosa Valley & Cardwell (1958) & 38.4508 & -122.6930 & 526789 & 4255673 & 7 & 8 & 13 & $\mathrm{k}$ & 1 & 61.0 & 161.2 & Sedimentary rocks only \\
\hline 137 & 7/9-15Q1 & Santa Rosa Valley & Cardwell (1958) & 38.4511 & -122.8425 & 513748 & 4255672 & 7 & 9 & 15 & $\mathrm{Q}$ & 1 & 22.9 & 61.0 & Hydrology data only; no geologic information \\
\hline 138 & 7/9-14K1 & Santa Rosa Valley & Cardwell (1958) & 38.4511 & -122.8222 & 515515 & 4255672 & 7 & 9 & 14 & $\mathrm{k}$ & 1 & 24.4 & 193.9 & Sedimentary rocks only \\
\hline 139 & 7/7-18L1 & Santa Rosa Valley & Cardwell (1958) & 38.4494 & -122.6775 & 528144 & 4255525 & 7 & 7 & 18 & $\mathrm{~L}$ & 1 & 60.0 & 49.1 & Sedimentary and volcanic rocks \\
\hline 140 & 7/9-16Q1A & Santa Rosa Valley & Cardwell (1958) & 38.4495 & -122.8594 & 512272 & 4255495 & 7 & 9 & 16 & $\mathrm{Q}$ & 1 & 68.6 & 81.7 & Hydrology data only; no geologic information \\
\hline 141 & 7/9-16Q1B & Santa Rosa Valley & Cardwell (1958) & 38.4495 & -122.8594 & 512272 & 4255495 & 7 & 9 & 16 & $\mathrm{Q}$ & 1 & 68.6 & 97.8 & Hydrology data only; no geologic information \\
\hline 142 & 7/9-13R1 & Santa Rosa Valley & Cardwell (1958) & 38.4493 & -122.7984 & 517589 & 4255476 & 7 & 8 & 13 & $\mathrm{R}$ & 1 & 21.3 & 114.3 & Sedimentary rocks only \\
\hline 143 & $7 / 8-17 \mathrm{~N} 1$ & Santa Rosa Valley & Cardwell (1958) & 38.4481 & -122.7757 & 519574 & 4255348 & 7 & 8 & 17 & $\mathrm{~N}$ & 1 & 25.9 & 165.8 & Sedimentary rocks only \\
\hline 144 & $7 / 9-21 B 1$ & Santa Rosa Valley & Cardwell (1958) & 38.4477 & -122.8598 & 512238 & 4255288 & 7 & 9 & 21 & B & 1 & 73.2 & 167.6 & Hydrology data only; no geologic information \\
\hline 145 & $7 / 7-18 \mathrm{R} 2$ & Santa Rosa Valley & Cardwell (1958) & 38.4451 & -122.6706 & 528747 & 4255049 & 7 & 7 & 18 & $\mathrm{R}$ & 2 & 64.0 & 62.8 & Sedimentary and volcanic rocks \\
\hline 146 & $7 / 8-24 \mathrm{~A} 2$ & Santa Rosa Valley & Cardwell (1958) & 38.4452 & -122.6869 & 527320 & 4255048 & 7 & 8 & 24 & A & 2 & 59.4 & 283.5 & Hydrology data only; no geologic information \\
\hline 147 & $7 / 7-18 \mathrm{~N} 1$ & Santa Rosa Valley & Cardwell (1958) & 38.4451 & -122.6825 & 527708 & 4255045 & 7 & 7 & 18 & $\mathrm{~N}$ & 1 & 63.0 & 42.7 & Sedimentary rocks only \\
\hline 148 & $7 / 8-21 \mathrm{C1}$ & Santa Rosa Valley & Cardwell (1958) & 38.4449 & -122.7515 & 521688 & 4255005 & 7 & 8 & 21 & c & 1 & 33.5 & 94.5 & Hydrology data only; no geologic information \\
\hline 149 & $7 / 8-20 C 1$ & Santa Rosa Valley & Cardwell (1958) & 38.4450 & -122.7725 & 519857 & 4255005 & 7 & 8 & 19 & $\mathrm{P}$ & 2 & 27.4 & 190.5 & Sedimentary rocks only \\
\hline 150 & $7 / 9-21 C 1$ & Santa Rosa Valley & Cardwell (1958) & 38.4450 & -122.8641 & 511858 & 4254991 & 7 & 9 & 21 & c & 1 & 68.6 & 99.1 & Sedimentary rocks only \\
\hline 151 & $7 / 8-24 \mathrm{~A} 3$ & Santa Rosa Valley & Cardwell (1958) & 38.4443 & -122.6869 & 527325 & 4254949 & 7 & 8 & 24 & A & 3 & 59.4 & 91.4 & Hydrology data only; no geologic information \\
\hline 152 & $7 / 9-23 F 1$ & Santa Rosa Valley & Cardwell (1958) & 38.4446 & -122.8265 & 515145 & 4254948 & 7 & 9 & 23 & $\mathrm{~F}$ & 1 & 4.9 & 185.9 & Sedimentary rocks only \\
\hline 153 & $7 / 8-20 \mathrm{D} 1$ & Santa Rosa Valley & Cardwell (1958) & 38.4441 & -122.7755 & 519594 & 4254908 & 7 & 8 & 20 & D & 1 & 27.4 & 147.5 & Sedimentary rocks only \\
\hline 154 & 7/8-24A5 & Santa Rosa Valley & Cardwell (1958) & 38.4435 & -122.6870 & 527313 & 4254858 & 7 & 8 & 24 & A & 5 & 57.9 & 88.7 & Hydrology data only; no geologic information \\
\hline 155 & 7/8-24A6 & Santa Rosa Valley & Cardwell (1958) & 38.4433 & -122.6868 & 527332 & 4254844 & 7 & 8 & 24 & A & 6 & 57.9 & 365.8 & Hydrology data only; no geologic information \\
\hline 156 & 7/7-14A1 & Santa Rosa Valley & Cardwell (1958) & 38.4430 & -122.5779 & 536833 & 4254843 & 7 & 7 & 24 & A & 1 & 172.2 & 189.6 & Volcanic rocks only \\
\hline 157 & 7/7-23A1 & Santa Rosa Valley & Cardwell (1958) & 38.4428 & -122.5939 & 535439 & 4254820 & 7 & 7 & 23 & A & 1 & 144.8 & 111.3 & Sedimentary and volcanic rocks \\
\hline 158 & $7 / 8-24 \mathrm{~A} 4$ & Santa Rosa Valley & Cardwell (1958) & 38.4428 & -122.6889 & 527148 & 4254785 & 7 & 8 & 24 & A & 1 & 59.4 & 304.8 & Sedimentary and volcanic rocks \\
\hline 159 & 7/7-24A1 & Santa Rosa Valley & Cardwell (1958) & 38.4421 & -122.5785 & 536784 & 4254750 & 7 & 7 & 24 & A & 1 & 172.2 & 189.6 & Sedimentary and volcanic rocks \\
\hline 160 & $7 / 9-21 \mathrm{G} 2$ & Santa Rosa Valley & Cardwell (1958) & 38.4426 & -122.8594 & 512267 & 4254724 & 7 & 9 & 21 & G & 2 & 62.5 & 73.2 & Hydrology data only; no geologic information \\
\hline 161 & 7/7-23A2 & Santa Rosa Valley & Cardwell (1958) & 38.4416 & -122.5949 & 535353 & 4254682 & 7 & 7 & 23 & A & 2 & 137.2 & 102.7 & Sedimentary rocks only \\
\hline 162 & $7 / 9-22 F 1$ & Santa Rosa Valley & Cardwell (1958) & 38.4420 & -122.8440 & 513617 & 4254667 & 7 & 9 & 22 & $\mathrm{~F}$ & 1 & 38.1 & 175.3 & Sedimentary rocks only \\
\hline 163 & $7 / 9-22 F 2$ & Santa Rosa Valley & Cardwell (1958) & 38.4418 & -122.8468 & 513367 & 4254642 & 7 & 9 & 22 & $\mathrm{~F}$ & 2 & 45.7 & 115.8 & Hydrology data only; no geologic information \\
\hline 164 & 7/9-21F1 & Santa Rosa Valley & Cardwell (1958) & 38.4416 & -122.8644 & 511832 & 4254615 & 7 & 9 & 21 & $\mathrm{~F}$ & 1 & 61.0 & 97.5 & Hydrology data only; no geologic information \\
\hline 165 & 7/9-21G3 & Santa Rosa Valley & Cardwell (1958) & 38.4414 & -122.8611 & 512125 & 4254598 & 7 & 9 & 21 & G & 3 & 65.5 & 124.4 & Sedimentary rocks only \\
\hline 166 & $7 / 7-23 G 1$ & Santa Rosa Valley & Cardwell (1958) & 38.4404 & -122.5986 & 535033 & 4254549 & 7 & 7 & 23 & G & 1 & 131.1 & 356.6 & Sedimentary and volcanic rocks \\
\hline 167 & 7/9-21G1 & Santa Rosa Valley & Cardwell (1958) & 38.4405 & -122.8590 & 512308 & 4254488 & 7 & 9 & 21 & G & 1 & 73.2 & 161.5 & Sedimentary rocks only \\
\hline 168 & $7 / 9-21 \mathrm{J1}$ & Santa Rosa Valley & Cardwell (1958) & 38.4401 & -122.8574 & 512444 & 4254445 & 7 & 9 & 21 & $\mathrm{~J}$ & 1 & 70.1 & 62.5 & Sedimentary rocks only \\
\hline 169 & 7/9-23K1 & Santa Rosa Valley & Cardwell (1958) & 38.4398 & -122.8230 & 515449 & 4254424 & 7 & 9 & 23 & $\mathrm{~K}$ & 1 & 25.9 & 61.0 & Hydrology data only; no geologic information \\
\hline
\end{tabular}


Appendix 1. Well Location Data. _Continued

[Site IDs corrspond to locations shown on Plate 1; Well number and area correspond to descriptions in the Water-Supply Paper listed; latitude and longitude geographic coordinates have a horizontal datum of North American Datum 1927; Easting and Northing coordinates are in Universa Transverse Mercator projection zone 10, North American Datum 1927; system of quarter-quarter section designation is explained in the Water-Supply Papers; leaders (--), well number not assigned]

\begin{tabular}{|c|c|c|c|c|c|c|c|c|c|c|c|c|c|c|c|}
\hline Site ID & Well number & Area & Water-Supply Paper & Latitude & Longitude & Easting & Northing & $\begin{array}{l}\text { Township } \\
\text { (N) }\end{array}$ & $\begin{array}{c}\text { Range } \\
\text { (W) }\end{array}$ & Section & $\begin{array}{c}\text { Quarter- } \\
\text { quarter } \\
\text { section } \\
\text { designation }\end{array}$ & $\begin{array}{c}\text { Well } \\
\text { number } \\
\text { within } \\
\text { quarter- } \\
\text { quarter } \\
\text { section }\end{array}$ & $\begin{array}{l}\text { Land } \\
\text { surface } \\
\text { elevation, in } \\
\text { meters }\end{array}$ & $\begin{array}{l}\text { Total } \\
\text { depth of } \\
\text { well, in } \\
\text { meters }\end{array}$ & Geologic units intersected \\
\hline 170 & 7/9-24L2 & Santa Rosa Valley & Cardwell (1958) & 38.4396 & -122.8085 & 516715 & 4254399 & 7 & 9 & 24 & $\mathrm{~L}$ & 2 & 29.0 & 100.6 & Hydrology data only; no geologic information \\
\hline 171 & 7/8-23L1 & Santa Rosa Valley & Cardwell (1958) & 38.4374 & -122.7172 & 524680 & 4254182 & 7 & 8 & 23 & $\mathrm{~L}$ & 1 & 47.2 & 91.4 & Sedimentary rocks only \\
\hline 172 & $7 / 8-24 K 1$ & Santa Rosa Valley & Cardwell (1958) & 38.4372 & -122.6954 & 526582 & 4254167 & 7 & 8 & 24 & $\mathrm{k}$ & 1 & 54.9 & 72.5 & Sedimentary rocks only \\
\hline 173 & 7/8-23M3 & Santa Rosa Valley & Cardwell (1958) & 38.4368 & -122.7184 & 524581 & 4254113 & 7 & 8 & 23 & M & 3 & 47.2 & 91.4 & Hydrology data only; no geologic information \\
\hline 174 & $7 / 8-22 \mathrm{~K} 1$ & Santa Rosa Valley & Cardwell (1958) & 38.4364 & -122.7318 & 523408 & 4254067 & 7 & 8 & 22 & $\mathrm{k}$ & 1 & 41.1 & 86.9 & Hydrology data only; no geologic information \\
\hline 175 & $7 / 8-23 \mathrm{M} 4$ & Santa Rosa Valley & Cardwell (1958) & 38.4362 & -122.7228 & 524191 & 4254049 & 7 & 8 & 23 & M & 4 & 45.7 & 389.8 & Sedimentary and volcanic rocks \\
\hline 176 & 7/8-19P1 & Santa Rosa Valley & Cardwell (1958) & 38.4361 & -122.7880 & 518506 & 4254018 & 7 & 8 & 19 & $\mathrm{P}$ & 1 & 25.9 & 74.7 & Sedimentary rocks only \\
\hline 177 & 7/8-20K1 & Santa Rosa Valley & Cardwell (1958) & 38.4359 & -122.7659 & 520429 & 4254000 & 7 & 8 & 20 & $\mathrm{k}$ & 1 & 29.9 & 190.8 & Sedimentary rocks only \\
\hline 178 & $7 / 9-24 J 1$ & Santa Rosa Valley & Cardwell (1958) & 38.4351 & -122.7988 & 517557 & 4253904 & 7 & 9 & 24 & $\mathrm{~J}$ & 1 & 28.0 & 632.8 & Sedimentary rocks only \\
\hline 179 & 7/8-22R2 & Santa Rosa Valley & Cardwell (1958) & 38.4348 & -122.7257 & 523942 & 4253882 & 7 & 8 & 22 & $\mathrm{R}$ & 2 & 44.2 & 62.5 & Hydrology data only; no geologic information \\
\hline 180 & 7/8-23M1 & Santa Rosa Valley & Cardwell (1958) & 38.4347 & -122.7194 & 524492 & 4253878 & 7 & 8 & 23 & M & 1 & 47.2 & 304.8 & Sedimentary rocks only \\
\hline 181 & $7 / 8-22 Q 1$ & Santa Rosa Valley & Cardwell (1958) & 38.4343 & -122.7294 & 523616 & 4253835 & 7 & 8 & 22 & Q & 1 & 42.7 & 109.7 & Hydrology data only; no geologic information \\
\hline 182 & 7/9-30A1 & Santa Rosa Valley & Cardwell (1958) & 38.4338 & -122.8910 & 509517 & 4253741 & 7 & 9 & 30 & A & 1 & 41.1 & 79.2 & Hydrology data only; no geologic information \\
\hline 183 & 7/8-23P1 & Santa Rosa Valley & Cardwell (1958) & 38.4334 & -122.7159 & 524801 & 4253736 & 7 & 8 & 23 & $\mathrm{P}$ & 1 & 45.7 & 81.1 & Sedimentary rocks only \\
\hline 184 & $7 / 6-19 \mathrm{R} 1$ & Santa Rosa Valley & Cardwell (1958) & 38.4325 & -122.5599 & 538410 & 4253687 & 7 & 6 & 19 & $\mathrm{R}$ & 1 & 146.1 & 91.4 & Sedimentary and volcanic rocks \\
\hline 185 & $7 / 6-19 \mathrm{~N} 1$ & Santa Rosa Valley & Cardwell (1958) & 38.4305 & -122.5737 & 537208 & 4253459 & 7 & 6 & 19 & $\mathrm{~N}$ & 1 & 142.1 & 45.4 & Sedimentary rocks only \\
\hline 186 & 7/9-25A1A & Santa Rosa Valley & Cardwell (1958) & 38.4308 & -122.8002 & 517441 & 4253428 & 7 & 9 & 25 & A & 1 & 24.4 & 26.2 & Sedimentary rocks only \\
\hline 187 & 7/9-25A1B & Santa Rosa Valley & Cardwell (1958) & 38.4308 & -122.8002 & 517441 & 4253428 & 7 & 9 & 25 & A & 1 & 24.4 & 78.0 & Sedimentary rocks only \\
\hline 188 & 7/9-30G2 & Santa Rosa Valley & Cardwell (1958) & 38.4293 & -122.8964 & 509040 & 4253246 & 7 & 9 & 30 & G & 2 & 61.0 & 61.0 & Hydrology data only; no geologic information \\
\hline 189 & 7/7-29D1 & Santa Rosa Valley & Cardwell (1958) & 38.4288 & -122.6662 & 529139 & 4253241 & 7 & 7 & 29 & D & 1 & 73.2 & 179.2 & Sedimentary and volcanic rocks \\
\hline 190 & 7/9-27E2 & Santa Rosa Valley & Cardwell (1958) & 38.4289 & -122.8487 & 513208 & 4253213 & 7 & 9 & 27 & $\mathrm{E}$ & 2 & 50.3 & 112.8 & Sedimentary rocks only \\
\hline 191 & 7/9-27F1 & Santa Rosa Valley & Cardwell (1958) & 38.4276 & -122.8472 & 513339 & 4253068 & 7 & 9 & 27 & $\mathrm{~F}$ & 1 & 45.7 & 61.0 & Hydrology data only; no geologic information \\
\hline 192 & $7 / 7-30 c 1$ & Santa Rosa Valley & Cardwell (1958) & 38.4272 & -122.6778 & 528126 & 4253063 & 7 & 7 & 30 & c & 1 & 77.7 & 136.6 & Sedimentary and volcanic rocks \\
\hline 193 & 7/9-28G1 & Santa Rosa Valley & Cardwell (1958) & 38.4275 & -122.8596 & 512260 & 4253046 & 7 & 9 & 28 & G & 1 & 36.6 & 79.2 & Sedimentary rocks only \\
\hline 194 & 7/9-28G2 & Santa Rosa Valley & Cardwell (1958) & 38.4272 & -122.8593 & 512284 & 4253022 & 7 & 9 & 28 & G & 2 & 35.1 & 73.2 & Sedimentary rocks only \\
\hline 195 & 7/9-27E1 & Santa Rosa Valley & Cardwell (1958) & 38.4265 & -122.8498 & 513115 & 4252936 & 7 & 9 & 27 & $\mathrm{E}$ & 1 & 44.2 & 79.2 & Sedimentary rocks only \\
\hline 196 & 7/9-30J1 & Santa Rosa Valley & Cardwell (1958) & 38.4264 & -122.8903 & 509573 & 4252922 & 7 & 9 & 30 & $\mathrm{~J}$ & 1 & 86.9 & 61.0 & Sedimentary rocks only \\
\hline 197 & 7/9-26M1 & Santa Rosa Valley & Cardwell (1958) & 38.4260 & -122.8332 & 514560 & 4252887 & 7 & 9 & 26 & M & 1 & 24.4 & 62.8 & Hydrology data only; no geologic information \\
\hline 198 & $7 / 8-28 F 1$ & Santa Rosa Valley & Cardwell (1958) & 38.4255 & -122.7547 & 521410 & 4252845 & 7 & 8 & 28 & $\mathrm{~F}$ & 1 & 30.5 & 20.1 & Sedimentary rocks only \\
\hline 199 & 7/9-28L1 & Santa Rosa Valley & Cardwell (1958) & 38.4243 & -122.8642 & 511859 & 4252692 & 7 & 9 & 28 & $\mathrm{~L}$ & 1 & 33.5 & 165.2 & Sedimentary rocks only \\
\hline 200 & 7/9-29J1 & Santa Rosa Valley & Cardwell (1958) & 38.4239 & -122.8724 & 511135 & 4252646 & 7 & 9 & 29 & $\mathrm{~J}$ & 1 & 36.6 & 121.9 & Hydrology data only; no geologic information \\
\hline 201 & 7/8-25L1 & Santa Rosa Valley & Cardwell (1958) & 38.4229 & -122.6987 & 526298 & 4252579 & 7 & 8 & 25 & L & 1 & 53.3 & 227.1 & Hydrology data only; no geologic information \\
\hline 202 & 7/8-29R1 & Santa Rosa Valley & Cardwell (1958) & 38.4213 & -122.7604 & 520913 & 4252382 & 7 & 8 & 29 & $\mathrm{R}$ & 1 & 28.3 & 207.9 & Sedimentary rocks only \\
\hline 203 & $7 / 8-29 \mathrm{R} 2$ & Santa Rosa Valley & Cardwell (1958) & 38.4211 & -122.7602 & 520935 & 4252360 & 7 & 8 & 29 & $\mathrm{R}$ & 2 & 28.7 & 244.1 & Hydrology data only; no geologic information \\
\hline 204 & $7 / 9-29 J 2$ & Santa Rosa Valley & Cardwell (1958) & 38.4210 & -122.8737 & 511028 & 4252328 & 7 & 9 & 29 & $\mathrm{~J}$ & 1 & 36.6 & 200.3 & Sedimentary rocks only \\
\hline 205 & $7 / 9-28 \mathrm{P} 1$ & Santa Rosa Valley & Cardwell (1958) & 38.4194 & -122.8663 & 511675 & 4252146 & 7 & 9 & 28 & $\mathrm{P}$ & 1 & 35.1 & 178.3 & Hydrology data only; no geologic information \\
\hline 206 & $7 / 8-29 J 1$ & Santa Rosa Valley & Cardwell (1958) & 38.4190 & -122.7617 & 520801 & 4252126 & 7 & 8 & 29 & $\mathrm{~J}$ & 1 & 28.7 & 213.4 & Sedimentary rocks only \\
\hline 207 & $7 / 8-25 R 1$ & Santa Rosa Valley & Cardwell (1958) & 38.4183 & -122.6894 & 527119 & 4252069 & 7 & 8 & 25 & $\mathrm{R}$ & 1 & 71.6 & 97.5 & Hydrology data only; no geologic information \\
\hline 208 & 7/9-33D2 & Santa Rosa Valley & Cardwell (1958) & 38.4182 & -122.8675 & 511568 & 4252023 & 7 & 9 & 33 & D & 2 & 47.2 & 91.4 & Hydrology data only; no geologic information \\
\hline 209 & 7/9-33А1 & Santa Rosa Valley & Cardwell (1958) & 38.4180 & -122.8534 & 512795 & 4252000 & 7 & 9 & 33 & A & 1 & 68.6 & 74.7 & Hydrology data only; no geologic information \\
\hline 210 & 7/9-31A1 & Santa Rosa Valley & Cardwell (1958) & 38.4161 & -122.8897 & 509634 & 4251779 & 7 & 9 & 31 & A & 1 & 125.0 & 73.2 & Hydrology data only; no geologic information \\
\hline 211 & $7 / 8-36 \mathrm{D} 2$ & Santa Rosa Valley & Cardwell (1958) & 38.4156 & -122.7045 & 525797 & 4251760 & 7 & 8 & 36 & D & 2 & 50.3 & 76.8 & Sedimentary and volcanic rocks \\
\hline 212 & 7/8-30P1 & Santa Rosa Valley & Cardwell (1958) & 38.4153 & -122.7899 & 518343 & 4251714 & 7 & 8 & 30 & $\mathrm{P}$ & 1 & 25.9 & 62.5 & Sedimentary rocks only \\
\hline 213 & $7 / 8-31 \mathrm{C} 1$ & Santa Rosa Valley & Cardwell (1958) & 38.4146 & -122.7911 & 518238 & 4251627 & 7 & 8 & 31 & c & 1 & 26.8 & 97.5 & Sedimentary rocks only \\
\hline
\end{tabular}


Appendix 1. Well Location Data. _Continued

[Site IDs corrspond to locations shown on Plate 1; Well number and area correspond to descriptions in the Water-Supply Paper listed; latitude and longitude geographic coordinates have a horizontal datum of North American Datum 1927; Easting and Northing coordinates are in Universa Transverse Mercator projection zone 10, North American Datum 1927; system of quarter-quarter section designation is explained in the Water-Supply Papers; leaders (--), well number not assigned]

\begin{tabular}{|c|c|c|c|c|c|c|c|c|c|c|c|c|c|c|c|}
\hline Site ID & Well number & Area & Water-Supply Paper & Latitude & Longitude & Easting & Northing & $\begin{array}{l}\text { Township } \\
\text { (N) }\end{array}$ & $\begin{array}{c}\text { Range } \\
\text { (W) }\end{array}$ & Section & $\begin{array}{l}\text { Quarter- } \\
\text { quarter } \\
\text { section } \\
\text { designation }\end{array}$ & $\begin{array}{c}\text { Well } \\
\text { number } \\
\text { within } \\
\text { quarter- } \\
\text { quarter } \\
\text { section }\end{array}$ & $\begin{array}{l}\text { Land } \\
\text { surface } \\
\text { elevation, in } \\
\text { meters }\end{array}$ & $\begin{array}{l}\text { Total } \\
\text { depth of } \\
\text { well, in } \\
\text { meters }\end{array}$ & Geologic units intersected \\
\hline 214 & $7 / 8-35 \mathrm{H} 1$ & Santa Rosa Valley & Cardwell (1958) & 38.4143 & -122.7050 & 525751 & 4251619 & 7 & 8 & 35 & $\mathrm{H}$ & 1 & 45.7 & 82.3 & Hydrology data only; no geologic information \\
\hline 215 & $7 / 9-34 \mathrm{G} 1$ & Santa Rosa Valley & Cardwell (1958) & 38.4145 & -122.8401 & 513963 & 4251613 & 7 & 9 & 34 & G & 1 & 59.4 & 109.1 & Sedimentary rocks only \\
\hline 216 & $7 / 9-32 F 2$ & Santa Rosa Valley & Cardwell (1958) & 38.4141 & -122.8799 & 510482 & 4251559 & 7 & 9 & 32 & $\mathrm{~F}$ & 2 & 120.4 & 111.3 & Hydrology data only; no geologic information \\
\hline 217 & 7/9-33F1 & Santa Rosa Valley & Cardwell (1958) & 38.4128 & -122.8658 & 511720 & 4251417 & 7 & 9 & 33 & $\mathrm{~F}$ & 1 & 50.3 & 61.0 & Sedimentary rocks only \\
\hline 218 & 7/9-35E1 & Santa Rosa Valley & Cardwell (1958) & 38.4127 & -122.8311 & 514746 & 4251417 & 7 & 9 & 35 & $\mathrm{E}$ & 1 & 45.7 & 77.1 & Hydrology data only; no geologic information \\
\hline 219 & 7/9-32E1 & Santa Rosa Valley & Cardwell (1958) & 38.4125 & -122.8878 & 509795 & 4251386 & 7 & 9 & 32 & $\mathrm{E}$ & 1 & 147.8 & 61.0 & Hydrology data only; no geologic information \\
\hline 220 & $7 / 9-36 \mathrm{H} 1$ & Santa Rosa Valley & Cardwell (1958) & 38.4116 & -122.8013 & 517350 & 4251296 & 7 & 9 & 36 & $\mathrm{H}$ & 1 & 25.9 & 173.1 & Sedimentary rocks only \\
\hline 221 & $7 / 6-32 F 1$ & Santa Rosa Valley & Cardwell (1958) & 38.4106 & -122.5515 & 539160 & 4251259 & 7 & 6 & 32 & $\mathrm{~F}$ & 1 & 121.0 & 57.9 & Sedimentary rocks only \\
\hline 222 & 7/9-34L1 & Santa Rosa Valley & Cardwell (1958) & 38.4110 & -122.8444 & 513582 & 4251227 & 7 & 9 & 34 & L & 1 & 57.9 & 96.0 & Sedimentary rocks only \\
\hline 223 & $7 / 9-36 \mathrm{~F} 1$ & Santa Rosa Valley & Cardwell (1958) & 38.4099 & -122.8073 & 516823 & 4251101 & 7 & 9 & 36 & $\mathrm{~F}$ & 1 & 25.9 & 82.3 & Sedimentary rocks only \\
\hline 224 & 7/9-31J1 & Santa Rosa Valley & Cardwell (1958) & 38.4098 & -122.8920 & 509428 & 4251083 & 7 & 9 & 31 & $\mathrm{~J}$ & 1 & 141.7 & 107.3 & Sedimentary rocks only \\
\hline 225 & $7 / 9-34 J 1$ & Santa Rosa Valley & Cardwell (1958) & 38.4096 & -122.8358 & 514335 & 4251070 & 7 & 9 & 34 & $\mathrm{~J}$ & 1 & 57.9 & 70.1 & Hydrology data only; no geologic information \\
\hline 226 & $7 / 7-32 \mathrm{G} 1$ & Santa Rosa Valley & Cardwell (1958) & 38.4092 & -122.6545 & 530162 & 4251064 & 7 & 7 & 32 & G & 1 & 99.1 & 122.8 & Sedimentary and volcanic rocks \\
\hline 227 & $7 / 9-34 J 2$ & Santa Rosa Valley & Cardwell (1958) & 38.4088 & -122.8349 & 514415 & 4250985 & 7 & 9 & 34 & $\mathrm{~J}$ & 2 & 53.3 & 106.7 & Hydrology data only; no geologic information \\
\hline 228 & 7/8-33м1 & Santa Rosa Valley & Cardwell (1958) & 38.4075 & -122.7553 & 521363 & 4250846 & 7 & 8 & 33 & M & 1 & 8.5 & 137.8 & Sedimentary rocks only \\
\hline 229 & 7/9-31Q1 & Santa Rosa Valley & Cardwell (1958) & 38.4075 & -122.8935 & 509303 & 4250829 & 7 & 9 & 31 & $\mathrm{Q}$ & 1 & 146.3 & 71.6 & Sedimentary rocks only \\
\hline 230 & 7/9-36M1 & Santa Rosa Valley & Cardwell (1958) & 38.4070 & -122.8121 & 516406 & 4250786 & 7 & 9 & 36 & M & 1 & 20.0 & 26.8 & Sedimentary rocks only \\
\hline 231 & $7 / 9-31 Q 3$ & Santa Rosa Valley & Cardwell (1958) & 38.4061 & -122.8942 & 509239 & 4250673 & 7 & 9 & 31 & $\mathrm{Q}$ & 3 & 143.3 & 76.2 & Hydrology data only; no geologic information \\
\hline 232 & 7/9-35R1 & Santa Rosa Valley & Cardwell (1958) & 38.4039 & -122.8200 & 515717 & 4250434 & 7 & 9 & 35 & $\mathrm{R}$ & 1 & 20.0 & 27.4 & Sedimentary rocks only \\
\hline 233 & 6/9-3A1 & Santa Rosa Valley & Cardwell (1958) & 38.3986 & -122.8381 & 514137 & 4249847 & 6 & 9 & 3 & A & 1 & 70.1 & 79.2 & Sedimentary rocks only \\
\hline 234 & $6 / 9-2 \mathrm{C} 1$ & Santa Rosa Valley & Cardwell (1958) & 38.3974 & -122.8273 & 515080 & 4249721 & 6 & 9 & 2 & c & 1 & 36.6 & 182.9 & Sedimentary rocks only \\
\hline 235 & 6/8-5E1 & Santa Rosa Valley & Cardwell (1958) & 38.3945 & -122.7761 & 519551 & 4249405 & 6 & 8 & 5 & $\mathrm{E}$ & 1 & 25.9 & 194.5 & Sedimentary rocks only \\
\hline 236 & $6 / 6-4 J 1$ & Santa Rosa Valley & Cardwell (1958) & 38.3929 & -122.5224 & 541711 & 4249310 & 6 & 6 & 4 & $\mathrm{~J}$ & 1 & 123.8 & 57.9 & Volcanic rocks only \\
\hline 237 & 6/7-3Q1 & Santa Rosa Valley & Cardwell (1958) & 38.3872 & -122.6176 & 533400 & 4248644 & 6 & 7 & 3 & Q & 1 & 169.2 & 127.1 & Sedimentary and volcanic rocks \\
\hline 238 & 6/8-7D1 & Santa Rosa Valley & Cardwell (1958) & 38.3860 & -122.7961 & 517806 & 4248462 & 6 & 8 & 7 & D & 1 & 21.3 & 109.7 & Sedimentary rocks only \\
\hline 239 & 6/8-12J1 & Santa Rosa Valley & Cardwell (1958) & 38.3791 & -122.6894 & 527130 & 4247716 & 6 & 8 & 12 & $\mathrm{~J}$ & 1 & 39.6 & 68.0 & Sedimentary rocks only \\
\hline 240 & 6/6-8N1 & Santa Rosa Valley & Cardwell (1958) & 38.3752 & -122.5522 & 539115 & 4247329 & 6 & 6 & 8 & $\mathrm{~N}$ & 1 & 98.5 & 149.4 & Sedimentary and volcanic rocks \\
\hline 241 & 6/6-17A1 & Santa Rosa Valley & Cardwell (1958) & 38.3720 & -122.5411 & 540090 & 4246984 & 6 & 6 & 17 & A & 1 & 110.6 & 45.7 & Sedimentary rocks only \\
\hline 242 & 6/8-17K1 & Santa Rosa Valley & Cardwell (1958) & 38.3621 & -122.7696 & 520131 & 4245814 & 6 & 8 & 17 & $\mathrm{~K}$ & 1 & 42.7 & 64.0 & Sedimentary rocks only \\
\hline 243 & 6/8-16R1A & Santa Rosa Valley & Cardwell (1958) & 38.3604 & -122.7436 & 522404 & 4245623 & 6 & 8 & 16 & $\mathrm{R}$ & 1 & 27.4 & 367.0 & Sedimentary and volcanic rocks \\
\hline 244 & 6/9-22A1 & Santa Rosa Valley & Cardwell (1958) & 38.3553 & -122.8349 & 514422 & 4245045 & 6 & 9 & 22 & A & 1 & 36.6 & 109.7 & Sedimentary rocks only \\
\hline 245 & 6/7-19D1 & Santa Rosa Valley & Cardwell (1958) & 38.3550 & -122.6833 & 527669 & 4245043 & 6 & 7 & 19 & D & 1 & 35.0 & 32.9 & Sedimentary rocks only \\
\hline 246 & $6 / 8-23 \mathrm{C} 1$ & Santa Rosa Valley & Cardwell (1958) & 38.3546 & -122.7144 & 524952 & 4244996 & 6 & 8 & 23 & c & 1 & 29.0 & 133.5 & Sedimentary rocks only \\
\hline 247 & $6 / 8-22 N 1$ & Santa Rosa Valley & Cardwell (1958) & 38.3448 & -122.7389 & 522815 & 4243898 & 6 & 8 & 22 & $\mathrm{~N}$ & 1 & 36.6 & 61.0 & Sedimentary rocks only \\
\hline 248 & 6/7-29P1 & Santa Rosa Valley & Cardwell (1958) & 38.3290 & -122.6595 & 529759 & 4242171 & 6 & 7 & 29 & $\mathrm{P}$ & 1 & 62.0 & 56.4 & Sedimentary and volcanic rocks \\
\hline 249 & $6 / 8-35 A 2$ & Santa Rosa Valley & Cardwell (1958) & 38.3276 & -122.7067 & 525637 & 4242002 & 6 & 8 & 35 & A & 2 & 33.5 & 201.2 & Sedimentary rocks only \\
\hline 250 & 6/8-36A1 & Santa Rosa Valley & Cardwell (1958) & 38.3253 & -122.6885 & 527231 & 4241744 & 6 & 8 & 36 & A & 1 & 35.1 & 211.8 & Sedimentary rocks only \\
\hline 251 & $6 / 7-31 \mathrm{H} 1$ & Santa Rosa Valley & Cardwell (1958) & 38.3249 & -122.6668 & 529124 & 4241707 & 6 & 7 & 31 & $\mathrm{H}$ & 1 & 48.8 & 90.8 & Sedimentary rocks only \\
\hline 252 & $6 / 7-31 \mathrm{G} 1$ & Santa Rosa Valley & Cardwell (1958) & 38.3235 & -122.6761 & 528312 & 4241549 & 6 & 7 & 31 & G & 1 & 41.1 & 154.8 & Sedimentary and volcanic rocks \\
\hline 253 & 6/8-33E1 & Santa Rosa Valley & Cardwell (1958) & 38.3210 & -122.7556 & 521365 & 4241252 & 6 & 8 & 33 & $\mathrm{E}$ & 1 & 59.4 & 65.5 & Sedimentary rocks only \\
\hline 254 & 6/7-31L1 & Santa Rosa Valley & Cardwell (1958) & 38.3195 & -122.6763 & 528294 & 4241104 & 6 & 7 & 31 & L & 1 & 39.0 & 24.1 & Sedimentary rocks only \\
\hline 255 & 6/7-32M1 & Santa Rosa Valley & Cardwell (1958) & 38.3193 & -122.6652 & 529264 & 4241094 & 6 & 7 & 32 & M & 1 & 56.4 & 354.5 & Sedimentary and volcanic rocks \\
\hline 256 & $6 / 7-31 \mathrm{J1}$ & Santa Rosa Valley & Cardwell (1958) & 38.3175 & -122.6713 & 528732 & 4240891 & 6 & 7 & 31 & $\mathrm{~J}$ & 1 & 41.1 & 85.3 & Sedimentary rocks only \\
\hline 257 & 5/7-6E1 & Petaluma Valley & Cardwell (1958) & 38.3108 & -122.6823 & 527774 & 4240140 & 5 & 7 & 6 & $\mathrm{E}$ & 1 & 33.4 & 35.1 & Sedimentary rocks only \\
\hline
\end{tabular}


Appendix 1. Well Location Data. _Continued

[Site IDs corrspond to locations shown on Plate 1; Well number and area correspond to descriptions in the Water-Supply Paper listed; latitude and longitude geographic coordinates have a horizontal datum of North American Datum 1927; Easting and Northing coordinates are in Universa Transverse Mercator projection zone 10, North American Datum 1927; system of quarter-quarter section designation is explained in the Water-Supply Papers; leaders (--), well number not assigned]

\begin{tabular}{|c|c|c|c|c|c|c|c|c|c|c|c|c|c|c|c|}
\hline Site ID & Well number & Area & Water-Supply Paper & Latitude & Longitude & Easting & Northing & $\begin{array}{l}\text { Township } \\
\text { (N) }\end{array}$ & $\begin{array}{l}\text { Range } \\
\text { (W) }\end{array}$ & Section & $\begin{array}{c}\text { Quarter- } \\
\text { quarter } \\
\text { section } \\
\text { designation }\end{array}$ & $\begin{array}{c}\text { Well } \\
\text { number } \\
\text { within } \\
\text { quarter- } \\
\text { quarter } \\
\text { section }\end{array}$ & $\begin{array}{l}\text { Land } \\
\text { surface } \\
\text { elevation, in } \\
\text { meters }\end{array}$ & $\begin{array}{l}\text { Total } \\
\text { depth of } \\
\text { well, in } \\
\text { meters }\end{array}$ & Geologic units intersected \\
\hline 258 & $5 / 8-1 \mathrm{H} 1$ & Petaluma Valley & Cardwell (1958) & 38.3101 & -122.6899 & 527115 & 4240058 & 5 & 8 & 1 & $\mathrm{H}$ & 1 & 37.0 & 70.7 & Sedimentary rocks only \\
\hline 259 & $5 / 7-5 K 1$ & Petaluma Valley & Cardwell (1958) & 38.3072 & -122.6532 & 530323 & 4239747 & 5 & 7 & 5 & $\mathrm{k}$ & 1 & 12.1 & 103.3 & Sedimentary rocks only \\
\hline 260 & $5 / 8-1 \mathrm{J1}$ & Petaluma Valley & Cardwell (1958) & 38.3071 & -122.6891 & 527186 & 4239729 & 5 & 8 & 1 & $\mathrm{~J}$ & 1 & 38.0 & 88.4 & Sedimentary rocks only \\
\hline 261 & 5/8-1L1 & Petaluma Valley & Cardwell (1958) & 38.3059 & -122.6967 & 526520 & 4239589 & 5 & 8 & 1 & $\mathrm{~L}$ & 1 & 57.2 & 77.1 & Sedimentary rocks only \\
\hline 262 & $5 / 8-13 B 1$ & Petaluma Valley & Cardwell (1958) & 38.2821 & -122.6901 & 527101 & 4236949 & 5 & 8 & 13 & B & 1 & 3.7 & 52.4 & Sedimentary rocks only \\
\hline 263 & 5/7-15K1 & Petaluma Valley & Cardwell (1958) & 38.2741 & -122.6196 & 533273 & 4236094 & 5 & 7 & 15 & $\mathrm{k}$ & 1 & 14.7 & 32.3 & Sedimentary rocks only \\
\hline 264 & $5 / 7-20 C 1$ & Petaluma Valley & Cardwell (1958) & 38.2679 & -122.6609 & 529661 & 4235384 & 5 & 7 & 20 & c & 1 & 2.7 & 209.7 & Sedimentary rocks only \\
\hline 265 & $5 / 7-19 \mathrm{~N} 1$ & Petaluma Valley & Cardwell (1958) & 38.2563 & -122.6817 & 527847 & 4234091 & 5 & 7 & 19 & $\mathrm{~N}$ & 1 & 4.0 & 54.9 & Sedimentary rocks only \\
\hline 266 & $5 / 7-25 \mathrm{~A} 1$ & Petaluma Valley & Cardwell (1958) & 38.2543 & -122.5735 & 537314 & 4233905 & 5 & 7 & 25 & A & 1 & 11.0 & 80.8 & Sedimentary rocks only \\
\hline 267 & $5 / 7-25 \mathrm{C} 1$ & Petaluma Valley & Cardwell (1958) & 38.2540 & -122.5826 & 536517 & 4233868 & 5 & 7 & 25 & c & 1 & 10.3 & 71.6 & Sedimentary rocks only \\
\hline 268 & $5 / 7-28 \mathrm{A3}$ & Petaluma Valley & Cardwell (1958) & 38.2540 & -122.6284 & 532511 & 4233851 & 5 & 7 & 28 & A & 3 & 10.7 & 85.3 & Sedimentary rocks only \\
\hline 269 & $5 / 7-28 \mathrm{H} 5$ & Petaluma Valley & Cardwell (1958) & 38.2525 & -122.6289 & 532470 & 4233694 & 5 & 7 & 28 & $\mathrm{H}$ & 5 & 3.2 & 142.6 & Sedimentary rocks only \\
\hline 270 & $5 / 7-28 \mathrm{H} 1$ & Petaluma Valley & Cardwell (1958) & 38.2512 & -122.6286 & 532498 & 4233548 & 5 & 7 & 28 & $\mathrm{H}$ & 1 & 4.6 & 147.2 & Sedimentary rocks only \\
\hline 271 & $5 / 7-26 \mathrm{R} 1$ & Petaluma Valley & Cardwell (1958) & 38.2442 & -122.5935 & 535573 & 4232787 & 5 & 7 & 26 & $\mathrm{R}$ & 1 & 4.0 & 254.5 & Sedimentary rocks and pre-Cenozoic rocks \\
\hline 272 & $5 / 7-35 \mathrm{H} 1$ & Petaluma Valley & Cardwell (1958) & 38.2354 & -122.5948 & 535460 & 4231808 & 5 & 7 & 35 & $\mathrm{H}$ & 1 & 3.0 & 165.2 & Sedimentary rocks only \\
\hline 273 & 5/7-3зм1 & Petaluma Valley & Cardwell (1958) & 38.2343 & -122.6438 & 531178 & 4231666 & 5 & 7 & 33 & M & 1 & 9.1 & 40.2 & Sedimentary rocks only \\
\hline 274 & 5/7-33Q1 & Petaluma Valley & Cardwell (1958) & 38.2301 & -122.6329 & 532134 & 4231199 & 5 & 7 & 33 & $\mathrm{Q}$ & 1 & 1.7 & 62.5 & Sedimentary rocks and pre-Cenozoic rocks \\
\hline 275 & 5/7-32R1 & Petaluma Valley & Cardwell (1958) & 38.2300 & -122.6486 & 530760 & 4231183 & 5 & 7 & 32 & $\mathrm{R}$ & 1 & 6.1 & 66.1 & Sedimentary rocks and pre-Cenozoic rocks \\
\hline 276 & 4/6-27L1 & Petaluma Valley & Cardwell (1958) & 38.1609 & -122.5132 & 542648 & 4223570 & 4 & 6 & 27 & $\mathrm{~L}$ & 1 & 1.5 & 152.4 & Sedimentary rocks only \\
\hline 277 & 6/6-10F1 & Santa Rosa Valley & Cardwell (1958) & 38.3805 & -122.5137 & 542472 & 4247939 & 6 & 6 & 10 & $\mathrm{~F}$ & 1 & 102.1 & 106.7 & Sedimentary and volcanic rocks \\
\hline 278 & 6/6-16J2 & Sonoma Valley & Kunkel and Upson (1960) & 38.3653 & -122.5236 & 541623 & 4246246 & 6 & 6 & 16 & $\mathrm{~J}$ & 2 & 79.2 & 85.3 & Sedimentary rocks only \\
\hline 279 & 6/6-22R3 & Sonoma Valley & Kunkel and Upson (1960) & 38.3463 & -122.5032 & 543412 & 4244152 & 6 & 6 & 22 & $\mathrm{R}$ & 3 & 57.9 & 127.4 & Sedimentary and volcanic rocks \\
\hline 280 & 6/6-26E1 & Sonoma Valley & Kunkel and Upson (1960) & 38.3384 & -122.4995 & 543740 & 4243271 & 6 & 6 & 26 & $\mathrm{E}$ & 1 & 54.9 & 92.7 & Sedimentary and volcanic rocks \\
\hline 281 & $6 / 6-34 \mathrm{C} 1$ & Sonoma Valley & Kunkel and Upson (1960) & 38.3284 & -122.5099 & 542835 & 4242162 & 6 & 6 & 34 & c & 1 & 61.0 & 152.4 & Sedimentary rocks only \\
\hline 282 & 5/5-8B1 & Sonoma Valley & Kunkel and Upson (1960) & 38.2989 & -122.4330 & 549579 & 4238920 & 5 & 5 & 8 & B & 1 & 44.2 & 24.4 & Sedimentary rocks only \\
\hline 283 & $5 / 5-8 \mathrm{~A} 1$ & Sonoma Valley & Kunkel and Upson (1960) & 38.2986 & -122.4308 & 549771 & 4238894 & 5 & 5 & 8 & A & 1 & 53.2 & 53.3 & Sedimentary and volcanic rocks \\
\hline 284 & 5/5-7A1 & Sonoma Valley & Kunkel and Upson (1960) & 38.2979 & -122.4477 & 548297 & 4238811 & 5 & 5 & 7 & A & 1 & 39.6 & 175.3 & Sedimentary and volcanic rocks \\
\hline 285 & $5 / 5-8 \mathrm{E} 1$ & Sonoma Valley & Kunkel and Upson (1960) & 38.2961 & -122.4459 & 548458 & 4238603 & 5 & 5 & 8 & $\mathrm{E}$ & 1 & 34.1 & 71.6 & Sedimentary and volcanic rocks \\
\hline 286 & 5/5-9M1 & Sonoma Valley & Kunkel and Upson (1960) & 38.2926 & -122.4253 & 550260 & 4238231 & 5 & 5 & 9 & M & 1 & 44.8 & 65.5 & Sedimentary and volcanic rocks \\
\hline 287 & $5 / 6-10 K 1$ & Sonoma Valley & Kunkel and Upson (1960) & 38.2918 & -122.5060 & 543203 & 4238100 & 5 & 6 & 10 & $\mathrm{k}$ & 1 & 61.0 & 72.8 & Sedimentary rocks only \\
\hline 288 & 5/5-9N2 & Sonoma Valley & Kunkel and Upson (1960) & 38.2863 & -122.4253 & 550263 & 4237534 & 5 & 5 & 9 & $\mathrm{~N}$ & 2 & 30.8 & 86.9 & Sedimentary rocks only \\
\hline 289 & $5 / 5-8 \mathrm{P} 1$ & Sonoma Valley & Kunkel and Upson (1960) & 38.2862 & -122.4405 & 548934 & 4237511 & 5 & 5 & 8 & $\mathrm{P}$ & 1 & 27.7 & 128.3 & Sedimentary and volcanic rocks \\
\hline 290 & 5/5-17B1 & Sonoma Valley & Kunkel and Upson (1960) & 38.2853 & -122.4334 & 549554 & 4237414 & 5 & 5 & 17 & B & 1 & 28.0 & 219.5 & Sedimentary rocks only \\
\hline 291 & $5 / 5-17 \mathrm{C} 4$ & Sonoma Valley & Kunkel and Upson (1960) & 38.2852 & -122.4380 & 549150 & 4237397 & 5 & 5 & 17 & c & 4 & 28.3 & 80.8 & Sedimentary rocks only \\
\hline 292 & $5 / 6-14 B 2$ & Sonoma Valley & Kunkel and Upson (1960) & 38.2840 & -122.4889 & 544703 & 4237241 & 5 & 6 & 14 & B & 2 & 29.0 & 61.0 & Sedimentary rocks only \\
\hline 293 & 5/5-16E1 & Sonoma Valley & Kunkel and Upson (1960) & 38.2812 & -122.4255 & 550244 & 4236960 & 5 & 5 & 16 & $\mathrm{E}$ & 1 & 24.1 & 119.8 & Sedimentary rocks only \\
\hline 294 & $5 / 6-13 F 1$ & Sonoma Valley & Kunkel and Upson (1960) & 38.2794 & -122.4752 & 545904 & 4236744 & 5 & 6 & 13 & $\mathrm{~F}$ & 1 & 18.9 & 33.5 & Sedimentary rocks only \\
\hline 295 & $5 / 6-13 F 2$ & Sonoma Valley & Kunkel and Upson (1960) & 38.2787 & -122.4768 & 545766 & 4236666 & 5 & 6 & 13 & $\mathrm{~F}$ & 2 & 20.1 & 32.0 & Sedimentary rocks only \\
\hline 296 & 5/5-17J1 & Sonoma Valley & Kunkel and Upson (1960) & 38.2778 & -122.4290 & 549948 & 4236582 & 5 & 5 & 17 & $\mathrm{~J}$ & 1 & 21.3 & 86.9 & Sedimentary rocks only \\
\hline 297 & $5 / 5-18 \mathrm{~K} 1$ & Sonoma Valley & Kunkel and Upson (1960) & 38.2751 & -122.4554 & 547640 & 4236274 & 5 & 5 & 18 & $\mathrm{k}$ & 1 & 14.9 & 39.0 & Sedimentary rocks only \\
\hline 298 & $5 / 5-17 \mathrm{~N} 1$ & Sonoma Valley & Kunkel and Upson (1960) & 38.2723 & -122.4427 & 548750 & 4235969 & 5 & 5 & 17 & $\mathrm{~N}$ & 1 & 13.7 & 144.8 & Sedimentary and volcanic rocks \\
\hline 299 & 5/5-17R2 & Sonoma Valley & Kunkel and Upson (1960) & 38.2721 & -122.4288 & 549968 & 4235955 & 5 & 5 & 17 & $\mathrm{R}$ & 2 & 17.7 & 109.4 & Sedimentary rocks only \\
\hline 300 & 5/5-21E1 & Sonoma Valley & Kunkel and Upson (1960) & 38.2656 & -122.4265 & 550173 & 4235230 & 5 & 5 & 21 & $\mathrm{E}$ & 1 & 12.2 & 76.2 & Sedimentary rocks only \\
\hline 301 & 5/6-24K1 & Sonoma Valley & Kunkel and Upson (1960) & 38.2635 & -122.4725 & 546148 & 4234972 & 5 & 6 & 24 & $\mathrm{~K}$ & 1 & 12.5 & 46.0 & Sedimentary rocks only \\
\hline
\end{tabular}


Appendix 1. Well Location Data. _Continued

[Site IDs corrspond to locations shown on Plate 1; Well number and area correspond to descriptions in the Water-Supply Paper listed; latitude and longitude geographic coordinates have a horizontal datum of North American Datum 1927; Easting and Northing coordinates are in Universa Transverse Mercator projection zone 10, North American Datum 1927; system of quarter-quarter section designation is explained in the Water-Supply Papers; leaders (--), well number not assigned]

\begin{tabular}{|c|c|c|c|c|c|c|c|c|c|c|c|c|c|c|c|}
\hline Site ID & Well number & Area & Water-Supply Paper & Latitude & Longitude & Easting & Northing & $\begin{array}{l}\text { Township } \\
\text { (N) }\end{array}$ & $\begin{array}{c}\text { Range } \\
\text { (W) }\end{array}$ & Section & $\begin{array}{c}\text { Quarter- } \\
\text { quarter } \\
\text { section } \\
\text { designation }\end{array}$ & $\begin{array}{c}\text { Well } \\
\text { number } \\
\text { within } \\
\text { quarter- } \\
\text { quarter } \\
\text { section }\end{array}$ & $\begin{array}{l}\text { Land } \\
\text { surface } \\
\text { elevation, in } \\
\text { meters }\end{array}$ & $\begin{array}{l}\text { Total } \\
\text { depth of } \\
\text { well, in } \\
\text { meters }\end{array}$ & Geologic units intersected \\
\hline 302 & 5/5-22L1 & Sonoma Valley & Kunkel and Upson (1960) & 38.2631 & -122.4040 & 552137 & 4234969 & 5 & 5 & 22 & $\mathrm{~L}$ & 1 & 48.8 & 170.7 & Sedimentary and volcanic rocks \\
\hline 303 & 5/5-19L5 & Sonoma Valley & Kunkel and Upson (1960) & 38.2629 & -122.4564 & 547556 & 4234921 & 5 & 5 & 19 & $\mathrm{~L}$ & 5 & 12.2 & 19.8 & Sedimentary rocks only \\
\hline 304 & $5 / 5-22\llcorner 2$ & Sonoma Valley & Kunkel and Upson (1960) & 38.2625 & -122.4014 & 552373 & 4234899 & 5 & 5 & 22 & $\mathrm{~L}$ & 2 & 55.5 & 64.6 & Sedimentary and volcanic rocks \\
\hline 305 & 5/5-19L1 & Sonoma Valley & Kunkel and Upson (1960) & 38.2607 & -122.4571 & 547498 & 4234678 & 5 & 5 & 19 & $\mathrm{~L}$ & 1 & 12.2 & 45.7 & Sedimentary rocks only \\
\hline 306 & $5 / 5-22 Q 1$ & Sonoma Valley & Kunkel and Upson (1960) & 38.2598 & -122.3992 & 552564 & 4234604 & 5 & 5 & 22 & $\mathrm{Q}$ & 1 & 54.9 & 165.2 & Sedimentary and volcanic rocks \\
\hline 307 & 5/5-20R1 & Sonoma Valley & Kunkel and Upson (1960) & 38.2599 & -122.4286 & 549987 & 4234601 & 5 & 5 & 20 & $\mathrm{R}$ & 1 & 11.3 & 153.6 & Sedimentary rocks only \\
\hline 308 & $5 / 5-21 R 1$ & Sonoma Valley & Kunkel and Upson (1960) & 38.2584 & -122.4142 & 551251 & 4234439 & 5 & 5 & 21 & $\mathrm{R}$ & 1 & 24.4 & 73.8 & Sedimentary rocks only \\
\hline 309 & 5/5-30E1 & Sonoma Valley & Kunkel and Upson (1960) & 38.2550 & -122.4606 & 547195 & 4234043 & 5 & 5 & 30 & $\mathrm{E}$ & 1 & 7.6 & 230.1 & Sedimentary and volcanic rocks \\
\hline 310 & $5 / 5-27 \mathrm{H} 1$ & Sonoma Valley & Kunkel and Upson (1960) & 38.2534 & -122.3936 & 553057 & 4233898 & 5 & 5 & 27 & $\mathrm{H}$ & 1 & 53.3 & 228.6 & Sedimentary and volcanic rocks \\
\hline 311 & 6/6-9R1 & Santa Rosa Valley & Cardwell (1958) & 38.2476 & -122.4160 & 551100 & 4233237 & 6 & 6 & 9 & $\mathrm{R}$ & 1 & 96.4 & 58.8 & Sedimentary and volcanic rocks \\
\hline 312 & 6/6-16B1 & Santa Rosa Valley & Cardwell (1958) & 38.2464 & -122.4177 & 550952 & 4233110 & 6 & 6 & 16 & B & 1 & 147.0 & 129.8 & Sedimentary rocks only \\
\hline 313 & $5 / 6-25 P 1$ & Sonoma Valley & Kunkel and Upson (1960) & 38.2436 & -122.4750 & 545940 & 4232771 & 5 & 6 & 25 & $\mathrm{P}$ & 1 & 11.0 & 52.1 & Sedimentary rocks only \\
\hline 314 & 5/5-31A1 & Sonoma Valley & Kunkel and Upson (1960) & 38.2403 & -122.4470 & 548398 & 4232410 & 5 & 5 & 31 & A & 1 & 3.0 & 124.4 & Sedimentary rocks only \\
\hline 315 & $5 / 5-31 \mathrm{H} 1$ & Sonoma Valley & Kunkel and Upson (1960) & 38.2397 & -122.4482 & 548287 & 4232353 & 5 & 5 & 31 & $\mathrm{H}$ & 1 & 2.1 & 61.9 & Sedimentary rocks only \\
\hline 316 & $4 / 5-3 \mathrm{C} 1$ & Sonoma Valley & Kunkel and Upson (1960) & 38.2294 & -122.4044 & 552128 & 4231228 & 4 & 5 & 3 & c & 1 & 9.1 & 79.6 & Sedimentary rocks only \\
\hline 317 & $4 / 5-14 \mathrm{D} 2$ & Sonoma Valley & Kunkel and Upson (1960) & 38.1974 & -122.3908 & 553348 & 4227691 & 4 & 5 & 14 & D & 2 & 0.0 & 493.8 & Sedimentary rocks only \\
\hline 318 & $4 / 5-14 \mathrm{D} 1$ & Sonoma Valley & Kunkel and Upson (1960) & 38.1974 & -122.3908 & 553348 & 4227688 & 4 & 5 & 14 & D & 1 & 0.0 & 164.6 & Sedimentary rocks only \\
\hline 319 & 4/5-14L1 & Sonoma Valley & Kunkel and Upson (1960) & 38.1908 & -122.3854 & 553820 & 4226960 & 4 & 5 & 14 & $\mathrm{~L}$ & 1 & 0.0 & 201.2 & Sedimentary rocks only \\
\hline 320 & $9 / 7-26 \mathrm{C} 1$ & Napa Valley & Kunkel and Upson (1960) & 38.6050 & -122.6070 & 534223 & 4272813 & 9 & 7 & 26 & c & 1 & 134.1 & 137.2 & Sedimentary and volcanic rocks \\
\hline 321 & 9/7-26G1 & Napa Valley & Kunkel and Upson (1960) & 38.6014 & -122.6001 & 534821 & 4272411 & 9 & 7 & 26 & G & 1 & 129.5 & 93.0 & Sedimentary and volcanic rocks \\
\hline 322 & 8/6-14R1 & Napa Valley & Kunkel and Upson (1960) & 38.5383 & -122.4862 & 544780 & 4265466 & 8 & 6 & 14 & $\mathrm{R}$ & 1 & 88.4 & 40.2 & Sedimentary and volcanic rocks \\
\hline 323 & 8/6-23M1 & Napa Valley & Kunkel and Upson (1960) & 38.5290 & -122.5005 & 543536 & 4264421 & 8 & 6 & 23 & M & 1 & 86.9 & 39.3 & Sedimentary rocks only \\
\hline 324 & 7/5-15A1 & Napa Valley & Kunkel and Upson (1960) & 38.4621 & -122.3960 & 552695 & 4257049 & 7 & 5 & 15 & A & 1 & 43.6 & 108.2 & Sedimentary rocks only \\
\hline 325 & $7 / 5-14 \mathrm{G} 1$ & Napa Valley & Kunkel and Upson (1960) & 38.4615 & -122.3810 & 554009 & 4256996 & 7 & 5 & 14 & G & 1 & 42.4 & 80.8 & Sedimentary and volcanic rocks \\
\hline 326 & 7/5-16ட1 & Napa Valley & Kunkel and Upson (1960) & 38.4559 & -122.4214 & 550481 & 4256356 & 7 & 5 & 16 & L & 1 & 52.1 & 67.4 & Sedimentary rocks only \\
\hline 327 & 7/5-17J1 & Napa Valley & Kunkel and Upson (1960) & 38.4549 & -122.4338 & 549402 & 4256229 & 7 & 5 & 17 & $\mathrm{~J}$ & 1 & 61.0 & 171.9 & Sedimentary and volcanic rocks \\
\hline 328 & $7 / 5-16 \mathrm{~N} 1$ & Napa Valley & Kunkel and Upson (1960) & 38.4540 & -122.4247 & 550197 & 4256135 & 7 & 5 & 16 & $\mathrm{~N}$ & 1 & 52.7 & 76.8 & Sedimentary rocks only \\
\hline 329 & 7/5-23ட1 & Napa Valley & Kunkel and Upson (1960) & 38.4436 & -122.3824 & 553894 & 4255010 & 7 & 5 & 23 & L & 1 & 36.6 & 45.7 & Sedimentary rocks only \\
\hline 330 & $7 / 5-27 \mathrm{D} 1$ & Napa Valley & Kunkel and Upson (1960) & 38.4340 & -122.4057 & 551867 & 4253931 & 7 & 5 & 27 & D & 1 & 51.8 & 125.0 & Sedimentary and volcanic rocks \\
\hline 331 & 7/4-30L1 & Napa Valley & Kunkel and Upson (1960) & 38.4258 & -122.3456 & 557123 & 4253055 & 7 & 4 & 30 & L & 1 & 34.1 & 52.1 & Sedimentary and volcanic rocks \\
\hline 332 & 7/4-31E1 & Napa Valley & Kunkel and Upson (1960) & 38.4170 & -122.3530 & 556481 & 4252070 & 7 & 4 & 31 & $\mathrm{E}$ & 1 & 27.4 & 82.9 & Sedimentary rocks only \\
\hline 333 & $7 / 5-36 \mathrm{~N} 1$ & Napa Valley & Kunkel and Upson (1960) & 38.4075 & -122.3719 & 554843 & 4251014 & 7 & 5 & 36 & $\mathrm{~N}$ & 1 & 43.0 & 31.7 & Sedimentary and volcanic rocks \\
\hline 334 & 6/5-1C3 & Napa Valley & Kunkel and Upson (1960) & 38.4060 & -122.3646 & 555477 & 4250851 & 6 & 5 & 1 & c & 3 & 35.1 & 38.1 & Sedimentary rocks only \\
\hline 335 & 6/4-5A1 & Napa Valley & Kunkel and Upson (1960) & 38.4043 & -122.3213 & 559259 & 4250683 & 6 & 4 & 5 & A & 1 & 32.6 & 97.2 & Sedimentary rocks and pre-Cenozoic rocks \\
\hline 336 & 6/4-5B1 & Napa Valley & Kunkel and Upson (1960) & 38.4028 & -122.3242 & 559005 & 4250514 & 6 & 4 & 5 & B & 1 & 32.6 & 17.7 & Sedimentary rocks and pre-Cenozoic rocks \\
\hline 337 & $6 / 4-5 B 2$ & Napa Valley & Kunkel and Upson (1960) & 38.4028 & -122.3243 & 559002 & 4250514 & 6 & 4 & 5 & B & 2 & 32.6 & 8.7 & Sedimentary rocks and pre-Cenozoic rocks \\
\hline 338 & 6/4-4E1 & Napa Valley & Kunkel and Upson (1960) & 38.3976 & -122.3154 & 559778 & 4249947 & 6 & 4 & 4 & $\mathrm{E}$ & 1 & 33.5 & 182.9 & Sedimentary and volcanic rocks \\
\hline 339 & $6 / 4-5 J 1$ & Napa Valley & Kunkel and Upson (1960) & 38.3957 & -122.3208 & 559311 & 4249734 & 6 & 4 & 5 & $\mathrm{~J}$ & 1 & 32.6 & 89.0 & Sedimentary rocks and pre-Cenozoic rocks \\
\hline 340 & 6/4-6R1 & Napa Valley & Kunkel and Upson (1960) & 38.3926 & -122.3370 & 557897 & 4249374 & 6 & 4 & 6 & $\mathrm{R}$ & 1 & 30.5 & 73.8 & Sedimentary rocks only \\
\hline 341 & $6 / 4-6 \mathrm{R} 2$ & Napa Valley & Kunkel and Upson (1960) & 38.3925 & -122.3370 & 557897 & 4249370 & 6 & 4 & 6 & $\mathrm{R}$ & 2 & 30.5 & 67.1 & Sedimentary and volcanic rocks \\
\hline 342 & 6/4-6P1 & Napa Valley & Kunkel and Upson (1960) & 38.3915 & -122.3468 & 557046 & 4249249 & 6 & 4 & 6 & $\mathrm{P}$ & 1 & 22.9 & 36.6 & Sedimentary rocks only \\
\hline 343 & $6 / 4-5 Q 1$ & Napa Valley & Kunkel and Upson (1960) & 38.3900 & -122.3228 & 559141 & 4249094 & 6 & 4 & 5 & Q & 1 & 19.8 & 50.9 & Sedimentary rocks and pre-Cenozoic rocks \\
\hline 344 & 6/4-17A1 & Napa Valley & Kunkel and Upson (1960) & 38.3729 & -122.3181 & 559562 & 4247199 & 6 & 4 & 17 & A & 1 & 20.4 & 76.2 & Sedimentary rocks only \\
\hline 345 & 6/4-18A3 & Napa Valley & Kunkel and Upson (1960) & 38.3729 & -122.3364 & 557966 & 4247189 & 6 & 4 & 18 & A & 3 & 25.0 & 222.5 & Sedimentary and volcanic rocks \\
\hline
\end{tabular}


Appendix 1. Well Location Data. _Continued

[Site IDs corrspond to locations shown on Plate 1; Well number and area correspond to descriptions in the Water-Supply Paper listed; latitude and longitude geographic coordinates have a horizontal datum of North American Datum 1927; Easting and Northing coordinates are in Universa Transverse Mercator projection zone 10, North American Datum 1927; system of quarter-quarter section designation is explained in the Water-Supply Papers; leaders (--), well number not assigned]

\begin{tabular}{|c|c|c|c|c|c|c|c|c|c|c|c|c|c|c|c|}
\hline Site ID & Well number & Area & Water-Supply Paper & Latitude & Longitude & Easting & Northing & $\begin{array}{l}\text { Township } \\
\text { (N) }\end{array}$ & $\begin{array}{l}\text { Range } \\
\text { (W) }\end{array}$ & Section & $\begin{array}{c}\text { Quarter- } \\
\text { quarter } \\
\text { section } \\
\text { designation }\end{array}$ & $\begin{array}{c}\text { Well } \\
\text { number } \\
\text { within } \\
\text { quarter- } \\
\text { quarter } \\
\text { section }\end{array}$ & $\begin{array}{l}\text { Land } \\
\text { surface } \\
\text { elevation, in } \\
\text { meters }\end{array}$ & $\begin{array}{l}\text { Total } \\
\text { depth of } \\
\text { well, in } \\
\text { meters }\end{array}$ & Geologic units intersected \\
\hline 346 & 6/4-15Q1 & Napa Valley & Kunkel and Upson (1960) & 38.3605 & -122.2863 & 562358 & 4245847 & 6 & 4 & 15 & Q & 1 & 20.4 & 92.4 & Sedimentary and volcanic rocks \\
\hline 347 & 6/4-15Q3 & Napa Valley & Kunkel and Upson (1960) & 38.3604 & -122.2900 & 562028 & 4245837 & 6 & 4 & 15 & $\mathrm{Q}$ & 3 & 19.8 & 99.7 & Sedimentary rocks only \\
\hline 348 & 6/4-22B3 & Napa Valley & Kunkel and Upson (1960) & 38.3593 & -122.2853 & 562444 & 4245713 & 6 & 4 & 22 & B & 3 & 26.2 & 72.2 & Sedimentary rocks only \\
\hline 349 & $6 / 4-22 B 2$ & Napa Valley & Kunkel and Upson (1960) & 38.3582 & -122.2862 & 562369 & 4245589 & 6 & 4 & 22 & B & 2 & 18.3 & 35.7 & Sedimentary rocks only \\
\hline 350 & 6/4-19B1 & Napa Valley & Kunkel and Upson (1960) & 38.3570 & -122.3436 & 557351 & 4245419 & 6 & 4 & 19 & B & 1 & 38.1 & 38.1 & Sedimentary and volcanic rocks \\
\hline 351 & $6 / 4-23 F 1$ & Napa Valley & Kunkel and Upson (1960) & 38.3550 & -122.2735 & 563474 & 4245248 & 6 & 4 & 23 & $\mathrm{~F}$ & 1 & 22.9 & 67.1 & Sedimentary and volcanic rocks \\
\hline 352 & 6/3-19M1 & Napa Valley & Kunkel and Upson (1960) & 38.3502 & -122.2416 & 566269 & 4244738 & 6 & 3 & 19 & M & 1 & 164.6 & 57.9 & Volcanic rocks only \\
\hline 353 & $6 / 4-23 J 1$ & Napa Valley & Kunkel and Upson (1960) & 38.3488 & -122.2635 & 564357 & 4244565 & 6 & 4 & 23 & $\mathrm{~J}$ & 1 & 26.5 & 213.4 & Sedimentary and volcanic rocks \\
\hline 354 & 6/4-21Q1 & Napa Valley & Kunkel and Upson (1960) & 38.3481 & -122.3073 & 560532 & 4244459 & 6 & 4 & 21 & $\mathrm{Q}$ & 1 & 20.1 & 33.5 & Sedimentary rocks only \\
\hline 355 & $6 / 4-27 \mathrm{C} 2$ & Napa Valley & Kunkel and Upson (1960) & 38.3441 & -122.2910 & 561957 & 4244028 & 6 & 4 & 27 & c & 2 & 12.2 & 73.2 & Sedimentary rocks only \\
\hline 356 & $6 / 4-30 C 1$ & Napa Valley & Kunkel and Upson (1960) & 38.3440 & -122.3471 & 557053 & 4243976 & 6 & 4 & 30 & c & 1 & 46.3 & 34.7 & Sedimentary rocks only \\
\hline 357 & $6 / 4-25 B 1$ & Napa Valley & Kunkel and Upson (1960) & 38.3431 & -122.2508 & 565475 & 4243948 & 6 & 4 & 25 & B & 1 & 48.8 & 39.3 & Sedimentary and volcanic rocks \\
\hline 358 & $6 / 4-25 \mathrm{~B} 2$ & Napa Valley & Kunkel and Upson (1960) & 38.3431 & -122.2541 & 565181 & 4243938 & 6 & 4 & 25 & B & 2 & 36.6 & 56.4 & Sedimentary and volcanic rocks \\
\hline 359 & 6/4-25D1 & Napa Valley & Kunkel and Upson (1960) & 38.3420 & -122.2597 & 564695 & 4243815 & 6 & 4 & 25 & D & 1 & 29.0 & 93.0 & Sedimentary and volcanic rocks \\
\hline 360 & $6 / 4-26 \mathrm{H} 1$ & Napa Valley & Kunkel and Upson (1960) & 38.3400 & -122.2634 & 564371 & 4243588 & 6 & 4 & 26 & $\mathrm{H}$ & 1 & 25.9 & 196.0 & Sedimentary and volcanic rocks \\
\hline 361 & $6 / 4-28 F 1$ & Napa Valley & Kunkel and Upson (1960) & 38.3386 & -122.3114 & 560178 & 4243405 & 6 & 4 & 28 & $\mathrm{~F}$ & 1 & 20.7 & 29.0 & Sedimentary rocks only \\
\hline 362 & $6 / 4-26 F 1$ & Napa Valley & Kunkel and Upson (1960) & 38.3373 & -122.2742 & 563435 & 4243282 & 6 & 4 & 26 & $\mathrm{~F}$ & 1 & 18.9 & 62.2 & Sedimentary and volcanic rocks \\
\hline 363 & $6 / 4-28 K 2$ & Napa Valley & Kunkel and Upson (1960) & 38.3369 & -122.3066 & 560605 & 4243212 & 6 & 4 & 28 & $\mathrm{k}$ & 2 & 18.9 & 47.2 & Sedimentary rocks only \\
\hline 364 & 6/4-26L2 & Napa Valley & Kunkel and Upson (1960) & 38.3350 & -122.2765 & 563233 & 4243026 & 6 & 4 & 26 & $\mathrm{~L}$ & 2 & 15.2 & 54.9 & Sedimentary and volcanic rocks \\
\hline 365 & 6/4-26L1 & Napa Valley & Kunkel and Upson (1960) & 38.3345 & -122.2753 & 563334 & 4242969 & 6 & 4 & 26 & $\mathrm{~L}$ & 1 & 16.2 & 75.9 & Sedimentary and volcanic rocks \\
\hline 366 & 6/4-27M1 & Napa Valley & Kunkel and Upson (1960) & 38.3338 & -122.2991 & 561260 & 4242882 & 6 & 4 & 27 & M & 1 & 16.8 & 32.9 & Sedimentary rocks only \\
\hline 367 & $6 / 4-26 \mathrm{~N} 1$ & Napa Valley & Kunkel and Upson (1960) & 38.3328 & -122.2802 & 562914 & 4242780 & 6 & 4 & 26 & $\mathrm{~N}$ & 1 & 9.8 & 45.7 & Sedimentary rocks only \\
\hline 368 & $6 / 4-27 N 1$ & Napa Valley & Kunkel and Upson (1960) & 38.3327 & -122.2987 & 561291 & 4242761 & 6 & 4 & 27 & $\mathrm{~N}$ & 1 & 15.2 & 43.0 & Sedimentary rocks only \\
\hline 369 & 6/4-28Q1 & Napa Valley & Kunkel and Upson (1960) & 38.3313 & -122.3041 & 560824 & 4242597 & 6 & 4 & 28 & Q & 1 & 17.4 & 24.7 & Sedimentary rocks only \\
\hline 370 & 6/4-34D2 & Napa Valley & Kunkel and Upson (1960) & 38.3297 & -122.2976 & 561392 & 4242419 & 6 & 4 & 34 & D & 2 & 13.1 & 115.8 & Sedimentary rocks only \\
\hline 371 & $6 / 4-36 \mathrm{C} 1$ & Napa Valley & Kunkel and Upson (1960) & 38.3292 & -122.2589 & 564779 & 4242390 & 6 & 4 & 36 & c & 1 & 16.8 & 136.2 & Sedimentary rocks only \\
\hline 372 & $6 / 4-35 \mathrm{G} 2$ & Napa Valley & Kunkel and Upson (1960) & 38.3247 & -122.2708 & 563738 & 4241892 & 6 & 4 & 35 & G & 2 & 11.3 & 78.6 & Sedimentary and volcanic rocks \\
\hline 373 & $6 / 4-35 G 3$ & Napa Valley & Kunkel and Upson (1960) & 38.3247 & -122.2709 & 563735 & 4241892 & 6 & 4 & 35 & G & 3 & 11.6 & 79.2 & Sedimentary and volcanic rocks \\
\hline 374 & $6 / 4-35 G 4$ & Napa Valley & Kunkel and Upson (1960) & 38.3246 & -122.2720 & 563634 & 4241873 & 6 & 4 & 35 & G & 4 & 8.2 & 76.2 & Sedimentary and volcanic rocks \\
\hline 375 & 6/4-33ட2 & Napa Valley & Kunkel and Upson (1960) & 38.3229 & -122.3120 & 560143 & 4241659 & 6 & 4 & 33 & $\mathrm{~L}$ & 2 & 22.9 & 61.6 & Sedimentary rocks only \\
\hline 376 & 6/4-35L2 & Napa Valley & Kunkel and Upson (1960) & 38.3225 & -122.2732 & 563534 & 4241644 & 6 & 4 & 35 & L & 2 & 7.6 & 79.2 & Sedimentary and volcanic rocks \\
\hline 377 & 6/4-36J1 & Napa Valley & Kunkel and Upson (1960) & 38.3218 & -122.2487 & 565672 & 4241582 & 6 & 4 & 36 & $\mathrm{~J}$ & 1 & 39.6 & 131.7 & Sedimentary rocks only \\
\hline 378 & 6/4-36L1 & Napa Valley & Kunkel and Upson (1960) & 38.3204 & -122.2564 & 565003 & 4241423 & 6 & 4 & 36 & $\mathrm{~L}$ & 1 & 18.3 & 74.1 & Sedimentary and volcanic rocks \\
\hline 379 & 6/4-32J1 & Napa Valley & Kunkel and Upson (1960) & 38.3208 & -122.3209 & 559369 & 4241420 & 6 & 4 & 32 & $\mathrm{~J}$ & 1 & 29.6 & 144.8 & Sedimentary rocks only \\
\hline 380 & 6/4-33M1 & Napa Valley & Kunkel and Upson (1960) & 38.3193 & -122.3142 & 559949 & 4241256 & 6 & 4 & 33 & M & 1 & 25.9 & 53.6 & Sedimentary rocks only \\
\hline 381 & $6 / 4-35 P 2$ & Napa Valley & Kunkel and Upson (1960) & 38.3182 & -122.2764 & 563257 & 4241159 & 6 & 4 & 35 & $\mathrm{P}$ & 2 & 6.7 & 32.0 & Sedimentary and volcanic rocks \\
\hline 382 & 6/4-32N1 & Napa Valley & Kunkel and Upson (1960) & 38.3180 & -122.3323 & 558370 & 4241107 & 6 & 4 & 32 & $\mathrm{~N}$ & 1 & 42.7 & 158.2 & Sedimentary rocks only \\
\hline 383 & $6 / 4-35 \mathrm{Q} 1$ & Napa Valley & Kunkel and Upson (1960) & 38.3164 & -122.2702 & 563799 & 4240966 & 6 & 4 & 35 & $\mathrm{Q}$ & 1 & 42.7 & 58.5 & Volcanic rocks only \\
\hline 384 & $6 / 4-35 R 1$ & Napa Valley & Kunkel and Upson (1960) & 38.3161 & -122.2677 & 564019 & 4240930 & 6 & 4 & 35 & $\mathrm{R}$ & 1 & 70.1 & 116.4 & Sedimentary and volcanic rocks \\
\hline 385 & $5 / 4-2 B 1$ & Napa Valley & Kunkel and Upson (1960) & 38.3139 & -122.2725 & 563599 & 4240689 & 5 & 4 & 2 & B & 1 & 21.3 & 61.6 & Sedimentary and volcanic rocks \\
\hline 386 & $5 / 4-1 \mathrm{G} 1$ & Napa Valley & Kunkel and Upson (1960) & 38.3103 & -122.2553 & 565105 & 4240294 & 5 & 4 & 1 & G & 1 & 43.3 & 23.8 & Sedimentary and volcanic rocks \\
\hline 387 & $5 / 4-5 \mathrm{H} 4$ & Napa Valley & Kunkel and Upson (1960) & 38.3095 & -122.3199 & 559458 & 4240171 & 5 & 4 & 5 & $\mathrm{H}$ & 4 & 28.3 & 22.9 & Sedimentary rocks only \\
\hline 388 & $5 / 3-6 \mathrm{M} 2$ & Napa Valley & Kunkel and Upson (1960) & 38.3075 & -122.2453 & 565986 & 4239998 & 5 & 3 & 6 & M & 2 & 41.1 & 19.8 & Sedimentary and volcanic rocks \\
\hline 389 & 5/3-6M1 & Napa Valley & Kunkel and Upson (1960) & 38.3069 & -122.2431 & 566181 & 4239928 & 5 & 3 & 6 & M & 1 & 38.1 & 89.9 & Sedimentary and volcanic rocks \\
\hline
\end{tabular}


Appendix 1. Well Location Data. _Continued

[Site IDs corrspond to locations shown on Plate 1; Well number and area correspond to descriptions in the Water-Supply Paper listed; latitude and longitude geographic coordinates have a horizontal datum of North American Datum 1927; Easting and Northing coordinates are in Universa Transverse Mercator projection zone 10, North American Datum 1927; system of quarter-quarter section designation is explained in the Water-Supply Papers; leaders (--), well number not assigned]

\begin{tabular}{|c|c|c|c|c|c|c|c|c|c|c|c|c|c|c|c|}
\hline Site ID & Well number & Area & Water-Supply Paper & Latitude & Longitude & Easting & Northing & $\begin{array}{l}\text { Township } \\
\text { (N) }\end{array}$ & $\begin{array}{l}\text { Range } \\
\text { (W) }\end{array}$ & Section & $\begin{array}{c}\text { Quarter- } \\
\text { quarter } \\
\text { section } \\
\text { designation }\end{array}$ & $\begin{array}{c}\text { Well } \\
\text { number } \\
\text { within } \\
\text { quarter- } \\
\text { quarter } \\
\text { section }\end{array}$ & $\begin{array}{l}\text { Land } \\
\text { surface } \\
\text { elevation, in } \\
\text { meters }\end{array}$ & $\begin{array}{l}\text { Total } \\
\text { depth of } \\
\text { well, in } \\
\text { meters }\end{array}$ & Geologic units intersected \\
\hline 390 & 5/4-3M1 & Napa Valley & Kunkel and Upson (1960) & 38.3062 & -122.2988 & 561306 & 4239817 & 5 & 4 & 3 & M & 1 & 6.7 & 263.7 & Sedimentary rocks only \\
\hline 391 & 5/3-5M2 & Napa Valley & Kunkel and Upson (1960) & 38.3057 & -122.2288 & 567427 & 4239805 & 5 & 3 & 5 & M & 2 & 73.2 & 41.1 & Sedimentary and volcanic rocks \\
\hline 392 & 5/3-6J1 & Napa Valley & Kunkel and Upson (1960) & 38.3053 & -122.2316 & 567186 & 4239761 & 5 & 3 & 6 & $\mathrm{~J}$ & 1 & 61.0 & 88.4 & Sedimentary and volcanic rocks \\
\hline 393 & $5 / 4-4 K 2$ & Napa Valley & Kunkel and Upson (1960) & 38.3049 & -122.3086 & 560452 & 4239663 & 5 & 4 & 4 & $\mathrm{k}$ & 2 & 19.5 & 30.5 & Sedimentary rocks only \\
\hline 394 & $5 / 3-5 N 3$ & Napa Valley & Kunkel and Upson (1960) & 38.3040 & -122.2252 & 567749 & 4239628 & 5 & 3 & 5 & $\mathrm{~N}$ & 3 & 137.2 & 58.5 & Sedimentary and volcanic rocks \\
\hline 395 & $5 / 3-5 \mathrm{P} 1$ & Napa Valley & Kunkel and Upson (1960) & 38.3040 & -122.2232 & 567922 & 4239624 & 5 & 3 & 5 & $\mathrm{P}$ & 1 & 152.4 & 137.2 & Sedimentary and volcanic rocks \\
\hline 396 & $5 / 4-5 R 1$ & Napa Valley & Kunkel and Upson (1960) & 38.3045 & -122.3197 & 559481 & 4239608 & 5 & 4 & 5 & $\mathrm{R}$ & 1 & 29.6 & 21.3 & Sedimentary and volcanic rocks \\
\hline 397 & $5 / 3-6 N 2$ & Napa Valley & Kunkel and Upson (1960) & 38.3039 & -122.2430 & 566188 & 4239598 & 5 & 3 & 6 & $\mathrm{~N}$ & 2 & 47.2 & 62.5 & Sedimentary and volcanic rocks \\
\hline 398 & $5 / 4-5 Q 2$ & Napa Valley & Kunkel and Upson (1960) & 38.3043 & -122.3220 & 559285 & 4239584 & 5 & 4 & 5 & Q & 2 & 35.7 & 45.7 & Sedimentary rocks only \\
\hline 399 & $5 / 4-1 R 1$ & Napa Valley & Kunkel and Upson (1960) & 38.3037 & -122.2501 & 565565 & 4239576 & 5 & 4 & 1 & $\mathrm{R}$ & 1 & 39.6 & 112.5 & Sedimentary and volcanic rocks \\
\hline 400 & $5 / 3-6 Q 1$ & Napa Valley & Kunkel and Upson (1960) & 38.3031 & -122.2360 & 566804 & 4239513 & 5 & 3 & 6 & Q & 1 & 49.7 & 69.5 & Sedimentary and volcanic rocks \\
\hline 401 & $5 / 3-5 \mathrm{~N} 1$ & Napa Valley & Kunkel and Upson (1960) & 38.3026 & -122.2265 & 567635 & 4239462 & 5 & 3 & 5 & $\mathrm{~N}$ & 1 & 73.2 & 41.1 & Sedimentary and volcanic rocks \\
\hline 402 & $5 / 3-6 \mathrm{~N} 1$ & Napa Valley & Kunkel and Upson (1960) & 38.3018 & -122.2467 & 565865 & 4239358 & 5 & 3 & 6 & $\mathrm{~N}$ & 1 & 36.6 & 36.6 & Sedimentary and volcanic rocks \\
\hline 403 & $5 / 3-6 N 3$ & Napa Valley & Kunkel and Upson (1960) & 38.3014 & -122.2446 & 566053 & 4239322 & 5 & 3 & 6 & $\mathrm{~N}$ & 3 & 36.6 & 44.5 & Sedimentary and volcanic rocks \\
\hline 404 & $5 / 4-3 Q 1$ & Napa Valley & Kunkel and Upson (1960) & 38.3013 & -122.2901 & 562075 & 4239282 & 5 & 4 & 3 & Q & 1 & 6.7 & 73.5 & Sedimentary rocks only \\
\hline 405 & $5 / 4-12 B 1$ & Napa Valley & Kunkel and Upson (1960) & 38.2996 & -122.2551 & 565134 & 4239109 & 5 & 4 & 12 & B & 1 & 36.6 & 45.7 & Sedimentary and volcanic rocks \\
\hline 406 & $5 / 3-8 C 1$ & Napa Valley & Kunkel and Upson (1960) & 38.2991 & -122.2222 & 568014 & 4239086 & 5 & 3 & 8 & c & 1 & 88.4 & 29.0 & Sedimentary and volcanic rocks \\
\hline 407 & $5 / 3-7 D 1$ & Napa Valley & Kunkel and Upson (1960) & 38.2987 & -122.2454 & 565982 & 4239015 & 5 & 3 & 7 & D & 1 & 38.1 & 44.2 & Sedimentary and volcanic rocks \\
\hline 408 & $5 / 4-9 D 1$ & Napa Valley & Kunkel and Upson (1960) & 38.2987 & -122.3162 & 559794 & 4238971 & 5 & 4 & 9 & D & 1 & 36.6 & 41.1 & Sedimentary and volcanic rocks \\
\hline 409 & 5/4-12E1 & Napa Valley & Kunkel and Upson (1960) & 38.2974 & -122.2631 & 564435 & 4238866 & 5 & 4 & 12 & $\mathrm{E}$ & 1 & 36.6 & 77.7 & Sedimentary and volcanic rocks \\
\hline 410 & $5 / 3-7$ E3 & Napa Valley & Kunkel and Upson (1960) & 38.2971 & -122.2466 & 565882 & 4238837 & 5 & 3 & 7 & $\mathrm{E}$ & 3 & 36.0 & 145.7 & Sedimentary and volcanic rocks \\
\hline 411 & 5/4-11E1 & Napa Valley & Kunkel and Upson (1960) & 38.2971 & -122.2799 & 562965 & 4238821 & 5 & 4 & 11 & $\mathrm{E}$ & 1 & 7.6 & 213.4 & Sedimentary rocks only \\
\hline 412 & $5 / 4-9 E 1$ & Napa Valley & Kunkel and Upson (1960) & 38.2966 & -122.3162 & 559797 & 4238734 & 5 & 4 & 9 & $\mathrm{E}$ & 1 & 48.8 & 115.8 & Sedimentary and volcanic rocks \\
\hline 413 & $5 / 4-12 J 1$ & Napa Valley & Kunkel and Upson (1960) & 38.2939 & -122.2500 & 565588 & 4238489 & 5 & 4 & 12 & $\mathrm{~J}$ & 1 & 23.8 & 92.4 & Sedimentary and volcanic rocks \\
\hline 414 & $5 / 4-9 \mathrm{~K} 2$ & Napa Valley & Kunkel and Upson (1960) & 38.2937 & -122.3071 & 560596 & 4238422 & 5 & 4 & 9 & $\mathrm{~K}$ & 2 & 12.8 & 32.9 & Sedimentary rocks only \\
\hline 415 & $5 / 4-11\llcorner 3$ & Napa Valley & Kunkel and Upson (1960) & 38.2935 & -122.2734 & 563536 & 4238421 & 5 & 4 & 11 & L & 3 & 7.6 & 21.0 & Sedimentary and volcanic rocks \\
\hline 416 & 5/4-9L1 & Napa Valley & Kunkel and Upson (1960) & 38.2917 & -122.3131 & 560069 & 4238201 & 5 & 4 & 9 & L & 1 & 24.4 & 78.6 & Sedimentary rocks only \\
\hline 417 & $5 / 4-9 Q 1$ & Napa Valley & Kunkel and Upson (1960) & 38.2895 & -122.3071 & 560596 & 4237954 & 5 & 4 & 9 & $\mathrm{Q}$ & 1 & 10.7 & 54.3 & Sedimentary rocks only \\
\hline 418 & $5 / 4-10 P 4$ & Napa Valley & Kunkel and Upson (1960) & 38.2874 & -122.2766 & 563268 & 4237745 & 5 & 4 & 10 & $\mathrm{P}$ & 4 & 7.6 & 91.4 & Sedimentary rocks only \\
\hline 419 & $5 / 4-14 \mathrm{C} 1$ & Napa Valley & Kunkel and Upson (1960) & 38.2852 & -122.2765 & 563278 & 4237497 & 5 & 4 & 14 & c & 1 & 5.2 & 67.1 & Sedimentary rocks only \\
\hline 420 & $5 / 4-15 \mathrm{C} 2$ & Napa Valley & Kunkel and Upson (1960) & 38.2844 & -122.2946 & 561692 & 4237403 & 5 & 4 & 15 & c & 2 & 6.7 & 26.4 & Sedimentary rocks only \\
\hline 421 & $5 / 3-17 \mathrm{C} 1$ & Napa Valley & Kunkel and Upson (1960) & 38.2838 & -122.2218 & 568066 & 4237383 & 5 & 3 & 17 & c & 1 & 182.9 & 79.6 & Volcanic rocks only \\
\hline 422 & 5/4-13E1 & Napa Valley & Kunkel and Upson (1960) & 38.2828 & -122.2632 & 564442 & 4237248 & 5 & 4 & 13 & $\mathrm{E}$ & 1 & 18.3 & 46.6 & Sedimentary rocks only \\
\hline 423 & $5 / 4-14 \mathrm{H} 3$ & Napa Valley & Kunkel and Upson (1960) & 38.2824 & -122.2672 & 564093 & 4237194 & 5 & 4 & 14 & $\mathrm{H}$ & 3 & 17.1 & 73.2 & Sedimentary rocks only \\
\hline 424 & $5 / 4-17 F 1$ & Napa Valley & Kunkel and Upson (1960) & 38.2813 & -122.3261 & 558943 & 4237031 & 5 & 4 & 17 & $\mathrm{~F}$ & 1 & 3.0 & 48.8 & Sedimentary rocks only \\
\hline 425 & 5/4-13L1 & Napa Valley & Kunkel and Upson (1960) & 38.2799 & -122.2590 & 564810 & 4236925 & 5 & 3 & 13 & L & 1 & 25.3 & 275.5 & Sedimentary and volcanic rocks \\
\hline 426 & 5/4-14L2 & Napa Valley & Kunkel and Upson (1960) & 38.2780 & -122.2748 & 563434 & 4236704 & 5 & 4 & 14 & $\mathrm{~L}$ & 2 & 4.9 & 99.1 & Sedimentary and volcanic rocks \\
\hline 427 & 5/4-14L1 & Napa Valley & Kunkel and Upson (1960) & 38.2780 & -122.2748 & 563434 & 4236701 & 5 & 4 & 14 & $\mathrm{~L}$ & 1 & 4.4 & 160.0 & Sedimentary and volcanic rocks \\
\hline 428 & $5 / 4-15 K 1$ & Napa Valley & Kunkel and Upson (1960) & 38.2777 & -122.2871 & 562353 & 4236662 & 5 & 4 & 15 & $\mathrm{k}$ & 1 & 1.8 & 356.0 & Sedimentary and volcanic rocks \\
\hline 429 & $5 / 4-14 \mathrm{P} 1$ & Napa Valley & Kunkel and Upson (1960) & 38.2758 & -122.2765 & 563284 & 4236454 & 5 & 4 & 14 & $\mathrm{P}$ & 1 & 3.0 & 182.9 & Sedimentary and volcanic rocks \\
\hline 430 & $5 / 4-15 \mathrm{P} 2$ & Napa Valley & Kunkel and Upson (1960) & 38.2728 & -122.2947 & 561692 & 4236107 & 5 & 4 & 15 & $\mathrm{P}$ & 2 & 3.0 & 370.0 & Sedimentary and volcanic rocks \\
\hline 431 & $5 / 4-21 \mathrm{~A} 1$ & Napa Valley & Kunkel and Upson (1960) & 38.2720 & -122.3033 & 560943 & 4236023 & 5 & 4 & 21 & A & 1 & 10.7 & 166.1 & Sedimentary and volcanic rocks \\
\hline 432 & 5/4-19J1 & Napa Valley & Kunkel and Upson (1960) & 38.2621 & -122.3387 & 557855 & 4234897 & 5 & 4 & 19 & $\mathrm{~J}$ & 1 & 32.0 & 30.5 & Sedimentary rocks only \\
\hline 433 & 5/4-19J2 & Napa Valley & Kunkel and Upson (1960) & 38.2615 & -122.3394 & 557793 & 4234826 & 5 & 4 & 19 & $\mathrm{~J}$ & 2 & 30.5 & 32.9 & Sedimentary rocks only \\
\hline
\end{tabular}


Appendix 1. Well Location Data. -Continued

[Site IDs corrspond to locations shown on Plate 1; Well number and area correspond to descriptions in the Water-Supply Paper listed; latitude and longitude geographic coordinates have a horizontal datum of North American Datum 1927; Easting and Northing coordinates are in Universa Transverse Mercator projection zone 10, North American Datum 1927; system of quarter-quarter section designation is explained in the Water-Supply Papers; leaders (--), well number not assigned]

\begin{tabular}{|c|c|c|c|c|c|c|c|c|c|c|c|c|c|c|c|}
\hline Site ID & Well number & Area & Water-Supply Paper & Latitude & Longitude & Easting & Northing & $\begin{array}{l}\text { Township } \\
\text { (N) }\end{array}$ & $\begin{array}{l}\text { Range } \\
\text { (W) }\end{array}$ & Section & $\begin{array}{c}\text { Quarter- } \\
\text { quarter } \\
\text { section } \\
\text { designation }\end{array}$ & $\begin{array}{c}\text { Well } \\
\text { number } \\
\text { within } \\
\text { quarter- } \\
\text { quarter } \\
\text { section }\end{array}$ & $\begin{array}{l}\text { Land } \\
\text { surface } \\
\text { elevation, in } \\
\text { meters }\end{array}$ & $\begin{array}{c}\text { Total } \\
\text { depth of } \\
\text { well, in } \\
\text { meters }\end{array}$ & Geologic units intersected \\
\hline 434 & 5/4-21P2 & Napa Valley & Kunkel and Upson (1960) & 38.2592 & -122.3135 & 560065 & 4234589 & 5 & 4 & 21 & $\mathrm{P}$ & 2 & 7.6 & 71.6 & Sedimentary rocks only \\
\hline 435 & $5 / 4-19 R 1$ & Napa Valley & Kunkel and Upson (1960) & 38.2582 & -122.3359 & 558106 & 4234467 & 5 & 4 & 19 & $\mathrm{R}$ & 1 & 38.1 & 41.1 & Sedimentary rocks only \\
\hline 436 & $5 / 4-19 Q 1$ & Napa Valley & Kunkel and Upson (1960) & 38.2579 & -122.3409 & 557666 & 4234424 & 5 & 4 & 19 & $\mathrm{Q}$ & 1 & 30.5 & 53.3 & Sedimentary rocks only \\
\hline 437 & 5/4-27A1 & Napa Valley & Kunkel and Upson (1960) & 38.2568 & -122.2832 & 562714 & 4234343 & 5 & 4 & 27 & A & 1 & 2.2 & 242.3 & Sedimentary and volcanic rocks \\
\hline 438 & 5/4-30B2 & Napa Valley & Kunkel and Upson (1960) & 38.2566 & -122.3430 & 557484 & 4234283 & 5 & 4 & 30 & B & 2 & 33.5 & 173.7 & Sedimentary and volcanic rocks \\
\hline 439 & $5 / 4-26 \mathrm{~B} 1$ & Napa Valley & Kunkel and Upson (1960) & 38.2560 & -122.2725 & 563651 & 4234262 & 5 & 4 & 26 & B & 1 & 6.1 & 438.9 & Sedimentary and volcanic rocks \\
\hline 440 & $5 / 4-26 \mathrm{E} 1$ & Napa Valley & Kunkel and Upson (1960) & 38.2553 & -122.2793 & 563054 & 4234184 & 5 & 4 & 26 & $\mathrm{E}$ & 1 & 2.4 & 245.7 & Sedimentary and volcanic rocks \\
\hline 441 & $5 / 4-27 \mathrm{H} 2$ & Napa Valley & Kunkel and Upson (1960) & 38.2546 & -122.2835 & 562692 & 4234105 & 5 & 4 & 27 & $\mathrm{H}$ & 2 & 2.4 & 262.1 & Sedimentary and volcanic rocks \\
\hline 442 & $5 / 5-26 \mathrm{G} 1$ & Napa Valley & Kunkel and Upson (1960) & 38.2546 & -122.3798 & 554261 & 4234042 & 5 & 5 & 26 & G & 1 & 86.9 & 125.0 & Sedimentary and volcanic rocks \\
\hline 443 & $5 / 4-27 \mathrm{H} 1$ & Napa Valley & Kunkel and Upson (1960) & 38.2533 & -122.2863 & 562445 & 4233951 & 5 & 4 & 27 & $\mathrm{H}$ & 1 & 2.4 & 373.7 & Sedimentary and volcanic rocks \\
\hline 444 & $5 / 4-29 F 1$ & Napa Valley & Kunkel and Upson (1960) & 38.2526 & -122.3267 & 558916 & 4233852 & 5 & 4 & 29 & $\mathrm{~F}$ & 1 & 28.3 & 32.3 & Sedimentary rocks only \\
\hline 445 & $5 / 4-27 K 2$ & Napa Valley & Kunkel and Upson (1960) & 38.2518 & -122.2887 & 562238 & 4233781 & 5 & 4 & 27 & $\mathrm{~K}$ & 2 & 2.4 & 139.0 & Sedimentary and volcanic rocks \\
\hline 446 & $5 / 4-26 \mathrm{M} 1$ & Napa Valley & Kunkel and Upson (1960) & 38.2500 & -122.2806 & 562947 & 4233593 & 5 & 4 & 26 & M & 1 & 2.3 & 286.2 & Sedimentary and volcanic rocks \\
\hline 447 & $5 / 4-27 K 1$ & Napa Valley & Kunkel and Upson (1960) & 38.2500 & -122.2868 & 562402 & 4233592 & 5 & 4 & 27 & $\mathrm{~K}$ & 1 & 2.4 & 84.1 & Sedimentary and volcanic rocks \\
\hline 448 & 5/4-26M2 & Napa Valley & Kunkel and Upson (1960) & 38.2493 & -122.2808 & 562931 & 4233513 & 5 & 4 & 26 & M & 2 & 1.0 & 207.9 & Sedimentary and volcanic rocks \\
\hline 449 & $5 / 4-29 K 1$ & Napa Valley & Kunkel and Upson (1960) & 38.2487 & -122.3261 & 558971 & 4233416 & 5 & 4 & 29 & $\mathrm{k}$ & 1 & 24.4 & 32.0 & Sedimentary rocks only \\
\hline 450 & $5 / 4-26 \mathrm{~N} 1$ & Napa Valley & Kunkel and Upson (1960) & 38.2481 & -122.2793 & 563067 & 4233380 & 5 & 4 & 26 & $\mathrm{~N}$ & 1 & 2.4 & 137.2 & Sedimentary and volcanic rocks \\
\hline 451 & $5 / 4-29 N 2$ & Napa Valley & Kunkel and Upson (1960) & 38.2477 & -122.3321 & 558446 & 4233302 & 5 & 4 & 26 & $\mathrm{~N}$ & 2 & 21.9 & 94.5 & Sedimentary rocks only \\
\hline 452 & $5 / 4-30 \mathrm{R} 1$ & Napa Valley & Kunkel and Upson (1960) & 38.2472 & -122.3355 & 558144 & 4233248 & 5 & 4 & 30 & $\mathrm{R}$ & 1 & 21.3 & 426.7 & Sedimentary rocks only \\
\hline 453 & $5 / 4-29 N 1$ & Napa Valley & Kunkel and Upson (1960) & 38.2471 & -122.3336 & 558315 & 4233234 & 5 & 4 & 29 & $\mathrm{~N}$ & 1 & 21.9 & 10.1 & Hydrology data only; no geologic information \\
\hline 454 & 5/4-29Q1 & Napa Valley & Kunkel and Upson (1960) & 38.2467 & -122.3236 & 559191 & 4233194 & 5 & 4 & 29 & $\mathrm{Q}$ & 1 & 23.5 & 51.8 & Sedimentary rocks only \\
\hline 455 & 5/4-29Q2 & Napa Valley & Kunkel and Upson (1960) & 38.2465 & -122.3225 & 559286 & 4233172 & 5 & 4 & 29 & Q & 2 & 21.3 & 45.4 & Sedimentary rocks only \\
\hline 456 & $5 / 4-27 R 1$ & Napa Valley & Kunkel and Upson (1960) & 38.2461 & -122.2858 & 562494 & 4233160 & 5 & 4 & 27 & $\mathrm{R}$ & 1 & 1.0 & 147.8 & Sedimentary and volcanic rocks \\
\hline 457 & 5/4-30P1 & Napa Valley & Kunkel and Upson (1960) & 38.2461 & -122.3470 & 557141 & 4233115 & 5 & 4 & 30 & $\mathrm{P}$ & 1 & 36.0 & 105.8 & Sedimentary rocks only \\
\hline 458 & 5/4-27Q1 & Napa Valley & Kunkel and Upson (1960) & 38.2457 & -122.2887 & 562241 & 4233113 & 5 & 4 & 27 & $\mathrm{Q}$ & 1 & 2.0 & 217.0 & Sedimentary rocks and pre-Cenozoic rocks \\
\hline 459 & $5 / 4-27 R 2$ & Napa Valley & Kunkel and Upson (1960) & 38.2450 & -122.2846 & 562602 & 4233036 & 5 & 4 & 27 & M & 1 & 1.5 & 100.6 & Sedimentary and volcanic rocks \\
\hline 460 & $5 / 4-35 R 4$ & Napa Valley & Kunkel and Upson (1960) & 38.2321 & -122.2667 & 564181 & 4231619 & 5 & 4 & 35 & $\mathrm{R}$ & 4 & 7.0 & 368.2 & Sedimentary rocks and pre-Cenozoic rocks \\
\hline 461 & $4 / 4-4 C 1$ & Napa Valley & Kunkel and Upson (1960) & 38.2297 & -122.3109 & 560314 & 4231316 & 4 & 4 & 4 & c & 1 & 3.7 & 24.4 & Sedimentary rocks only \\
\hline 462 & $4 / 4-5 C 1$ & Napa Valley & Kunkel and Upson (1960) & 38.2293 & -122.3274 & 558871 & 4231262 & 4 & 4 & 5 & c & 1 & 9.1 & 47.2 & Sedimentary rocks only \\
\hline 463 & $4 / 4-16 \mathrm{D} 1$ & Napa Valley & Kunkel and Upson (1960) & 38.1985 & -122.3170 & 559804 & 4227859 & 4 & 4 & 16 & D & 1 & 0.0 & 70.4 & Sedimentary rocks only \\
\hline 464 & 4/4-16E1 & Napa Valley & Kunkel and Upson (1960) & 38.1937 & -122.3166 & 559840 & 4227316 & 4 & 4 & 16 & $\mathrm{E}$ & 1 & 0.0 & 93.3 & Sedimentary rocks only \\
\hline
\end{tabular}


Appendix 2. Hydrologic data.

[Site IDs and well number correspond to locations in Appendix 1; leaders (--), not reported]

\begin{tabular}{|c|c|c|c|c|c|c|c|c|c|c|c|c|c|c|c|c|}
\hline Site ID & Well number & $\begin{array}{l}\text { Reported geologic unit } \\
\text { present in water interval' }\end{array}$ & $\begin{array}{c}\text { Simplified } \\
\text { geologic unit } \\
\text { present in water } \\
\text { intervalt }^{2}\end{array}$ & $\begin{array}{l}\text { Reported lithology in water- } \\
\text { bearing interval| }\end{array}$ & $\begin{array}{l}\text { Interprotede lithologic class in } \\
\text { water-bearing interval| }\end{array}$ & Well type ${ }^{5}$ & $\begin{array}{l}\text { Casing } \\
\text { diameter, in } \\
\text { inchese }\end{array}$ & $\begin{array}{l}\text { Date Water level } \\
\text { Measured? }\end{array}$ & $\begin{array}{l}\text { Water level } \\
\text { distance below } \\
\text { land surface } \\
\text { datum, in ft }\end{array}$ & $\begin{array}{c}\text { Discharge, in } \\
\text { galmin }\end{array}$ & $\begin{array}{l}\text { Pump test } \\
\text { duration, in } \\
\text { hours }\end{array}$ & $\begin{array}{l}\text { Bailling or } \\
\text { pumping } \\
\text { rate, in } \\
\text { gal/min }\end{array}$ & $\begin{array}{l}\text { Drawdown, in } \\
\mathrm{ft}^{9}\end{array}$ & $\begin{array}{c}\text { Temperature, in } \\
{ }^{\circ} \mathrm{F}\end{array}$ & Use & Comment \\
\hline $\begin{array}{l}1 \\
2 \\
3 \\
4 \\
5 \\
6 \\
7 \\
7\end{array}$ & 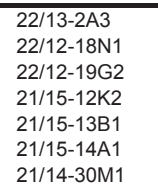 & $\begin{array}{l}\text { Q." } \\
\text { Qaal } \\
\text { Qal, Qt (?) } \\
\text { Qal } \\
\text { Qt } \\
\text { Qal }\end{array}$ & $\begin{array}{l}\text { Qal } \\
\text { Qal } \\
\text { Qal, QTc } \\
\text { Qal } \\
\text { Qal } \\
\text { Qal }\end{array}$ & $\begin{array}{l}-- \\
\text { soil } \\
\text { gravel and yellow clay } \\
\text { yellow clay } \\
\text { surface soil } \\
\text { yellow clay and gravel } \\
\text { blue clay }\end{array}$ & $\begin{array}{l}\text { Sand and clay } \\
\text { Clay and gravel } \\
\text { Clay } \\
\text { Sand and clay } \\
\text { Clay and gravel } \\
\text { Clay }\end{array}$ & $\begin{array}{l}\mathrm{D} \\
\mathrm{DC} \\
\mathrm{DR}, \mathrm{G} \\
\mathrm{DC} \\
\mathrm{DR}, \mathrm{G} \\
\mathrm{DC} \\
\text { Dug }\end{array}$ & $\begin{array}{c}-\overline{14} \\
12-10 \\
8 \\
12-8 \\
8 \\
60\end{array}$ & $\begin{array}{l}1 / 1 / 1948 \\
4 / 3 / 1952 \\
10 / 30 / 1952 \\
9 / 11 / 1940 \\
6 / 11 / 1951 \\
3 / 15 / 1951 \\
11 / 19 / 1952\end{array}$ & $\begin{array}{c}2.84 \\
31.4 \\
16 \\
\text { Flowing } \\
3 \\
16.2\end{array}$ & $\begin{array}{l}\overline{-} \\
\overline{-} \\
\overline{-} \\
- \\
-\end{array}$ & $\begin{array}{l}-- \\
-- \\
-- \\
60 \\
-- \\
--\end{array}$ & $\begin{array}{c}150 \\
150 \\
-\overline{7} \\
700 \\
10 \\
--\end{array}$ & $\begin{array}{c}-- \\
50 \\
-- \\
1 \\
18 \\
38 \\
--\end{array}$ & $\begin{array}{l}\overline{-} \\
\overline{-} \\
\overline{-} \\
- \\
-\end{array}$ & $\begin{array}{l}\text { Stock } \\
\text { Irrigation } \\
\text { Abandoned } \\
\text { Public supply } \\
\text { Domestic } \\
--\end{array}$ & $\begin{array}{l}-- \\
-- \\
-- \\
-- \\
-- \\
-\end{array}$ \\
\hline $\begin{array}{l}8 \\
9\end{array}$ & $\begin{array}{l}\begin{array}{l}21 / 14-31 J 1 \\
18 / 13-18 \mathrm{H} 1\end{array}\end{array}$ & $\stackrel{\text { Qal }}{--}$ & Qal & yellow clay and pea gravel & Clay and gravel & $\begin{array}{l}\mathrm{DR}, \mathrm{G} \\
\mathrm{DR}, \mathrm{G}\end{array}$ & $\begin{array}{l}10 \\
10\end{array}$ & $\begin{array}{l}\text { 10/23/1993 } \\
6 / 9 / 1953\end{array}$ & 12.02 & - & -- & $\begin{array}{c}-- \\
952\end{array}$ & $\ddot{90}$ & $-\overline{-} .0$ & $\begin{array}{l}\text { Irigation } \\
\text { Irrigation }\end{array}$ & - \\
\hline $\begin{array}{l}10 \\
11 \\
12 \\
13 \\
14\end{array}$ & $\begin{array}{l}\text { 18/13-19B1 } \\
18813-2022 \\
11813-3141 \\
17711-331 \\
16112 \mathrm{~W}-34 \mathrm{~N} 2\end{array}$ & $\begin{array}{l}\text { Qal } \\
\text { Tp } \\
\text { Tp } \\
\text { Qt (?) }\end{array}$ & $\begin{array}{l}\text { Qaal } \\
T p \\
T p \\
\text { Qal } \\
--\end{array}$ & $\begin{array}{l}\text { sand and gravel } \\
\text { yellow clay } \\
\text { yellow clay and gravel } \\
\text { yellow clay }\end{array}$ & $\begin{array}{l}\text { Sand (or sandstone) and } \\
\text { gravel } \\
\text { Clay } \\
\text { Clay and gravel } \\
\text { Clay } \\
--\end{array}$ & $\begin{array}{l}D R, G \\
D C C \\
D C \\
D R \\
D\end{array}$ & $\begin{array}{c}12-10 \\
8 \\
8 \\
8\end{array}$ & $\begin{array}{l}7 / 14 / 1954 \\
7 / 14 / 1955 \\
9 / 28 / 1939 \\
9 / 23 / 1952 \\
1 / 1 / 1953\end{array}$ & $\begin{array}{c}182.5 \\
3 \\
26 \\
13\end{array}$ & $\begin{array}{l}- \\
- \\
-\end{array}$ & $\begin{array}{l}\overline{-} \\
\overline{-} \\
-\end{array}$ & $\begin{array}{l}190 \\
15 \\
10 \\
5 \\
-\end{array}$ & $\begin{array}{c}210 \\
- \\
144 \\
--\end{array}$ & $\begin{array}{c}67.0 \\
-- \\
64.0 \\
--\end{array}$ & $\begin{array}{l}\text { Industrial, } \\
\text { irrigation } \\
\text { Irrigation } \\
\text { Domestic } \\
\text { Unused } \\
--\end{array}$ & $\bar{z}-$ \\
\hline $\begin{array}{l}15 \\
16\end{array}$ & $\begin{array}{l}14 / 12-5 K 1 \\
14 / 12-26 \mathrm{H}\end{array}$ & Qyal & Qal & $\begin{array}{l}\text { yellow sand, clay and } \\
\text { gravel }\end{array}$ & $\begin{array}{l}\text { Sand (or sandstone) and } \\
\text { gravel }\end{array}$ & $\mathrm{DC}$ & 8 & $11 / 8 / 1952$ & 38 & $\because$ & - & $\begin{array}{l}5 \\
--\end{array}$ & $\begin{array}{l}56 \\
--\end{array}$ & - & $\begin{array}{l}\text { Domestic, } \\
\text { irrigation } \\
--\end{array}$ & $-\overline{-}$ \\
\hline $\begin{array}{l}17 \\
18\end{array}$ & $\begin{array}{l}\text { 13/11-8H1 } \\
13 / 11-18 \mathrm{D} 1\end{array}$ & $\begin{array}{l}\text { Tp } \\
\text { Qal }\end{array}$ & $\begin{array}{l}\text { Tp } \\
\text { Qal }\end{array}$ & $\begin{array}{l}\text { medium gravelly brown soil } \\
\text { sand }\end{array}$ & $\begin{array}{l}\text { Clay, sand, and gravel } \\
\text { Sand }\end{array}$ & $\begin{array}{l}\mathrm{DC} \\
\mathrm{DR}, \mathrm{G}\end{array}$ & $\begin{array}{c}8 \\
16 \\
60 \text { (Dug) } 8\end{array}$ & $\begin{array}{l}11 / 1 / 1936 \\
8 / 23 / 1950\end{array}$ & $\begin{array}{l}10 \\
15\end{array}$ & - & -- & $\begin{array}{l}75 \\
100\end{array}$ & $\begin{array}{c}70 \\
5\end{array}$ & - & Irrigation & - \\
\hline $\begin{array}{l}19 \\
20\end{array}$ & $\begin{array}{l}13 / 111-18 \mathrm{M} 1 \\
1311-22 \mathrm{G}\end{array}$ & Qal & Qal & topsoil & Sand and clay & Dug, DC & (DC) & 6/23/1953 & 9.4 & -- & -- & $\begin{array}{l}550 \\
100\end{array}$ & -- & -- & Irrigation, stock & - \\
\hline 21 & $\begin{array}{l}13 / 11-2 G \mathrm{G} \\
13 / 11-21 L 2\end{array}$ & $\overline{\text { Qal }}$ & Qal & gravel & $\overline{\text { Gravel }}$ & $\overline{\mathrm{DR}, \mathrm{G}}$ & $\overline{12}$ & $8 / 14 / 1950$ & 44 & - & 72 & 550 & 155 & $=$ & Unused & - \\
\hline 22 & $\begin{array}{l}\begin{array}{l}131 / 11-19 \mathrm{QQ} 1 \\
11 / 10-17 \mathrm{R} 1\end{array}\end{array}$ & Qäl & Qal & brown sitt, moist & Sand and clay & $\begin{array}{l}\mathrm{DR} \\
\mathrm{DC}\end{array}$ & $\begin{array}{l}14 \\
6\end{array}$ & $\begin{array}{l}1 / 1 / 1949 \\
5 / 26 / 1950\end{array}$ & $\overline{15}$ & -- & - & $\begin{array}{l}900 \\
10\end{array}$ & $\overline{15}$ & $\overline{-}$ & $\begin{array}{l}\text { Irrigatition } \\
\text { Domestic }\end{array}$ & $\overline{-}$ \\
\hline 25 & $\begin{array}{l}\text { 11/110-28L1 } \\
11 / 10-33 \mathrm{~A}\end{array}$ & $\ddot{\text { Qal }}$ & $\overline{\text { Qal }}$ & $\overline{\text { gravel (Dry) }}$ & $\overline{\text { Gravel }}$ & $\begin{array}{l}\text { DC } \\
\text { Dug }\end{array}$ & $\begin{array}{l}16 \\
72\end{array}$ & $\begin{array}{l}\text { 1/1/1/1952 } \\
10 / 19 / 1951\end{array}$ & $\overline{11}$ & - & $\overline{-}$ & 1000 & $\overline{8}$ & $\overline{-}$ & $\begin{array}{l}\text { Irrigation } \\
\text { Industrial }\end{array}$ & $\overline{-}$ \\
\hline $\begin{array}{l}26 \\
27\end{array}$ & $\begin{array}{l}\text { 10/11-12Q1 } \\
10 / 9-26 L 1\end{array}$ & $\begin{array}{l}\text { Qal } \\
\text { Qal, QTge }\end{array}$ & $\begin{array}{l}\text { Qal } \\
\text { Qal, QTC }\end{array}$ & $\begin{array}{l}\text { gravel and sand } \\
\text { topsoil } \\
\text { bottom in soff clav and }\end{array}$ & $\begin{array}{l}\text { Sand (or sandstone) and } \\
\text { gravel } \\
\text { Sand and clay }\end{array}$ & $\begin{array}{l}\mathrm{DR} \\
\mathrm{DR}, \mathrm{G}\end{array}$ & $\begin{array}{l}10 \\
10\end{array}$ & $\begin{array}{l}11 / 5 / 1953 \\
7 / 19 / 1950\end{array}$ & $\begin{array}{l}8.68 \\
8.61\end{array}$ & -- & -- & 240 & $\overline{90}$ & 64.0 & $\begin{array}{l}\text { Irrigation } \\
\text { Irrigation }\end{array}$ & - \\
\hline $\begin{array}{l}28 \\
29\end{array}$ & $\begin{array}{l}9 / 10-2 B 3 \\
9 / 10-2 B 1\end{array}$ & Qal, Qt, QTge (?) & Qal, QTc & 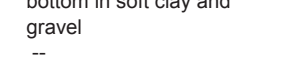 & Clay and gravel & $\begin{array}{l}\mathrm{DC} \\
\mathrm{DR}, \mathrm{G}\end{array}$ & $\begin{array}{l}12 \\
12\end{array}$ & $\begin{array}{l}1 / 15 / 1954 \\
1 / 1 / 1950\end{array}$ & 244.3 & $\begin{array}{l}-- \\
-\end{array}$ & $\overline{-}$ & $\begin{array}{c}240 \\
--\end{array}$ & 45 & $\begin{array}{l}-- \\
--\end{array}$ & $\begin{array}{l}\text { Unused } \\
\text { Irrigation }\end{array}$ & $\overline{-}$ \\
\hline 30 & 9/9-4E1 & Qal, KJ(?) & Qal & topsoil & $\begin{array}{l}\text { Sand and clay } \\
\text { Sand (or sandstone) and }\end{array}$ & $\mathrm{DR}, \mathrm{G}$ & 6 & 4/2/1952 & 4.04 & -- & -- & - & -- & 60.0 & $\begin{array}{l}\text { Unused } \\
\text { Domestic, }\end{array}$ & - \\
\hline $\begin{array}{l}31 \\
32\end{array}$ & $\begin{array}{l}\text { 9/8-7Q1 } \\
9 / 9-21 R 1\end{array}$ & Qal, QTge & Qal, QTc & blue sand and large gravel & gravel & $\begin{array}{l}\mathrm{DR}, \mathrm{G} \\
\mathrm{DC}\end{array}$ & $\begin{array}{c}12 \\
6 \\
8\end{array}$ & $\begin{array}{l}7 / 20 / 1950 \\
1 / 1 / 1941\end{array}$ & $\begin{array}{l}23.29 \\
-\overline{2}\end{array}$ & $\begin{array}{l}-- \\
--\end{array}$ & $\begin{array}{l}-- \\
--\end{array}$ & 250 & $\begin{array}{c}195 \\
--\end{array}$ & $\begin{array}{l}-- \\
--\end{array}$ & $\begin{array}{l}\text { irrigation, stock } \\
\text { Abandoned }\end{array}$ & - \\
\hline 33 & 9/9-33Е2 & Qal & Qal & yellow clay & Clay & $\mathrm{DC}$ & 8 & $5 / 16 / 1952$ & 20 & -- & - & 400 & 2 & -- & $\begin{array}{l}\text { Irrigation } \\
\text { Domestic. }\end{array}$ & - \\
\hline $\begin{array}{l}34 \\
35\end{array}$ & $\begin{array}{l}\text { 8/9-19K1 } \\
8 / 10-20 \mathrm{M} 3\end{array}$ & $\overline{\text { Qal }}$ & $\overline{\text { Qal }}$ & $\begin{array}{l}- \\
\text { clay, yellow, and gravel }\end{array}$ & Clay and gravel & $\begin{array}{l}\mathrm{DC} \\
\mathrm{DC}\end{array}$ & $\begin{array}{l}8 \\
12\end{array}$ & $\begin{array}{l}\text { 1/1/19941 } \\
3 / 1 / 1940\end{array}$ & $\overline{10}$ & - & $\overline{-}$ & $\begin{array}{l}30 \\
20\end{array}$ & $\overline{0}$ & - & $\begin{array}{l}\text { irrigation, } \\
\text { Domestic }\end{array}$ & - \\
\hline 36 & 8/10-29D1 & - & -- & -- & -- & $\mathrm{DR}, \mathrm{G}$ & 8 & 1/1/1951 & - & - & - & 110 & 50 & - & $\begin{array}{l}\text { Domestic, } \\
\text { irrigation }\end{array}$ & - \\
\hline $\begin{array}{l}37 \\
38 \\
39 \\
40 \\
41 \\
42 \\
43 \\
44 \\
45\end{array}$ & $\begin{array}{l}8 / 10-29 H 2 \\
8 / 10-29 N 1 \\
8 / 9-3 A 1 \\
8 / 9-32 E 3 \\
7 / 10-7 D 1 \\
7 / 11-11111 \\
7 / 11-17 J 1 \\
8 / 9-171 \\
8 / 9-3 P 1\end{array}$ & $\begin{array}{l}\text { Qal } \\
\text { Qal } \\
\text { Qt } \\
-- \\
-- \\
\text { Qal } \\
\text { Qal } \\
\text { QTge } \\
\text { Qyal }\end{array}$ & $\begin{array}{l}\text { Qal } \\
\text { Qal } \\
\text { Qal } \\
-- \\
- \\
\text { Qal } \\
\text { Qal } \\
\text { QTC } \\
\text { Qal }\end{array}$ & $\begin{array}{l}\text { top clay and sand } \\
\text { blue clay } \\
\text { yellow clay } \\
-- \\
- \\
\text { sand } \\
\text { blue clay and gravel } \\
\text { yellow clay } \\
\text { black soil } \\
\text { loose vellow sand and }\end{array}$ & $\begin{array}{l}\text { Sand (or sandstone) and } \\
\text { gravel } \\
\text { Clay } \\
\text { Clay } \\
-- \\
-- \\
\text { Sand } \\
\text { Clay and gravel } \\
\text { Clay } \\
\text { Sand and clay } \\
\text { Sand (or sandstone) and }\end{array}$ & $\begin{array}{l}\mathrm{D} \\
\mathrm{DC} \\
\mathrm{DC} \\
\mathrm{DC} \\
\mathrm{D} \\
\mathrm{DC} \\
\mathrm{D} \\
\mathrm{D} \\
\mathrm{D}\end{array}$ & $\begin{array}{c}16 \\
8 \\
8 \\
12 \\
12 \\
12 \\
12 \\
6 \\
10\end{array}$ & $\begin{array}{l}10 / 15 / 1953 \\
11 / 28 / 1952 \\
7 / 11 / 1952 \\
1 / 1 / 1953 \\
7 / 11 / 1950 \\
9 / 121 / 1951 \\
10 / 151 / 1953 \\
3 / 27 / 1951 \\
5 / 25 / 1950\end{array}$ & $\begin{array}{c}21.19 \\
15 \\
10 \\
-- \\
-- \\
26.01 \\
1.97 \\
69.7 \\
0.72\end{array}$ & $\begin{array}{l}-\overline{-} \\
\overline{-} \\
- \\
- \\
- \\
- \\
-\end{array}$ & $\begin{array}{l}\overline{-} \\
\overline{-} \\
- \\
- \\
- \\
-\end{array}$ & $\begin{array}{c}200 \\
200 \\
43 \\
60 \\
-- \\
-- \\
-- \\
12 \\
90\end{array}$ & $\begin{array}{c}-\overline{14} \\
53 \\
50 \\
- \\
500 \\
150 \\
28 \\
63\end{array}$ & $\begin{array}{c}-\overline{-} \\
60.0 \\
-\overline{-} \\
- \\
- \\
- \\
-\overline{0} \\
65.0\end{array}$ & $\begin{array}{l}\text { Public supply } \\
\text { Domestic } \\
\text { Irrigation } \\
\text { Abandoned } \\
\text { Public supply } \\
\text { Abandoned } \\
\text { Irrigation } \\
\text { Domestic } \\
\text { Irrigation, stock }\end{array}$ & $\begin{array}{l}\overline{-} \\
\overline{-} \\
- \\
- \\
- \\
- \\
-\end{array}$ \\
\hline 46 & $8 / 9-9 \mathrm{H} 1$ & Qyal & Qal & $\begin{array}{l}\text { loose yellow sand and } \\
\text { gravel }\end{array}$ & $\begin{array}{l}\text { Sand (or sandstone) and } \\
\text { gravel }\end{array}$ & D & 10 & $5 / 25 / 1950$ & 12.23 & -- & -- & 350 & 3 & 64.0 & Irrigation & -- \\
\hline
\end{tabular}


Appendix 2. Hydrologic data. - Continued

[Site IDs and well number correspond to locations in Appendix 1; leaders (--), not reported]

\begin{tabular}{|c|c|c|c|c|c|c|c|c|c|c|c|c|c|c|c|c|}
\hline Site ID & Well number & $\begin{array}{l}\text { Reported geologic unit } \\
\text { present in water interval' }\end{array}$ & $\begin{array}{l}\text { Simplified } \\
\text { geologic unit } \\
\text { present in water } \\
\text { interval }^{2}\end{array}$ & $\begin{array}{l}\text { Reported lithology in water- } \\
\text { bearing interval }\end{array}$ & $\begin{array}{l}\text { Interpreteded lithologic class in } \\
\text { water-bearing interval }\end{array}$ & Well type $^{5}$ & $\begin{array}{l}\text { Casing } \\
\text { diameter, in } \\
\text { inches }^{6}\end{array}$ & $\begin{array}{l}\text { Date Water level } \\
\text { Measured }^{\ominus}\end{array}$ & $\begin{array}{l}\text { Water level } \\
\text { distance below } \\
\text { land surface } \\
\text { datum, in ft }\end{array}$ & $\begin{array}{c}\text { Discharge, in } \\
\text { gal/min }\end{array}$ & $\begin{array}{l}\text { Pump test } \\
\text { duration, in } \\
\text { hours }\end{array}$ & $\begin{array}{l}\text { Bailing or } \\
\text { pumping } \\
\text { rate, in } \\
\text { gal/min }\end{array}$ & $\begin{array}{c}\text { Drawdown, in } \\
\mathrm{ft}^{9}\end{array}$ & Temperature, in & Use & Comment \\
\hline $\begin{array}{l}47 \\
48 \\
49 \\
50 \\
51\end{array}$ & $\begin{array}{l}8 / 9-10 R 1 \\
89-1111 \\
891501 \\
89-1513 C 1 \\
8 / 9-14 L 1\end{array}$ & $\begin{array}{l}\text { QTge } \\
- \text { Qyal, QTge (?) } \\
\text { Qyal, QTge }\end{array}$ & $\begin{array}{l}\text { QTc } \\
-- \\
\text { QTc } \\
\text { QTc } \\
--\end{array}$ & \begin{tabular}{l}
-- \\
\hdashline topsoil \\
yellow clay \\
-
\end{tabular} & $\begin{array}{l}- \\
-- \\
\text { Sand and clay } \\
\text { Clay }\end{array}$ & $\begin{array}{l}D \\
D \\
D \\
D, G \\
D\end{array}$ & $\begin{array}{l}10 \\
8 \\
10 \\
10 \\
8\end{array}$ & $\begin{array}{l}\text { 1/1/10/1950 } \\
1 / 1 / 1948 \\
4 / 26 / 1950 \\
661 / 1950 \\
11 / 9 / 1950\end{array}$ & $\begin{array}{c}80.15 \\
- \\
8 \\
12 \\
13\end{array}$ & $\begin{array}{c}200 \\
\ddot{-} \\
-- \\
-\end{array}$ & $\begin{array}{l}\because \\
\ddot{-}\end{array}$ & $\begin{array}{l}200 \\
120 \\
75 \\
100 \\
220\end{array}$ & $\begin{array}{l}32 \\
-15 \\
88 \\
88\end{array}$ & $\begin{array}{c}- \\
\ddot{z} \\
66.0\end{array}$ & $\begin{array}{l}\text { Domestic, dairy, } \\
\text { irrigation } \\
\text { IIrigation } \\
\text { Irrigation } \\
\text { Public supply } \\
\text { Irrigation }\end{array}$ & $\begin{array}{l}\overline{-} \\
\ddot{-}\end{array}$ \\
\hline $\begin{array}{l}52 \\
53 \\
54 \\
55 \\
56 \\
57 \\
58 \\
59\end{array}$ & $\begin{array}{l}\text { 8/8-17L1 } \\
8 / 9-14 \mathrm{P} 1 \\
8 / 9-13 \mathrm{~N} 1 \\
8 / 8-20 \mathrm{~B} 2 \\
8 / / 8-20 \mathrm{D} 1 \\
8 / 9-23 \mathrm{G} 1 \\
8 / 9-24 \mathrm{E} 1 \\
8 / 9-23 \mathrm{~L} 1\end{array}$ & $\begin{array}{l}\text { QTge } \\
-- \\
\text { QTge } \\
\overline{\text { QTge }} \\
-- \\
\text { Qyal (?), QTge } \\
\text { QTge }\end{array}$ & $\begin{array}{l}\text { QTc } \\
-- \\
\text { QTC } \\
-- \\
\text { QTc } \\
\overline{\text { Qal, QTc }} \\
\text { QTc }\end{array}$ & $\begin{array}{l}\text { clay, yellow, and gravel; } \\
\text { water at } 103 \text { feet } \\
-- \\
\text { gravel } \\
-- \\
-- \\
-- \\
-- \\
\text { coarse gravel }\end{array}$ & $\begin{array}{l}\text { Clay and gravel } \\
-- \\
\text { Gravel } \\
-- \\
-- \\
-- \\
-- \\
\text { Gravel }\end{array}$ & $\begin{array}{l}\text { D } \\
\text { D, G } \\
D \\
D \\
D \\
D, G \\
D \\
D, G\end{array}$ & $\begin{array}{c}8 \\
10 \\
12 \\
8 \\
10 \\
10 \\
12-10 \\
12\end{array}$ & 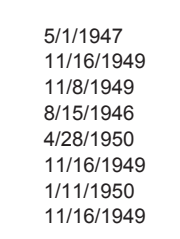 & $\begin{array}{c}103 \\
10.68 \\
27.42 \\
35 \\
70 \\
4.48 \\
3.09 \\
28.54\end{array}$ & $\begin{array}{l}6 \\
- \\
- \\
- \\
- \\
- \\
-\end{array}$ & $\begin{array}{l}- \\
- \\
- \\
- \\
- \\
-\end{array}$ & $\begin{array}{l}10 \\
400 \\
425 \\
20 \\
200 \\
200 \\
600 \\
750\end{array}$ & $\begin{array}{l}132 \\
110 \\
105 \\
120 \\
150 \\
70 \\
60 \\
69\end{array}$ & $\begin{array}{c}- \\
- \\
- \\
- \\
- \\
- \\
65.0\end{array}$ & $\begin{array}{l}\text { Domestic } \\
\text { IIrrigation } \\
\text { Irigation } \\
\text { Domestic } \\
\text { Irrigation } \\
\text { Irrigation } \\
\text { Irrigation } \\
\text { IIrigation }\end{array}$ & $\begin{array}{l}- \\
- \\
- \\
- \\
- \\
- \\
-\end{array}$ \\
\hline $\begin{array}{l}60 \\
61 \\
62\end{array}$ & $\begin{array}{l}8 / 8-19 \mathrm{M} 1 \\
8 / 8-19 \mathrm{~N} 1 \\
8 / 8-20 \mathrm{Q} 1\end{array}$ & $\begin{array}{l}\text { QTge } \\
--\end{array}$ & $\begin{array}{l}\text { QTC } \\
--\end{array}$ & $\begin{array}{l}\text { yellow sandy clay } \\
--\end{array}$ & $\begin{array}{l}\text { Clay and sand (or sandstone) } \\
-- \\
--\end{array}$ & $\begin{array}{l}\mathrm{D}, \mathrm{G} \\
\mathrm{D}, \mathrm{G} \\
\mathrm{D}, \mathrm{G}\end{array}$ & $\begin{array}{l}12 \\
10 \\
10\end{array}$ & $\begin{array}{l}\text { 10/19/1950 } \\
4 / 19 / 1951 \\
6 / 1 / 1950\end{array}$ & $\begin{array}{c}23.27 \\
12 \\
20\end{array}$ & $=$ & $\because$ & $\begin{array}{l}280 \\
290 \\
300\end{array}$ & $\begin{array}{l}70 \\
51 \\
10\end{array}$ & $\begin{array}{r}68.0 \\
-0.0 \\
65.0\end{array}$ & $\begin{array}{l}\text { Domestic, } \\
\text { industrial } \\
\text { Irrigation } \\
\text { Irrigation }\end{array}$ & $\begin{array}{l}-- \\
-\end{array}$ \\
\hline $\begin{array}{l}63 \\
64\end{array}$ & $\begin{array}{l}8 / 9-26 \mathrm{D} 1 \\
8 / 8-29 \mathrm{C} 1\end{array}$ & $\overline{\text { Qyal, QTge }}$ & $\overline{\text { QTC }}$ & yellow clay & $\ddot{\text { Clay }}$ & $\begin{array}{l}D \\
D\end{array}$ & $\begin{array}{l}12 \\
6\end{array}$ & $\begin{array}{l}2 / 1 / 1948 \\
11 / 21 / 1950\end{array}$ & $\begin{array}{l}20 \\
30\end{array}$ & 450 & - & $\begin{array}{l}485 \\
20\end{array}$ & $\begin{array}{l}70 \\
10\end{array}$ & 66.0 & $\begin{array}{l}\text { Domessic, } \\
\text { irrigation, } \\
\text { Domestic }\end{array}$ & - \\
\hline 65 & 8/8-29D1 & Qyal, QTge & QTC & sandy blue clay & Clay and sand (or sandstone) & D & 6 & 1/4/1951 & 8 & - & - & 30 & 16 & - & Domestic & - \\
\hline 66 & $8 / 8-30 \mathrm{G} 1$ & - & - & - & -- & D & 8 & $8 / 15 / 1950$ & 15 & - & - & 40 & 65 & - & $\begin{array}{l}\text { irrigation } \\
\text { ing }\end{array}$ & -- \\
\hline $\begin{array}{l}67 \\
68 \\
69 \\
70\end{array}$ & $\begin{array}{l}\text { 8/8-30L1 } \\
\text { 8/9-27K1 } \\
\text { 8/8-30N1 } \\
\text { 8/8-30N2 }\end{array}$ & $\begin{array}{l}\text { QTge } \\
\text { QTge, QTm } \\
\text { QTge } \\
\text { QTge }\end{array}$ & $\begin{array}{l}\text { QTc } \\
\text { QTc, Twg } \\
\text { QTc } \\
\text { QTc }\end{array}$ & $\begin{array}{l}\text { clay, sandy, blue } \\
\text { clay hard, yellow } \\
\text { clay, yellow, and gravel } \\
\text { clay, yellow, and gravel }\end{array}$ & $\begin{array}{l}\text { Clay and sand (or sandstone) } \\
\text { Clay } \\
\text { Clay and gravel } \\
\text { Clay and gravel }\end{array}$ & $\begin{array}{l}\text { D } \\
D \\
D, G \\
D, G\end{array}$ & $\begin{array}{c}12 \\
12 \\
12 \\
12-8\end{array}$ & $\begin{array}{l}10 / 21 / 1944 \\
11 / 16 / 1949 \\
8 / 17 / 1942 \\
3 / 27 / 1951\end{array}$ & $\begin{array}{c}32 \\
16.1 \\
23 \\
21.71\end{array}$ & $\begin{array}{c}500 \\
800 \\
480 \\
-\end{array}$ & $\begin{array}{l}\bar{z} \\
\overline{-}\end{array}$ & $\begin{array}{l}400 \\
-00 \\
400 \\
--\end{array}$ & $\begin{array}{l}69 \\
- \\
89 \\
--\end{array}$ & $\begin{array}{c}-- \\
\overline{72.0} \\
89.0\end{array}$ & $\begin{array}{l}\text { Industrial } \\
\text { Irrigation } \\
\text { Industrial } \\
\text { Unused }\end{array}$ & $\begin{array}{l}- \\
\overline{-} \\
-\end{array}$ \\
\hline $\begin{array}{l}71 \\
72 \\
73 \\
74 \\
75 \\
76 \\
77 \\
78 \\
79\end{array}$ & $\begin{array}{l}8 / 8-31 \mathrm{~B} 1 \\
8 / 9-33 \mathrm{G} 1 \\
8 / 9-36 \mathrm{G} 1 \\
8 / 9-36 \mathrm{~K} 1 \\
8 / 9-36 \mathrm{~L} 1 \\
88 / 9-33 \mathrm{JJ} \\
8 / 9-35 \mathrm{M} 1 \\
8 / / 9-35 \mathrm{~K} 1 \\
8 / 9-34 \mathrm{R} 1\end{array}$ & $\begin{array}{l}-- \\
\overline{\text { QTge, QTm }} \\
\overline{\text { QTge }} \\
-- \\
\text { Qyal, QTge, QTm } \\
\text { QTge } \\
---\end{array}$ & $\begin{array}{l}- \\
-\overline{Q T C}, \text { Twg } \\
-\overline{Q T C} \\
\overline{-} \\
\overline{\text { Qal, QTc }} \\
\text { QTc } \\
--\end{array}$ & $\begin{array}{l}-- \\
-- \\
-- \\
-\overline{b l u e ~ c l a y ~} \\
-- \\
-\overline{-l a y, ~ b l u e ~} \\
--\end{array}$ & $\begin{array}{l}-- \\
-- \\
-- \\
-\overline{C l a y} \\
-- \\
-\overline{C l a y} \\
--\end{array}$ & $\begin{array}{l}D \\
D \\
D \\
D \\
D \\
D \\
D \\
D \\
D \\
D \\
D\end{array}$ & $\begin{array}{c}8 \\
- \\
12 \\
12 \\
12-8 \\
12 \\
12 \\
12 \\
12\end{array}$ & $\begin{array}{l}5 / 3 / 1947 \\
1 / 1 / 11949 \\
11 / 171 / 1949 \\
11 / 1771 / 149 \\
11 / 18 / 1949 \\
1 / 1 / 1945 \\
11 / 16 / 1949 \\
11 / 161 / 1499 \\
1 / 1 / 1933\end{array}$ & $\begin{array}{c}22 \\
22 \\
16.08 \\
45.98 \\
35.5 \\
-- \\
15.18 \\
28.3 \\
-\end{array}$ & $\begin{array}{r}-- \\
- \\
- \\
500 \\
450 \\
-- \\
800 \\
300 \\
900\end{array}$ & $\begin{array}{l}- \\
- \\
- \\
- \\
- \\
-\end{array}$ & $\begin{array}{c}50 \\
-- \\
750 \\
7- \\
70 \\
-- \\
-\overline{90}\end{array}$ & $\begin{array}{c}31 \\
-- \\
-- \\
75 \\
90 \\
-- \\
-- \\
-- \\
35\end{array}$ & $\begin{array}{l}-- \\
-- \\
-- \\
- \\
- \\
- \\
--\end{array}$ & $\begin{array}{l}\text { irmigation } \\
\text { Unused } \\
\text { Irrigation } \\
\text { Irrigation } \\
\text { Irigation } \\
\text { Unused } \\
\text { Irrigation } \\
\text { Irrigation } \\
\text { Irrigation } \\
\text { Domestic, dairy, }\end{array}$ & $\begin{array}{l}- \\
- \\
- \\
- \\
- \\
- \\
- \\
-\end{array}$ \\
\hline $\begin{array}{l}80 \\
81 \\
82 \\
83\end{array}$ & $\begin{array}{l}8 / 8-34 \mathrm{Q} 1 \\
8 / 9-36 \mathrm{P} 1 \\
7 / / 9-2 \mathrm{C} 1 \\
8 / 9-34 \mathrm{R} 2\end{array}$ & $\begin{array}{l}-\overline{-} \\
\text { Qyal } \\
\text { Qyal, QTge, QTm } \\
\text { Qyal, QTge (?) }\end{array}$ & $\begin{array}{l}-\overline{-} \\
\text { Qal } \\
\text { Qal, QTc } \\
\text { QTC }\end{array}$ & $\begin{array}{l}-\overline{-} \\
\text { clay, blue } \\
\text { topsoil }\end{array}$ & $\begin{array}{l}-\overline{C l a y} \\
-- \\
\text { Sand and clay }\end{array}$ & $\begin{array}{l}\mathrm{D} \\
\mathrm{D} \\
\mathrm{D} \\
\mathrm{D}, \mathrm{G}\end{array}$ & $\begin{array}{c}8-6 \\
12-10 \\
12 \\
12\end{array}$ & $\begin{array}{l}1 / 1 / 1948 \\
10 / 5 / 1949 \\
11 / 166 / 1949 \\
10 / 1 / 1949\end{array}$ & $\begin{array}{c}-- \\
22.56 \\
14.01 \\
9\end{array}$ & $\begin{array}{c}-\bar{c} \\
650 \\
1000 \\
-\end{array}$ & $\begin{array}{l}- \\
-\end{array}$ & $\begin{array}{l}- \\
- \\
-\end{array}$ & $\begin{array}{c}-- \\
150 \\
-- \\
-\end{array}$ & $\begin{array}{l}- \\
- \\
-\end{array}$ & $\begin{array}{l}\text { Domestic, dariry, } \\
\text { irrigation } \\
\text { Irrigation } \\
\text { Irrigation } \\
\text { Irrigation }\end{array}$ & $\begin{array}{l}- \\
- \\
--\end{array}$ \\
\hline $\begin{array}{l}84 \\
85 \\
86\end{array}$ & $\begin{array}{l}7 / 8-3 C 1 \\
7 / 8-483 \\
7 / 7-5 F 1\end{array}$ & 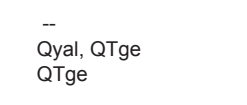 & $\begin{array}{l}-\overline{\text { QTC }} \\
\text { QTC }\end{array}$ & $\begin{array}{l}- \\
\text { gravel, cemented } \\
\text { clay, yellow, and gravel }\end{array}$ & $\begin{array}{l}-- \\
\text { Gravel } \\
\text { Clay and gravel }\end{array}$ & $\begin{array}{l}D \\
D \\
D \\
D\end{array}$ & $\begin{array}{l}8 \\
8 \\
8\end{array}$ & $\begin{array}{l}8 / 3 / 1946 \\
9 / 23 / 1941 \\
5 / 1 / 1948\end{array}$ & $\begin{array}{l}11 \\
33.8 \\
71\end{array}$ & $\begin{array}{l}- \\
-\end{array}$ & $\begin{array}{l}- \\
-\end{array}$ & $\begin{array}{l}10 \\
120 \\
4\end{array}$ & $\begin{array}{c}189 \\
72.7 \\
59\end{array}$ & $\begin{array}{l}- \\
-\end{array}$ & $\begin{array}{l}\text { Domestic, stock } \\
\text { Irrigation } \\
\text { Domestic }\end{array}$ & $\overline{-}$ \\
\hline $\begin{array}{l}87 \\
88\end{array}$ & $\begin{array}{l}777-552 \\
7 / 9-3 E 1\end{array}$ & $\overline{\text { QTm }}$ & $\overline{\text { Twg }}$ & bilue sandstone & $\overline{\text { Sandstone }}$ & $\begin{array}{l}\mathrm{D} \\
\mathrm{D}\end{array}$ & $\begin{array}{c}8 \\
12-8\end{array}$ & $\begin{array}{l}\text { 3/299/1951 } \\
\text { 11/18/1949 }\end{array}$ & $\begin{array}{l}27.26 \\
41.45\end{array}$ & $\begin{array}{l}6 \\
5\end{array}$ & $\because$ & - & $\stackrel{50}{--}$ & $\because$ & $\begin{array}{l}\text { irrigation, stock } \\
\text { Domestic }\end{array}$ & $=$ \\
\hline $\begin{array}{l}89 \\
90\end{array}$ & $\begin{array}{l}777-6 G 1 \\
7 / 8-6 \mathrm{H} 2\end{array}$ & $\begin{array}{l}\text { QTge } \\
\text { QTge }\end{array}$ & $\begin{array}{l}\text { QTc } \\
\text { QTc }\end{array}$ & $\begin{array}{l}\text { fine sand } \\
\text { brown clay }\end{array}$ & $\begin{array}{l}\text { Sand } \\
\text { Clay }\end{array}$ & $\begin{array}{l}\mathrm{D}, \mathrm{G} \\
\mathrm{D}, \mathrm{G}\end{array}$ & $\begin{array}{c}8 \\
10 \\
72 \text { (Dug) } 8\end{array}$ & $\begin{array}{l}\text { 6/5/1951 } \\
3 / 15 / 1951\end{array}$ & $\begin{array}{l}12.73 \\
9.18\end{array}$ & $=$ & $\overline{12}$ & $\begin{array}{l}40 \\
125\end{array}$ & $\begin{array}{l}22 \\
100\end{array}$ & $\overline{-}$ & $\begin{array}{l}\text { irriestion, } \\
\text { Irrigation }\end{array}$ & 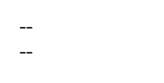 \\
\hline 91 & 7/9-3M1 & QTm & Twg & -- & -- & Dug, D & (D) & 4/1/1949 & 35 & 12 & - & 17 & - & - & Domestic & -- \\
\hline
\end{tabular}


Appendix 2. Hydrologic data. - Continued

[Site IDs and well number correspond to locations in Appendix 1; leaders (--), not reported]

\begin{tabular}{|c|c|c|c|c|c|c|c|c|c|c|c|c|c|c|c|c|}
\hline Site ID & Well number & $\begin{array}{l}\text { Reported geologic unit } \\
\text { present in water interval' }\end{array}$ & $\begin{array}{c}\text { Simplified } \\
\text { geologic unit } \\
\text { present in water } \\
\text { interval| }^{2}\end{array}$ & $\begin{array}{l}\text { Reported lithology in water- } \\
\text { bearing interval| }\end{array}$ & $\begin{array}{l}\text { Interpretede lithologic class in } \\
\text { water-bearing interval }\end{array}$ & Well type ${ }^{5}$ & $\begin{array}{c}\text { Casing } \\
\text { diameter, in }^{\text {diater }} \\
\text { inches }^{8}\end{array}$ & $\begin{array}{l}\text { Date Water level } \\
\text { Measured }^{7}\end{array}$ & $\begin{array}{l}\text { Water level } \\
\text { distance below } \\
\text { land surface } \\
\text { datum, in ft }\end{array}$ & $\begin{array}{c}\text { Discharge, in } \\
\text { gal/min }\end{array}$ & $\begin{array}{l}\text { Pump test } \\
\text { duration, in } \\
\text { hours }\end{array}$ & $\begin{array}{l}\text { Bailing or } \\
\text { pumping } \\
\text { rate, in } \\
\text { gal/min }\end{array}$ & $\begin{array}{l}\text { Drawdown, in } \\
\mathrm{ft}^{9}\end{array}$ & $\begin{array}{c}\text { Temperature, in } \\
{ }^{\circ}\end{array}$ & Use & Comment \\
\hline $\begin{array}{l}92 \\
93\end{array}$ & $\begin{array}{l}7 / 7-5 \mathrm{~N} 1 \\
717-8 \mathrm{~A} 1\end{array}$ & $\begin{array}{l}\text { Qyal, QTge (?) } \\
\text { QTge }\end{array}$ & $\begin{array}{l}\text { QTC } \\
\text { QTc }\end{array}$ & fine gravel & Gravel & $\begin{array}{l}\mathrm{D} \\
\mathrm{D}\end{array}$ & $\begin{array}{l}8 \\
8\end{array}$ & $\begin{array}{l}\text { 6/21/1950 } \\
5 / 1 / 1947\end{array}$ & $\begin{array}{l}9.4 \\
128\end{array}$ & -- & - & $\stackrel{35}{--}$ & 50 & - & $\begin{array}{l}\text { Domestic, } \\
\text { irrirazition } \\
\text { Domestic }\end{array}$ & $\overline{-}$ \\
\hline 94 & $7 / 9-4 \mathrm{~N} 1$ & QTm & Twg & clay and sand, dry & Clay and sand (or sandstone) & D & 8 & $4 / 18 / 1951$ & 15.83 & $\ldots$ & $\ldots$ & 6 & -- & -- & Domestic & -- \\
\hline 95 & $7 / 9-5 \mathrm{~N} 1$ & QTm & Twg & sand, hard yellow & Sand & $\mathrm{D}$ & 12 & $\begin{array}{l}7 / 20 / 1950 \\
7 / 2 / 1950\end{array}$ & 32.62 & 77 & -- & 74 & 100 & -- & $\begin{array}{l}\text { Industrial } \\
\text { Intial }\end{array}$ & -- \\
\hline $\begin{array}{l}96 \\
97\end{array}$ & $\begin{array}{l}717-9033 \\
777-9022\end{array}$ & $\overline{\text { OTae }}$ & Öтс & & $\overline{\text { Clav sand and gravel }}$ & D & 8 & $\begin{array}{l}11 / 26 / 1948 \\
11 / 12019090\end{array}$ & 11 & -- & -- & 20 & 154 & -- & Domestic & -- \\
\hline $\begin{array}{l}97 \\
98\end{array}$ & $\begin{array}{l}717-9022 \\
717-8 A 2\end{array}$ & QTge & QTC & sandy clay and gravel & Clay, sand, and gravel & $\begin{array}{l}D \\
D\end{array}$ & $\begin{array}{l}6 \\
8\end{array}$ & $\begin{array}{l}11 / 30 / 1950 \\
11 / 3 / 1948\end{array}$ & 15 & $--\overline{6}$ & -- & $\begin{array}{l}10 \\
30\end{array}$ & $\begin{array}{l}40 \\
117\end{array}$ & - & $\begin{array}{l}\text { Domestic } \\
\text { Domestic }\end{array}$ & - \\
\hline 99 & $717-9 \mathrm{D} 1$ & QTge & QTC & $\overline{-}$ & -- & $\begin{array}{l}D \\
D\end{array}$ & $\begin{array}{l}8 \\
8\end{array}$ & $\begin{array}{l}11 / 3 / 1948 \\
8 / 17 / 1950\end{array}$ & $\begin{array}{l}1.4 \\
32\end{array}$ & 6.5 & -- & $\begin{array}{l}30 \\
15\end{array}$ & $\begin{array}{l}117 \\
60\end{array}$ & -- & $\begin{array}{l}\text { Domestic } \\
\text { Domestic }\end{array}$ & - \\
\hline 100 & $7 / 8-901$ & QTge & QTC & -- & & D & $\begin{array}{l}0 \\
10\end{array}$ & $\begin{array}{l}8 / 1 / 13950 \\
12 / 13 / 1949\end{array}$ & $\begin{array}{c}32 \\
12.83\end{array}$ & $-\overline{150}$ & -- & 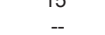 & -. & -- & $\begin{array}{l}\text { Irrigation, stock } \\
\text { Intes }\end{array}$ & -- \\
\hline 101 & 7/9-7G1 & KJu & KJu & (cased to $40 \mathrm{ft}$ ) & No data & $\mathrm{D}$ & 6 & $7 / 20 / 1950$ & 150 & - & -- & 8 & -- & -- & Domestic, dairy & -- \\
\hline 102 & 7/8-11G1 & QTge & QTC & -- & - & D & 8 & $2 / 1 / 1949$ & 90 & - & -- & 7 & 155 & -- & Domestic & -- \\
\hline $\begin{array}{l}103 \\
104\end{array}$ & $\begin{array}{l}717-862 \\
710-0 \times 1\end{array}$ & QTge & QTC & -- & $-\bar{c}$ & $\mathrm{D}$ & 10 & $5 / 26 / 1950$ & 6.64 & -- & -- & 50 & -- & -- & Dairy, irrigation & -- \\
\hline 105 & $7 / 8-12 \mathrm{H} 1$ & $\overline{\text { Tsv }}$ & $\bar{T} \bar{T}$ & dry sand & Sand & $\stackrel{D}{D}$ & $\begin{array}{l}7 \\
6\end{array}$ & $\begin{array}{l}12 / 1 / 1938 \\
2 / 27 / 1950\end{array}$ & $\begin{array}{l}46 \\
75\end{array}$ & 5 & - & $\overline{15}$ & $\overline{50}$ & -- & Domestic & -- \\
\hline 106 & 7/8-12E2 & -- & I- & - & - & $\begin{array}{l}D \\
D\end{array}$ & $\begin{array}{c}6 \\
12\end{array}$ & $\begin{array}{l}2 / 2 / 1950 \\
1 / 1 / 1948\end{array}$ & -15 & 300 & -- & $\begin{array}{l}15 \\
450\end{array}$ & $\begin{array}{l}50 \\
171\end{array}$ & -- & $\begin{array}{l}\text { Domestic } \\
\text { Public supply }\end{array}$ & -- \\
\hline 107 & 7/9-10E1 & -- & - & -- & - & $\mathrm{D}$ & 8 & 7/14/1950 & 46.75 & - & -- & -- & -- & 62.0 & -- & -- \\
\hline $\begin{array}{l}108 \\
109\end{array}$ & $\begin{array}{l}7 / 7-8 \mathrm{M} 2 \\
777-7 F 2\end{array}$ & $-\overline{-}$ 佂 & - & $\overline{-}$ & -- & $D_{G}-a-b$ & 8 & $\begin{array}{l}12 / 8 / 1949 \\
1 / 11 / 1950\end{array}$ & 54 & $\overline{-}$ & -- & 8 & 66 & - & Domestic & -- \\
\hline $\begin{array}{l}109 \\
110\end{array}$ & $\begin{array}{l}717-7722 \\
7 / 9-8 J 1\end{array}$ & $\because$ & -- & yellow sand & Sand & $\begin{array}{l}\mathrm{D}, \mathrm{G} \\
\mathrm{D}\end{array}$ & $\begin{array}{l}8 \\
8\end{array}$ & $\begin{array}{l}1 / 1 / 11150 \\
8 / 10 / 1950\end{array}$ & 52.04 & $-\overline{-}$ & $-\overline{-}$ & 20 & 70 & $\because$ & $\begin{array}{l}\text { Domestic } \\
\text { Domestic }\end{array}$ & $\overline{-}$ \\
\hline 111 & $\begin{array}{l}799-8 \mathrm{JJ1} \\
7 / 8-11 \mathrm{~K} 1\end{array}$ & QTge & OTC & yellow sand & Sand & $\begin{array}{l}D \\
D\end{array}$ & $\begin{array}{l}8 \\
8 \\
8\end{array}$ & $\begin{array}{l}8 / 10 / 1950 \\
7 / 2 / 1941\end{array}$ & $\begin{array}{c}52.04 \\
100\end{array}$ & $\overline{--}$ & -- & 22 & -- & -- & $\begin{array}{l}\text { Domestic } \\
\text { Domestic }\end{array}$ & -- \\
\hline 112 & $7 / 8-10 \mathrm{~L} 1$ & QTge & $\begin{array}{l}\text { QTC } \\
\text { QTC }\end{array}$ & -- & $\overline{--}$ & D & $\begin{array}{l}8 \\
12\end{array}$ & $\begin{array}{l}1 / 1 / 13411949 \\
1049\end{array}$ & 21.6 & 337 & -- & 276 & 47.5 & -- & $\begin{array}{l}\text { Domestic } \\
\text { Unused }\end{array}$ & -- \\
\hline 113 & $7 / 9-8 \mathrm{~K} 1$ & -5 & - & yellow sand and clay & Sand and clay & D & $8-6$ & $9 / 21 / 1949$ & 90 & - & -- & 3 & 200 & -- & Domestic & -- \\
\hline 114 & $7 / 8-12 \mathrm{~K} 1$ & Tsv & Tv & -- & - & D & 5 & 11/14/1949 & 165 & -- & -- & 8 & 13 & -- & Domestic & -- \\
\hline 115 & $7 / 8-12 Q 2$ & -- & - & -- & -- & $\mathrm{D}$ & 8 & $\begin{array}{l}11 / 13 / 1949 \\
2 / 15 / 951\end{array}$ & 123 & - & -- & 20 & 27 & -- & Domestic & -- \\
\hline $\begin{array}{l}116 \\
117\end{array}$ & $\begin{array}{l}7 / 7-8 \mathrm{P} 2 \\
7 / 7-8 \mathrm{~L} 1\end{array}$ & -- & -- & $\because$ & -- & $\begin{array}{l}\mathrm{D} \\
\mathrm{D}\end{array}$ & $\begin{array}{l}6 \\
8\end{array}$ & $\begin{array}{l}2 / 15 / 1951 \\
1 / 1 / 1949\end{array}$ & $\begin{array}{c}50 \\
--\end{array}$ & $\overline{-}$ & -- & $\begin{array}{c}9 \\
12\end{array}$ & $\begin{array}{l}60 \\
90\end{array}$ & $\begin{array}{l}-- \\
--\end{array}$ & $\begin{array}{l}\text { Domestic } \\
\text { Public supply }\end{array}$ & -- \\
\hline 118 & 7/8-9R1 & QTge & QTC & clay sandy brown & Clay and sand (or sandstone) & D & 10 & 8/1/1948 & 9 & -- & -- & 100 & 20 & -- & Irrigation & -- \\
\hline 119 & $7 / 8-14 \mathrm{~A} 1$ & Qyal, QTge & QTC & & Sand and clay & $\mathrm{D}$ & 8 & 4/11/1951 & 0.07 & -- & - & 23 & 160 & - & Unused & - \\
\hline 120 & $7 / 7-16 \mathrm{~A} 1$ & QTge & QTC & - & & D & $8-6$ & 3/6/1951 & 80 & - & - & 30 & 5 & -- & Domestic & -- \\
\hline 121 & $7 / 7-15 \mathrm{C} 1$ & & Qal & boulders & Gravel & D & -- & $5 / 26 / 1950$ & 4 & 1 & -- & 20 & 160 & -- & $\begin{array}{l}\text { Domestic, } \\
\text { irrigation }\end{array}$ & -- \\
\hline 122 & 7/7-17A1 & Qyal, Qoal (?) & Qal & boulders and gravel & Gravel & $\mathrm{D}$ & 12 & 6/1/1947 & 84 & -- & - & 485 & 64 & - & 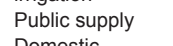 & - \\
\hline 123 & 7/9-15E2 & & -- & -- & & D & 8 & 1/1/1945 & -- & -- & -- & -- & - & -- & $\begin{array}{l}\text { Domestic } \\
\text { prrigtion }\end{array}$ & -- \\
\hline 124 & $7 / 8-14 \mathrm{D} 1$ & Qyal, QTge & QTC & clay & Clay & D & 12 & 10/1/1945 & 12 & -- & - & 270 & 56 & -- & $\begin{array}{l}\text { Irrigation, public } \\
\text { supply }\end{array}$ & - \\
\hline 125 & 7/8-14E1 & QTge & QTC & -- & - & D & 12 & 12/20/1949 & 22.5 & 127 & -- & -- & -- & -- & $\begin{array}{l}\text { Irrigation, public } \\
\text { supply }\end{array}$ & -- \\
\hline 126 & 7/9-18J1 & $\mathrm{KJu}$ & $\mathrm{KJu}$ & -- & -- & D & 10 & $8 / 8 / 1951$ & $\begin{array}{l}22.5 \\
13.6\end{array}$ & -- & -- & -- & -- & -- & $\begin{array}{l}\text { Suppiy } \\
\text { Unused }\end{array}$ & -- \\
\hline 127 & 7/8-18M1 & QTge & QTC & -- & $-\bar{c}$ & $\mathrm{D}, \mathrm{G}$ & 12 & 4/29/1952 & 0.99 & -- & -- & $-\overline{0}$ & $\ddot{13}$ & -- & Irrigation & -- \\
\hline 128 & $\begin{array}{l}\text { 7/9-13M1 } \\
\text { 788-144M1 }\end{array}$ & ÖTae & $\ddot{\text { Öс }}$ & blue clay & Clay & $D$ & $12-8$ & $10 / 5 / 1949$ & $\begin{array}{l}26.02 \\
2000\end{array}$ & 200 & - & 40 & 13 & - & $\begin{array}{l}\text { Irrigation, dairy } \\
\text { rIsud, }\end{array}$ & -- \\
\hline $\begin{array}{l}129 \\
130\end{array}$ & $\begin{array}{l}7 / 8-14 \mathrm{M} 1 \\
7 / 8-13 \mathrm{~J} 1 \mathrm{~B}\end{array}$ & QTge & Q- & $=$ & -- & D & 10 & 6/20/1951 & 22.98 & $\because$ & -- & $\overline{50}$ & $\overline{61}$ & 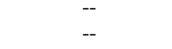 & $\begin{array}{l}\text { Unused } \\
--\end{array}$ & $\because$ \\
\hline 131 & $7 / 8-18 \mathrm{~K} 1$ & QTge(?) & QTC & sand and gravel & $\begin{array}{l}\text { Sand (or sandstone) and } \\
\text { gravel }\end{array}$ & $D, G$ & 10 & $5 / 25 / 1950$ & 8 & -- & -- & 100 & 15 & -- & Irrigation & -- \\
\hline 132 & 7/9-18K1 & $\mathrm{KJu}$ & KJu & (reported "dry hole") & No data & $\mathrm{D}, \mathrm{G}$ & 6 & 8/8/1951 & 7.1 & -- & -- & -- & $-\overline{-}$ & & Unused & -- \\
\hline 133 & 7/9-16L1 & QTm & Twg & - & -- & $\mathrm{D}$ & $8-6$ & $2 / 9 / 1950$ & 65 & -- & - & 3 & 10 & -- & Domestic & - \\
\hline $\begin{array}{l}134 \\
135\end{array}$ & 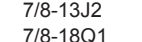 & Tsv (?) & Tv & $-\overline{-}$ & $-\overline{-}$ & $\begin{array}{l}D \\
D\end{array}$ & $\overline{12}$ & 5/27/1947 & 7 & - & $-\overline{-}$ & 60 & 30 & $\overline{-}$ & $\begin{array}{l}\text { Irrigation } \\
\text { IIriation }\end{array}$ & - \\
\hline 136 & 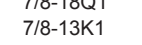 & QTge & QTC & - & - & D & $\begin{array}{l}12 \\
12\end{array}$ & 4/20/1950 & 6.77 & - & -- & $=$ & $=$ & Warm & $\begin{array}{l}\text { IIfrigation } \\
\text { Irrigation }\end{array}$ & - \\
\hline 137 & 7/9-15Q1 & $\ldots$ & -- & -- & $\ldots$ & $\mathrm{D}$ & 8 & approx. 1920 & & .- & -- & -- & -- & -- & $\begin{array}{l}\text { Industrial, } \\
\text { domestic }\end{array}$ & .- \\
\hline 138 & 7/9-14K1 & QTge & QTC & sand & Sand & $\mathrm{D}, \mathrm{G}$ & $16-12$ & $10 / 18 / 1949$ & 18.6 & 625 & -- & 750 & 53 & -- & Irrigation & -- \\
\hline $\begin{array}{l}139 \\
140\end{array}$ & $\begin{array}{l}777-18 \mathrm{~L} 1 \\
779 .-1601 \mathrm{~A}\end{array}$ & Qyal, Qoal (?), QTge & Qal, QTc & clay, yellow, and gravel & Clay and gravel & $\begin{array}{l}D \\
D \\
D\end{array}$ & $\begin{array}{c}12 \\
8\end{array}$ & 9/1/1947 & 31 & 550 & - & $\begin{array}{l}100 \\
20\end{array}$ & $\begin{array}{l}16 \\
22\end{array}$ & - & $\begin{array}{l}\text { Irrigation } \\
\text { Industrial }\end{array}$ & - \\
\hline $\begin{array}{l}140 \\
141\end{array}$ & 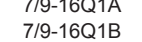 & 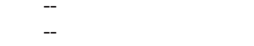 & $\overline{-}$ & - & 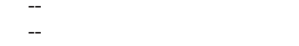 & $\begin{array}{l}D \\
D\end{array}$ & $\begin{array}{l}8 \\
8\end{array}$ & $\overline{7 / 3 / 1949}$ & $\overline{76}$ & $\overline{-}$ & $=$ & 20 & 22 & $\overline{-}$ & Industrial & -- \\
\hline 142 & 7/9-13R1 & -- & - & -- & -- & D & 12 & $5 / 1 / 1948$ & 3 & 350 & - & 355 & 178 & -- & Irrigation & -- \\
\hline
\end{tabular}


Appendix 2. Hydrologic data. - Continued

[Site IDs and well number correspond to locations in Appendix 1; leaders (--), not reported]

\begin{tabular}{|c|c|c|c|c|c|c|c|c|c|c|c|c|c|c|c|c|}
\hline Site ID & Well number & $\begin{array}{l}\text { Reported geologic unit } \\
\text { present in water interval' }\end{array}$ & $\begin{array}{l}\text { Simplified } \\
\text { geologic unit } \\
\text { present in water } \\
\text { intervall }^{2}\end{array}$ & $\begin{array}{l}\text { Reported lithology in water- } \\
\text { bearing interval" }\end{array}$ & $\begin{array}{l}\text { Interpreteded lithologic class in } \\
\text { water-bearing interval }\end{array}$ & Well type ${ }^{5}$ & $\begin{array}{l}\text { Casing } \\
\text { diameter, in } \\
\text { inches }{ }^{6}\end{array}$ & $\begin{array}{l}\text { Date Water level } \\
\text { Measured }\end{array}$ & $\begin{array}{l}\text { Water level } \\
\text { distance below } \\
\text { land surface } \\
\text { datum, in ft }\end{array}$ & $\begin{array}{c}\text { Discharge, in } \\
\text { gal/min }\end{array}$ & $\begin{array}{l}\text { Pump test } \\
\text { duration, in } \\
\text { hours }\end{array}$ & $\begin{array}{c}\text { Bailling or } \\
\text { pumping } \\
\text { rate, in } \\
\text { gal/min }\end{array}$ & $\begin{array}{c}\text { Drawdown, in } \\
\mathrm{ft}^{9}\end{array}$ & $\begin{array}{c}\text { Temperature, in } \\
{ }_{\mathrm{F}}\end{array}$ & Use & Comment \\
\hline 143 & $7 / 8-17 \mathrm{~N} 1$ & Qyal & Qal & soft sandstone, gravel & $\begin{array}{l}\text { Sand (or sandstone) and } \\
\text { gravel }\end{array}$ & D & 12 & 11/30/1949 & 13.75 & -- & $\ldots$ & $\ldots$ & $\ldots$ & & Irriqation & 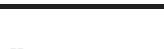 \\
\hline 144 & $7 / 9-21 \mathrm{~B} 1$ & - & -- & -- & & D & $8-7$ & & 10.10 & -- & -- & -- & & -- & Abandoned & - \\
\hline 145 & 7/7-18R2 & Tsv & Tv & volcanic rock, soft & Ash or tuff & D & 12 & 10/1/1949 & 78 & -- & -- & 700 & -- & & Irrigation & -- \\
\hline 146 & $7 / 8-24 \mathrm{~A} 2$ & Qyal, QTge, Tsv (?) & Qal, QTc & -- & -- & D & $14-5.75$ & $5 / 26 / 1949$ & 159 & -- & -- & - & - & 84.0 & $\begin{array}{l}\text { Public supply } \\
\text { Iriagtion public }\end{array}$ & - \\
\hline 147 & $7 / 7-18 \mathrm{~N} 1$ & - & -- & -- & -- & D & -- & 1/1/1946 & -- & 230 & -- & -- & -- & - & $\begin{array}{l}\text { IIfrigation, puobic } \\
\text { supply }\end{array}$ & - \\
\hline 148 & 7/8-21C1 & -- & - & -- & & & & 1/1/1/1951 & -- & & & & -- & -- & Industrial & - \\
\hline 149 & $7 / 8-20 \mathrm{C} 1$ & - & - & -- & - & D & $12-10$ & 11/30/1949 & 14.89 & -- & -- & 320 & 73 & -- & Irrigation & - \\
\hline 150 & 7/9-21C1 & -- & - & -- & - & D & 8 & 1/1/1/1944 & -- & -- & -- & -- & - & -- & Domestic & - \\
\hline 151 & $\begin{array}{l}\text { 7/8-24A3 } \\
779-231\end{array}$ & $-\overline{O T m}$ & 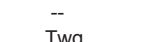 & -- & $\overline{\text { Sandstone }}$ & $\mathrm{D}$ & $12-10$ & $1 / 1 / 1904$ & --2 & 278 & -- & -- & - & 70.0 & Public supply & - \\
\hline $\begin{array}{l}152 \\
153\end{array}$ & $\begin{array}{l}7 / 9-23 \mathrm{~T} 1 \\
7 / 8-20 \mathrm{D} 1\end{array}$ & $\begin{array}{l}\text { QTm } \\
\text { QTge }\end{array}$ & $\begin{array}{l}\text { Twg } \\
\text { QTc }\end{array}$ & $\begin{array}{l}\text { blue coarse sandstone } \\
---\end{array}$ & Sandstone & $\begin{array}{l}\mathrm{D} \\
\mathrm{D}, \mathrm{G}\end{array}$ & $\begin{array}{l}12-10-8 \\
10\end{array}$ & 6/20/1951 & 78.3 & 325 & -- & $\begin{array}{l}400 \\
100\end{array}$ & 85 & 73.0 & $\begin{array}{l}\text { Irrigation } \\
\text { Ifrigation }\end{array}$ & - \\
\hline 154 & $\begin{array}{l}7 / 88-2001 \\
7 / 8-24 A 5\end{array}$ & $\begin{array}{l}\text { Qlge } \\
\text { QTge }\end{array}$ & $\begin{array}{l}\text { QIC } \\
\text { QTC }\end{array}$ & $\overline{-}$ & $-\overline{-}$ & $\begin{array}{l}\text { D, G } \\
\text { D }\end{array}$ & 10 & $\begin{array}{l}6 / 217 / 1950 \\
7 / 10 / 1949\end{array}$ & $\begin{array}{l}12 \\
105\end{array}$ & 382 & -- & 100 & 20 & 66.0 & Public supply & -- \\
\hline 155 & $7 / 8-24 A 6$ & Qyal (?), QTge, Tsv (?) & Qal, QTc & -- & -- & $\mathrm{D}, \mathrm{G}$ & $30-16$ & $7 / 3 / 1950$ & 59 & - & -- & 1994 & 138.5 & - & $\begin{array}{l}\text { Public supply } \\
\text { Puble }\end{array}$ & - \\
\hline 156 & 7/7-14A1 & Tsv & Tv & rock, soft, brown & Ash or tuff & D & 12 & $7 / 5 / 1950$ & 73.25 & - & -- & - & -- & - & $\begin{array}{l}\text { supply } \\
\text { surabuic }\end{array}$ & -- \\
\hline 157 & 7/7-23A1 & QTge & QTc & clay and boulders & Clay and gravel & $\mathrm{D}$ & 8 & 11/3/1944 & 25 & 10 to 15 & - & 27 & 170 & -- & Irrigation & - \\
\hline $\begin{array}{l}158 \\
159\end{array}$ & $\begin{array}{l}7 / 8-24 A 4 \\
7 / 7-241\end{array}$ & QTge (?), QTm & QTc, Twg & sand solidified & Sandstone & $\mathrm{D}$ & $16-10$ & 5/26/1949 & 240 & 1004 & - & -- & -- & 86.0 & Public supply & - \\
\hline $\begin{array}{l}159 \\
160\end{array}$ & $\begin{array}{l}71-2447 \\
7 / 9-21 \mathrm{G} 2\end{array}$ & -- & -- & -- & -- & D & 8 & $\overline{1 / 1 / 1947}$ & $=$ & -- & $-\overline{-}$ & - & -- & $=$ & Domestic & $-\overline{-}$ \\
\hline 161 & 7/7-23A2 & Qyal, QTge & QTC & clay, yellow, and gravel & Clay and gravel & D & 8 & $5 / 1 / 1948$ & 6 & 6 to 8 & -- & 32 & 207 & - & Irrigation & -- \\
\hline 162 & $\begin{array}{l}7 / 9-22 F 1 \\
7 / 9-2252\end{array}$ & QTm & Twg & $\begin{array}{l}\text { fine sand } \\
\text { (Nonefrations) }\end{array}$ & Sand & $\mathrm{D}, \mathrm{G}$ & 10 & 8/30/1951 & 70.4 & - & - & 666 & 120 & - & Irrigation & - \\
\hline & 7/9-22F2 & & & (No perforations) & No data & D & 10 & 8/30/1951 & 53.78 & -- & -- & -- & - & - & $\begin{array}{l}\text { Domestic } \\
\text { Domestic, }\end{array}$ & -- \\
\hline 164 & 7/9-21F1 & QTm & Twg & - & -- & D & $9-5-3$ & 5/1/1942 & 48 & - & -- & 22 & 75 & 64.0 & irrigation & -- \\
\hline 165 & $7 / 9-21 \mathrm{G} 3$ & & $-\overline{-}$ & -- & -- & D & $8-5$ & 8/6/1946 & 62 & -- & -- & 8 & 40 & -- & Domestic, stock & - \\
\hline 166 & $7 / 7-23 \mathrm{G} 1$ & Qyal, Qoal (?), QTge & Qal, QTc & clay, yellow & Clay & D & 12.5 & $6 / 22 / 1950$ & 6.25 & -- & - & -- & - & -- & Unused & - \\
\hline 167 & 7/9-21G1 & QTm & Twg & sand, yellow & Sand & $\mathrm{D}$ & 8 & $2 / 1 / 1942$ & 88 & 6 & -- & 4 & 52 & -- & $\begin{array}{l}\text { Domestic } \\
\text { Domestitis }\end{array}$ & - \\
\hline 168 & 7/99-2111 & - & - & - & - & D & 7 & - & - & - & -- & -- & -- & - & $\begin{array}{l}\text { Domessic } \\
\text { Domestic, }\end{array}$ & -- \\
\hline 169 & 7/9-23K1 & QTge, QTm & QTc, Twg & -- & -- & D & -- & 11/28/1941 & 27 & -- & -- & 300 & -- & -- & irrigation & -- \\
\hline $\begin{array}{l}170 \\
171\end{array}$ & 7/9-24L2 & QTge, QTm (?) & QTc, Twg & -- & -- & $\mathrm{D}, \mathrm{G}$ & 10 & 4/18/1951 & $\begin{array}{l}33.3 \\
792 \\
392-l\end{array}$ & -- & - & 350 & 35 & $\ddot{60}$ & Irrigation & - \\
\hline $\begin{array}{l}1 / 1 \\
172\end{array}$ & $\begin{array}{l}7 / 8-2331 \\
7 / 8-24 k 1\end{array}$ & Qige & QIC & -- & -- & $\begin{array}{l}D \\
D\end{array}$ & $\begin{array}{l}10-8 \\
8-6\end{array}-1$ & $\begin{array}{l}19 / 194 / 1950 \\
12 / 19 / 9\end{array}$ & $\begin{array}{l}19.2 \\
60\end{array}$ & $-\overline{-}$ & $-\overline{-}$ & $\overline{8}$ & $\overline{160}$ & 66.0 & $\begin{array}{l}\text { Industrial } \\
\text { Domestic }\end{array}$ & $\overline{-}$ \\
\hline 173 & $7 / 8-23 \mathrm{M} 3$ & 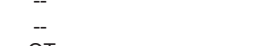 & $=$ & -- & -- & D & 10 & 11/21/1949 & 27.8 & $=$ & - & - & - & - & Unused & - \\
\hline 174 & $7 / 8-22 \mathrm{~K} 1$ & QTge & QTc & -. & -- & D & 12 & 1/23/1950 & 14.99 & 125 & -- & -- & -. & -- & Irrigation & -- \\
\hline 175 & 7/8-23M4 & Qyal, Qoal (?), QTge & Qal, QTC & gravel & Gravel & D & 14 & $6 / 5 / 1951$ & 52.12 & - & -- & - & - & -- & Unused & -- \\
\hline 176 & 7/8-19P1 & QTge & QTc & sand; gravel & $\begin{array}{l}\text { Sand (or sandstone) and } \\
\text { gravel }\end{array}$ & D & 8 & 11/30/1949 & 21.56 & -- & -- & 94 & 39 & -- & - & - \\
\hline 177 & $7 / 8-20 K 1$ & QTge & QTC & yellow clay & Clay & D & 12 & 10/4/1949 & 20.4 & 450 & - & - & - & -- & Irrigation & -- \\
\hline 178 & $\begin{array}{l}7 / 9-2411 \\
7 / 8-2082\end{array}$ & $-\overline{-}$ & $-\overline{-}$ & -- & - & R & 12 & $-\overline{1}$ & $\ddot{41}$ & 90 & - & -- & -- & -- & - & - \\
\hline & & & & clay vellow sandy & Clay and cand (ro sandston) & & & 101111090 & & & & & & & & \\
\hline $\begin{array}{l}180 \\
181\end{array}$ & $\begin{array}{l}7 / 8-23311 \\
7 / 8-22 Q 1\end{array}$ & QTm (?), QTge (?) & Q- $-\mathrm{C}, \mathrm{Twg}$ & clay, yellow, sandy & Clay and sand (or sandstone) & $\begin{array}{l}\mathrm{D}, \mathrm{G} \\
\mathrm{D}\end{array}$ & $\begin{array}{c}12-10 \\
12\end{array}$ & $\begin{array}{l}10 / 1 / 1 / 1949 \\
1 / 1 / 1925\end{array}$ & 190 & 125 & 24- & 295 & 201.7 & -- & Irrigation & $\overline{-}-$ \\
\hline 182 & 7/9-30A1 & QTm & Twg & -- & -- & D & 12 & 4/12/1944 & 10 & - & -- & 75 & -- & - & Domestic & -- \\
\hline 183 & 7/8-23P1 & - & -- & -- & -- & D & & & 16 & -- & - & 43 & 40 & -- & Industrial & -- \\
\hline 184 & 7/6-19R1 & -- & $-\overline{-}$ & -- & -- & D & 8 & $1 / 1 / 1950$ & & 100 & - & -- & 80 & -- & IIrigation & - \\
\hline 185 & $7 / 6-19 \mathrm{~N} 1$ & Qyal, QTge (?) & QTC & brown silt & Sand and clay & D & 10 & 3/29/1951 & 5 & 7 & - & - & 80 & -- & Domestic & -- \\
\hline 186 & 7/9-25A1A & - & - & -- & -- & $-\overline{0}$ & $-\overline{8}$ & $\begin{array}{l}2 / 2 / 1950 \\
\text { L }\end{array}$ & 9.15 & -- & - & 50 & 30 & -- & Domestic, dairy & - \\
\hline $\begin{array}{l}187 \\
188\end{array}$ & $\begin{array}{l}7 / 9-25 \mathrm{~A} 1 \mathrm{~B} \\
7 / 9-30 \mathrm{G} 2\end{array}$ & $\ddot{\mathrm{TTm}}$ & $\bar{T}-\overline{0}$ & $-\overline{-}-$ & Nondata & $\begin{array}{c}\mathrm{D} \\
\mathrm{D}\end{array}$ & $\begin{array}{c}8 \\
12\end{array}$ & $\begin{array}{l}4 / 18 / 1951 \\
7 / 6 / 950\end{array}$ & $\begin{array}{l}7.34 \\
3587\end{array}$ & - & - & 100 & 30 & -- & & - \\
\hline $\begin{array}{l}188 \\
189\end{array}$ & $\begin{array}{l}7 / 7-30 \mathrm{G} 2 \\
71 /-29 \mathrm{D} 1\end{array}$ & $\begin{array}{l}\text { QTm } \\
\text { Qval QTae (?) }\end{array}$ & $\begin{array}{l}\text { Iwg } \\
\text { OTC }\end{array}$ & $\begin{array}{l}\text { (cased to } 1144 \mathrm{ft} . \text { ) } \\
\text { loose gravel }\end{array}$ & No data & $D_{D}^{D}$ & $\begin{array}{c}12 \\
8\end{array}$ & $\begin{array}{l}7 / 7 / 1950 \\
4 / 18 / 1950\end{array}$ & $\begin{array}{l}35.87 \\
4655\end{array}$ & -- & $-\overline{-}$ & $\overline{-}$ & $\overline{-}$ & -- & $\begin{array}{l}\text { Unused } \\
\text { Unciontion }\end{array}$ & - \\
\hline 190 & 7/9-27E2 & -- & Qic & loose gravel & Gravel & $D, G$ & 8 & $\begin{array}{l}5 / 1 / 1951 \\
5 / 1050\end{array}$ & $\begin{array}{c}40.00 \\
9\end{array}$ & -- & - & 300 & 100 & -- & Industrial & - \\
\hline 191 & 7/9-27F1 & QTm & Twg & - & -- & D & 8 & 6/23/1950 & 10.62 & - & -- & 100 & - & - & Industrial & -- \\
\hline 192 & $\begin{array}{l}777-30 C 1 \\
7\end{array}$ & QTge, QTm (?) & QTc, Twg & boulders, hard & Gravel & $\mathrm{D}, \mathrm{G}$ & 8 & 7/23/1951 & 55 & $-\overline{5}$ & -- & 200 & 120 & -- & $\begin{array}{l}\text { Public supply } \\
\text { plustria }\end{array}$ & -- \\
\hline
\end{tabular}


Appendix 2. Hydrologic data. - Continued

[Site IDs and well number correspond to locations in Appendix 1; leaders (--), not reported]

\begin{tabular}{|c|c|c|c|c|c|c|c|c|c|c|c|c|c|c|c|c|}
\hline Site ID & Well number & $\begin{array}{l}\text { Reported geologic unit } \\
\text { present in water interval' }\end{array}$ & $\begin{array}{c}\text { Simplified } \\
\text { geologic unit } \\
\text { present in water }^{\text {interval }}\end{array}$ & $\begin{array}{l}\text { Reported lithology in water- } \\
\text { bearing interval| }\end{array}$ & $\begin{array}{l}\text { Interpretede lithologic class in } \\
\text { water-bearing interval }\end{array}$ & Well type ${ }^{5}$ & $\begin{array}{c}\text { Casing } \\
\text { diameter, in } \\
\text { inches }^{6}\end{array}$ & $\begin{array}{l}\text { Date Water level } \\
\text { Measured }\end{array}$ & $\begin{array}{l}\text { Water level } \\
\text { distance below } \\
\text { land surface } \\
\text { datum, in ft }\end{array}$ & $\begin{array}{c}\text { Discharge, in } \\
\text { galmmin }\end{array}$ & $\begin{array}{l}\text { Pump test } \\
\text { duration, in } \\
\text { hours }\end{array}$ & $\begin{array}{l}\text { Bailling or } \\
\text { pumping } \\
\text { rate, in } \\
\text { ga/min }\end{array}$ & $\begin{array}{c}\text { Drawdown, in } \\
\mathrm{ft}^{9}\end{array}$ & $\begin{array}{l}\text { Temperature, in } \\
{ }_{{ }_{F}}\end{array}$ & Use & Comment \\
\hline 194 & $7 / 9-28 \mathrm{G} 2$ & - & -- & -- & $-\overline{-}$ & $\begin{array}{c}D \\
D\end{array}$ & $\begin{array}{c}8 \\
8\end{array}$ & $4 / 17 / 1951$ & 4 & -- & -- & -- & -- & -- & $\begin{array}{l}\text { Industrial } \\
\text { Indstivatis }\end{array}$ & -- \\
\hline $\begin{array}{l}195 \\
196\end{array}$ & $\begin{array}{l}\text { 7/9-27E1 } \\
\text { 7/9-30J1 }\end{array}$ & - & - & $\overline{-}$ & $\overline{-}$ & D & $\begin{array}{l}8 \\
8\end{array}$ & $\overline{1 / 1 / 1948}$ & $=$ & $=$ & $=$ & $\begin{array}{l}-- \\
--\end{array}$ & - & -- & $\begin{array}{l}\text { Industrial } \\
--\end{array}$ & $=$ \\
\hline 197 & 7/9-26M1 & QTm & Twg & -- & -- & $\mathrm{D}$ & 8 & 4/12/1950 & 19.93 & 17 & -- & & 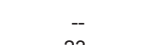 & - & Domestic, stock & - \\
\hline 198 & $7 / 8-28 \mathrm{~F} 1$ & QTge & QTC & $\begin{array}{l}\text { hardpan } \\
\text { sandstone } 220 \mathrm{ft} \text { of surface }\end{array}$ & Clay and gravel & D & 8 & 12/19/1949 & 9.03 & -- & -- & 25 & 23 & -- & Domestic & -- \\
\hline 199 & 7/9-28L1 & QTm & Twg & $\begin{array}{l}\text { casing) } \\
\text { coll }\end{array}$ & Sandstone & D & 12 & $8 / 30 / 1951$ & 130.8 & - & - & -- & -- & 70.0 & Irrigation & -- \\
\hline $\begin{array}{l}200 \\
201 \\
202 \\
203\end{array}$ & $\begin{array}{l}\text { 7/9-29J1 } \\
\text { 7/8-25L1 } \\
\text { 7/8-29R1 } \\
7 / 8-29 R 2\end{array}$ & $\begin{array}{l}\text { QTm } \\
-- \\
-- \\
--\end{array}$ & $\begin{array}{l}\text { Twg } \\
- \\
-\end{array}$ & $\begin{array}{l}\text { uncased below } 60 \mathrm{ft} \\
-- \\
- \\
\text { blue sandstone with } \\
\text { occasional hard streaks of }\end{array}$ & $\begin{array}{l}\text { No data } \\
- \\
--\end{array}$ & $\begin{array}{l}\mathrm{D} \\
\mathrm{D}, \mathrm{G} \\
\mathrm{D} \\
\mathrm{D}\end{array}$ & $\begin{array}{c}12 \\
8 \\
12-10 \\
12\end{array}$ & $\begin{array}{l}7 / 6 / 1950 \\
6 / 15 / 1951 \\
10 / 4 / 1949 \\
1 / 1 / 1943\end{array}$ & $\begin{array}{l}55.95 \\
10.65 \\
20.86 \\
--\end{array}$ & $\begin{array}{l}300 \\
-\overline{-} \\
480 \\
--\end{array}$ & $\begin{array}{l}-- \\
- \\
- \\
-\end{array}$ & $\begin{array}{l}100 \\
450 \\
--\end{array}$ & $\begin{array}{c}7 \overline{7} \\
90 \\
90\end{array}$ & $\begin{array}{l}\ddot{-} \\
-\end{array}$ & $\begin{array}{l}\text { Domestic, dairy, } \\
\text { iririgation, stock } \\
\text { Unused } \\
\text { Public supply } \\
\text { Abandoned }\end{array}$ & $\begin{array}{l}- \\
-- \\
--\end{array}$ \\
\hline 204 & $7 / 9-29 \mathrm{~J} 2$ & QTm & Twg & $\begin{array}{l}\text { hard gray sandstone } \\
\text { hats on }\end{array}$ & Sandstone & D & 12 & 8/30/1951 & 118.5 & 200 & -- & -- & -- & 70.0 & Irrigation & -- \\
\hline 205 & 7/9-28P1 & QTm & Twg & Uncased below $100 \mathrm{ft}$ & No data & D & & 4/17/1951 & 2 & 30 & -- & 400 & 150 & 69.0 & Irrigation & -- \\
\hline $\begin{array}{l}206 \\
207\end{array}$ & $\begin{array}{l}7 / 8-29 J 1 \\
788-25 \mathrm{R} 1\end{array}$ & QTge & QTC & clay, blue, some gravel & Clay and gravel & $\begin{array}{l}\mathrm{D}, \mathrm{G} \\
\mathrm{D}\end{array}$ & $\begin{array}{c}12-10 \\
8\end{array}$ & $\begin{array}{l}\text { 10/4/1949 } \\
1 / 1 / 1947\end{array}$ & $\begin{array}{l}22.14 \\
-\end{array}$ & 450 & - & 450 & 192 & -- & $\begin{array}{l}\text { Public supply } \\
\text { Domestic }\end{array}$ & - \\
\hline 208 & 7/9-33D2 & QTm & Twg & - & -- & $\mathrm{D}$ & 12 & 4/117/1951 & 18.2 & - & - & - & - & 61.0 & $\begin{array}{l}\text { Irigation } \\
\text { Intion }\end{array}$ & - \\
\hline $\begin{array}{l}209 \\
210\end{array}$ & 7/9-33A1 & QTm & Twg & - & - & $\mathrm{D}$ & ${ }_{8}^{7} 6$ & $4 / 13 / 1950$ & 36 & - & -- & $-\overline{1}$ & -- & -- & Unused & -- \\
\hline 210 & 7/9-31A1 & QTm & Twg & cemented gravel, first & - & D & $8-6$ & 2/1/11956 & 85 & - & - & 13 & 85 & - & Domestic & - \\
\hline $\begin{array}{l}211 \\
212\end{array}$ & $\begin{array}{l}7 / 8-36 \mathrm{D} 2 \\
78-30 \mathrm{P} 1\end{array}$ & $\begin{array}{l}\text { Qyal, QTge } \\
\text { OTae }\end{array}$ & $\begin{array}{l}\text { QTC } \\
\text { OTC }\end{array}$ & $\begin{array}{l}\text { water at } 77 \text { feet } \\
\text { hardan }\end{array}$ & $\begin{array}{l}\text { Gravel } \\
\text { Clay and aravel }\end{array}$ & D & $\begin{array}{l}8 \\
8\end{array}$ & $\begin{array}{l}\text { 6/24/1946 } \\
12 / 21 / 1994\end{array}$ & $\begin{array}{c}42 \\
1045\end{array}$ & -- & -- & $\begin{array}{l}42 \\
160\end{array}$ & 8 & - & $\begin{array}{l}\text { Domestic } \\
\text { frication stock }\end{array}$ & - \\
\hline $\begin{array}{l}212 \\
213\end{array}$ & $\begin{array}{l}18-3031 \\
78-31 \mathrm{C} 1\end{array}$ & $\begin{array}{l}\text { QTTe } \\
\text { QTge }\end{array}$ & $\begin{array}{l}\text { QTC } \\
\text { QTC }\end{array}$ & hardpan & $\begin{array}{l}\text { Clay and gravel } \\
-\end{array}$ & $\begin{array}{l}D \\
D\end{array}$ & $\begin{array}{c}8 \\
12-10\end{array}$ & $\begin{array}{l}12 / 21 / 19949 \\
8 / 11 / 1950\end{array}$ & $\begin{array}{l}10.45 \\
14.4\end{array}$ & 250 & -- & ${ }^{160}$ & $\begin{array}{c}33 \\
--\end{array}$ & - & $\begin{array}{l}\text { Irrigation, stock } \\
\text { Unused }\end{array}$ & -- \\
\hline 214 & $7 / 8-35 \mathrm{H} 1$ & QTge & QTC & -- & -- & $\mathrm{D}$ & $12-18$ & 1/1/1946 & 42 & -- & -- & 33 & 24 & -- & $\begin{array}{l}\text { Domestic, } \\
\text { irrigation, stock }\end{array}$ & -- \\
\hline $\begin{array}{l}215 \\
216\end{array}$ & $\begin{array}{l}\text { 7/9-34G1 } \\
7 / 9-32 \mathrm{~F} 2\end{array}$ & $=$ & $\ddot{-}$ & $=$ & - & $\begin{array}{l}D \\
D\end{array}$ & $\begin{array}{l}8-6 \\
8-6\end{array}$ & $\begin{array}{l}\text { 4/11/1951 } \\
1 / 1 / 1946\end{array}$ & $\begin{array}{l}40 \\
--\end{array}$ & $\overline{7}$ & -- & $\begin{array}{c}26 \\
--\end{array}$ & $\begin{array}{l}30 \\
--\end{array}$ & - & $\begin{array}{l}\text { Domesstic } \\
\text { Domestic }\end{array}$ & - \\
\hline 217 & $7 / 9-33 F 1$ & QTm & Twq & sand sandstone & Sandstone & $D$ & 8 & $4 / 13 / 1950$ & 37 & -- & $\ldots$ & -- & $\ldots$ & $\ldots$ & $\begin{array}{l}\text { Domestic, } \\
\text { inriagiton }\end{array}$ & $\ldots$ \\
\hline 218 & 作 & QTm & . & salu, santustivite & - & $\mathrm{D}$ & 8 & $41 / 211950$ & $\begin{array}{l}5.1 \\
59\end{array}$ & $\ldots$ & $\ldots$ & $\ldots$ & $\ldots$ & $\ldots$ & Domestic & $\ldots$ \\
\hline 219 & 7/9-32E1 & QTm & Twg & (Uncased below $6 \mathrm{ft}$ ) & No data & $\mathrm{D}$ & 8 & 7/1/1945 & 180 & -- & -- & -- & -- & - & Domestic & - \\
\hline 220 & $7 / 9-36 \mathrm{H} 1$ & $\begin{array}{l}\text { QTge } \\
\text { QTge }\end{array}$ & QTC & yellow clay & Clay & $\mathrm{D}, \mathrm{G}$ & 12 & 7/114/1950 & 15 & -- & - & 100 & 40 & - & $\begin{array}{l}\text { Irrigation } \\
\text { Intion }\end{array}$ & -- \\
\hline 221 & $7 / 6-32 F 1$ & Qyal, QTge & QTC & tule mud & Clay and sand (or sandstone) & D & 12 & 10/27/1950 & 2.6 & -- & -- & 180 & 67 & - & Irrigation, unused & - \\
\hline 222 & 7/9-34L1 & -- & -- & -- & -- & D & $8-6$ & 4/13/1950 & 28.32 & -- & - & & -- & - & $\begin{array}{l}\text { Donnesic, } \\
\text { irrigation }\end{array}$ & -- \\
\hline $\begin{array}{l}{ }_{223}^{223} \\
224 \mathrm{l} \mathrm{l}\end{array}$ & $\begin{array}{l}\text { 7/9-36F1 } \\
7 / 9-31 \mathrm{~J} 1\end{array}$ & QTge & QTC & clay and mixed gravel & $\begin{array}{l}\text { Clay and gravel } \\
--\end{array}$ & $\begin{array}{l}\mathrm{D}, \\
\mathrm{D},\end{array}$ & $\begin{array}{c}10 \\
6\end{array}$ & $\begin{array}{l}10 / 4 / 1949 \\
1 / 1 / 1950\end{array}$ & 31.35 & $\begin{array}{c}460 \\
--\end{array}$ & $\overline{-}$ & $\begin{array}{l}550 \\
10\end{array}$ & $\begin{array}{c}26 \\
100\end{array}$ & - & $\begin{array}{l}\text { Irrigation } \\
\text { Domestic }\end{array}$ & $\because$ \\
\hline 225 & $7 / 9-34 \mathrm{~J} 1$ & QTm & Twg & (cased to $204 \mathrm{ft}$ ) & No data & $D$ & 8 & $6 / 23 / 1950$ & 53.1 & -- & -- & $\ldots$ & $\ldots$ & -- & $\begin{array}{l}\text { Domestic, } \\
\text { industrial }\end{array}$ & $\ldots$ \\
\hline 226 & $7 / 7-32 \mathrm{G} 1$ & Qyal & Qal & gravel & Gravel & $\mathrm{D}, \mathrm{G}$ & $12-8$ & $4 / 18 / 1959$ & 4 & 150 & - & .- & -. & 74.0 & Irrigation & - \\
\hline 227 & $7 / 9-34 \mathrm{~J} 2$ & & & & & & & 7/25/1946 & 39 & -- & -- & 20 & 50 & -- & Public supply & \\
\hline 228 & 7/8-33M1 & QTge & QTC & clay & Clay & $\mathrm{D}, \mathrm{G}$ & 10 & $11 / 2 / 1950$ & 20 & -- & -- & 500 & 195 & -- & Public supply & -- \\
\hline 229 & 7/9-31Q1 & $\ddot{0} \overline{0}$ & $\ddot{0}$ & -- & $-\bar{s}$ & $\mathrm{D}$ & 7 & 4/1/1944 & 70 & -- & - & 8 & $-\overline{-}$ & - & Domestic & -- \\
\hline 230 & 7/9-36M1 & QTge & QTc & clay, blue & Clay & $\mathrm{D}$ & 8 & 8/1/1937 & 11 & -- & -- & 33 & 34 & -- & Industrial & - \\
\hline $\begin{array}{l}231 \\
232\end{array}$ & $\begin{array}{l}\text { 7/9-31Q3 } \\
799-35 \mathrm{R} 1\end{array}$ & $\begin{array}{l}\text { QTm } \\
\text { QTm }\end{array}$ & $\begin{array}{l}\text { Twg } \\
\text { Twg }\end{array}$ & $\begin{array}{l}\text { (Uncased below } 8 \mathrm{ft.} \text { ) } \\
\text { soil }\end{array}$ & $\begin{array}{l}\text { No data } \\
\text { Sand and clav }\end{array}$ & D & ${ }_{10}^{8}$ & $\begin{array}{l}9 / 21 / 1949 \\
441 / 21150\end{array}$ & $\begin{array}{l}145 \\
0.05\end{array}$ & - & -- & 5 & 205 & - & Domestic & -- \\
\hline 233 & 6/9-3A1 & QIm & - I $^{2}$ & Soll & Sand and clay & D & $\begin{array}{l}10 \\
8\end{array}$ & $\begin{array}{l}1 / 1 / 1 / 1946 \\
1\end{array}$ & -0. & - & $=$ & $\overline{17}$ & -- & - & $\begin{array}{l}\text { Unumed } \\
\text { Domestic }\end{array}$ & - \\
\hline 234 & $6 / 9-2-21$ & $\mathrm{QTm}$ & Twg & sandstone & Sandstone & D & 12 & 4/16/1951 & 70.72 & - & - & -- & -- & - & Public supply & - \\
\hline 235 & $6 / 8-5 E 1$ & QTge & QTC & $\begin{array}{l}\text { coarse, brown sand } \\
\text { volcanic conglomerate }\end{array}$ & Sand & $\mathrm{D}, \mathrm{G}$ & $12-8$ & $5 / 22 / 1951$ & 10 & - & - & 600 & 140 & 67.0 & $\begin{array}{l}\text { Irrigation } \\
\text { Domestic, }\end{array}$ & -- \\
\hline 236 & $6 / 6-4 J 1$ & Tsv & Tv & formation & Ash or tuff & & 10 & 6/6/1949 & 7 & -- & -- & 280 & 80 & 72.0 & irrigation, stock & - \\
\hline $\begin{array}{l}237 \\
238\end{array}$ & $\begin{array}{l}\begin{array}{l}6 / 7-331 \\
6 / 8-7 D 1\end{array} \\
6\end{array}$ & $\begin{array}{l}\text { QTge } \\
--\end{array}$ & QTC & clay & Clay & $\begin{array}{l}\mathrm{D}, \mathrm{G} \\
\mathrm{D}\end{array}$ & $\begin{array}{c}12-10 \\
12\end{array}$ & $\begin{array}{l}\text { 4/1/9/1950 } \\
1 / 1 / 949\end{array}$ & ${ }_{-5}^{5}$ & 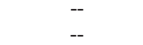 & 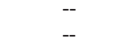 & $.1200,1500$ & 55 & $\overline{-}$ & $\begin{array}{l}\text { Irrigation } \\
\text { Irrigation }\end{array}$ & - \\
\hline
\end{tabular}


Appendix 2. Hydrologic data. - Continued

[Site IDs and well number correspond to locations in Appendix 1; leaders (--), not reported]

\begin{tabular}{|c|c|c|c|c|c|c|c|c|c|c|c|c|c|c|c|c|}
\hline Site ID & Well number & $\begin{array}{l}\text { Reported geologic unit } \\
\text { present in water interval' }\end{array}$ & $\begin{array}{l}\text { Simplified } \\
\text { geologic unit } \\
\text { present in water } \\
\text { interval| }\end{array}$ & $\begin{array}{l}\text { Reported lithology in water- } \\
\text { bearing interval| }\end{array}$ & $\begin{array}{l}\text { Interpreted lithologic class in } \\
\text { water-bearing interval }\end{array}$ & Well type $^{5}$ & $\begin{array}{l}\text { Casing } \\
\text { diameter, in } \\
\text { inches }{ }^{6}\end{array}$ & $\begin{array}{l}\text { Date Water level } \\
\text { Measured }\end{array}$ & $\begin{array}{l}\text { Water level } \\
\text { distance below } \\
\text { land surface } \\
\text { datum, in ft }\end{array}$ & $\begin{array}{c}\text { Discharge, in } \\
\text { gal/min }\end{array}$ & $\begin{array}{l}\text { Pump test } \\
\text { duration, in } \\
\text { hours }\end{array}$ & $\begin{array}{l}\text { Bailling or } \\
\text { pumping } \\
\text { rate, in } \\
\text { gal/min }\end{array}$ & $\underset{\mathrm{ft}^{9}}{\text { Drawdown in }}$ & $\begin{array}{c}\text { Temperature, in } \\
{ }^{\circ} \mathrm{F}\end{array}$ & Use & Comment \\
\hline $\begin{array}{l}239 \\
240\end{array}$ & $\begin{array}{l}6 / 8-12 \mathrm{J1} \\
6 / 6-8 \mathrm{~N} 1\end{array}$ & $\begin{array}{l}\text { QTge } \\
\text { QTge }\end{array}$ & $\begin{array}{l}\text { QTC } \\
\text { QTC }\end{array}$ & $\begin{array}{l}\text { sandy, yellow clay } \\
\text { boulders }\end{array}$ & \multirow{3}{*}{$\begin{array}{l}\text { Clay and sand (or sandstone) } \\
\text { Gravel } \\
\text { Sand (or sandstone) and } \\
\text { gravel } \\
\text { Sand (or sandstone) and } \\
\text { gravel }\end{array}$} & $\begin{array}{l}\mathrm{D} \\
\mathrm{D}\end{array}$ & $\begin{array}{l}8 \\
12\end{array}$ & $\begin{array}{l}6 / 1 / 1939 \\
7 / 26 / 1950\end{array}$ & $\begin{array}{c}20 \\
98.45\end{array}$ & $-\overline{-}$ & - & $\begin{array}{l}30 \\
--\end{array}$ & $\begin{array}{l}40 \\
--\end{array}$ & -- & $\begin{array}{l}\text { Domestic } \\
\text { Irrigation }\end{array}$ & - \\
\hline 241 & 6/6-17A1 & QTge & QTC & dirt and boulders & & D & 8 & 6/30/1949 & 18 & - & - & 10 & 72 & - & Domestic & - \\
\hline 242 & 6/8-17K1 & QTm & Twg & sand, yellow, and gravel & & D & 10 & 2/9/1950 & 49.65 & -- & -- & - & - & - & Dairy, irrigation & - \\
\hline $\begin{array}{l}243 \\
244 \\
245 \\
246\end{array}$ & $\begin{array}{l}6 / 8-16 \mathrm{R} 1 \mathrm{~A} \\
6 / 9-22 \mathrm{~A} 1 \\
6 / 7-19 \mathrm{D} 1 \\
6 / 8-23 \mathrm{C} 1\end{array}$ & $\begin{array}{l}\text { QTm (?) } \\
\text { QTm } \\
\text { QTge }\end{array}$ & $\begin{array}{l}\text { Twg } \\
\text { Twg } \\
\text { QTC }\end{array}$ & $\begin{array}{l}\text { sandy yellow clay } \\
\text { topsoil, sandy loam } \\
\text { clay, blue }\end{array}$ & $\begin{array}{l}\text { Clay and sand (or sandstone) } \\
\text { Sand and clay } \\
\text { Clay }\end{array}$ & $\begin{array}{l}D \\
D \\
D \\
D \\
D\end{array}$ & $\begin{array}{c}12 \\
10 \\
8\end{array}$ & $\begin{array}{l}6 / 14 / 1950 \\
6 / 6 / 1951 \\
6 / 1 / 1949 \\
1 / 1 / 1931\end{array}$ & $\begin{array}{c}130 \\
1.4 \\
9 \\
-\end{array}$ & $\begin{array}{c}-- \\
5.5 \\
-- \\
-\end{array}$ & $\begin{array}{l}\overline{-} \\
\overline{-}\end{array}$ & $\begin{array}{c}315 \\
85 \\
75 \\
-\end{array}$ & $\begin{array}{l}152 \\
200 \\
45 \\
-\end{array}$ & $\begin{array}{l}\overline{-} \\
\ddot{-}\end{array}$ & $\begin{array}{l}\text { Irrigation } \\
\text { Irrigation } \\
\text { Irrigation } \\
\text { Abandoned }\end{array}$ & $\begin{array}{l}-- \\
\overline{-} \\
-\end{array}$ \\
\hline $\begin{array}{l}247 \\
248 \\
249\end{array}$ & $\begin{array}{l}6 / 8-22 \mathrm{~N} 1 \\
667-29511 \\
6 / 8-35 \mathrm{~A} 2\end{array}$ & $\begin{array}{l}\text { QTm } \\
\text { Tsv } \\
\text { Qtal }\end{array}$ & $\begin{array}{l}\text { Twg } \\
\text { Tv } \\
\text { Qal }\end{array}$ & \multirow{2}{*}{$\begin{array}{l}\text { rock } \\
\text { clay, yellow } \\
\text { clay, yellow, and gravel; } \\
\text { mixed }\end{array}$} & $\begin{array}{l}\text { Sand (or sandstone) and } \\
\text { gravel } \\
\text { Ash or tuff } \\
\text { Clay }\end{array}$ & $\begin{array}{l}\mathrm{D} \\
\mathrm{D} \\
\mathrm{D}\end{array}$ & $\begin{array}{l}8-7 \\
10 \\
12\end{array}$ & $\begin{array}{l}\text { 3/1/1942 } \\
8 / 3 / 1944 \\
12 / 9 / 1949\end{array}$ & $\begin{array}{r}25 \\
2.5 \\
8.33\end{array}$ & $\ddot{-}-$ & $\overline{-}-$ & $\begin{array}{l}38 \\
51 \\
-\end{array}$ & $\begin{array}{c}33 \\
12.5 \\
--\end{array}$ & $\ddot{-}-$ & $\begin{array}{l}\text { Domestic } \\
\text { Domestic } \\
\text { Public supply }\end{array}$ & $\overline{-}-$ \\
\hline 250 & 6/8-36A1 & Qyal, QTm & Qal, Twg & & Clay and gravel & D & 14-12 & $11 / 1 / 1935$ & 8 & 600 & -- & 375 & 137 & - & \multirow{2}{*}{$\begin{array}{l}\text { Irrigation } \\
\text { Domestic, } \\
\text { irrigation, stock } \\
\text { Irrigation }\end{array}$} & - \\
\hline $\begin{array}{l}251 \\
252\end{array}$ & $\begin{array}{l}6 / 7-31 \mathrm{H} 1 \\
6 / 7-31 \mathrm{G1}\end{array}$ & $\begin{array}{l}\text { Qyal, QTge } \\
\text { QTge }\end{array}$ & $\begin{array}{l}\text { QTC } \\
\text { QTC }\end{array}$ & $\begin{array}{l}\text { clay, yellow, and gravel } \\
\text { clay, yellow, and gravel }\end{array}$ & $\begin{array}{l}\text { Clay and gravel } \\
\text { Clay and gravel }\end{array}$ & $\begin{array}{l}\mathrm{D} \\
\mathrm{D}\end{array}$ & $\begin{array}{l}8 \\
12\end{array}$ & $\begin{array}{l}\text { 11/29/1949 } \\
11 / 29 / 1949\end{array}$ & $\begin{array}{l}40.44 \\
21.62\end{array}$ & $\begin{array}{l}80 \\
--\end{array}$ & - & $\overline{75}$ & $\overline{60}$ & -- & & - \\
\hline $\begin{array}{l}253 \\
254 \\
255 \\
256\end{array}$ & $\begin{array}{l}\text { 6/8-33E1 } \\
667-3111 \\
6677-32 \mathrm{M} 1 \\
6 / 7-31 \mathrm{~J} 1\end{array}$ & $\begin{array}{l}\text { Tp } \\
\text { Qyal, QTge } \\
\text { Qyal, TQge } \\
\text { Qyal, QTge }\end{array}$ & $\begin{array}{l}\text { Tp } \\
\text { QTc } \\
\text { QTc } \\
\text { QTC }\end{array}$ & $\begin{array}{l}\text { clay, sandy, yellow } \\
\text { clay, yellow, and gravel } \\
\text { clay, yellow, and gravel } \\
\text { yellow clay } \\
\text { sand, yellow; and gravel; }\end{array}$ & $\begin{array}{l}\text { Clay and sand (or sandstone) } \\
\text { Clay and gravel } \\
\text { Clay and gravel } \\
\text { Clay }\end{array}$ & $\begin{array}{l}D \\
D \\
D \\
D\end{array}$ & $\begin{array}{l}8 \\
8 \\
12 \\
12\end{array}$ & $\begin{array}{l}6 / 1 / 1945 \\
6 / 1 / 1948 \\
11 / 22211949 \\
11 / 30 / 1949\end{array}$ & $\begin{array}{c}12 \\
13 \\
24.49 \\
27.01\end{array}$ & $\begin{array}{c}-- \\
- \\
- \\
125\end{array}$ & $\begin{array}{l}-\overline{-} \\
-\overline{-}\end{array}$ & $\begin{array}{c}5 \\
20 \\
70 \\
--\end{array}$ & $\begin{array}{c}68 \\
54 \\
200 \\
-\end{array}$ & $\begin{array}{l}-\ddot{-} \\
-\end{array}$ & $\begin{array}{l}\text { Domestic, stock } \\
\text { Stock } \\
\text { Unused } \\
\text { Irrigation }\end{array}$ & $\begin{array}{l}-- \\
-- \\
-\end{array}$ \\
\hline 257 & 5/7-6E1 & Qyal, QTm & Qal, Twg & water & Sand & $\mathrm{D}$ & 8 & 6/1/1949 & 37 & 20 & -- & 23 & 26 & - & \multirow{4}{*}{$\begin{array}{l}\text { Domestic, stock } \\
\text { Domestit, public } \\
\text { supply } \\
\text { Domestic } \\
\text { Domestic } \\
\text { Domestic } \\
\text { Stock } \\
\text { Domestic } \\
\text { Irrigation, stock } \\
\text { Domestic, dairy, } \\
\text { stock }\end{array}$} & - \\
\hline $\begin{array}{l}258 \\
259 \\
260 \\
261\end{array}$ & $\begin{array}{l}5 / 8-1 \mathrm{H} 1 \\
5 / 7-5 \mathrm{~K} 1 \\
5 / 8-1 \mathrm{J1} \\
5 / 8-1 \mathrm{~L} 1\end{array}$ & $\begin{array}{l}\text { Qyal, QTm } \\
\text { QTm, Tp } \\
\text { QTm } \\
\text { QTm }\end{array}$ & $\begin{array}{l}\text { Qal, Twg } \\
\text { Twg, Tp } \\
\text { Twg } \\
\text { Twg }\end{array}$ & $\begin{array}{l}\text { clay, blue } \\
\text { clay, blue } \\
\text { clay, blue } \\
\text { clay, blue }\end{array}$ & $\begin{array}{l}\text { Clay } \\
\text { Clay } \\
\text { Clay } \\
\text { Clay }\end{array}$ & $\begin{array}{l}D \\
D \\
D \\
D\end{array}$ & $\begin{array}{l}8 \\
8 \\
8 \\
8\end{array}$ & $\begin{array}{l}12 / 8 / 1949 \\
4 / 1 / 1947 \\
2 / 16 / 1950 \\
10 / 1 / 1945\end{array}$ & $\begin{array}{c}126.56 \\
128 \\
33.97 \\
70\end{array}$ & $\begin{array}{l}-- \\
17 \\
30 \\
--\end{array}$ & $\begin{array}{l}- \\
-\end{array}$ & $\begin{array}{l}-- \\
17 \\
18 \\
5\end{array}$ & $\begin{array}{c}-- \\
51 \\
120 \\
30\end{array}$ & $\begin{array}{l}-- \\
- \\
--\end{array}$ & & $\begin{array}{l}- \\
-\end{array}$ \\
\hline $\begin{array}{l}262 \\
263 \\
264\end{array}$ & $\begin{array}{l}5 / 8-13 B 1 \\
57 /-1511 \\
5 / 7-20 C 1\end{array}$ & $\begin{array}{l}\text { Qyal, QTm } \\
\text { Tp } \\
\text { Qyal, Qoal (?) }\end{array}$ & $\begin{array}{l}\text { Qal, Twg } \\
T p \\
\text { Qal }\end{array}$ & $\begin{array}{l}\text { sand; first water at } 22 \text { feet } \\
\text { yellow clay } \\
\text { clay }\end{array}$ & $\begin{array}{l}\text { Sand } \\
\text { Clay } \\
\text { Clay }\end{array}$ & $\begin{array}{l}\mathrm{D} \\
\mathrm{D} \\
\mathrm{D}, \mathrm{G}\end{array}$ & $\frac{8}{-}$ & $\begin{array}{l}8 / 7 / 1940 \\
11 / 1 / 1939 \\
10 / 11 / 1949\end{array}$ & $\begin{array}{c}12 \\
34 \\
8.04\end{array}$ & $\begin{array}{l}-- \\
--\end{array}$ & $\overline{-}-$ & $\begin{array}{l}25 \\
40 \\
650\end{array}$ & $\begin{array}{c}36 \\
31 \\
140\end{array}$ & $\overline{-}-$ & & $\begin{array}{l}-- \\
-\end{array}$ \\
\hline 265 & $5 / 7-19 \mathrm{~N} 1$ & QTm & Twg & sand & Sand & D & 8 & 3/1/1950 & 4.37 & -- & -- & 18 & 37 & - & & - \\
\hline $\begin{array}{l}266 \\
267 \\
268 \\
269\end{array}$ & $\begin{array}{l}5 / 7-25 \mathrm{~A} 1 \\
5 / 7-25 \mathrm{C} 1 \\
5 / 7-28 \mathrm{~A} 3 \\
5 / 7-28 \mathrm{H} 5\end{array}$ & $\begin{array}{l}\text { Tp } \\
\text { Tp } \\
\text { Qyal, Qoal } \\
--\end{array}$ & $\begin{array}{l}\mathrm{Tp} \\
\mathrm{Tp} \\
\text { Qal } \\
-\end{array}$ & $\begin{array}{l}\text { blue clay } \\
\text { clay, yellow, and gravel } \\
--\end{array}$ & $\begin{array}{l}-- \\
\text { Clay } \\
\text { Clay and gravel } \\
--\end{array}$ & $\begin{array}{l}D \\
D \\
D \\
D \\
D\end{array}$ & $\begin{array}{c}10-6 \\
8 \\
14 \\
14\end{array}$ & $\begin{array}{l}11 / 1 / 1949 \\
4 / 5 / 1951 \\
4 / 5 / 1951 \\
--\end{array}$ & $\begin{array}{c}126.18 \\
123 \\
15.79 \\
--\end{array}$ & $\begin{array}{l}-\overline{-} \\
-\overline{-}\end{array}$ & $\begin{array}{l}-- \\
-\overline{-}\end{array}$ & $\begin{array}{l}3 \\
- \\
44 \\
132\end{array}$ & $\begin{array}{c}12 \\
117 \\
117 \\
62\end{array}$ & $\begin{array}{l}\ddot{-}- \\
-\overline{-}\end{array}$ & $\begin{array}{l}\text { Domestic, stock } \\
\text { Public supply } \\
\text { Public suppply } \\
\text { Public supply }\end{array}$ & $\begin{array}{l}-- \\
-- \\
--\end{array}$ \\
\hline 270 & $5 / 7-28 \mathrm{H} 1$ & Qyal, Qoal & Qal & clay, sandy, yellow & Clay and sand (or sandstone) & D & 12-10 & $4 / 5 / 1951$ & 21.5 & - & -- & - & -- & - & Public supply & - \\
\hline 271 & $5 / 7-26 \mathrm{R} 1$ & Qyal, Qoal & Qal & $\begin{array}{l}\text { sandy, coarse, and gravel } \\
\text { sand, coarse streaks in }\end{array}$ & $\begin{array}{l}\text { gavel (sanosione) and } \\
\text { gravel }\end{array}$ & $\mathrm{D}, \mathrm{G}$ & $28-12$ & 2/2/1950 & 32.93 & - & - & 300 & 140 & - & Unused & - \\
\hline $\begin{array}{l}272 \\
273 \\
274\end{array}$ & $\begin{array}{l}5 / 7-35 \mathrm{H} 1 \\
5 / 7-33 \mathrm{M} 1 \\
5 / 7-33 \mathrm{Q} 1\end{array}$ & $\begin{array}{l}\text { Qyal, Qoal (?) } \\
\text { QTm } \\
\text { Qyal }\end{array}$ & $\begin{array}{l}\text { Qal } \\
\text { Twg } \\
\text { Qal }\end{array}$ & $\begin{array}{l}\text { yellow clay } \\
\text { clay, blue, and gravel } \\
\text { sand and clay, yellow }\end{array}$ & $\begin{array}{l}\text { Sand and clay } \\
\text { Clay and gravel } \\
\text { Clay }\end{array}$ & $\begin{array}{l}D \\
D \\
D \\
D\end{array}$ & $\begin{array}{c}28-12 \\
8 \\
10-8\end{array}$ & $\begin{array}{l}6 / 30 / 1948 \\
10 / 25 / 1947 \\
3 / 24 / 1950\end{array}$ & $\begin{array}{c}75 \\
40 \\
19.37\end{array}$ & $\begin{array}{l}-- \\
--\end{array}$ & $\begin{array}{l}-- \\
--\end{array}$ & $\begin{array}{l}-- \\
37 \\
55\end{array}$ & $\begin{array}{c}-\overline{6} \\
140\end{array}$ & $\begin{array}{l}-- \\
--\end{array}$ & $\begin{array}{l}\text { Public supply } \\
\text { Unused } \\
\text { Irrigation }\end{array}$ & $\begin{array}{l}-- \\
-\end{array}$ \\
\hline $\begin{array}{l}275 \\
276 \\
277\end{array}$ & $\begin{array}{l}5 / 7-32 R 1 \\
4 / 6-271 \\
6 / 6-10 F 1\end{array}$ & $\begin{array}{l}\text { Tsv } \\
\text { Qyal, Qoal (?), QTge } \\
\text { QTge }\end{array}$ & $\begin{array}{l}\text { TV } \\
\text { Qal, QTc } \\
\text { QTC }\end{array}$ & $\begin{array}{l}\text { rock, decomposed, yellow } \\
\text { hardpan and boulders } \\
\text { diltit gravel and clay yellow- }\end{array}$ & $\begin{array}{l}\text { Sandstone } \\
\overline{\text { Clay and gravel }}\end{array}$ & $\begin{array}{l}D \\
D \\
D R\end{array}$ & $\begin{array}{c}-- \\
10-8 \\
6\end{array}$ & $\begin{array}{l}11 / 22 / 1949 \\
2 / 22 / 1950 \\
6 / 1 / 1947\end{array}$ & $\begin{array}{l}25.6 \\
0.13 \\
31\end{array}$ & $\overline{-}$ & $\ddot{-}$ & $\begin{array}{l}110 \\
16 \\
300\end{array}$ & $\begin{array}{c}92 \\
119 \\
119\end{array}$ & $\frac{-}{67.0}$ & $\begin{array}{l}\text { Public supply } \\
\text { Unused } \\
\text { Domestic }\end{array}$ & $\overline{-}$ \\
\hline 278 & 6/6-16J2 & QTge & QTC & $\begin{array}{l}\text { mixture } \\
\text { matide }\end{array}$ & Clay, sand, and gravel & $\mathrm{DR}$ & 6 & $5 / 1 / 1947$ & Flowing & 3 & -- & $500 \mathrm{gph}$ & 70 & -- & Domestic & - \\
\hline
\end{tabular}


Appendix 2. Hydrologic data. - Continued

[Site IDs and well number correspond to locations in Appendix 1; leaders (--), not reported]

\begin{tabular}{|c|c|c|c|c|c|c|c|c|c|c|c|c|c|c|c|c|}
\hline Site ID & Well number & $\begin{array}{l}\text { Reported geologic unit } \\
\text { present in water interval' }\end{array}$ & $\begin{array}{l}\text { Simplified } \\
\text { geologic unit } \\
\text { present in water } \\
\text { intervall }^{2}\end{array}$ & $\begin{array}{l}\text { Reported lithology in water- } \\
\text { bearing interval| }\end{array}$ & $\begin{array}{l}\text { Interpreted lithologic class in } \\
\text { water-bearing interval }\end{array}$ & Well type ${ }^{5}$ & $\begin{array}{l}\text { Casing } \\
\text { diameter, in } \\
\text { inches }{ }^{6}\end{array}$ & $\begin{array}{l}\text { Date Water level } \\
\text { Measured }^{7}\end{array}$ & $\begin{array}{l}\text { Water level } \\
\text { distance below } \\
\text { land surface } \\
\text { datum, in ft }\end{array}$ & $\underset{\text { Discharge, in }}{\text { gal/min }}$ & $\begin{array}{l}\text { Pump test } \\
\text { duration, in } \\
\text { hours }\end{array}$ & $\begin{array}{l}\text { Bailling or } \\
\text { pumping } \\
\text { rate, in } \\
\text { gal/min }\end{array}$ & $\begin{array}{c}\text { Drawdown, in } \\
\mathrm{ft}^{9}\end{array}$ & 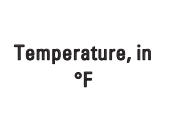 & Use & Comment \\
\hline 279 & 6/6-22R3 & -- & -- & -- & -- & $\mathrm{DR}$ & 8 & 1/1/1946 & -- & -- & -- & & - & -- & $\begin{array}{l}\text { Domestic, } \\
\text { iririgation }\end{array}$ & -- \\
\hline 280 & 6/6-26E1 & Qoal, QTge & Qal, QTc & soil & Sand and clay & $\mathrm{DR}$ & 10 & 10/1/1948 & 4 & -- & -- & 100 & 96 & -- & Domestic & -- \\
\hline $\begin{array}{l}281 \\
282\end{array}$ & $\begin{array}{l}\text { 6/6-34C1 } \\
5 / 5-8 \mathrm{~B} 1\end{array}$ & $\begin{array}{l}\text { QTge } \\
\text { Qoal }\end{array}$ & $\begin{array}{l}\text { QTc } \\
\text { Qal }\end{array}$ & $\begin{array}{l}\text { Clay, yellow } \\
\text { dirt and gravel }\end{array}$ & $\begin{array}{l}\text { Clay } \\
\text { Clay, sand, and gravel }\end{array}$ & $\begin{array}{l}\mathrm{DR} \\
\mathrm{DR}\end{array}$ & $\begin{array}{l}10 \\
6 \\
6\end{array}$ & $\begin{array}{l}7 / 3 / 1951 \\
2 / 1 / 1942\end{array}$ & $\begin{array}{ll}14.84 \\
22\end{array}$ & -- & - & 150 & $\overline{48}$ & - & IIrigation & - \\
\hline & $5 / 5-8 B 1$ & & Qal & dirt and gravel & Clay, sand, and gravel & $\mathrm{DR}$ & 6 & $2 / 7 / 1942$ & 22 & -- & -- & $150 \mathrm{gph}$ & 48 & - & $\begin{array}{l}\text { Domessit } \\
\text { Domestic, }\end{array}$ & -- \\
\hline 283 & 5/5-8A1 & Qoal & Qal & boulder and clay & Clay and gravel & $\mathrm{DR}$ & 6 & 9/1/1946 & 17 & - & -- & $700 \mathrm{gph}$ & 83 & - & $\begin{array}{l}\text { irrigation } \\
\text { Domestic. }\end{array}$ & - \\
\hline 284 & 5/5-7A1 & - & - & - & - & $\mathrm{DR}$ & 12 & - & -- & -- & -- & - & - & -- & $\begin{array}{l}\text { industrial } \\
\text { industiat }\end{array}$ & - \\
\hline 285 & $5 / 5-8 E 1$ & .- & -. & - & -- & $\mathrm{DR}$ & 12 & 1/1/1946 & .- & _- & $\ldots$ & -- & -- & .- & $\begin{array}{l}\text { Domestit, } \\
\text { industrial }\end{array}$ & $\ldots$ \\
\hline 286 & 5/5-9M1 & Qoal & Qal & "hardpan" & Clay and gravel & $\mathrm{DR}$ & 6 & $1 / 1 / 1949$ & 27 & -- & -- & $800 \mathrm{gph}$ & 27 & -- & Domestic & -- \\
\hline 287 & $5 / 6-10 \mathrm{~K} 1$ & Qoal & Qal & "hardpan" and boulders & Clay and gravel & $\mathrm{DR}$ & 8 & 12/1/1949 & 28 & -- & -- & $900 \mathrm{gph}$ & 112 & - & Domestic & -- \\
\hline 288 & $5 / 5-9 \mathrm{~N} 2$ & Qoal, Qh & Qal, QTc & soil & Sand and clay & DR & 6 & 11/1/1/1947 & Flowing & -- & - & $800 \mathrm{gph}$ & 90 & - & Domestic & - \\
\hline 289 & $\begin{array}{l}5 / 5-8 \mathrm{P} 1 \\
5517 \mathrm{~B} 1\end{array}$ & Qoal & Qal & \multirow{3}{*}{$\begin{array}{l}\text { hardpan and clay } \\
\text { clay, yellow } \\
\text { gravel and boulders, water } \\
\text { bearing }\end{array}$} & $\begin{array}{l}\text { Clay and gravel } \\
\text { Clay and razael }\end{array}$ & DR & 8 & 6/1/1947 & 12 & -- & -- & 3,000 gph & 88 & - & Domestic & - \\
\hline $\begin{array}{l}290 \\
291\end{array}$ & $\begin{array}{l}5 / 5-17 \mathrm{~B} 1 \\
5 / 5-17 \mathrm{C} 4\end{array}$ & $\begin{array}{l}\text { Qoal } \\
\text { Qh }\end{array}$ & $\begin{array}{l}\text { Qal } \\
\text { QTC }\end{array}$ & & $\begin{array}{l}\text { Clay and gravel } \\
\text { Clay }\end{array}$ & $\begin{array}{l}\mathrm{DR} \\
-\end{array}$ & 12 & $\begin{array}{l}1 / 1 / 1952 \\
8 / 15 / 1951\end{array}$ & $\begin{array}{l}6.07 \\
59\end{array}$ & $\overline{-}$ & $-\overline{-}$ & $\overline{20}$ & 39 & $\overline{-}-$ & $\begin{array}{l}\text { Irrigation } \\
\text { Irrigation }\end{array}$ & - \\
\hline 292 & $5 / 6-14 \mathrm{~B} 2$ & Qyal & Qal & & Gravel & $\mathrm{DR}$ & 12 & 4/1/1947 & 22 & - & - & 365 & 88 & - & IIrigation & -- \\
\hline 293 & 5/5-16E1 & Qoal, Qh & Qal, QTc & rock & Gravel & $\mathrm{DR}$ & 8 & 6/1/1948 & 21 & -- & -- & $1,100 \mathrm{gph}$ & 129 & -- & $\begin{array}{l}\text { Domestic, } \\
\text { irrigation }\end{array}$ & -. \\
\hline $\begin{array}{l}294 \\
295\end{array}$ & $\begin{array}{l}5 / 6-13 F 1 \\
5 / 6-13 F 2\end{array}$ & $\begin{array}{l}\text { Qyal, Qool } \\
\text { Qyal, Qoal }\end{array}$ & $\begin{array}{l}\text { Qal } \\
\text { Qal }\end{array}$ & $\begin{array}{l}\text { clay } \\
\text { clay }\end{array}$ & $\begin{array}{l}\text { Clay } \\
\text { Clay }\end{array}$ & $\begin{array}{l}\mathrm{DR} \\
\mathrm{DR}\end{array}$ & $\begin{array}{l}14 \\
14\end{array}$ & $\begin{array}{l}12 / 1 / 1949 \\
12 / 1 / 1949\end{array}$ & $\begin{array}{l}17 \\
18\end{array}$ & - & - & $\begin{array}{l}500 \\
400\end{array}$ & $\begin{array}{l}46 \\
57\end{array}$ & -- & $\begin{array}{l}\text { IIrigation } \\
\text { Irrigation }\end{array}$ & -- \\
\hline 296 & 5/5-17J1 & Qoal & Qal & \multirow{2}{*}{$\begin{array}{l}\text { clay, sandy } \\
\text { dirt, gravel and clay, yellow- } \\
\text { mixture }\end{array}$} & Clay and sand (or sandstone) & -- & -- & 9/1/1945 & 14 & -- & -- & $975 \mathrm{gph}$ & 66 & -- & Domestic & -- \\
\hline 297 & 5/5-18K1 & Qoal & Qal & & Clay, sand, and gravel & DR & 8 & 81/1/1947 & 17 & -- & -- & $900 \mathrm{gph}$ & 73 & -- & Domestic & -- \\
\hline 298 & $5 / 5-17 \mathrm{~N} 1$ & -- & -- & & & $\mathrm{DR}$ & 8 & 1/1/1930 & -- & -- & - & r & -- & -- & Domestic & - \\
\hline 299 & 5/5-17R2 & Qoal, Qh & Qal, QTc & clay, gray & Clay & $\mathrm{DR}$ & 6 & 5//1/1948 & 10 & -- & - & $100 \mathrm{gph}$ & 60 & - & Domestic & - \\
\hline 300 & 5/5-21E1 & & QTC & clay, blue & Clay & $\mathrm{DR}$ & 8 & 4/6/1950 & 4.6 & -- & -- & -- & -- & -- & $\begin{array}{l}\text { irrigation, } \\
\text { inter }\end{array}$ & -- \\
\hline 301 & $5 / 6-24 \mathrm{~K} 1$ & Qyal, Qoal & Qal & $\begin{array}{l}\text { gravel. } \\
\text { gate }\end{array}$ & Gravel & $\mathrm{DR}$ & - & 5/17/1950 & 8.61 & -- & - & - & - & - & $\begin{array}{l}\text { Irrigation } \\
\text { Intion }\end{array}$ & - \\
\hline 302 & 5/5-22L1 & Qh & QTC & soil & Sand and clay & DR & 8 & $3 / 1 / 1950$ & Flowing & -- & -- & -- & - & -- & Domestic & - \\
\hline $\begin{array}{l}303 \\
304\end{array}$ & $\begin{array}{l}5 / 5-19 L 5 \\
5 / 5-22212\end{array}$ & Qoal & $\begin{array}{l}\text { Qal } \\
\text { OTc }\end{array}$ & $\begin{array}{l}\text { hardpan and sand } \\
\text { soil }\end{array}$ & $\begin{array}{l}\text { Clay and gravel } \\
\text { Sand and clay }\end{array}$ & DR & 6 & $\begin{array}{l}10 / 1 / 1942 \\
3 / 31 / 1950\end{array}$ & $\begin{array}{l}21 \\
\text { Flowing }\end{array}$ & -- & -- & $950 \mathrm{gph}$ & -- & -- & Domestic & - \\
\hline 304 & 5/5-22L2 & Qh & QTc & & Sand and clay & - & -- & 3/31/1950 & Flowing & -- & -- & - & - & - & $\begin{array}{l}\text { Irrigation } \\
\text { Domestic. }\end{array}$ & - \\
\hline $\begin{array}{l}305 \\
306\end{array}$ & $\begin{array}{l}5 / 5-19\llcorner 1 \\
5 / 5-22 Q 1\end{array}$ & $\begin{array}{l}\text { Qoal, Qh (?) } \\
\text { Qh }\end{array}$ & $\begin{array}{l}\text { Qal, QTc } \\
\text { QTC }\end{array}$ & $\begin{array}{l}\text { hardpan and clay } \\
\text { soil }\end{array}$ & $\begin{array}{l}\text { Clay and gravel } \\
\text { Sand and clay }\end{array}$ & $\begin{array}{l}\mathrm{DR} \\
\mathrm{DR}\end{array}$ & $\begin{array}{l}12 \\
12\end{array}$ & $\begin{array}{l}11 / 1 / 1948 \\
4 / 5 / 1950\end{array}$ & $\begin{array}{c}10 \\
\text { Flowing }\end{array}$ & -- & -- & $\begin{array}{l}450 \\
--\end{array}$ & $\begin{array}{l}80 \\
--\end{array}$ & -- & $\begin{array}{l}\text { irrigation } \\
\text { Irrigation }\end{array}$ & - \\
\hline 307 & $5 / 5-20 \mathrm{R} 1$ & Qoal & Qal & sand and dirt & Sand and clay & $\mathrm{DR}$ & 8 & 3/28/1950 & 2.48 & -- & -- & $350 \mathrm{gph}$ & - & -- & Domestic & $\begin{array}{l}\text { Reported to flow when } \\
\text { drilled }\end{array}$ \\
\hline 308 & $5 / 5-21 R 1$ & Qoal & Qal & \multirow{5}{*}{$\begin{array}{l}\text { clay, sandy } \\
\text { soil } \\
\text { soil } \\
\text { clay, yellow, and gravel; } \\
\text { water at } 70 \text { feet } \\
\text { clay and rock } \\
\text { soil }\end{array}$} & Clay and sand (or sandstone) & $\mathrm{DR}$ & 8 & 11/1/1/1948 & 30 & - & - & \multirow{3}{*}{300} & -- & - & \multirow{3}{*}{$\begin{array}{l}\text { Domestic } \\
\text { Irrigation } \\
\text { Dairy }\end{array}$} & - \\
\hline 309 & $5 / 5-30 \mathrm{E} 1$ & & - & & Sand and clay & DR & 12 & 2/1/1951 & Flowing & - & -- & & -- & - & & - \\
\hline 310 & $5 / 5-27 \mathrm{H} 1$ & Qh & QTC & & Sand and clay & $\mathrm{DR}$ & 12 & $1 / 26 / 1950$ & Flowing & -- & -- & & -- & 80.5 & & -- \\
\hline 311 & 6/6-9R1 & QTge & QTC & & Clay and gravel & D & 8 & 12/1/1947 & 46 & - & -- & 30 & 34 & -- & Domestic & - \\
\hline $\begin{array}{l}312 \\
313\end{array}$ & $\begin{array}{l}6 / 6-16 \mathrm{~B} 1 \\
5 / 6-25 \mathrm{P} 1\end{array}$ & $\begin{array}{l}\text { QTge } \\
\text { Qoal }\end{array}$ & $\begin{array}{l}\text { QTc } \\
\text { Qal }\end{array}$ & & $\begin{array}{l}\text { Clay and gravel } \\
\text { Sand and clay }\end{array}$ & $\begin{array}{l}\mathrm{D} \\
\mathrm{DR}\end{array}$ & $\begin{array}{l}5 \\
8\end{array}$ & $\begin{array}{l}\text { 10/30/1941 } \\
10 / 5 / 1946\end{array}$ & $\begin{array}{l}40 \\
\text { Flowing }\end{array}$ & - & $-\overline{-}$ & $\begin{array}{l}7 \\
600 \mathrm{gph}\end{array}$ & $\begin{array}{c}210 \\
--\end{array}$ & $\begin{array}{l}73.0 \\
--\end{array}$ & $\begin{array}{l}\text { Domestic } \\
\text { Domestic }\end{array}$ & - \\
\hline 314 & 5/5-31A 1 & Qyal (?) & Qal & - & - & DR & 8 & 9/1/1927 & Flowing & -- & -- & - & -- & - & Domestic, stock & -- \\
\hline $\begin{array}{l}315 \\
316\end{array}$ & $\begin{array}{l}5 / 5-31 \mathrm{H} 1 \\
4 / 5-3 \mathrm{C} 1\end{array}$ & Qyal (?) & Qal & "muck," blue & $\begin{array}{l}\text { Clay and sand (or sandstone) } \\
--\end{array}$ & $\begin{array}{l}D R \\
D R\end{array}$ & $\begin{array}{l}6 \\
7\end{array}$ & $\begin{array}{l}\text { 10/1/1948 } \\
1 / 1 / 1945\end{array}$ & 9 & $\overline{-}$ & - & $450 \mathrm{gph}$ & $\stackrel{116}{--}$ & $\overline{-}$ & $\begin{array}{l}\text { Domestic } \\
\text { Irrigation }\end{array}$ & - \\
\hline 317 & $4 / 5-14 \mathrm{D} 2$ & - & -- & peat and silt & Clay and sand (or sandstone) & -- & -- & 9/13/1951 & 23 & - & - & 395 & 140 & -- & Public supply & -- \\
\hline 318 & 4/5-14D1 & -- & -- & $\begin{array}{l}\text { clay loue } \\
\text { dark "surface" mud and }\end{array}$ & & DR & - & 3/16/1950 & 10.96 & -- & -- & 43 & 61 & -- & Public supply & - \\
\hline 319 & 4/5-14L1 & $-\overline{-}$ & $-\overline{-}$ & peat & Clay and sand (or sandstone) & DR & - & 3/16/1950 & 1.35 & - & - & -- & -- & $-\overline{-}$ & Public supply & - \\
\hline
\end{tabular}


Appendix 2. Hydrologic data. - Continued

[Site IDs and well number correspond to locations in Appendix 1; leaders (--), not reported]

\begin{tabular}{|c|c|c|c|c|c|c|c|c|c|c|c|c|c|c|c|c|}
\hline Site ID & Well number & $\begin{array}{l}\text { Reported geologic unit } \\
\text { present in water interval' }\end{array}$ & $\begin{array}{c}\text { Simplified } \\
\text { geologic unit } \\
\text { present in water }^{\text {intervall }}\end{array}$ & $\begin{array}{l}\text { Reported lithology in water- } \\
\text { bearing interval }\end{array}$ & $\begin{array}{l}\text { Interpreted lithologic class in } \\
\text { water-bearing interval }\end{array}$ & Well type ${ }^{5}$ & $\begin{array}{c}\text { Casing } \\
\text { diameter, in } \\
\text { Inches }^{8}\end{array}$ & $\begin{array}{l}\text { Date Water level } \\
\text { Measured? }\end{array}$ & $\begin{array}{l}\text { Water level } \\
\text { distance below } \\
\text { land surface } \\
\text { datum, in } \mathrm{ft}\end{array}$ & $\begin{array}{c}\text { Discharge in } \\
\text { galmmin }\end{array}$ & $\begin{array}{l}\text { Pump test } \\
\text { duration, in } \\
\text { hours }\end{array}$ & $\begin{array}{c}\text { Bailing or } \\
\text { pumping } \\
\text { rate, in } \\
\text { gal/min }\end{array}$ & $\begin{array}{l}\text { Drawdown, in } \\
\mathrm{ft}^{9}\end{array}$ & $\begin{array}{c}\text { Temperature, in } \\
{ }^{\circ} \mathrm{F}\end{array}$ & Use & Comment \\
\hline 321 & 9/7-26G1 & Qyal & Qal & surface & Sand and clay & $\overline{D R}$ & 12 & 10/6/1949 & Flowing & -- & -- & -- & -- & 89.0 & Unused & - \\
\hline $\begin{array}{l}322 \\
323\end{array}$ & $\begin{array}{l}\text { 8/6-14R1 } \\
8 / 6-23 \mathrm{M} 1\end{array}$ & $\begin{array}{l}\text { Qyal } \\
\text { Qyal, Qoal }\end{array}$ & Qal & $\begin{array}{l}\text { soil } \\
\text { clay and boulders }\end{array}$ & $\begin{array}{l}\text { Sand and clay } \\
\text { Clay and gravel }\end{array}$ & $\begin{array}{c}D R \\
D R \\
D R\end{array}$ & $\begin{array}{l}8 \\
8\end{array}$ & $\begin{array}{l}11 / 28 / 1949 \\
10 / 31 / 1949\end{array}$ & $\begin{array}{l}8.28 \\
58.63\end{array}$ & $\because$ & $\because$ & $\overline{1}$ & - & 65.0 & Domestic & - \\
\hline 324 & $7 / 5-15 \mathrm{~A} 1$ & & Qal & $\begin{array}{l}\text { clay, boulders; some water } \\
\text { at } 15 \mathrm{ft.}\end{array}$ & Clay and gravel & DR & 10 & $3 / 28 / 1934$ & &.- & -- & 300 & -- & 71.0 & Irrigation & -. \\
\hline 325 & $7 / 5-14 \mathrm{G} 1$ & Qyal, Qoal & Qal & clay, yellow & Clay & $\mathrm{DR}$ & & $11 / 9 / 1949$ & 11.26 & -- & -- & - & -- & 68.5 & $\begin{array}{l}\text { Irrigation } \\
\text { Inton }\end{array}$ & - \\
\hline 326 & $7 / 5-16\llcorner 1$ & Qyal, Qoal & Qal & clay, yellow; and boulders & Clay and gravel & $\mathrm{DR}$ & 8 & 10/4/1949 & 35 & -- & - & 36 & -- & -- & Domestic & -- \\
\hline 327 & $7 / 5-17 \mathrm{J1}$ & Tsv & Ty & rock, broken, water & Ash or tuff & $\mathrm{DR}$ & $\ldots$ & 10/4/1949 & 607 & $\ldots$ & $\ldots$ & $\ldots$ & $\ldots$ & $\ldots$ & $\begin{array}{l}\text { Domestic, } \\
\text { ivriagtion }\end{array}$ & 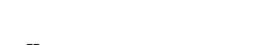 \\
\hline $\begin{array}{l}327 \\
328\end{array}$ & $7 / 5-16 \mathrm{~N} 1$ & $\begin{array}{l}\text { IIV } \\
\text { Qyal, Qoal }\end{array}$ & Qal & $\begin{array}{l}\text { rock, rokoken, water } \\
\text { clay and boulders }\end{array}$ & $\begin{array}{l}\text { Ass or turt } \\
\text { Clay and gravel }\end{array}$ & $\mathrm{DR}$ & -- & $\begin{array}{l}10 / 4 / 1949 \\
10 / 4 / 1949\end{array}$ & $\begin{array}{l}0.17 \\
59\end{array}$ & - & -- & -- & -- & -- & Domestic & -- \\
\hline 329 & $7 / 5-23\llcorner 1$ & Qyal, Qoal & Qal & $\begin{array}{l}\text { soil } \\
\text { soil butices }\end{array}$ & Sand and clay & $\mathrm{DR}$ & $=$ & $10 / 14 / 1949$ & 7.66 & - & - & - & - & $=$ & $\begin{array}{l}\text { Irrigation } \\
\text { Inter }\end{array}$ & - \\
\hline 330 & $\begin{array}{l}7 / 5-27 \mathrm{D} 1 \\
7\end{array}$ & -- & - & - & -- & $D R$ & 8 & $\begin{array}{l}1 / 1 / 1929 \\
-1 / 1745\end{array}$ & - & - & - & - & - & -- & Abandoned & - \\
\hline $\begin{array}{l}331 \\
332\end{array}$ & $\begin{array}{l}\text { 7/4-30L1 } \\
\text { 7/4-31E1 }\end{array}$ & $\ddot{\text { Qyal Qoal }}$ & $\overline{\text { Qal }}$ & $\bar{c}$ clay & $\overline{\text { Clay }}$ & $\begin{array}{l}\mathrm{DR} \\
\mathrm{DR}\end{array}$ & $\begin{array}{c}8 \\
12\end{array}$ & $\begin{array}{l}\text { 12/20/1949 } \\
9 / 30 / 949\end{array}$ & $\overline{9.26}$ & - & - & - & -- & 62.0 & Ünused & - \\
\hline 333 & $7 / 5-36 \mathrm{~N} 1$ & & Qal & clay, brown & & $\mathrm{DR}$ & 8 & 10/13/1949 & 4.17 & $\ldots$ & $\ldots$ & 15 & 6 & $\ldots$ & $\begin{array}{l}\text { Domestic, } \\
\text { irivation }\end{array}$ & $\ldots$ \\
\hline 334 & $6 / 5-1 \mathrm{C3}$ & Qoal & Qal & $\begin{array}{l}\text { Clayy, brown } \\
\text { clay boulders, vellow }\end{array}$ & $\begin{array}{l}\text { Clay } \\
\text { Clay and gravel }\end{array}$ & $\begin{array}{l}D R \\
D R\end{array}$ & $\begin{array}{l}8 \\
8\end{array}$ & $\begin{array}{l}10 / 131 / 19499 \\
12 / 2 / 1949\end{array}$ & $\begin{array}{l}4.111 \\
20.9\end{array}$ & -- & -- & 15 & -. & 60.0 & $\begin{array}{l}\text { irrigation } \\
\text { Domestic }\end{array}$ & $\overline{-}$ \\
\hline 335 & $6 / 4-5 A 1$ & Qyal, Qoal & Qal & $\begin{array}{l}\text { Clay, bunders, veliow } \\
\text { hardpan and clay }\end{array}$ & Clay and gravel & $\mathrm{DR}$ & 16 & $12 / 16 / 1949$ & 7.23 & $=$ & $=$ & - & - & -- & Domestic & - \\
\hline $\begin{array}{l}336 \\
327\end{array}$ & 6/4-5B1 & - & - & - & -- & DR & -- & 1/1/1916 & - & - & - & - & - & - & Abandoned & -- \\
\hline 337 & 6/4-5B2 & & & & & & & & & & & & & -- & Abandoned & \\
\hline $\begin{array}{l}338 \\
339\end{array}$ & $\begin{array}{l}6 / 4-4 E 1 \\
6 / 4-5 J 1\end{array}$ & Qyal & Qal & clay, yellow and boulders & Clay and gravel & $\begin{array}{l}D R \\
D R\end{array}$ & 16 & $\begin{array}{l}\text { 12/6/1949 } \\
1 / 1 / 1916\end{array}$ & 12.04 & -- & $=$ & - & - & $\because$ & $\begin{array}{l}\text { Unused } \\
\text { Abandoned }\end{array}$ & $\begin{array}{l}\text { Reported to be a dry hole } \\
\text { Well destroyed }\end{array}$ \\
\hline 340 & 6/4-6R1 & Qyal & Qal & clay, yellow & Clay & $\mathrm{DR}$ & 26 & 7/27/1948 & 32 & -- & -. & 15 & 15 & -. & $\begin{array}{l}\text { Domestic, dairy, } \\
\text { stock }\end{array}$ & -- \\
\hline & G146P2 & & & & & & & $12 / 6 / 1950$ & & - & 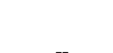 & & $-t$ & & Domestic, dairy, & \\
\hline $\begin{array}{l}341 \\
342\end{array}$ & 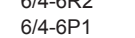 & $\begin{array}{l}\text { Qyal, Qoal } \\
\text { Qyal, Qoal }\end{array}$ & Qal & $\begin{array}{l}\text { Clay, yellow } \\
\text { topsoil }\end{array}$ & $\begin{array}{l}\text { Clay } \\
\text { Sand and clay }\end{array}$ & $\begin{array}{l}\mathrm{DR} \\
\mathrm{DR}\end{array}$ & $\begin{array}{l}12 \\
12\end{array}$ & $\begin{array}{l}1 / 2 / 1 / 1950 \\
12 / 1949\end{array}$ & $\begin{array}{l}10.9 \\
12\end{array}$ & -- & -- & $\begin{array}{l}400 \\
400-125\end{array}$ & 26 & 63.0 & $\begin{array}{l}\text { irrigation, stock } \\
\text { IIriagation }\end{array}$ & $\overline{-}$ \\
\hline 343 & $6 / 4-501$ & Qyal, Qoal & Qal & soil & Sand and clay & $\mathrm{DR}$ & - & $12 / 6 / 1949$ & 8.38 & - & - & - & - & -- & Unused & - \\
\hline 344 & 6/4-17A1 & Qyal, Qoal & Qal & clay, yellow, and gravel & Clay and gravel & $\mathrm{DR}$ & 12 & 10/19/1949 & 16.62 & -- & -- & 250 & -- & -- & Irrigation & -- \\
\hline 345 & 6/4-18A3 & Qyal & Qal & & & $\mathrm{DR}$ & 12 & 3/27/1952 & 12.15 & - & -- & -- & - & 66.5 & Irrigation & -- \\
\hline 346 & $6 / 4-15 Q 1$ & Qoal & Qal & clay and boulders & Clay and gravel & $\mathrm{DR}$ & 8 & 10/13/1949 & 27 & -- & -- & -- & -- & 68.0 & Domestic, stock & -- \\
\hline 347 & $6 / 4-15 Q 3$ & Qoal, Qh (?) & Qal, QTc & clay, and boulders & Clay and gravel & $\mathrm{DR}$ & 8 & $5 / 22 / 1948$ & 12 & -- & -- & -- & -- & -- & Domestic & -- \\
\hline 348 & 6/4-22B3 & - & -- & -- & -- & DR & 8 & 1/1/1948 & -- & -- & -- & -- & -- & -- & Domestic & -- \\
\hline $\begin{array}{l}349 \\
350\end{array}$ & $\begin{array}{l}6 / 4-22 \mathrm{~B} 2 \\
6 / 4-19 \mathrm{~B} 1\end{array}$ & 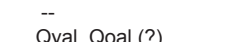 & $\overline{\mathrm{Q}}$ & c- & $\bar{c}$ Clay and aravel & $\begin{array}{l}\mathrm{DR} \\
\mathrm{DR}\end{array}$ & 8 & $\begin{array}{l}1 / 1 / 1948 \\
3 / 27 / 1952\end{array}$ & 20.68 & $-\overline{-}$ l & $-\overline{-}$ & $=$ & $=$ & $=$ & Domestic & $\overline{-}$ \\
\hline $\begin{array}{l}350 \\
351\end{array}$ & $\begin{array}{l}6 / 4-19 \mathrm{BB} 1 \\
6 / 4-23 \mathrm{~F} 1\end{array}$ & $\begin{array}{l}\text { Qyal, Qoal (?) } \\
\text { Oh }\end{array}$ & $\begin{array}{l}\text { Qal } \\
\text { QTc }\end{array}$ & $\begin{array}{l}\text { lay and boulders } \\
\text { rock, gray }\end{array}$ & $\begin{array}{l}\text { Clay and gravel } \\
\text { Ash or tuff }\end{array}$ & $\begin{array}{l}\mathrm{DR} \\
\mathrm{DR}\end{array}$ & 12 & $\begin{array}{l}3 / 27 / 1952 \\
2 / 111950\end{array}$ & $\begin{array}{l}20.68 \\
3394\end{array}$ & $-\overline{-}$ & $-\overline{-}$ & 20 & -- & $-\overline{-}$ l & Stock & Pump dry october 1949 \\
\hline 352 & 6/3-19M1 & $\begin{array}{l}\text { Qh } \\
\text { Tsv }\end{array}$ & $\begin{array}{l}\text { QIV } \\
\text { TV }\end{array}$ & $\begin{array}{l}\text { lock, gray } \\
\text { pumice }\end{array}$ & $\begin{array}{l}\text { Ash or tuff } \\
\text { Ash or tuff }\end{array}$ & $\mathrm{DR}$ & $\overline{8}$ & $\begin{array}{l}\text { 2/17/1950 } \\
4 / 25 / 1949\end{array}$ & $\begin{array}{c}39.94 \\
20\end{array}$ & -- & -- & 10 & 127 & -- & Domestic & formp ary ociover IS49 \\
\hline 353 & $6 / 4-23 \mathrm{~J} 1$ & $\mathrm{Qh}$ & QTC & $\begin{array}{l}\text { soil } \\
\text { soilce }\end{array}$ & Sand and clay & $\mathrm{DR}$ & 18 & $2 / 1 / 1950$ & Flowing & -- & - & 665 & 66 & 85.0 & $\begin{array}{l}\text { Irrigation } \\
\text { Intes }\end{array}$ & -- \\
\hline 354 & 6/4-21Q1 & Qyal & Qal & clay & Clay & $\mathrm{DR}$ & 8 & 6/1/1918 & 13.4 & -- & -- & -- & -- & -- & Abandoned & Well destroyed \\
\hline 355 & $6 / 4-27 \mathrm{C} 2$ & $\ddot{0}$ & $-\overline{0}$ & -- & - & $\mathrm{DR}$ & -- & 1/1/1950 & -- & - & -- & $1 / 2$ & -- & -- & Abandoned & -- \\
\hline $\begin{array}{l}356 \\
357\end{array}$ & 6/4-30C1 & Qoal & Qal & clay & Clay & DR & 8 & $1 / 1 / 1928$ & 31 & -- & -- & -- & - & -- & Domestic & -- \\
\hline $\begin{array}{l}357 \\
358\end{array}$ & $\begin{array}{l}6 / 4-25 B 1 \\
6 / 4-25 B 2\end{array}$ & Tsv & Tv & tuff, blue & Ash or tuff & $\begin{array}{l}D R \\
D R\end{array}$ & 8 & $\begin{array}{l}2 / 9 / 1950 \\
1 / 1 / 1946\end{array}$ & 45 & $=$ & $=$ & - & - & -- & & -- \\
\hline 359 & $6 / 4-25 \mathrm{D} 1$ & Oh & QTC & sandstone, yellow, soft & Sandstone & $\mathrm{DR}$ & 10 & $6 / 1 / 1918$ & Flowing & $\ldots$ & $\ldots$ & $\ldots$ & $\ldots$ & $\ldots$ & $\begin{array}{l}\text { Public swimming } \\
\text { pool }\end{array}$ & .. \\
\hline 360 & $6 / 4-26 \mathrm{H} 1$ & $\mathrm{Qh}$ & QTC & soil & Sand and clay & $\mathrm{DR}$ & - & 3/23/1950 & Flowing & - & - & - & - & 75.0 & Domestic & - \\
\hline $\begin{array}{l}361 \\
362\end{array}$ & $\begin{array}{l}6 / 4-28 F 1 \\
6 / 4-26 F\end{array}$ & Qoal & Qal & clay, yellow & Clay & $\begin{array}{l}D R \\
D R \\
D R\end{array}$ & $\begin{array}{l}8 \\
6\end{array}$ & $\begin{array}{l}2 / 8 / 1950 \\
1 / 1 / 1944\end{array}$ & 3.03 & -- & -- & -- & - & - & $\begin{array}{l}\text { Domestic } \\
\text { Domestic }\end{array}$ & - \\
\hline 363 & 6/4-28K2 & Qoal & Qal & clay, yellow & Clay & $\mathrm{DR}$ & 8 & $7 / 1 / 1950$ & 30 & -- & -- & -- & -- & -- & Domestic, stock & -- \\
\hline 364 & $6 / 4-2612$ & Qoal & Qal & clay & Clay & $D R$ & 6 & $2 / 1 / 1950$ & 1211 & $\ldots$ & $\ldots$ & $\ldots$ & $\ldots$ & $\ldots$ & $\begin{array}{l}\text { Domestic, } \\
\text { irrigation }\end{array}$ & $\ldots$ \\
\hline 365 & $\begin{array}{l}6 / 4-26 L 1 \\
6 / 427\end{array}$ & - & - & - & - & $\mathrm{DR}$ & 6 & $1 / 1 / 1946$ & - & - & - & - & -- & -- & Domestic & -- \\
\hline 366 & 6/4-27M1 & -- & $-\overline{0}$ & $-\overline{-}$ & $-\bar{c}$ & DR & 8 & $\begin{array}{l}1 / 1 / 1948 \\
1 / 121 / 909\end{array}$ & -- & - & - & - & -- & - & Domestic & - \\
\hline
\end{tabular}


Appendix 2. Hydrologic data. - Continued

[Site IDs and well number correspond to locations in Appendix 1; leaders (--), not reported]

\begin{tabular}{|c|c|c|c|c|c|c|c|c|c|c|c|c|c|c|c|c|}
\hline Site ID & Well number & $\begin{array}{l}\text { Reported geologic unit } \\
\text { present in water interval' }\end{array}$ & $\begin{array}{l}\text { Simplified } \\
\text { geologic unit } \\
\text { present in water } \\
\text { intervall }^{2}\end{array}$ & $\begin{array}{l}\text { Reported lithology in water- } \\
\text { bearing interval }\end{array}$ & $\begin{array}{l}\text { Interpreteded lithologic class in } \\
\text { water-bearing interval| }\end{array}$ & Well type & $\begin{array}{l}\text { Casing } \\
\text { diameter, in } \\
\text { inches } s^{6}\end{array}$ & $\begin{array}{l}\text { Date Water level } \\
\text { Measured }^{7}\end{array}$ & $\begin{array}{l}\text { Water level } \\
\text { distance below } \\
\text { land surface } \\
\text { datum, in ft }\end{array}$ & $\begin{array}{c}\text { Discharge, in } \\
\text { galmin }\end{array}$ & $\begin{array}{l}\text { Pump test } \\
\text { duration, in } \\
\text { hours }\end{array}$ & $\begin{array}{l}\text { Bailling or } \\
\text { pumping } \\
\text { rate, in } \\
\text { gal/min }\end{array}$ & $\underset{\mathrm{ft}}{\text { Drawdown, in }}$ & $\begin{array}{c}\text { Temperature, in } \\
{ }_{\mathrm{F}}\end{array}$ & Use & Comment \\
\hline 368 & 6/4-27N1 & Qoal & Qal & -- & - & $\mathrm{DR}$ & 8 & 11/21/1949 & 24.12 & - & - & - & - & - & Domestic, stock & - \\
\hline 369 & 6/4-28Q1 & Qoal & Qal & -- & $-\bar{c}$ & DR & - & 1/1/19930 & 20 & -- & - & - & - & - & Unused & - \\
\hline $\begin{array}{l}370 \\
371\end{array}$ & $\begin{array}{l}\text { 6/4-34D2 } \\
664-36 \mathrm{C} 1\end{array}$ & Qoal & Qal & clay, yellow & Clay & DR & 8 & $\begin{array}{l}10 / 12 / 1949 \\
41 / 1948\end{array}$ & 13.72 & -- & - & -- & -- & - & $\begin{array}{l}\text { Unused } \\
\text { Uriciontipon }\end{array}$ & -- \\
\hline 372 & $\begin{array}{l}6 / 4-36 \mathrm{CC} 1 \\
6 / 4-35 \mathrm{G} 2\end{array}$ & Qyal (?) & Qal & $-\bar{x}$ & -- & $\begin{array}{l}D R \\
D R\end{array}$ & ${ }_{8}^{10}$ & $\begin{array}{l}4 / 1 / 1948 \\
1 / 1 / 1948\end{array}$ & 9.9 & $\overline{--}$ & -- & 150 & 113 & $\overline{-}$ & $\begin{array}{l}\text { Irrigatiton } \\
\text { Domestic }\end{array}$ & $\overline{-}$ \\
\hline 373 & $6 / 4-3563$ & Qoal & Qal & surface & Sand and clay & $D R$ & 6 & $1 / 1 / 31 / 1950$ & 8.11 & - & - & - & - & - & Domestic & -- \\
\hline $\begin{array}{l}374 \\
375\end{array}$ & $\begin{array}{l}6 / 4-35 \mathrm{G} 4 \\
6 / 4-33\llcorner 2\end{array}$ & Qoal & Qal & surface & Sand and clay & $\begin{array}{l}\mathrm{DR} \\
\mathrm{DR}\end{array}$ & $\begin{array}{l}8 \\
8\end{array}$ & $\begin{array}{l}3 / 27 / 1952 \\
1 / 1 / 1931\end{array}$ & 2.64 & -- & -- & 50 & 30 & -- & $\begin{array}{l}\text { irrigation, } \\
\text { Domestic }\end{array}$ & - \\
\hline 376 & 6/4-35ட2 & -- & -- & -- & -- & $\mathrm{DR}$ & 8 & 1/12/1950 & -- & -- & -- & -- & -- & -- & Domestic, stock & -- \\
\hline $\begin{array}{l}377 \\
378\end{array}$ & $\begin{array}{l}\text { 6/4-36J1 } \\
\text { 6/4-36L1 }\end{array}$ & - & -- & $\begin{array}{l}\text { boulders and conglomerate } \\
--\end{array}$ & $\begin{array}{l}\text { Clay, sand, and gravel } \\
\text {-- }\end{array}$ & $\begin{array}{l}\mathrm{DR} \\
\mathrm{DR}\end{array}$ & $\frac{8}{--}$ & $\begin{array}{l}2 / 2 / 1950 \\
1 / 1 / 1948\end{array}$ & 41.85 & -- & -- & - & - & $-\overline{63.0}$ & $\begin{array}{l}\text { Domestic } \\
\text { Domestic }\end{array}$ & - \\
\hline $\begin{array}{l}379 \\
380 \\
381\end{array}$ & $\begin{array}{l}6 / 4-3251 \\
6 / 4-3311 \\
6 / 4-35 P 2\end{array}$ & $\begin{array}{l}\text { Qyal, Qool } \\
\text { Qyal, Qool } \\
\text { Qyal, Qoal }\end{array}$ & $\begin{array}{l}\text { Qal } \\
\text { Qal } \\
\text { Qal }\end{array}$ & $\begin{array}{l}\text { clay, yellow, sand and } \\
\text { gravel } \\
\text { clay, yellow } \\
\text { sand loam }\end{array}$ & $\begin{array}{l}\text { Clay, sand, and gravel } \\
\text { Clay } \\
\text { Clay and gravel }\end{array}$ & $\begin{array}{l}D R \\
D R \\
D R\end{array}$ & $\begin{array}{l}8 \\
8 \\
8\end{array}$ & $\begin{array}{l}10 / 12 / 1949 \\
10 / 12194949 \\
6 / 11 / 1950\end{array}$ & $\begin{array}{l}43.68 \\
13.64 \\
17.76\end{array}$ & $\overline{-}$ & $\overline{-}$ & $\overline{-}$ & $\overline{-}$ & $\overline{-}$ & $\begin{array}{l}\text { Domestic } \\
\text { Unused } \\
\text { Irrigation }\end{array}$ & $\overline{-}-$ \\
\hline 382 & $6 / 4-32 \mathrm{~N} 1$ & KJu & KJu & shale, blue & Clay and sand (or sandstone) & $\mathrm{DR}$ & 8 & 10/11/1949 & 49.03 & -- & -- & - & -- & -- & Unused & - \\
\hline 383 & 6/4-3501 & -- & -- & -- & -- & DR & 8 & 1/1/1937 & -- & .- & -- & 50 & -- & .- & $\begin{array}{l}\text { Domesstio, } \\
\text { irrigation }\end{array}$ & -- \\
\hline 384 & $6 / 4-35 R 1$ & Tsv & Tv & volcanic rock, hard, red & Ash or tuff & DR & 6 & 2/8/1950 & 150 & - & - & 10 & -- & -- & Domestic & -- \\
\hline 385 & $5 / 4-2 B 1$ & -- & - & -- & -- & $\mathrm{DR}$ & -- & - تими & -- & -- & - & -- & -- & -- & Domestic & -- \\
\hline $\begin{array}{l}386 \\
387\end{array}$ & $\begin{array}{l}5 / 4-1 \mathrm{G} 1 \\
5 / 4-5 \mathrm{H} 4\end{array}$ & $\overline{-}$ & $\overline{-}$ & $-\overline{-}$ & $=$ & $\begin{array}{l}\mathrm{DR} \\
\mathrm{DR}\end{array}$ & 8 & 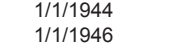 & - & $\overline{-}$ & $\overline{-}$ & $\overline{-}$ & $\overline{-}$ & $\overline{-}$ & $\begin{array}{l}\text { Domesstic } \\
\text { Domestic }\end{array}$ & $\overline{-}$ \\
\hline 388 & 5/3-6M2 & Tsv & Tv & rock, blue & Ash or tuff & $\mathrm{DR}$ & 6 & 1/30/1950 & 48.52 & -- & -- & -- & -- & -- & Domestic & $\begin{array}{l}\text { Water reported to have } \\
\text { strong "sulfur" tasste. }\end{array}$ \\
\hline $\begin{array}{l}389 \\
390\end{array}$ & $\begin{array}{l}5 / 3-6 \mathrm{M} 1 \\
5 / 4-3 \mathrm{M} 1\end{array}$ & $\overline{-}$ & -- & -- & -- & $\begin{array}{l}\mathrm{DR} \\
\mathrm{DR}\end{array}$ & 8 & $\frac{1 / 1 / 1930}{-}$ & $\overline{--}$ & $\overline{-}-$ & -- & - & -- & - & \multirow{2}{*}{$\begin{array}{l}\text { Domestic, stock } \\
\text { Abandoned } \\
\text { Domestic, } \\
\text { irrigation }\end{array}$} & \multirow{2}{*}{$\begin{array}{l}-\overline{-} \\
\text { Water Reported to have } \\
\text { high iron content. }\end{array}$} \\
\hline 391 & 5/3-5M2 & -- & -- & -- & -- & $\mathrm{DR}$ & 8 & 1/1/1948 & -- & -- & -- & -- & -- & -- & & \\
\hline 392 & $5 / 3-6 / 1$ & Tsv & Tv & $\begin{array}{l}\text { diatomite } \\
\text { dav }\end{array}$ & Clay and sand (or sandstone) & DR & 10 & $5 / 22 / 1951$ & 27.36 & -- & -- & $\ddot{24}$ & 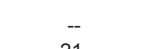 & -- & - & \multirow{3}{*}{$\begin{array}{l}-- \\
\text { Water Reported to have } \\
\text { high iron content. }\end{array}$} \\
\hline 393 & $5 / 4-4 K 2$ & Qyal, Qoal & Qal & clay, yellow & Clay & $\mathrm{DR}$ & 8 & 12/18/1948 & 20 & -- & -- & 24 & 21 & -- & Domestic & \\
\hline 394 & $5 / 3-5 N 3$ & Tsv & Tv & rock and lava & Ash or tuff & $\mathrm{DR}$ & 8 & 6/1/1951 & 81.8 & -- & -- & -- & -- & -- & Domestic & \\
\hline 395 & 5/3-5P1 & .- & -- & -- & -- & $\mathrm{DR}$ & .- & 1/1/1941 & & $\ldots$ & $\ldots$ & $\ldots$ & .- & .- & $\begin{array}{l}\text { Domestio, } \\
\text { irrigation }\end{array}$ & \multirow{3}{*}{ 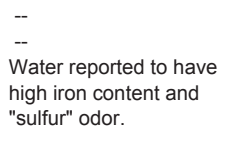 } \\
\hline 396 & $5 / 4-5 R 1$ & Qyal, Qoal & Qal & clay, yellow & Clay & $\mathrm{DR}$ & 8 & $6 / 1 / 1951$ & 10.04 & -- & - & - & - & -- & Domestic & \\
\hline 397 & $5 / 3-6 \mathrm{~N} 2$ & Tsv & Tv & chalk rock (diatomite) & Clay and sand (or sandstone) & $\mathrm{DR}$ & 8 & 12/1/1945 & 21 & 18 & -- & - & - & 64.0 & Domestic & \\
\hline 398 & $5 / 4-5 Q 2$ & Qyal & Qal & clay, blue, and silt & Clay and sand (or sandstone) & $\mathrm{DR}$ & 8 & $3 / 16 / 1951$ & 6.94 & -- & -- & 5 & -- & -- & Domestic & -. \\
\hline 399 & $5 / 4-1 R 1$ & $\therefore$ & -- & - & - & DR & 6 & 1/1/1946 & $-\overline{-}$ r & $-\overline{-}$ & $-\overline{-}$ & -- & $-\overline{-}$ - & $-\overline{-}$ & $\begin{array}{l}\text { Domestic } \\
\text { Domestitis }\end{array}$ & - \\
\hline 400 & 5/3-6Q1 & -- & -- & -- & -- & & & 1/1/1/1950 & - & - & - & -- & - & -- & $\begin{array}{l}\text { Domessic } \\
\text { Domestic, }\end{array}$ & - \\
\hline 401 & $5 / 3-5 N 1$ & -- & - & - & -- & DR & 8 & 1/1/1948 & - & - & - & -- & - & $\because$ & irrigation & - \\
\hline 402 & $5 / 3-6 \mathrm{~N} 1$ & Tsv & Tv & rock, blue & Ash or tuff & $\mathrm{DR}$ & 6 & 1/11/1950 & 52.84 & - & - & -- & - & 66.0 & Domestic, dairy & - \\
\hline 403 & $5 / 3-6 \mathrm{~N} 3$ & Tsv & Tv & rock and clay & Clay and gravel & DR & $-\overline{8}$ & 2/9/1950 & $\begin{array}{l}2.32 \\
30.37\end{array}$ & -- & -- & -- & -- & -- & Domestic & - \\
\hline $\begin{array}{l}404 \\
405\end{array}$ & 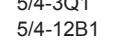 & $\begin{array}{l}\text { Qyal } \\
\text { Tsv }\end{array}$ & Trv & $\begin{array}{l}\text { dug well not described } \\
\text { rock, hard }\end{array}$ & $\begin{array}{l}\text { No data } \\
\text { Ash or tuff }\end{array}$ & $\begin{array}{l}\mathrm{DR} \\
\mathrm{DR}\end{array}$ & $\frac{8}{6}$ & $\begin{array}{l}2 / 10 / 1948 \\
3 / 14 / 4950\end{array}$ & $\begin{array}{l}39.31 \\
29.3\end{array}$ & $=-$ & $\overline{--}$ & $-\overline{-}$ & -- & -- & $\begin{array}{l}\text { Irligation } \\
\text { Domestic }\end{array}$ & -- \\
\hline 406 & $5 / 3-8 \mathrm{C} 1$ & Tsv & Tv & soil & Sand and clay & $\mathrm{DR}$ & 6 & $2 / 15 / 1950$ & Flowing & -- & -- & - & -- & -- & Domestic & - \\
\hline 407 & $5 / 3-7 \mathrm{D} 1$ & $-\overline{-}$ & - & - & & DR & 10 & 1/1/1/1950 & - & - & - & - & -- & - & Domestic & -- \\
\hline 408 & $\begin{array}{l}5 / 4-9 \mathrm{D} 1 \\
5\end{array}$ & $\begin{array}{l}\text { Tsv } \\
\text { Tsv }\end{array}$ & $\begin{array}{l}\text { Tv } \\
\text { Tv }\end{array}$ & $\begin{array}{l}\text { tuff } \\
\text { tuff brown }\end{array}$ & $\begin{array}{l}\text { Ash or tuff } \\
\text { Ash or tuff }\end{array}$ & $\begin{array}{l}\mathrm{DR} \\
\mathrm{DR}\end{array}$ & $\begin{array}{l}6 \\
8\end{array}$ & $2 / 2 / 1950$ & 25.32 & -- & -- & $-\overline{-}$ & -- & -- & Domestic & - \\
\hline 409 & 5/4-12E1 & & & tuff, brown & Ash or tuff & DR & 8 & 12/30/1949 & 30 & & & & -- & -- & Domestic & -- \\
\hline
\end{tabular}


Appendix 2. Hydrologic data. - Continued

[Site IDs and well number correspond to locations in Appendix 1; leaders (--), not reported]

\begin{tabular}{|c|c|c|c|c|c|c|c|c|c|c|c|c|c|c|c|c|}
\hline Site ID & Well number & $\begin{array}{l}\text { Reported geologic unit } \\
\text { present in water interval' }\end{array}$ & $\begin{array}{c}\text { Simplified } \\
\text { geologic unit } \\
\text { present in water }^{\text {interval| }}\end{array}$ & $\begin{array}{l}\text { Reported lithology in water- } \\
\text { bearing interval }\end{array}$ & $\begin{array}{l}\text { Interpretede lithologic class in } \\
\text { water-bearing interval }\end{array}$ & Well type ${ }^{5}$ & $\begin{array}{l}\text { Casing } \\
\text { diameter, in } \\
\text { inches }{ }^{8}\end{array}$ & $\begin{array}{l}\text { Date Water level } \\
\text { Measured }^{7}\end{array}$ & $\begin{array}{l}\text { Water level } \\
\text { distance below } \\
\text { land surface } \\
\text { datum, in ft }\end{array}$ & $\begin{array}{c}\text { Discharge in } \\
\text { galmin }\end{array}$ & $\begin{array}{l}\text { Pump test } \\
\text { duration, in } \\
\text { hours }\end{array}$ & $\begin{array}{c}\text { Bailing or } \\
\text { pumping } \\
\text { rate, in } \\
\text { gal/minn }\end{array}$ & $\begin{array}{l}\text { Drawdown, in } \\
\mathrm{ft}^{9}\end{array}$ & $\begin{array}{c}\text { Temperature, in } \\
{ }^{\circ} \mathrm{F}\end{array}$ & Use & Comment \\
\hline 410 & 5/3-7E3 & -- & -. & -- & -- & $\mathrm{DR}$ & 12 & $2 / 19 / 1900$ & & 35 & -- & -- & 17 & -- & Domestic & $\begin{array}{l}\text { High iron and } \mathrm{H} 2 \mathrm{~S} \\
\text { reported }\end{array}$ \\
\hline 411 & 5/4-11E1 & Qyal, Qoal & Qal & clay & Clay & -- & - & $6 / 1 / 1918$ & Flowing & - & - & - & -- & - & Abandoned & \\
\hline 412 & 5/4-9E1 & Tsy & Ty & lava & Basalt & $\mathrm{DP}$ & 12 & $1 / 1 / 1923$ & 40 & $\ldots$ & 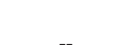 & 150 & 140 & 680 & $\begin{array}{l}\text { Domestic, } \\
\text { irrication }\end{array}$ & $\begin{array}{l}\text { Water reported to have } \\
\text { high inontont }\end{array}$ \\
\hline $\begin{array}{l}412 \\
413\end{array}$ & $\begin{array}{l}5 / 4-4-12 \sqrt{2} \\
5 / 4-12\end{array}$ & $\begin{array}{l}\text { ISv } \\
\text { Tsv }\end{array}$ & $\operatorname{TV}_{\mathrm{TV}} \mathrm{r} \mathrm{c}$ & $\begin{array}{l}\text { lava } \\
\text { clay }\end{array}$ & $\begin{array}{l}\text { Basayt } \\
\text { Clay }\end{array}$ & DR & $\begin{array}{c}12 \\
8\end{array}$ & $\begin{array}{l}1 / 1 / 17 / 1950 \\
1 / 1950\end{array}$ & $\begin{array}{l}40 \\
31.97\end{array}$ & -- & -- & 150 & 140 & 68.0 & $\begin{array}{l}\text { Irfigation } \\
\text { Domestic }\end{array}$ & \\
\hline 414 & 5/4-9K2 & Qyal, Qoal & Qal & surface & Sand and clay & $\mathrm{DR}$ & 108 & $2 / 16 / 1950$ & 5.3 & -- & -- & -- & - & -- & $\begin{array}{l}\text { Irrigation } \\
\text { In }\end{array}$ & -- \\
\hline 415 & $5 / 4-11\llcorner 3$ & & Qal & sand and clay & Sand and clay & $\mathrm{DR}$ & 8 & 6/1/1951 & 27.19 & -- & -- & -- & -- & -- & Domestic & -- \\
\hline 416 & 5/4-9L1 & -- & -- & - & -- & $\mathrm{DR}$ & - & 1/1/1929 & -- & -- & -- & -- & -- & -- & Domestic & -- \\
\hline 417 & $5 / 4-9 \mathrm{Q} 1$ & -- & -- & $-\overline{-}$ & -- & DR & 6 & 1/1/1946 & -- & -- & -- & -- & -- & -- & Domestic & -- \\
\hline $\begin{array}{l}418 \\
419\end{array}$ & $\begin{array}{l}5 / 4-10 \mathrm{PP} 4 \\
5 / 4-14 \mathrm{C} 1\end{array}$ & Qoal & $\overline{\text { Qal }}$ & clay, yellow & $\overline{\text { Clay }}$ & $\begin{array}{l}\mathrm{DR} \\
\mathrm{DR}\end{array}$ & - & $\overline{12 / 2 / 1949}$ & 12.86 & -- & - & - & - & -- & $\begin{array}{l}\text { Abandoned } \\
\text { Domestic dairy }\end{array}$ & -- \\
\hline $\begin{array}{l}419 \\
420\end{array}$ & $\begin{array}{l}5 / 4-14 \mathrm{C} 1 \\
5 / 4-15 \mathrm{C} 2\end{array}$ & Qoal & Qal & clay, yellow & Clay & $\begin{array}{l}\text { DR } \\
D R\end{array}$ & $\overline{8}$ & $\begin{array}{l}12 / 2 / 1949 \\
2 / 2 / 1951\end{array}$ & $\begin{array}{l}12.86 \\
16.02\end{array}$ & - & 24 & $\overline{15}$ & - & -- & $\begin{array}{l}\text { Domestic, dairy } \\
\text { Irrigation }\end{array}$ & -- \\
\hline 421 & $\begin{array}{l}\text { S/4-10C2 } \\
5 / 3-17 C 1\end{array}$ & Tsv & $\overline{\mathrm{TV}}$ & rock, hard & Ash or tuff & $\mathrm{DR}$ & -. & $\begin{array}{l}9 / 21 / 1948 \\
9\end{array}$ & 100 & -- & 24. & -. & -- & -- & Domestic & -- \\
\hline 422 & $5 / 4-13 E 1$ & Qoal & Qal & hardpan & Clay and gravel & $D R$ & -- & 2/2/1950 & 35.2 & -- & -- & -- & -- & -- & Domestic & -- \\
\hline $\begin{array}{l}423 \\
424\end{array}$ & $\begin{array}{l}5 / 4-14 \mathrm{H} 3 \\
5 / 4-17 \mathrm{~F} 1\end{array}$ & Qoal & Qal & clay & Clay & $\begin{array}{l}\mathrm{DR} \\
\mathrm{DR}\end{array}$ & $\begin{array}{l}8 \\
6\end{array}$ & $\begin{array}{l}2 / 2 / 1950 \\
1 / 1 / 1938\end{array}$ & 57.83 & -- & -- & -- & $\because$ & -- & $\begin{array}{l}\text { Public supply } \\
\text { Domestic }\end{array}$ & -- \\
\hline 425 & 5/4-13L1 & Qoal & Qal & clay, blue, sandy & Clay and sand (or sandstone) & $\mathrm{DR}$ & 15 & 10/17/1942 & 123.4 & $\ldots$ & $\ldots$ & 225 & 40 & $\ldots$ & Irrigation & .- \\
\hline 426 & $5 / 4-14 \mathrm{~L} 2$ & Qoal & Qal & $\begin{array}{l}\text { gravel and clay } \\
\text { clay blue gravel: and }\end{array}$ & Clay and gravel & $\mathrm{DR}$ & 8 & 6/1/1918 & Flowing & -- & -- & - & - & -- & $\begin{array}{l}\text { Abandoned } \\
\text { Public supoly }\end{array}$ & -- \\
\hline 427 & $5 / 4-14\llcorner 1$ & Qoal & Qal & boulders & Clay and gravel & $\mathrm{DR}$ & 20 & 10/17/1942 & 56.8 & - & - & - & - & - & $\begin{array}{l}\text { irrigation } \\
\text { ingly }\end{array}$ & - \\
\hline 428 & $5 / 4-15 \mathrm{~K} 1$ & Qyal, Qoal & Qal & clay, blue & Clay & $\mathrm{DR}$ & 12 & $12 / 21 / 1949$ & 48.31 & - & - & - & -- & - & $\begin{array}{l}\text { Unused } \\
\text { Unusice }\end{array}$ & -- \\
\hline 429 & 5/4-14P1 & Qoal & Qal & boulders & Gravel & $\mathrm{DR}$ & 14 & 1/4/1950 & 65 & -- & -- & 430 & 11 & 80.0 & $\begin{array}{l}\text { ubirc supply, } \\
\text { irrigation }\end{array}$ & -- \\
\hline 430 & $5 / 4-15 P 2$ & - & - & - & - & $\mathrm{DR}$ & - & 1/1/1926 & - & -- & - & - & -- & -- & Unused & -- \\
\hline 431 & $5 / 4-21 \mathrm{~A} 1$ & -- & -- & -- & - & $\mathrm{DR}$ & 12 & 12/23/1949 & -- & -- & -- & 400 & - & -- & Stock, irrigation & -- \\
\hline $\begin{array}{l}432 \\
433\end{array}$ & $\begin{array}{l}5 / 4-19 \mathrm{~J} 1 \\
5 / 4-19 \mathrm{~J} 2\end{array}$ & $\begin{array}{l}\text { Qt } \\
Q \mathrm{Qt}\end{array}$ & $\begin{array}{l}\text { Qal } \\
\text { Qal }\end{array}$ & $\begin{array}{l}\text { soil } \\
\text { clay, blue }\end{array}$ & $\begin{array}{l}\text { Sand and clay } \\
\text { Clay }\end{array}$ & $\begin{array}{l}\mathrm{DR} \\
\mathrm{DR}\end{array}$ & $\begin{array}{l}6 \\
8\end{array}$ & $\begin{array}{l}1 / 31 / 1950 \\
1 / 31 / 1950\end{array}$ & $\begin{array}{l}2.44 \\
2.64\end{array}$ & -- & -- & $\begin{array}{l}-- \\
--\end{array}$ & -- & -- & $\begin{array}{l}\text { Domestic } \\
\text { Domestic }\end{array}$ & -- \\
\hline $\begin{array}{l}434 \\
435\end{array}$ & $\begin{array}{l}5 / 4-21 \mathrm{P} 2 \\
554-19 \mathrm{R} 1\end{array}$ & Qt & Qal & $\begin{array}{l}\text { shale, black } \\
\text { soil }\end{array}$ & Clay and sand (or sandstone) & $\begin{array}{l}\mathrm{DR} \\
\mathrm{DR}\end{array}$ & 8 & 3/26/1952 & $\begin{array}{l}15.67 \\
0\end{array}$ & -- & -- & - & - & -- & $\begin{array}{l}\text { Domestic } \\
\text { Unused }\end{array}$ & -- \\
\hline 436 & 5/4-19Q1 & Qyal & Qal & silt black & Clay and sand (or sandstone) & $\mathrm{DR}$ & 8 & 1/27/1950 & 16.46 & .- & .- & -- & -. & -- & -- & -- \\
\hline 437 & $5 / 4-27 \mathrm{~A} 1$ & Qoal & Qal & soil and aravel & $\begin{array}{l}\text { Sand (or sandstone) and } \\
\text { gravel }\end{array}$ & $\mathrm{DR}$ & 12 & $9 / 21 / 1920$ & Flowing & $\ldots$ & $\ldots$ & $\ldots$ & $\ldots$ & $\ldots$ & Unused & $\ldots$ \\
\hline & 5 & & & Sit blo & Chen & ל קח & 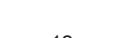 & (15) & $-\mathrm{s}$ & & & 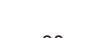 & & 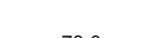 & Domestic, dairy, & \\
\hline 年38 & $\begin{array}{l}5 / 4-30 \mathrm{~B} 2 \\
55-26 \mathrm{~B} 1\end{array}$ & Qyal & Qal & silt, black & Clay and sand (or sandstone) & $\begin{array}{l}\mathrm{DR} \\
\mathrm{DR}\end{array}$ & 12 & 1/10/1947 & Flowing & -- & - & 30 & - & 73.0 & stock & - \\
\hline${ }_{440}^{439}$ & $\begin{array}{l}\text { /54-26B? } \\
5 / 4-26 \mathrm{E} 1\end{array}$ & Qoal & $\ddot{\text { Qal }}$ & clay blue, and gravel & $\bar{c}$ cay and aravel & $\begin{array}{l}\mathrm{DR} \\
\mathrm{DR}\end{array}$ & 20 & 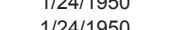 & 72.74 & - & - & - & - & 92.0 & Public supply & -- \\
\hline 441 & $5 / 4-27 \mathrm{H} 2$ & Qyal & Qal & clay, yellow; and gravel & $\begin{array}{l}\text { Clay and gravel } \\
\text { Clay and gravel }\end{array}$ & $\begin{array}{l}\text { DR } \\
D R\end{array}$ & $\begin{array}{l}15.5 \\
12\end{array}$ & 11/24/1950 & 62.52 & - & -- & - & -- & 82.0 & $\begin{array}{l}\text { Public supply } \\
\text { Puts }\end{array}$ & - \\
\hline 442 & $5 / 5-26 \mathrm{G} 1$ & Tsv & Tv & rock, blue tuff, water & $\begin{array}{l}\text { Clay and gravel } \\
\text { Sandstone }\end{array}$ & $\begin{array}{l}\mathrm{DR} \\
\mathrm{DR}\end{array}$ & $\begin{array}{c}12 \\
6\end{array}$ & $\begin{array}{l}1 / 24 / 1950 \\
1 / 26 / 1950\end{array}$ & 64.13 & - & - & - & - & 68.0 & Puolic suppiy & - \\
\hline 443 & $5 / 4-27 \mathrm{H} 1$ & & & -- & Sanastone & $\mathrm{DR}$ & $\begin{array}{l}0 \\
16\end{array}$ & $1 / 1 / 1 / 1923$ & $3 r .4$ & - & - & - & - & - & s. & - \\
\hline 444 & $5 / 4-29 F 1$ & $\mathrm{Qh}$ & QTC & clay blue & Clay & $\mathrm{DR}$ & $\begin{array}{c}10 \\
8\end{array}$ & $2 / 2 / 1951$ & 58.72 & -- & -- & -- & -- & -- & $\begin{array}{l}\text { Unusea } \\
\text { Domestic }\end{array}$ & -- \\
\hline 445 & $5 / 4-27 \mathrm{~K} 2$ & Qyal, Qoal & Qal & & Sand and clay & $D R$ & 12 & $\begin{array}{l}9 / 5 / 1919 \\
9 / 219\end{array}$ & Flowing & - & - & -- & - & - & Abandoned & -- \\
\hline 446 & $5 / 4-26 \mathrm{M} 1$ & Qyal, Qoal & Qal & clay, yellow, and gravel & Clay and gravel & $\mathrm{DR}$ & 15.5 & 3/23/1942 & 35.3 & -- & -- & -- & -- & -- & Unused & \\
\hline 447 & $5 / 4-27 K 1$ & Qyal, Qoal & Qal & adobe, black & Sand and clay & DR & 12 & 1/1/1919 & Flowing & -- & -- & -- & -- & -- & Abandoned & Well Plugged in 1931 \\
\hline 448 & $\begin{array}{l}\text { 5/4-26M2 } \\
5 / 4-29 k 1\end{array}$ & Qyal & Qal & soil & Sand and clay & $\begin{array}{l}\text { DR } \\
D R\end{array}$ & 20 & $\begin{array}{l}1 / 24 / 1950 \\
1 / 11 / 139\end{array}$ & 2.17 & - & - & - & -- & - & $\begin{array}{l}\text { Unused } \\
\text { hand }\end{array}$ & Well probably plugged \\
\hline $\begin{array}{l}449 \\
450\end{array}$ & $\begin{array}{l}5 / 4-29 \mathrm{~K} 1 \\
5 / 4-26 \mathrm{~N} 1\end{array}$ & Qoal & Qal & clay and gravel & $\overline{\text { Clay and gravel }}$ & $\begin{array}{l}D R \\
D R\end{array}$ & $\overline{20}$ & $\begin{array}{l}1 / 1 / 11939 \\
1 / 24 / 1950\end{array}$ & --.5 & - & -- & -- & -- & - & $\begin{array}{l}\text { Abandoned } \\
\text { Unused }\end{array}$ & Well destroyed \\
\hline 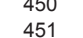 & $\begin{array}{l}5 / 4-26 \mathrm{~N} 1 \\
5 / 4-29 \mathrm{~N} 2\end{array}$ & Qoal & Qal & clay and gravel & $\begin{array}{l}\text { Clay and gravel } \\
\text {-- }\end{array}$ & & 20 & 1/24/1950 & 40.5 & -- & -- & -- & -- & $\overline{-}$ & Unused & $\overline{-}$ \\
\hline 452 & 5/4-30R1 & Qal, Qh & Qal, QTc & clay, yellow & Clay & $\mathrm{DR}$ & 12 & $1 / 26 / 1950$ & Flowing & -- & -- & -- & -- & 68.0 & Unused & -- \\
\hline 453 & $5 / 4-29 \mathrm{~N} 1$ & & & - & -- & Dug & - & $1 / 26 / 1950$ & 13.91 & -- & -- & -- & -- & - & - & -- \\
\hline 454 & 5/4-29Q1 & -- & -- & -- & -- & DR & - & 1/1/1946 & -- & -- & -- & -- & -- & -- & Abandoned & Well destroyed \\
\hline 455 & $5 / 4-29 Q 2$ & -- & -- & -- & -- & DR & 8 & $\begin{array}{l}1 / 1 / 1946 \\
\end{array}$ & -- & -- & -- & -- & -- & -- & Domestic & - \\
\hline $\begin{array}{l}456 \\
457\end{array}$ & $\begin{array}{l}5 / 4-27 \mathrm{R} 1 \\
5 / 4-30 \mathrm{P} 1\end{array}$ & $\overline{\mathrm{oh}}$ & $\ddot{\text { QTC }}$ & surface & Sand and clay & $\begin{array}{l}\mathrm{DR} \\
\mathrm{DR}\end{array}$ & $\begin{array}{l}20 \\
10\end{array}$ & $\begin{array}{l}5 / 71 / 1931 \\
1 / 26 / 1950\end{array}$ & $10-25$ & -- & 270 & -- & $-\overline{-}$ & -- & $\begin{array}{l}\text { Unused } \\
\end{array}$ & - \\
\hline $\begin{array}{l}45 / \\
458\end{array}$ & $\begin{array}{l}\text { SI4-30PT } \\
5 / 4-27 Q 1\end{array}$ & Qyal & $\begin{array}{l}\text { QIC } \\
\text { Qal }\end{array}$ & $\begin{array}{l}\text { surrace } \\
\text { hard gravel, salt water }\end{array}$ & $\begin{array}{l}\text { Sand and clay } \\
\text { Gravel }\end{array}$ & DR & $\begin{array}{l}10 \\
8\end{array}$ & $\begin{array}{l}1 / 26 / 191 / 190 \\
12 / 29\end{array}$ & $\begin{array}{l}10.25 \\
1851\end{array}$ & - & -- & -- & - & - & $\begin{array}{l}\text { Unused } \\
\text { Unused }\end{array}$ & -- \\
\hline $\begin{array}{l}450 \\
459\end{array}$ & $5 / 4-27 R 2$ & - & Qal & nard gravel, salt water & Gravel & DR & $\begin{array}{l}8 \\
10\end{array}$ & $\begin{array}{l}1 / 21 / 1119309 \\
1 / 1930\end{array}$ & 18.51 & -- & -- & -- & -- & $-\overline{-}$ & $\begin{array}{l}\text { Unusea } \\
\text { Unused }\end{array}$ & -- \\
\hline
\end{tabular}


Appendix 2. Hydrologic data. - Continued

[Site IDs and well number correspond to locations in Appendix 1; leaders (--), not reported]

\begin{tabular}{|c|c|c|c|c|c|c|c|c|c|c|c|c|c|c|c|c|}
\hline Site ID & Well number & $\begin{array}{l}\text { Reported geologic unit } \\
\text { present in water interval' }\end{array}$ & $\begin{array}{c}\text { Simplified } \\
\text { geologic unit } \\
\text { present in water }^{\text {interval| }}\end{array}$ & $\begin{array}{l}\text { Reported lithology in water- } \\
\text { bearing interval }\end{array}$ & $\begin{array}{l}\text { Interprotede lithologic class in } \\
\text { water-bearing interval }\end{array}$ & Well type ${ }^{5}$ & $\begin{array}{c}\text { Casing } \\
\text { diameter, in } \\
\text { inches }^{8}\end{array}$ & $\begin{array}{l}\text { Date Water level } \\
\text { Measured? }\end{array}$ & $\begin{array}{l}\text { Water level } \\
\text { distance below } \\
\text { land surface } \\
\text { datum, in ft }\end{array}$ & $\begin{array}{c}\text { Discharge, in } \\
\text { gal/min }\end{array}$ & $\begin{array}{l}\text { Pump test } \\
\text { duration, in } \\
\text { hours }\end{array}$ & $\begin{array}{l}\text { Bailing or } \\
\text { pumping } \\
\text { rate, in } \\
\text { gal/min }\end{array}$ & $\begin{array}{l}\text { Drawdown, in } \\
\mathrm{ft}^{9}\end{array}$ & $\begin{array}{c}\text { Temperature, in } \\
{ }^{\circ}{ }^{\circ}\end{array}$ & Use & Comment \\
\hline $\begin{array}{l}460 \\
461 \\
462\end{array}$ & $\begin{array}{l}5 / 4-35 R 4 \\
4 / 4-4 C 1 \\
4 / 4-5 C 1\end{array}$ & Qh (?) & $\overline{Q T C}$ & clay, yellow & Clay & $\begin{array}{l}\mathrm{DR} \\
\mathrm{DR} \\
--\end{array}$ & 8 & $\begin{array}{l}1 / 1 / 1948 \\
1 / 11 / 1950 \\
1 / 1 / 1947\end{array}$ & 16.41 & 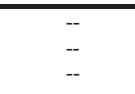 & - & $\begin{array}{l}\overline{-} \\
-\overline{-}\end{array}$ & - & $\begin{array}{l}\overline{-} \\
\overline{-}\end{array}$ & $\begin{array}{l}\text { Unused } \\
\text { Domestic } \\
\text { Domestic }\end{array}$ & $\begin{array}{l}\overline{-} \\
--\end{array}$ \\
\hline $\begin{array}{l}463 \\
464\end{array}$ & $\begin{array}{l}4 / 4-16 \mathrm{D} 1 \\
4 / 4-16 \mathrm{E} 1\end{array}$ & Qyal, Qoal & Qal & tule mud & Clay and sand (or sandstone) & $\overline{\mathrm{DR}}$ & $\overline{10}$ & $\begin{array}{l}1 / 11 / 1950 \\
1 / 1 / 1950\end{array}$ & 0.42 & $\begin{array}{l}-- \\
-\end{array}$ & $\begin{array}{l}-- \\
-\end{array}$ & - & -- & -- & $\begin{array}{l}\text { Domestic, stock } \\
\text { Domestic }\end{array}$ & -- \\
\hline
\end{tabular}

'Geologic units listed as published in the three Water-Supply Papers.

${ }^{2}$ Geologic unit abbreviations explained in table 2 .

${ }^{3}$ Lithology transcribed exactly from driller's des

Assignment of interpreted lithologic class explained in table 1

D, drilied well, melid unknown; DC, drilled by cabletool method; DR, drilled by rotary method; Dug, dug well; G, gravel-packed well. Multiple abbreviations shown where necessary.

casing size was used, the largest and smallest casing size used are listed. For dug wells, the largest dimension of the rectangular opening is listed

Date format is in Month/Day/Year.
${ }^{8}$ Rate for some wells is given in gallons per hour (gph).

'Drawdown defined as the difference, in feet, between static water level and pumping water level at end of pumping period 
Appendix 3. Lithologic data.

[Site IDs and well number correspond to locations in Appendix 1, a site ID and well number is listed for each drilled interval for a particular drill hole. For convenience, the site ID and well numbers are repeated at the shallowest drilled interval for each well. leaders (--), not reported]

\begin{tabular}{|c|c|c|c|c|c|c|c|c|c|c|c|c|c|}
\hline Site ID & Well number & $\begin{array}{l}\text { Site ID, } \\
\text { top of } \\
\text { well }\end{array}$ & $\begin{array}{l}\text { Well number, } \\
\text { top of well }\end{array}$ & $\begin{array}{l}\text { Top of } \\
\text { interval, in } \\
\text { feet }\end{array}$ & $\begin{array}{l}\text { Base of } \\
\text { interval, in } \\
\text { feet }\end{array}$ & $\begin{array}{l}\text { Top of } \\
\text { interval, in } \\
\text { m }\end{array}$ & $\begin{array}{c}\text { Base of } \\
\text { interval, in } \\
\mathrm{m}\end{array}$ & Reported lithologic unit' ${ }^{1}$ & Interpreted lithologic class ${ }^{2}$ & $\begin{array}{c}\text { Reported } \\
\text { degree of } \\
\text { cementation or } \\
\text { induration }^{3} \\
\end{array}$ & $\begin{array}{c}\text { Fossils } \\
\text { reported in } \\
\text { interval }^{4}\end{array}$ & $\begin{array}{c}\text { Organic } \\
\text { matter } \\
\text { reported in } \\
\text { interval }^{5}\end{array}$ & $\begin{array}{l}\text { Reported stratigraphic top of geologic } \\
\text { unit }^{6}\end{array}$ \\
\hline 1 & $22 / 13-2 \mathrm{~A} 3$ & 1 & $22 / 13-2 A 3$ & 0 & 146 & 0.0 & 44.5 & gravel and boulders & Gravel & & -- & -- & Qal \\
\hline 1 & $22 / 13-2 \mathrm{~A} 3$ & & & 146 & 146 & 44.5 & 44.5 & bedrock & Undifferentiated basement & hard & -- & -- & KJu \\
\hline 2 & $22 / 12-18 \mathrm{~N} 1$ & 2 & 22/12-18N1 & 0 & 9 & 0.0 & 2.7 & soil & Sand and clay & - & -- & -- & Qal \\
\hline 2 & $22 / 12-18 \mathrm{~N} 1$ & & & 9 & 11 & 2.7 & 3.4 & gravel & Gravel & -- & -- & -- & \\
\hline 2 & $22 / 12-18 \mathrm{~N} 1$ & & & 11 & 22 & 3.4 & 6.7 & clay, gravelly & Clay and gravel & -- & -- & -- & \\
\hline 2 & 22/12-18N1 & & & 22 & 23 & 6.7 & 7.0 & gravel & Gravel & -- & -- & -- & \\
\hline 2 & $22 / 12-18 \mathrm{~N} 1$ & & & 23 & 31 & 7.0 & 9.4 & clay, gravelly & Clay and gravel & -- & -- & -- & \\
\hline 2 & $22 / 12-18 \mathrm{~N} 1$ & & & 31 & 35 & 9.4 & $\begin{array}{l}10.7 \\
10.7 x-1\end{array}$ & gravel & Gravel & -- & -- & -- & \\
\hline 2 & $22 / 12-18 \mathrm{~N} 1$ & & & 35 & 58 & 10.7 & 17.7 & clay & Clay & -- & -- & -- & \\
\hline 2 & $22 / 12-18 \mathrm{~N} 1$ & & & 58 & 63 & 17.7 & 19.2 & gravel & Gravel & -- & -- & -- & \\
\hline 2 & $22 / 12-18 \mathrm{~N} 1$ & & & 63 & 97 & 19.2 & 29.6 & clay, gravelly & Clay and gravel & -- & -- & -- & \\
\hline 2 & $22 / 12-18 \mathrm{~N} 1$ & & & 97 & 101 & 29.6 & 30.8 & gravel & Gravel & -- & -- & -- & \\
\hline 2 & $22 / 12-18 \mathrm{~N} 1$ & & & 101 & 114 & 30.8 & 34.7 & clay gravelly & Clay and gravel & -- & -- & -- & \\
\hline 2 & $22 / 12-18 \mathrm{~N} 1$ & & & 114 & 116 & 34.7 & 35.4 & gravel & Gravel & -- & -- & -- & \\
\hline 2 & $22 / 12-18 \mathrm{~N} 1$ & & & 116 & 180 & 35.4 & 54.9 & clay gravel & Clay and gravel & -- & -- & -- & \\
\hline 2 & $22 / 12-18 \mathrm{~N} 1$ & & & 180 & 183 & 54.9 & 55.8 & gravel & Gravel & -- & -- & -- & \\
\hline 2 & $22 / 12-18 \mathrm{~N} 1$ & & & 183 & 274 & 55.8 & 83.5 & clay & Clay & -- & -- & -- & \\
\hline 2 & 22/12-18N1 & & & 274 & 293 & 83.5 & 89.3 & $\begin{array}{l}\text { clay, hard, sandy }\end{array}$ & Clay and sand (or sandstone) & hard & -- & -- & \\
\hline 2 & $22 / 12-18 \mathrm{~N} 1$ & & & 293 & 295 & 89.3 & 89.9 & rocks & Gravel & hard & -- & -- & \\
\hline 2 & $22 / 12-18 \mathrm{~N} 1$ & & & 295 & 359 & 89.9 & 109.4 & gravel and clay & Clay and gravel & -- & -- & -- & \\
\hline 2 & $22 / 12-18 \mathrm{~N} 1$ & & & 359 & 361 & 109.4 & 110.0 & gravel & Gravel & -- & -- & -- & \\
\hline 2 & $22 / 12-18 \mathrm{~N} 1$ & & & 361 & 452 & 110.0 & 137.8 & gravel and clay & Clay and gravel & -- & -- & -- & \\
\hline 2 & $22 / 12-18 \mathrm{~N} 1$ & & & 452 & 452 & 137.8 & 137.8 & rock & Gravel & hard & -- & -- & \\
\hline 3 & $22 / 12-19 G 2$ & 3 & 22/12-19G2 & 0 & 10 & 0.0 & 3.0 & surface soil and yellow clay & Sand and clay & -- & -- & -- & Qal \\
\hline 3 & $22 / 12-19 G 2$ & & & 10 & 20 & 3.0 & 6.1 & yellow clay and gravel & Clay and gravel & -- & -- & -- & \\
\hline 3 & $22 / 12-19 \mathrm{G} 2$ & & & 20 & 30 & 6.1 & 9.1 & clay & Clay & -- & -- & -- & \\
\hline 3 & 22/12-19G2 & & & 30 & 40 & 9.1 & 12.2 & gravel and yellow clay & Clay and gravel & -- & -- & -- & \\
\hline 3 & $22 / 12-19 G 2$ & & & 40 & 50 & 12.2 & 15.2 & coarse sand & Sand & -- & -- & -- & \\
\hline 3 & 22/12-19G2 & & & 50 & 70 & 15.2 & 21.3 & yellow clay & Clay & -- & -- & -- & \\
\hline 3 & $22 / 12-19 G 2$ & & & 70 & 90 & 21.3 & 27.4 & sand and blue clay & Sand and clay & -- & -- & -- & \\
\hline 3 & $22 / 12-19 \mathrm{G} 2$ & & & 90 & 120 & 27.4 & 36.6 & coarse sand & Sand & -- & -- & -- & \\
\hline 3 & 22/12-19G2 & & & 120 & 130 & 36.6 & 39.6 & pea gravel & Gravel & -- & -- & -- & \\
\hline 3 & $22 / 12-19 \mathrm{G} 2$ & & & 130 & 160 & 39.6 & 48.8 & sand and gravel & Sand (or sandstone) and gravel & -- & -- & -- & \\
\hline 3 & 22/12-19G2 & & & 160 & 170 & 48.8 & 51.8 & gravel & Gravel & -- & -- & -- & \\
\hline 3 & $22 / 12-19 G 2$ & & & 170 & 190 & 51.8 & 57.9 & gray clay & Clay & -- & -- & -- & \\
\hline 3 & $22 / 12-19 G 2$ & & & 190 & 200 & 57.9 & 61.0 & yellow clay & Clay & -- & -- & -- & \\
\hline 3 & 22/12-19G2 & & & 200 & 210 & 61.0 & 64.0 & sandy clay & Clay and sand (or sandstone) & -- & -- & -- & \\
\hline 3 & $22 / 12-19 \mathrm{G} 2$ & & & 210 & 230 & 64.0 & 70.1 & gravel, fine & Gravel & -- & -- & -- & \\
\hline 3 & 22/12-19G2 & & & 230 & 240 & 70.1 & 73.2 & sand with streaks of blue clay & Sand and clay & -- & -- & -- & \\
\hline 3 & $22 / 12-19 \mathrm{G} 2$ & & & 240 & 250 & 73.2 & 76.2 & yellow clay and gravel & Clay and gravel & -- & -- & -- & \\
\hline 3 & $22 / 12-19 G 2$ & & & 250 & 260 & 76.2 & 79.2 & sand and gravel, small & Sand (or sandstone) and gravel & -- & -- & -- & \\
\hline 3 & $22 / 12-19 \mathrm{G} 2$ & & & 260 & 270 & 79.2 & 82.3 & $\begin{array}{l}\text { blue sand, fine } \\
\text { sing }\end{array}$ & Sand & -- & -- & -- & \\
\hline 3 & $22 / 12-19 \mathrm{G} 2$ & & & 270 & 280 & 82.3 & 85.3 & fine blue sand and gravel & Sand (or sandstone) and gravel & -- & -- & -- & \\
\hline 3 & $22 / 12-19 \mathrm{G} 2$ & & & 280 & 300 & 85.3 & 91.4 & yellow clay and gravel & Clay and gravel & -- & -- & -- & \\
\hline 3 & $22 / 12-19 \mathrm{G} 2$ & & & 300 & 310 & 91.4 & 94.5 & blue clay & Clay & - & -- & -- & \\
\hline 3 & 22/12-19G2 & & & 310 & 320 & 94.5 & 97.5 & blue sandy clay & Clay and sand (or sandstone) & -- & -- & -- & \\
\hline 3 & $22 / 12-19 G 2$ & & & 320 & 330 & 97.5 & 100.6 & streaked blue clay and gravel & Clay and gravel & -- & -- & -- & \\
\hline 3 & $22 / 12-19 G 2$ & & & 330 & 340 & 100.6 & 103.6 & gray clay and gravel & Clay and gravel & -- & -- & -- & \\
\hline 3 & 22/12-19G2 & & & 340 & 380 & 103.6 & 115.8 & coarse sand and gravel & Sand (or sandstone) and gravel & -- & -- & -- & \\
\hline 3 & $22 / 12-19 \mathrm{G} 2$ & & & 380 & 390 & 115.8 & 118.9 & gravel & Gravel & -- & -- & -- & \\
\hline 3 & 22/12-19G2 & & & 390 & 410 & 118.9 & 125.0 & blue clay and gravel & Clay and gravel & -- & -- & -- & \\
\hline 3 & 22/12-19G2 & & & 410 & 420 & 125.0 & 128.0 & brown clay & Clay & -- & -- & -- & QTc (?) \\
\hline 3 & 22/12-19G2 & & & 420 & 430 & 128.0 & 131.1 & blue clay & Clay & -- & -- & -- & \\
\hline 3 & $22 / 12-19 G 2$ & & & 430 & 440 & 131.1 & 134.1 & fine gravel & Gravel & -- & -- & -- & \\
\hline 3 & $22 / 12-19 G 2$ & & & 440 & 450 & 134.1 & 137.2 & yellow clay and fine gravel & Clay and gravel & -- & -- & -- & \\
\hline 3 & 22/12-19G2 & & & 450 & 460 & 137.2 & 140.2 & hard red rock & Gravel & hard & -- & -- & \\
\hline 3 & $22 / 12-19 \mathrm{G} 2$ & & & 460 & 480 & 140.2 & 146.3 & broken rock, shattered & Gravel & hard & -- & -- & \\
\hline 3 & 22/12-19G2 & & & 480 & 500 & 146.3 & 152.4 & blue clay and coarse gravel & Clay and gravel &.- & -- & -- & \\
\hline 3 & $22 / 12-19 G 2$ & & & 500 & 508 & 152.4 & 154.8 & yellow clay & Clay & -- & -- & -- & \\
\hline 4 & 21/15-12K2 & 4 & 21/15-12K2 & 0 & 3 & 0.0 & 0.9 & soil & Sand and clay & -- & -- & -- & Qal and Qt (?) \\
\hline 4 & $21 / 15-12 \mathrm{~K} 2$ & & & 3 & 21 & 0.9 & 6.4 & yellow clay & Clay & -- & -- & -- & \\
\hline
\end{tabular}


Appendix 3. Lithologic data. - Continued

ISite IDs and well number correspond to locations in Appendix 1, a site ID and well number is listed for each drilled interval for a particular drill hole. For convenience, the site ID and well numbers are repeated at the shallowest drilled interval for each well. leaders

\begin{tabular}{|c|c|c|c|c|c|c|c|c|c|c|c|c|c|}
\hline Site ID & Well number & $\begin{array}{l}\text { Site ID, } \\
\text { top of } \\
\text { well }\end{array}$ & $\begin{array}{l}\text { Well number, } \\
\text { top of well }\end{array}$ & $\begin{array}{l}\text { Top of } \\
\text { interval, in } \\
\text { feet }\end{array}$ & $\begin{array}{l}\text { Base of } \\
\text { interval, in } \\
\text { feet }\end{array}$ & $\begin{array}{c}\text { Top of } \\
\text { interval, in } \\
\mathrm{m}\end{array}$ & $\begin{array}{c}\text { Base of } \\
\text { interval, in } \\
\text { m }\end{array}$ & Reported lithologic unit' & Interpreted lithologic class ${ }^{2}$ & $\begin{array}{c}\text { Reported } \\
\text { degree of } \\
\text { cementation or } \\
\text { induration }^{3} \\
\end{array}$ & $\begin{array}{c}\text { Fossils } \\
\text { reported in } \\
\text { interval }^{4}\end{array}$ & $\begin{array}{c}\text { Organic } \\
\text { matter } \\
\text { reported in } \\
\text { interval }^{5}\end{array}$ & $\begin{array}{l}\text { Reported stratigraphic top of geologic } \\
\text { unit }^{6}\end{array}$ \\
\hline 4 & 21/15-12K2 & & & 21 & 37 & 6.4 & 11.3 & yellow clay and gravel & Clay and gravel & -- & -- & -- & \\
\hline 4 & 21/15-12K2 & & & 37 & 50 & 11.3 & 15.2 & blue clay & Clay & -- & -- & -- & \\
\hline 4 & 21/15-12K2 & & & 50 & 125 & 15.2 & 38.1 & blue clay and gravel (water) & Clay and gravel & -- & -- & -- & \\
\hline 4 & 21/15-12K2 & & & 125 & 129 & 38.1 & 39.3 & gravel & Gravel & -- & -- & -- & \\
\hline 4 & 21/15-12K2 & & & 129 & 180 & 39.3 & 54.9 & blue clay and gravel & Clay and gravel & -- & -- & -- & \\
\hline 4 & 21/15-12K2 & & & 180 & 201 & 54.9 & 61.3 & yellow clay & Clay & -- & -- & -- & \\
\hline 4 & 21/15-12K2 & & & 201 & 250 & 61.3 & 76.2 & blue shale & Clay and sand (or sandstone) & cemented & -- & -- & $\mathrm{KJu}$ \\
\hline 5 & 21/15-13В1 & 5 & 21/15-13B1 & 0 & 10 & 0.0 & 3.0 & surface soil & Sand and clay & -- & -- & -- & Qal \\
\hline 5 & 21/15-13B1 & & & 10 & 40 & 3.0 & 12.2 & coarse sand & Sand & -- & -- & -- & \\
\hline 5 & 21/15-13B1 & & & 40 & 60 & 12.2 & 18.3 & pea gravel & Gravel & -- & -- & -- & \\
\hline 5 & 21/15-13B1 & & & 60 & 80 & 18.3 & 24.4 & gray sand & Sand & -- & -- & -- & \\
\hline 5 & 21/15-13B1 & & & 80 & 120 & 24.4 & 36.6 & gray sand and gravel & Sand (or sandstone) and gravel & -- & -- & -- & \\
\hline 5 & 21/15-13В1 & & & 120 & 130 & 36.6 & 39.6 & hard shale & Clay and sand (or sandstone) & hard & -- & -- & Qt \\
\hline 5 & 21/15-13B1 & & & 130 & 140 & 39.6 & 42.7 & cemented gravel & Gravel & cemented & -- & -- & \\
\hline 5 & 21/15-13B1 & & & 140 & 170 & 42.7 & 51.8 & brown clay and large gravel & Clay and gravel & -- & -- & -- & \\
\hline 5 & 21/15-13B1 & & & 170 & 190 & 51.8 & 57.9 & pea gravel & Gravel & -- & -- & -- & \\
\hline 5 & 21/15-13B1 & & & 190 & 220 & 57.9 & 67.1 & blue clay and gravel & Clay and gravel & -- & -- & -- & \\
\hline 5 & 21/15-13B1 & & & 220 & 250 & 67.1 & 76.2 & green sand & Sand & -- & -- & -- & \\
\hline 5 & 21/15-13B1 & & & 250 & 280 & 76.2 & 85.3 & $\begin{array}{l}\text { blue clay and green sand } \\
\text { hard blue rock with streaks of }\end{array}$ & Clay and sand (or sandstone) & -- & -- & -- & \\
\hline 5 & 21/15-13B1 & & & 280 & 320 & 85.3 & 97.5 & sand & Sandstone & hard & -- & -- & \\
\hline 5 & 21/15-13B1 & & & 320 & 330 & 97.5 & 100.6 & blue clay with large gravel & Clay and gravel & -- & -- & -- & \\
\hline 5 & 21/15-13B1 & & & 330 & 340 & 100.6 & 103.6 & red rock, hard & Undifferentiated basement & hard & -- & -- & $\mathrm{KJu}$ \\
\hline 5 & 21/15-13B1 & & & 340 & 350 & 103.6 & 106.7 & porous red volcanic rock & Undifferentiated basement & hard & -- & -- & \\
\hline 5 & 21/15-13B1 & & & 350 & 370 & 106.7 & 112.8 & red volcanic rock & Undifferentiated basement & hard & -- & -- & \\
\hline 5 & 21/15-13B1 & & & 370 & 400 & 112.8 & 121.9 & black basalt rock & Undifferentiated basement & hard & -- & -- & \\
\hline 5 & 21/15-13В1 & & & 400 & 440 & 121.9 & 134.1 & red volcanic rock & Undifferentiated basement & hard & -- & -- & \\
\hline 5 & 21/15-13B1 & & & 440 & 500 & 134.1 & 152.4 & red and black rock & Undifferentiated basement & hard & -- & -- & \\
\hline 5 & 21/15-13B1 & & & 500 & 528 & 152.4 & 160.9 & black and green rock, hard & Undifferentiated basement & hard & -- & -- & \\
\hline 6 & 21/15-14A1 & 6 & 21/15-14A1 & 0 & 2 & 0.0 & 0.6 & surface soil & Sand and clay & -- & -- & -- & Qt \\
\hline 6 & 21/15-14A1 & & & 2 & 25 & 0.6 & 7.6 & yellow clay and gravel & Clay and gravel & -- & -- & -- & 2. \\
\hline 6 & 21/15-14A1 & & & 25 & 29 & 7.6 & 8.8 & blue clay & Clay & -- & -- & -- & \\
\hline 6 & 21/15-14A1 & & & 29 & 49 & 8.8 & 14.9 & yellow clay and gravel & Clay and gravel & -- & -- & -- & \\
\hline 7 & 21/14-30M1 & 7 & 21/14-30M1 & 0 & 1 & 0.0 & 0.3 & topsoil & Sand and clay & -- & -- & -- & Qal \\
\hline 7 & 21/14-30M1 & & & 1 & 15 & 0.3 & 4.6 & yellow clay and pea gravel & Clay and gravel & -- & -- & -- & \\
\hline 7 & 21/14-30M1 & & & 15 & 18 & 4.6 & 5.5 & 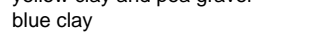 & Clay & -- & -- & -- & \\
\hline 7 & 21/14-30M1 & & & 18 & 23 & 5.5 & 7.0 & gravel & Gravel & -- & -- & -- & \\
\hline 8 & $21 / 14-31 \mathrm{J1}$ & 8 & 21/14-31J1 & 0 & 10 & 0.0 & 3.0 & topsoil and yellow clay & Sand and clay & -- & -- & -- & Qal \\
\hline 8 & 21/14-31J1 & & & 10 & 20 & 3.0 & 6.1 & yellow clay and pea gravel & Clay and gravel & -- & -- & -- & \\
\hline 8 & $21 / 14-31 J 1$ & & & 20 & 30 & 6.1 & 9.1 & blue clay & Clay & -- & -- & -- & \\
\hline 8 & 21/14-31J1 & & & 30 & 50 & 9.1 & 15.2 & coarse sand & Sand & -- & -- & -- & \\
\hline 8 & $21 / 14-31 \mathrm{~J} 1$ & & & 50 & 80 & 15.2 & 24.4 & sand and fine gravel & Sand (or sandstone) and gravel & -- & -- & -- & \\
\hline 8 & $21 / 14-3131$ & & & 80 & 100 & 24.4 & 30.5 & blue sand & Sand & -- & -- & -- & \\
\hline 8 & $21 / 14-31 J 1$ & & & 100 & 110 & 30.5 & 33.5 & green sandy silt & Clay and sand (or sandstone) & -- & -- & -- & \\
\hline 8 & $21 / 14-31 \mathrm{J1}$ & & & 110 & 130 & 33.5 & 39.6 & red and black rock & Undifferentiated basement & hard & -- & -- & $\mathrm{KJu}$ \\
\hline 8 & 21/14-31J1 & & & 130 & 140 & 39.6 & 42.7 & blue and black rock & Undifferentiated basement & hard & -- & -- & \\
\hline & & & & & & & & blue and black rock with salt white & & & & & \\
\hline 8 & 21/14-31J1 & & & 140 & 405 & 42.7 & 123.4 & ashy streaks & Undifferentiated basement & hard & -- & -- & \\
\hline 9 & 18/13-18H1 & 9 & 18/13-18H1 & 0 & 25 & 0.0 & 7.6 & surface soil & Sand and clay & -- & -- & -- & Qal and QTc (?) \\
\hline 9 & 18/13-18H1 & & & 25 & 30 & 7.6 & 9.1 & brown clay & Clay & -- & -- & -- & \\
\hline 9 & 18/13-18H1 & & & 30 & 40 & 9.1 & 12.2 & coarse sand & Sand & -- & -- & -- & \\
\hline 9 & 18/13-18H1 & & & 40 & 68 & 12.2 & 20.7 & blue clay & Clay & -- & -- & -- & \\
\hline 9 & 18/13-18H1 & & & 68 & 250 & 20.7 & 76.2 & coarse & Clay and gravel & -- & -- & -- & \\
\hline 9 & 18/13-18H1 & & & 250 & 305 & 76.2 & 93.0 & coarse gravel and blue clay & Clay and gravel & -- & -- & -- & \\
\hline 9 & 18/13-18H1 & & & 305 & 332 & 93.0 & 101.2 & blue clay & Clay & -- & -- & -- & \\
\hline 9 & 18/13-18H1 & & & 332 & 354 & 101.2 & 107.9 & blue clay with gravel & Clay and gravel & -- & -- & -- & \\
\hline 10 & 18/13-19B1 & 10 & 18/13-19B1 & 0 & 14 & 0.0 & 4.3 & topsoil & Sand and clay & -- & -- & -- & Qal and QTc (?) \\
\hline 10 & 18/13-19B1 & & & 14 & 16 & 4.3 & 4.9 & fine sand & Sand & -- & -- & -- & \\
\hline 10 & 18/13-19B1 & & & 16 & 23 & 4.9 & 7.0 & clay & Clay & -- & -- & -- & \\
\hline 10 & 18/13-19B1 & & & 23 & 29 & 7.0 & 8.8 & blue clay & Clay & -- & -- & -- & \\
\hline 10 & 18/13-19B1 & & & 29 & 36 & 8.8 & 11.0 & coarse sand & Sand & -- & -- & -- & \\
\hline
\end{tabular}


Appendix 3. Lithologic data. - Continued

[Site IDs and well number correspond to locations in Appendix 1, a site ID and well number is listed for each drilled interval for a particular drill hole. For convenience, the site ID and well numbers are repeated at the shallowest drilled interval for each well. leaders

\begin{tabular}{|c|c|c|c|c|c|c|c|c|c|c|c|c|c|}
\hline Site ID & Well number & $\begin{array}{l}\text { Site ID, } \\
\text { top of } \\
\text { well }\end{array}$ & $\begin{array}{l}\text { Well number, } \\
\text { top of well }\end{array}$ & $\begin{array}{l}\text { Top of } \\
\text { interval, in } \\
\text { feet }\end{array}$ & $\begin{array}{l}\text { Base of } \\
\text { interval, in } \\
\text { feet }\end{array}$ & $\begin{array}{c}\text { Top of } \\
\text { interval, in } \\
\mathrm{m}\end{array}$ & $\begin{array}{c}\text { Base of } \\
\text { interval, in } \\
\text { m }\end{array}$ & Reported lithologic unit' & Interpreted lithologic class ${ }^{2}$ & $\begin{array}{c}\text { Reported } \\
\text { degree of } \\
\text { cementation or } \\
\text { induration }^{3} \\
\end{array}$ & $\begin{array}{l}\text { Fossils } \\
\text { reported in } \\
\text { interval }^{4}\end{array}$ & $\begin{array}{c}\text { Organic } \\
\text { matter } \\
\text { reported in } \\
\text { interval }^{5}\end{array}$ & $\begin{array}{l}\text { Reported stratigraphic top of geologic } \\
\text { unit }^{6}\end{array}$ \\
\hline 10 & 18/13-19B1 & & & 36 & 38 & 11.0 & 11.6 & blue clay & Clay & -- & -- & -- & \\
\hline 10 & 18/13-19B1 & & & 38 & 79 & 11.6 & 24.1 & coarse gravel & Gravel & -- & -- & -- & \\
\hline 10 & 18/13-19B1 & & & 79 & 82 & 24.1 & 25.0 & blue clay & Clay & -- & -- & -- & \\
\hline 10 & 18/13-19B1 & & & 82 & 152 & 25.0 & 46.3 & coarse gravel & Gravel & -- & -- & -- & \\
\hline 10 & 18/13-19B1 & & & 152 & 174 & 46.3 & 53.0 & blue clay & Clay & -- & -- & -- & \\
\hline 10 & 18/13-19B1 & & & 174 & 196 & 53.0 & 59.7 & sand and gravel & Sand (or sandstone) and gravel & -- & -- & -- & \\
\hline 10 & 18/13-19B1 & & & 196 & 282 & 59.7 & 86.0 & blue clay & Clay & -- & -- & -- & \\
\hline 10 & 18/13-19B1 & & & 282 & 289 & 86.0 & 88.1 & sand and gravel & Sand (or sandstone) and gravel & -- & -- & -- & \\
\hline 10 & 18/13-19B1 & & & 289 & 320 & 88.1 & 97.5 & blue sand, coarse & Sand & -- & -- & -- & \\
\hline 10 & 18/13-19B1 & & & 320 & 345 & 97.5 & 105.2 & brown sand, coarse & Sand & -- & -- & -- & \\
\hline 10 & 18/13-19B1 & & & 345 & 350 & 105.2 & 106.7 & gray sand, coarse & Sand & -- & -- & -- & \\
\hline 10 & 18/13-19B1 & & & 350 & 362 & 106.7 & 110.3 & multicolored sand, coarse & Sand & -- & -- & -- & \\
\hline 10 & 18/13-19B1 & & & 362 & 372 & 110.3 & 113.4 & yellow sandy clay & Clay and sand (or sandstone) & -- & -- & -- & \\
\hline 10 & 18/13-19B1 & & & 372 & 410 & 113.4 & 125.0 & blue clay & Clay & -- & -- & -- & \\
\hline 10 & 18/13-19B1 & & & 410 & 430 & 125.0 & 131.1 & coarse brown sand & Sand & -- & -- & -- & \\
\hline 10 & 18/13-19B1 & & & 430 & 444 & 131.1 & 135.3 & brown sandy clay with gravel & Clay, sand, and gravel & -- & -- & -- & \\
\hline 10 & 18/13-19B1 & & & 444 & 454 & 135.3 & 138.4 & blue clay and gravel & Clay and gravel & -- & -- & -- & \\
\hline 11 & 18/13-20P2 & 11 & 18/13-20P2 & 0 & 14 & 0.0 & 4.3 & yellow clay & Clay & -- & -- & -- & QTC \\
\hline 11 & 18/13-20P2 & & & 14 & 22 & 4.3 & 6.7 & water gravel & Clay and gravel & -- & -- & -- & \\
\hline 11 & 18/13-20P2 & & & 22 & 55 & 6.7 & 16.8 & blue clay & Clay & -- & -- & -- & \\
\hline 11 & 18/13-20P2 & & & 55 & 60 & 16.8 & 18.3 & $\begin{array}{l}\text { water gravel ( } 50 \mathrm{gpm}) \\
\text { blue clay (buried coniferous forest }\end{array}$ & Gravel & -- & -- & -- & \\
\hline 11 & 18/13-20P2 & & & 60 & 72 & 18.3 & 21.9 & at $72 \mathrm{ft}$.) & Clay & -- & -- & -- & \\
\hline 12 & 18/13-31A1 & 12 & 18/13-31A1 & 0 & 2 & 0.0 & 0.6 & soil & Sand and clay & -- & -- & -- & QTC \\
\hline 12 & 18/13-31A1 & & & 2 & 34 & 0.6 & 10.4 & yellow clay and gravel & Clay and gravel & -- & -- & -- & \\
\hline 12 & 18/13-31A1 & & & 34 & 37 & 10.4 & 11.3 & blue clay and gravel & Clay and gravel & -- & -- & -- & \\
\hline 12 & 18/13-31A1 & & & 37 & 39 & 11.3 & 11.9 & blue clay, sticky & Clay & -- & -- & -- & \\
\hline 12 & 18/13-31A1 & & & 39 & 51 & 11.9 & 15.5 & (no record) & No data & -- & -- & -- & \\
\hline 12 & 18/13-31A1 & & & 51 & 60 & 15.5 & 18.3 & blue clay & Clay & -- & -- & -- & \\
\hline 12 & 18/13-31A1 & & & 60 & 67 & 18.3 & 20.4 & blue clay and gravel & Clay and gravel & -- & -- & -- & \\
\hline 12 & 18/13-31A1 & & & 67 & 79 & 20.4 & 24.1 & blue clay & Clay & -- & -- & -- & \\
\hline 12 & 18/13-31A1 & & & 79 & 80 & 24.1 & 24.4 & blue clay and gravel & Clay and gravel & -- & -- & -- & \\
\hline 12 & 18/13-31A1 & & & 80 & 100 & 24.4 & 30.5 & blue clay & Clay & -- & -- & -- & \\
\hline 12 & 18/13-31A1 & & & 100 & 124 & 30.5 & 37.8 & yellow cemented gravel & Clay, sand, and gravel & cemented & -- & -- & \\
\hline 12 & 18/13-31A1 & & & 124 & 151 & 37.8 & 46.0 & blue clay & Clay & -- & -- & -- & \\
\hline 12 & 18/13-31A1 & & & 151 & 158 & 46.0 & 48.2 & yellow clay and gravel & Clay and gravel & -- & -- & -- & \\
\hline 12 & 18/13-31A1 & & & 158 & 187 & 48.2 & 57.0 & blue clay & Clay & -- & -- & -- & \\
\hline 12 & 18/13-31A1 & & & 187 & 192 & 57.0 & 58.5 & blue clay and gravel & Clay and gravel & -- & -- & -- & \\
\hline 12 & 18/13-31A1 & & & 192 & 200 & 58.5 & 61.0 & blue clay and boulders & Clay and gravel & -- & -- & -- & \\
\hline 12 & 18/13-31A1 & & & 200 & 214 & 61.0 & 65.2 & yellow clay and gravel & Clay and gravel & -- & -- & -- & \\
\hline 12 & 18/13-31A1 & & & 214 & 240 & 65.2 & 73.2 & blue clay and gravel & Clay and gravel & -- & -- & -- & \\
\hline 12 & 18/13-31A1 & & & 240 & 250 & 73.2 & 76.2 & blue clay & Clay & -- & -- & -- & \\
\hline 12 & 18/13-31A1 & & & 250 & 260 & 76.2 & 79.2 & yellow clay & Clay & -- & -- & -- & \\
\hline 12 & 18/13-31A1 & & & 260 & 285 & 79.2 & 86.9 & blue clay & Clay & -- & -- & -- & \\
\hline 12 & 18/13-31A1 & & & 285 & 287 & 86.9 & 87.5 & yellow sand and gravel & Sand (or sandstone) and gravel & -- & -- & -- & \\
\hline 12 & 18/13-31A1 & & & 287 & 289 & 87.5 & 88.1 & yellow clay & Clay & -- & -- & -- & \\
\hline 13 & 17/11-33D1 & 13 & 17/11-33D1 & 0 & 2.5 & 0.0 & 0.8 & topsoil & Sand and clay & -- & -- & -- & Qal \\
\hline 13 & 17/11-33D1 & & & 2.5 & 17 & 0.8 & 5.2 & yellow clay & Clay & -- & -- & -- & Qt (?) \\
\hline 13 & 17/11-33D1 & & & 17 & 375 & 5.2 & 114.3 & blue clay & Clay & -- & -- & -- & QTm \\
\hline 13 & 17/11-33D1 & & & 375 & 430 & 114.3 & 131.1 & rock & Undifferentiated basement & hard & -- & -- & KJu \\
\hline 13 & 17/11-33D1 & & & 430 & 431 & 131.1 & 131.4 & hard formation & Undifferentiated basement & hard & -- & -- & \\
\hline 13 & 17/11-33D1 & & & 431 & 459 & 131.4 & 139.9 & shale & Undifferentiated basement & cemented & -- & -- & \\
\hline 13 & 17/11-33D1 & & & 459 & 570 & 139.9 & 173.7 & hard blue rock & Undifferentiated basement & hard & -- & -- & \\
\hline 13 & 17/11-33D1 & & & 570 & 617 & 173.7 & 188.1 & $\begin{array}{l}\text { rock } \\
\text { hard blue rock (Interval from } 375 \\
\text { to } 658 \text { ft. further described as }\end{array}$ & Undifferentiated basement & hard & -- & -- & \\
\hline 13 & 17/11-33D1 & & & 617 & 658 & 188.1 & 200.6 & $\begin{array}{l}\text { gray fairly coarse textured rock in } \\
\text { hard and soft layers) } \\
\text { yellow-brown gravelly clay, } 1 \text { to } 3 \\
\text { in anqular gravel small }\end{array}$ & Undifferentiated basement & hard & -- & -- & \\
\hline 14 & $16 / 12 \mathrm{~W}-34 \mathrm{~N}:$ & 14 & $16 / 12 W-34 N$ & 0 & 14.5 & 0.0 & 4.4 & $\begin{array}{l}\text { In. angular gravel, small } \\
\text { percentage of sand }\end{array}$ & Clay and gravel & -- & -- & -- & Qt \\
\hline
\end{tabular}


Appendix 3. Lithologic data. - Continued

[Site IDs and well number correspond to locations in Appendix 1, a site ID and well number is listed for each drilled interval for a particular drill hole. For convenience, the site ID and well numbers are repeated at the shallowest drilled interval for each well. leaders (--), not reported]

\begin{tabular}{|c|c|c|c|c|c|c|c|c|c|c|c|c|c|}
\hline Site ID & Well number & $\begin{array}{l}\text { Site ID, } \\
\text { top of } \\
\text { well }\end{array}$ & $\begin{array}{l}\text { Well number, } \\
\text { top of well }\end{array}$ & $\begin{array}{l}\text { Top of } \\
\text { interval, in } \\
\text { feet }\end{array}$ & $\begin{array}{l}\text { Base of } \\
\text { interval, in } \\
\text { feet }\end{array}$ & $\begin{array}{c}\text { Top of } \\
\text { interval, in } \\
m\end{array}$ & $\begin{array}{c}\text { Base of } \\
\text { interval, in } \\
m\end{array}$ & Reported lithologic unit' ${ }^{\prime}$ & Interpreted lithologic class ${ }^{2}$ & $\begin{array}{l}\text { Reported } \\
\text { degree of } \\
\text { cementation or } \\
\text { induration } \\
\end{array}$ & $\begin{array}{l}\text { Fossils } \\
\text { reported in } \\
\text { interval }^{4}\end{array}$ & $\begin{array}{c}\text { Organic } \\
\text { matter } \\
\text { reported in } \\
\text { interval }^{5}\end{array}$ & $\begin{array}{l}\text { Reported stratigraphic top of geologic } \\
\text { unit }^{6}\end{array}$ \\
\hline 14 & $16 / 12 \mathrm{~W}-34 \mathrm{~N} 2$ & & & 14.5 & 18 & 4.4 & 5.5 & $\begin{array}{l}\text { brown sandy gravel, small percent } \\
\text { clay } \\
\text { brown sandy gravel, } 1 \text { to } 4 \text { in. } \\
\text { angular to round, small }\end{array}$ & Clay, sand, and gravel & -- & -- & -- & \\
\hline 14 & $16 / 12 \mathrm{~W}-34 \mathrm{~N} 2$ & & & 18 & 25 & 5.5 & 7.6 & $\begin{array}{l}\text { percentage of clay } \\
\text { olive-drab sandy clay, well }\end{array}$ & Clay, sand, and gravel & -- & -- & -- & \\
\hline 14 & $16 / 12 \mathrm{~W}-34 \mathrm{~N} 2$ & & & 25 & 30.5 & 7.6 & 9.3 & consolidated & Sand and clay & -- & -- & -- & QTm \\
\hline 14 & $16 / 12 \mathrm{~W}-34 \mathrm{~N} 2$ & & & 30.5 & 32 & 9.3 & 9.8 & $\begin{array}{l}\text { blue clayey sand, very tight } \\
\text { brown sandy clay, well } \\
\text { consolidated, occasional streaks } \\
\text { of blue sand, and small }\end{array}$ & Sand and clay & -- & -- & -- & \\
\hline 14 & $16 / 12 \mathrm{~W}-34 \mathrm{~N} 2$ & & & 32 & 39 & 9.8 & 11.9 & $\begin{array}{l}\text { percentage of small pebbles } \\
\text { blue gravelly sand, } 1 \text { to } 2 \text { in. }\end{array}$ & Clay, sand, and trace gravel & -- & -- & -- & \\
\hline 14 & $16 / 12 \mathrm{~W}-34 \mathrm{~N} 2$ & & & 39 & 40 & 11.9 & 12.2 & $\begin{array}{l}\text { angular to round } \\
\text { blue sandy gravel, loosely } \\
\text { cemented, } 1 \text { to } 3 \text { in. angular to }\end{array}$ & Sand (or sandstone) and gravel & -- & -- & -- & \\
\hline 14 & $16 / 12 \mathrm{~W}-34 \mathrm{~N} 2$ & & & 40 & 57 & 12.2 & 17.4 & $\begin{array}{l}\text { round } \\
\text { blue gravel conglomerate, very }\end{array}$ & Clay, sand, and gravel & -- & -- & -- & \\
\hline 14 & $16 / 12 \mathrm{~W}-34 \mathrm{~N} 2$ & & & 57 & 58 & 17.4 & 17.7 & $\begin{array}{l}\text { well consolidated } \\
\text { blue sandy gravel, loosely } \\
\text { cemented } 1 \text { to } 3 \text { in anqular to }\end{array}$ & Gravel & cemented & -- & -- & \\
\hline 14 & $16 / 12 \mathrm{~W}-34 \mathrm{~N} 2$ & & & 58 & 65 & 17.7 & 19.8 & $\begin{array}{l}\text { round } \\
\text { blue clayey sand with some }\end{array}$ & Clay, sand, and gravel & -- & -- & -- & \\
\hline 14 & $16 / 12 \mathrm{~W}-34 \mathrm{~N} 2$ & & & 65 & 68.5 & 19.8 & 20.9 & $\begin{array}{l}\text { pebbles } \\
\text { blue sandy clayey gravel, } 1 \text { to } 3 \\
\text { in. angular to round, consolidated }\end{array}$ & Clay, sand, and gravel & -- & -- & -- & \\
\hline 14 & 16/12W-34N2 & & & 68.5 & 76 & 20.9 & 23.2 & $\begin{array}{l}\text { with clayey matrix } \\
\text { blue gravelly clayey sand, } 1 \text { to } 4\end{array}$ & Clay, sand, and gravel & -- & -- & -- & \\
\hline 14 & $16 / 12 \mathrm{~W}-34 \mathrm{~N} 2$ & & & 76 & 77 & 23.2 & 23.5 & in. angular to round & Clay, sand, and gravel & -- & -- & -- & \\
\hline & & & & & & & & $\begin{array}{l}\text { blue clayey sand, well- } \\
\text { consolidated, occasional streaks }\end{array}$ & & & & & \\
\hline 14 & $16 / 12 \mathrm{~W}-34 \mathrm{~N} 2$ & & & 77 & 83.5 & 23.5 & 25.5 & $\begin{array}{l}\text { of olive-drab fine sandy clay } \\
\text { blue gravelly clayey sand, well- }\end{array}$ & Sand and clay & -- & -- & -- & \\
\hline 14 & $16 / 12 \mathrm{~W}-34 \mathrm{~N} 2$ & & & 83.5 & 85 & 25.5 & 25.9 & $\begin{array}{l}\text { consolidated } \\
\text { blue clayey sand, with streaks of } \\
\text { pebbles and streaks of olive-drab }\end{array}$ & Clay, sand, and gravel & -- & -- & -- & \\
\hline 14 & $16 / 12 \mathrm{~W}-34 \mathrm{~N} 2$ & & & 85 & 88.5 & 25.9 & 27.0 & $\begin{array}{l}\text { fine sandy clay } \\
\text { olive-drab sandy clay, extremely }\end{array}$ & Sand and clay & -- & -- & -- & \\
\hline 14 & $16 / 12 \mathrm{~W}-34 \mathrm{~N} 2$ & & & 88.5 & 93 & 27.0 & 28.3 & $\begin{array}{l}\text { well consolidated } \\
\text { olive-drab clayey sand, extremely }\end{array}$ & Sand and clay & -- & -- & -- & \\
\hline 14 & $16 / 12 \mathrm{~W}-34 \mathrm{~N} 2$ & & & 93 & 94 & 28.3 & 28.7 & $\begin{array}{l}\text { well consolidated } \\
\text { blue clayey sand with streaks of }\end{array}$ & Sand and clay & -- & -- & -- & \\
\hline 14 & $16 / 12 \mathrm{~W}-34 \mathrm{~N} 2$ & & & 94 & 96.5 & 28.7 & 29.4 & olive-drab clay & Sand and clay & -- & -- & -- & \\
\hline 14 & $16 / 12 \mathrm{~W}-34 \mathrm{~N} 2$ & & & 96.5 & 97.5 & 29.4 & 29.7 & blue clayey sand with pebbles & Clay, sand, and gravel & -- & -- & -- & \\
\hline 14 & $16 / 12 \mathrm{~W}-34 \mathrm{~N} 2$ & & & 97.5 & 99 & 29.7 & 30.2 & $\begin{array}{l}\text { blue clayey gravelly sand } \\
\text { blue sandy gravel, loosely } \\
\text { consolidated, } 1 \text { to } 4 \text { in. angular to }\end{array}$ & Sand (or sandstone) and gravel & -- & -- & -- & \\
\hline 14 & $16 / 12 \mathrm{~W}-34 \mathrm{~N} 2$ & & & 99 & 107 & 30.2 & 32.6 & $\begin{array}{l}\text { round } \\
\text { blue sandy clayey gravel, } 1 \text { to } 3 \\
\text { in. angular to round, well- } \\
\text { consolidated, clay matrix (losing }\end{array}$ & Clay, sand, and gravel & -- & -- & -- & \\
\hline 14 & $16 / 12 \mathrm{~W}-34 \mathrm{~N} 2$ & & & 107 & 154 & 32.6 & 46.9 & $\begin{array}{l}\text { water in hole) } \\
\text { blue sandy clayey gravel, } 1 \text { to } 2 \\
\text { in. angular to round, well- }\end{array}$ & Clay, sand, and gravel & -- & -- & -- & \\
\hline 14 & $16 / 12 \mathrm{~W}-34 \mathrm{~N} 2$ & & & 154 & 171.5 & 46.9 & 52.3 & $\begin{array}{l}\text { consolidated, clayey matrix } \\
\text { blue clayey gravelly sand, streaks } \\
\text { of olive-drab clayey sand, well- }\end{array}$ & Clay, sand, and gravel & -- & -- & -- & \\
\hline 14 & $16 / 12 \mathrm{~W}-34 \mathrm{~N} 2$ & & & 171.5 & 173 & 52.3 & 52.7 & consolidated & Clay, sand, and gravel & -- & -- & -- & \\
\hline
\end{tabular}


Appendix 3. Lithologic data. - Continued

ISite IDs and well number correspond to locations in Appendix 1, a site ID and well number is listed for each drilled interval for a particular drill hole. For convenience, the site ID and well numbers are repeated at the shallowest drilled interval for each well. leaders (--) not reported]

\begin{tabular}{|c|c|c|c|c|c|c|c|c|c|c|c|c|c|}
\hline Site ID & Well number & $\begin{array}{l}\text { Site ID, } \\
\text { top of } \\
\text { well }\end{array}$ & $\begin{array}{l}\text { Well number, } \\
\text { top of well }\end{array}$ & $\begin{array}{l}\text { Top of } \\
\text { interval, in } \\
\text { feet }\end{array}$ & $\begin{array}{l}\text { Base of } \\
\text { interval, in } \\
\text { feet }\end{array}$ & $\begin{array}{c}\text { Top of } \\
\text { interval, in } \\
\mathrm{m}\end{array}$ & $\begin{array}{c}\text { Base of } \\
\text { interval, in } \\
\mathrm{m}\end{array}$ & Reported lithologic unit' ${ }^{1}$ & Interpreted lithologic class ${ }^{2}$ & $\begin{array}{c}\text { Reported } \\
\text { degree of } \\
\text { cementation or } \\
\text { induration }^{3} \\
\end{array}$ & $\begin{array}{c}\text { Fossils } \\
\text { reported in } \\
\text { interval }^{4}\end{array}$ & $\begin{array}{c}\text { Organic } \\
\text { matter } \\
\text { reported in } \\
\text { interval }^{5} \\
\end{array}$ & $\begin{array}{l}\text { Reported stratigraphic top of geologic } \\
\text { unit }^{6}\end{array}$ \\
\hline 14 & $16 / 12 \mathrm{~W}-34 \mathrm{~N} 2$ & & & 173 & 176.5 & 52.7 & 53.8 & $\begin{array}{l}\text { olive-drab clayey sand, well- } \\
\text { consolidated, occasional streaks } \\
\text { of blue sandy clay }\end{array}$ & Sand and clay & -- & -- & -- & \\
\hline 14 & $16 / 12 \mathrm{~W}-34 \mathrm{~N} 2$ & & & 176.5 & 178.5 & 53.8 & 54.4 & $\begin{array}{l}\text { blue sandy clay with occasional } \\
\text { streaks of olive-drab clayey sand }\end{array}$ & Clay and sand (or sandstone) & -- & -- & -- & \\
\hline 14 & $16 / 12 \mathrm{~W}-34 \mathrm{~N} 2$ & & & 178.5 & 179.5 & 54.4 & 54.7 & $\begin{array}{l}\text { blue clayey sand with pebbles } \\
\text { blue sandy clayey gravel, well- }\end{array}$ & Clay, sand, and gravel & -- & -- & -- & \\
\hline 14 & $16 / 12 W-34 N 2$ & & & 179.5 & 195.5 & 54.7 & 59.6 & $\begin{array}{l}\text { consolidated, clay matrix, } 1 \text { to } 3 \\
\text { in. angular to round } \\
\text { blue sandy clayey gravel well- } \\
\text { consolidated, clayey matrix, } 1 \text { to } 2 \\
\text { in. angular to round. some }\end{array}$ & Clay, sand, and gravel & -- & - & -- & \\
\hline 14 & $16 / 12 \mathrm{~W}-34 \mathrm{~N} 2$ & & & 195.5 & 210.5 & 59.6 & 64.2 & conglomerate in last $3.5 \mathrm{ft}$. & Clay, sand, and gravel & -- & -- & -- & \\
\hline 15 & 14/12-5K1 & 15 & 14/12-5K1 & 0 & 2 & 0.0 & 0.6 & surface & Sand and clay & -- & -- & -- & Qyal \\
\hline 15 & 14/12-5K1 & & & 2 & 15 & 0.6 & 4.6 & clay, sand, and gravel & Clay, sand, and gravel & -- & -- & -- & \\
\hline 15 & 14/12-5K1 & & & 15 & 20 & 4.6 & 6.1 & boulders and clay & Clay and gravel & -- & -- & -- & \\
\hline 15 & $14 / 12-5 \mathrm{~K} 1$ & & & 20 & 38 & 6.1 & 11.6 & yellow sand, clay and gravel & Sand (or sandstone) and gravel & -- & -- & -- & \\
\hline 15 & $14 / 12-5 K 1$ & & & 38 & 45 & 11.6 & 13.7 & yellow clay, hard & Clay & hard & -- & -- & \\
\hline 15 & 14/12-5K1 & & & 45 & 60 & 13.7 & 18.3 & yellow cemented gravel & Clay, sand, and gravel & cemented & -- & -- & \\
\hline 15 & 14/12-5K1 & & & 60 & 64 & 18.3 & 19.5 & yellow sand & Sand & -- & -- & -- & \\
\hline 15 & $14 / 12-5 K 1$ & & & 64 & 78 & 19.5 & 23.8 & yellow cemented gravel & Clay, sand, and gravel & cemented & -- & -- & \\
\hline 15 & $14 / 12-5 K 1$ & & & 78 & 84 & 23.8 & 25.6 & blue clay and gravel & Clay and gravel & -- & -- & -- & \\
\hline 15 & $14 / 12-5 \mathrm{~K} 1$ & & & 84 & 94 & 25.6 & 28.7 & blue rock & Undifferentiated basement & hard & -- & -- & $\mathrm{KJu}$ \\
\hline 16 & $14 / 12-26 \mathrm{H}$ & 16 & $14 / 12-26 \mathrm{H}$ & 0 & 10 & 0.0 & 3.0 & sand & Sand & -- & -- & -- & Qal \\
\hline 16 & $14 / 12-26 \mathrm{H}$ & & & 10 & 17 & 3.0 & 5.2 & loose gravel & Gravel & -- & -- & -- & \\
\hline 16 & $14 / 12-26 \mathrm{H}$ & & & 17 & 25 & 5.2 & 7.6 & yellow sandy clay gravel & Clay, sand, and gravel & -- & -- & -- & \\
\hline 16 & $14 / 12-26 \mathrm{H}$ & & & 25 & 55 & 7.6 & 16.8 & cemented gravel, hard & Gravel & cemented & -- & -- & \\
\hline 16 & $14 / 12-26 \mathrm{H}$ & & & 55 & 62 & 16.8 & 18.9 & soft gravel & Gravel & -- & -- & -- & \\
\hline 16 & $14 / 12-26 \mathrm{H}$ & & & 62 & 65 & 18.9 & 19.8 & hard & Gravel & hard & -- & -- & \\
\hline 17 & 13/11-8H1 & 17 & 7 13/11-8H1 & 0 & 10 & 0.0 & 3.0 & medium gravelly brown soil & Clay, sand, and gravel & -- & -- & -- & QTC \\
\hline 17 & $13 / 11-8 \mathrm{H} 1$ & Nr & 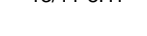 & 10 & 30 & 3.0 & 9.1 & fine gravelly yellow clay & Sand (or sandstone) and gravel & -- & -- & -- & 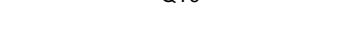 \\
\hline 17 & 13/11-8H1 & & & 30 & 32 & 9.1 & 9.8 & blue sand; water & Sand & -- & -- & -- & \\
\hline 17 & $13 / 11-8 \mathrm{H} 1$ & & & 32 & 46 & 9.8 & 14.0 & yellow sandy clay & Clay and sand (or sandstone) & -- & -- & -- & \\
\hline 17 & 13/11-8H1 & & & 46 & 50 & 14.0 & 15.2 & fine blue sand, water & Sand & -- & -- & -- & \\
\hline 17 & 13/11-8H1 & & & 50 & 77 & 15.2 & 23.5 & sedimentary lake debris & Clay and sand (or sandstone) & -- & -- & -- & \\
\hline 17 & $13 / 11-8 \mathrm{H} 1$ & & & 77 & 97 & 23.5 & 29.6 & large blue gravel; water & Gravel & -- & -- & -- & \\
\hline 17 & $13 / 11-8 \mathrm{H} 1$ & & & 97 & 147 & 29.6 & 44.8 & blue sedimentary sandy clay & Clay and sand (or sandstone) & -- & -- & -- & \\
\hline 17 & 13/11-8H1 & & & 147 & 152 & 44.8 & 46.3 & decayed vegetation & No data & -- & -- & plants & \\
\hline 17 & 13/11-8H1 & & & 152 & 166 & 46.3 & 50.6 & blue packed coarse gravel & Gravel & -- & -- & -- & \\
\hline 17 & $13 / 11-8 \mathrm{H} 1$ & & & 166 & 168 & 50.6 & 51.2 & fine blue sand and gravel; water & Sand (or sandstone) and gravel & -- & -- & -- & \\
\hline 17 & 13/11-8H1 & & & 168 & 178 & 51.2 & 54.3 & light blue sandstone & Sandstone & cemented & -- & -- & \\
\hline 17 & $13 / 11-8 \mathrm{H} 1$ & & & 178 & 187 & 54.3 & 57.0 & large brown gravel; water & Gravel & -- & -- & -- & \\
\hline 18 & 13/11-18D1 & 18 & 3 13/11-18D1 & 0 & 2 & 0.0 & 0.6 & topsoil & Sand and clay & -- & -- & -- & Qal \\
\hline 18 & 13/11-18D1 & & & 2 & 10 & 0.6 & 3.0 & yellow clay & Clay & -- & -- & -- & \\
\hline 18 & 13/11-18D1 & & & 10 & 25 & 3.0 & 7.6 & sand & Sand & -- & -- & -- & \\
\hline 18 & 13/11-18D1 & & & 25 & 51 & 7.6 & 15.5 & blue gravel, coarse & Gravel & -- & -- & -- & \\
\hline 18 & 13/11-18D1 & & & 51 & 55 & 15.5 & 16.8 & gravel & Gravel & -- & -- & -- & \\
\hline 18 & 13/11-18D1 & & & 55 & 61 & 16.8 & 18.6 & blue clay and gravel & Clay and gravel & -- & -- & -- & \\
\hline 19 & 13/11-18M1 & 19 & 9 13/11-18M1 & 0 & 22 & 0.0 & 6.7 & topsoil & Sand and clay & -- & -- & -- & Qal \\
\hline 19 & 13/11-18M1 & & & 22 & 63 & 6.7 & 19.2 & gravel & Gravel & -- & -- & -- & \\
\hline 19 & 13/11-18M1 & & & 63 & 63 & 19.2 & 19.2 & blue clay & Clay & -- & -- & -- & \\
\hline 20 & $13 / 11-22 \mathrm{G}$ & 20 & 13/11-22G & 0 & 1 & 0.0 & 0.3 & soil & Sand and clay & -- & -- & -- & Qal and QTc \\
\hline 20 & $13 / 11-22 G$ & & & 1 & 20 & 0.3 & 6.1 & brown clay and gravel & Clay and gravel & -- & -- & -- & \\
\hline 20 & $13 / 11-22 G$ & & & 20 & 32 & 6.1 & 9.8 & sandy blue clay & Clay and sand (or sandstone) & -- & -- & -- & \\
\hline 20 & $13 / 11-22 \mathrm{G}$ & & & 32 & 35 & 9.8 & 10.7 & gravel, some water & Gravel & -- & -- & -- & \\
\hline 20 & $13 / 11-22 \mathrm{G}$ & & & 35 & 50 & 10.7 & 15.2 & sandy blue clay & Clay and sand (or sandstone) & -- & -- & -- & \\
\hline 20 & $13 / 11-22 \mathrm{G}$ & & & 50 & 53 & 15.2 & 16.2 & gravel & Gravel & -- & -- & -- & \\
\hline 20 & 13/11-22G & & & 53 & 78 & 16.2 & 23.8 & blue clay & Clay & -- & -- & -- & \\
\hline 20 & $13 / 11-22 \mathrm{G}$ & & & 78 & 83 & 23.8 & 25.3 & gravel & Gravel & -- & -- & -- & \\
\hline 20 & $13 / 11-22 G$ & & & 83 & 191 & 25.3 & 58.2 & blue clay & Clay & -- & -- & -- & \\
\hline
\end{tabular}


Appendix 3. Lithologic data. - Continued

[Site IDs and well number correspond to locations in Appendix 1, a site ID and well number is listed for each drilled interval for a particular drill hole. For convenience, the site ID and well numbers are repeated at the shallowest drilled interval for each well. leaders $1-$

\begin{tabular}{|c|c|c|c|c|c|c|c|c|c|c|c|c|c|}
\hline Site ID & Well number & $\begin{array}{l}\text { Site ID, } \\
\text { top of } \\
\text { well }\end{array}$ & $\begin{array}{l}\text { Well number, } \\
\text { top of well }\end{array}$ & $\begin{array}{l}\text { Top of } \\
\text { interval, in } \\
\text { feet }\end{array}$ & $\begin{array}{l}\text { Base of } \\
\text { interval, in } \\
\text { feet }\end{array}$ & $\begin{array}{c}\text { Top of } \\
\text { interval, in } \\
\mathrm{m}\end{array}$ & $\begin{array}{c}\text { Base of } \\
\text { interval, in } \\
\mathrm{m}\end{array}$ & Reported lithologic unit' & Interpreted lithologic class ${ }^{2}$ & $\begin{array}{c}\text { Reported } \\
\text { degree of } \\
\text { cementation or } \\
\text { induration }^{3} \\
\end{array}$ & $\begin{array}{c}\text { Fossils } \\
\text { reported in } \\
\text { interval }^{4}\end{array}$ & $\begin{array}{c}\text { Organic } \\
\text { matter } \\
\text { reported in } \\
\text { interval }^{5}\end{array}$ & $\begin{array}{l}\text { Reported stratigraphic top of geologic } \\
\text { unit }^{6}\end{array}$ \\
\hline 20 & $13 / 11-22 \mathrm{G}$ & & & 191 & 202 & 58.2 & 61.6 & good gravel & Gravel & -- & -- & -- & \\
\hline 20 & 13/11-22G & & & 202 & 204 & 61.6 & 62.2 & blue clay & Clay & -- & -- & -- & \\
\hline 20 & 13/11-22G & & & 204 & 205 & 62.2 & 62.5 & bedrock & Gravel & hard & -- & -- & $\mathrm{KJu}$ \\
\hline 21 & 13/11-21L2 & 21 & 13/11-21L2 & 0 & 8 & 0.0 & 2.4 & soil & Sand and clay & -- & -- & -- & Qal \\
\hline 21 & 13/11-21L2 & & & 8 & 18 & 2.4 & 5.5 & yellow clay & Clay & -- & -- & -- & \\
\hline 21 & 13/11-21L2 & & & 18 & 26 & 5.5 & 7.9 & sand, coarse & Sand & -- & -- & -- & \\
\hline 21 & 13/11-21L2 & & & 26 & 34 & 7.9 & 10.4 & blue clay & Clay & -- & -- & -- & \\
\hline 21 & 13/11-21L2 & & & 34 & 46 & 10.4 & 14.0 & gravel & Gravel & -- & -- & -- & \\
\hline 21 & 13/11-21L2 & & & 46 & 53 & 14.0 & 16.2 & clay, broken & Clay & -- & -- & -- & QTm \\
\hline 21 & 13/11-21L2 & & & 53 & 78 & 16.2 & 23.8 & gravel & Gravel & -- & -- & -- & \\
\hline 21 & 13/11-21L2 & & & 78 & 88 & 23.8 & 26.8 & clay, broken & Clay & -- & -- & -- & \\
\hline 21 & 13/11-21L2 & & & 88 & 96 & 26.8 & 29.3 & sand and gravel & Sand (or sandstone) and gravel & -- & -- & -- & \\
\hline 21 & 13/11-21L2 & & & 96 & 106 & 29.3 & 32.3 & clay, soft & Clay & -- & -- & -- & \\
\hline 21 & 13/11-21L2 & & & 106 & 112 & 32.3 & 34.1 & broken gravel and sand & Sand (or sandstone) and gravel & -- & -- & -- & \\
\hline 21 & 13/11-21L2 & & & 112 & 150 & 34.1 & 45.7 & hard clay & Clay & hard & -- & -- & \\
\hline 21 & 13/11-21L2 & & & 150 & 165 & 45.7 & 50.3 & boulders, rough & Gravel & -- & -- & -- & \\
\hline 21 & 13/11-21L2 & & & 165 & 169 & 50.3 & 51.5 & hard clay & Clay & hard & -- & -- & KJu \\
\hline 21 & 13/11-21L2 & & & 169 & 200 & 51.5 & 61.0 & hard blue shale & Clay and sand (or sandstone) & hard & -- & -- & \\
\hline 21 & 13/11-21L2 & & & 200 & 202 & 61.0 & 61.6 & gravel & Gravel & -- & -- & -- & \\
\hline 21 & 13/11-21L2 & & & 202 & 220 & 61.6 & 67.1 & blue shale and rock & Gravel & cemented & -- & -- & \\
\hline 22 & 13/11-19Q1 & 22 & 13/11-19Q1 & 0 & 3 & 0.0 & 0.9 & surface & Sand and clay & -- & -- & -- & Qal and QTm \\
\hline 22 & 13/11-19Q1 & & & 3 & 14 & 0.9 & 4.3 & gravel, large, and sand & Sand (or sandstone) and gravel & -- & -- & -- & \\
\hline 22 & 13/11-19Q1 & & & 14 & 40 & 4.3 & 12.2 & gravel & Gravel & -- & -- & -- & \\
\hline 22 & 13/11-19Q1 & & & 40 & 52 & 12.2 & 15.8 & gravel, large & Gravel & -- & -- & -- & \\
\hline 22 & 13/11-19Q1 & & & 52 & 54 & 15.8 & 16.5 & clay, blue & Clay & -- & .- & .- & \\
\hline 22 & 13/11-19Q1 & & & 54 & 75 & 16.5 & 22.9 & gravel, large blue & Gravel & -- & -- & -- & \\
\hline 22 & 13/11-19Q1 & & & 75 & 79 & 22.9 & 24.1 & clay, blue, and gravel & Clay and gravel & -- & -- & -- & \\
\hline 22 & 13/11-19Q1 & & & 79 & 100 & 24.1 & 30.5 & clay, sticky blue & Clay & -- & -- & -- & \\
\hline 22 & 13/11-19Q1 & & & 100 & 114 & 30.5 & 34.7 & gravel & Gravel & -- & -- & -- & \\
\hline 23 & 11/10-17R1 & 23 & 11/10-17R1 & 0 & 2 & 0.0 & 0.6 & surface solid & Sand and clay & -- & -- & -- & Qal \\
\hline 23 & 11/10-17R1 & & & 2 & 12 & 0.6 & 3.7 & yellow sand and scattered gravel & Sand (or sandstone) and gravel & -- & -- & -- & \\
\hline 23 & 11/10-17R1 & & & 12 & 18 & 3.7 & 5.5 & brown silt, moist & Sand and clay & -- & -- & -- & \\
\hline 23 & 11/10-17R1 & & & 18 & 39 & 5.5 & 11.9 & loose gravel and yellow sand & Sand (or sandstone) and gravel & -- & -- & -- & \\
\hline 23 & 11/10-17R1 & & & 39 & 41 & 11.9 & 12.5 & hard blue rock, broken & Undifferentiated basement & hard & -- & -- & $\mathrm{KJu}$ \\
\hline 24 & 11/10-28L1 & 24 & 11/10-28L1 & 0 & 18.5 & 0.0 & 5.6 & river gravel & Gravel & -- & -- & -- & Qal \\
\hline 24 & 11/10-28L1 & & & 18.5 & 20 & 5.6 & 6.1 & bedrock & Gravel & hard & -- & -- & KJu \\
\hline 25 & $11 / 10-33 \mathrm{~A}$ & 25 & 11/10-33A & 0 & 12 & 0.0 & 3.7 & gravel (dry) & Gravel & -- & -- & -- & Qal \\
\hline 25 & $11 / 10-33 \mathrm{~A}$ & & & 12 & 20 & 3.7 & 6.1 & gravel (water) & Gravel & -- & -- & -- & \\
\hline 25 & 11/10-33A & & & 20 & 40 & 6.1 & 12.2 & shale & Clay and sand (or sandstone) & cemented & -- & -- & KJu \\
\hline 26 & 10/11-12Q1 & 26 & 10/11-12Q1 & 0 & 10 & 0.0 & 3.0 & gravel and sand & Sand (or sandstone) and gravel & -- & -- & -- & Qal \\
\hline 26 & 10/11-12Q1 & & & 10 & 20 & 3.0 & 6.1 & gravel and small boulders & Gravel & -- & -- & -- & \\
\hline 26 & 10/11-12Q1 & & & 20 & 38 & 6.1 & 11.6 & big rocks and gravel & Gravel & -- & -- & -- & \\
\hline 26 & 10/11-12Q1 & & & 38 & 42 & 11.6 & 12.8 & clay and brown gravel & Clay and gravel & -- & -- & -- & \\
\hline 26 & 10/11-12Q1 & & & 42 & 47 & 12.8 & 14.3 & large gravel & Gravel & -- & -- & -- & \\
\hline 26 & 10/11-12Q1 & & & 47 & 55 & 14.3 & 16.8 & blue clay & Clay & -- & -- & -- & \\
\hline 27 & 10/9-26L1 & 27 & 10/9-26L1 & 0 & 12 & 0.0 & 3.7 & topsoil & Sand and clay & -- & -- & -- & Qal and QTge \\
\hline 27 & 10/9-26L1 & & & 12 & 37 & 3.7 & 11.3 & sand and small gravel & Sand (or sandstone) and gravel & -- & -- & -- & \\
\hline 27 & 10/9-26L1 & & & 37 & 44 & 11.3 & 13.4 & tight clay, yellow & Clay & -- & -- & -- & \\
\hline 27 & 10/9-26L1 & & & 44 & 51 & 13.4 & 15.5 & medium small gravel & Gravel & -- & -- & -- & \\
\hline 27 & 10/9-26L1 & & & 51 & 80 & 15.5 & 24.4 & sticky clay & Clay & -- & -- & -- & \\
\hline 27 & 10/9-26L1 & & & 80 & 87 & 24.4 & 26.5 & sand & Sand & -- & -- & -- & \\
\hline 27 & 10/9-26L1 & & & 87 & 109 & 26.5 & 33.2 & blue clay & Clay & -- & -- & -- & \\
\hline 27 & 10/9-26L1 & & & 109 & 119 & 33.2 & 36.3 & clay and mixed sand & Clay and sand (or sandstone) & -- & -- & -- & \\
\hline 27 & 10/9-26L1 & & & 119 & 127 & 36.3 & 38.7 & tight mica sand & Sand & -- & -- & -- & \\
\hline 27 & 10/9-26L1 & & & 127 & 134 & 38.7 & 40.8 & sand and small gravel & Sand (or sandstone) and gravel & -- & -- & -- & \\
\hline 27 & 10/9-26L1 & & & 134 & 142 & 40.8 & 43.3 & pea gravel and sand & Sand (or sandstone) and gravel & -- & -- & -- & \\
\hline 27 & 10/9-26L1 & & & 142 & 145 & 43.3 & 44.2 & pea gravel & Gravel & -- & -- & -- & \\
\hline 27 & 10/9-26L1 & & & 145 & 150 & 44.2 & 45.7 & clay & Clay & -- & -- & -- & \\
\hline 27 & 10/9-26L1 & & & 150 & 155 & 45.7 & 47.2 & yellow sand and gravel & Sand (or sandstone) and gravel & -- & -- & -- & \\
\hline 27 & 10/9-26L1 & & & 155 & 163 & 47.2 & 49.7 & sandy yellow clay & Clay and sand (or sandstone) & -- & -- & -- & \\
\hline
\end{tabular}


Appendix 3. Lithologic data. - Continued

[Site IDs and well number correspond to locations in Appendix 1, a site ID and well number is listed for each drilled interval for a particular drill hole. For convenience, the site ID and well numbers are repeated at the shallowest drilled interval for each well.

\begin{tabular}{|c|c|c|c|c|c|c|c|c|c|c|c|c|c|}
\hline Site ID & Well number & $\begin{array}{l}\text { Site ID, } \\
\text { top of } \\
\text { well }\end{array}$ & $\begin{array}{l}\text { Well number, } \\
\text { top of well }\end{array}$ & $\begin{array}{c}\text { Top of } \\
\text { interval, in } \\
\text { feet }\end{array}$ & $\begin{array}{c}\text { Base of } \\
\text { interval, in } \\
\text { feet }\end{array}$ & $\begin{array}{c}\text { Top of } \\
\text { interval, in } \\
m\end{array}$ & $\begin{array}{c}\text { Base of } \\
\text { interval, in } \\
\mathrm{m}\end{array}$ & Reported lithologic unit ${ }^{1}$ & Interpreted lithologic class ${ }^{2}$ & $\begin{array}{c}\text { Reported } \\
\text { degree of } \\
\text { cementation or } \\
\text { induration } \\
\end{array}$ & $\begin{array}{c}\text { Fossils } \\
\text { reported in } \\
\text { interval }^{4}\end{array}$ & $\begin{array}{c}\text { Organic } \\
\text { matter } \\
\text { reported in } \\
\text { interval }^{5}\end{array}$ & $\begin{array}{l}\text { Reported stratigraphic top of geologic } \\
\text { unit }^{6}\end{array}$ \\
\hline 27 & 10/9-26L1 & & & 163 & 170 & 49.7 & 51.8 & gray clay & Clay & -- & -- & -- & \\
\hline 27 & 10/9-26L1 & & & 170 & 172 & 51.8 & 52.4 & gravel & Gravel & -- & -- & -- & \\
\hline 27 & 10/9-26L1 & & & 172 & 192 & 52.4 & 58.5 & sandy soil & Sand and clay & -- & -- & -- & \\
\hline 27 & 10/9-26L1 & & & 192 & 210 & 58.5 & 64.0 & gray clay and sand & Clay and sand (or sandstone) & -- & -. & -- & \\
\hline 27 & 10/9-26L1 & & & 210 & 216 & 64.0 & 65.8 & gravel & Gravel & -- & -- & -- & \\
\hline 27 & 10/9-26L1 & & & 216 & 220 & 65.8 & 67.1 & gray mud & Clay and sand (or sandstone) & -- & -- & -- & \\
\hline 27 & 10/9-26L1 & & & 220 & 224 & 67.1 & 68.3 & gravel & Gravel & -- & -- & -- & \\
\hline 27 & 10/9-26L1 & & & 224 & 240 & 68.3 & 73.2 & gravel and blue mud & Clay, sand, and gravel & -- & -- & -- & \\
\hline 27 & 10/9-26L1 & & & 240 & 244 & 73.2 & 74.4 & $\begin{array}{l}\text { gray mud } \\
\text { gice }\end{array}$ & Clay and sand (or sandstone) & -- & -- & - & \\
\hline 28 & 9/10-2B3 & 28 & 9/10-2B3 & 0 & 18 & 0.0 & 5.5 & clay and silt & Clay and sand (or sandstone) & -- & -. & -- & Qal and Qt and QTge (?) \\
\hline 28 & 9/10-2B3 & & & 18 & 30 & 5.5 & 9.1 & gravel & Gravel & -- & -- & -- & \\
\hline 28 & 9/10-2B3 & & & 30 & 50 & 9.1 & 15.2 & mostly clay and clay and gravel & Clay and gravel & -- & -- & -- & \\
\hline 28 & 9/10-2B3 & & & 50 & 56 & 15.2 & 17.1 & gravel (6 ft. thick) near $55 \mathrm{ft}$. & Gravel & -- & -- & -- & \\
\hline 28 & 9/10-2B3 & & & 56 & 160 & 17.1 & 48.8 & (not logged) & No data & -- & -- & -- & \\
\hline 28 & 9/10-2B3 & & & 160 & 167 & 48.8 & 50.9 & gravel $(7 \mathrm{ft}$. thick) near $160 \mathrm{ft}$. & Gravel & .- & .. & .. & \\
\hline 28 & 9/10-2B3 & & & 167 & 285 & 50.9 & 86.9 & bottom in soft clay and gravel & Clay and gravel & -- & -- & -- & \\
\hline 29 & 9/10-2B1 & 29 & 9/10-2B1 & 0 & 17 & 0.0 & 5.2 & topsoil & Sand and clay & -- & -- & -- & Qal \\
\hline 29 & 9/10-2B1 & & & 17 & 23 & 5.2 & 7.0 & sand & Sand & -- & -- & -- & \\
\hline 29 & 9/10-2B1 & & & 23 & 37 & 7.0 & 11.3 & clay & Clay & -- & -- & -- & \\
\hline 29 & 9/10-2B1 & & & 37 & 43 & 11.3 & 13.1 & gravel, pea & Gravel & -- & -- & -- & \\
\hline 29 & 9/10-2B1 & & & 43 & 49 & 13.1 & 14.9 & boulders, large (to $1 \mathrm{ft.}$, diameter) & Gravel & -- & .- & .- & \\
\hline 29 & 9/10-2B1 & & & 49 & 73 & 14.9 & 22.3 & shale (hard blue clay) & Clay and sand (or sandstone) & hard & .. & -- & \\
\hline 29 & 9/10-2B1 & & & 73 & 87 & 22.3 & 26.5 & sticky gumbo (like rubber) & Clay and sand (or sandstone) & -. & -. & -.- & \\
\hline 29 & 9/10-2B1 & & & 87 & 89 & 26.5 & 27.1 & sand and clay, mixed & Sand and clay & -- & -- & -- & \\
\hline 29 & 9/10-2B1 & & & 89 & 99 & 27.1 & 30.2 & gravel & Gravel & -- & -- & -- & \\
\hline 29 & 9/10-2B1 & & & 99 & 107 & 30.2 & 32.6 & shale and serpentine & Undifferentiated basement & cemented & -- & -- & KJu \\
\hline 30 & 9/9-4E1 & 30 & 9/9-4E1 & 0 & 12 & 0.0 & 3.7 & $\begin{array}{l}\text { topsoil } \\
\text { to }\end{array}$ & Sand and clay & - & -. & - & Qal and KJu(?) \\
\hline 30 & 9/9-4E1 & & & 12 & 18 & 3.7 & 5.5 & sand and small gravel & Sand (or sandstone) and gravel & -- & -. & -- & \\
\hline 30 & 9/9-4E1 & & & 18 & 37 & 5.5 & 11.3 & clay and mixed gravel & Clay and gravel & -- & -- & -- & \\
\hline 30 & 9/9-4E1 & & & 37 & 76 & 11.3 & 23.2 & clay & Clay & -- & -- & -- & \\
\hline 30 & 9/9-4E1 & & & 76 & 81 & 23.2 & 24.7 & $\begin{array}{l}\text { clay and mixed boulders } \\
\text {. }\end{array}$ & Clay and gravel & -- & -- & -- & \\
\hline 30 & 9/9-4E1 & & & 81 & 89 & 24.7 & 27.1 & shale & Clay and sand (or sandstone) & cemented & -- & -- & \\
\hline 30 & 9/9-4E1 & & & 89 & 96 & 27.1 & 29.3 & rock & Gravel & hard & -- & -- & \\
\hline 30 & 9/9-4E1 & & & 96 & 98 & 29.3 & 29.9 & clay & Clay & -- & -- & -- & \\
\hline 30 & 9/9-4E1 & & & 98 & 104 & 29.9 & 31.7 & rock & Gravel & hard & -- & -- & \\
\hline 30 & 9/9-4E1 & & & 104 & 108 & 31.7 & 32.9 & clay & Clay & -- & -- & -- & \\
\hline 30 & 9/9-4E1 & & & 108 & 112 & 32.9 & 34.1 & clay, boulders & Clay and gravel & -- & -- & -- & \\
\hline 30 & 9/9-4E1 & & & 112 & 116 & 34.1 & 35.4 & boulders, small gravel, and sand & Sand (or sandstone) and gravel & .. & .- & .- & \\
\hline 30 & 9/9-4E1 & & & 116 & 117 & 35.4 & 35.7 & shale & Clay and sand (or sandstone) & cemented & -- & -- & \\
\hline 31 & 9/8-7Q1 & 31 & 9/8-7Q1 & 0 & 4 & 0.0 & 1.2 & topsoil & Sand and clay & -- & -- & -- & Qal and QTge \\
\hline 31 & 9/8-7Q1 & & & 4 & 19 & 1.2 & 5.8 & brown clay & Clay & -- & -- & -.- & \\
\hline 31 & 9/8-7Q1 & & & 19 & 40 & 5.8 & 12.2 & blue sand and large gravel & Sand (or sandstone) and gravel & -- & -- & -- & \\
\hline 31 & 9/8-7Q1 & & & 40 & 75 & 12.2 & 22.9 & blue clay and sand & Clay and sand (or sandstone) & -- & - & -- & \\
\hline 31 & 9/8-7Q1 & & & 75 & 92 & 22.9 & 28.0 & soft blue clay & Clay & -- & -- & -- & \\
\hline 31 & 9/8-7Q1 & & & 92 & 130 & 28.0 & 39.6 & hard blue clay & Clay & hard & -- & -- & \\
\hline 31 & 9/8-7Q1 & & & 130 & 147 & 39.6 & 44.8 & soft blue sand & Sand & -. & -. & -.- & \\
\hline 31 & 9/8-7Q1 & & & 147 & 160 & 44.8 & 48.8 & hard blue shale & Clay and sand (or sandstone) & hard & -. & -- & \\
\hline 31 & 9/8-7Q1 & & & 160 & 200 & 48.8 & 61.0 & soft sand & Sand & -- & -- & -- & \\
\hline 31 & 9/8-7Q1 & & & 200 & 476 & 61.0 & 145.1 & soft blue sand, and shale streaks & Sand and clay & cemented & -- & -- & \\
\hline 31 & 9/8-7Q1 & & & 476 & 490 & 145.1 & 149.4 & blue clay & Clay & -- & .- & -- & \\
\hline 32 & 9/9-21R1 & 32 & 9/9-21R1 & 0 & 20 & 0.0 & 6.1 & sediment & Sand & -- & -- & -- & Qal \\
\hline 32 & 9/9-21R1 & & & 20 & 30 & 6.1 & 9.1 & gravel & Gravel & -- & -- & -- & \\
\hline 32 & 9/9-21R1 & & & 30 & 36 & 9.1 & 11.0 & gravel and boulders & Gravel & -- & -- & -- & \\
\hline 32 & 9/9-21R1 & & & 36 & 41 & 11.0 & 12.5 & boulders and clay & Clay and gravel & -- & -- & -- & \\
\hline 32 & 9/9-21R1 & & & 41 & 55 & 12.5 & 16.8 & boulders & Gravel & -- & -- & -- & \\
\hline 32 & 9/9-21R1 & & & 55 & 113 & 16.8 & 34.4 & blue clay & Clay & -- & -- & -- & \\
\hline 32 & 9/9-21R1 & & & 113 & 138 & 34.4 & 42.1 & gravel & Gravel & -- & -- & -- & \\
\hline 32 & 9/9-21R1 & & & 138 & 154 & 42.1 & 46.9 & blue clay & Clay & -. & -. & -- & \\
\hline
\end{tabular}


Appendix 3. Lithologic data. - Continued

[Site IDs and well number correspond to locations in Appendix 1, a site ID and well number is listed for each drilled interval for a particular drill hole. For convenience, the site ID and well numbers are repeated at the shallowest drilled interval for each well. leaders

\begin{tabular}{|c|c|c|c|c|c|c|c|c|c|c|c|c|c|}
\hline Site ID & Well number & $\begin{array}{l}\text { Site ID, } \\
\text { top of } \\
\text { well }\end{array}$ & $\begin{array}{l}\text { Well number, } \\
\text { top of well }\end{array}$ & $\begin{array}{c}\text { Top of } \\
\text { interval, in } \\
\text { feet }\end{array}$ & $\begin{array}{l}\text { Base of } \\
\text { interval, in } \\
\text { feet }\end{array}$ & $\begin{array}{c}\text { Top of } \\
\text { interval, in } \\
\mathrm{m}\end{array}$ & $\begin{array}{c}\text { Base of } \\
\text { interval, in } \\
\mathrm{m}\end{array}$ & Reported lithologic unit' & Interpreted lithologic class ${ }^{2}$ & $\begin{array}{c}\text { Reported } \\
\text { degree of } \\
\text { cementation or } \\
\text { induration }^{3} \\
\end{array}$ & $\begin{array}{c}\text { Fossils } \\
\text { reported in } \\
\text { interval }^{4}\end{array}$ & $\begin{array}{c}\text { Organic } \\
\text { matter } \\
\text { reported in } \\
\text { interval }^{5}\end{array}$ & $\begin{array}{l}\text { Reported stratigraphic top of geologic } \\
\text { unit }^{6}\end{array}$ \\
\hline 32 & 9/9-21R1 & & & 154 & 164 & 46.9 & 50.0 & brown shale & Clay and sand (or sandstone) & cemented & -- & -- & \\
\hline 32 & 9/9-21R1 & & & 164 & 167 & 50.0 & 50.9 & blue clay & Clay & -- & -- & -- & \\
\hline 32 & 9/9-21R1 & & & 167 & 170 & 50.9 & 51.8 & brown clay & Clay & -- & -- & -- & \\
\hline 32 & 9/9-21R1 & & & 170 & 173 & 51.8 & 52.7 & blue clay & Clay & -- & -- & -- & \\
\hline 32 & 9/9-21R1 & & & 173 & 177.5 & 52.7 & 54.1 & shale & Clay and sand (or sandstone) & cemented & -- & -- & $\mathrm{KJu}$ \\
\hline 33 & 9/9-33Е2 & 33 & 9/9-33Е2 & 0 & 27 & 0.0 & 8.2 & yellow clay & Clay & -- & -- & -- & Qal \\
\hline 33 & 9/9-33E2 & & & 27 & 53 & 8.2 & 16.2 & gravel and coarse sand & Gravel & -- & -- & -- & \\
\hline 33 & 9/9-33E2 & & & 53 & 66 & 16.2 & 20.1 & gravel & Gravel & -- & -- & -- & \\
\hline 34 & 8/9-19K1 & 34 & 8/9-19K1 & 0 & 4 & 0.0 & 1.2 & soil & Sand and clay & -- & -- & -- & Qal \\
\hline 34 & 8/9-19K1 & & & 4 & 25 & 1.2 & 7.6 & clay, brown & Clay & -- & -- & -- & \\
\hline 34 & 8/9-19K1 & & & 25 & 62 & 7.6 & 18.9 & blue clay and sand & Clay and sand (or sandstone) & -- & -- & -- & \\
\hline 34 & 8/9-19K1 & & & 62 & 63 & 18.9 & 19.2 & gravel & Gravel & -- & -- & -- & \\
\hline 34 & 8/9-19K1 & & & 63 & 64 & 19.2 & 19.5 & serpentine rock & Undifferentiated basement & -- & -- & -- & $\mathrm{KJu}$ \\
\hline 35 & 8/10-20M3 & 35 & 8/10-20M3 & 0 & 2 & 0.0 & 0.6 & soil & Sand and clay & -- & -- & -- & Qal \\
\hline 35 & 8/10-20M3 & & & 2 & 6 & 0.6 & 1.8 & gravel & Gravel & -- & -- & -- & \\
\hline 35 & 8/10-20M3 & & & 6 & 30 & 1.8 & 9.1 & clay, yellow, and gravel & Clay and gravel & -- & -- & -- & \\
\hline 35 & 8/10-20M3 & & & 30 & 47 & 9.1 & 14.3 & gravel & Gravel & -- & -- & -- & \\
\hline 35 & 8/10-20M3 & & & 47 & 50 & 14.3 & 15.2 & clay, blue, soft & Clay & -- & -- & -- & \\
\hline 35 & 8/10-20M3 & & & 50 & 55 & 15.2 & 16.8 & gravel & Gravel & -- & -- & -- & \\
\hline 35 & 8/10-20M3 & & & 55 & 59 & 16.8 & 18.0 & redwood log & Clay and sand (or sandstone) & -- & -- & wood & \\
\hline 35 & 8/10-20M3 & & & 59 & 65 & 18.0 & 19.8 & mud, blue & Clay and sand (or sandstone) & -- & -- & -- & \\
\hline 35 & 8/10-20M3 & & & 65 & 84 & 19.8 & 25.6 & clay, blue, soft & Clay & -- & -- & -- & \\
\hline 35 & 8/10-20M3 & & & 84 & 110 & 25.6 & 33.5 & clay, yellow, soft, and gravel & Clay and gravel & -- & -- & -- & \\
\hline 35 & 8/10-20M3 & & & 110 & 120 & 33.5 & 36.6 & gravel & Gravel & -- & -- & -- & \\
\hline 35 & 8/10-20M3 & & & 120 & 123 & 36.6 & 37.5 & clay, yellow and gravel & Clay and gravel & -- & -- & -- & \\
\hline 36 & 8/10-29D1 & 36 & 8/10-29D1 & 0 & 30 & 0.0 & 9.1 & sand & Sand & -- & -- & -- & Qal \\
\hline 36 & 8/10-29D1 & & & 30 & 120 & 9.1 & 36.6 & gravel & Gravel & -- & -- & -- & \\
\hline 36 & 8/10-29D1 & & & 120 & 183 & 36.6 & 55.8 & basalt & Undifferentiated basement & -- & -- & -- & $\mathrm{KJu}$ \\
\hline 37 & $8 / 10-29 \mathrm{H} 2$ & 37 & 8/10-29H2 & 0 & 23 & 0.0 & 7.0 & top clay and sand & Sand and clay & -- & -- & -- & Qal \\
\hline 37 & $8 / 10-29 \mathrm{H} 2$ & & & 23 & 28 & 7.0 & 8.5 & top clay and sand & Sand and clay & -- & -- & -- & \\
\hline 37 & $8 / 10-29 \mathrm{H} 2$ & & & 28 & 48 & 8.5 & 14.6 & gravel & Gravel & -- & -- & -- & \\
\hline 37 & $8 / 10-29 \mathrm{H} 2$ & & & 48 & 56 & 14.6 & 17.1 & sand and gravel & Sand (or sandstone) and gravel & -- & -- & -- & \\
\hline 37 & 8/10-29H2 & & & 56 & 70 & 17.1 & 21.3 & fine gravel & Gravel & -- & -- & -- & \\
\hline 37 & $8 / 10-29 \mathrm{H} 2$ & & & 70 & 74 & 21.3 & 22.6 & coarse gravel & Gravel & -- & -- & -- & \\
\hline 37 & $8 / 10-29 \mathrm{H} 2$ & & & 74 & 76 & 22.6 & 23.2 & fine gravel & Gravel & -- & -- & -- & \\
\hline 37 & $8 / 10-29 \mathrm{H} 2$ & & & 76 & 107 & 23.2 & 32.6 & gravel & Gravel & -- & -- & -- & \\
\hline 37 & $8 / 10-29 \mathrm{H} 2$ & & & 107 & 111 & 32.6 & 33.8 & fine gravel & Gravel & -- & -- & -- & \\
\hline 37 & $8 / 10-29 \mathrm{H} 2$ & & & 111 & 115 & 33.8 & 35.1 & $\begin{array}{l}\text { sand gravel } \\
\text { gray shale and gravel (reported by }\end{array}$ & Sand (or sandstone) and gravel & -- & -- & -- & \\
\hline 37 & $8 / 10-29 \mathrm{H} 2$ & & & 115 & 125 & 35.1 & 38.1 & observer to be bedrock) & Undifferentiated basement & cemented & -- & -- & KJu \\
\hline 38 & 8/10-29N1 & 38 & 8/10-29N1 & 0 & 12 & 0.0 & 3.7 & sandy yellow loam & Sand and clay & -- & -- & -- & Qal \\
\hline 38 & 8/10-29N1 & & & 12 & 18 & 3.7 & 5.5 & blue clay & Clay & -- & -- & -- & \\
\hline 38 & 8/10-29N1 & & & 18 & 30 & 5.5 & 9.1 & gravel & Gravel & -- & -- & -- & \\
\hline 38 & 8/10-29N1 & & & 30 & 35 & 9.1 & 10.7 & blue clay & Clay & -- & -- & -- & \\
\hline 38 & 8/10-29N1 & & & 35 & 40 & 10.7 & 12.2 & yellow clay & Clay & -- & -- & -- & \\
\hline 38 & 8/10-29N1 & & & 40 & 43 & 12.2 & 13.1 & blue clay & Clay & -- & -- & -- & \\
\hline 38 & 8/10-29N1 & & & 43 & 72 & 13.1 & 21.9 & green clay & Clay & -- & -- & -- & \\
\hline 38 & 8/10-29N1 & & & 72 & 75 & 21.9 & 22.9 & blue sand & Sand & -- & -- & -- & \\
\hline 38 & 8/10-29N1 & & & 75 & 79 & 22.9 & 24.1 & blue sand and clay & Sand and clay & -- & -- & -- & \\
\hline 38 & $8 / 10-29 N 1$ & & & 79 & 88 & 24.1 & 26.8 & blue sand and gravel & Sand (or sandstone) and gravel & -- & -- & -- & \\
\hline 38 & 8/10-29N1 & & & 88 & 90 & 26.8 & 27.4 & blue sand & Sand & -- & -- & -- & \\
\hline 39 & 8/9-31A1 & 39 & 8/9-31A1 & 0 & 4 & 0.0 & 1.2 & black loam & Sand and clay & -- & -- & -- & Qt \\
\hline 39 & 8/9-31A1 & & & 4 & 17 & 1.2 & 5.2 & yellow clay & Clay & -- & -- & -- & \\
\hline 39 & 8/9-31A1 & & & 17 & 20 & 5.2 & 6.1 & yellow sand and gravel & Sand (or sandstone) and gravel & -- & -- & -- & \\
\hline 39 & 8/9-31A1 & & & 20 & 30 & 6.1 & 9.1 & gravel & Gravel & -- & -- & -- & \\
\hline 39 & 8/9-31A1 & & & 30 & 34 & 9.1 & 10.4 & yellow sand and gravel & Sand (or sandstone) and gravel & -- & -- & -- & \\
\hline 39 & 8/9-31A1 & & & 34 & 72 & 10.4 & 21.9 & black sandy clay & Clay and sand (or sandstone) & -- & -- & -- & \\
\hline 39 & 8/9-31A1 & & & 72 & 74 & 21.9 & 22.6 & blue sand and clay & Sand and clay & -- & -- & -- & QTm \\
\hline 39 & 8/9-31A1 & & & 74 & 110 & 22.6 & 33.5 & blue sandy clay & Clay and sand (or sandstone) & -- & -- & -- & \\
\hline 39 & 8/9-31A1 & & & 110 & 130 & 33.5 & 39.6 & blue sand & Sand & -- & -- & -- & \\
\hline 39 & 8/9-31A1 & & & 130 & 235 & 39.6 & 71.6 & blue sandstone & Sandstone & cemented & -- & -- & \\
\hline
\end{tabular}


Appendix 3. Lithologic data. - Continued

ISite IDs and well number correspond to locations in Appendix 1, a site ID and well number is listed for each drilled interval for a particular drill hole. For convenience, the site ID and well numbers are repeated at the shallowest drilled interval for each well.

\begin{tabular}{|c|c|c|c|c|c|c|c|c|c|c|c|c|c|}
\hline Site ID & Well number & $\begin{array}{l}\text { Site ID, } \\
\text { top of } \\
\text { well }\end{array}$ & $\begin{array}{l}\text { Well number, } \\
\text { top of well }\end{array}$ & $\begin{array}{c}\text { Top of } \\
\text { interval, in } \\
\text { feet }\end{array}$ & $\begin{array}{c}\text { Base of } \\
\text { interval, in } \\
\text { feet }\end{array}$ & $\begin{array}{c}\text { Top of } \\
\text { interval, in } \\
m\end{array}$ & $\begin{array}{c}\text { Base of } \\
\text { interval, in } \\
\mathrm{m}\end{array}$ & Reported lithologic unit ${ }^{1}$ & Interpreted lithologic class ${ }^{2}$ & $\begin{array}{c}\text { Reported } \\
\text { degree of } \\
\text { cementation or } \\
\text { induration } \\
\end{array}$ & $\begin{array}{c}\text { Fossils } \\
\text { reported in } \\
\text { interval }^{4}\end{array}$ & $\begin{array}{c}\text { Organic } \\
\text { matter } \\
\text { reported in } \\
\text { interval }^{5}\end{array}$ & $\begin{array}{l}\text { Reported stratigraphic top of geologic } \\
\text { unit }^{6}\end{array}$ \\
\hline $\begin{array}{l}39 \\
39\end{array}$ & $\begin{array}{l}\text { 8/9-31A1 } \\
\text { 8/9-31A1 }\end{array}$ & & & $\begin{array}{l}235 \\
260\end{array}$ & $\begin{array}{l}260 \\
325\end{array}$ & $\begin{array}{l}71.6 \\
79.2\end{array}$ & $\begin{array}{l}79.2 \\
99.1\end{array}$ & $\begin{array}{l}\text { blue clay } \\
\text { blue sandstone }\end{array}$ & $\begin{array}{l}\text { Clay } \\
\text { Sandstone }\end{array}$ & $\begin{array}{c}-- \\
\text { cemented }\end{array}$ & $\overline{--}$ & -- & \\
\hline 40 & $\begin{array}{l}\text { O//9-3IA1 } \\
8 / 9-32 E 3\end{array}$ & 40 & 8/9-32E3 & $\begin{array}{l}260 \\
0\end{array}$ & $\begin{array}{l}325 \\
3\end{array}$ & 0.0 & $\begin{array}{l}9.1 \\
0.9\end{array}$ & $\begin{array}{l}\text { liue sandstone } \\
\text { black loam }\end{array}$ & $\begin{array}{l}\text { Sandstone } \\
\text { Sand and clay }\end{array}$ & $\begin{array}{c}\text { cemented } \\
\text {-- }\end{array}$ & $\begin{array}{l}-- \\
-\end{array}$ & -- & Qt \\
\hline 40 & 8/9-32E3 & & & 3 & 31 & 0.9 & 9.4 & river silt, brown & Sand and clay & -- & -- & -- & \\
\hline 40 & 8/9-32E3 & & & 31 & 37 & 9.4 & 11.3 & yellow sand and gravel & Sand (or sandstone) and gravel & -- & -- & -- & \\
\hline 40 & 8/9-32E3 & & & 37 & 43 & 11.3 & 13.1 & yellow clay and gravel & Clay and gravel & -- & -- & -- & \\
\hline 40 & 8/9-32E 3 & & & 43 & 56 & 13.1 & 17.1 & yellow clay & Clay & -- & -- & -- & \\
\hline 40 & 8/9-32E3 & & & 56 & 60 & 17.1 & 18.3 & black sand and clay & Sand and clay & -- & -- & -- & \\
\hline 40 & 8/9-32E3 & & & 60 & 83 & 18.3 & 25.3 & blue sandstone & Sandstone & cemented & -- & -- & QTm \\
\hline 41 & 7/10-7D1 & 41 & 7/10-7D1 & 0 & 27 & 0.0 & 8.2 & clay and sand & Clay and sand (or sandstone) & -- & -. & -- & Qal \\
\hline 41 & 7/10-7D1 & & & 27 & 40 & 8.2 & 12.2 & sand and gravel & Sand (or sandstone) and gravel & -- & -- & -- & \\
\hline 41 & 7/10-7D1 & & & 40 & 55 & 12.2 & 16.8 & gravel & Gravel & -- & -- & -- & \\
\hline 41 & 7/10-7D1 & & & 55 & 67 & 16.8 & 20.4 & sand and gravel & Sand (or sandstone) and gravel & -- & -- & -- & \\
\hline 41 & 7/10-7D1 & & & 67 & 71 & 20.4 & 21.6 & $\begin{array}{l}\text { gravel } \\
\text { la gravel }\end{array}$ & Gravel & - & - & - & \\
\hline 41 & 7/10-7D1 & & & 71 & 80 & 21.6 & 24.4 & sand and gravel & Sand (or sandstone) and gravel & -- & -- & -- & \\
\hline 41 & 7/10-7D1 & & & 80 & 84 & 24.4 & 25.6 & $\begin{array}{l}\text { gravel } \\
\text { gataver }\end{array}$ & Gravel & -. & -- & -- & \\
\hline 41 & 7/10-7D1 & & & 84 & 94 & 25.6 & 28.7 & sand and gravel & Sand (or sandstone) and gravel & -- & -- & -- & \\
\hline 41 & 7/10-7D1 & & & 94 & 120 & 28.7 & 36.6 & coarse gravel & Gravel & -- & -- & -- & \\
\hline 42 & 7/11-11J1 & 42 & 7/11-11J1 & 0 & 3 & 0.0 & 0.9 & surface & Sand and clay & -- & -- & -- & Qal \\
\hline 42 & 7/11-11J1 & & & 3 & 102 & 0.9 & 31.1 & sand & Sand & -- & -- & -- & \\
\hline 42 & 7/11-11J1 & & & 102 & 107 & 31.1 & 32.6 & boulders and yellow clay & Clay and gravel & -. & -. & -- & \\
\hline 42 & 7/111-11J1 & & & 107 & 136 & 32.6 & 41.5 & gravel, free & Gravel & -- & -- & -- & \\
\hline 43 & 7/11-17J1 & 43 & 7/11-17J1 & 0 & 146 & 0.0 & 44.5 & blue clay and gravel & Clay and gravel & .. & -. & -- & Qal \\
\hline 43 & 7/11-17J1 & & & 146 & 148 & 44.5 & 45.1 & serpentine & Undifferentiated basement & .. & -. & .- & KJu \\
\hline 44 & 8/9-1M1 & 44 & 8/9-1M1 & 0 & 6 & 0.0 & 1.8 & surface soil & Sand and clay & -. & -- & -- & QTge \\
\hline 44 & 8/9-1M1 & & & 6 & 90 & 1.8 & 27.4 & yellow clay & Clay & -- & -- & -- & \\
\hline 44 & 8/9-1M1 & & & 90 & 95 & 27.4 & 29.0 & gravel, first water & Gravel & -. & -- & -- & \\
\hline 44 & 8/9-1M1 & & & 95 & 162 & 29.0 & 49.4 & yellow clay & Clay & - & -. & - & \\
\hline 44 & 8/9-1M1 & & & 162 & 250 & 49.4 & 76.2 & blue clay & Clay & .. & -- & -.- & \\
\hline 44 & 8/9-1M1 & & & 250 & 252 & 76.2 & 76.8 & sand and gravel & Sand (or sandstone) and gravel & .. & -. & -.- & \\
\hline 44 & 8/9-1M1 & & & 252 & 261 & 76.8 & 79.6 & blue clay & Clay & -- & -- & -- & \\
\hline 44 & 8/9-1M1 & & & 261 & 262 & 79.6 & 79.9 & sand and gravel & Sand (or sandstone) and gravel & -- & -- & -- & \\
\hline 44 & 8/9-1M1 & & & 262 & 281 & 79.9 & 85.6 & $\begin{array}{l}\text { blue clay } \\
\text { antive }\end{array}$ & Clay & - & -- & - & \\
\hline 44 & 8/9-1M1 & & & 281 & 282 & 85.6 & 86.0 & sand and gravel & Sand (or sandstone) and gravel & .. & .- & .- & \\
\hline 44 & 8/9-1M1 & & & 282 & 298 & 86.0 & 90.8 & blue clay & Clay & -. & -- & -- & \\
\hline 44 & 8/9-1M1 & & & 298 & 300 & 90.8 & 91.4 & sand and gravel & Sand (or sandstone) and gravel & -- & -- & -- & \\
\hline 44 & 8/9-1M1 & & & 300 & 315 & 91.4 & 96.0 & blue clay & Clay & -. & -- & -- & \\
\hline 45 & 8/9-3P1 & 45 & 8/9-3P1 & 0 & 10 & 0.0 & 3.0 & black soil & Sand and clay & -- & -- & -- & Qyal \\
\hline 45 & 8/9-3P1 & & & 10 & 20 & 3.0 & 6.1 & hardpan, yellow & Clay and gravel & hard & -- & .- & QTge \\
\hline 45 & 8/9-3P1 & & & 20 & 35 & 6.1 & 10.7 & yellow clay & Clay & -- & .- & .- & \\
\hline 45 & 8/9-3P1 & & & 35 & 40 & 10.7 & 12.2 & yellow sand and gravel & Sand (or sandstone) and gravel & -- & -- & -- & \\
\hline 45 & 8/9-3P1 & & & 40 & 52 & 12.2 & 15.8 & yellow gravel & Sand (or sandstone) and gravel & -- & -. & -.- & \\
\hline 45 & 8/9-3P1 & & & 52 & 74 & 15.8 & 22.6 & blue sandy clay & Clay and sand (or sandstone) & -- & -- & -.- & \\
\hline 45 & 8/9-3P1 & & & 74 & 85 & 22.6 & 25.9 & gravel and clay & Clay and gravel & -- & -- & -- & \\
\hline 45 & 8/9-3P1 & & & 85 & 90 & 25.9 & 27.4 & $\begin{array}{l}\text { blue sand } \\
\text { blay }\end{array}$ & Sand & .- & -- & -- & \\
\hline 45 & 8/9-3P1 & & & 90 & 118 & 27.4 & 36.0 & blue clay & Clay & -- & -- & -- & \\
\hline 46 & 8/9-9H1 & 46 & 8/9-9H1 & 0 & 2 & 0.0 & 0.6 & surface soil & Sand and clay & -- & -. & .- & Qyal \\
\hline 46 & 8/9-9H1 & & & 2 & 38 & 0.6 & 11.6 & loose yellow sand and gravel & Sand (or sandstone) and gravel & -- & -- & -.- & \\
\hline 46 & 8/9-9H1 & & & 38 & 40 & 11.6 & 12.2 & hard yellow cemented gravel & Gravel & cemented & .- & .. & Qoal and QTge \\
\hline 47 & 8/9-10R1 & 47 & 8/9-10R1 & 0 & 400 & 0.0 & 121.9 & no data & No data & -- & -- & -. & QTge \\
\hline 48 & 8/9-11P1 & 48 & 8/9-11P1 & 0 & 4 & 0.0 & 1.2 & topsoil & Sand and clay & -- & -- & -- & QTge \\
\hline 48 & $8 / 9-11 \mathrm{P} 1$ & & & 4 & 12 & 1.2 & 3.7 & gravel & Gravel & -. & -- & -- & \\
\hline 48 & 8/9-11P1 & & & 12 & 40 & 3.7 & 12.2 & blue clay & Clay & -- & -- & -- & \\
\hline 48 & $8 / 9-11 \mathrm{P} 1$ & & & 40 & 150 & 12.2 & 45.7 & gravel & Gravel & -- & -- & .- & \\
\hline 49 & 8/9-15D1 & 49 & 8/9-15D1 & 0 & 12 & 0.0 & 3.7 & topsoil & Sand and clay & -- & -- & -- & Qyal and QTge (?) \\
\hline 49 & 8/9-15D1 & & & 12 & 21 & 3.7 & 6.4 & sand, fine & Sand & -- & -- & -- & \\
\hline 49 & 8/9-15D1 & & & 21 & 29 & 6.4 & 8.8 & gravel and sand & Sand (or sandstone) and gravel & -- & -- & -- & \\
\hline 49 & 8/9-15D1 & & & 29 & 37 & 8.8 & 11.3 & grave, coarse & Gravel & .- & -- & -. & \\
\hline 49 & 8/9-15D1 & & & 37 & 48 & 11.3 & 14.6 & clay & Clay & -. & -- & -. & \\
\hline 49 & 8/9-15D1 & & & 48 & 63 & 14.6 & 19.2 & sticky clay, blue & Clay & -- & -- & -- & \\
\hline 49 & 8/9-15D1 & & & 63 & 66 & 19.2 & 20.1 & pea gravel & Gravel & -. & -- & -- & \\
\hline
\end{tabular}


Appendix 3. Lithologic data. - Continued

[Site IDs and well number correspond to locations in Appendix 1, a site ID and well number is listed for each drilled interval for a particular drill hole. For convenience, the site ID and well numbers are repeated at the shallowest drilled interval for each we

\begin{tabular}{|c|c|c|c|c|c|c|c|c|c|c|c|c|c|}
\hline Site ID & Well number & $\begin{array}{l}\text { Site ID, } \\
\text { top of } \\
\text { well }\end{array}$ & $\begin{array}{l}\text { Well number, } \\
\text { top of well }\end{array}$ & $\begin{array}{c}\text { Top of } \\
\text { interval, in } \\
\text { feet }\end{array}$ & $\begin{array}{c}\text { Base of } \\
\text { interval, in } \\
\text { feet }\end{array}$ & $\begin{array}{c}\text { Top of } \\
\text { interval, in } \\
m\end{array}$ & $\begin{array}{c}\text { Base of } \\
\text { interval, in } \\
\mathrm{m}\end{array}$ & Reported lithologic unit ${ }^{1}$ & Interpreted lithologic class ${ }^{2}$ & $\begin{array}{c}\text { Reported } \\
\text { degree of } \\
\text { cementation or } \\
\text { induration } \\
\end{array}$ & $\begin{array}{c}\text { Fossils } \\
\text { reported in } \\
\text { interval }^{4}\end{array}$ & $\begin{array}{c}\text { Organic } \\
\text { matter } \\
\text { reported in } \\
\text { interval }^{5}\end{array}$ & $\begin{array}{l}\text { Reported stratigraphic top of geologic } \\
\text { unit }^{6}\end{array}$ \\
\hline 49 & 8/9-15D1 & & & 66 & 87 & 20.1 & 26.5 & pure blue gumbo, tight & Clay & -- & -- & -- & \\
\hline 49 & 8/9-15D1 & & & 87 & 120 & 26.5 & 36.6 & quicksand, tight, packed & Sand and clay & -- & -- & -- & \\
\hline 49 & 8/9-15D1 & & & 120 & 126 & 36.6 & 38.4 & clay & Clay & -- & -- & -- & \\
\hline 49 & 8/9-15D1 & & & 126 & 133 & 38.4 & 40.5 & sand and gravel & Sand (or sandstone) and gravel & -- & -- & -- & \\
\hline 49 & 8/9-15D1 & & & 133 & 134 & 40.5 & 40.8 & hard clay & Clay & hard & -- & -- & \\
\hline 50 & 8/9-13C1 & 50 & 8/9-13C1 & 0 & 15 & 0.0 & 4.6 & yellow clay & Clay & -- & -- & -- & Qyal and QTge \\
\hline 50 & $8 / 9-13 \mathrm{C} 1$ & & & 15 & 21 & 4.6 & 6.4 & gravel & Gravel & -- & -- & -- & \\
\hline 50 & 8/9-13C1 & & & 21 & 65 & 6.4 & 19.8 & yellow clay & Clay & -- & -- & -- & \\
\hline 50 & $8 / 9-13 \mathrm{C} 1$ & & & 65 & 170 & 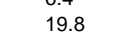 & 51.8 & yellow clay and gravel & Clay and gravel & -- & -. & - & \\
\hline 50 & 8/9-13C1 & & & 170 & 197 & 51.8 & 60.0 & gravel & Gravel & -- & -- & -- & \\
\hline 50 & 8/9-13C1 & & & 197 & 202 & 60.0 & 61.6 & yellow clay & Clay & -. & -- & -- & \\
\hline 51 & 8/9-14L1 & 51 & 8/9-14L1 & 0 & 53 & 0.0 & 16.2 & no data & No data & -- & -- & -- & -- \\
\hline 51 & 8/9-14L1 & & & 53 & 58 & 16.2 & 17.7 & loose gravel & Gravel & -- & -- & -- & QTge \\
\hline 51 & 8/9-14L1 & & & 58 & 83 & 17.7 & 25.3 & no data & No data & -- & -- & -- & \\
\hline 51 & 8/9-14L1 & & & 83 & 89 & 25.3 & 27.1 & cemented gravel & Gravel & cemented & -- & -- & \\
\hline 51 & 8/9-14L1 & & & 89 & 106 & 27.1 & 32.3 & no data & No data & - & -- & -.- & \\
\hline 51 & 8/9-14L1 & & & 106 & 109 & 32.3 & 33.2 & loose gravel & Gravel & -- & -- & -- & \\
\hline 51 & 8/9-14L1 & & & 109 & 242 & 33.2 & 73.8 & no data & No data & -- & -. & -- & \\
\hline 51 & 8/9-14L1 & & & 242 & 253 & 73.8 & 77.1 & pea gravel & Gravel & -- & -. & -- & \\
\hline 51 & 8/9-14L1 & & & 253 & 265 & 77.1 & 80.8 & no data & No data & -- & -- & -- & \\
\hline 52 & 8/8-17L1 & 52 & 8/8-17L1 & 0 & 30 & 0.0 & 9.1 & clay, sandy, and gravel & Clay, sand, and gravel & - & - & -. & QTge \\
\hline 52 & 8/8-17L1 & & & 30 & 62 & 9.1 & 18.9 & clay, yellow, and gravel & 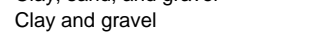 & -- & -- & -- & \\
\hline 52 & 8/8-17L1 & & & 62 & 74 & 18.9 & 22.6 & $\begin{array}{l}\text { clay, brown } \\
\text { clay yellow and aravel: water at }\end{array}$ & Clay & -- & -- & -- & \\
\hline 52 & 8/8-17L1 & & & 74 & 156 & 22.6 & 47.5 & 103 feet & Clay and gravel & -- & -- & -. & \\
\hline 52 & 8/8-17L1 & & & 156 & 170 & 47.5 & 51.8 & clay, blue & Clay & -- & -- & -- & \\
\hline 52 & 8/8-17L1 & & & 170 & 222 & 51.8 & 67.7 & clay, sandy gray, and gravel & Clay, sand, and gravel & -- & -- & -- & \\
\hline 52 & 8/8-17L1 & & & 222 & 248 & 67.7 & 75.6 & $\begin{array}{l}\text { clay, brown, and gravel } \\
\text { late }\end{array}$ & $\begin{array}{l}\text { Clay and gravel } \\
\text { Caris }\end{array}$ & - & - & - & \\
\hline 52 & 8/8-17L1 & & & 248 & 257 & 75.6 & 78.3 & $\begin{array}{l}\text { clay, blue } \\
\text { gravel; top } 3 \text { fee good gravel, }\end{array}$ & Clay & -- & -- & -- & \\
\hline 52 & 8/8-17L1 & & & 257 & 274 & 78.3 & 83.5 & then clay, then fair gravel & Clay and gravel & -- & -- & -- & \\
\hline 52 & 8/8-17L1 & & & 274 & 278 & 83.5 & 84.7 & clay, yellow, and gravel & Clay and gravel & -- & -- & -- & \\
\hline 53 & 8/9-14P1 & 53 & 8/9-14P1 & 0 & 11 & 0.0 & 3.4 & 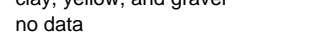 & $\begin{array}{l}\text { No data } \\
\text { Navel }\end{array}$ & - & -- & - & -- \\
\hline 53 & 8/9-14P1 & & & 11 & 16 & 3.4 & 4.9 & sand and gravel & Sand (or sandstone) and gravel & -- & -- & -- & QTge \\
\hline 53 & 8/9-14P1 & & & 16 & 19 & 4.9 & 5.8 & no data & No data & -- & -- & -.- & \\
\hline 53 & 8/9-14P1 & & & 19 & 37 & 5.8 & 11.3 & large gravel & Gravel & -- & -- & -- & \\
\hline 53 & 8/9-14P1 & & & 37 & 60 & 11.3 & 18.3 & no data & No data & -- & -- & -- & \\
\hline 53 & $8 / 9-14 \mathrm{P} 1$ & & & 60 & 65 & 18.3 & 19.8 & sand & Sand & -- & -- & -- & \\
\hline 53 & 8/9-14P1 & & & 65 & 118 & 19.8 & 36.0 & no data & No data & .- & -. & .. & \\
\hline 53 & 8/9-14P1 & & & 118 & 120 & 36.0 & 36.6 & gravel & Gravel & .- & -- & -- & \\
\hline 53 & 8/9-14P1 & & & 120 & 176 & 36.6 & 53.6 & no data & No data & -- & -- & -- & \\
\hline 53 & 8/9-14P1 & & & 176 & 184 & 53.6 & 56.1 & fine gravel & Gravel & -- & -- & -- & \\
\hline 53 & 8/9-14P1 & & & 184 & 253 & 56.1 & 77.1 & no data & No data & -- & -- & .- & \\
\hline 53 & 8/9-14P1 & & & 253 & 264 & 77.1 & 80.5 & pea gravel & Gravel & -- & -- & -- & \\
\hline 54 & 8/9-13N1 & 54 & 8/9-13N1 & 0 & 6 & 0.0 & 1.8 & soil & Sand and clay & -- & -- & -. & QTge \\
\hline 54 & 8/9-13N1 & & & 6 & 14 & 1.8 & 4.3 & hardpan & Clay and gravel & hard & -- & -- & \\
\hline 54 & 8/9-13N1 & & & 14 & 21 & 4.3 & 6.4 & gravel, water & Gravel & -- & -- & .- & \\
\hline 54 & 8/9-13N1 & & & 21 & 40 & 6.4 & 12.2 & gravel & Gravel & -- & -- & -- & \\
\hline 54 & 8/9-13N1 & & & 40 & 70 & 12.2 & 21.3 & clay, sandy & Clay and sand (or sandstone) & -- & .- & .. & \\
\hline 54 & 8/9-13N1 & & & 70 & 74 & 21.3 & 22.6 & gravel, water & Gravel & -- & -- & -- & \\
\hline 54 & 8/9-13N1 & & & 74 & 82 & 22.6 & 25.0 & clay, blue & Clay & -- & -- & -- & \\
\hline 54 & 8/9-13N1 & & & 82 & 86 & 25.0 & 26.2 & gravel, water & Gravel & -- & -- & -. & \\
\hline 54 & 8/9-13N1 & & & 86 & 98 & 26.2 & 29.9 & clay & Clay & -- & -- & .- & \\
\hline 54 & 8/9-13N1 & & & 98 & 110 & 29.9 & 33.5 & sand and gravel, water & Sand (or sandstone) and gravel & -- & -- & -- & \\
\hline 54 & 8/9-13N1 & & & 110 & 130 & 33.5 & 39.6 & clay & Clay & -- & -- & -- & \\
\hline 54 & 8/9-13N1 & & & 130 & 136 & 39.6 & 41.5 & sand, clay, and gravel; water & Clay, sand, and gravel & -- & -- & -. & \\
\hline 54 & 8/9-13N1 & & & 136 & 152 & 41.5 & 46.3 & clay & Clay & -. & -- & - & \\
\hline 54 & 8/9-13N1 & & & 152 & 168 & 46.3 & 51.2 & gravel, water & Gravel & -- & -- & - & \\
\hline 54 & 8/9-13N1 & & & 168 & 172 & 51.2 & 52.4 & clay, blue & Clay & .- & -- & .. & \\
\hline 54 & $8 / 9-13 N 1$ & & & 172 & 184 & 52.4 & 56.1 & gravel, water & Gravel & -- & -- & -- & \\
\hline 54 & 8/9-13N1 & & & 184 & 208 & 56.1 & 63.4 & shale, blue & Clay and sand (or sandstone) & cemented & -. & -. & \\
\hline
\end{tabular}


Appendix 3. Lithologic data. - Continued

[Site IDs and well number correspond to locations in Appendix 1, a site ID and well number is listed for each drilled interval for a particular drill hole. For convenience, the site ID and well numbers are repeated at the shallowest drilled interval for each well. leaderst.

\begin{tabular}{|c|c|c|c|c|c|c|c|c|c|c|c|c|c|}
\hline Site ID & Well number & $\begin{array}{l}\text { Site ID, } \\
\text { top of } \\
\text { well }\end{array}$ & $\begin{array}{l}\text { Well number, } \\
\text { top of well }\end{array}$ & $\begin{array}{c}\text { Top of } \\
\text { interval, in } \\
\text { feet }\end{array}$ & $\begin{array}{c}\text { Base of } \\
\text { interval, in } \\
\text { feet }\end{array}$ & $\begin{array}{c}\text { Top of } \\
\text { interval, in } \\
m\end{array}$ & $\begin{array}{c}\text { Base of } \\
\text { interval, in } \\
\mathrm{m}\end{array}$ & Reported lithologic unit ${ }^{1}$ & Interpreted lithologic class ${ }^{2}$ & $\begin{array}{c}\text { Reported } \\
\text { degree of } \\
\text { cementation or } \\
\text { induration } \\
\end{array}$ & $\begin{array}{l}\text { Fossils } \\
\text { reported in } \\
\text { interval }^{4}\end{array}$ & $\begin{array}{c}\text { Organic } \\
\text { matter } \\
\text { reported in } \\
\text { interval }^{5}\end{array}$ & $\begin{array}{l}\text { Reported stratigraphic top of geologic } \\
\text { unit }\end{array}$ \\
\hline 55 & 8/8-20B2 & 55 & 8/8-20B2 & 0 & 202 & 0.0 & 61.6 & no data & No data & -- & -- & -- & -- \\
\hline 55 & 8/8-20B2 & & & 202 & 227 & 61.6 & 69.2 & sand clay and gravel; gravel & Clay, sand, and gravel & -- & -- & -- & QTge \\
\hline 55 & 8/8-20B2 & & & 227 & 231 & 69.2 & 70.4 & no data & No data & -- & -- & -- & \\
\hline 56 & 8/8-20D1 & 56 & 8/8-20D1 & 0 & 260 & 0.0 & 79.2 & no data & No data & -- & -- & -- & QTge \\
\hline 57 & 8/9-23G1 & 57 & 8/9-23G1 & 0 & 18 & 0.0 & 5.5 & no data & No data & -- & -- & -- & -. \\
\hline 57 & 8/9-23G1 & & & 18 & 28 & 5.5 & 8.5 & gravel & Gravel & -- & -- & -- & QTge \\
\hline 57 & 8/9-23G1 & & & 28 & 37 & 8.5 & 11.3 & no data & No data & -- & -- & -- & \\
\hline 57 & 8/9-23G1 & & & 37 & 52 & 11.3 & 15.8 & sand; pea gravel & Sand (or sandstone) and gravel & -- & -- & -- & \\
\hline 57 & 8/9-23G1 & & & 52 & 79 & 15.8 & 24.1 & no data & No data & -- & -- & -- & \\
\hline 57 & $8 / 9-23 G 1$ & & & 79 & 95 & 24.1 & 29.0 & fine gravel & Gravel & -- & -. & -- & \\
\hline 57 & 8/9-23G1 & & & 95 & 128 & 29.0 & 39.0 & no data & No data & -- & -- & -- & \\
\hline 57 & 8/9-23G1 & & & 128 & 138 & 39.0 & 42.1 & sand and gravel & Sand (or sandstone) and gravel & -- & -- & -- & \\
\hline 57 & $8 / 9-23 G 1$ & & & 138 & 200 & 42.1 & 61.0 & no data & No data & -- & -- & -- & \\
\hline 57 & 8/9-23G1 & & & 200 & 223 & 61.0 & 68.0 & gravel & Gravel & -- & -- & -- & \\
\hline 57 & 8/9-23G1 & & & 223 & 276 & 68.0 & 84.1 & no data & No data & -- & $\ldots$ & - & \\
\hline 57 & $8 / 9-23 \mathrm{G} 1$ & & & 276 & 280 & 84.1 & 85.3 & gravel & Gravel & -- & -- & -- & \\
\hline 57 & 8/9-23G1 & & & 280 & 440 & 85.3 & 134.1 & no data & No data & -- & -- & -- & \\
\hline 58 & 8/9-24E1 & 58 & 8/9-24E1 & 0 & 330 & 0.0 & 100.6 & no data & No data & -- & -- & -- & Qyal (?) and QTge \\
\hline 59 & 8/9-23L1 & 59 & 8/9-23L1 & 0 & 4 & 0.0 & 1.2 & topsoil & Sand and clay & -- & -- & -- & QTge \\
\hline 59 & 8/9-23L1 & & & 4 & 12 & 1.2 & 3.7 & soil and white clay & Sand and clay & -- & -- & -- & \\
\hline 59 & 8/9-23L1 & & & 12 & 15 & 3.7 & 4.6 & sand and clay & Sand and clay & -- & -- & -- & \\
\hline 59 & $8 / 9-23\llcorner 1$ & & & 15 & 25 & 4.6 & 7.6 & sand, blue & Sand & -- & -- & -- & \\
\hline 59 & 8/9-23L1 & & & 25 & 51 & 7.6 & 15.5 & coarse gravel & Gravel & -- & -- & -- & \\
\hline 59 & 8/9-23L1 & & & 51 & 56 & 15.5 & 17.1 & blue clay & Clay & -- & .- & -- & \\
\hline 59 & 8/9-23L1 & & & 56 & 70 & 17.1 & 21.3 & coarse gravel & Gravel & -- & -- & -- & \\
\hline 59 & 8/9-23L1 & & & 70 & 74 & 21.3 & 22.6 & blue clay & Clay & -- & -- & -- & \\
\hline 59 & $8 / 9-23\llcorner 1$ & & & 74 & 85 & 22.6 & 25.9 & coarse gravel & Gravel & -- & -- & -- & \\
\hline 59 & 8/9-23L1 & & & 85 & 107 & 25.9 & 32.6 & blue clay, mixed gravel & Clay and gravel & -- & -- & -- & \\
\hline 59 & 8/9-23L1 & & & 107 & 171 & 32.6 & 52.1 & soft blue clay & Clay & -- & -- & -- & \\
\hline 59 & 8/9-23L1 & & & 171 & 224 & 52.1 & 68.3 & hard blue shale & Clay and sand (or sandstone) & hard & -- & -- & \\
\hline 59 & 8/9-23L1 & & & 224 & 306 & 68.3 & 93.3 & soft blue sandy shale & Clay and sand (or sandstone) & cemented & -- & -- & \\
\hline 59 & $8 / 9-23\llcorner 1$ & & & 306 & 351 & 93.3 & 107.0 & gravel & Gravel & -- & -- & -- & \\
\hline 59 & 8/9-23L1 & & & 351 & 354 & 107.0 & 107.9 & blue clay & Clay & -- & -- & -- & \\
\hline 59 & $8 / 9-23\llcorner 1$ & & & 354 & 364 & 107.9 & 110.9 & blue shale & Clay and sand (or sandstone) & cemented & -- & -- & \\
\hline 59 & 8/9-23L1 & & & 364 & 376 & 110.9 & 114.6 & sandy blue clay & Clay and sand (or sandstone) & -- & -- & -- & \\
\hline 59 & 8/9-23L1 & & & 376 & 403 & 114.6 & 122.8 & coarse sand and washed shale & Clay and sand (or sandstone) & cemented & -- & -- & \\
\hline 59 & 8/9-23ட1 & & & 403 & 429 & 122.8 & 130.8 & blue clay & Clay & -- & -- & -- & \\
\hline 60 & 8/8-19M1 & 60 & 8/8-19M1 & 0 & 2 & 0.0 & 0.6 & $\begin{array}{l}\text { fine soil } \\
\text { forl }\end{array}$ & Sand and clay & -- & -- & -- & QTge \\
\hline 60 & 8/8-19M1 & & & 2 & 27 & 0.6 & 8.2 & yellow sandy clay & Clay and sand (or sandstone) & - & -- & -- & Qige \\
\hline 60 & 8/8-19M1 & & & 27 & 37 & 8.2 & 11.3 & yellow sand and fine gravel & Sand (or sandstone) and gravel & -- & -- & -- & \\
\hline 60 & 8/8-19M1 & & & 37 & 45 & 11.3 & 13.7 & coarse packed gravel & Gravel & -- & -- & -- & \\
\hline 60 & 8/8-19M1 & & & 45 & 65 & 13.7 & 19.8 & yellow sandy clay & Clay and sand (or sandstone) & -- & -- & -- & \\
\hline 60 & 8/8-19M1 & & & 65 & 90 & 19.8 & 27.4 & coarse gravelly clay & Clay and gravel & -- & -- & -- & \\
\hline 60 & 8/8-19M1 & & & 90 & 115 & 27.4 & 35.1 & medium packed brown clay & Clay & -- & -- & -- & \\
\hline 60 & 8/8-19M1 & & & 115 & 130 & 35.1 & 39.6 & small boulders & Gravel & -- & -- & -- & \\
\hline 60 & 8/8-19M1 & & & 130 & 160 & 39.6 & 48.8 & yellow clay and fine gravel & Clay and gravel & -- & -- & -. & \\
\hline 60 & 8/8-19M1 & & & 160 & 185 & 48.8 & 56.4 & fine gravel and brown sand & Sand (or sandstone) and gravel & -- & -- & -- & \\
\hline 60 & 8/8-19M1 & & & 185 & 205 & 56.4 & 62.5 & small gravelly brown clay & Clay and gravel & -- & -- & -- & \\
\hline 60 & 8/8-19M1 & & & 205 & 235 & 62.5 & 71.6 & coarse packed brown gravel & Gravel & -- & -- & -- & \\
\hline 60 & 8/8-19M1 & & & 235 & 255 & 71.6 & 77.7 & medium brown gravel & Gravel & .- & -- & .- & \\
\hline 60 & 8/8-19M1 & & & 255 & 260 & 77.7 & 79.2 & large packed gravel & Gravel & -- & -- & -- & \\
\hline 61 & $8 / 8-19 \mathrm{~N} 1$ & 61 & 8/8-19N1 & 0 & 27 & 0.0 & 8.2 & $\begin{array}{l}\text { no data } \\
\text { packed fine sandy gravel; coarse }\end{array}$ & No data & -- & -- & -- & -- \\
\hline 61 & $8 / 8-19 \mathrm{~N} 1$ & & & 27 & 45 & 8.2 & 13.7 & packed gravel & Sand (or sandstone) and gravel & -- & -- & -. & QTge \\
\hline 61 & $8 / 8-19 \mathrm{~N} 1$ & & & 45 & 105 & 13.7 & 32.0 & no data & No data & -- & -- & -- & \\
\hline 61 & 8/8-19N1 & & & 105 & 120 & 32.0 & 36.6 & "small bouldery" sand & Sand (or sandstone) and gravel & -- & -- & -- & \\
\hline 61 & $8 / 8-19 \mathrm{~N} 1$ & & & 120 & 150 & 36.6 & 45.7 & no data & No data & -- & -- & -- & \\
\hline 61 & $8 / 8-19 \mathrm{~N} 1$ & & & 150 & 175 & 45.7 & 53.3 & fine gravel and coarse sand & Sand (or sandstone) and gravel & -- & -- & -- & \\
\hline 61 & 8/8-19N1 & & & 175 & 200 & 53.3 & 61.0 & no data & No data & -- & -- & -- & \\
\hline 61 & $8 / 8-19 \mathrm{~N} 1$ & & & 200 & 249 & 61.0 & 75.9 & packed coarse gravelly sand & Sand (or sandstone) and gravel & -. & -- & -- & \\
\hline 62 & 8/8-20Q1 & 62 & 8/8-20Q1 & 0 & 10 & 0.0 & 3.0 & topsoil & Sand and clay & -- & -- & -- & Qyal \\
\hline
\end{tabular}


Appendix 3. Lithologic data. - Continued

[Site IDs and well number correspond to locations in Appendix 1, a site ID and well number is listed for each drilled interval for a particular drill hole. For convenience, the site ID and well numbers are repeated at the shallowest drilled interval for each well. lea

\begin{tabular}{|c|c|c|c|c|c|c|c|c|c|c|c|c|c|}
\hline Site ID & Well number & $\begin{array}{l}\text { Site ID, } \\
\text { top of } \\
\text { well }\end{array}$ & $\begin{array}{l}\text { Well number, } \\
\text { top of well }\end{array}$ & $\begin{array}{c}\text { Top of } \\
\text { interval, in } \\
\text { feet }\end{array}$ & $\begin{array}{c}\text { Base of } \\
\text { interval, in } \\
\text { feet }\end{array}$ & $\begin{array}{c}\text { Top of } \\
\text { interval, in } \\
m\end{array}$ & $\begin{array}{c}\text { Base of } \\
\text { interval, in } \\
\mathrm{m}\end{array}$ & Reported lithologic unit ${ }^{1}$ & Interpreted lithologic class ${ }^{2}$ & $\begin{array}{c}\text { Reported } \\
\text { degree of } \\
\text { cementation or } \\
\text { induration }^{3} \\
\end{array}$ & $\begin{array}{c}\text { Fossils } \\
\text { reported in } \\
\text { interval }^{4}\end{array}$ & $\begin{array}{c}\text { Organic } \\
\text { matter } \\
\text { reported in } \\
\text { interval }^{5}\end{array}$ & $\begin{array}{l}\text { Reported stratigraphic top of geologic } \\
\text { unit }^{6}\end{array}$ \\
\hline 62 & 8/8-20Q1 & & & 10 & 20 & 3.0 & 6.1 & yellow clay & Clay & -- & -- & -- & QTge \\
\hline 62 & 8/8-20Q1 & & & 20 & 122 & 6.1 & 37.2 & gravel and some clay & Sand (or sandstone) and gravel & -- & -- & -- & \\
\hline 62 & 8/8-20Q1 & & & 122 & 237 & 37.2 & 72.2 & gravel and sand & Sand (or sandstone) and gravel & -- & -- & -- & \\
\hline 62 & 8/8-20Q1 & & & 237 & 295 & 72.2 & 89.9 & $\begin{array}{l}\text { gravel } \\
\text { gan sarlu }\end{array}$ & Gravel & -- & - & -- & \\
\hline 62 & 8/8-20Q1 & & & 295 & 308 & 89.9 & 93.9 & yellow clay & Clay & -- & -- & -- & \\
\hline 62 & 8/8-20Q1 & & & 308 & 312 & 93.9 & 95.1 & boulders and clay & Clay and gravel & -- & -- & -- & \\
\hline 63 & 8/9-26D1 & 63 & 8/9-26D1 & 0 & 240 & 0.0 & 73.2 & no data & No data & -- & -- & -- & -- \\
\hline 63 & 8/9-26D1 & & & 240 & 280 & 73.2 & 85.3 & sandy clay and gravel; gravel & Clay, sand, and gravel & -- & -- & -- & QTge \\
\hline 63 & 8/9-26D1 & & & 280 & 394 & 85.3 & 120.1 & $\begin{array}{l}\text { no data } \\
\text { nata }\end{array}$ & No data & -- & -- & - & \\
\hline 63 & 8/9-26D1 & & & 394 & 462 & 120.1 & 140.8 & clay and gravel; gravel & Clay and gravel & -- & -. & -- & \\
\hline 63 & 8/9-26D1 & & & 462 & 464 & 140.8 & 141.4 & no data & No data & -- & -- & -- & \\
\hline 64 & $8 / 8-29 \mathrm{Cl}$ & 64 & $8 / 8-29 \mathrm{C} 1$ & 0 & 2 & 0.0 & 0.6 & soil & Sand and clay & -- & -- & -- & Qyal and QTge \\
\hline 64 & $8 / 8-29 \mathrm{C} 1$ & & & 2 & 20 & 0.6 & 6.1 & yellow clay & Clay & -- & -- & -- & \\
\hline 64 & $8 / 8-29 \mathrm{C} 1$ & & & 20 & 25 & 6.1 & 7.6 & blue clay & Clay & -- & -- & -- & \\
\hline 64 & $8 / 8-29 \mathrm{C} 1$ & & & 25 & 60 & 7.6 & 18.3 & yellow clay & Clay & -- & -- & -- & \\
\hline 64 & 8/8-29C1 & & & 60 & 68 & 18.3 & 20.7 & gravel & Gravel & -- & -- & -- & \\
\hline 65 & 8/8-29D1 & 65 & 8/8-29D1 & 0 & 24 & 0.0 & 7.3 & sandy blue clay & Clay and sand (or sandstone) & -- & -- & -- & Qyal and QTge \\
\hline 65 & 8/8-29D1 & & & 24 & 50 & 7.3 & 15.2 & sandy red clay & Clay and sand (or sandstone) & -- & -- & -- & \\
\hline 65 & 8/8-29D1 & & & 50 & 56 & 15.2 & 17.1 & red sand & Sand & -- & -- & -- & \\
\hline 65 & 8/8-29D1 & & & 56 & 57 & 17.1 & 17.4 & blue clay & Clay & -- & -- & -- & \\
\hline 66 & 8/8-30G1 & 66 & 8/8-30G1 & 57 & 84 & 17.4 & 25.6 & old well & No data & - & - & - & Qyal and QTge \\
\hline 66 & 8/8-30G1 & & & 84 & 92 & 25.6 & 28.0 & yellow gravel & Gravel & -- & -- & -- & \\
\hline 66 & 8/8-30G1 & & & 92 & 110 & 28.0 & 33.5 & yellow clay & Clay & -- & -- & -. & \\
\hline 66 & 8/8-30G1 & & & 110 & 115 & 33.5 & 35.1 & yellow gravel & Gravel & -- & -- & .- & \\
\hline 66 & 8/8-30G1 & & & 115 & 122 & 35.1 & 37.2 & gray clay & Clay & -- & -- & -- & \\
\hline 66 & 8/8-30G1 & & & 122 & 140 & 37.2 & 42.7 & yellow sand and gravel & Sand (or sandstone) and gravel & -- & -- & -- & \\
\hline 67 & 8/8-30L1 & 67 & 8/8-30L1 & 0 & 3 & 0.0 & 0.9 & soil & Sand and clay & -- & -- & -- & QTge \\
\hline 67 & 8/8-30L1 & & & 3 & 17 & 0.9 & 5.2 & hardpan & Clay and gravel & hard & - & - & \\
\hline 67 & 8/8-30L1 & & & 17 & 43 & 5.2 & 13.1 & clay, sandy, blue & Clay and sand (or sandstone) & -. & -- & -.- & \\
\hline 67 & 8/8-30L1 & & & 43 & 90 & 13.1 & 27.4 & clay, yellow & Clay & -. & -- & -- & \\
\hline 67 & 8/8-30L1 & & & 90 & 107 & 27.4 & 32.6 & clay, yellow, and gravel & Clay and gravel & -- & -- & -- & \\
\hline 67 & 8/8-30L1 & & & 107 & 124 & 32.6 & 37.8 & gravel & Gravel & -- & -- & -- & \\
\hline 67 & 8/8-30L1 & & & 124 & 135 & 37.8 & 41.1 & $\begin{array}{l}\text { clay, blue, and gravel } \\
\text { gravel, coarse, blue, and some }\end{array}$ & Clay and gravel & -- & -- & -- & \\
\hline 67 & 8/8-30L1 & & & 135 & 145 & 41.1 & 44.2 & clay & Sand (or sandstone) and gravel & -- & -- & -.- & \\
\hline 67 & 8/8-30L1 & & & 145 & 188 & 44.2 & 57.3 & gravel and clay & Sand (or sandstone) and gravel & -- & -- & -- & \\
\hline 67 & 8/8-30L1 & & & 188 & 204 & 57.3 & 62.2 & $\begin{array}{l}\text { clay, blue } \\
\text { s. }\end{array}$ & Clay & -- & -- & -- & \\
\hline 67 & 8/8-30L1 & & & 204 & 245 & 62.2 & 74.7 & clay, yellow, and gravel & Clay and gravel & -- & -- & -- & \\
\hline 67 & 8/8-30L1 & & & 245 & 268 & 74.7 & 81.7 & clay, sandy, yellow, and gravel & Sand (or sandstone) and gravel & .- & -. & .. & \\
\hline 67 & $8 / 8-30\llcorner 1$ & & & 268 & 285 & 81.7 & 86.9 & $\begin{array}{l}\text { clay, sandy, blue } \\
\text { lat }\end{array}$ & Clay and sand (or sandstone) & .- & -. & .- & \\
\hline 67 & 8/8-30L1 & & & 285 & 325 & 86.9 & 99.1 & clay, blue and gravel & Clay and gravel & -- & -- & -- & \\
\hline 67 & 8/8-30L1 & & & 325 & 340 & 99.1 & 103.6 & clay, sandy, blue & Clay and sand (or sandstone) & -- & -- & -- & \\
\hline 67 & 8/8-30L1 & & & 340 & 345 & 103.6 & 105.2 & clay, blue, and gravel & Clay and gravel & -- & -- & -.- & \\
\hline 67 & 8/8-30L1 & & & 345 & 375 & 105.2 & 114.3 & clay, sandy, blue & Clay and sand (or sandstone) & -- & -- & -- & \\
\hline 67 & 8/8-30L1 & & & 375 & 463 & 114.3 & 141.1 & clay, sandy, blue, and gravel & $\begin{array}{l}\text { Clay, sand, and gravel } \\
\text { lo }\end{array}$ & - & -- & -- & \\
\hline 68 & 8/9-27K1 & 68 & 8/9-27K1 & 0 & 6 & 0.0 & 1.8 & soil & Sand and clay & -- & -- & -- & Qyal \\
\hline 68 & $8 / 9-27 \mathrm{~K} 1$ & & & 6 & 50 & 1.8 & 15.2 & clay hard, yellow & Clay & hard & -- & -- & QTge and QTm \\
\hline 68 & $8 / 9-27 \mathrm{~K} 1$ & & & 50 & 52 & 15.2 & 15.8 & sand and gravel, water & Sand (or sandstone) and gravel & -. & -- & -.- & \\
\hline 68 & $8 / 9-27 \mathrm{~K} 1$ & & & 52 & 303 & 15.8 & 92.4 & blue clay & Clay & -- & .- & .. & \\
\hline 68 & $8 / 9-27 \mathrm{~K} 1$ & & & 303 & 333 & 92.4 & 101.5 & gravel and clam shells, water & Gravel & -- & shells & -- & \\
\hline 69 & 8/8-30N1 & 69 & 8/8-30N1 & 0 & 2 & 0.0 & 0.6 & soil & Sand and clay & -- & -- & -- & QTge \\
\hline 69 & 8/8-30N1 & & & 2 & 19 & 0.6 & 5.8 & hardpan & Clay and gravel & hard & -. & -- & \\
\hline 69 & 8/8-30N1 & & & 19 & 21 & 5.8 & 6.4 & clay yellow & Clay & -- & -- & -- & \\
\hline 69 & $8 / 8-30 N 1$ & & & 21 & 40 & 6.4 & 12.2 & clay, yellow, and gravel & Clay and gravel & -- & -- & -- & \\
\hline 69 & 8/8-30N1 & & & 40 & 60 & 12.2 & 18.3 & clay, yellow & Clay & -- & -- & -- & \\
\hline 69 & 8/8-30N1 & & & 60 & 95 & 18.3 & 29.0 & clay, yellow, and gravel & Clay and gravel & -- & -- & -. & \\
\hline 69 & 8/8-30N1 & & & 95 & 98 & 29.0 & 29.9 & clay, blue, and gravel & Clay and gravel & -- & -- & -- & \\
\hline 69 & 8/8-30N1 & & & 98 & 115 & 29.9 & 35.1 & clay, blue & Clay & .- & -- & .. & \\
\hline 69 & 8/8-30N1 & & & 115 & 119 & 35.1 & 36.3 & clay, yellow, and gravel & Clay and gravel & -- & -- & .. & \\
\hline 69 & 8/8-30N1 & & & 119 & 125 & 36.3 & 38.1 & gravel & Gravel & - & -- & -- & \\
\hline 69 & 8/8-30N1 & & & 125 & 185 & 38.1 & 56.4 & gravel and clay & Sand (or sandstone) and gravel & -. & -- & -- & \\
\hline
\end{tabular}


Appendix 3. Lithologic data. - Continued

[Site IDs and well number correspond to locations in Appendix 1, a site ID and well number is listed for each drilled interval for a particular drill hole. For convenience, the site ID and well numbers are repeated at the shallowest drilled interval for each well. leaders

\begin{tabular}{|c|c|c|c|c|c|c|c|c|c|c|c|c|c|}
\hline Site ID & Well number & $\begin{array}{l}\text { Site ID, } \\
\text { top of } \\
\text { well }\end{array}$ & $\begin{array}{c}\text { Well number, } \\
\text { top of well }\end{array}$ & $\begin{array}{c}\text { Top of } \\
\text { interval, in } \\
\text { feet }\end{array}$ & $\begin{array}{c}\text { Base of } \\
\text { interval, in } \\
\text { feet }\end{array}$ & $\begin{array}{c}\text { Top of } \\
\text { interval, in } \\
m\end{array}$ & $\begin{array}{c}\text { Base of } \\
\text { interval, in } \\
\mathrm{m}\end{array}$ & Reported lithologic unit ${ }^{1}$ & Interpreted lithologic class ${ }^{2}$ & $\begin{array}{c}\text { Reported } \\
\text { degree of } \\
\text { cementation or } \\
\text { induration } \\
\end{array}$ & $\begin{array}{c}\text { Fossils } \\
\text { reported in } \\
\text { interval }^{4}\end{array}$ & $\begin{array}{c}\text { Organic } \\
\text { matter } \\
\text { reported in } \\
\text { interval }^{5}\end{array}$ & $\begin{array}{l}\text { Reported stratigraphic top of geologic } \\
\text { unit }^{6}\end{array}$ \\
\hline 69 & 8/8-30N1 & & & 185 & 223 & 56.4 & 68.0 & gravel and clay, yellow & Clay and gravel & -- & -- & -- & \\
\hline 69 & 8/8-30N1 & & & 223 & 231 & 68.0 & 70.4 & clay, blue & Clay & -- & -- & -- & \\
\hline 69 & 8/8-30N1 & & & 231 & 239 & 70.4 & 72.8 & gravel, cemented & Gravel & cemented & -- & -- & \\
\hline 69 & 8/8-30N1 & & & 239 & 253 & 72.8 & 77.1 & $\begin{array}{l}\text { clay, blue } \\
\text { lat }\end{array}$ & Clay & - & -- & -- & \\
\hline 69 & 8/8-30N1 & & & 253 & 330 & 77.1 & 100.6 & gravel and clay, blue & Clay and gravel & -- & .- & -- & \\
\hline 69 & 8/8-30N1 & & & 330 & 350 & 100.6 & 106.7 & clay, sandy, and gravel & Clay, sand, and gravel & -- & -- & -- & \\
\hline 69 & 8/8-30N1 & & & 350 & 405 & 106.7 & 123.4 & clay, blue & Clay & -- & -- & -- & \\
\hline 69 & 8/8-30N1 & & & 405 & 410 & 123.4 & 125.0 & clay, sandy, blue, and gravel & Clay, sand, and gravel & -- & -- & -- & \\
\hline 69 & 8/8-30N1 & & & 410 & 450 & 125.0 & 137.2 & $\begin{array}{l}\text { clay and gravel } \\
\text { and }\end{array}$ & Clay and gravel & -- & -- & - & \\
\hline 69 & 8/8-30N1 & & & 450 & 455 & 137.2 & 138.7 & clay, blue & Clay & -- & -- & -- & \\
\hline 70 & 8/8-30N2 & 70 & 0 8/8-30N2 & 0 & 4 & 0.0 & 1.2 & soil & Sand and clay & -- & -- & -- & QTge \\
\hline 70 & 8/8-30N2 & & & 4 & 55 & 1.2 & 16.8 & clay, yellow, and gravel & Clay and gravel & -- & -- & -- & \\
\hline 70 & 8/8-30N2 & & & 55 & 62 & 16.8 & 18.9 & clay, sandy yellow & Sand and clay & -- & -- & -- & \\
\hline 70 & 8/8-30N2 & & & 62 & 175 & $\begin{array}{l}18.9 \\
18.9-\ln (x)\end{array}$ & 53.3 & clay and gravel & Clay and gravel & -- &.- & - & \\
\hline 70 & 8/8-30N2 & & & 175 & 177 & 53.3 & 53.9 & clay, blue & Clay & -- & -. & -- & \\
\hline 70 & 8/8-30N2 & & & 177 & 183 & 53.9 & 55.8 & gravel & Gravel & -- & -- & -- & \\
\hline 70 & 8/8-30N2 & & & 183 & 190 & 55.8 & 57.9 & clay, blue & Clay & -- & -- & -- & \\
\hline 70 & 8/8-30N2 & & & 190 & 260 & 57.9 & 79.2 & clay, blue and gravel & Clay and gravel & -- & -. & -- & \\
\hline 70 & 8/8-30N2 & & & 260 & 283 & 79.2 & 86.3 & clay, sandy, blue and gravel & Clay, sand, and gravel & -- & -. & -- & \\
\hline 70 & 8/8-30N2 & & & 283 & 340 & 86.3 & 103.6 & $\begin{array}{l}\text { clay, blue, and gravel } \\
\text {. }\end{array}$ & $\begin{array}{l}\text { Clay and gravel } \\
\text {. }\end{array}$ & -- & -- & -- & \\
\hline 70 & 8/8-30N2 & & & 340 & 348 & 103.6 & 106.1 & clay, blue and coarse gravel & Clay and gravel & -. & -. & -- & \\
\hline 70 & 8/8-30N2 & & & 348 & 375 & 106.1 & 114.3 & clay, blue, and gravel & Clay and gravel & -- & -- & -- & \\
\hline 70 & 8/8-30N2 & & & 375 & 390 & 114.3 & 118.9 & clay, yellow, and gravel & Clay and gravel & -- & -. & -- & \\
\hline 70 & 8/8-30N2 & & & 390 & 425 & 118.9 & 129.5 & clay, blue & Clay & -- & -. & -.- & \\
\hline 70 & 8/8-30N2 & & & 425 & 430 & 129.5 & 131.1 & clay, blue, and gravel & Clay and gravel & -- & -- & -- & \\
\hline 70 & 8/8-30N2 & & & 430 & 436 & 131.1 & 132.9 & clay, sandy, blue & Clay, sand, and gravel & -- & -- & -- & \\
\hline 70 & 8/8-30N2 & & & 436 & 580 & 132.9 & 176.8 & clay, blue & Clay & -- & -- & -- & \\
\hline 70 & 8/8-30N2 & & & 580 & 585 & 176.8 & 178.3 & clay, blue, and some gravel & Clay and gravel & - & - & - & \\
\hline 70 & 8/8-30N2 & & & 585 & 600 & 178.3 & 182.9 & clay, blue & Clay & -- & -- & -- & \\
\hline 71 & 8/8-31B1 & 71 & 1 8/8-31B1 & 0 & 118 & 0.0 & 36.0 & no data & No data & -- & -- & -.- & -- \\
\hline 71 & 8/8-31B1 & & & 118 & 206 & 36.0 & 62.8 & gravel; clay and gravel & Sand (or sandstone) and gravel & -- & -- & -- & QTge \\
\hline 71 & 8/8-31B1 & & & 206 & 210 & 62.8 & 64.0 & no data & No data & -- & -- & -- & \\
\hline 72 & 8/9-33G1 & 72 & $28 / 9-33 \mathrm{G} 1$ & 0 & 358 & 0.0 & 109.1 & (Untested hole) & No data & - & -- & - & \\
\hline 73 & $8 / 9-36 \mathrm{G} 1$ & 73 & $3 \quad 8 / 9-36 \mathrm{G} 1$ & 0 & 642 & 0.0 & 195.7 & no data & No data & -- & -- & -- & QTge and QTm \\
\hline 74 & 8/9-36K1 & 74 & $4 \quad 8 / 9-36 K 1$ & 0 & 1325 & 0.0 & 403.9 & no data & No data & -- & -- & -- & -- \\
\hline 75 & 8/9-36L1 & 75 & 5 8/9-36ட1 & 0 & 27 & 0.0 & 8.2 & blue clay & Clay & -- & -- & -- & Qyal \\
\hline 75 & 8/9-36L1 & & & 27 & 33 & 8.2 & 10.1 & gravel & Gravel & -- & -- & -.. & \\
\hline 75 & 8/9-36L1 & & & 33 & 430 & 10.1 & 131.1 & blue clay & Clay & -- & -- & -- & QTge \\
\hline 75 & 8/9-36L1 & & & 430 & 455 & 131.1 & 138.7 & gravel & Gravel & -- & -- & .. & \\
\hline 75 & 8/9-36L1 & & & 455 & 520 & 138.7 & 158.5 & blue clay & Clay & -- & -- & -- & \\
\hline 75 & 8/9-36L1 & & & 520 & 535 & 158.5 & 163.1 & gravel & Gravel & -- & -- & -- & \\
\hline 75 & 8/9-36L1 & & & 535 & 565 & 163.1 & 172.2 & blue clay & Clay & -- & -- & -.- & \\
\hline 76 & 8/9-33J1 & 76 & 6 8/9-33J1 & 0 & 7 & 0.0 & 2.1 & adobe & Sand and clay & .- & -- & -.- & Qyal \\
\hline 76 & 8/9-33J1 & & & 7 & 20 & 2.1 & 6.1 & hardpan & Clay and gravel & hard & -- & -- & QTm \\
\hline 76 & 8/9-33J1 & & & 20 & 55 & 6.1 & 16.8 & clay, sandy water & Clay and sand (or sandstone) & -- & -- & -- & \\
\hline 76 & 8/9-33J1 & & & 55 & 62 & 16.8 & 18.9 & blue clay & Clay & -- & -- & -- & \\
\hline 76 & $8 / 9-33 \mathrm{J1}$ & & & 62 & 71 & 18.9 & 21.6 & gravel water & Gravel & -- & -- & -- & \\
\hline 76 & 8/9-33J1 & & & 71 & 101 & 21.6 & 30.8 & blue clay and gravel, hard & Clay and gravel & hard & -- & -.- & \\
\hline 76 & $8 / 9-33 \mathrm{J1}$ & & & 101 & 106 & 30.8 & 32.3 & blue clay and gravel & Clay and gravel & -- & -. & -- & \\
\hline 76 & $8 / 9-33 J 1$ & & & 106 & 129 & 32.3 & 39.3 & yellow clay and gravel & Clay and gravel & -- & -- & -- & \\
\hline 76 & $8 / 9-3331$ & & & 129 & 150 & 39.3 & 45.7 & blue clay and gravel & Clay and gravel & -- & -- & -- & \\
\hline 76 & $8 / 9-33 \mathrm{J1}$ & & & 150 & 156 & 45.7 & 47.5 & decomposed brown rock & Ash or tuff & -- & -- & -- & \\
\hline 76 & 8/9-33J1 & & & 156 & 190 & 47.5 & 57.9 & decomposed rock & Ash or tuff & -- & -- & -- & \\
\hline 76 & 8/9-33J1 & & & 190 & 195 & 57.9 & 59.4 & yellow clay & Clay & -- & -- & -- & \\
\hline 76 & 8/9-33J1 & & & 195 & 220 & 59.4 & 67.1 & blue clay and gravel & Clay and gravel & -- & -- & -- & \\
\hline 76 & $8 / 9-33 \mathrm{~s} 1$ & & & 220 & 268 & 67.1 & 81.7 & cemented gravel & Gravel & cemented & -- & -- & \\
\hline 76 & $8 / 9-33 \mathrm{J1}$ & & & 268 & 285 & 81.7 & 86.9 & cemented gravel, hard & Gravel & cemented & -- & -- & \\
\hline 76 & $8 / 9-33 \mathrm{J1}$ & & & 285 & 325 & 86.9 & 99.1 & hard rock and shale & Clay, sand, and gravel & hard & .- & .. & $\mathrm{KJu}$ \\
\hline 77 & 8/9-35M1 & 77 & 7 8/9-35M1 & 0 & 475 & 0.0 & 144.8 & no data & No data & -- & -- & -. & Qyal and QTge and QTm \\
\hline 78 & $8 / 9-35 K 1$ & 78 & 8 8/9-35K1 & 0 & 28 & 0.0 & 8.5 & clay, yellow & Clay & -- & -- & -- & QTge \\
\hline 78 & 8/9-35K1 & & & 28 & 33 & 8.5 & 10.1 & clay, blue & Clay & .- & -. & .- & \\
\hline
\end{tabular}


Appendix 3. Lithologic data. - Continued

ISite IDs and well number correspond to locations in Appendix 1, a site ID and well number is listed for each drilled interval for a particular drill hole. For convenience, the site ID and well numbers are repeated at the shallowest drilled interval for each well. leaders $(-1$

\begin{tabular}{|c|c|c|c|c|c|c|c|c|c|c|c|c|c|}
\hline Site ID & Well number & $\begin{array}{l}\text { Site ID, } \\
\text { top of } \\
\text { well }\end{array}$ & $\begin{array}{l}\text { Well number, } \\
\text { top of well }\end{array}$ & $\begin{array}{c}\text { Top of } \\
\text { interval, in } \\
\text { feet }\end{array}$ & $\begin{array}{c}\text { Base of } \\
\text { interval, in } \\
\text { feet }\end{array}$ & $\begin{array}{c}\text { Top of } \\
\text { interval, in } \\
m\end{array}$ & $\begin{array}{c}\text { Base of } \\
\text { interval, in } \\
\mathrm{m}\end{array}$ & Reported lithologic unit ${ }^{1}$ & Interpreted lithologic class ${ }^{2}$ & $\begin{array}{c}\text { Reported } \\
\text { degree of } \\
\text { cementation or } \\
\text { induration }^{3}\end{array}$ & $\begin{array}{l}\text { Fossils } \\
\text { reported in } \\
\text { interval }^{4}\end{array}$ & $\begin{array}{c}\text { Organic } \\
\text { matter } \\
\text { reported in } \\
\text { interval }^{5}\end{array}$ & $\begin{array}{l}\text { Reported stratigraphic top of geologic } \\
\text { unit }^{6}\end{array}$ \\
\hline 78 & $8 / 9-35 \mathrm{~K} 1$ & & & 33 & 37 & 10.1 & 11.3 & sand, blue, and gravel & Sand (or sandstone) and gravel & -- & -- & -- & \\
\hline 78 & 8/9-35K1 & & & 37 & 50 & 11.3 & 15.2 & clay, blue & Clay & -- & -- & -- & \\
\hline 78 & 8/9-35K1 & & & 50 & 72 & 15.2 & 21.9 & gravel, cement; water and gas & Gravel & cemented & -- & -- & \\
\hline 78 & 8/9-35K1 & & & 72 & 104 & 21.9 & 31.7 & clay, sandy, blue & Clay and sand (or sandstone) & -- & -- & -- & \\
\hline 78 & 8/9-35K1 & & & 104 & 106 & 31.7 & 32.3 & gravel, cement & Gravel & cemented & -- & -- & \\
\hline 78 & 8/9-35K1 & & & 106 & 128 & 32.3 & 39.0 & clay, blue & Clay & -- & -- & -- & \\
\hline 78 & 8/9-35K1 & & & 128 & 140 & 39.0 & 42.7 & clay, sandy, blue & Clay and sand (or sandstone) & -- & -- & -- & \\
\hline 78 & 8/9-35K1 & & & 140 & 160 & 42.7 & 48.8 & clay, tough, blue & Clay & -- & -- & -- & \\
\hline 78 & 8/9-35K1 & & & 160 & 198 & 48.8 & 60.4 & clay, sandy, blue & Clay and sand (or sandstone) & -- & -- & -- & \\
\hline 78 & 8/9-35K1 & & & 198 & 208 & 60.4 & 63.4 & gravel, cement & Gravel & cemented & -- & -- & \\
\hline 78 & 8/9-35K1 & & & 208 & 211 & 63.4 & 64.3 & clay, blue & Clay & -- & -- & -- & \\
\hline 78 & 8/9-35K1 & & & 211 & 230 & 64.3 & 70.1 & clay, sandy, blue & Clay and sand (or sandstone) & -- & -- & -- & \\
\hline 78 & $8 / 9-35 K 1$ & & & 230 & 232 & 70.1 & 70.7 & gravel, cement & Gravel & cemented & -- & -- & \\
\hline 78 & 8/9-35K1 & & & 232 & 236 & 70.7 & 71.9 & clay, blue & Clay & -- & -- & -. & QTm \\
\hline 78 & 8/9-35K1 & & & 236 & 240 & 71.9 & $\begin{array}{l}1.9 \\
73.2\end{array}$ & $\begin{array}{l}\text { clay, sandy, blue } \\
\text { cand }\end{array}$ & Clay and sand (or sandstone) & -. & -. & -. & \\
\hline 78 & 8/9-35K1 & & & 240 & 268 & 73.2 & 81.7 & clay, blue & Clay & -- & - & -- & \\
\hline 78 & 8/9-35K1 & & & 268 & 270 & 81.7 & 82.3 & sandstone & Sandstone & cemented & -- & -- & \\
\hline 78 & 8/9-35K1 & & & 270 & 280 & 82.3 & 85.3 & clay, blue, and gravel & Clay and gravel & -- & -- & -- & \\
\hline 78 & 8/9-35K1 & & & 280 & 320 & 85.3 & 97.5 & sandstone & Sandstone & cemented & -- & -. & \\
\hline 78 & 8/9-35K1 & & & 320 & 352 & $\begin{array}{l}05.5 \\
97.5\end{array}$ & 107.3 & clay, blue, tough & Clay & -. & -- & -. & \\
\hline 78 & $8 / 9-35 \mathrm{~K} 1$ & & & 352 & 360 & 107.3 & 109.7 & clay, blue, soft & Clay & -- & -- & -- & \\
\hline 78 & $8 / 9-35 \mathrm{~K} 1$ & & & 360 & 362 & 109.7 & 110.3 & gravel, cement & Gravel & cemented & -- & -- & \\
\hline 78 & 8/9-35K1 & & & 362 & 378 & 110.3 & 115.2 & clay, blue, soft & Clay & -- & -- & -- & \\
\hline 78 & 8/9-35K1 & & & 378 & 389 & 115.2 & 118.6 & sandstone & Sandstone & cemented & -- & -- & \\
\hline 78 & 8/9-35K1 & & & 389 & 398 & 118.6 & 121.3 & clay, soft, blue & Clay & -- & -.- & .- & \\
\hline 78 & 8/9-35K1 & & & 398 & 410 & 121.3 & 125.0 & gravel, cement & Gravel & cemented & -- & -- & \\
\hline 78 & 8/9-35K1 & & & $\begin{array}{l}390 \\
410\end{array}$ & 425 & 125.0 & 129.5 & sandstone & Sandstone & cemented & -- &.- & \\
\hline 78 & 8/9-35K1 & & & 425 & 434 & 129.5 & 132.3 & clay, blue & Clay & -- & - & -- & \\
\hline 78 & 8/9-35K1 & & & 434 & 442 & 132.3 & 134.7 & clay, soft, blue & Clay & -- & -. & -- & \\
\hline 78 & 8/9-35K1 & & & 442 & 462 & 134.7 & 140.8 & sandstone & Sandstone & cemented & ..- & .. & \\
\hline 78 & 8/9-35K1 & & & 462 & 465 & 140.8 & 141.7 & clay, soft, blue & Clay & -- & -- & -- & \\
\hline 78 & 8/9-35K1 & & & 465 & 473 & 141.7 & 144.2 & sandstone, hard & Sandstone & hard & -- & .. & \\
\hline 78 & 8/9-35K1 & & & 473 & 490 & 144.2 & 149.4 & gravel, cement & Gravel & cemented & -- & -- & \\
\hline 78 & 8/9-35K1 & & & 490 & 496 & 149.4 & 151.2 & sand and gravel & Sand (or sandstone) and gravel & - & - & - & \\
\hline 78 & $8 / 9-35 K 1$ & & & 496 & 498 & 151.2 & 151.8 & clay, blue & Clay & -- & -- & -- & \\
\hline 79 & 8/9-34R1 & 79 & 9 8/9-34R1 & 0 & 260 & 0.0 & 79.2 & $\begin{array}{l}\text { clay, blue and yellow; contains } \\
\text { gravel } \\
\text { clean sand and gravel, clam and }\end{array}$ & Clay and gravel & -. & -- & -- & Qyal and QTge (?) \\
\hline 79 & 8/9-34R1 & & & 260 & 320 & 79.2 & 97.5 & snail shells & Sand (or sandstone) and gravel & -- & shells & .- & QTm \\
\hline 79 & 8/9-34R1 & & & 320 & 357 & 97.5 & 108.8 & $\begin{array}{l}\text { fine sand; includes shells } \\
\text {. }\end{array}$ & Sand & -- & shells & -- & \\
\hline 79 & 8/9-34R1 & & & 357 & 360 & 108.8 & 109.7 & clay & Clay & .. & -- & .. & \\
\hline 80 & 8/8-34Q1 & 80 & 0 8/8-34Q1 & 0 & 285 & 0.0 & 86.9 & no data & No data & -- & -- & -- & QTge \\
\hline 81 & $8 / 9-36 \mathrm{P} 1$ & 81 & $18 / 9-36 \mathrm{P} 1$ & 0 & 9 & 0.0 & 2.7 & soil & Sand and clay & -- & -- & -- & Qyal \\
\hline $\begin{array}{ll}81 \\
81\end{array}$ & $8 / 9-36 \mathrm{P} 1$ & & & 9 & 50 & 2.7 & 15.2 & clay, blue & Clay & -. & -- & -. & \\
\hline 81 & 8/9-36P1 & & & 50 & 57 & 15.2 & 17.4 & yellow & Clay & -- & -- & -- & \\
\hline 81 & 8/9-36P1 & & & 57 & 62 & 17.4 & $\begin{array}{l}1.4 \\
18.9\end{array}$ & gravel & Gravel & .- & -- & .. & \\
\hline 81 & 8/9-36P1 & & & 62 & 80 & 18.9 & 24.4 & clay, yellow & Clay & -- & -- & -- & QTge \\
\hline 81 & $8 / 9-36 \mathrm{P} 1$ & & & 80 & 141 & 24.4 & 43.0 & $\begin{array}{l}\text { clay, blue } \\
\text { lat }\end{array}$ & Clay & -- & -- & -- & \\
\hline 81 & 8/9-36P1 & & & 141 & 147 & 43.0 & 44.8 & pea gravel & Gravel & -- & -- & -- & \\
\hline 81 & 8/9-36P1 & & & 147 & 210 & 44.8 & 64.0 & clay blue & Clay & -- & -- & -. & \\
\hline 81 & 8/9-36P1 & & & 210 & 217 & 64.0 & 66.1 & $\begin{array}{l}\text { clay, yellow } \\
\text { cla }\end{array}$ & Clay & -- & -- & .- & \\
\hline 81 & 8/9-36P1 & & & 217 & 455 & 66.1 & 138.7 & $\begin{array}{l}\text { clay, blue } \\
\text { clus }\end{array}$ & Clay & - & -- & -- & \\
\hline 81 & $8 / 9-36 \mathrm{P} 1$ & & & 455 & 463 & 138.7 & 141.1 & clay, blue, sandy & Clay and sand (or sandstone) & -- & -- & -. & \\
\hline 81 & 8/9-36P1 & & & 463 & 631 & 141.1 & 192.3 & clay blue & Clay & .- & -. & .. & \\
\hline 81 & 8/9-36P1 & & & 631 & 650 & 192.3 & 198.1 & clay, blue sandy & Clay and sand (or sandstone) & - & -- & -. & \\
\hline 81 & 8/9-36P1 & & & 650 & 678 & 198.1 & 206.7 & $\begin{array}{l}\text { clay, sandy } \\
\text { cantion }\end{array}$ & Clay and sand (or sandstone) & -. & -. & .. & \\
\hline 81 & $8 / 9-36 \mathrm{P} 1$ & & & 678 & 711 & 206.7 & 216.7 & clay, blue & Clay & -- & -- & -- & \\
\hline 81 & $8 / 9-36 \mathrm{P} 1$ & & & 711 & 730 & 216.7 & 222.5 & sand, coarse, and pea gravel & Sand (or sandstone) and gravel & - & - & -. & \\
\hline 81 & 8/9-36P1 & & & 730 & 765 & 222.5 & 233.2 & clay, blue & Clay & -- & -. & -- & QTm \\
\hline 81 & $8 / 9-36 \mathrm{P} 1$ & & & 765 & 804 & 233.2 & 245.1 & sand, gray & Sand & - & -. & -. & \\
\hline 81 & 8/9-36P1 & & & 804 & 808 & 245.1 & 246.3 & clay, blue & Clay & -- & -- & -- & \\
\hline
\end{tabular}


Appendix 3. Lithologic data. - Continued

[Site IDs and well number correspond to locations in Appendix 1, a site ID and well number is listed for each drilled interval for a particular drill hole. For convenience, the site ID and well numbers are repeated at the shallowest drilled interval for each well. leaders

\begin{tabular}{|c|c|c|c|c|c|c|c|c|c|c|c|c|c|}
\hline Site ID & Well number & $\begin{array}{l}\text { Site ID, } \\
\text { top of } \\
\text { well }\end{array}$ & $\begin{array}{c}\text { Well number, } \\
\text { top of well }\end{array}$ & $\begin{array}{c}\text { Top of } \\
\text { interval, in } \\
\text { feet }\end{array}$ & $\begin{array}{c}\text { Base of } \\
\text { interval, in } \\
\text { feet }\end{array}$ & $\begin{array}{c}\text { Top of } \\
\text { interval, in } \\
m\end{array}$ & $\begin{array}{c}\text { Base of } \\
\text { interval, in } \\
\mathrm{m}\end{array}$ & Reported lithologic unit ${ }^{1}$ & Interpreted lithologic class ${ }^{2}$ & $\begin{array}{c}\text { Reported } \\
\text { degree of } \\
\text { cementation or } \\
\text { induration }^{3} \\
\end{array}$ & $\begin{array}{l}\text { Fossils } \\
\text { reported in } \\
\text { interval }^{4}\end{array}$ & $\begin{array}{c}\text { Organic } \\
\text { matter } \\
\text { reported in } \\
\text { interval }^{5}\end{array}$ & $\begin{array}{l}\text { Reported stratigraphic top of geologic } \\
\text { unit }^{6}\end{array}$ \\
\hline 81 & 8/9-36P1 & & & 808 & 835 & 246.3 & 254.5 & clay, sandy & Clay and sand (or sandstone) & -- & -- & -- & \\
\hline 81 & 8/9-36P1 & & & 835 & 850 & 254.5 & 259.1 & clay, blue & Clay & -- & -- & -- & \\
\hline 81 & 8/9-36P1 & & & 850 & 880 & 259.1 & 268.2 & sand, gray; sand running bad & Sand & -- & -- & -- & \\
\hline 81 & 8/9-36P1 & & & 880 & 910 & 268.2 & 277.4 & $\begin{array}{l}\text { clay, blue } \\
\text { sand }\end{array}$ & Clay & -- & -- & -- & \\
\hline 81 & 8/9-36P1 & & & 910 & 994 & 277.4 & 303.0 & gravel & Gravel & -- & -- & -- & QTge \\
\hline 81 & 8/9-36P1 & & & 994 & 1005 & 303.0 & 306.3 & sand & Sand & -- & -- & -- & QTm \\
\hline 81 & $8 / 9-36 \mathrm{P} 1$ & & & 1005 & 1010 & 306.3 & 307.8 & sand and clam shells & Sand & -- & shells & -- & \\
\hline 81 & $8 / 9-36 \mathrm{P} 1$ & & & 1010 & 1020 & 307.8 & 310.9 & clay, blue & Clay & -- & -- & -- & \\
\hline & & & & & & & & $\begin{array}{l}\text { basalt(?) probably dark massive } \\
\text { well-cemented fine-grained }\end{array}$ & & & & & \\
\hline 81 & 8/9-36P1 & & & 1020 & 1025 & 310.9 & 312.4 & sandstone & Basalt & hard & -- & -- & \\
\hline 81 & 8/9-36P1 & & & 1025 & 1048 & 312.4 & 319.4 & fossil formation & Clay and sand (or sandstone) & -- & fossils (?) & -- & \\
\hline 81 & 8/9-36P1 & & & 1048 & 1050 & 319.4 & 320.0 & Petaluma Valley & Clay and sand (or sandstone) & -- & -- & -- & \\
\hline 82 & $7 / 9-2 \mathrm{C} 1$ & 82 & $27 / 9-2 \mathrm{C} 1$ & 0 & 610 & 0.0 & 185.9 & no data & No data & -- & -- & -- & Qyal and QTge and QTm \\
\hline 83 & 8/9-34R2 & 83 & 3 8/9-34R2 & 0 & 20 & 0.0 & 6.1 & topsoil & Sand and clay & -- & -. & -- & Qyal and QTge (?) \\
\hline 83 & 8/9-34R2 & & & 20 & 36 & 6.1 & 11.0 & topsoil & Sand and clay & -- & -- & -- & Gy ar and Lige (?) \\
\hline 83 & 8/9-34R2 & & & 36 & 70 & 11.0 & 21.3 & pea gravel & Gravel & -- & -- & -- & \\
\hline 83 & 8/9-34R2 & & & 70 & 108 & 21.3 & 32.9 & clay, blue, sandy & Clay and sand (or sandstone) & -- & -- & -- & \\
\hline 83 & 8/9-34R2 & & & 108 & 118 & 32.9 & 36.0 & clay, yellow & Clay & -- & -- & -- & \\
\hline 83 & 8/9-34R2 & & & 118 & 128 & 36.0 & 39.0 & gravel, small & Gravel & -- & -- & -- & \\
\hline 83 & 8/9-34R2 & & & 128 & 155 & 39.0 & 47.2 & mixed clay and small gravel & Gravel & - & - & -- & \\
\hline 83 & 8/9-34R2 & & & 155 & 159 & 47.2 & 48.5 & gravel, small, and tules & Gravel & -- & -- & tule & \\
\hline 83 & 8/9-34R2 & & & 159 & 190 & 48.5 & 57.9 & clay, sandy & Clay and sand (or sandstone) & -- & -- & -- & QTm \\
\hline 83 & 8/9-34R2 & & & 190 & 210 & 57.9 & 64.0 & sand, fine & Sand & -- & -- & -- & \\
\hline 83 & 8/9-34R2 & & & 210 & 230 & 64.0 & 70.1 & sand and tules & Sand & -- & -- & tule & \\
\hline 83 & 8/9-34R2 & & & 230 & 260 & 70.1 & 79.2 & gravel, small & Gravel & -- & -- & -- & \\
\hline 83 & 8/9-34R2 & & & 260 & 280 & 79.2 & 85.3 & gravel, pea & Gravel & -- & -- & -- & \\
\hline 83 & 8/9-34R2 & & & 280 & 300 & 85.3 & 91.4 & $\begin{array}{l}\text { gravel, small, and tules } \\
\text { garas }\end{array}$ & Gravel & - & -- & tule & \\
\hline 83 & 8/9-34R2 & & & 300 & 340 & 91.4 & 103.6 & shells and gravel & Sand (or sandstone) and gravel & -- & shells & -- & \\
\hline 83 & $8 / 9-34 R 2$ & & & 340 & 350 & 103.6 & 106.7 & shells & Sand & -- & shells & -.- & \\
\hline 83 & $8 / 9-34 R 2$ & & & 350 & 410 & 106.7 & 125.0 & clay, sand, and shells & Clay and sand (or sandstone) & -- & shells & -- & \\
\hline 83 & 8/9-34R2 & & & 410 & 485 & 125.0 & 147.8 & sand, small gravel, and shells & Sand (or sandstone) and gravel & -- & shells & -- & \\
\hline 83 & $8 / 9-34 \mathrm{R} 2$ & & & 485 & 491 & 147.8 & 149.7 & clay, hard, blue & Clay & hard & -- & - & \\
\hline 84 & $7 / 8-3 \mathrm{CC}$ & 84 & $4 \quad 7 / 8-3 C 1$ & 0 & 47 & 0.0 & 14.3 & no data & No data & -. & -- & .- & -- \\
\hline 84 & $7 / 8-3 \mathrm{C} 1$ & & & 47 & 115 & 14.3 & 35.1 & clay and gravel & Clay and gravel & -- & -- & -.- & QTge \\
\hline 84 & $7 / 8-3 \mathrm{C} 1$ & & & 115 & 235 & 35.1 & 71.6 & no data & No data & -- & -- & -. & \\
\hline 85 & 7/8-4B3 & 85 & $5 \quad 7 / 8-4 B 3$ & 0 & 4 & 0.0 & 1.2 & topsoil & Sand and clay & -- & -- & .- & Qyal and QTge \\
\hline 85 & $7 / 8-4 \mathrm{~B} 3$ & & & 4 & 10 & 1.2 & 3.0 & clay, sand & Clay and sand (or sandstone) & -- & -- & -- & \\
\hline 85 & 7/8-4B3 & & & 10 & 13 & 3.0 & 4.0 & clay, yellow, and gravel & Clay and gravel & -- & -- & .. & \\
\hline 85 & $7 / 8-4 \mathrm{~B} 3$ & & & 13 & 25 & 4.0 & 7.6 & gravel & Gravel & .. & -. & .- & \\
\hline 85 & 7/8-4B3 & & & 25 & 49 & 7.6 & 14.9 & gravel, cemented & Gravel & cemented & -- & -- & \\
\hline 85 & 7/8-4B3 & & & 49 & 54 & 14.9 & 16.5 & clay, yellow, and sandy gravel & Clay, sand, and gravel & -. & -- & -.- & \\
\hline 85 & 7/8-4B3 & & & 54 & 70 & 16.5 & 21.3 & clay, sandy, yellow & Clay and sand (or sandstone) & -- & -- & -- & \\
\hline 85 & 7/8-4B3 & & & 70 & 85 & 21.3 & 25.9 & clay, blue & Clay & -- & -- & -- & \\
\hline 85 & $7 / 8-4 \mathrm{~B} 3$ & & & 85 & 95 & 25.9 & 29.0 & gravel, yellow cemented & Gravel & cemented & -- & -- & \\
\hline 85 & 7/8-4B3 & & & 95 & 118 & 29.0 & 36.0 & clay, sandy, blue & Clay and sand (or sandstone) & -- & -- & -- & \\
\hline 85 & 7/8-4B3 & & & 118 & 120 & 36.0 & 36.6 & sandy, blue, and gravel & Clay, sand, and gravel & -- & -- & -- & \\
\hline 85 & 7/8-4B3 & & & 120 & 125 & 36.6 & 38.1 & clay, blue & Clay & -- & -- & -- & \\
\hline 85 & 7/8-4B3 & & & 125 & 134 & 38.1 & 40.8 & clay, sandy, yellow & Clay and sand (or sandstone) & -- & -- & .. & \\
\hline 85 & 7/8-4B3 & & & 134 & 150 & 40.8 & 45.7 & clay, yellow, and gravel & Clay and gravel & -- & -- & -- & \\
\hline 85 & 7/8-4B3 & & & 150 & 152 & 45.7 & 46.3 & gravel, yellow, cemented & Gravel & cemented & -- & -- & \\
\hline 86 & $7 / 7-5 \mathrm{~F} 1$ & 86 & $6 \quad 7 / 7-5 F 1$ & 0 & 2 & 0.0 & 0.6 & loam, black & Sand and clay & -- & -- & -- & QTge \\
\hline 86 & $7 / 7-5 \mathrm{~F} 1$ & & & 2 & 4 & 0.6 & 1.2 & & Gravel & hard & -- & -- & \\
\hline 86 & $7 / 7-5 F 1$ & & & 4 & 47 & 12 & 14.3 & $\begin{array}{l}\text { clay, yellow, and gravel; water at } \\
12 \text { feet }\end{array}$ & Clay and gravel &.- & .- & $\ldots$ & \\
\hline 86 & $7 / 7-5 F 1$ & & & $\begin{array}{l}4 \\
47\end{array}$ & $\begin{array}{l}41 \\
52\end{array}$ & $\begin{array}{l}1.2 \\
14.3\end{array}$ & $\begin{array}{l}15.3 \\
15.8\end{array}$ & $\begin{array}{l}12 \text { reet } \\
\text { gravel }\end{array}$ & Gravel & -- & -- & -. & \\
\hline 86 & $7 / 7-5 \mathrm{~F} 1$ & & & 52 & 72 & 15.8 & 21.9 & clay, yellow, and gravel & Clay and gravel & - & -- & -- & \\
\hline 86 & 7/7-5F1 & & & 72 & 74 & 21.9 & 22.6 & gravel, water & Gravel & -- & -- & .. & \\
\hline 86 & $7 / 7-5 \mathrm{~F} 1$ & & & 74 & 97 & 22.6 & 29.6 & clay, yellow, and gravel & Clay and gravel & -- & -- & -. & \\
\hline 86 & $7 / 7-5 \mathrm{~F} 1$ & & & 97 & 117 & 29.6 & 35.7 & clay, yellow & Clay & -- & -- & -- & \\
\hline 86 & 7/7-5F1 & & & 117 & 129 & 35.7 & 39.3 & clay, yellow, and gravel & Clay and gravel & -. & -- & -- & \\
\hline
\end{tabular}


Appendix 3. Lithologic data. - Continued

[Site IDs and well number correspond to locations in Appendix 1, a site ID and well number is listed for each drilled interval for a particular drill hole. For convenience, the site ID and well numbers are repeated at the shallowest drilled interval for each well. lea

\begin{tabular}{|c|c|c|c|c|c|c|c|c|c|c|c|c|c|}
\hline Site ID & Well number & $\begin{array}{l}\text { Site ID, } \\
\text { top of } \\
\text { well }\end{array}$ & $\begin{array}{c}\text { Well number, } \\
\text { top of well }\end{array}$ & $\begin{array}{c}\text { Top of } \\
\text { interval, in } \\
\text { feet }\end{array}$ & $\begin{array}{c}\text { Base of } \\
\text { interval, in } \\
\text { feet }\end{array}$ & $\begin{array}{c}\text { Top of } \\
\text { interval, in } \\
m\end{array}$ & $\begin{array}{c}\text { Base of } \\
\text { interval, in } \\
\mathrm{m}\end{array}$ & Reported lithologic unit ${ }^{1}$ & Interpreted lithologic class ${ }^{2}$ & $\begin{array}{c}\text { Reported } \\
\text { degree of } \\
\text { cementation or } \\
\text { induration }^{3}\end{array}$ & $\begin{array}{c}\text { Fossils } \\
\text { reported in } \\
\text { interval }^{4}\end{array}$ & $\begin{array}{c}\text { Organic } \\
\text { matter } \\
\text { reported in } \\
\text { interval }^{5}\end{array}$ & $\begin{array}{l}\text { Reported stratigraphic top of geologic } \\
\text { unit }^{6}\end{array}$ \\
\hline 86 & $7 / 7-5 \mathrm{~F} 1$ & & & 129 & 157 & 39.3 & 47.9 & gravel, water & Gravel & -- & -- & -- & \\
\hline 86 & $7 / 7-5 \mathrm{~F} 1$ & & & 157 & 164 & 47.9 & 50.0 & clay, yellow, and gravel & Clay and gravel & -- & -- & -- & \\
\hline 87 & $7 / 7-5 F 2$ & 87 & $7 / 7-5 F 2$ & 0 & 65 & 0.0 & 19.8 & no data & No data & -- & -- & -- & -- \\
\hline 87 & $7 / 7-5 F 2$ & & & 65 & 75 & 19.8 & 22.9 & packed gravel & Gravel & -- & -- & -- & QTge \\
\hline 87 & $7 / 7-5 F 2$ & & & 160 & 175 & 48.8 & 53.3 & medium packed gravel & Gravel & -- & -- & -- & \\
\hline 87 & $7 / 7-5 F 2$ & & & 175 & 275 & 53.3 & 83.8 & no data & No data & -- & -- & -- & \\
\hline 88 & 7/9-3Е1 & 88 & 7/9-3E1 & 0 & 4 & 0.0 & 1.2 & soil & Sand and clay & -- & -- & -. & QTm \\
\hline 88 & 7/9-3E1 & & & 4 & 37 & 1.2 & 11.3 & yellow clay & Clay & -- & -- & -- & \\
\hline 88 & 7/9-3E1 & & & 37 & 55 & 11.3 & 16.8 & blue sandstone & Sandstone & cemented & -- & -- & \\
\hline 88 & 7/9-3E1 & & & 55 & 63 & 16.8 & 19.2 & $\begin{array}{l}\text { clam shells, blue } \\
\text { clam shells and gravel (probably }\end{array}$ & Sand & -- & shells & -- & \\
\hline 88 & 7/9-3E1 & & & 63 & 130 & 19.2 & 39.6 & in sand matrix) & Sand (or sandstone) and gravel & -- & shells & -- & \\
\hline 89 & $7 / 7-6 \mathrm{G} 1$ & 89 & $7 / 7-6 \mathrm{G} 1$ & 0 & 3 & 0.0 & 0.9 & topsoil & Sand and clay & -- & -- & -- & QTge \\
\hline 89 & 7/7-6G1 & & & 3 & 30 & 0.9 & 9.1 & fine sand & Sand & -- & .. & -- & \\
\hline 89 & $7 / 7-6 \mathrm{G} 1$ & & & 30 & 41 & 9.1 & $\begin{array}{l}.1 \\
12.5\end{array}$ & blue clay with streaks of gravel & Clay and gravel & -- & -. & -- & \\
\hline 89 & $7 / 7-6 \mathrm{G} 1$ & & & 41 & 87 & 12.5 & 26.5 & sand and gravel & Sand (or sandstone) and gravel & -- & -- & -- & \\
\hline 89 & $7 / 7-6 \mathrm{G} 1$ & & & 87 & 108 & 26.5 & 32.9 & sand and gravel & Sand (or sandstone) and gravel & -- & .. & -- & \\
\hline 89 & $7 / 7-6 \mathrm{G} 1$ & & & 108 & 124 & 32.9 & 37.8 & blue clay & Clay & -- & -- & -- & \\
\hline 89 & $7 / 7-6 \mathrm{G} 1$ & & & 124 & 141 & 37.8 & 43.0 & sand and gravel & Sand (or sandstone) and gravel & -- & -. & -- & \\
\hline 89 & $7 / 7-6 \mathrm{G} 1$ & & & 141 & $\begin{array}{l}171 \\
172\end{array}$ & 43.0 & 52.4 & $\begin{array}{l}\text { blue clay } \\
\text { and }\end{array}$ & Clay & -. & -. & -- & \\
\hline 89 & 7/7-6G1 & & & 172 & 203 & 52.4 & 61.9 & clay with streaks of gravel & Clay and gravel & -- & -- & -- & \\
\hline 89 & $7 / 7-6 \mathrm{G} 1$ & & & 203 & 229 & 61.9 & 69.8 & blue clay & Clay & -- & -. & -- & \\
\hline 89 & $7 / 7-6 \mathrm{G} 1$ & & & 229 & 243 & 69.8 & 74.1 & sandy clay & Clay and sand (or sandstone) & -- & -- & -- & \\
\hline 89 & $7 / 7-6 \mathrm{G} 1$ & & & 243 & 310 & 74.1 & 94.5 & $\begin{array}{l}\text { blue clay } \\
\text { bay }\end{array}$ & Clay & -- & -. & -- & \\
\hline 89 & $7 / 7-6 \mathrm{G} 1$ & & & 310 & 333 & 94.5 & 101.5 & sand and gravel & Sand (or sandstone) and gravel & -- & ..- & .- & \\
\hline 89 & $7 / 7-6 \mathrm{G} 1$ & & & 333 & 338 & 101.5 & 103.0 & blue clay & Clay & -- & -- & -- & \\
\hline 90 & $7 / 8-6 \mathrm{H} 2$ & 90 & $7 / 8-6 \mathrm{H} 2$ & 0 & 2 & 0.0 & 0.6 & soil & Sand and clay & -- & -- & .- & QTge \\
\hline 90 & $7 / 8-6 \mathrm{H} 2$ & & & 2 & 8 & 0.6 & 2.4 & hardpan, gravelly & Clay and gravel & hard & -- & -- & \\
\hline 90 & $7 / 8-6 \mathrm{H} 2$ & & & 8 & 26 & 2.4 & 7.9 & brown clay & Clay & -- & .. & .- & \\
\hline 90 & $\begin{array}{l}7 / 8-6 \mathrm{~Hz} \\
7 / 8-6 \mathrm{H} 2\end{array}$ & & & $\begin{array}{l}0 \\
26\end{array}$ & $\begin{array}{l}20 \\
31\end{array}$ & $\begin{array}{l}.4 .4 \\
7.9\end{array}$ & $\begin{array}{l}9.9 \\
9.4\end{array}$ & loose boulders & Gravel & -. & -- & -- & \\
\hline 90 & $7 / 8-6 \mathrm{H} 2$ & & & 31 & 60 & 9.4 & 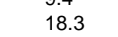 & brown sandy clay & Clay and sand (or sandstone) & -- & - & -- & \\
\hline 90 & $7 / 8-6 \mathrm{H} 2$ & & & 60 & 82 & 18.3 & 25.0 & blue sandstone & Sandstone & cemented & -- & -- & \\
\hline 90 & $7 / 8-6 \mathrm{H} 2$ & & & 82 & 103 & 25.0 & 31.4 & blue clay & Clay & -- & -- & -- & \\
\hline 90 & $7 / 8-6 \mathrm{H} 2$ & & & 103 & 110 & 31.4 & 33.5 & blue hard clay & Clay & hard & -- & - & \\
\hline 90 & $7 / 8-6 \mathrm{H} 2$ & & & 110 & 125 & 33.5 & 38.1 & brown and blue clay & Clay & -- & -- & -- & \\
\hline 90 & $7 / 8-6 \mathrm{H} 2$ & & & 125 & 167 & 38.1 & 50.9 & gravelly clay & Clay and gravel & .. & .. & .. & \\
\hline 90 & $7 / 8-6 \mathrm{H} 2$ & & & 167 & 232 & 50.9 & 70.7 & small boulder blue clay & Clay and gravel & -- & -- & -- & \\
\hline 90 & $7 / 8-6 \mathrm{H} 2$ & & & 232 & 235 & 70.7 & 71.6 & small loose boulders & Gravel & -- & -- & -- & \\
\hline 90 & $\begin{array}{l}7 / 8-6 \mathrm{H} 2 \\
-102\end{array}$ & & & 235 & 275 & 71.6 & $\begin{array}{r}1.0 \\
83.8\end{array}$ & brown clay & Clay & -- & -- & - & \\
\hline 90 & $7 / 8-6 \mathrm{H} 2$ & & & 275 & 295 & 83.8 & 89.9 & blue gravelly clay & Clay and gravel & -- & -- & -- & \\
\hline 90 & $7 / 8-6 \mathrm{H} 2$ & & & 295 & 297 & 89.9 & 90.5 & gray loose sandstone & Sandstone & cemented & .. & $\ldots$ & QTm \\
\hline 90 & $7 / 8-6 \mathrm{H} 2$ & & & 297 & 330 & 90.5 & 100.6 & blue hard sandstone & Sandstone & hard & - & -- & \\
\hline 90 & $7 / 8-6 \mathrm{H} 2$ & & & 330 & 342 & 100.6 & 104.2 & blue clay & Clay & -- & -- & -- & \\
\hline 90 & $\begin{array}{l}7 / 8-6 \mathrm{H} 2 \\
7 / 2\end{array}$ & & & 342 & 352 & 104.2 & 107.3 & gray sandstone & Sandstone & cemented & -- & -. & \\
\hline 90 & $7 / 8-6 \mathrm{H} 2$ & & & 352 & 356 & 107.3 & 108.5 & blue gravelly clay & Clay and gravel & -- & -- & - & QTge \\
\hline 90 & $7 / 8-6 \mathrm{H} 2$ & & & 356 & 397 & 108.5 & 121.0 & blue gravelly clay & Clay and gravel & - & -- & - & \\
\hline 91 & 7/9-3M1 & 91 & 7/9-3M1 & 0 & 61 & 0.0 & 18.6 & old dug well & No data & -- & -- & -- & QTm \\
\hline 91 & 7/9-3M1 & & & 61 & 72 & 18.6 & 21.9 & clay, blue, sandy & Clay and sand (or sandstone) & -- & -- & -- & \\
\hline 91 & 7/9-3M1 & & & 72 & 78 & 21.9 & 23.8 & clay, sticky, blue & Clay & -- & -- & -- & \\
\hline 91 & 7/9-3M1 & & & 78 & 94 & 23.8 & 28.7 & clay, sandy, blue & Clay and sand (or sandstone) & -- & -- & -- & \\
\hline 91 & 7/9-3M1 & & & 94 & 102 & 28.7 & 31.1 & clay, blue, sticky & Clay & - & -- & -. & \\
\hline 91 & 7/9-3M1 & & & 102 & 106 & 31.1 & 32.3 & clay, blue, sandy & Clay and sand (or sandstone) & -- & -- & -- & \\
\hline 91 & 7/9-3M1 & & & 106 & 125 & 32.3 & 38.1 & sandstone & Sandstone & cemented & -- & -- & \\
\hline 91 & 7/9-3M1 & & & 125 & 127 & 38.1 & 38.7 & clay, blue, sandy & Clay and sand (or sandstone) & -- & -- & -- & \\
\hline 91 & 7/9-3M1 & & & 127 & 131 & 38.7 & 39.9 & gravel & Gravel & -. & -- & -- & \\
\hline 92 & $7 / 7-5 \mathrm{~N} 1$ & 92 & $7 / 7-5 \mathrm{~N} 1$ & 0 & 4 & 0.0 & 1.2 & soil adobe & Sand and clay & -. & -- & -. & Qyal and QTge (?) \\
\hline 92 & $7 / 7-5 \mathrm{~N} 1$ & & & 4 & 10 & 1.2 & 3.0 & $\begin{array}{l}\text { fine gravel } \\
\text { f }\end{array}$ & Gravel & - & -- & -- & \\
\hline 92 & $7 / 7-5 \mathrm{~N} 1$ & & & 10 & 22 & 3.0 & 6.7 & boulder and yellow sand & Sand (or sandstone) and gravel &.- & - & - & \\
\hline 92 & $7 / 7-5 \mathrm{~N} 1$ & & & 22 & 45 & 6.7 & 13.7 & sandy yellow clay & Clay and sand (or sandstone) & -- & -- & -- & \\
\hline 92 & $7 / 7-5 \mathrm{~N} 1$ & & & 45 & 55 & 13.7 & 16.8 & large yellow gravel hardpan & Clay and gravel & hard & -. & -- & \\
\hline 92 & $7 / 7-5 \mathrm{~N} 1$ & & & 55 & 57 & 16.8 & 17.4 & brown sand vein & Sand & -- & -- & -- & \\
\hline
\end{tabular}


Appendix 3. Lithologic data. - Continued

[Site IDs and well number correspond to locations in Appendix 1, a site ID and well number is listed for each drilled interval for a particular drill hole. For convenience, the site ID and well numbers are repeated at the shallowest drilled interval for each well. leaders

\begin{tabular}{|c|c|c|c|c|c|c|c|c|c|c|c|c|c|}
\hline Site ID & Well number & $\begin{array}{l}\text { Site ID, } \\
\text { top of } \\
\text { well }\end{array}$ & $\begin{array}{l}\text { Well number, } \\
\text { top of well }\end{array}$ & $\begin{array}{l}\text { Top of } \\
\text { interval, in } \\
\text { feet }\end{array}$ & $\begin{array}{l}\text { Base of } \\
\text { interval, in } \\
\text { feet }\end{array}$ & $\begin{array}{c}\text { Top of } \\
\text { interval, in } \\
m\end{array}$ & $\begin{array}{c}\text { Base of } \\
\text { interval, in } \\
m\end{array}$ & Reported lithologic unit' & Interpreted lithologic class ${ }^{2}$ & $\begin{array}{c}\text { Reported } \\
\text { degree of } \\
\text { cementation or } \\
\text { induration }^{3}\end{array}$ & $\begin{array}{l}\text { Fossils } \\
\text { reported in } \\
\text { interval }^{4}\end{array}$ & $\begin{array}{c}\text { Organic } \\
\text { matter } \\
\text { reported in } \\
\text { interval }^{5}\end{array}$ & $\begin{array}{l}\text { Reported stratigraphic top of geologic } \\
\text { unit }^{6}\end{array}$ \\
\hline 92 & $7 / 7-5 \mathrm{~N} 1$ & & & 57 & 72 & 17.4 & 21.9 & medium gravelly clay & Clay and gravel & -- & -- & -- & \\
\hline 92 & $7 / 7-5 \mathrm{~N} 1$ & & & 72 & 86 & 21.9 & 26.2 & large brown packed gravel & Gravel & -- & -- & -- & \\
\hline 92 & $7 / 7-5 \mathrm{~N} 1$ & & & 86 & 88 & 26.2 & 26.8 & brown coarse sand & Sand & -- & .- & -- & \\
\hline 93 & $7 / 7-8 \mathrm{~A} 1$ & 93 & 7/7-8A1 & 0 & 385 & 0.0 & 117.3 & no data & No data & -- & .- & -- & QTge \\
\hline 94 & $7 / 9-4 \mathrm{~N} 1$ & 94 & 7/9-4N1 & 0 & 4 & 0.0 & 1.2 & soil & Sand and clay & -- & -- & -- & QTm \\
\hline 94 & $7 / 9-4 \mathrm{~N} 1$ & & & 4 & 30 & 1.2 & 9.1 & clay and sand, dry & Clay and sand (or sandstone) & -- & -- & -- & \\
\hline 94 & $7 / 9-4 \mathrm{~N} 1$ & & & 30 & 55 & 9.1 & 16.8 & sand, soft, yellow & Sand & -- & .- & .- & \\
\hline 94 & $7 / 9-4 \mathrm{~N} 1$ & & & 55 & 96 & 16.8 & 29.3 & sand, soft, blue & Sand & -- & -- & -- & \\
\hline 94 & $7 / 9-4 \mathrm{~N} 1$ & & & 96 & 120 & 29.3 & 36.6 & sandstone, hard, blue & Sandstone & hard & .- & -- & \\
\hline 95 & $7 / 9-5 \mathrm{~N} 1$ & 95 & $7 / 9-5 \mathrm{~N} 1$ & 0 & 3 & 0.0 & 0.9 & 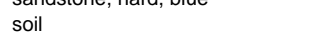 & Sand and clay & -- & -- & -- & QTm \\
\hline 95 & $7 / 9-5 \mathrm{~N} 1$ & & & 3 & 68 & 0.9 & 20.7 & sand, hard yellow & Sand & hard & -- & -- & \\
\hline 95 & $7 / 9-5 \mathrm{~N} 1$ & & & 68 & 150 & 20.7 & 45.7 & clay, blue, and sandstone & Clay and sand (or sandstone) & cemented & .- & .- & \\
\hline 95 & $7 / 9-5 \mathrm{~N} 1$ & & & 150 & 170 & 45.7 & 51.8 & sandstone, blue & Sandstone & cemented & -- & -- & \\
\hline 95 & 7/9-5N1 & & & 170 & 205 & 51.8 & 62.5 & clay, blue, sand sandstone & Clay and sand (or sandstone) & cemented & -- & -- & \\
\hline 95 & $7 / 9-5 \mathrm{~N} 1$ & & & 205 & 220 & $\begin{array}{l}62.0 \\
62.5\end{array}$ & $\begin{array}{l}62.5 \\
67.1\end{array}$ & sandstone, blue & $\begin{array}{l}\text { Sandstone } \\
\text { Sal }\end{array}$ & $\begin{array}{l}\text { cemented } \\
\text { ced }\end{array}$ & -- & -- & \\
\hline 95 & $7 / 9-5 \mathrm{~N} 1$ & & & 220 & 250 & 67.1 & 76.2 & $\begin{array}{l}\text { clay, blue, and sandstone }\end{array}$ & Clay and sand (or sandstone) & cemented & -- & -- & \\
\hline 95 & 7/9-5N1 & & & 250 & 260 & 76.2 & 79.2 & sandstone, blue & Sandstone & cemented & -- & -- & \\
\hline 95 & $7 / 9-5 \mathrm{~N} 1$ & & & 260 & 282 & 79.2 & 86.0 & clay, blue, and sandstone & Clay and sand (or sandstone) & cemented & -- & -- & \\
\hline 95 & 7/9-5N1 & & & 282 & 287 & 86.0 & 87.5 & clamshells in sandstone & Sandstone & cemented & shells & -- & \\
\hline 95 & $7 / 9-5 \mathrm{~N} 1$ & & & 287 & 292 & 87.5 & 89.0 & bedrock, KJu & Undifferentiated basement & hard & -- & -- & KJu \\
\hline 96 & 7/7-9D3 & 96 & 7/7-9D3 & 0 & 70 & 0.0 & 21.3 & $\begin{array}{l}\text { no data } \\
\text { sandy clay and gravel; cemented }\end{array}$ & No data & - & -- & -- & -- \\
\hline 96 & 7/7-9D3 & & & 70 & 130.0 & 21.3 & 39.6 & gravel & Clay, sand, and gravel & cemented & -- & -- & QTge \\
\hline 96 & 7/7-9D3 & & & 130.0 & 241.0 & 39.6 & 73.5 & no data & No data & - & -- & -- & \\
\hline 96 & 7/7-9D3 & & & 241.0 & 243.0 & 73.5 & 74.1 & gravel & Gravel & .- & .- & .- & \\
\hline 96 & 7/7-9D3 & & & 243.0 & 252.0 & 74.1 & 76.8 & no data & No data & -- & -- & -- & \\
\hline 96 & 7/7-9D3 & & & 252.0 & 256.0 & 76.8 & 78.0 & cemented gravel & Gravel & cemented & .- & .- & \\
\hline 96 & 7/7-9D3 & & & 256.0 & 271.0 & 78.0 & 82.6 & no data & No data & - & -- & -- & \\
\hline 96 & 7/7-9D3 & & & 271.0 & 275.0 & 82.6 & 83.8 & sandy clay, some gravel & Clay, sand, and trace gravel & -- & -- & -- & \\
\hline 96 & 7/7-9D3 & & & 275.0 & 289.0 & 83.8 & 88.1 & no data & No data & -. & .- & .- & \\
\hline 96 & 7/7-9D3 & & & 289.0 & 309.0 & 88.1 & 94.2 & cemented gravel & Gravel & cemented & -- & -- & \\
\hline 96 & 7/7-9D3 & & & 309.0 & 319.0 & 94.2 & 97.2 & no data & No data & - & .- & .- & \\
\hline 97 & 7/7-9D2 & 97 & 7/7-9D2 & 0 & 1 & 0.0 & 0.3 & surface & Sand and clay & -- & -- & -- & QTge \\
\hline 97 & 7/7-9D2 & & & 1 & 15 & 0.3 & 4.6 & sandy clay and gravel & Clay, sand, and gravel & .- & .- & .- & \\
\hline 97 & 7/7-9D2 & & & 15 & 25 & 4.6 & 7.6 & yellow sandy clay & Clay and sand (or sandstone) & .- & .- & .- & \\
\hline 97 & 7/7-9D2 & & & 25 & 90 & 7.6 & 27.4 & $\begin{array}{l}\text { yellow clay and gravel } \\
\text { y nat }\end{array}$ & $\begin{array}{l}\text { Clay and gravel } \\
\text { (a) }\end{array}$ & -- & -- & -- & \\
\hline 97 & 7/7-9D2 & & & 90 & 130 & 27.4 & 39.6 & yellow clay, soft & Clay & -- & -- & -- & \\
\hline 97 & 7/7-9D2 & & & 130 & 160 & 39.6 & 48.8 & yellow clay, sticky & Clay & -- & -- & -- & \\
\hline 97 & $7 / 7-9 \mathrm{D} 2$ & & & 160 & 185 & $\begin{array}{r}39.0 \\
48.8\end{array}$ & $\begin{array}{l}40.0 \\
56.4\end{array}$ & $\begin{array}{l}\text { cement gravel } \\
\text { comy }\end{array}$ & Gravel & cemented & -- & -- & \\
\hline 97 & 7/7-9D2 & & & 185 & 205 & 56.4 & 62.5 & yellow clay and gravel, sticky & Clay and gravel & - & -- & -- & \\
\hline 97 & 7/7-9D2 & & & 205 & 210 & 62.5 & 64.0 & $\begin{array}{l}\text { hard gravel } \\
\text { s. }\end{array}$ & Gravel & hard & .- & .- & \\
\hline 97 & 7/7-9D2 & & & 210 & 241 & 64.0 & 73.5 & yellow clay and gravel & Clay and gravel & - & -- & -- & \\
\hline 97 & 7/7-9D2 & & & 241 & 245 & 73.5 & 74.7 & $\begin{array}{l}\text { loose gravel } \\
\text { lataver }\end{array}$ & Gravel & -- & -- & -- & \\
\hline 97 & $7 / 7-9 \mathrm{D} 2$ & & & 245 & 250 & $\begin{array}{l}74.5 \\
74.7 x-10\end{array}$ & 76.2 & yellow sticky clay & Clay & -- & -- & -- & \\
\hline 97 & 7/7-9D2 & & & 250 & 255 & 76.2 & 77.7 & soft yellow sandy clay & Clay and sand (or sandstone) & -- & -- & -- & \\
\hline 97 & 7/7-9D2 & & & 255 & 263 & 77.7 & 80.2 & gray clay and gravel & $\begin{array}{l}\text { Clay and gravel } \\
\text { (a) }\end{array}$ & .- & .- & .- & \\
\hline 97 & 7/7-9D2 & & & 263 & 267 & 80.2 & 81.4 & yellow clay & Clay & -- & -- & -- & \\
\hline 97 & 7/7-9D2 & & & 267 & 275 & 81.4 & 83.8 & hard clay and gravel & Clay and gravel & hard & -- & -- & \\
\hline 97 & $7 / 7-9 \mathrm{D} 2$ & & & 275 & 280 & $\begin{array}{l}81.4 \\
83.8\end{array}$ & $\begin{array}{l}83.0 \\
85.3\end{array}$ & $\begin{array}{l}\text { loose gravel } \\
\text { lovel }\end{array}$ & Gravel & -. & -- & -- & \\
\hline 97 & 7/7-9D2 & & & 280 & 300 & 85.3 & 91.4 & hard gravel & Gravel & hard & -- & -- & \\
\hline 97 & $7 / 7-9 \mathrm{D} 2$ & & & 300 & 307 & 91.4 & $\begin{array}{l}91.4 \\
93.6\end{array}$ & light blue mud & Sand and clay & malu & -- & -- & \\
\hline 97 & 7/7-9D2 & & & 307 & 312 & 93.6 & 95.1 & hard gravel and clay & Clay and gravel & hard & -- & -- & \\
\hline 97 & 7/7-9D2 & & & 312 & 320 & 95.1 & 97.5 & $\begin{array}{l}\text { soft gray clay } \\
\text { latal }\end{array}$ & Clay & -- & -- & -- & \\
\hline 97 & 7/7-9D2 & & & 320 & 323 & 97.5 & 98.5 & hard yellow clay & Clay & hard & .- & .- & \\
\hline 98 & $7 / 7-8 \mathrm{~A} 2$ & 98 & 7/7-8A2 & 0 & 210 & 0.0 & 64.0 & no data & No data & -. & -- & -- & -- \\
\hline 98 & $7 / 7-8 \mathrm{~A} 2$ & & & 210 & 217 & 64.0 & 66.1 & cemented gravel, gravel & Gravel & cemented & -- & -- & QTge \\
\hline 98 & $7 / 7-8 \mathrm{~A} 2$ & & & 217 & 224 & 66.1 & 68.3 & no data & No data & - & -- & -- & \\
\hline 98 & $7 / 7-8 \mathrm{~A} 2$ & & & 224 & 228 & 68.3 & 69.5 & clay and gravel & Clay and gravel & -- & -- & -- & \\
\hline 98 & $7 / 7-8 \mathrm{~A} 2$ & & & 228 & 230 & 69.5 & 70.1 & no data & No data & .- & .- & .- & \\
\hline 99 & $7 / 7-9 \mathrm{D} 1$ & 99 & 7/7-9D1 & 0 & 35 & 0.0 & 10.7 & no data & No data & .- & .- & -- & QTge \\
\hline 99 & 7/7-9D1 & & & 35 & 45 & 10.7 & 13.7 & fine packed gravel and sand & Sand (or sandstone) and gravel & -- & -- & -- & \\
\hline
\end{tabular}


Appendix 3. Lithologic data. - Continued

[Site IDs and well number correspond to locations in Appendix 1, a site ID and well number is listed for each drilled interval for a particular drill hole. For convenience, the site ID and well numbers are repeated at the shallowest drilled interval for each well. leaders

\begin{tabular}{|c|c|c|c|c|c|c|c|c|c|c|c|c|c|}
\hline Site ID & Well number & $\begin{array}{l}\text { Site ID, } \\
\text { top of } \\
\text { well }\end{array}$ & $\begin{array}{l}\text { Well number, } \\
\text { top of well }\end{array}$ & $\begin{array}{l}\text { Top of } \\
\text { interval, in } \\
\text { feet }\end{array}$ & $\begin{array}{l}\text { Base of } \\
\text { interval, in } \\
\text { feet }\end{array}$ & $\begin{array}{c}\text { Top of } \\
\text { interval, in } \\
m\end{array}$ & $\begin{array}{c}\text { Base of } \\
\text { interval, in } \\
m\end{array}$ & Reported lithologic unit' & Interpreted lithologic class ${ }^{2}$ & $\begin{array}{c}\text { Reported } \\
\text { degree of } \\
\text { cementation or } \\
\text { induration } \\
\end{array}$ & $\begin{array}{c}\text { Fossils } \\
\text { reported in } \\
\text { interval }^{4}\end{array}$ & $\begin{array}{c}\text { Organic } \\
\text { matter } \\
\text { reported in } \\
\text { interval }^{5}\end{array}$ & $\begin{array}{l}\text { Reported stratigraphic top of geologic } \\
\text { unit }^{6}\end{array}$ \\
\hline 99 & 7/7-9D1 & & & 45 & 230 & 13.7 & 70.1 & no data & No data & -- & -- & -- & \\
\hline 99 & 7/7-9D1 & & & 230 & 245 & 70.1 & 74.7 & coarse gravelly sand & Sand (or sandstone) and gravel & -- & -- & -- & \\
\hline 99 & 7/7-9D1 & & & 230 & 245 & 70.1 & 74.7 & no data & No data & -- & -- & -- & \\
\hline 100 & $7 / 8-9 \mathrm{D} 1$ & 100 & $7 / 8-9 D 1$ & 0 & 200 & 0.0 & 61.0 & no data & No data & -- & -- & -- & QTge \\
\hline 101 & $7 / 9-7 \mathrm{G} 1$ & 101 & $7 / 9-7 \mathrm{G} 1$ & 0 & 331 & 0.0 & 100.9 & (cased to 40ft.) & No data & -- & -- & -- & $\mathrm{KJu}$ \\
\hline 102 & $7 / 8-11 \mathrm{G} 1$ & 102 & $7 / 8-11 \mathrm{G} 1$ & 0 & 237 & 0.0 & 72.2 & no data & No data & -- & -- & -- & QTge \\
\hline 103 & $7 / 7-8 \mathrm{G} 2$ & 103 & 7/7-8G2 & 0 & 425 & 0.0 & 129.5 & no data & No data & -- & -- & -- & QTge \\
\hline 104 & 7/9-9F1 & 104 & 7/9-9F1 & 0 & 3 & 0.0 & 0.9 & soil & Sand and clay & -- & .- & -- & Sige \\
\hline 104 & 7/9-9F1 & & & 3 & 5 & 0.9 & 1.5 & clay & Clay & -- & -- & -- & \\
\hline 104 & 7/9-9F1 & & & 5 & 49 & 1.5 & 14.9 & dry sand & Sand & -- & -- & -- & \\
\hline 104 & 7/9-9F1 & & & 49 & 110 & 14.9 & 33.5 & wet sand & Sand & -- & -- & -- & \\
\hline 104 & 7/9-9F1 & & & 110 & 115 & 33.5 & 35.1 & white clay & Clay & -- & -- & -- & \\
\hline 104 & 7/9-9F1 & & & 115 & 180 & 35.1 & 54.9 & sand, soft & Sand & -- & -- & -- & \\
\hline 104 & 7/9-9F1 & & & 180 & 186 & 54.9 & 56.7 & yellow clay & Clay & -- & -- & -- & \\
\hline 104 & 7/9-9F1 & & & 186 & 220 & 56.7 & 67.1 & blue sandstone & Sandstone & cemented & -- & -- & QTm \\
\hline 104 & $7 / 9-9 F 1$ & & & 220 & 226 & 67.1 & 68.9 & blue sand, soft (water) & Sand & -- & -- & -- & \\
\hline 105 & $7 / 8-12 \mathrm{H} 1$ & 105 & 7/8-12H1 & 0 & 160 & 0.0 & 48.8 & no data & No data & -- & -- & -- & Tsv \\
\hline 105 & $7 / 8-12 \mathrm{H} 1$ & & & 160 & 184 & 48.8 & 56.1 & perforated intervals & No data & -- & -- & -- & \\
\hline 105 & $7 / 8-12 \mathrm{H} 1$ & & & 184 & 208 & 56.1 & 63.4 & no data & No data & -- & -- & -- & \\
\hline 105 & $7 / 8-12 \mathrm{H} 1$ & & & 208 & 255 & 63.4 & 77.7 & perforated intervals & No data & -- & -- & -- & \\
\hline 106 & $7 / 8-12 \mathrm{E} 2$ & 106 & 7/8-12E2 & 0 & 80 & 0.0 & 24.4 & no data & No data & -- & -- & -- & Tsv \\
\hline 106 & 7/8-12E2 & & & 80 & 298 & 24.4 & 90.8 & perforated intervals & No data & -- & -- & -- & \\
\hline 106 & 7/8-12E2 & & & 298 & 300 & 90.8 & 91.4 & no data & No data & -- & -- & -- & \\
\hline 107 & 7/9-10E1 & 107 & 7/9-10E1 & 160 & 100 & 48.8 & 30.5 & sandstone, sand & Sand (or sandstone) and gravel & cemented & -- & -- & QTm \\
\hline 108 & $7 / 7-8 \mathrm{M} 2$ & 108 & 7/7-8M2 & 0 & 211 & 0.0 & 64.3 & no data & No data & -- & -- & -- & -- \\
\hline 109 & $7 / 7-7 F 2$ & 109 & $7 / 7-7 F 2$ & 0 & 4 & 0.0 & 1.2 & topsoil & Sand and clay & -- & -- & -- & Tsv \\
\hline 109 & 7/7-7F2 & & & 4 & 36 & 1.2 & 11.0 & black volcanic rock & Basalt & hard & -- & -- & \\
\hline 109 & $7 / 7-7 F 2$ & & & 36 & 42 & 11.0 & 12.8 & boulders & Gravel & -- & -- & -- & \\
\hline 109 & $7 / 7-7 F 2$ & & & 42 & 130 & 12.8 & 39.6 & black volcanic rock & Basalt & hard & -. & -- & \\
\hline 109 & $7 / 7-7 F 2$ & & & 130 & 155 & 39.6 & 47.2 & boulders with red volcanic ash & Ash or tuff & -- & -- & -- & \\
\hline 109 & 7/7-7F2 & & & 155 & 216 & 47.2 & 65.8 & black volcanic rock & Basalt & hard & -- & -- & \\
\hline 109 & 7/7-7F2 & & & 216 & 221 & 65.8 & 67.4 & red volcanic ash with boulders & Ash or tuff & -- & -- & -- & \\
\hline 109 & $7 / 7-7 F 2$ & & & 221 & 234 & 67.4 & 71.3 & red volcanic ash & Ash or tuff & -- & -- & -- & \\
\hline 109 & $7 / 7-7 F 2$ & & & 234 & 283 & 71.3 & 86.3 & black volcanic rock & Basalt & hard & -- & -- & \\
\hline 109 & 7/7-7F2 & & & 283 & 314 & 86.3 & 95.7 & red volcanic ash & Ash or tuff & -- & -- & -- & \\
\hline 109 & $7 / 7-7 F 2$ & & & 314 & 320 & 95.7 & 97.5 & black volcanic rock & Basalt & hard & -- & -- & \\
\hline 110 & $7 / 9-8 \mathrm{~J} 1$ & 110 & 7/9-8J1 & 0 & 16 & 0.0 & 4.9 & soil & Sand and clay & -- & -- & -- & \\
\hline 110 & $7 / 9-8 J 1$ & & & 16 & 55 & 4.9 & 16.8 & yellow sand & Sand & -- & -- & -- & \\
\hline 110 & 7/9-8J1 & & & 55 & 105 & 16.8 & 32.0 & yellow-red sticky clay & Clay & -- & -- & -- & \\
\hline 110 & 7/9-8J1 & & & 105 & 132 & 32.0 & 40.2 & water, sand & Sand & -- & -- & -- & \\
\hline 110 & $7 / 9-8 \mathrm{~J} 1$ & & & 132 & 160 & 40.2 & 48.8 & yellow sticky clay & Clay & -- & -- & -- & \\
\hline 110 & 7/9-8J1 & & & 160 & 168 & 48.8 & 51.2 & yellow and blue sandstone & Sandstone & cemented & -- & -- & QTm \\
\hline 110 & 7/9-8J1 & & & 168 & 205 & 51.2 & 62.5 & blue sandstone & Sandstone & cemented & -- & -- & \\
\hline 110 & $7 / 9-8 \mathrm{~J} 1$ & & & 205 & 240 & 62.5 & 73.2 & water, sandstone & Sandstone & cemented & -- & -- & \\
\hline 110 & $7 / 9-8 J 1$ & & & 240 & 245 & 73.2 & 74.7 & sticky blue sandstone & Sandstone & cemented & -- & -- & \\
\hline 110 & $7 / 9-8 \mathrm{~J} 1$ & & & 245 & 250 & 74.7 & 76.2 & blue sandstone; washed gravel & Sand (or sandstone) and gravel & cemented & -- & -- & \\
\hline 111 & $7 / 8-11 \mathrm{~K} 1$ & 111 & 7/8-11K1 & 0 & 238 & 0.0 & 72.5 & no data & No data & -- & -- & -- & QTge \\
\hline 111 & $7 / 8-11 \mathrm{~K} 1$ & & & 238 & 254 & 72.5 & 77.4 & sandstone & Sandstone & cemented & -- & -- & \\
\hline 112 & 7/8-10L1 & 112 & 7/8-10L1 & 0 & 100 & 0.0 & 30.5 & $\begin{array}{l}\text { no data } \\
\text { "light gravel" and some coarse }\end{array}$ & No data & -- & -- & -- & QTge \\
\hline 112 & 7/8-10L1 & & & 100 & 114 & 30.5 & 34.7 & sand & Sand (or sandstone) and gravel & -- & -- & -- & \\
\hline 112 & 7/8-10L1 & & & 114 & 212 & 34.7 & 64.6 & no data & No data & -- & -- & -- & \\
\hline 113 & $7 / 9-8 \mathrm{~K} 1$ & 113 & 7/9-8K1 & 0 & 3 & 0.0 & 0.9 & soil & Sand and clay & -- & -- & -- & \\
\hline 113 & $7 / 9-8 K 1$ & & & 3 & 60 & 0.9 & 18.3 & yellow clay & Clay & -- & -- & -- & \\
\hline 113 & 7/9-8K1 & & & 60 & 140 & 18.3 & 42.7 & yellow sand and clay & Sand and clay & -- & -- & -- & \\
\hline 113 & $7 / 9-8 \mathrm{~K} 1$ & & & 140 & 164 & 42.7 & 50.0 & clay, blue hard & Clay & hard & -. & -- & \\
\hline 113 & 7/9-8K1 & & & 164 & 200 & 50.0 & 61.0 & blue clay & Clay & -- & -- & -- & \\
\hline 113 & 7/9-8K1 & & & 200 & 230 & 61.0 & 70.1 & blue sand & Sand & -- & -- & -- & \\
\hline 113 & 7/9-8K1 & & & 230 & 240 & 70.1 & 73.2 & yellow sand & Sand & -. & -- & -.- & \\
\hline 113 & 7/9-8K1 & & & 240 & 260 & 73.2 & 79.2 & sandstone & Sandstone & cemented & -- & -- & \\
\hline 113 & 7/9-8K1 & & & 260 & 276 & 79.2 & 84.1 & sandstone and clay & Sandstone & cemented & -- & -- & \\
\hline
\end{tabular}


Appendix 3. Lithologic data. - Continued

[Site IDs and well number correspond to locations in Appendix 1, a site ID and well number is listed for each drilled interval for a particular drill hole. For convenience, the site ID and well numbers are repeated at the shallowest drilled interval for each well. leaders

\begin{tabular}{|c|c|c|c|c|c|c|c|c|c|c|c|c|c|}
\hline Site ID & Well number & $\begin{array}{l}\text { Site ID, } \\
\text { top of } \\
\text { well }\end{array}$ & $\begin{array}{l}\text { Well number, } \\
\text { top of well }\end{array}$ & $\begin{array}{l}\text { Top of } \\
\text { interval, in } \\
\text { feet }\end{array}$ & $\begin{array}{l}\text { Base of } \\
\text { interval, in } \\
\text { feet }\end{array}$ & $\begin{array}{c}\text { Top of } \\
\text { interval, in } \\
\mathrm{m}\end{array}$ & $\begin{array}{l}\text { Base of } \\
\text { interval, in } \\
\mathrm{m}\end{array}$ & Reported lithologic unit' & Interpreted lithologic class ${ }^{2}$ & $\begin{array}{c}\text { Reported } \\
\text { degree of } \\
\text { cementation or } \\
\text { induration }^{3} \\
\end{array}$ & $\begin{array}{c}\text { Fossils } \\
\text { reported in } \\
\text { interval }^{4}\end{array}$ & $\begin{array}{c}\text { Organic } \\
\text { matter } \\
\text { reported in } \\
\text { interval }^{5}\end{array}$ & $\begin{array}{l}\text { Reported stratigraphic top of geologic } \\
\text { unit }^{6}\end{array}$ \\
\hline 113 & 7/9-8K1 & & & 276 & 350 & 84.1 & 106.7 & sandstone & Sandstone & cemented & -- & -- & QTm \\
\hline 114 & 7/8-12K1 & 114 & 7/8-12K1 & 0 & 273 & 0.0 & 83.2 & $\begin{array}{l}\text { no data } \\
\text { sand, clay, and gravel; sand and }\end{array}$ & No data & -- & -- & -- & Tsv \\
\hline 114 & 7/8-12K1 & & & 273 & 347 & 83.2 & 105.8 & $\begin{array}{l}\text { rock } \\
\text { sandy clay and gravel; clay and }\end{array}$ & Sand (or sandstone) and gravel & -- & -- & -- & \\
\hline 115 & 7/8-12Q2 & 115 & 7/8-12Q2 & 125 & 95 & 38.1 & 29.0 & gravel & Clay, sand, and gravel & -- & -- & -- & Tsv \\
\hline 116 & $7 / 7-8 \mathrm{P} 2$ & 116 & $7 / 7-8 \mathrm{P} 2$ & 233 & 250 & 71.0 & 76.2 & volcanic ash & Ash or tuff & -- & -- & -- & Tsv \\
\hline 117 & 7/7-8L1 & 117 & 7/7-8L1 & 0 & 25 & 0.0 & 7.6 & yellow clay and boulders & Clay and gravel & -- & -- & -- & QTge \\
\hline 117 & 7/7-8L1 & & & 25 & 75 & 7.6 & 22.9 & yellow clay and gravel & Clay and gravel & -- & -- & -- & \\
\hline 117 & 7/7-8L1 & & & 75 & 80 & 22.9 & 24.4 & yellow clay and boulders & Clay and gravel & -- & -- & -- & \\
\hline 117 & 7/7-8L1 & & & 80 & 172 & 24.4 & 52.4 & yellow clay and gravel & Clay and gravel & -- & -- & -- & \\
\hline 117 & 7/7-8L1 & & & 172 & 177 & 52.4 & 53.9 & yellow clay and boulders & Clay and gravel & -- & -- & -- & \\
\hline 117 & 7/7-8L1 & & & 177 & 178 & 53.9 & 54.3 & yellow clay and gravel & Clay and gravel & -- & -- & -- & \\
\hline 117 & 7/7-8L1 & & & 178 & 184 & 54.3 & 56.1 & hard boulders & Gravel & hard & -- & -- & \\
\hline 117 & 7/7-8L1 & & & 184 & 208 & 56.1 & 63.4 & rock & Ash or tuff & hard & -- & -- & Tsv \\
\hline 117 & 7/7-8L1 & & & 208 & 223 & 63.4 & 68.0 & red volcanic ash & Ash or tuff & -- & -- & -- & \\
\hline 117 & 7/7-8L1 & & & 223 & 278 & 68.0 & 84.7 & hard volcanic rock & Ash or tuff & hard & -- & -- & \\
\hline 118 & 7/8-9R1 & 118 & 7/8-9R1 & 0 & 2 & 0.0 & 0.6 & topsoil & Sand and clay & -- & -- & -- & QTge \\
\hline 118 & 7/8-9R1 & & & 2 & 3 & 0.6 & 0.9 & clay, yellow, and gravel & Clay and gravel & -- & -- & -- & \\
\hline 118 & 7/8-9R1 & & & 3 & 6 & 0.9 & 1.8 & hardpan & Clay and gravel & hard & -- & -- & \\
\hline 118 & 7/8-9R1 & & & 6 & 20 & 1.8 & 6.1 & clay sandy brown & Clay and sand (or sandstone) & -- & -- & -- & \\
\hline 118 & 7/8-9R1 & & & 20 & 28 & 6.1 & 8.5 & clay, blue, and gravel & Clay and gravel & -- & -- & -- & \\
\hline 118 & 7/8-9R1 & & & 28 & 32 & 8.5 & 9.8 & sand and gravel & Sand (or sandstone) and gravel & -- & -- & -- & \\
\hline 118 & 7/8-9R1 & & & 32 & 35 & 9.8 & 10.7 & clay, gray & Clay & -- & -- & -- & \\
\hline 118 & 7/8-9R1 & & & 35 & 50 & 10.7 & 15.2 & sand and gravel & Sand (or sandstone) and gravel & -- & -- & -- & \\
\hline 118 & 7/8-9R1 & & & 50 & 118 & 15.2 & 36.0 & clay, yellow, and gravel & Clay and gravel & -- & -- & -- & \\
\hline 118 & 7/8-9R1 & & & 118 & 136 & 36.0 & 41.5 & small loose boulders & Gravel & -- & -- & -- & \\
\hline 118 & 7/8-9R1 & & & 136 & 139 & 41.5 & 42.4 & gravel & Gravel & -- & -- & -- & \\
\hline 118 & 7/8-9R1 & & & 139 & 144 & 42.4 & 43.9 & clay, creamy & Clay & -- & -- & -- & \\
\hline 118 & 7/8-9R1 & & & 144 & 162 & 43.9 & 49.4 & clay, brown & Clay & -- & -- & -- & \\
\hline 118 & $7 / 8-9 \mathrm{R} 1$ & & & 162 & 165 & 49.4 & 50.3 & gravel & Gravel & -- & -- & -- & \\
\hline 118 & 7/8-9R1 & & & 165 & 180 & 50.3 & 54.9 & clay, brown & Clay & -- & -- & -- & \\
\hline 118 & 7/8-9R1 & & & 180 & 187 & 54.9 & 57.0 & gravel & Gravel & -- & -- & -- & \\
\hline 119 & 7/8-14A1 & 119 & 7/8-14A1 & 0 & 3 & 0.0 & 0.9 & adobe & Sand and clay & -- & -- & -- & Qyal and QTge \\
\hline 119 & 7/8-14A1 & & & 3 & 18 & 0.9 & 5.5 & ash, black & Ash or tuff & -- & -- & -- & \\
\hline 119 & 7/8-14A1 & & & 18 & 25 & 5.5 & 7.6 & ash, yellow & Ash or tuff & -- & -- & -- & \\
\hline 119 & 7/8-14A1 & & & 25 & 137 & 7.6 & 41.8 & ash, brown & Ash or tuff & -- & -- & -- & \\
\hline 119 & 7/8-14A1 & & & 137 & 153 & 41.8 & 46.6 & clay, blue & Clay & -- & -- & -- & \\
\hline 119 & 7/8-14A1 & & & 153 & 185 & 46.6 & 56.4 & clay, yellow & Clay & -- & -- & -- & \\
\hline 119 & 7/8-14A1 & & & 185 & 270 & 56.4 & 82.3 & clay, blue & Clay & -- & -- & -- & \\
\hline 119 & 7/8-14A1 & & & 270 & 350 & 82.3 & 106.7 & clay, blue and gravel & Clay and gravel & -- & -- & -- & \\
\hline 119 & 7/8-14A1 & & & 350 & 360 & 106.7 & 109.7 & clay, blue & Clay & -- & -- & -- & \\
\hline 119 & 7/8-14A1 & & & 360 & 470 & 109.7 & 143.3 & rock & Ash or tuff & hard & -- & -- & Tsv \\
\hline 119 & 7/8-14A1 & & & 470 & 489 & 143.3 & 149.0 & clay, blue & Clay & -- & -- & -- & \\
\hline 119 & 7/8-14A1 & & & 489 & 548 & 149.0 & 167.0 & clay, blue, and rock & Clay and gravel & -- & -- & -- & \\
\hline 119 & 7/8-14A1 & & & 548 & 621 & 167.0 & 189.3 & rock & Ash or tuff & hard & -- & -- & \\
\hline 119 & 7/8-14A1 & & & 621 & 640 & 189.3 & 195.1 & clay & Clay & -- & -- & -- & \\
\hline 119 & 7/8-14A1 & & & 640 & 671 & 195.1 & 204.5 & rock & Ash or tuff & hard & -- & -- & \\
\hline 119 & 7/8-14A1 & & & 671 & 899 & 204.5 & 274.0 & clay & Clay & -- & -- & -- & \\
\hline 119 & 7/8-14A1 & & & 899 & 901 & 274.0 & 274.6 & rock & Ash or tuff & hard & -- & -- & \\
\hline 120 & 7/7-16A1 & 120 & 7/7-16A1 & 0 & 200 & 0.0 & 61.0 & no record & No data & -- & -- & -- & QTge \\
\hline 120 & 7/7-16A1 & & & 200 & 207 & 61.0 & 63.1 & loose gravel boulders & Gravel & -- & -- & -- & \\
\hline 120 & 7/7-16A1 & & & 207 & 230 & 63.1 & 70.1 & yellow clay and gravel & Clay and gravel & -. & -- & -- & \\
\hline 120 & 7/7-16A1 & & & 230 & 235 & 70.1 & 71.6 & hard gravel and clay & Clay and gravel & hard & -- & -- & \\
\hline 120 & 7/7-16A1 & & & 235 & 243 & 71.6 & 74.1 & soft gravel and yellow clay & Clay and gravel & - & -- & -- & \\
\hline 120 & 7/7-16A1 & & & 243 & 249 & 74.1 & 75.9 & hard yellow clay and gravel & Clay and gravel & hard & -- & -- & \\
\hline 120 & 7/7-16A1 & & & 249 & 253 & 75.9 & 77.1 & soft, sandy yellow clay & Clay and sand (or sandstone) & -- & -- & -- & \\
\hline 120 & 7/7-16A1 & & & 253 & 255 & 77.1 & 77.7 & hard yellow clay & Clay & hard & -- & -- & \\
\hline 120 & 7/7-16A1 & & & 255 & 262 & 77.7 & 79.9 & soft, sandy yellow clay & Clay and sand (or sandstone) & -- & -- & -- & \\
\hline 120 & 7/7-16A1 & & & 262 & 263 & 79.9 & 80.2 & hard yellow clay & Clay & hard & -- & -- & \\
\hline 121 & 7/7-15C1 & 121 & $7 / 7-15 \mathrm{C} 1$ & 0 & 1 & 0.0 & 0.3 & soil & Sand and clay & -- & -- & -- & Qyal \\
\hline
\end{tabular}


Appendix 3. Lithologic data. - Continued

[Site IDs and well number correspond to locations in Appendix 1, a site ID and well number is listed for each drilled interval for a particular drill hole. For convenience, the site ID and well numbers are repeated at the shallowest drilled interval for each well. leaderst.

\begin{tabular}{|c|c|c|c|c|c|c|c|c|c|c|c|c|c|}
\hline Site ID & Well number & $\begin{array}{l}\text { Site ID, } \\
\text { top of } \\
\text { well }\end{array}$ & $\begin{array}{l}\text { Well number, } \\
\text { top of well }\end{array}$ & $\begin{array}{c}\text { Top of } \\
\text { interval, in } \\
\text { feet }\end{array}$ & $\begin{array}{l}\text { Base of } \\
\text { interval, in } \\
\text { feet }\end{array}$ & $\begin{array}{c}\text { Top of } \\
\text { interval, in } \\
\mathrm{m}\end{array}$ & $\begin{array}{c}\text { Base of } \\
\text { interval, in } \\
\mathrm{m}\end{array}$ & Reported lithologic unit' & Interpreted lithologic class ${ }^{2}$ & $\begin{array}{c}\text { Reported } \\
\text { degree of } \\
\text { cementation or } \\
\text { induration }^{3} \\
\end{array}$ & $\begin{array}{c}\text { Fossils } \\
\text { reported in } \\
\text { interval }^{4}\end{array}$ & $\begin{array}{c}\text { Organic } \\
\text { matter } \\
\text { reported in } \\
\text { interval }^{5}\end{array}$ & $\begin{array}{l}\text { Reported stratigraphic top of geologic } \\
\text { unit }^{6}\end{array}$ \\
\hline 121 & $7 / 7-15 \mathrm{C} 1$ & & & 1 & 20 & 0.3 & 6.1 & boulders & Gravel & -- & -- & -- & \\
\hline 121 & $7 / 7-15 \mathrm{C} 1$ & & & 20 & 26 & 6.1 & 7.9 & clay yellow & Clay & -- & -- & -- & QTge \\
\hline 121 & $7 / 7-15 \mathrm{C} 1$ & & & 26 & 48 & 7.9 & 14.6 & yellow clay, hard, sandy & Clay and sand (or sandstone) & hard & -- & -- & \\
\hline 121 & $7 / 7-15 \mathrm{C} 1$ & & & 48 & 54 & 14.6 & 16.5 & boulders & Gravel & -- & -- & -- & \\
\hline 121 & $7 / 7-15 \mathrm{C} 1$ & & & 54 & 108 & 16.5 & 32.9 & clay, yellow; water at 55 feet et & Clay & -- & -- & -- & \\
\hline 121 & $7 / 7-15 \mathrm{C} 1$ & & & 108 & 119 & 32.9 & 36.3 & yellow clay and gravel & Clay and gravel & -- & -- & -- & \\
\hline 121 & $7 / 7-15 \mathrm{C} 1$ & & & 119 & 139 & 36.3 & 42.4 & yellow clay and gravel & Clay and gravel & -- & -- & -- & \\
\hline 121 & $7 / 7-15 \mathrm{C} 1$ & & & 139 & 160 & 42.4 & 48.8 & boulders & Gravel & -- & -- & -- & \\
\hline 121 & $7 / 7-15 \mathrm{C} 1$ & & & 160 & 179 & 48.8 & 54.6 & yellow clay and boulders & Clay and gravel & -- & -- & -- & \\
\hline 121 & $7 / 7-15 \mathrm{C} 1$ & & & 179 & 189 & 54.6 & 57.6 & boulders, yellow clay, and gravel & Clay and gravel & -- & -- & -- & \\
\hline 121 & $7 / 7-15 \mathrm{C} 1$ & & & 189 & 211 & 57.6 & 64.3 & clay and boulders & Clay and gravel & -- & -- & -- & \\
\hline 121 & $7 / 7-15 \mathrm{C} 1$ & & & 211 & 258 & 64.3 & 78.6 & boulders and rock & Gravel & hard & -- & -- & \\
\hline 121 & $7 / 7-15 \mathrm{C} 1$ & & & 258 & 277 & 78.6 & 84.4 & yellow clay and boulders & Clay and gravel & -- & -- & -- & \\
\hline 121 & $7 / 7-15 \mathrm{C} 1$ & & & 277 & 289 & 84.4 & 88.1 & yellow clay and gravel & Clay and gravel & -- & -- & -- & \\
\hline 121 & $7 / 7-15 \mathrm{C} 1$ & & & 289 & 293 & 88.1 & 89.3 & boulders & Gravel & -- & -- & -- & \\
\hline 121 & $7 / 7-15 \mathrm{C} 1$ & & & 293 & 309 & 89.3 & 94.2 & yellow clay and boulders & Clay and gravel & -- & -- & -- & \\
\hline 121 & $7 / 7-15 \mathrm{C} 1$ & & & 309 & 312 & 94.2 & 95.1 & red clay and gravel & Clay and gravel & -- & -- & -- & \\
\hline 121 & $7 / 7-15 \mathrm{C} 1$ & & & 312 & 322 & 95.1 & 98.1 & $\begin{array}{l}\text { red clay } \\
\text { white sandy clay and gravel; well } \\
\text { flowed } 20 \text { gallons per hour at } 342\end{array}$ & Clay & -- & -- & -- & \\
\hline 121 & $7 / 7-15 \mathrm{C} 1$ & & & 322 & 342 & 98.1 & 104.2 & feet & Clay, sand, and gravel & -- & -- & -- & \\
\hline 121 & $7 / 7-15 \mathrm{C} 1$ & & & 342 & 345 & 104.2 & 105.2 & white sandy clay & Clay and sand (or sandstone) & -- & -- & -- & \\
\hline 121 & $7 / 7-15 \mathrm{C} 1$ & & & 345 & 363 & 105.2 & 110.6 & gravel and sand & Sand (or sandstone) and gravel & -- & -- & -- & \\
\hline 121 & $7 / 7-15 \mathrm{C} 1$ & & & 363 & 397 & 110.6 & 121.0 & sandy clay and gravel & Clay, sand, and gravel & -- & -- & -- & \\
\hline 122 & 7/7-17A1 & 122 & 7/7-17A1 & 0 & 3 & 0.0 & 0.9 & $\begin{array}{l}\text { topsoil } \\
\text { clay, yellow, and gravel; small }\end{array}$ & Sand and clay & -- & -- & -- & Qyal and Qoal (?) \\
\hline 122 & 7/7-17A1 & & & 3 & 44 & 0.9 & 13.4 & boulders & Clay and gravel & -- & -- & -- & \\
\hline 122 & 7/7-17A1 & & & 44 & 48 & 13.4 & 14.6 & gravel & Gravel & -- & -- & -- & \\
\hline 122 & 7/7-17A1 & & & 48 & 76 & 14.6 & 23.2 & clay, yellow, and gravel & Clay and gravel & -- & -- & -- & \\
\hline 122 & $7 / 7-17 \mathrm{~A} 1$ & & & 76 & 85 & 23.2 & 25.9 & boulders and gravel & Gravel & -- & -- & -- & \\
\hline 122 & 7/7-17A1 & & & 85 & 90 & 25.9 & 27.4 & rock & Ash or tuff & hard & -- & -- & Tsv \\
\hline 122 & $7 / 7-17 \mathrm{~A} 1$ & & & 90 & 96 & 27.4 & 29.3 & rock, basalt & Basalt & -- & -- & -- & \\
\hline 122 & 7/7-17A1 & & & 96 & 110 & 29.3 & 33.5 & ash, red, volcanic & Ash or tuff & -- & -- & -- & \\
\hline 122 & 7/7-17A1 & & & 110 & 145 & 33.5 & 44.2 & ash, red, volcanic, and gravel & Ash or tuff & -- & -- & -- & \\
\hline 122 & 7/7-17A1 & & & 145 & 165 & 44.2 & 50.3 & clay, yellow, and rock & Clay and gravel & -- & -- & -- & \\
\hline 122 & 7/7-17A1 & & & 165 & 205 & 50.3 & 62.5 & ash, red, volcanic, and boulders & Ash or tuff & -- & -- & -- & \\
\hline 122 & $7 / 7-17 \mathrm{~A} 1$ & & & 205 & 213 & 62.5 & 64.9 & rock, black & Basalt & hard & -- & -- & \\
\hline 122 & 7/7-17A1 & & & 213 & 258 & 64.9 & 78.6 & ash, red, volcanic, and boulders & Ash or tuff & -- & -- & -- & \\
\hline 122 & 7/7-17A1 & & & 258 & 263 & 78.6 & 80.2 & rock, black & Basalt & hard & -- & -- & \\
\hline 122 & 7/7-17A1 & & & 263 & 268 & 80.2 & 81.7 & ash, red, volcanic, and boulders & Ash or tuff & -- & -- & -- & \\
\hline 122 & 7/7-17A1 & & & 268 & 270 & 81.7 & 82.3 & rock, black & Basalt & hard & -- & -- & \\
\hline 122 & 7/7-17A1 & & & 270 & 276 & 82.3 & 84.1 & rock, blue & Sandstone & hard & -- & -- & \\
\hline 122 & 7/7-17A1 & & & 276 & 280 & 84.1 & 85.3 & ash, red, volcanic, and boulders & Ash or tuff & -- & -- & -- & \\
\hline 122 & $7 / 7-17 \mathrm{~A} 1$ & & & 280 & 307 & 85.3 & 93.6 & rock, red, volcanic & Ash or tuff & hard & -- & -- & \\
\hline 122 & 7/7-17A1 & & & 307 & 364 & 93.6 & 110.9 & rock & Ash or tuff & hard & -- & -- & \\
\hline 122 & 7/7-17A1 & & & 364 & 392 & 110.9 & 119.5 & clay, yellow, and gravel & Clay and gravel & -- & -- & -- & \\
\hline 122 & 7/7-17A1 & & & 392 & 419 & 119.5 & 127.7 & gravel, blue cement, and boulders & Gravel & cemented & -- & -- & \\
\hline 122 & 7/7-17A1 & & & 419 & 425 & 127.7 & 129.5 & sandstone, blue & Sandstone & cemented & -- & -- & QTm \\
\hline 122 & 7/7-17A1 & & & 425 & 495 & 129.5 & 150.9 & sandstone, blue, and boulders & Sand (or sandstone) and gravel & cemented & -- & -- & \\
\hline 122 & 7/7-17A1 & & & 495 & 505 & 150.9 & 153.9 & sandstone, blue & Sandstone & cemented & -- & -- & \\
\hline 122 & 7/7-17A1 & & & 505 & 522 & 153.9 & 159.1 & clay, blue, and gravel & Clay and gravel & -- & -- & -- & \\
\hline 122 & 7/7-17A1 & & & 522 & 523 & 159.1 & 159.4 & rock, black & Basalt & hard & -- & -- & Tsv and QTm \\
\hline 122 & 7/7-17A1 & & & 523 & 531 & 159.4 & 161.8 & rock & Ash or tuff & hard & -- & -- & \\
\hline 122 & 7/7-17A1 & & & 531 & 538 & 161.8 & 164.0 & clay, blue, and gravel & Clay and gravel & -- & -- & -. & \\
\hline 122 & 7/7-17A1 & & & 538 & 560 & 164.0 & 170.7 & rock, blue & Sandstone & hard & -- & -- & \\
\hline 122 & 7/7-17A1 & & & 560 & 565 & 170.7 & 172.2 & rock, red, volcanic & Ash or tuff & hard & -- & -- & \\
\hline 122 & 7/7-17A1 & & & 565 & 640 & 172.2 & 195.1 & rock, blue & Sandstone & hard & -- & -- & \\
\hline 122 & $7 / 7-17 \mathrm{~A} 1$ & & & 640 & 656 & 195.1 & 199.9 & rock, red, volcanic & Ash or tuff & hard & -- & -- & \\
\hline 122 & 7/7-17A1 & & & 656 & 669 & 199.9 & 203.9 & rock, black & Basalt & hard & -- & -- & \\
\hline
\end{tabular}


Appendix 3. Lithologic data. - Continued

ISite IDs and well number correspond to locations in Appendix 1, a site ID and well number is listed for each drilled interval for a particular drill hole. For convenience, the site ID and well numbers are repeated at the shallowest drilled

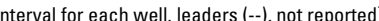

\begin{tabular}{|c|c|c|c|c|c|c|c|c|c|c|c|c|c|}
\hline Site ID & Well number & $\begin{array}{l}\text { Site ID, } \\
\text { top of } \\
\text { well }\end{array}$ & $\begin{array}{l}\text { Well number, } \\
\text { top of well }\end{array}$ & $\begin{array}{l}\text { Top of } \\
\text { interval, in } \\
\text { feet }\end{array}$ & $\begin{array}{l}\text { Base of } \\
\text { interval, in } \\
\text { feet }\end{array}$ & $\begin{array}{c}\text { Top of } \\
\text { interval, in } \\
\mathrm{m}\end{array}$ & $\begin{array}{c}\text { Base of } \\
\text { interval, in } \\
\mathrm{m}\end{array}$ & Reported lithologic unit' & Interpreted lithologic class ${ }^{2}$ & $\begin{array}{c}\text { Reported } \\
\text { degree of } \\
\text { cementation or } \\
\text { induration }^{3} \\
\end{array}$ & $\begin{array}{c}\text { Fossils } \\
\text { reported in } \\
\text { interval }^{4}\end{array}$ & $\begin{array}{c}\text { Organic } \\
\text { matter } \\
\text { reported in } \\
\text { interval }^{5}\end{array}$ & $\begin{array}{l}\text { Reported stratigraphic top of geologic } \\
\text { unit }^{6}\end{array}$ \\
\hline & & & & & & & & $\begin{array}{l}\text { rock, blue, and clamshells (much } \\
\text { of blue rock reported is probably }\end{array}$ & & & & & \\
\hline 122 & 7/7-17A1 & & & 669 & 675 & 203.9 & 205.7 & sandstone) & Sandstone & cemented & shells & shells & \\
\hline 122 & 7/7-17A1 & & & 675 & 747 & 205.7 & 227.7 & rock, blue & Sandstone & hard & -- & -- & \\
\hline 122 & 7/7-17A1 & & & 747 & 765 & 227.7 & 233.2 & rock, blue, little red & Sandstone & hard & -- & -- & \\
\hline 122 & 7/7-17A1 & & & 765 & 768 & 233.2 & 234.1 & rock, blue & Sandstone & hard & -- & -- & \\
\hline 122 & 7/7-17A1 & & & 768 & 783 & 234.1 & 238.7 & rock, blue, volcanic & Ash or tuff & hard & -- & -- & \\
\hline 122 & 7/7-17A1 & & & 783 & 807 & 238.7 & 246.0 & rock, red, hard & Ash or tuff & hard & -- & -- & \\
\hline 122 & 7/7-17A1 & & & 807 & 841 & 246.0 & 256.3 & rock, blue & Sandstone & hard & -- & -- & \\
\hline 122 & 7/7-17A1 & & & 841 & 846 & 256.3 & 257.9 & blue and red & Sandstone & -- & -- & -- & \\
\hline 123 & 7/9-15E2 & 123 & 7/9-15E2 & 0 & 215 & 0.0 & 65.5 & no data & No data & -- & -- & -- & -- \\
\hline 123 & 7/9-15E2 & & & 215 & 220 & 65.5 & 67.1 & sand and shells & Sand & -- & shells & -- & QTm \\
\hline 124 & 7/8-14D1 & 124 & 7/8-14D1 & 0 & 4 & 0.0 & 1.2 & soil & Sand and clay & -- & -- & -- & Qyal and QTge \\
\hline 124 & 7/8-14D1 & & & 4 & 17 & 1.2 & 5.2 & clay & Clay & -- & -- & -- & \\
\hline 124 & 7/8-14D1 & & & 17 & 20 & 5.2 & 6.1 & gravel & Gravel & -- & -- & -- & \\
\hline 124 & 7/8-14D1 & & & 20 & 85 & 6.1 & 25.9 & clay, yellow & Clay & -- & -- & -- & \\
\hline 124 & 7/8-14D1 & & & 85 & 95 & 25.9 & 29.0 & gravel, cemented & Gravel & cemented & -- & -- & \\
\hline 124 & 7/8-14D1 & & & 95 & 146 & 29.0 & 44.5 & clay, yellow & Clay & -- & -- & -- & \\
\hline 124 & 7/8-14D1 & & & 146 & 162 & 44.5 & 49.4 & gravel & Gravel & -- & -- & -- & \\
\hline 124 & 7/8-14D1 & & & 162 & 188 & 49.4 & 57.3 & clay and gravel & Clay and gravel & -- & -- & -- & \\
\hline 124 & 7/8-14D1 & & & 188 & 200 & 57.3 & 61.0 & gravel, free & Gravel & -- & -- & -- & \\
\hline 124 & $7 / 8-14 \mathrm{D} 1$ & & & 200 & 250 & 61.0 & 76.2 & clay and gravel mix & Clay and gravel & -- & -- & -- & \\
\hline 124 & 7/8-14D1 & & & 250 & 265 & 76.2 & 80.8 & clay, sandy & Clay and sand (or sandstone) & -- & -- & -- & \\
\hline 124 & 7/8-14D1 & & & 265 & 285 & 80.8 & 86.9 & gravel, cemented, hard & Gravel & cemented & -- & -- & \\
\hline 124 & 7/8-14D1 & & & 285 & 300 & 86.9 & 91.4 & $\begin{array}{l}\text { clay and gravel, hard } \\
\text { cols }\end{array}$ & Clay and gravel & hard & -- & -- & \\
\hline 125 & 7/8-14E1 & 125 & 7/8-14E1 & 0 & 761 & 0.0 & 232.0 & no data & No data & -- & -- & -- & QTge \\
\hline 126 & 7/9-18J1 & 126 & 7/9-18J1 & 0 & 650 & 0.0 & 198.1 & no data & No data & -- & -- & -- & $\mathrm{KJu}$ \\
\hline 127 & 7/8-18M1 & 127 & 7/8-18M1 & 0 & 640 & 0.0 & 195.1 & no data & No data & -- & -- & -- & QTge \\
\hline 128 & 7/9-13M1 & 128 & 7/9-13М1 & 0 & 2 & 0.0 & 0.6 & topsoil & Sand and clay & -- & -- & -- & \\
\hline 128 & 7/9-13M1 & & & 2 & 10 & 0.6 & 3.0 & yellow clay and gravel & Clay and gravel & -- & -- & -- & \\
\hline 128 & 7/9-13M1 & & & 10 & 25 & 3.0 & 7.6 & yellow clay & Clay & -- & -- & -- & \\
\hline 128 & 7/9-13M1 & & & 25 & 40 & 7.6 & 12.2 & blue clay & Clay & -- & -- & -- & \\
\hline 128 & 7/9-13M1 & & & 40 & 52 & 12.2 & 15.8 & sandy blue clay & Clay and sand (or sandstone) & -- & -- & -- & \\
\hline 128 & 7/9-13M1 & & & 52 & 55 & 15.8 & 16.8 & blue sand & Sand & -- & -- & -- & \\
\hline 128 & 7/9-13M1 & & & 55 & 80 & 16.8 & 24.4 & blue clay and gravel & Clay and gravel & -- & -- & -- & \\
\hline 128 & 7/9-13M1 & & & 80 & 235 & 24.4 & 71.6 & blue clay & Clay & -- & -- & -- & \\
\hline 128 & 7/9-13M1 & & & 235 & 316 & 71.6 & 96.3 & sandy blue clay & Clay and sand (or sandstone) & -- & -- & -- & QTm \\
\hline 129 & 7/8-14M1 & 129 & 7/8-14M1 & 0 & 227.8 & 0.0 & 69.4 & no data & No data & -- & -- & -- & QTge \\
\hline 130 & 7/8-13J1B & 130 & $7 / 8-13 \mathrm{J1B}$ & 0 & 148 & 0.0 & 45.1 & no data & No data & .- & -- & -- & QTge(?) \\
\hline 130 & 7/8-13J1B & & & 148 & 253 & 45.1 & 77.1 & perforated intervals & No data & -- & -- & -- & \\
\hline 131 & 7/8-18K1 & 131 & 7/8-18K1 & 0 & 20 & 0.0 & 6.1 & sand and gravel & Sand (or sandstone) and gravel & -- & -- & -- & QTge(?) \\
\hline 131 & 7/8-18K1 & & & 20 & 34 & 6.1 & 10.4 & no data & No data & -- & -- & -- & 2igc(:) \\
\hline 131 & $7 / 8-18 \mathrm{~K} 1$ & & & 34 & 40 & 10.4 & 12.2 & no data & No data & -- & -- & -- & \\
\hline 131 & 7/8-18K1 & & & 40 & 42 & 12.2 & 12.8 & gravel & Gravel & -- & -- & -- & \\
\hline 131 & 7/8-18K1 & & & 42 & 330 & 12.8 & 100.6 & no data & No data & -- & -- & -- & \\
\hline 131 & 7/8-18K1 & & & 330 & 416 & 100.6 & 126.8 & gravel & Gravel & -- & -- & -- & \\
\hline 131 & 7/8-18K1 & & & 416 & 420 & 126.8 & 128.0 & no data & No data & -- & -- & -- & \\
\hline 132 & 7/9-18K1 & 132 & 7/9-18K1 & 0 & 266 & 0.0 & 81.1 & (reported "dry hole") & No data & -- & -- & -- & KJu (?) \\
\hline 133 & 7/9-16L1 & 133 & 7/9-16L1 & 0 & 255 & 0.0 & 77.7 & no data & No data & -- & -- & -- & QTm \\
\hline 134 & $7 / 8-13 \mathrm{~J} 2$ & 134 & $7 / 8-13 \mathrm{~J} 2$ & 0 & 188 & 0.0 & 57.3 & no data & No data & -- & -- & -- & Tsv (?) \\
\hline 134 & $7 / 8-1332$ & & & 188 & 206 & 57.3 & 62.8 & perforated intervals & No data & -- & -- & -- & \\
\hline 134 & 7/8-13J2 & & & 206 & 210 & 62.8 & 64.0 & no data & No data & -- & -- & -- & \\
\hline 135 & 7/8-18Q1 & 135 & 7/8-18Q1 & 0 & 50 & 0.0 & 15.2 & no data & No data & -- & -- & -- & -- \\
\hline 135 & 7/8-18Q1 & & & 50 & 55 & 15.2 & 16.8 & sandy clay and gravel & Clay, sand, and gravel & -- & -- & -- & QTge \\
\hline 135 & 7/8-18Q1 & & & 55 & 130 & 16.8 & 39.6 & no data & No data & -- & -- & -- & \\
\hline 135 & 7/8-18Q1 & & & 130 & 153 & 39.6 & 46.6 & cemented gravel, sand and clay & Gravel & cemented & -- & -- & \\
\hline 135 & 7/8-18Q1 & & & 153 & 192 & 46.6 & 58.5 & no data & No data & -- & -- & -- & \\
\hline 135 & 7/8-18Q1 & & & 192 & 198 & 58.5 & 60.4 & coarse cemented gravel & Gravel & cemented & -- & -- & \\
\hline 135 & 7/8-18Q1 & & & 198 & 222 & 60.4 & 67.7 & no data & No data & - & -- & -- & \\
\hline 135 & 7/8-18Q1 & & & 222 & 236 & 67.7 & 71.9 & clay and gravel & Clay and gravel & -- & -- & -- & \\
\hline 135 & 7/8-18Q1 & & & 236 & 881 & 71.9 & 268.5 & no data & No data & -- & -- & -- & \\
\hline
\end{tabular}


Appendix 3. Lithologic data. - Continued

[Site IDs and well number correspond to locations in Appendix 1, a site ID and well number is listed for each drilled interval for a particular drill hole. For convenience, the site ID and well numbers are repeated at the shallowest drilled interval for each well. leaders $(-1$

\begin{tabular}{|c|c|c|c|c|c|c|c|c|c|c|c|c|c|}
\hline Site ID & Well number & $\begin{array}{c}\text { Site ID, } \\
\text { top of } \\
\text { well }\end{array}$ & $\begin{array}{l}\text { Well number, } \\
\text { top of well }\end{array}$ & $\begin{array}{c}\text { Top of } \\
\text { interval, in } \\
\text { feet }\end{array}$ & $\begin{array}{l}\text { Base of } \\
\text { interval, in } \\
\text { feet }\end{array}$ & $\begin{array}{c}\begin{array}{c}\text { Top of } \\
\text { interval, in } \\
\mathrm{m}\end{array} \\
\end{array}$ & $\begin{array}{c}\text { Base of } \\
\text { interval, in } \\
\mathrm{m}\end{array}$ & Reported lithologic unit' & Interpreted lithologic class ${ }^{2}$ & $\begin{array}{c}\text { Reported } \\
\text { degree of } \\
\text { cementation or } \\
\text { induration }^{3} \\
\end{array}$ & $\begin{array}{c}\text { Fossils } \\
\text { reported in } \\
\text { interval }^{4}\end{array}$ & $\begin{array}{c}\text { Organic } \\
\text { matter } \\
\text { reported in } \\
\text { interval }^{5}\end{array}$ & $\begin{array}{l}\text { Reported stratigraphic top of geologic } \\
\text { unit }^{6}\end{array}$ \\
\hline 136 & 7/8-13K1 & 136 & 7/8-13K1 & 0 & 129 & 0.0 & 39.3 & no data & No data & -- & -- & -- & QTge \\
\hline 136 & 7/8-13K1 & & & 129 & 135 & 39.3 & 41.1 & cemented gravel & Clay and gravel & cemented & -- & -- & \\
\hline 136 & 7/8-13K1 & & & 135 & 272 & 41.1 & 82.9 & $\begin{array}{l}\text { no data } \\
\text { clay and gravel; cemented gravel; }\end{array}$ & No data & -- & -- & -- & \\
\hline 136 & 7/8-13K1 & & & 272 & 431 & 82.9 & 131.4 & coarse gravel; clay & Clay and gravel & cemented & -- & -- & \\
\hline 136 & $7 / 8-13 \mathrm{~K} 1$ & & & 431 & 529 & 131.4 & 161.2 & no data & No data & -- & -- & -- & \\
\hline 137 & 7/9-15Q1 & 137 & 7/9-15Q1 & 0 & 200 & 0.0 & 61.0 & no data & No data & -- & -- & -- & QTm \\
\hline 138 & 7/9-14K1 & 138 & 7/9-14K1 & 0 & 2 & 0.0 & 0.6 & topsoil & Sand and clay & -- & -- & -- & QTge \\
\hline 138 & $7 / 9-14 K 1$ & & & 2 & 16 & 0.6 & 4.9 & clay and sand & Clay and sand (or sandstone) & -- & -- & -- & \\
\hline 138 & $7 / 9-14 \mathrm{~K} 1$ & & & 16 & 20 & 4.9 & 6.1 & sand & Sand & -- & -- & -- & \\
\hline 138 & $7 / 9-14 \mathrm{~K} 1$ & & & 20 & 23 & 6.1 & 7.0 & clay, hard & Clay & hard & -- & -- & \\
\hline 138 & $7 / 9-14 \mathrm{~K} 1$ & & & 23 & 35 & 7.0 & 10.7 & sand & Sand & -- & -- & -- & \\
\hline 138 & 7/9-14K1 & & & 35 & 40 & 10.7 & 12.2 & gravel, very small; water & Gravel & -- & -- & -- & \\
\hline 138 & 7/9-14K1 & & & 40 & 62 & 12.2 & 18.9 & clay, sticky blue & Clay & -- & -- & -- & \\
\hline 138 & $7 / 9-14 \mathrm{~K} 1$ & & & 62 & 86 & 18.9 & 26.2 & clay and gravel mix & Clay and gravel & -- & -- & -- & \\
\hline 138 & $7 / 9-14 \mathrm{~K} 1$ & & & 86 & 106 & 26.2 & 32.3 & clay and gravel mix & Clay and gravel & -- & -- & -- & \\
\hline 138 & 7/9-14K1 & & & 106 & 138 & 32.3 & 42.1 & clay and sand mix & Clay and sand (or sandstone) & -- & -- & -- & \\
\hline 138 & 7/9-14K1 & & & 138 & 208 & 42.1 & 63.4 & sand, blue & Sand & -- & -- & -- & QTm \\
\hline 138 & 7/9-14K1 & & & 208 & 257 & 63.4 & 78.3 & sand, blue and some gravel & Sand (or sandstone) and gravel & -- & -- & -- & \\
\hline 138 & $7 / 9-14 \mathrm{~K} 1$ & & & 257 & 296 & 78.3 & 90.2 & sand and gravel, small & Sand (or sandstone) and gravel & -- & -- & -- & \\
\hline 138 & 7/9-14K1 & & & 296 & 358 & 90.2 & 109.1 & gravel, small & Gravel & -- & -- & -- & \\
\hline 138 & 7/9-14K1 & & & 358 & 560 & 109.1 & 170.7 & shells and gravel mix & Sand (or sandstone) and gravel & -- & shells & -- & \\
\hline 138 & 7/9-14K1 & & & 560 & 570 & 170.7 & 173.7 & gravel, fine & Gravel & -- & -- & -- & \\
\hline 138 & $7 / 9-14 \mathrm{~K} 1$ & & & 570 & 584 & 173.7 & 178.0 & sand and shells & Sand & -- & shells & -- & \\
\hline 138 & $7 / 9-14 \mathrm{~K} 1$ & & & 584 & 636 & 178.0 & 193.9 & shale & Clay and sand (or sandstone) & cemented & -- & -- & \\
\hline 139 & $7 / 7-18\llcorner 1$ & 139 & $7 / 7-18\llcorner 1$ & 0 & 36 & 0.0 & 11.0 & clay, yellow, and gravel & Clay and gravel & -- & -- & -- & Qyal and Qoal (?) and QTge \\
\hline 139 & 7/7-18L1 & & & 36 & 78 & 11.0 & 23.8 & gravel & Gravel & -- & -- & -- & \\
\hline 139 & $7 / 7-18 \mathrm{~L} 1$ & & & 78 & 157 & 23.8 & 47.9 & clay, blue, and gravel & Clay and gravel & -- & -- & -- & \\
\hline 139 & 7/7-18L1 & & & 157 & 161 & 47.9 & 49.1 & rock & Ash or tuff & hard & -- & -- & Tsv \\
\hline 140 & 7/9-16Q1A & 140 & 7/9-16Q1A & 0 & 268 & 0.0 & 81.7 & no data & No data & -- & -- & -- & QTm \\
\hline 141 & 7/9-16Q1B & 141 & 7/9-16Q1B & 0 & 321 & 0.0 & 97.8 & no data & No data & -- & -- & -- & -- \\
\hline 142 & 7/9-13R1 & 142 & 7/9-13R1 & 0 & 37 & 0.0 & 11.3 & no data & No data & -- & -- & -- & -- \\
\hline 142 & 7/9-13R1 & & & 37 & 46 & 11.3 & 14.0 & sand and gravel; clay and gravel & Clay, sand, and gravel & -- & -- & -- & QTge \\
\hline 142 & 7/9-13R1 & & & 46 & 70 & 14.0 & 21.3 & no data & No data & -- & -- & -- & \\
\hline 142 & 7/9-13R1 & & & 70 & 81 & 21.3 & 24.7 & clay and gravel; gravel & Clay and gravel & -- & -- & -- & \\
\hline 142 & 7/9-13R1 & & & 81 & 94 & 24.7 & 28.7 & no data & No data & -- & -- & -- & \\
\hline 142 & $7 / 9-13 R 1$ & & & 94 & 120 & 28.7 & 36.6 & clay and gravel & Clay and gravel & -- & -- & -- & \\
\hline 142 & 7/9-13R1 & & & 120 & 130 & 36.6 & 39.6 & no data & No data & -- & -- & -- & \\
\hline 142 & 7/9-13R1 & & & 130 & 170 & 39.6 & 51.8 & clay and gravel & Clay and gravel & -- & -- & -- & \\
\hline 142 & 7/9-13R1 & & & 170 & 264 & 51.8 & 80.5 & no data & No data & -- & -- & -- & \\
\hline 142 & $7 / 9-13 R 1$ & & & 264 & 275 & 80.5 & 83.8 & clay and gravel & Clay and gravel & -- & -- & -- & \\
\hline 142 & 7/9-13R1 & & & 275 & 330 & 83.8 & 100.6 & no data & No data & -- & -- & -- & \\
\hline 142 & 7/9-13R1 & & & 330 & 375 & 100.6 & 114.3 & sandy clay and gravel; gravel & Clay, sand, and gravel & -- & -- & -- & \\
\hline 143 & 7/8-17N1 & 143 & 7/8-17N1 & 0 & 3 & 0.0 & 165.8 & no data & No data & -- & -- & -- & Qyal \\
\hline 143 & $7 / 8-17 \mathrm{~N} 1$ & & & 3 & 36.0 & 0.9 & 11.0 & soft sandstone, gravel & Sand (or sandstone) and gravel & cemented & -- & -- & Qyal \\
\hline 143 & $7 / 8-17 \mathrm{~N} 1$ & & & 36.0 & 52.0 & 11.0 & 15.8 & no data & No data & -- & -- & -- & \\
\hline 143 & $7 / 8-17 \mathrm{~N} 1$ & & & 52.0 & 62.0 & 15.8 & 18.9 & gravel & Gravel & -- & -- & -- & QTge \\
\hline 143 & $7 / 8-17 \mathrm{~N} 1$ & & & 62.0 & 162.0 & 18.9 & 49.4 & no data & No data & -- & -- & -- & \\
\hline 143 & $7 / 8-17 \mathrm{~N} 1$ & & & 162.0 & 164.0 & 49.4 & 50.0 & cemented gravel & Gravel & cemented & -- & -- & \\
\hline 143 & $7 / 8-17 \mathrm{~N} 1$ & & & 164.0 & 234.0 & 50.0 & 71.3 & $\begin{array}{l}\text { no data } \\
\text { sandy clay, soft streaks with }\end{array}$ & No data & -- & -- & -- & \\
\hline 143 & $7 / 8-17 \mathrm{~N} 1$ & & & 234.0 & 268.0 & 71.3 & 81.7 & gravel; cemented gravel. & Clay, sand, and trace gravel & cemented & -- & -- & \\
\hline 143 & $7 / 8-17 \mathrm{~N} 1$ & & & 268.0 & 376.0 & 81.7 & 114.6 & no data & No data & -- & -- & -- & \\
\hline 143 & $7 / 8-17 \mathrm{~N} 1$ & & & 376.0 & 384.0 & 114.6 & 117.0 & cemented gravel & Gravel & cemented & -- & -- & \\
\hline 143 & $7 / 8-17 \mathrm{~N} 1$ & & & 384.0 & 424.0 & 117.0 & 129.2 & no data & No data & -- & -- & -- & \\
\hline 143 & 7/8-17N1 & & & 424.0 & 430.0 & 129.2 & 131.1 & cemented gravel & Gravel & cemented & -- & -. & \\
\hline 143 & $7 / 8-17 \mathrm{~N} 1$ & & & 430.0 & 506.0 & 131.1 & 154.2 & no data & No data & -- & -- & -- & \\
\hline 143 & $7 / 8-17 \mathrm{~N} 1$ & & & 506.0 & 516.0 & 154.2 & 157.3 & sandstone & Sandstone & cemented & -- & -. & \\
\hline 143 & $7 / 8-17 \mathrm{~N} 1$ & & & 516.0 & 544.0 & 157.3 & 165.8 & no data & No data & -- & -- & -- & \\
\hline 144 & $7 / 9-21 \mathrm{~B} 1$ & 144 & 7/9-21B1 & 0 & 550 & 0.0 & 167.6 & no data & No data & -- & -- & -- & QTm \\
\hline 145 & 7/7-18R2 & 145 & $7 / 7-18 \mathrm{R} 2$ & 0 & 4 & 0.0 & 1.2 & adobe & Sand and clay & -- & -- & -- & oal (?) \\
\hline
\end{tabular}


Appendix 3. Lithologic data. - Continued

ISite IDs and well number correspond to locations in Appendix 1, a site ID and well number is listed for each drilled interval for a particular drill hole. For convenience, the site ID and well numbers are repeated at the shallowest drilled interval for each well. leaderst.

\begin{tabular}{|c|c|c|c|c|c|c|c|c|c|c|c|c|c|}
\hline Site ID & Well number & $\begin{array}{l}\text { Site ID, } \\
\text { top of } \\
\text { well }\end{array}$ & $\begin{array}{l}\text { Well number, } \\
\text { top of well }\end{array}$ & $\begin{array}{c}\text { Top of } \\
\text { interval, in } \\
\text { feet }\end{array}$ & $\begin{array}{c}\text { Base of } \\
\text { interval, in } \\
\text { feet }\end{array}$ & $\begin{array}{c}\text { Top of } \\
\text { interval, in } \\
m\end{array}$ & $\begin{array}{c}\text { Base of } \\
\text { interval, in } \\
\mathrm{m}\end{array}$ & Reported lithologic unit ${ }^{1}$ & Interpreted lithologic class ${ }^{2}$ & $\begin{array}{c}\text { Reported } \\
\text { degree of } \\
\text { cementation or } \\
\text { induration }^{3}\end{array}$ & $\begin{array}{c}\text { Fossils } \\
\text { reported in } \\
\text { interval }^{4}\end{array}$ & $\begin{array}{c}\text { Organic } \\
\text { matter } \\
\text { reported in } \\
\text { interval }^{5}\end{array}$ & $\begin{array}{l}\text { Reported stratigraphic top of geologic } \\
\text { unit }^{6}\end{array}$ \\
\hline 145 & $7 / 7-18 \mathrm{R} 2$ & & & 4 & 20 & 1.2 & 6.1 & clay, blue & Clay & -- & -- & -- & \\
\hline 145 & $7 / 7-18 \mathrm{R} 2$ & & & 20 & 30 & 6.1 & 9.1 & clay, sandy, blue, some gravel & Clay, sand, and trace gravel & -- & -- & -- & \\
\hline 145 & 7/7-18R2 & & & 30 & 35 & 9.1 & 10.7 & clay, sandy, blue, and gravel & Clay, sand, and gravel & -- & -. & -- & \\
\hline 145 & $7 / 7-18 \mathrm{R} 2$ & & & 35 & 40 & 10.7 & 12.2 & $\begin{array}{l}\text { gravel and wood } \\
\text { and }\end{array}$ & Gravel & - & -- & wood & \\
\hline 145 & $7 / 7-18 \mathrm{R} 2$ & & & 40 & 41 & 12.2 & 12.5 & sand and gravel & Sand (or sandstone) and gravel & -- & -- & -- & \\
\hline 145 & 7/7-18R2 & & & 41 & 50 & 12.5 & 15.2 & clay, blue & Clay & -- & -- & -- & \\
\hline 145 & $7 / 7-18 \mathrm{R} 2$ & & & 50 & 65 & 15.2 & 19.8 & gravel, blue, cemented & Gravel & cemented & -- & -- & \\
\hline 145 & $7 / 7-18 \mathrm{R} 2$ & & & 65 & 88 & 19.8 & 26.8 & volcanic rock, soft & Ash or tuff & -. & -- & -.- & Tsv \\
\hline 145 & $7 / 7-18 \mathrm{R} 2$ & & & 88 & 90 & 26.8 & 27.4 & volcanic rock, hard & Ash or tuff & hard & -- & -.- & \\
\hline 145 & 7/7-18R2 & & & 90 & 123 & 27.4 & 37.5 & volcanic rock, streaks of soft & Ash or tuff & -- & -- & -- & \\
\hline 145 & 7/7-18R2 & & & 123 & 206 & 37.5 & 62.8 & volcanic rock & Ash or tuff & -- & -- & -- & \\
\hline 146 & $7 / 8-24 \mathrm{~A} 2$ & 146 & 7/8-24A2 & 0 & 930 & 0.0 & 283.5 & no data & No data & -- & -- & -- & Qyal and QTge and Tsv (?) \\
\hline 147 & $7 / 7-18 \mathrm{~N} 1$ & 147 & 7/7-18N1 & 0 & 11 & 0.0 & 3.4 & soil & Sand and clay & -- & -- & -.- & Qyal and Qoal (?) and QTge \\
\hline 147 & $7 / 7-18 \mathrm{~N} 1$ & & & 11 & 28 & 3.4 & 8.5 & clay, yellow & Clay & -. & -- & -. & \\
\hline 147 & $7 / 7-18 \mathrm{~N} 1$ & & & 28 & 30 & 8.5 & 9.1 & rock & Gravel & hard & -- & -- & \\
\hline 147 & $7 / 7-18 \mathrm{~N} 1$ & & & 30 & 50 & 9.1 & 15.2 & gravel, free & Gravel & -- & -- & .- & \\
\hline 147 & $7 / 7-18 \mathrm{~N} 1$ & & & 50 & 65 & 15.2 & 19.8 & gravel & Gravel & -- & -- & -- & \\
\hline 147 & $7 / 7-18 \mathrm{~N} 1$ & & & 65 & 69 & 19.8 & 21.0 & sand, gray & Sand & .- & -. & .- & \\
\hline 147 & 7/7-18N1 & & & 69 & 75 & 21.0 & 22.9 & clay, blue & Clay & -- & .. & -. & \\
\hline 147 & $7 / 7-18 \mathrm{~N} 1$ & & & 75 & 78 & 22.9 & 23.8 & clay, blue & Clay & -- & -- & -- & \\
\hline 147 & $7 / 7-18 \mathrm{~N} 1$ & & & 78 & 80 & 23.8 & 24.4 & gravel & Gravel & -- & -- & -- & \\
\hline 147 & $7 / 7-18 \mathrm{~N} 1$ & & & 80 & 87 & 24.4 & 26.5 & clay and rock & Clay and gravel & -- & -- & -- & \\
\hline 147 & $7 / 7-18 \mathrm{~N} 1$ & & & 87 & 108 & 26.5 & 32.9 & clay, sandy, blue & Clay and sand (or sandstone) & -- & -. & -- & \\
\hline 147 & 7/7-18N1 & & & 108 & 111 & 32.9 & 33.8 & gravel & Gravel & .- & .. & .. & \\
\hline 147 & $7 / 7-18 \mathrm{N1}$ & & & 111 & 115 & 33.8 & 35.1 & clay, sandy & Clay and sand (or sandstone) & -. & -. & -. & \\
\hline 147 & $7 / 7-18 \mathrm{~N} 1$ & & & 115 & 133 & 35.1 & 40.5 & gravel & Gravel & -- & -- & -- & \\
\hline 147 & $7 / 7-18 \mathrm{~N} 1$ & & & 133 & 140 & 40.5 & 42.7 & clay and rock & Clay and gravel & -- & -- & -. & \\
\hline 148 & $7 / 8-21 \mathrm{C1}$ & 148 & 7/8-21C1 & 0 & 310 & 0.0 & 94.5 & $\begin{array}{l}\text { no data } \\
\text { nock }\end{array}$ & No data & - & - & -. & QTge \\
\hline 149 & $7 / 8-2001$ & 149 & $7 / 8-20 C 1$ & 0 & 77 & 0.0 & 23.5 & no data & No data & .- & -. & .. & -- \\
\hline 149 & $7 / 8-2001$ & & & 77 & 80 & 23.5 & 24.4 & gravel & Gravel & .- & -. & .. & QTge \\
\hline 149 & $7 / 8-2001$ & & & 80 & 230 & 24.4 & 70.1 & no data & No data & -- & -- & -- & \\
\hline 149 & $7 / 8-2001$ & & & 230 & 231 & 70.1 & 70.4 & gravel & Gravel & -- & -- & -- & \\
\hline 149 & $7 / 8-2001$ & & & 231 & 539 & 70.4 & 164.3 & no data & No data & - & -- & - & \\
\hline 149 & $7 / 8-2001$ & & & 539 & 542 & 164.3 & 165.2 & gravel & Gravel & .- & .- & .. & \\
\hline 149 & $7 / 8-2001$ & & & 542 & 580 & 165.2 & 176.8 & no data & No data & -- & -. & .. & \\
\hline 149 & $7 / 8-2001$ & & & 580 & 582 & 176.8 & 177.4 & gravel & Gravel & -- & -- & - & \\
\hline 149 & $7 / 8-20 \mathrm{C} 1$ & & & 582 & 591 & 177.4 & 180.1 & no data & No data & -- & -- & -- & \\
\hline 149 & $7 / 8-20 \mathrm{C} 1$ & & & 591 & 623 & 180.1 & 189.9 & gravel & Sand and clay & .. & .. & .- & \\
\hline 149 & $7 / 8-20 \mathrm{C} 1$ & & & 623 & 625 & 189.9 & 190.5 & no data & $\begin{array}{l}\text { No data } \\
\text { lata }\end{array}$ & - & - & -. & \\
\hline 150 & $7 / 9-21 \mathrm{C} 1$ & 150 & $7 / 9-21 \mathrm{C} 1$ & 0 & 290 & 0.0 & 88.4 & no data & No data & -- & -- & - & -- \\
\hline 150 & $7 / 9-21 \mathrm{C1}$ & & & 290 & 325 & 88.4 & 99.1 & sandstone & Sandstone & cemented & -- & -. & QTm \\
\hline 151 & 7/8-24A3 & 151 & $7 / 8-24 A 3$ & 0 & 300 & 0.0 & 91.4 & no data & No data & -- & -. & .. & Qyal and QTge \\
\hline 152 & 7/9-23F1 & 152 & 7/9-23F1 & 0 & 6 & 0.0 & 1.8 & black adobe soil & Sand and clay & -- & -- & -- & \\
\hline 152 & 7/9-23F1 & & & 6 & 12 & 1.8 & 3.7 & brown clay & Clay & -- & -. & -. & \\
\hline 152 & 7/9-23F1 & & & 12 & 15 & 3.7 & 4.6 & brown gravelly clay & Clay and gravel & -- & -- & -- & \\
\hline 152 & $7 / 9-23 F 1$ & & & 15 & 35 & 4.6 & 10.7 & blue sandstone & Sandstone & cemented & -- & -. & QTm \\
\hline 152 & $7 / 9-23 F 1$ & & & $\begin{array}{l}15 \\
35\end{array}$ & $\begin{array}{l}35 \\
37\end{array}$ & $\begin{array}{l}4.0 \\
10.7\end{array}$ & 11.3 & blue sand, water & Sand & - & -- & -- & QIm \\
\hline 152 & $7 / 9-23 F 1$ & & & 37 & 48 & 11.3 & 14.6 & blue sandy clay & Clay and sand (or sandstone) & -- & -- & -. & \\
\hline 152 & $7 / 9-23 F 1$ & & & 48 & 50 & 14.6 & 15.2 & blue sand and quicksand & 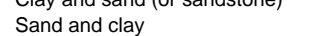 & -- & .- & .. & \\
\hline 152 & $7 / 9-23 F 1$ & & & 50 & 58 & 15.2 & 17.7 & blue coarse sandstone & Sandstone & cemented & -- & -- & \\
\hline 152 & $7 / 9-23 F 1$ & & & 58 & 65 & 17.7 & 19.8 & gray quicksand & Sand and clay & - & - & -. & \\
\hline 152 & 7/9-23F1 & & & 65 & 70 & 19.8 & 21.3 & blue sandy sedimentary clay & Clay and sand (or sandstone) & -- & -- & -- & \\
\hline 152 & $7 / 9-23 F 1$ & & & 70 & 98 & 21.3 & 29.9 & blue coarse sandstone & Sandstone & cemented & $\ldots$ & .. & \\
\hline 152 & $7 / 9-23 F 1$ & & & 98 & 114 & 29.9 & 34.7 & blue fine sandstone & Sandstone & cemented & -- & -- & \\
\hline 152 & 7/9-23F1 & & & 114 & 120 & 34.7 & 36.6 & $\begin{array}{l}\text { blue sand and quicksand } \\
\text { blue coarse sand and gravel with }\end{array}$ & Sand and clay & -- & -- & -- & \\
\hline 152 & $7 / 9-23 F 1$ & & & 120 & 170 & 36.6 & 51.8 & $\begin{array}{l}\text { quicksand } \\
\text { blue coarse sand and oyster }\end{array}$ & Clay, sand, and gravel & -- & -- & -- & \\
\hline 152 & $7 / 9-23 F 1$ & & & 170 & 212 & 51.8 & 64.6 & shells & Sand & -- & shells & -- & \\
\hline 152 & $7 / 9-23 F 1$ & & & 212 & 225 & 64.6 & 68.6 & fine gravel and quicksand & Sand (or sandstone) and gravel & -- & -- & -- & \\
\hline
\end{tabular}


Appendix 3. Lithologic data. - Continued

[Site IDs and well number correspond to locations in Appendix 1, a site ID and well number is listed for each drilled interval for a particular drill hole. For convenience, the site ID and well numbers are repeated at the shallowest drilled interval for each well. leaders $(-1$

\begin{tabular}{|c|c|c|c|c|c|c|c|c|c|c|c|c|c|}
\hline Site ID & Well number & $\begin{array}{l}\text { Site ID, } \\
\text { top of } \\
\text { well }\end{array}$ & $\begin{array}{l}\text { Well number, } \\
\text { top of well }\end{array}$ & $\begin{array}{c}\text { Top of } \\
\text { interval, in } \\
\text { feet }\end{array}$ & $\begin{array}{l}\text { Base of } \\
\text { interval, in } \\
\text { feet }\end{array}$ & $\begin{array}{c}\text { Top of } \\
\text { interval, in } \\
m\end{array}$ & $\begin{array}{c}\text { Base of } \\
\text { interval, in } \\
\mathrm{m}\end{array}$ & Reported lithologic unit' ${ }^{1}$ & Interpreted lithologic class ${ }^{2}$ & $\begin{array}{c}\text { Reported } \\
\text { degree of } \\
\text { cementation or } \\
\text { induration }^{3} \\
\end{array}$ & $\begin{array}{c}\text { Fossils } \\
\text { reported in } \\
\text { interval }^{4}\end{array}$ & $\begin{array}{c}\text { Organic } \\
\text { matter } \\
\text { reported in } \\
\text { interval }^{5}\end{array}$ & $\begin{array}{l}\text { Reported stratigraphic top of geologic } \\
\text { unit }^{6}\end{array}$ \\
\hline 152 & $7 / 9-23 F 1$ & & & 225 & 310 & 68.6 & 94.5 & $\begin{array}{l}\text { oyster shell and gravelly } \\
\text { sandstone }\end{array}$ & Sand (or sandstone) and gravel & cemented & shells & 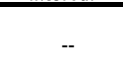 & \\
\hline 152 & $7 / 9-23 F 1$ & & & 310 & 320 & 94.5 & 97.5 & medium gray sand, water & Sand & -- & -- & -- & \\
\hline 152 & 7/9-23F1 & & & 320 & 420 & 97.5 & 128.0 & coarse gray sandstone and shells & Sandstone & cemented & shells & -- & \\
\hline 152 & $7 / 9-23 F 1$ & & & 420 & 425 & 128.0 & 129.5 & dark gray sand, water & Sand & -- & -- & -- & \\
\hline 152 & $7 / 9-23 F 1$ & & & 425 & 590 & 129.5 & 179.8 & gray sandstone and shells & Sandstone & cemented & shells & -- & \\
\hline 152 & $7 / 9-23 F 1$ & & & 590 & 610 & 179.8 & 185.9 & medium beach gray sand, water & Sand & -- & -- & -- & \\
\hline 153 & $7 / 8-20 D 1$ & 153 & 7/8-20D1 & 0 & 190 & 0.0 & 57.9 & no data & No data & -- & -- & -- & QTge \\
\hline 153 & $7 / 8-20 \mathrm{D} 1$ & & & 190 & 294 & 57.9 & 89.6 & clay and gravel & Clay and gravel & -- & -- & -- & \\
\hline 153 & 7/8-20D1 & & & 294 & 446 & 89.6 & 135.9 & no data & No data & -- & -- & -- & \\
\hline 153 & $7 / 8-2001$ & & & 446 & 476 & 135.9 & 145.1 & cemented gravel. & Gravel & cemented & -- & -- & \\
\hline 153 & 7/8-20D1 & & & 476 & 484 & 145.1 & 147.5 & no data & No data & -- & -- & -- & \\
\hline 154 & 7/8-24A5 & 154 & 7/8-24A5 & 0 & 50 & 0.0 & 15.2 & no data & No data & -- & -- & -- & -- \\
\hline 154 & 7/8-24A5 & & & 50 & 170 & 15.2 & 51.8 & perforated intervals & No data & -- & -- & -- & QTge \\
\hline 154 & 7/8-24A5 & & & 170 & 186 & 51.8 & 56.7 & no data & No data & -. & -- & -- & \\
\hline 154 & 7/8-24A5 & & & 186 & 288 & 56.7 & 87.8 & perforated intervals & No data & -- & -- & -- & \\
\hline 154 & 7/8-24A5 & & & 288 & 291 & 87.8 & 88.7 & no data & No data & -- & -- & -- & \\
\hline 155 & 7/8-24A6 & 155 & 7/8-24A6 & 0 & 1200.0 & 0.0 & 365.8 & no data & No data & -- & -- & -- & Qyal (?) and QTge and Tsv (?) \\
\hline 156 & 7/7-14A1 & 156 & 7/7-14A1 & 0 & 120 & 0.0 & 36.6 & rock, soft, brown & Ash or tuff & hard & -- & -- & Tsv \\
\hline 156 & 7/7-14A1 & 100 & $1 / T-14 A \perp$ & 120 & 208 & 36.6 & 63.4 & soft red rock & Ash or tuff & -- & - & -- & ISV \\
\hline 156 & 7/7-14A1 & & & 208 & 222 & 63.4 & 67.7 & dark brown rock & Ash or tuff & -- & -- & -- & \\
\hline 156 & 7/7-14A1 & & & 222 & 281 & 67.7 & 85.6 & hard red rock with many seams & Ash or tuff & hard & -- & -- & \\
\hline 156 & $7 / 7-14 \mathrm{~A} 1$ & & & 281 & 434 & 85.6 & 132.3 & $\begin{array}{l}\text { hard brown rock with many seams } \\
\text { soft yellow-brown rock with many }\end{array}$ & Ash or tuff & hard & -- & -- & \\
\hline 156 & 7/7-14A1 & & & 434 & 488 & 132.3 & 148.7 & seams & Ash or tuff & -- & -- & -- & \\
\hline 156 & 7/7-14A1 & & & 488 & 580 & 148.7 & 176.8 & hard red rock, very seamy & Ash or tuff & hard & -- & -- & \\
\hline 156 & 7/7-14A1 & & & 580 & 620 & 176.8 & 189.0 & harder red rock, very seamy & Ash or tuff & hard & -- & -- & \\
\hline 156 & 7/7-14A1 & & & 620 & 622 & 189.0 & 189.6 & very hard red rock, very seamy & Ash or tuff & hard & -- & -- & \\
\hline 157 & $7 / 7-23 \mathrm{~A} 1$ & 157 & 7/7-23A1 & 0 & 3 & 0.0 & 0.9 & topsoil & Sand and clay & -- & -- & -- & QTge \\
\hline 157 & 7/7-23A1 & & & 3 & 18 & 0.9 & 5.5 & boulders & Gravel & -- & -- & -- & \\
\hline 157 & 7/7-23A1 & & & 18 & 30 & 5.5 & 9.1 & clay and boulders & Clay and gravel & -- & -- & -- & \\
\hline 157 & 7/7-23A1 & & & 30 & 63 & 9.1 & 19.2 & $\begin{array}{l}\text { clay and rock } \\
\text { lack }\end{array}$ & Clay and gravel & -- & -- & -- & \\
\hline 157 & $7 / 7-23 \mathrm{~A} 1$ & & & 63 & 73 & 19.2 & 22.3 & rock & Ash or tuff & hard & -- & -- & \\
\hline 157 & 7/7-23A1 & & & 73 & 94 & 22.3 & 28.7 & clay and gravel & Clay and gravel & -- & -- & -- & \\
\hline 157 & 7/7-23A1 & & & 94 & 168 & 28.7 & 51.2 & gravel cemented & Gravel & cemented & -- & -- & \\
\hline 157 & $7 / 7-23 A 1$ & & & 168 & 190 & 51.2 & 57.9 & cemented gravel and boulders & Gravel & cemented & -- & -- & \\
\hline 157 & $7 / 7-23 \mathrm{~A} 1$ & & & 190 & 207 & 57.9 & 63.1 & blue clay and boulders & Clay and gravel & -. & -- & -- & \\
\hline 157 & 7/7-23A1 & & & 207 & 250 & 63.1 & 76.2 & red clay and boulders & Clay and gravel & -- & -- & -- & \\
\hline 157 & 7/7-23A1 & & & 250 & 258 & 76.2 & 78.6 & sandy blue clay and gravel & Clay, sand, and gravel & -- & - & -- & \\
\hline 157 & $7 / 7-23 \mathrm{~A} 1$ & & & 258 & 270 & 78.6 & 82.3 & gravel & Gravel & -. & -- & -- & \\
\hline 157 & 7/7-23A1 & & & 270 & 273 & 82.3 & 83.2 & gravel & Gravel & -- & -- & -- & \\
\hline 157 & 7/7-23A1 & & & 273 & 300 & 83.2 & 91.4 & blue clay and gravel & Clay and gravel & -- & -- & -- & \\
\hline 157 & 7/7-23A1 & & & 300 & 303 & 91.4 & 92.4 & clay and rock & Clay and gravel & -- & - & - & \\
\hline 157 & 7/7-23A1 & & & 303 & 320 & 92.4 & 97.5 & cemented gravel & Gravel & cemented & -- & -. & \\
\hline 157 & 7/7-23A1 & & & 320 & 337 & 97.5 & 102.7 & blue sand and gravel & Sand (or sandstone) and gravel & -- & -- & -- & \\
\hline 157 & 7/7-23A1 & & & 337 & 340 & 102.7 & 103.6 & blue clay and gravel & Clay and gravel & -- & -- & -- & \\
\hline 157 & 7/7-23A1 & & & 340 & 365 & 103.6 & 111.3 & sandy yellow clay and gravel & Clay, sand, and gravel & -- & -- & -- & \\
\hline 158 & $7 / 8-24 \mathrm{~A} 4$ & 158 & 7/8-24A4 & 0 & 5 & 0.0 & 1.5 & topsoil & Sand and clay & -- & -- & -- & Qyal and Qoal (?) and QTge \\
\hline 158 & 7/8-24A4 & & & 5 & 17 & 1.5 & 5.2 & hardpan & Clay and gravel & hard & -- & .- & \\
\hline 158 & $7 / 8-24 A 4$ & & & 17 & 22 & 5.2 & 6.7 & clay, blue & Clay & -- & -- & -- & \\
\hline 158 & 7/8-24A4 & & & 22 & 26 & 6.7 & 7.9 & sand and gravel, first water & Sand (or sandstone) and gravel & -- & -- & -- & \\
\hline 158 & 7/8-24A4 & & & 26 & 30 & 7.9 & 9.1 & blue clay & Clay & -. & -. & -- & \\
\hline 158 & $7 / 8-24 A 4$ & & & 30 & 50 & 9.1 & 15.2 & gravel and boulders & Gravel & -- & -- & -- & \\
\hline 158 & 7/8-24A4 & & & 50 & 56 & 15.2 & 17.1 & $\begin{array}{l}\text { clay and gravel } \\
\text { lats }\end{array}$ & Clay and gravel & -- & -- & -- & \\
\hline 158 & 7/8-24A4 & & & 56 & 74 & 17.1 & 22.6 & gravel and boulders & Gravel & -- & -- & -- & \\
\hline 158 & 7/8-24A4 & & & 74 & 90 & 22.6 & 27.4 & gravel, cemented & Gravel & cemented & -- & -- & \\
\hline 158 & 7/8-24A4 & & & 90 & 101 & 27.4 & 30.8 & solidified sand and gravel & Sand (or sandstone) and gravel & -- & -.- & -- & \\
\hline 158 & 7/8-24A4 & & & 101 & 126 & 30.8 & 38.4 & blue clay & Clay & -- & -- & -- & \\
\hline
\end{tabular}


Appendix 3. Lithologic data. - Continued

[Site IDs and well number correspond to locations in Appendix 1, a site ID and well number is listed for each drilled interval for a particular drill hole. For convenience, the site ID and well numbers are repeated at the shallowest drilled interval for each well. leaders $1-$

\begin{tabular}{|c|c|c|c|c|c|c|c|c|c|c|c|c|c|}
\hline Site ID & Well number & $\begin{array}{l}\text { Site ID, } \\
\text { top of } \\
\text { well }\end{array}$ & $\begin{array}{l}\text { Well number, } \\
\text { top of well }\end{array}$ & $\begin{array}{c}\text { Top of } \\
\text { interval, in } \\
\text { feet }\end{array}$ & $\begin{array}{c}\text { Base of } \\
\text { interval, in } \\
\text { feet }\end{array}$ & $\begin{array}{c}\text { Top of } \\
\text { interval, in } \\
m\end{array}$ & $\begin{array}{c}\text { Base of } \\
\text { interval, in } \\
\mathrm{m}\end{array}$ & Reported lithologic unit ${ }^{1}$ & Interpreted lithologic class ${ }^{2}$ & $\begin{array}{c}\text { Reported } \\
\text { degree of } \\
\text { cementation or } \\
\text { induration }^{3}\end{array}$ & $\begin{array}{c}\text { Fossils } \\
\text { reported in } \\
\text { interval }^{4}\end{array}$ & $\begin{array}{c}\text { Organic } \\
\text { matter } \\
\text { reported in } \\
\text { interval }^{5}\end{array}$ & $\begin{array}{l}\text { Reported stratigraphic top of geologic } \\
\text { unit }^{6}\end{array}$ \\
\hline 158 & $7 / 8-24 \mathrm{~A} 4$ & & & 126 & 132 & 38.4 & 40.2 & coarse gravel & Gravel & -- & -- & -- & \\
\hline 158 & $7 / 8-24 \mathrm{~A} 4$ & & & 132 & 142 & 40.2 & 43.3 & clay and gravel, boulders & Clay and gravel & -- & -- & -- & \\
\hline 158 & $7 / 8-24 A 4$ & & & 142 & 146 & 43.3 & 44.5 & loose gravel & Gravel & -- & -. & -- & \\
\hline 158 & $7 / 8-24 \mathrm{~A} 4$ & & & 146 & 163 & 44.5 & 49.7 & sand and gravel & Sand (or sandstone) and gravel & -- & -- & -- & QTge (?) and QTm \\
\hline 158 & 7/8-24A4 & & & 163 & 181 & 49.7 & 55.2 & volcanic gravel & Gravel & -- & -- & -- & \\
\hline 158 & 7/8-24A4 & & & 181 & 196 & 55.2 & 59.7 & solidified sand & Sand & -- & -- & -- & \\
\hline 158 & 7/8-24A4 & & & 196 & 207 & 59.7 & 63.1 & basalt and boulders & Basalt & -- & -. & -- & \\
\hline 158 & $7 / 8-24 \mathrm{~A} 4$ & & & 207 & 227 & 63.1 & 69.2 & sand and gravel solidified & Sand (or sandstone) and gravel & -- & -- & -- & \\
\hline 158 & $7 / 8-24 \mathrm{~A} 4$ & & & 227 & 239 & 69.2 & 72.8 & gravel and boulders & Gravel & -- & -- & -- & \\
\hline 158 & $7 / 8-24 \mathrm{~A} 4$ & & & 239 & 252 & 72.8 & 76.8 & sand solidified & Sandstone & -- &.- & -- & \\
\hline 158 & 7/8-24A4 & & & 252 & 255 & 76.8 & 77.7 & gravel and boulders & Gravel & -- & -- & -- & \\
\hline 158 & 7/8-24A4 & & & 255 & 261 & 77.7 & 79.6 & sand and gravel solidified & Sand (or sandstone) and gravel & -- & -- & -- & \\
\hline 158 & $7 / 8-24 \mathrm{~A} 4$ & & & 261 & 327 & 79.6 & 99.7 & cemented gravel & Gravel & cemented & -- & -- & \\
\hline 158 & 7/8-24A4 & & & 327 & 334 & 99.7 & 101.8 & brown clay & Clay & -- & -- & -- & \\
\hline 158 & $7 / 8-24 \mathrm{~A} 4$ & & & 334 & $\begin{array}{l}304 \\
363\end{array}$ & 101.8 & 110.6 & gravel & Gravel & -. & -. & -- & \\
\hline 158 & 7/8-24A4 & & & 363 & 391 & 110.6 & 119.2 & blue clay, sticky & Clay & -- & - & -- & \\
\hline 158 & 7/8-24A4 & & & 391 & 428 & 119.2 & 130.5 & gravel and boulders, cemented & Gravel & cemented & -. & -- & \\
\hline 158 & $7 / 8-24 \mathrm{~A} 4$ & & & 428 & 441 & 130.5 & 134.4 & black shale & Clay and sand (or sandstone) & cemented & -- & -- & \\
\hline 158 & 7/8-24A4 & & & 441 & 462 & 134.4 & 140.8 & gravel, cemented & Gravel & cemented & -- & -- & \\
\hline $\begin{array}{l}150 \\
158\end{array}$ & $7 / 8-24 \mathrm{~A} 4$ & & & 462 & 530 & $\begin{array}{l}143.4 \\
140.8\end{array}$ & $\begin{array}{l}161.5 \\
161.5\end{array}$ & clay and boulders & Clay and gravel & -. & -- & -- & \\
\hline 158 & $7 / 8-24 \mathrm{~A} 4$ & & & 530 & 554 & 161.5 & 168.9 & boulders, cemented & Gravel & cemented & -- & -- & \\
\hline 158 & $7 / 8-24 \mathrm{~A} 4$ & & & 554 & 606 & 168.9 & 184.7 & blue clay, very tough and sticky & Clay & -. & -. & -. & \\
\hline 158 & $7 / 8-24 A 4$ & & & 606 & 642 & 184.7 & 195.7 & volcanic ash & Ash or tuff & -- & -- & -- & Tsv \\
\hline 158 & 7/8-24A4 & & & 642 & 692 & 195.7 & 210.9 & lava and basalt trap & Basalt & -- & -. & -- & \\
\hline 158 & 7/8-24A4 & & & 692 & 702 & 210.9 & 214.0 & blue rock & Sandstone & hard & ..- & .- & \\
\hline 158 & $7 / 8-24 \mathrm{~A} 4$ & & & 702 & 709 & 214.0 & 216.1 & volcanic ash, red & Ash or tuff & -- & -- & -- & \\
\hline 158 & $7 / 8-24 \mathrm{~A} 4$ & & & 709 & 734 & 216.1 & 223.7 & rock, blue & Sandstone & hard & .- & .. & \\
\hline 158 & 7/8-24A4 & & & 734 & 745 & 223.7 & 227.1 & $\begin{array}{l}\text { clay, blue, and boulders }\end{array}$ & Clay and gravel & -- & -- & -- & \\
\hline 158 & 7/8-24A4 & & & 745 & 755 & 227.1 & 230.1 & rock & Ash or tuff & hard & .. & -- & \\
\hline 158 & 7/8-24A4 & & & 755 & 770 & 230.1 & 234.7 & clay, blue & Clay & - & .. & .. & \\
\hline 158 & $7 / 8-24 \mathrm{~A} 4$ & & & 770 & 810 & 234.7 & 246.9 & gravel cemented & Gravel & cemented & - & -- & \\
\hline 158 & 7/8-24A4 & & & 810 & 842 & 246.9 & 256.6 & limestone & Clay and sand (or sandstone) & -- & -- & -. & \\
\hline 158 & 7/8-24A4 & & & 842 & 850 & 256.6 & 259.1 & sandstone & Sandstone & cemented & -- & -- & \\
\hline 158 & $7 / 8-24 \mathrm{~A} 4$ & & & 850 & 888 & 259.1 & 270.7 & volcanic clay, red & Ash or tuff & - & $\ldots$ & - & \\
\hline 158 & $7 / 8-24 \mathrm{~A} 4$ & & & 888 & 931 & 270.7 & 283.8 & rock, blue & Sandstone & hard & -. & .- & \\
\hline 158 & 7/8-24A4 & & & 931 & 935 & 283.8 & 285.0 & volcanic ash, red & Ash or tuff & -.- & .. & .. & \\
\hline 158 & $7 / 8-24 \mathrm{~A} 4$ & & & 935 & 940 & 285.0 & 286.5 & clay, red, and boulders & Clay and gravel & -- & -- & -- & \\
\hline 158 & $7 / 8-24 \mathrm{~A} 4$ & & & 940 & 975 & 286.5 & 297.2 & rock & Ash or tuff & hard & -- & -- & \\
\hline 158 & $7 / 8-24 \mathrm{~A} 4$ & & & 975 & 982 & 297.2 & 299.3 & clay, blue, and boulders & Clay and gravel & -.- & -. & - & \\
\hline 158 & $7 / 8-24 \mathrm{~A} 4$ & & & 982 & 998 & 299.3 & 304.2 & clay, blue and gray & Clay & -- & -- & -- & \\
\hline 158 & $7 / 8-24 \mathrm{~A} 4$ & & & 998 & 1000 & 304.2 & 304.8 & $\begin{array}{l}\text { rock } \\
\text { soil (described in log of well } 7 / 7 \text { - } \\
24 \mathrm{~B} 1 \text { as vellow clay and boulders, }\end{array}$ & Ash or tuff & hard & -- & - & \\
\hline & 7172401 & & & & 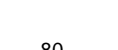 & & & $1-18$ feet, and brown clay and & & & & & \\
\hline $\begin{array}{l}159 \\
159\end{array}$ & $\begin{array}{l}7 / 7-24 \mathrm{~A} 1 \\
717-24 \mathrm{~A} 1\end{array}$ & 159 & 7/7-24A1 & $\begin{array}{l}0 \\
80\end{array}$ & $\begin{array}{l}80 \\
120\end{array}$ & $\begin{array}{l}0.0 \\
24.4\end{array}$ & & gravel, 18-90 feet) & $\begin{array}{l}\text { Clay, sand, and gravel } \\
\text { Ash or tuff }\end{array}$ & $\begin{array}{c}-- \\
\text { hard }\end{array}$ & -- & - & $\begin{array}{l}\text { QTge } \\
\text { Tsv }\end{array}$ \\
\hline $\begin{array}{l}159 \\
159\end{array}$ & $\begin{array}{l}\text { 7/7-24A1 } \\
7 / 7-24 \mathrm{~A} 1\end{array}$ & & & $\begin{array}{l}80 \\
120\end{array}$ & $\begin{array}{l}120 \\
208\end{array}$ & $\begin{array}{l}24.4 \\
36.6\end{array}$ & $\begin{array}{l}36.6 \\
63.4\end{array}$ & $\begin{array}{l}\text { rock, soft, brown } \\
\text { soft red rock }\end{array}$ & $\begin{array}{l}\text { Ash or tuff } \\
\text { Ash or tuff }\end{array}$ & $\begin{array}{c}\text { hard } \\
\text {-. }\end{array}$ & -- & $\overline{-}$ & $1 \mathrm{sV}$ \\
\hline 159 & $7 / 7-24 \mathrm{~A} 1$ & & & 208 & 222 & 63.4 & 67.7 & dark brown rock & Ash or tuff & -- & -. & -- & \\
\hline 159 & 7/7-24A1 & & & 222 & 281 & 67.7 & 85.6 & hard red rock with many seams & Ash or tuff & -- & -- & -- & \\
\hline 159 & $7 / 7-24 \mathrm{~A} 1$ & & & 281 & 434 & 85.6 & 132.3 & $\begin{array}{l}\text { hard brown rock with many seams } \\
\text { soft yellow-brown rock with many }\end{array}$ & Ash or tuff & hard & -- & -- & \\
\hline 159 & 7/7-24A1 & & & 434 & 488 & 132.3 & 148.7 & seams & Ash or tuff & -- & -- & -. & \\
\hline 159 & 7/7-24A1 & & & 488 & 580 & 148.7 & 176.8 & hard red rock, very seamy & Ash or tuff & -- & -- & -- & \\
\hline 159 & 7/7-24A1 & & & 580 & 620 & 176.8 & 189.0 & harder red rock, very seamy & Ash or tuff & hard & - & -- & \\
\hline 159 & 7/7-24A1 & & & 620 & 622 & 189.0 & 189.6 & very hard red rock, very seamy & Ash or tuff & hard & -. & -. & \\
\hline 160 & $7 / 9-21 \mathrm{G} 2$ & 160 & 7/9-21G2 & 0 & 240 & 0.0 & 73.2 & no data & No data & -. & .- & .. & -- \\
\hline 161 & $7 / 7-23 \mathrm{~A} 2$ & 161 & $7 / 7-23 \mathrm{~A} 2$ & 0 & 2 & 0.0 & 0.6 & topsoil & Sand and clay & -. & - & - & Qyal and QTge \\
\hline 161 & 7/7-23A2 & & & 2 & 179 & 0.6 & 54.6 & clay, yellow, and gravel & Clay and gravel & -- & -- & -- & \\
\hline 161 & $7 / 7-23 \mathrm{~A} 2$ & & & 179 & 202 & 54.6 & 61.6 & $\begin{array}{l}\text { clay, blue, and gravel } \\
\text { one }\end{array}$ & Clay and gravel & -- & -. & -- & \\
\hline 161 & 7/7-23A2 & & & 202 & 250 & 61.6 & 76.2 & clay, yellow, and gravel & Clay and gravel & -- & -- & -- & \\
\hline
\end{tabular}


Appendix 3. Lithologic data. - Continued

[Site IDs and well number correspond to locations in Appendix 1, a site ID and well number is listed for each drilled interval for a particular drill hole. For convenience, the site ID and well numbers are repeated at the shallowest drilled interval for each well. leaderst.

\begin{tabular}{|c|c|c|c|c|c|c|c|c|c|c|c|c|c|}
\hline Site ID & Well number & $\begin{array}{l}\text { Site ID, } \\
\text { top of } \\
\text { well }\end{array}$ & $\begin{array}{l}\text { Well number, } \\
\text { top of well }\end{array}$ & $\begin{array}{l}\text { Top of } \\
\text { interval, in } \\
\text { feet }\end{array}$ & $\begin{array}{l}\text { Base of } \\
\text { interval, in } \\
\text { feet }\end{array}$ & $\begin{array}{c}\text { Top of } \\
\text { interval, in } \\
m\end{array}$ & $\begin{array}{c}\text { Base of } \\
\text { interval, in } \\
\mathrm{m}\end{array}$ & Reported lithologic unit' & Interpreted lithologic class ${ }^{2}$ & $\begin{array}{c}\text { Reported } \\
\text { degree of } \\
\text { cementation or } \\
\text { induration }^{3}\end{array}$ & $\begin{array}{l}\text { Fossils } \\
\text { reported in } \\
\text { interval }^{4}\end{array}$ & $\begin{array}{c}\text { Organic } \\
\text { matter } \\
\text { reported in } \\
\text { interval }^{5}\end{array}$ & $\begin{array}{l}\text { Reported stratigraphic top of geologic } \\
\text { unit }^{6}\end{array}$ \\
\hline 161 & $717-23 \mathrm{~A} 2$ & & & 250 & 256 & 76.2 & 78.0 & gravel & Gravel & -- & -- & -- & \\
\hline 161 & $7 / 7-23 \mathrm{~A} 2$ & & & 256 & 280 & 78.0 & 85.3 & clay, yellow, and gravel & Clay and gravel & -- & -- & -- & \\
\hline 161 & 7/7-23A2 & & & 280 & 304 & 85.3 & 92.7 & clay, yellow & Clay & -- & -- & -- & \\
\hline 161 & $7 / 7-23 A 2$ & & & 304 & 337 & 92.7 & 102.7 & $\begin{array}{l}\text { clay, yellow, and gravel } \\
\text { chas }\end{array}$ & Clay and gravel & -- & -- & -- & \\
\hline 162 & 7/9-22F1 & 162 & 7/9-22F1 & 0 & 42 & 0.0 & 12.8 & no data & No data & .- & .- & .- & .- \\
\hline 162 & 7/9-22F1 & & & 42 & 257 & 12.8 & 78.3 & fine sand & Sand & -- & -- & -- & QTm \\
\hline 162 & $7 / 9-22 F 1$ & & & 257 & 383 & 78.3 & 116.7 & no data & No data & -- & -- & -- & \\
\hline 162 & $7 / 9-22 F 1$ & & & 383 & 400 & 116.7 & 121.9 & small boulders with shells & Gravel & .- & shells & -- & \\
\hline 162 & $7 / 9-22 F 1$ & & & 400 & 481 & 121.9 & 146.6 & no data & No data & -- & -- & -- & \\
\hline 162 & 7/9-22F1 & & & 481 & 512 & 146.6 & 156.1 & small boulders with shells & Gravel & -- & shells & -- & \\
\hline 162 & 7/9-22F1 & & & 512 & 517 & 156.1 & 157.6 & no data & No data & -- & -- & -- & \\
\hline 162 & 7/9-22F1 & & & 517 & 575 & 157.6 & 175.3 & fine gravel; coarse gravel & Gravel & -- & -- & -- & \\
\hline 163 & 7/9-22F2 & 163 & 7/9-22F2 & 0 & 380 & 0.0 & 115.8 & (no perforations) & No data & .- & -- & -- & QTm \\
\hline 164 & 7/9-21F1 & 164 & 7/9-21F1 & 0 & 320 & 0.0 & 97.5 & no data & No data & .- & .- & .- & OTm \\
\hline 165 & 7/9-21G3 & 165 & 7/9-21G3 & 0 & 390 & 0.0 & 118.9 & no data & No data & -- & -- & -- & -- \\
\hline 165 & $7 / 9-21 \mathrm{G} 3$ & & & 390 & 408 & 118.9 & 124.4 & sandstone & Sandstone & cemented & -- & .- & $\mathrm{QTm}$ \\
\hline 166 & $7 / 7-23 \mathrm{G} 1$ & 166 & 7/7-23G1 & 0 & 58 & 0.0 & 17.7 & clay, yellow & Clay & -- & -- & -- & Qyal and Qoal (?) and QTge \\
\hline 166 & 7/7-23G1 & & & 58 & 86 & 17.7 & 26.2 & blue shale & Clay and sand (or sandstone) & cemented & .- & .- & \\
\hline 166 & 7/7-23G1 & & & 86 & 115 & 26.2 & 35.1 & yellow clay & Clay & -. & .- & .- & \\
\hline 166 & 7/7-23G1 & & & 115 & 126 & 35.1 & 38.4 & blue clay & Clay & -- & -- & -- & \\
\hline 166 & $7 / 7-23 \mathrm{G} 1$ & & & 126 & 146 & 38.4 & 44.5 & blue clay & Clay & -- & -- & -- & \\
\hline 166 & 7/7-23G1 & & & 146 & 150 & 44.5 & 45.7 & blue shale and gravel & Clay, sand, and gravel & cemented & -- & -- & \\
\hline 166 & 7/7-23G1 & & & 150 & 155 & 45.7 & 47.2 & $\begin{array}{l}\text { blue shale } \\
\text { braver }\end{array}$ & Clay and sand (or sandstone) & cemented & -- & -- & \\
\hline 166 & 7/7-23G1 & & & 155 & 214 & 47.2 & 65.2 & shale and gravel & Clay and gravel & cemented & .- & .- & \\
\hline 166 & $7 / 7-23 \mathrm{G} 1$ & & & 214 & 245 & 65.2 & 74.7 & $\begin{array}{l}\text { shale and sand } \\
\text { sand }\end{array}$ & Clay and sand (or sandstone) & cemented & -- & -- & \\
\hline 166 & $7 / 7-23 \mathrm{G} 1$ & & & 245 & 265 & 74.7 & 80.8 & yellow clay and coarse gravel & Clay and gravel & -- & -- & -- & \\
\hline 166 & 7/7-23G1 & & & 265 & 278 & 80.8 & 84.7 & hardpan boulders & Clay and gravel & hard & -- & -- & \\
\hline 166 & $7 / 7-23 \mathrm{G} 1$ & & & 278 & 300 & 84.7 & $\begin{array}{l}94.1 \\
91.4\end{array}$ & shale rock & Clay and sand (or sandstone) & cemented & -- & -- & \\
\hline 166 & 7/7-23G1 & & & 300 & 302 & 91.4 & 92.0 & blue shale & Clay and sand (or sandstone) & cemented & -- & -- & \\
\hline 166 & 7/7-23G1 & & & 302 & 345 & 92.0 & 105.2 & shale rock & Clay and sand (or sandstone) & cemented & -- & -- & \\
\hline 166 & 7/7-23G1 & & & 345 & 349 & 105.2 & 106.4 & blue shale & Clay and sand (or sandstone) & cemented & -- & -- & \\
\hline 166 & 7/7-23G1 & & & 349 & 370 & 106.4 & 112.8 & volcanic clay and rock & Ash or tuff & -- & -- & -- & \\
\hline 166 & 7/7-23G1 & & & 370 & 379 & 112.8 & 115.5 & gravel cement & Gravel & cemented & -- & -. & \\
\hline 166 & 7/7-23G1 & & & 379 & 417 & 115.5 & 127.1 & gravel and shale & Clay and gravel & cemented & -- & -- & \\
\hline 166 & 7/7-23G1 & & & 417 & 445 & 127.1 & 135.6 & blue shale & Clay and sand (or sandstone) & cemented & .- & .- & \\
\hline 166 & $7 / 7-23 \mathrm{G} 1$ & & & 445 & 457 & 135.6 & 139.3 & yellow clay & Clay & -- & -- & -- & \\
\hline 166 & 7/7-23G1 & & & 457 & 510 & 139.3 & 155.4 & blue shale & Clay and sand (or sandstone) & cemented & -- & -- & \\
\hline 166 & 7/7-23G1 & & & 510 & 515 & 155.4 & $\begin{array}{l}153.4 \\
157.0\end{array}$ & blue clay & Clay & .- & -- & -. & \\
\hline 166 & 7/7-23G1 & & & 515 & 561 & 157.0 & 171.0 & shale & Clay and sand (or sandstone) & cemented & -- & -- & \\
\hline 166 & $7 / 7-23 \mathrm{G} 1$ & & & 561 & 576 & 171.0 & 175.6 & shale rock & Clay and sand (or sandstone) & $\begin{array}{l}\text { cemented } \\
\text { comed }\end{array}$ & -- & -- & \\
\hline 166 & 7/7-23G1 & & & 576 & 584 & 175.6 & 178.0 & very hard rock and gravel & Ash or tuff & hard & -- & -- & \\
\hline 166 & 7/7-23G1 & & & 584 & 645 & 178.0 & 196.6 & shale formation & Clay and sand (or sandstone) & cemented & -- & -- & \\
\hline $\begin{array}{l}100 \\
166\end{array}$ & 7/7-23G1 & & & $\begin{array}{l}584 \\
645\end{array}$ & 672 & 196.6 & $\begin{array}{l}204.8 \\
204.8\end{array}$ & gravel cement very hard & Gravel & $\begin{array}{l}\text { cemented } \\
\text { conten }\end{array}$ & -- & -- & \\
\hline 166 & 7/7-23G1 & & & 672 & 840 & 204.8 & 256.0 & no record & No data & -- & -- & -- & \\
\hline 166 & $7 / 7-23 \mathrm{G} 1$ & & & 840 & 870 & 256.0 & 265.2 & coarse gravel & Gravel & -- & -- & -- & \\
\hline 166 & 7/7-23G1 & & & 870 & 885 & 265.2 & 269.7 & boulders, sand, and gravel & Sand (or sandstone) and gravel & -- & -- & -- & \\
\hline 166 & 7/7-23G1 & & & 885 & 910 & 269.7 & 277.4 & boulders and gravel & Gravel & -- & -- & -- & \\
\hline 166 & 7/7-23G1 & & & 910 & 985 & 277.4 & 300.2 & fractured basalt rock & Basalt & hard & .- & .- & Tsv \\
\hline 166 & 7/7-23G1 & & & 985 & 1005 & 300.2 & 306.3 & blue shale and gravel & Clay, sand, and gravel & cemented & -- & -- & \\
\hline 166 & 7/7-23G1 & & & 1005 & 1015 & 306.3 & 309.4 & boulders & Gravel & -- & -- & .- & \\
\hline 166 & 7/7-23G1 & & & 1015 & 1025 & 309.4 & 312.4 & gray shale and boulders & Clay and gravel & cemented & -- & -- & \\
\hline 166 & 7/7-23G1 & & & 1025 & 1055 & 312.4 & 321.6 & $\begin{array}{l}\text { fine gravel } \\
\text { fould }\end{array}$ & Gravel & -- & -- & -- & \\
\hline 166 & 7/7-23G1 & & & 1055 & 1065 & 321.6 & 324.6 & boulders and fine gravel & Gravel & .- & .- & .- & \\
\hline 166 & 7/7-23G1 & & & 1065 & 1085 & 324.6 & 330.7 & sandy clay & Clay and sand (or sandstone) & -- & -. & -- & \\
\hline 166 & 7/7-23G1 & & & 1085 & 1090 & 330.7 & 332.2 & boulders, lime & Gravel & .- & -- & .- & \\
\hline 166 & 7/7-23G1 & & & 1090 & 1100 & 332.2 & 335.3 & sandy clay & Clay and sand (or sandstone) & -- & -- & -- & \\
\hline 166 & 7/7-23G1 & & & 1100 & 1170 & 335.3 & 356.6 & gray shale & Clay and sand (or sandstone) & cemented & -- & -- & \\
\hline 167 & 7/9-21G1 & 167 & 7/9-21G1 & 0 & 2 & 0.0 & 0.6 & soil, sandy & Sand and clay & -. & .- & .- & QTm \\
\hline 167 & $7 / 9-21 \mathrm{G} 1$ & & & 2 & 56 & 0.6 & 17.1 & clay, yellow, sandy & Clay and sand (or sandstone) & -. & -- & -- & \\
\hline 167 & 7/9-21G1 & & & 56 & 173 & 17.1 & 52.7 & sand, yellow & Sand & -- & -- & -- & \\
\hline 167 & 7/9-21G1 & & & 173 & 185 & 52.7 & 56.4 & clay, yellow, sandy & Clay and sand (or sandstone) & -- & -- & -- & \\
\hline
\end{tabular}


Appendix 3. Lithologic data. - Continued

[Site IDs and well number correspond to locations in Appendix 1, a site ID and well number is listed for each drilled interval for a particular drill hole. For convenience, the site ID and well numbers are repeated at the shallowest drilled interval for each well. leaders

\begin{tabular}{|c|c|c|c|c|c|c|c|c|c|c|c|c|c|}
\hline Site ID & Well number & $\begin{array}{l}\text { Site ID, } \\
\text { top of } \\
\text { well }\end{array}$ & $\begin{array}{l}\text { Well number, } \\
\text { top of well }\end{array}$ & $\begin{array}{l}\text { Top of } \\
\text { interval, in } \\
\text { feet }\end{array}$ & $\begin{array}{l}\text { Base of } \\
\text { interval, in } \\
\text { feet }\end{array}$ & $\begin{array}{c}\text { Top of } \\
\text { interval, in } \\
\mathrm{m}\end{array}$ & $\begin{array}{c}\text { Base of } \\
\text { interval, in } \\
\mathrm{m}\end{array}$ & Reported lithologic unit' & Interpreted lithologic class ${ }^{2}$ & $\begin{array}{c}\text { Reported } \\
\text { degree of } \\
\text { cementation or } \\
\text { induration }^{3} \\
\end{array}$ & $\begin{array}{l}\text { Fossils } \\
\text { reported in } \\
\text { interval }^{4}\end{array}$ & $\begin{array}{c}\text { Organic } \\
\text { matter } \\
\text { reported in } \\
\text { interval }^{5}\end{array}$ & $\begin{array}{l}\text { Reported stratigraphic top of geologic } \\
\text { unit }^{6}\end{array}$ \\
\hline 167 & $7 / 9-21 \mathrm{G1}$ & & & 185 & 186 & 56.4 & 56.7 & clay, blue, sandy & Clay and sand (or sandstone) & -- & -- & -- & \\
\hline 167 & 7/9-21G1 & & & 186 & 205 & 56.7 & 62.5 & sand, blue & Sand & -- & -- & -- & \\
\hline 167 & 7/9-21G1 & & & 205 & 244 & 62.5 & 74.4 & sand, blue, and clam shells & Sand & -- & shells & -- & \\
\hline 167 & 7/9-21G1 & & & 244 & 270 & 74.4 & 82.3 & $\begin{array}{l}\text { sand, blue } \\
\text { sullo }\end{array}$ & Sand & -- & -- & -- & \\
\hline 167 & $7 / 9-21 \mathrm{G} 1$ & & & 270 & 316 & 82.3 & 96.3 & sand, blue, and clam shells & Sand & -- & shells & -- & \\
\hline 167 & 7/9-21G1 & & & 316 & 463 & 96.3 & 141.1 & sand, blue & Sand & -- & -. & -- & \\
\hline 167 & $7 / 9-21 \mathrm{G} 1$ & & & 463 & 471 & 141.1 & 143.6 & sand, blue, and clam shells & Sand & -- & shells & -- & \\
\hline 167 & 7/9-21G1 & & & 471 & 496 & 143.6 & 151.2 & sandstone, blue & Sandstone & cemented & - & -- & \\
\hline 167 & $7 / 9-21 \mathrm{G} 1$ & & & 496 & 499 & 151.2 & 152.1 & sand, yellow & Sand & - & -- & -- & \\
\hline 167 & $7 / 9-21 \mathrm{G} 1$ & & & 499 & 504 & 152.1 & 153.6 & sandstone, blue & Sandstone & cemented & -- & -- & \\
\hline 167 & 7/9-21G1 & & & 504 & 514 & 153.6 & 156.7 & sand, yellow & Sand & -- & -- & -- & \\
\hline 167 & $7 / 9-21 \mathrm{G} 1$ & & & 514 & 530 & 156.7 & 161.5 & clay, blue, sandy & Clay and sand (or sandstone) & -- & .- & .- & \\
\hline 168 & 7/9-21J1 & 168 & 7/9-21J1 & 0 & 195 & 0.0 & 59.4 & sandstone & Sandstone & cemented & -- & -- & QTm \\
\hline 168 & 7/9-21J1 & & & 195 & 205 & 59.4 & 62.5 & sandstone & Sandstone & cemented & -- & -- & $\mathrm{QTm}$ \\
\hline 169 & 7/9-23К1 & 169 & 7/9-23K1 & 0 & 200 & 0.0 & 61.0 & no data & No data & -- & -- & -- & QTge and QTm \\
\hline 170 & 7/9-24L2 & 170 & 7/9-24L2 & 0 & 330 & 0.0 & 100.6 & no data & No data & -- & -- & -- & QTge and QTm (?) \\
\hline 171 & 7/8-23L1 & 171 & 7/8-23L1 & 0 & 48 & 0.0 & 14.6 & no data & No data & -- & -- & -- & -- \\
\hline 171 & 7/8-23L1 & & & 48 & 52 & 14.6 & 15.8 & gravel & Gravel & -- & -- & -- & QTge \\
\hline 171 & 7/8-23L1 & & & 52 & 132 & 15.8 & 40.2 & no data & No data & -- & -- & -- & \\
\hline 171 & 7/8-23L1 & & & 132 & 133 & 40.2 & 40.5 & gravel & Gravel & -- & -- & -- & \\
\hline 171 & 7/8-23L1 & & & 133 & 273 & 40.5 & 83.2 & no data & No data & -- & -- & -- & \\
\hline 171 & 7/8-23L1 & & & 273 & 278 & 83.2 & 84.7 & gravel & Gravel & -- & -- & -- & \\
\hline 171 & 7/8-23L1 & & & 278 & 300 & 84.7 & 91.4 & no data & No data & -- & -- & -- & \\
\hline 172 & $7 / 8-24 \mathrm{~K} 1$ & 172 & $7 / 8-24 \mathrm{~K} 1$ & 0 & 234 & 0.0 & 71.3 & no data & No data & -- & -- & -- & -- \\
\hline 172 & 7/8-24K1 & & & 234 & 236 & 71.3 & 71.9 & coarse sand and gravel & Sand (or sandstone) and gravel & -- & -- & -- & QTge \\
\hline 172 & 7/8-24K1 & & & 236 & 238 & 71.9 & 72.5 & no data & No data & .- & -- & -- & \\
\hline 173 & 7/8-23M3 & 173 & 7/8-23M3 & 0 & 300 & 0.0 & 91.4 & no data & No data & -- & -- & -- & -- \\
\hline 174 & $7 / 8-22 \mathrm{~K} 1$ & 174 & $7 / 8-22 \mathrm{~K} 1$ & 0 & 285 & 0.0 & 86.9 & no data & No data & .- & -- & -- & QTge \\
\hline 175 & 7/8-23M4 & 175 & 7/8-23M4 & 0 & 24 & 0.0 & 7.3 & clay, sticky & Clay & -- & -- & -- & Qyal and Qoal (?) and QTge \\
\hline 175 & 7/8-23M4 & & & 24 & 32 & 7.3 & 9.8 & cemented gravel & Gravel & cemented & -- & -- & \\
\hline 175 & 7/8-23M4 & & & 32 & 43 & 9.8 & 13.1 & clay & Clay & -- & -- & -- & \\
\hline 175 & 7/8-23M4 & & & 43 & 58 & 13.1 & 17.7 & gravel & Gravel & -- & -- & -- & \\
\hline 175 & 7/8-23M4 & & & 58 & 65 & 17.7 & 19.8 & clay & Clay & -- & -- & -- & \\
\hline 175 & 7/8-23M4 & & & 65 & 135 & 19.8 & 41.1 & clay and gravel & Clay and gravel & -- & -- & .- & \\
\hline 175 & 7/8-23M4 & & & 135 & 145 & 41.1 & 44.2 & blue clay & Clay & -- & -- & -- & \\
\hline 175 & 7/8-23M4 & & & 145 & 153 & 44.2 & 46.6 & clay and gravel & Clay and gravel & -- & -- & -- & \\
\hline 175 & 7/8-23M4 & & & 153 & 156 & 46.6 & 47.5 & sandstone & Sandstone & cemented & -- & -- & QTge (?) and QTm (?) \\
\hline 175 & 7/8-23M4 & & & 156 & 202 & 47.5 & 61.6 & clay with gravel & Clay and gravel & -- & -- & -- & \\
\hline 175 & 7/8-23M4 & & & 202 & 210 & 61.6 & 64.0 & $\begin{array}{l}\text { sandstone } \\
\text { hard and soft clay and gravel }\end{array}$ & Sandstone & cemented & -- & -- & \\
\hline 175 & 7/8-23M4 & & & 210 & 303 & 64.0 & 92.4 & layers & Clay and gravel & hard & -- & -- & \\
\hline 175 & $7 / 8-23 M 4$ & & & 303 & 310 & 92.4 & 94.5 & $\begin{array}{l}\text { sandstone } \\
\text { hard and soft clay with gravel }\end{array}$ & Sandstone & cemented & -- & -- & \\
\hline 175 & 7/8-23M4 & & & 310 & 412 & 94.5 & 125.6 & layers & Clay and gravel & hard & -- & -- & \\
\hline 175 & 7/8-23M4 & & & 412 & 423 & 125.6 & 128.9 & $\begin{array}{l}\text { sandstone } \\
\text { blue and yellow clay with small }\end{array}$ & Sandstone & cemented & -- & -- & \\
\hline 175 & 7/8-23M4 & & & 423 & 575 & 128.9 & 175.3 & amounts of gravel & Clay and gravel & .- & -- & .- & \\
\hline 175 & 7/8-23M4 & & & 575 & 590 & 175.3 & 179.8 & volcanic ash & Ash or tuff & -- & -- & -- & \\
\hline 175 & 7/8-23M4 & & & 590 & 650 & 179.8 & 198.1 & blue shale & Clay and sand (or sandstone) & cemented & -- & -- & \\
\hline 175 & 7/8-23M4 & & & 650 & 655 & 198.1 & 199.6 & clay and gravel & Clay and gravel & -- & -- & -- & \\
\hline 175 & 7/8-23M4 & & & 655 & 670 & 199.6 & 204.2 & $\begin{array}{l}\text { volcanic rock } \\
\text { blue clay, shale, and minor }\end{array}$ & Ash or tuff & -- & -- & -- & Tsv (?) and QTm (?) \\
\hline 175 & 7/8-23M4 & & & 670 & 805 & 204.2 & 245.4 & amounts of gravel & Clay, sand, and trace gravel & cemented & -- & -- & \\
\hline 175 & 7/8-23M4 & & & 805 & 820 & 245.4 & 249.9 & lava rock & Basalt & hard & -- & -- & \\
\hline 175 & 7/8-23M4 & & & 820 & 830 & 249.9 & 253.0 & blue clay & Clay & -- & -- & -- & \\
\hline 175 & 7/8-23M4 & & & 830 & 835 & 253.0 & 254.5 & lava rock & Basalt & hard & -- & -- & \\
\hline 175 & $7 / 8-23 \mathrm{M} 4$ & & & 835 & 842 & 254.5 & 256.6 & $\begin{array}{l}\text { clay and lava } \\
\text { blue clay with "iron" deposit 850- }\end{array}$ & Clay and gravel & -- & -- & -- & \\
\hline 175 & 7/8-23M4 & & & 842 & 878 & 256.6 & 267.6 & 854 & Clay & -- & -- & -- & \\
\hline 175 & 7/8-23M4 & & & 878 & 883 & 267.6 & 269.1 & cement gravel, water & Gravel & cemented & -- & -- & \\
\hline 175 & 7/8-23M4 & & & 883 & 920 & 269.1 & 280.4 & blue clay with gravel & Clay and gravel & -- & -- & -- & \\
\hline
\end{tabular}


Appendix 3. Lithologic data. - Continued

ISite IDs and well number correspond to locations in Appendix 1, a site ID and well number is listed for each drilled interval for a particular drill hole. For convenience, the site ID and well numbers are repeated at the shallowest drilled interval for each well. leaders $(-1$

\begin{tabular}{|c|c|c|c|c|c|c|c|c|c|c|c|c|c|}
\hline Site ID & Well number & $\begin{array}{l}\text { Site ID, } \\
\text { top of } \\
\text { well }\end{array}$ & $\begin{array}{l}\text { Well number, } \\
\text { top of well }\end{array}$ & $\begin{array}{c}\text { Top of } \\
\text { interval, in } \\
\text { feet }\end{array}$ & $\begin{array}{c}\text { Base of } \\
\text { interval, in } \\
\text { feet }\end{array}$ & $\begin{array}{c}\text { Top of } \\
\text { interval, in } \\
m\end{array}$ & $\begin{array}{c}\text { Base of } \\
\text { interval, in } \\
\mathrm{m}\end{array}$ & Reported lithologic unit ${ }^{1}$ & Interpreted lithologic class ${ }^{2}$ & $\begin{array}{c}\text { Reported } \\
\text { degree of } \\
\text { cementation or } \\
\text { induration }^{3}\end{array}$ & $\begin{array}{c}\text { Fossils } \\
\text { reported in } \\
\text { interval }^{4}\end{array}$ & $\begin{array}{c}\text { Organic } \\
\text { matter } \\
\text { reported in } \\
\text { interval }^{5}\end{array}$ & $\begin{array}{l}\text { Reported stratigraphic top of geologic } \\
\text { unit }^{6}\end{array}$ \\
\hline 175 & $7 / 8-23 \mathrm{M} 4$ & & & 920 & 925 & 280.4 & 281.9 & $\begin{array}{l}\text { clay, sand, and gravel } \\
\text { blue clay with some gravel and }\end{array}$ & Clay, sand, and gravel & -- & -- & -- & \\
\hline 175 & 7/8-23M4 & & & 925 & 970 & 281.9 & 295.7 & rock & Clay and gravel & hard & -- & -- & \\
\hline 175 & $7 / 8-23 \mathrm{M} 4$ & & & 970 & 981 & 295.7 & 299.0 & $\begin{array}{l}\text { hard yellow clay } \\
\text { clay, gravel, sand, limestone(?), }\end{array}$ & Clay & hard & -- & -- & \\
\hline 175 & $7 / 8-23 \mathrm{M} 4$ & & & 981 & 1279 & 299.0 & 389.8 & and lava & Clay, sand, and gravel & -- & -- & -- & \\
\hline 176 & $7 / 8-19 \mathrm{P} 1$ & 176 & 7/8-19P1 & 0 & 20 & 0.0 & 6.1 & no data & No data & -- & -- & -- & -- \\
\hline 176 & $7 / 8-19 \mathrm{P} 1$ & & & 20 & 32 & 6.1 & 9.8 & sand; gravel & Sand (or sandstone) and gravel & -- & -- & -- & QTge \\
\hline 176 & $7 / 8-19 \mathrm{P} 1$ & & & 32 & 45 & 9.8 & 13.7 & no data & No data & -- & -- & -- & \\
\hline 176 & $7 / 8-19 \mathrm{P} 1$ & & & 45 & 65 & $\begin{array}{l}3.0 \\
13.7\end{array}$ & 19.8 & gravel; cemented gravel & Gravel & cemented & -- & -- & \\
\hline 176 & $7 / 8-19 \mathrm{P} 1$ & & & 65 & 150 & 19.8 & 45.7 & no data & No data & -- & -- & -- & \\
\hline 176 & 7/8-19P1 & & & 150 & 180 & 45.7 & 54.9 & sand, gravel; sand, sediment & Sand (or sandstone) and gravel & -- & -. & -- & QTm \\
\hline 176 & $7 / 8-19 \mathrm{P} 1$ & & & 180 & 219 & 54.9 & 66.8 & no data & No data & -- & -- & -- & \\
\hline 176 & $7 / 8-19 \mathrm{P} 1$ & & & 219 & 235 & 66.8 & 71.6 & gravel & Gravel & -- & -. & -- & QTge \\
\hline 176 & $7 / 8-19 \mathrm{P} 1$ & & & 235 & 245 & 71.6 & 74.7 & no data & No data & -. & -. & -- & Qige \\
\hline 177 & $7 / 8-20 K 1$ & 177 & 7/8-20K1 & 0 & 4 & 0.0 & 1.2 & soil & Sand and clay & -- & - & -- & Qyal \\
\hline 177 & $7 / 8-20 K 1$ & & & 4 & 16 & 1.2 & 4.9 & yellow clay and gravel & Clay and gravel & -. & -- & -- & QTge \\
\hline 177 & $7 / 8-20 K 1$ & & & 16 & 35 & 4.9 & 10.7 & yellow clay & Clay & -- & -- & -- & \\
\hline 177 & $7 / 8-20 \mathrm{~K} 1$ & & & 35 & 60 & 10.7 & 18.3 & blue clay & Clay & -- & -- & -.- & \\
\hline 177 & $7 / 8-20 K 1$ & & & 60 & 65 & 18.3 & $\begin{array}{l}19.3 \\
19.8\end{array}$ & yellow clay and gravel & Clay and gravel & -. & -- & -- & \\
\hline 177 & $7 / 8-20 K 1$ & & & 65 & 79 & 19.8 & 24.1 & $\begin{array}{l}\text { yellow clay } \\
\text { lation }\end{array}$ & Clay & -- & -- & -- & \\
\hline 177 & $7 / 8-20 K 1$ & & & 79 & 84 & 24.1 & 25.6 & $\begin{array}{l}\text { gravel } \\
\text { gray }\end{array}$ & Gravel & -- & -. & .- & \\
\hline 177 & $7 / 8-20 K 1$ & & & 84 & 99 & 25.6 & 30.2 & sandy yellow clay & Clay and sand (or sandstone) & -- & -- & -- & \\
\hline 177 & 7/8-20K1 & & & 99 & 110 & 30.2 & 33.5 & $\begin{array}{l}\text { blue clay } \\
\text { nats }\end{array}$ & Clay & .- & ..- & .. & \\
\hline 177 & $7 / 8-20 \mathrm{~K} 1$ & & & 110 & 120 & 33.5 & 36.6 & gravel & Gravel & -- & ..- & .- & \\
\hline 177 & $7 / 8-20 K 1$ & & & 120 & 123 & 36.6 & 37.5 & yellow cement & Clay, sand, and gravel & cemented & -- & -- & \\
\hline 177 & $7 / 8-20 K 1$ & & & 123 & 145 & 37.5 & 44.2 & yellow clay & Clay & -- & -. & .. & \\
\hline 177 & $7 / 8-20 \mathrm{~K} 1$ & & & 145 & 170 & 44.2 & 51.8 & blue clay & Clay & -- & -- & -- & \\
\hline 177 & $7 / 8-20 K 1$ & & & 170 & 173 & 51.8 & 52.7 & yellow sand and gravel & Sand (or sandstone) and gravel & -- & -. & -- & \\
\hline 177 & $7 / 8-20 K 1$ & & & 173 & 185 & 52.7 & 56.4 & yellow clay & Clay & .- & .- & .. & \\
\hline 177 & $7 / 8-20 K 1$ & & & 185 & 198 & 56.4 & 60.4 & sandy yellow clay & Clay and sand (or sandstone) & -. & -- & -- & \\
\hline 177 & $7 / 8-20 K 1$ & & & 198 & 215 & 60.4 & 65.5 & blue clay & Clay & -- & -- & -- & \\
\hline 177 & $7 / 8-20 K 1$ & & & 215 & 256 & 65.5 & 78.0 & yellow clay & Clay & -- & -- & -- & \\
\hline 177 & $7 / 8-20 K 1$ & & & 256 & 308 & 78.0 & 93.9 & $\begin{array}{l}\text { blue clay } \\
\text { bla }\end{array}$ & Clay & - & -- & - & \\
\hline 177 & $7 / 8-20 K 1$ & & & 308 & 324 & 93.9 & 98.8 & blue clay and little mixed gravel & Clay and gravel & -- & -- & -- & \\
\hline 177 & $7 / 8-20 K 1$ & & & 324 & 462 & 98.8 & 140.8 & blue clay & Clay & .. & .. & .. & \\
\hline 177 & $7 / 8-20 K 1$ & & & 462 & 471 & 140.8 & 143.6 & sandy blue clay & Clay and sand (or sandstone) & -- & -- & -- & \\
\hline 177 & $7 / 8-20 \mathrm{~K} 1$ & & & 471 & 557 & 143.6 & 169.8 & blue clay and gravel, mixed & Clay and gravel & -- & -- & -- & \\
\hline 177 & $7 / 8-20 \mathrm{~K} 1$ & & & 557 & 615 & $\begin{array}{l}169.8 \\
169.8\end{array}$ & 187.5 & blue clay and gravel, mixed & Clay and gravel & -- & -- & - & \\
\hline 177 & 7/8-20K1 & & & 615 & 626 & 187.5 & 190.8 & blue clay and little gravel & Clay and gravel & .- & -. & .. & \\
\hline 178 & $7 / 9-24 J 1$ & 178 & 7/9-24J1 & 0 & 38 & 0.0 & 11.6 & brown, sandy clay & Clay and sand (or sandstone) & -- & .. & $\ldots$ & QTge \\
\hline 178 & $7 / 9-24 J 1$ & & & 38 & 45 & 11.6 & 13.7 & brown, sandy clay & Clay and sand (or sandstone) & -- & -- & -- & \\
\hline 178 & $7 / 9-24 J 1$ & & & 45 & 82 & 13.7 & 25.0 & blue sand and clay & Sand and clay & -- & -- & -- & \\
\hline 178 & $7 / 9-24 J 1$ & & & 82 & 86 & 25.0 & 26.2 & black clay & Clay & -- & -- & -- & \\
\hline 178 & $7 / 9-24 J 1$ & & & 86 & 130 & 26.2 & 39.6 & blue sand and clay & Sand and clay & -- & -- & -- & \\
\hline 178 & $7 / 9-24 J 1$ & & & 130 & 135 & 39.6 & 41.1 & gray sand and gravel & Sand (or sandstone) and gravel & .- & -- & .. & \\
\hline 178 & $7 / 9-24 J 1$ & & & 135 & 160 & 41.1 & 48.8 & gray sand and clay & Sand and clay & - & -- & -- & \\
\hline 178 & $7 / 9-24 J 1$ & & & 160 & 171 & 48.8 & 52.1 & brown and blue clay & Clay & -- & -- & -- & \\
\hline 178 & $7 / 9-24 J 1$ & & & 171 & 195 & 52.1 & 59.4 & sand and gravel & Sand (or sandstone) and gravel & .- & .- & .. & \\
\hline 178 & $7 / 9-24 J 1$ & & & 195 & 255 & 59.4 & 77.7 & blue clay and blue sand & Clay and sand (or sandstone) & -- & -- & -. & QTm \\
\hline 178 & $7 / 9-24 J 1$ & & & 255 & 279 & 77.7 & 85.0 & hard sand and clay & Sand and clay & hard & -- &.- & \\
\hline 178 & $7 / 9-24 J 1$ & & & 279 & 285 & 85.0 & 86.9 & coarse gravel & Gravel & -- & -- & -- & \\
\hline 178 & $7 / 9-24 J 1$ & & & 285 & 370 & 86.9 & 112.8 & blue clay and blue sand & Clay and sand (or sandstone) & -- & -- & -- & \\
\hline 178 & $7 / 9-24 J 1$ & & & 370 & 543 & $\begin{array}{l}8.9 \\
112.8\end{array}$ & $\begin{array}{l}11.0 \\
165.5\end{array}$ & blue clay and shale & Clay and sand (or sandstone) & cemented & -- & -- & \\
\hline 178 & $7 / 9-24 J 1$ & & & 543 & 565 & 165.5 & 172.2 & blue sand and shale & Sand and clay & cemented & -- & -- & \\
\hline 178 & $7 / 9-24 J 1$ & & & 565 & 575 & 172.2 & 175.3 & $\begin{array}{l}\text { brown shale } \\
\text { balle }\end{array}$ & Clay and sand (or sandstone) & cemented & -. & .. & \\
\hline 178 & $7 / 9-24 J 1$ & & & 575 & 615 & 175.3 & 187.5 & blue shale and sand & Clay and sand (or sandstone) & cemented & -- & -- & \\
\hline 178 & $7 / 9-24 J 1$ & & & 615 & 695 & 187.5 & 211.8 & shale and boulders & Clay and gravel & cemented & .. & .. & OTge (?) \\
\hline 178 & $7 / 9-24 J 1$ & & & 695 & 705 & 211.8 & 214.9 & shale and showing of wood & Clay and sand (or sandstone) & cemented & -- & wood & \\
\hline 178 & $7 / 9-24 J 1$ & & & 705 & 740 & 214.9 & 225.6 & hard shale & Clay and sand (or sandstone) & hard & -. & -- & \\
\hline 178 & $7 / 9-24 J 1$ & & & 740 & 760 & 225.6 & 231.6 & shale with brown sandy streaks & Clay and sand (or sandstone) & cemented & -- & -- & \\
\hline
\end{tabular}


Appendix 3. Lithologic data. - Continued

ISite IDs and well number correspond to locations in Appendix 1, a site ID and well number is listed for each drilled interval for a particular drill hole. For convenience, the site ID and well numbers are repeated at the shallowest drilled interval for each well. leaderst.

\begin{tabular}{|c|c|c|c|c|c|c|c|c|c|c|c|c|c|}
\hline Site ID & Well number & $\begin{array}{l}\text { Site ID, } \\
\text { top of } \\
\text { well }\end{array}$ & $\begin{array}{l}\text { Well number, } \\
\text { top of well }\end{array}$ & $\begin{array}{c}\text { Top of } \\
\text { interval, in } \\
\text { feet }\end{array}$ & $\begin{array}{l}\text { Base of } \\
\text { interval, in } \\
\text { feet }\end{array}$ & $\begin{array}{c}\text { Top of } \\
\text { interval, in } \\
\mathrm{m}\end{array}$ & $\begin{array}{c}\text { Base of } \\
\text { interval, in } \\
\mathrm{m}\end{array}$ & Reported lithologic unit' & Interpreted lithologic class ${ }^{2}$ & $\begin{array}{c}\text { Reported } \\
\text { degree of } \\
\text { cementation or } \\
\text { induration }^{3} \\
\end{array}$ & $\begin{array}{c}\text { Fossils } \\
\text { reported in } \\
\text { interval }^{4}\end{array}$ & $\begin{array}{c}\text { Organic } \\
\text { matter } \\
\text { reported in } \\
\text { interval }^{5}\end{array}$ & $\begin{array}{l}\text { Reported stratigraphic top of geologic } \\
\text { unit }^{6}\end{array}$ \\
\hline 178 & $7 / 9-24 \mathrm{~J} 1$ & & & 760 & 800 & 231.6 & 243.8 & black coarse gravel & Gravel & -- & -- & -- & \\
\hline 178 & $7 / 9-24 \mathrm{~J} 1$ & & & 800 & 826 & 243.8 & 251.8 & $\begin{array}{l}\text { shale and boulders } \\
\text { shale and limestone streaks, }\end{array}$ & Clay and gravel & cemented & -- & -- & \\
\hline 178 & $7 / 9-24 \mathrm{~J} 1$ & & & 826 & 920 & 251.8 & 280.4 & shells & Clay and sand (or sandstone) & cemented & shells & -- & QTm \\
\hline 178 & $7 / 9-24 \mathrm{~J} 1$ & & & 920 & 970 & 280.4 & 295.7 & black gravel and sand & Sand (or sandstone) and gravel & -- & -- & -- & \\
\hline 178 & $7 / 9-24 J 1$ & & & 970 & 1190 & 295.7 & 362.7 & shale and limestone streaks & Clay and sand (or sandstone) & cemented & -- & -- & \\
\hline 178 & $7 / 9-24 J 1$ & & & 1190 & 1220 & 362.7 & 371.9 & very hard shale and limestone & Clay and sand (or sandstone) & hard & -- & -- & \\
\hline 178 & $7 / 9-24 J 1$ & & & 1220 & 1265 & 371.9 & 385.6 & sand, shale, and boulders & Clay, sand, and gravel & cemented & -- & -- & \\
\hline 178 & $7 / 9-24 J 1$ & & & 1265 & 1280 & 385.6 & 390.1 & small black boulders, sand & Sand (or sandstone) and gravel & -- & -- & -- & \\
\hline 178 & $7 / 9-24 J 1$ & & & 1280 & 1468 & 390.1 & 447.4 & sand and shale with lime streaks & Sand and clay & cemented & -- & -- & \\
\hline 178 & $7 / 9-24 \mathrm{J1}$ & & & 1468 & 1475 & 447.4 & 449.6 & sandy shale and wood & Clay and sand (or sandstone) & cemented & -- & wood & \\
\hline 178 & $7 / 9-24 \mathrm{J1}$ & & & 1475 & 1520 & 449.6 & 463.3 & hard black shale and sand & Clay and sand (or sandstone) & hard & -- & -- & \\
\hline 178 & $7 / 9-24 \mathrm{J1}$ & & & 1520 & 1555 & 463.3 & 474.0 & very hard shale & Clay and sand (or sandstone) & hard & -- & -- & \\
\hline 178 & $7 / 9-24 J 1$ & & & 1555 & 1574 & 474.0 & 479.8 & blue sand and brown shale & Sand and clay & cemented & -- & -- & \\
\hline 178 & $7 / 9-24 J 1$ & & & 1574 & 1666 & 479.8 & 507.8 & clay and limestone streaks & Clay and sand (or sandstone) & -- & -- & -- & \\
\hline 178 & $7 / 9-24 J 1$ & & & 1666 & 1686 & 507.8 & 513.9 & blue sand and shale & Sand and clay & cemented & -- & -- & \\
\hline 178 & $7 / 9-24 J 1$ & & & 1686 & 1879 & 513.9 & 572.7 & shale and limestone shelves & Clay and sand (or sandstone) & cemented & -- & -- & \\
\hline 178 & $7 / 9-24 J 1$ & & & 1879 & 1905 & 572.7 & 580.6 & blue shale and hard black shale & Clay and sand (or sandstone) & hard & -- & -- & \\
\hline 178 & $7 / 9-24 \mathrm{~J} 1$ & & & 1905 & 1955 & 580.6 & 595.9 & blue shale and limestone & Clay, sand, and gravel & cemented & -- & -- & \\
\hline 178 & $7 / 9-24 \mathrm{~J} 1$ & & & 1955 & 2053 & 595.9 & 625.8 & $\begin{array}{l}\text { blue sandy shale, limestone shells } \\
\text { Interbedded sand and limestone } \\
\text { in wafer-like sections, } 1 / 2 \text { - } 1 \text { inch }\end{array}$ & Clay and sand (or sandstone) & cemented & shells & -- & \\
\hline 178 & $7 / 9-24 \mathrm{~J} 1$ & & & 2053 & 2076 & 625.8 & 632.8 & thick & Clay and sand (or sandstone) & -- & -- & -- & \\
\hline 179 & $7 / 8-22 \mathrm{R} 2$ & 179 & 7/8-22R2 & 0 & 205 & 0.0 & 62.5 & no data & No data & -- & -- & -- & -- \\
\hline 180 & 7/8-23M1 & 180 & 7/8-23M1 & 0 & 6 & 0.0 & 1.8 & soil, black, adobe & Sand and clay & -- & -- & -- & Qyal and Qoal (?) and QTge \\
\hline 180 & $7 / 8-23 \mathrm{M} 1$ & & & 6 & 24 & 1.8 & 7.3 & clay, yellow & Clay & -- & -- & -- & \\
\hline 180 & $7 / 8-23 \mathrm{M} 1$ & & & 24 & 54 & 7.3 & 16.5 & $\begin{array}{l}\text { sand and gravel } \\
\text { clay, yellow; include sand and }\end{array}$ & Sand (or sandstone) and gravel & -- & -- & -- & \\
\hline 180 & 7/8-23M1 & & & 54 & 106 & 16.5 & 32.3 & gravel streaks & Clay, sand, and gravel & -- & -- & -- & \\
\hline 180 & 7/8-23M1 & & & 106 & 120 & 32.3 & 36.6 & gravel, pea & Gravel & -- & -- & -- & \\
\hline 180 & 7/8-23M1 & & & 120 & 156 & 36.6 & 47.5 & clay, gray & Clay & -- & -- & -- & \\
\hline 180 & $7 / 8-23 \mathrm{M} 1$ & & & 156 & 216 & 47.5 & 65.8 & clay, yellow, sandy & Clay and sand (or sandstone) & -- & -- & -- & QTm (?) and QTge (?) \\
\hline 180 & $7 / 8-23 \mathrm{M} 1$ & & & 216 & 320 & 65.8 & 97.5 & clay, yellow, sandy & Clay and sand (or sandstone) & -- & -- & -- & \\
\hline 180 & 7/8-23м1 & & & 320 & 335 & 97.5 & 102.1 & sand and gravel & Sand (or sandstone) and gravel & -- & -- & -- & \\
\hline 180 & 7/8-23M1 & & & 335 & 356 & 102.1 & 108.5 & clay, yellow, sandy & Clay and sand (or sandstone) & -- & -- & -- & \\
\hline 180 & $7 / 8-23 \mathrm{M} 1$ & & & 356 & 380 & 108.5 & 115.8 & clay, yellow & Clay & -- & -- & -- & \\
\hline 180 & 7/8-23M1 & & & 380 & 442 & 115.8 & 134.7 & $\begin{array}{l}\text { clay, yellow, sandy; includes } \\
\text { gravel streaks } \\
\text { clay, yellow; includes gravel }\end{array}$ & Clay, sand, and gravel & -- & -- & -- & \\
\hline 180 & 7/8-23м1 & & & 442 & 500 & 134.7 & 152.4 & streaks & Clay and gravel & -- & -- & -- & \\
\hline 180 & 7/8-23м1 & & & 500 & 530 & 152.4 & 161.5 & sand, brown & Sand & -- & -- & -- & \\
\hline 180 & 7/8-23M1 & & & 530 & 627 & 161.5 & 191.1 & sand and pea gravel & Sand (or sandstone) and gravel & -- & -- & -- & \\
\hline 180 & $7 / 8-23 \mathrm{M} 1$ & & & 627 & 700 & 191.1 & 213.4 & clay, yellow & Clay & -- & -- & -- & \\
\hline 180 & $7 / 8-23 \mathrm{M} 1$ & & & 700 & 751 & 213.4 & 228.9 & clay, blue, sandy & Clay and sand (or sandstone) & -- & -- & -- & \\
\hline 180 & 7/8-23M1 & & & 751 & 814 & 228.9 & 248.1 & sand & Sand & -- & -- & -- & \\
\hline 180 & 7/8-23M1 & & & 814 & 955 & 248.1 & 291.1 & clay, blue, sandy & Clay and sand (or sandstone) & -- & -- & -- & \\
\hline 180 & 7/8-23M1 & & & 955 & 970 & 291.1 & 295.7 & sand & Sand & -- & -- & -- & \\
\hline 180 & $7 / 8-23 \mathrm{M} 1$ & & & 970 & 1000 & 295.7 & 304.8 & shale, blue & Clay and sand (or sandstone) & cemented & -- & -- & \\
\hline 181 & 7/8-22Q1 & 181 & 7/8-22Q1 & 0 & 360 & 0.0 & 109.7 & no data & No data & -- & -- & -- & -- \\
\hline 182 & 7/9-30A1 & 182 & 7/9-30A1 & 0 & 260 & 0.0 & 79.2 & no data & No data & -- & -- & -- & QTm \\
\hline 183 & $7 / 8-23 \mathrm{P} 1$ & 183 & 7/8-23P1 & 0 & 90 & 0.0 & 27.4 & no data & No data & -- & -- & -- & -- \\
\hline 183 & $7 / 8-23 \mathrm{P} 1$ & & & 90 & 95 & 27.4 & 29.0 & clay and gravel & Clay and gravel & -- & -- & -- & QTge \\
\hline 183 & $7 / 8-23 \mathrm{P} 1$ & & & 95 & 116 & 29.0 & 35.4 & $\begin{array}{l}\text { no data } \\
\text { clay and gravel; cemented gravel; }\end{array}$ & No data & -- & -- & -- & \\
\hline 183 & 7/8-23P1 & & & 116 & 138 & 35.4 & 42.1 & gravel & Clay and gravel & cemented & -- & -- & QTge \\
\hline 183 & $7 / 8-23 P 1$ & & & 138 & 258 & 42.1 & 78.6 & no data & No data & -- & -- & -- & \\
\hline 183 & $7 / 8-23 \mathrm{P} 1$ & & & 258 & 264 & 78.6 & 80.5 & clay and gravel & Clay and gravel & -- & -- & -- & QTge \\
\hline 183 & $7 / 8-23 P 1$ & & & 264 & 266 & 80.5 & 81.1 & no data & No data & -- & -- & -- & \\
\hline 184 & $7 / 6-19 R 1$ & 184 & $7 / 6-19 R 1$ & 0 & 3 & 0.0 & 0.9 & soil, small, brown, gravelly & Sand and clay & -- & -- & -- & (?) and QTge \\
\hline
\end{tabular}


Appendix 3. Lithologic data. - Continued

ISite IDs and well number correspond to locations in Appendix 1, a site ID and well number is listed for each drilled interval for a particular drill hole. For convenience, the site ID and well numbers are repeated at the shallowest drilled interval for each well. leaders

\begin{tabular}{|c|c|c|c|c|c|c|c|c|c|c|c|c|c|}
\hline Site ID & Well number & $\begin{array}{l}\text { Site ID, } \\
\text { top of } \\
\text { well }\end{array}$ & $\begin{array}{l}\text { Well number, } \\
\text { top of well }\end{array}$ & $\begin{array}{c}\text { Top of } \\
\text { interval, in } \\
\text { feet }\end{array}$ & $\begin{array}{c}\text { Base of } \\
\text { interval, in } \\
\text { feet }\end{array}$ & $\begin{array}{c}\text { Top of } \\
\text { interval, in } \\
m\end{array}$ & $\begin{array}{c}\text { Base of } \\
\text { interval, in } \\
\mathrm{m}\end{array}$ & Reported lithologic unit ${ }^{1}$ & Interpreted lithologic class ${ }^{2}$ & $\begin{array}{c}\text { Reported } \\
\text { degree of } \\
\text { cementation or } \\
\text { induration }^{3}\end{array}$ & $\begin{array}{c}\text { Fossils } \\
\text { reported in } \\
\text { interval }^{4}\end{array}$ & $\begin{array}{c}\text { Organic } \\
\text { matter } \\
\text { reported in } \\
\text { interval }^{5}\end{array}$ & $\begin{array}{l}\text { Reported stratigraphic top of geologic } \\
\text { unit }^{6}\end{array}$ \\
\hline $\begin{array}{l}184 \\
184\end{array}$ & $\begin{array}{l}\text { 7/6-19R1 } \\
7 / 6-19 \mathrm{R} 1\end{array}$ & & & $\begin{array}{l}3 \\
35\end{array}$ & $\begin{array}{l}35 \\
40\end{array}$ & $\begin{array}{l}0.9 \\
10.7\end{array}$ & $\begin{array}{l}10.7 \\
12.2\end{array}$ & $\begin{array}{l}\text { medium yellow gravelly clay } \\
\text { fine packed gravel }\end{array}$ & $\begin{array}{l}\text { Clay and gravel } \\
\text { Gravel }\end{array}$ & $\begin{array}{l}- \\
--\end{array}$ & $\begin{array}{l}-- \\
-.\end{array}$ & -- & \\
\hline 184 & 7/6-19R1 & & & 40 & 110 & 12.2 & 33.5 & medium gravelly yellow clay & Clay & -- & - & -- & \\
\hline 184 & 7/6-19R1 & & & 110 & 120 & 33.5 & 36.6 & $\begin{array}{l}\text { small amount of water } \\
\text { s. }\end{array}$ & Gravel & -- & -- & -- & \\
\hline 184 & $7 / 6-19 R 1$ & & & 120 & 200 & 36.6 & 61.0 & $\begin{array}{l}\text { soft yellow shaley rock } \\
\text { small yellow boulders and coarse }\end{array}$ & Clay and sand (or sandstone) & -- & -- & -- & \\
\hline 184 & $7 / 6-19 \mathrm{R} 1$ & & & 200 & 220 & 61.0 & 67.1 & sand, water strata & Sand (or sandstone) and gravel & -- & -- & -- & \\
\hline 184 & $7 / 6-19 R 1$ & & & 220 & 260 & 67.1 & 79.2 & $\begin{array}{l}\text { hard yellow volcanic ash } \\
\text { blue volcanic ash, water in a large }\end{array}$ & Ash or tuff & hard & -- & -- & \\
\hline 184 & $7 / 6-19 \mathrm{R} 1$ & & & 260 & 300 & 79.2 & 91.4 & quantity & Ash or tuff & -- & .- & -. & Tsv (?) \\
\hline 185 & $7 / 6-19 \mathrm{~N} 1$ & 185 & 7/6-19N1 & 0 & 1 & 0.0 & 0.3 & soil & Sand and clay & -- & -- & -- & Qyal and QTge (?) \\
\hline 185 & $7 / 6-19 \mathrm{~N} 1$ & & & 1 & 4 & 0.3 & 1.2 & gravel, coarse yellow & Gravel & -- & -- & -- & \\
\hline 185 & 7/6-19N1 & & & 4 & 6 & 1.2 & 1.8 & brown silt & Sand and clay & -- & -- & -- & \\
\hline 185 & 7/6-19N1 & & & 6 & 21 & 1.8 & 6.4 & yellow clay and small gravel & Clay and gravel & -- & -- & -- & \\
\hline 185 & $7 / 6-19 \mathrm{~N} 1$ & & & 21 & 25 & 6.4 & 7.6 & $\begin{array}{l}\text { coarse packed gravel } \\
\text { cold }\end{array}$ & Gravel & -- & -- & -- & \\
\hline 185 & $7 / 6-19 \mathrm{~N} 1$ & & & 25 & 28 & 7.6 & 8.5 & sandy yellow clay & Clay and sand (or sandstone) & -- & -- & -- & \\
\hline 185 & $7 / 6-19 \mathrm{~N} 1$ & & & 28 & 34 & 8.5 & 10.4 & $\begin{array}{l}\text { coarse gravel and yellow clay } \\
\text { blue clay and small gravel water }\end{array}$ & Sand (or sandstone) and gravel & -- & -- & -- & \\
\hline 185 & $7 / 6-19 \mathrm{~N} 1$ & & & 34 & 37 & 10.4 & 11.3 & strata & Clay and gravel & -- & -- & -. & \\
\hline 185 & $7 / 6-19 \mathrm{~N} 1$ & & & 37 & 57 & 11.3 & 17.4 & coarse gravelly yellow clay & Clay and gravel & -- & -- & -- & \\
\hline 185 & 7/6-19N1 & & & 57 & 87 & 17.4 & 26.5 & small boulders and yellow clay & Clay and gravel & - & - & -- & \\
\hline 185 & 7/6-19N1 & & & 87 & 112 & 26.5 & 34.1 & coarse gravelly yellow clay & Clay and gravel & -- & -- & -- & \\
\hline 185 & $7 / 6-19 \mathrm{~N} 1$ & & & 112 & 118 & 34.1 & 36.0 & blue clay and small boulders & Clay and gravel & .- & .- & .- & \\
\hline 185 & $7 / 6-19 \mathrm{~N} 1$ & & & 118 & 125 & 36.0 & 38.1 & small boulders and yellow clay & Clay and gravel & -- & .- & .- & \\
\hline 185 & $7 / 6-19 \mathrm{~N} 1$ & & & 125 & 130 & 38.1 & 39.6 & yellow shaley rock & Clay and sand (or sandstone) & -- & -- & -- & \\
\hline 185 & 7/6-19N1 & & & 130 & 140 & 39.6 & 42.7 & coarse gravelly yellow clay & Clay and gravel & -- & -- & -- & \\
\hline 185 & 7/6-19N1 & & & 140 & 145 & 42.7 & 44.2 & fine gravelly yellow clay & Sand (or sandstone) and gravel & -- & -- & -- & \\
\hline 185 & 7/6-19N1 & & & 145 & 149 & 44.2 & 45.4 & coarse packed gravel water strata & Gravel & .- & .- & .. & \\
\hline 186 & 7/9-25A1A & 186 & 7/9-25A1A & 0 & 20 & 0.0 & 6.1 & no data & No data & -- & -- & -- & -- \\
\hline 186 & 7/9-25A1A & & & 20 & 30 & 6.1 & 9.1 & $\begin{array}{l}\text { clay and gravel } \\
\text {. }\end{array}$ & Clay and gravel & -- & -- & -- & QTge \\
\hline 186 & $7 / 9-25 \mathrm{~A} 1 \mathrm{~A}$ & & & 30 & 65 & 9.1 & 19.8 & no data & No data & -- & -- & -.- & \\
\hline 186 & 7/9-25A1A & & & 65 & 85 & 19.8 & 25.9 & gravel & Gravel & -. & .- & .. & \\
\hline 186 & 7/9-25A1A & & & 85 & 86 & 25.9 & 26.2 & no data & No data & -- & -- & -- & \\
\hline 187 & 7/9-25A1B & 187 & 7/9-25A1B & 0 & 90 & 0.0 & 27.4 & no data & No data & -- & -- & -- & -- \\
\hline 187 & 7/9-25A1B & & & 90 & 106 & 27.4 & 32.3 & sand & Sand & -- & -- & -- & QTm \\
\hline 187 & $7 / 9-25 \mathrm{~A} 1 \mathrm{~B}$ & & & 106 & 190 & 32.3 & 57.9 & no data & No data & -- & -.- & .- & \\
\hline 187 & 7/9-25A1B & & & 190 & 206 & 57.9 & 62.8 & sand & Sand & -- & -- & -- & \\
\hline 187 & 7/9-25A1B & & & 206 & 256 & 62.8 & 78.0 & no data & No data & -- & .. & .. & \\
\hline 188 & $7 / 9-30 \mathrm{G} 2$ & 188 & 7/9-30G2 & 0 & 200 & 0.0 & 61.0 & (cased to $114 \mathrm{ft}$ ) & No data & -- & -- & -- & QTm \\
\hline 189 & $7 / 7-29 \mathrm{D} 1$ & 189 & 7/7-29D1 & 0 & 15 & 0.0 & 4.6 & $\begin{array}{l}\text { adobe and yellow clay } \\
\text { clay yellow, becoming sandy at }\end{array}$ & Sand and clay & -- & -- & -- & Qyal and QTge (?) \\
\hline 189 & 7/7-29D1 & & & 15 & 40 & 4.6 & 12.2 & bottom & Clay and sand (or sandstone) & -- & -- & -- & \\
\hline 189 & $7 / 7-29 \mathrm{D} 1$ & & & 40 & 60 & 12.2 & 18.3 & loose gravel & Gravel & -- & -- & -- & \\
\hline 189 & 7/7-29D1 & & & 60 & 130 & 18.3 & 39.6 & blue shale, hard streak of sand & Clay and sand (or sandstone) & hard & -- & -- & \\
\hline 189 & $7 / 7-29 \mathrm{D} 1$ & & & 130 & 160 & 39.6 & 48.8 & $\begin{array}{l}\text { sand and gravel } \\
\text { blue shale, fine sand, and gravel; }\end{array}$ & Sand (or sandstone) and gravel & -- & -- & -- & \\
\hline 189 & 7/7-29D1 & & & 160 & 180 & 48.8 & 54.9 & $\begin{array}{l}\text { bottom basaltic boulders as in } \\
\text { order } \\
\text { basalt, shows vesicular under }\end{array}$ & Clay and sand (or sandstone) & cemented & -- & -. & Tsv \\
\hline 189 & $7 / 7-29 \mathrm{D} 1$ & & & 180 & 260 & 54.9 & 79.2 & $\begin{array}{l}\text { glass } \\
\text { top of basalt, drills very hard first }\end{array}$ & Basalt & -- & -- & - & \\
\hline 189 & $7 / 7-29 \mathrm{D} 1$ & & & 180 & 180 & 54.9 & 54.9 & foot & Basalt & hard & .- & .. & \\
\hline 189 & $7 / 7-29 \mathrm{D} 1$ & & & 260 & 270 & 79.2 & 82.3 & basalt, dense, hard drilling & Basalt & hard & -- & -- & \\
\hline 189 & $7 / 7-29 \mathrm{D} 1$ & & & 270 & 300 & 82.3 & 91.4 & basalt, vesicular & Basalt & -- & -- & -- & \\
\hline 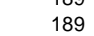 & 7/7-29D1 & & & 300 & 310 & 91.4 & $\begin{array}{l}1.4 \\
94.5\end{array}$ & red ash & Ash or tuff & - & -- & -- & \\
\hline 189 & 7/7-29D1 & & & 310 & 340 & 94.5 & 103.6 & basalt, vesicular & Basalt & -- & -- & -- & \\
\hline $\begin{array}{l}189 \\
189\end{array}$ & $7 / 7-29 \mathrm{D} 1$ & & & 340 & 350 & 103.6 & 106.7 & $\begin{array}{l}\text { basalt, hard drilling } \\
\text { basalt, drilled like fractured, but it }\end{array}$ & Basalt & hard & - & - & \\
\hline 189 & $7 / 7-29 D 1$ & & & 350 & 400 & 106.7 & 121.9 & is vesicular under glass & Basalt & .- & -- & -- & \\
\hline
\end{tabular}


Appendix 3. Lithologic data. - Continued

[Site IDs and well number correspond to locations in Appendix 1, a site ID and well number is listed for each drilled interval for a particular drill hole. For convenience, the site ID and well numbers are repeated at the shallowest drilled interval for each well teatersth not

\begin{tabular}{|c|c|c|c|c|c|c|c|c|c|c|c|c|c|}
\hline Site ID & Well number & $\begin{array}{l}\text { Site ID, } \\
\text { top of } \\
\text { well }\end{array}$ & $\begin{array}{l}\text { Well number, } \\
\text { top of well }\end{array}$ & $\begin{array}{l}\text { Top of } \\
\text { interval, in } \\
\text { feet }\end{array}$ & $\begin{array}{c}\text { Base of } \\
\text { interval, in } \\
\text { feet }\end{array}$ & $\begin{array}{c}\text { Top of } \\
\text { interval, in } \\
\mathrm{m}\end{array}$ & $\begin{array}{c}\text { Base of } \\
\text { interval, in } \\
\mathrm{m}\end{array}$ & Reported lithologic unit' & Interpreted lithologic class ${ }^{2}$ & $\begin{array}{c}\text { Reported } \\
\text { degree of } \\
\text { cementation or } \\
\text { induration }^{3} \\
\end{array}$ & $\begin{array}{c}\text { Fossils } \\
\text { reported in } \\
\text { interval }^{4}\end{array}$ & $\begin{array}{c}\text { Organic } \\
\text { matter } \\
\text { reported in } \\
\text { interval }^{5}\end{array}$ & $\begin{array}{l}\text { Reported stratigraphic top of geologic } \\
\text { unit }^{6}\end{array}$ \\
\hline 189 & 7/7-29D1 & & & 400 & 410 & 121.9 & 125.0 & $\begin{array}{l}\text { red vesicular lava } \\
\text { gray ash, appears to have had } \\
\text { stream action; drilled a foot per }\end{array}$ & Basalt & -- & -- & -- & \\
\hline 189 & 7/7-29D1 & & & 410 & 540 & 125.0 & 164.6 & minute & Ash or tuff & -- & -- & -- & \\
\hline 189 & $7 / 7-29 D 1$ & & & 540 & 550 & 164.6 & 167.6 & basalt, very hard & Basalt & hard & -- & -- & \\
\hline 189 & 7/7-29D1 & & & 550 & 570 & 167.6 & 173.7 & basalt, vesicular & Basalt & -- & -- & -- & \\
\hline 189 & 7/7-29D1 & & & 570 & 588 & 173.7 & 179.2 & basalt, dense & Basalt & -- & -- & -- & \\
\hline 190 & 7/9-27E2 & 190 & 7/9-27E2 & 0 & 47 & 0.0 & 14.3 & no data & No data & -- & -- & -- & -- \\
\hline 190 & 7/9-27E2 & & & 47 & 90 & 14.3 & 27.4 & sand and gravel; quicksand. & Sand (or sandstone) and gravel & -- & -- & -- & \\
\hline 190 & 7/9-27E2 & & & 90 & 370 & 27.4 & 112.8 & sandstone; gravel and sand. & Sand (or sandstone) and gravel & cemented & -- & -- & QTm \\
\hline 191 & 7/9-27F1 & 191 & 7/9-27F1 & 0 & 200 & 0.0 & 61.0 & no data & No data & -- & -- & -- & QTm \\
\hline 192 & $7 / 7-30 \mathrm{C} 1$ & 192 & $7 / 7-30 \mathrm{C} 1$ & 0 & 5 & 0.0 & 1.5 & topsoil, adobe & Sand and clay & -- & -- & -- & QTge and QTm (?) \\
\hline 192 & $7 / 7-30 \mathrm{C} 1$ & & & 5 & 75 & 1.5 & 22.9 & boulders, hard & Gravel & hard & -- & -- & \\
\hline 192 & $7 / 7-30 C 1$ & & & 75 & 93 & 22.9 & 28.3 & coarse sand & Sand & -- & -- & -- & \\
\hline 192 & $7 / 7-30 \mathrm{C} 1$ & & & 93 & 98 & 28.3 & 29.9 & blue clay & Clay & -- & -- & -- & \\
\hline 192 & $7 / 7-30 \mathrm{C} 1$ & & & 98 & 122 & 29.9 & 37.2 & rock & Ash or tuff & hard & - & -- & \\
\hline 192 & $7 / 7-30 \mathrm{C} 1$ & & & 122 & 138 & 37.2 & 42.1 & coarse sand & Sand & -- & -- & peat & \\
\hline 192 & $7 / 7-30 \mathrm{C} 1$ & & & 138 & 140 & 42.1 & 42.7 & blue clay & Clay & -- & -- & -- & \\
\hline 192 & $7 / 7-30 \mathrm{C} 1$ & & & 140 & 148 & 42.7 & 45.1 & $\begin{array}{l}\text { rock } \\
\text { blue clay with streaks of sand and }\end{array}$ & Ash or tuff & hard & -- & -- & \\
\hline 192 & $7 / 7-30 \mathrm{C} 1$ & & & 148 & 310 & 45.1 & 94.5 & gravel & Clay, sand, and gravel & -- & -- & -- & \\
\hline 192 & 7/7-30C1 & & & 310 & 320 & 94.5 & 97.5 & $\begin{array}{l}\text { coarse sand } \\
\text { blue clay with streaks of sand and }\end{array}$ & Sand & -- & -- & -- & \\
\hline 192 & $7 / 7-30 \mathrm{C} 1$ & & & 320 & 342 & 97.5 & 104.2 & gravel & Clay, sand, and gravel & -- & -- & -- & \\
\hline 192 & $7 / 7-30 \mathrm{C} 1$ & & & 342 & 360 & 104.2 & 109.7 & coarse sand & Sand & -- & -- & -- & \\
\hline 192 & $7 / 7-30 \mathrm{C} 1$ & & & 360 & 365 & 109.7 & 111.3 & blue clay & Clay & -- & -- & -- & \\
\hline 192 & $7 / 7-30 \mathrm{C} 1$ & & & 365 & 375 & 111.3 & 114.3 & blue sandstone with shells & Sandstone & cemented & shells & -- & \\
\hline 192 & $7 / 7-30 C 1$ & & & 375 & 377 & 114.3 & 114.9 & $\begin{array}{l}\text { blue clay } \\
\text { gravel, sand, streaks of }\end{array}$ & Clay & -- & -- & -- & \\
\hline 192 & $7 / 7-30 \mathrm{C} 1$ & & & 377 & 385 & 114.9 & 117.3 & sandstone with shells & Sand (or sandstone) and gravel & cemented & shells & -- & \\
\hline 192 & $7 / 7-30 C 1$ & & & 385 & 397 & 117.3 & 121.0 & coarse sand & Sand & -- & -- & -- & \\
\hline 192 & $7 / 7-30 \mathrm{C} 1$ & & & 397 & 400 & 121.0 & 121.9 & blue clay & Clay & -- & -- & -- & \\
\hline 192 & $7 / 7-30 C 1$ & & & 400 & 408 & 121.9 & 124.4 & coarse sand & Sand & -- & -- & -- & \\
\hline 192 & $7 / 7-30 \mathrm{C} 1$ & & & 408 & 410 & 124.4 & 125.0 & blue clay & Clay & -- & -- & -- & \\
\hline 192 & $7 / 7-30 \mathrm{C} 1$ & & & 410 & 422 & 125.0 & 128.6 & brown clay & Clay & -- & -- & -- & \\
\hline 192 & $7 / 7-30 C 1$ & & & 422 & 442 & 128.6 & 134.7 & blue clay & Clay & -- & -- & -- & \\
\hline 192 & $7 / 7-30 \mathrm{C} 1$ & & & 442 & 448 & 134.7 & 136.6 & hard rock (probably basalt) & Basalt & hard & -- & -- & Tsv \\
\hline 193 & 7/9-28G1 & 193 & $7 / 9-28 \mathrm{G} 1$ & 0 & 90 & 0.0 & 27.4 & no data & No data & -- & -- & -- & -- \\
\hline 193 & $7 / 9-28 \mathrm{G} 1$ & & & 90 & 260 & 27.4 & 79.2 & sandstone & Sandstone & cemented & -- & -- & QTm \\
\hline 194 & 7/9-28G2 & 194 & $7 / 9-28 \mathrm{G} 2$ & 0 & 40 & 0.0 & 12.2 & $\begin{array}{l}\text { no data } \\
\text { sandstone; sandstone and clam }\end{array}$ & No data & -- & -- & -- & -- \\
\hline 194 & 7/9-28G2 & & & 40 & 240 & 12.2 & 73.2 & shells & Sandstone & cemented & shells & -- & QTm \\
\hline 195 & 7/9-27E1 & 195 & 7/9-27E1 & 0 & 12 & 0.0 & 3.7 & $\begin{array}{l}\text { no data } \\
\text { sandstone and clam shells; sand }\end{array}$ & No data & -- & -- & -- & -- \\
\hline 195 & 7/9-27E1 & & & 12 & 260 & 3.7 & 79.2 & and gravel; sand. & Sand (or sandstone) and gravel & cemented & shells & -- & QTm \\
\hline 196 & 7/9-30J1 & 196 & 7/9-30J1 & 0 & 160 & 0.0 & 48.8 & no data & No data & -- & -- & -- & -- \\
\hline 196 & 7/9-30J1 & & & 160 & 200 & 48.8 & 61.0 & sandstone & Sandstone & cemented & -- & -- & QTm \\
\hline 197 & 7/9-26M1 & 197 & 7/9-26M1 & 0 & 206 & 0.0 & 62.8 & no data & No data & -- & -- & -- & QTm \\
\hline 198 & $7 / 8-28 \mathrm{~F} 1$ & 198 & $7 / 8-28 \mathrm{~F} 1$ & 0 & 5 & 0.0 & 1.5 & adobe & Sand and clay & -- & -- & -- & QTge \\
\hline 198 & $7 / 8-28 F 1$ & & & 5 & 15 & 1.5 & 4.6 & $\begin{array}{l}\text { hardpan } \\
\text { clay, sticky brown, and gravel; }\end{array}$ & Clay and gravel & hard & -- & -- & \\
\hline 198 & $7 / 8-28 \mathrm{~F} 1$ & & & 15 & 30 & 4.6 & 9.1 & water at 15 feet & Clay and gravel & -- & -- & -- & \\
\hline 198 & $7 / 8-28 \mathrm{~F} 1$ & & & 30 & 55 & 9.1 & 16.8 & blue clay & Clay & -- & -- & -- & \\
\hline 198 & $7 / 8-28 \mathrm{~F} 1$ & & & 55 & 64 & 16.8 & 19.5 & gravel & Gravel & -- & -- & -- & \\
\hline 198 & $7 / 8-28 F 1$ & & & 64 & 66 & 19.5 & 20.1 & $\begin{array}{l}\text { brown clay } \\
\text { sandstone( } 20 \mathrm{ft} \text { of surface }\end{array}$ & Clay & -- & -- & -- & \\
\hline 199 & 7/9-28L1 & 199 & 7/9-28L1 & 5 & 542 & 1.5 & 165.2 & casing) & Sandstone & cemented & -- & -- & QTm \\
\hline 200 & $7 / 9-29 \mathrm{~J} 1$ & 200 & $7 / 9-29 \mathrm{JI}$ & 0 & 400 & 0.0 & 121.9 & uncased below $60 \mathrm{ft}$ & No data & -- & -- & -- & QTm \\
\hline 201 & 7/8-25ட1 & 201 & 7/8-25L1 & 0 & 270 & 0.0 & 82.3 & no data & No data & -- & -- & -- & -- \\
\hline 201 & 7/8-25L1 & & & 270 & 370 & 82.3 & 112.8 & perforated intervals & No data & -- & -- & -- & Tsv (?) \\
\hline 201 & $7 / 8-25\llcorner 1$ & & & 370 & 545 & 112.8 & 166.1 & no data & No data & -- & -- & -- & \\
\hline
\end{tabular}


Appendix 3. Lithologic data. - Continued

[Site IDs and well number correspond to locations in Appendix 1, a site ID and well number is listed for each drilled interval for a particular drill hole. For convenience, the site ID and well numbers are repeated at the shallowest drilled interval for each well. leaders

\begin{tabular}{|c|c|c|c|c|c|c|c|c|c|c|c|c|c|}
\hline Site ID & Well number & $\begin{array}{l}\text { Site ID, } \\
\text { top of } \\
\text { well }\end{array}$ & $\begin{array}{l}\text { Well number, } \\
\text { top of well }\end{array}$ & $\begin{array}{l}\text { Top of } \\
\text { interval, in } \\
\text { feet }\end{array}$ & $\begin{array}{l}\text { Base of } \\
\text { interval, in } \\
\text { feet }\end{array}$ & $\begin{array}{c}\text { Top of } \\
\text { interval, in } \\
m\end{array}$ & $\begin{array}{c}\text { Base of } \\
\text { interval, in } \\
\mathrm{m}\end{array}$ & Reported lithologic unit' & Interpreted lithologic class ${ }^{2}$ & $\begin{array}{c}\text { Reported } \\
\text { degree of } \\
\text { cementation or } \\
\text { induration }\end{array}$ & $\begin{array}{l}\text { Fossils } \\
\text { reported in } \\
\text { interval }^{4}\end{array}$ & $\begin{array}{l}\text { Organic } \\
\text { matter } \\
\text { reported in } \\
\text { interval }^{5}\end{array}$ & $\begin{array}{l}\text { Reported stratigraphic top of geologic } \\
\text { unit }^{6}\end{array}$ \\
\hline 201 & $7 / 8-25\llcorner 1$ & & & 545 & 745 & 166.1 & 227.1 & perforated intervals & No data & -- & -- & -- & QTge \\
\hline 202 & $7 / 8-29 \mathrm{R} 1$ & 202 & 7/8-29R1 & 0 & 110 & 0.0 & 33.5 & no data & No data & -- & -- & -- & -. \\
\hline 202 & $7 / 8-29 R 1$ & & & 110 & 120 & 33.5 & 36.6 & cemented gravel & Gravel & cemented & -- & -- & QTge \\
\hline 202 & 7/8-29R1 & & & 120 & 130 & 36.6 & 39.6 & no data & No data & - & -- & -- & \\
\hline 202 & $7 / 8-29 \mathrm{R} 1$ & & & 130 & 145 & 39.6 & 44.2 & clay, gravel, boulders & Clay and gravel & -- & -- & -- & \\
\hline 202 & 7/8-29R1 & & & 145 & 150 & 44.2 & 45.7 & no data & No data & -- & -- & -- & \\
\hline 202 & $7 / 8-29 \mathrm{R} 1$ & & & 150 & 157 & 45.7 & 47.9 & sandy clay and gravel & Clay, sand, and gravel & -- & -- & -- & \\
\hline 202 & $7 / 8-29 R 1$ & & & 157 & 175 & 47.9 & 53.3 & no data & No data & -- & -- & -- & \\
\hline 202 & $7 / 8-29 \mathrm{R} 1$ & & & 175 & 197 & 53.3 & 60.0 & clay and gravel & Clay and gravel & -- & -- & -- & \\
\hline 202 & $7 / 8-29 \mathrm{R} 1$ & & & 197 & 236 & 60.0 & 71.9 & no data & $\begin{array}{l}\text { No data } \\
\text { latave }\end{array}$ & -- & -- & -- & \\
\hline 202 & 7/8-29R1 & & & 236 & 253 & 71.9 & 77.1 & cemented gravel & Gravel & cemented & -- & -- & \\
\hline 202 & $7 / 8-29 \mathrm{R} 1$ & & & 253 & 650 & 77.1 & 198.1 & no data & No data & -- & -- & -- & \\
\hline 202 & $7 / 8-29 R 1$ & & & 650 & 679 & 198.1 & 207.0 & fine free gravel; sandy sediment & Sand (or sandstone) and gravel & -- & -- & -- & \\
\hline 202 & $7 / 8-29 \mathrm{R} 1$ & & & 679 & 682 & 207.0 & 207.9 & no data & No data & -- & -- & -- & \\
\hline 203 & $7 / 8-29 R 2$ & 203 & 7/8-29R2 & 0 & 801 & 0.0 & 244.1 & no data & No data & -- & -- & -- & .- \\
\hline 204 & $7 / 9-29 \mathrm{~J} 2$ & 204 & 7/9-29J2 & 0 & 23 & 0.0 & 7.0 & topsoil; sandy loam & Sand and clay & -- & -- & -- & Qyal \\
\hline 204 & $7 / 9-29 \mathrm{~J} 2$ & & & 23 & 46 & 7.0 & 14.0 & $\begin{array}{l}\text { blue clay } \\
\text { blue sandstone with occasional } \\
\text { hard streaks of hard gray }\end{array}$ & Clay & -- & -- & -- & \\
\hline 204 & $7 / 9-29 \mathrm{~J} 2$ & & & 46 & 499 & 14.0 & 152.1 & sandstone & Sandstone & hard & .- & .- & QTm \\
\hline 204 & $7 / 9-29 \mathrm{~J} 2$ & & & 499 & 519 & 152.1 & 158.2 & blue clay & Clay & -. & .- & -- & \\
\hline 204 & $7 / 9-2932$ & & & 519 & 532 & 158.2 & 162.2 & hard sand rock & Sandstone & hard & .- & .- & \\
\hline 204 & $7 / 9-29 \mathrm{~J} 2$ & & & 532 & 548 & 162.2 & 167.0 & sandy blue clay & Clay and sand (or sandstone) & -- & -- & -- & \\
\hline 204 & 7/9-29J2 & & & 548 & 580 & 167.0 & 176.8 & blue sand with shells & Sand & -- & shells & -- & \\
\hline 204 & $7 / 9-29 \mathrm{Jj}$ & & & 580 & 657 & 176.8 & 200.3 & $\begin{array}{l}\text { blue sandstone } \\
\text { blis }\end{array}$ & Sandstone & cemented & -- & -- & \\
\hline 205 & $7 / 9-28 \mathrm{P} 1$ & 205 & 7/9-28P1 & 0 & 585 & 0.0 & 178.3 & Uncased below $100 \mathrm{ft}$ ) & No data & - & -- & -- & QTm \\
\hline 206 & $7 / 8-29 \mathrm{J1}$ & 206 & $7 / 8-29 \mathrm{J1}$ & 0 & 4 & 0.0 & 1.2 & topsoil and sediment & Sand and clay & -- & -- & -- & QTge \\
\hline 206 & $7 / 8-29 J 1$ & & & 4 & 10 & 1.2 & 3.0 & 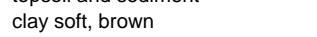 & Clay & -- & -- & -- & \\
\hline 206 & $7 / 8-29 J 1$ & & & 10 & 12 & 3.0 & 3.7 & sand, brown, coarse & Sand & .- & .- & .- & \\
\hline 206 & $7 / 8-29 J 1$ & & & 12 & 16 & 3.7 & 4.9 & sand black, fine & Sand & .- & .- & .- & \\
\hline 206 & $7 / 8-29 J 1$ & & & 16 & 29 & 4.9 & 8.8 & $\begin{array}{l}\text { clay, blue, some gravel } \\
\text { chent }\end{array}$ & Clay and gravel & -- & -- & -- & \\
\hline 206 & $7 / 8-29 J 1$ & & & 29 & 50 & 8.8 & 15.2 & clay, soft blue & Clay & -- & -- & -- & \\
\hline 206 & $7 / 8-29 \mathrm{J1}$ & & & 50 & 54 & 15.2 & 16.5 & $\begin{array}{l}\text { gravel, tight, cemented } \\
\text { gravel tight: clay sticky: shale. }\end{array}$ & Gravel & cemented & -- & -- & \\
\hline 206 & $7 / 8-29 J 1$ & & & 54 & 65 & 16.5 & 19.8 & $\begin{array}{l}\text { glavel, ugin, clay, sucky, sidale, } \\
\text { black }\end{array}$ & Clay and gravel & cemented & .- & .- & \\
\hline 206 & $7 / 8-29 J 1$ & & & 65 & 71 & 19.8 & 21.6 & gravel & Gravel & -. & .- & .- & \\
\hline 206 & $7 / 8-29 J 1$ & & & 71 & 75 & 21.6 & 22.9 & $\begin{array}{l}\text { clay } \\
\text { gravel, small, and sand; shale, }\end{array}$ & Clay & -- & -- & -- & \\
\hline 206 & $7 / 8-29 J 1$ & & & 75 & 79 & 22.9 & 24.1 & black; good & Clay, sand, and gravel & cemented & .- & -- & \\
\hline 206 & $7 / 8-29 \mathrm{J1}$ & & & 79 & 84 & 24.1 & 25.6 & $\begin{array}{l}\text { clay } \\
\text { gravel, medium, loose; shale, }\end{array}$ & Clay & -- & -- & -- & \\
\hline 206 & $7 / 8-29 J 1$ & & & 84 & 86 & 25.6 & 26.2 & black, fair & Clay and gravel & cemented & -- & .- & \\
\hline 206 & $7 / 8-29 J 1$ & & & 86 & 92 & 26.2 & 28.0 & clay, hard & Clay & hard & -- & -- & \\
\hline 206 & $7 / 8-29 J 1$ & & & 92 & 95 & 28.0 & 29.0 & clay and gravel & Clay and gravel & -- & -- & -- & \\
\hline 206 & $7 / 8-29 J 1$ & & & 95 & 100 & 29.0 & 30.5 & clay & Clay & .- & .- & .- & \\
\hline 206 & $7 / 8-29 J 1$ & & & 100 & 103 & 30.5 & 31.4 & gravel, small, free & Gravel & -. & .- & .- & \\
\hline 206 & $7 / 8-29 \mathrm{~J} 1$ & & & 103 & 110 & 31.4 & 33.5 & clay, hard & Clay & hard & -- & -- & \\
\hline 206 & $7 / 8-29 J 1$ & & & 110 & 115 & 33.5 & 35.1 & clay and gravel & Clay and gravel & -- & -- & -- & \\
\hline 206 & $7 / 8-29 J 1$ & & & 115 & 121 & $\begin{array}{l}3.5 \\
35.1\end{array}$ & $\begin{array}{l}35.1 \\
36.9\end{array}$ & $\begin{array}{l}\text { clay } \\
\text { clu gravel }\end{array}$ & $\begin{array}{l}\text { Clay } \\
\text { Clin gravel }\end{array}$ & -- & -- & -- & \\
\hline 206 & $7 / 8-29 \mathrm{~J} 1$ & & & 121 & 128 & 36.9 & 39.0 & clay and some gravel & Clay and gravel & -- & -- & -- & \\
\hline 206 & $7 / 8-29 J 1$ & & & 128 & 131 & 39.0 & $\begin{array}{l}39.0 \\
39.9\end{array}$ & gravel and clay; poor & Clay and gravel & -- & .- & - & \\
\hline 206 & $7 / 8-29 \mathrm{J1}$ & & & 131 & 136 & 39.9 & 41.5 & gravel, light, with clay & Clay and gravel & -- & -- & -- & \\
\hline 206 & $7 / 8-29 J 1$ & & & 136 & 138 & 41.5 & 42.1 & gravel, light, with clay & Clay and gravel & -- & -- & -- & \\
\hline 206 & 7/8-29J1 & & & 138 & 184 & 42.1 & 56.1 & clay, sticky and hard & Clay & hard & .- & .- & \\
\hline 206 & $7 / 8-29 J 1$ & & & 184 & 188 & 56.1 & 57.3 & $\begin{array}{l}\text { clay, soft } \\
\text { lath }\end{array}$ & Clay & -- & -- & -- & \\
\hline 206 & $7 / 8-29 \mathrm{J1}$ & & & 188 & 189 & 57.3 & 57.6 & gravel, loose; fair & Gravel & -- & -- & .- & \\
\hline 206 & $7 / 8-29 J 1$ & & & 189 & 191 & 57.6 & 58.2 & clay, soft & Clay & .- & .- & .- & \\
\hline 206 & $7 / 8-29 \mathrm{J1}$ & & & 191 & 227 & 58.2 & 69.2 & $\begin{array}{l}\text { clay, hard } \\
\text { gravel, small, and sand; good }\end{array}$ & Clay & hard & -- & - & \\
\hline 206 & $7 / 8-29 J 1$ & & & 227 & 234 & 69.2 & 71.3 & $\begin{array}{l}\text { colors; good } \\
\text { colus }\end{array}$ & Sand (or sandstone) and gravel & .- & -- & .- & \\
\hline 206 & $7 / 8-29 \mathrm{~J} 1$ & & & 234 & 236 & 71.3 & 71.9 & gravel and clay; poor & Clay and gravel & -- & -- & -- & \\
\hline
\end{tabular}


Appendix 3. Lithologic data. - Continued

ISite IDs and well number correspond to locations in Appendix 1, a site ID and well number is listed for each drilled interval for a particular drill hole. For convenience, the site ID and well numbers are repeated at the shallowest drilled

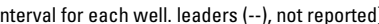

\begin{tabular}{|c|c|c|c|c|c|c|c|c|c|c|c|c|c|}
\hline Site ID & Well number & $\begin{array}{l}\text { Site ID, } \\
\text { top of } \\
\text { well }\end{array}$ & $\begin{array}{l}\text { Well number, } \\
\text { top of well }\end{array}$ & $\begin{array}{l}\text { Top of } \\
\text { interval, in } \\
\text { feet }\end{array}$ & $\begin{array}{l}\text { Base of } \\
\text { interval, in } \\
\text { feet }\end{array}$ & $\begin{array}{c}\text { Top of } \\
\text { interval, in } \\
m\end{array}$ & $\begin{array}{c}\text { Base of } \\
\text { interval, in } \\
\mathrm{m}\end{array}$ & Reported lithologic unit ${ }^{1}$ & Interpreted lithologic class ${ }^{2}$ & $\begin{array}{c}\text { Reported } \\
\text { degree of } \\
\text { cementation or } \\
\text { induration } \\
\end{array}$ & $\begin{array}{l}\text { Fossils } \\
\text { reported in }^{\text {interval }}{ }^{4}\end{array}$ & $\begin{array}{c}\text { Organic } \\
\text { matter } \\
\text { reported in } \\
\text { interval }^{5}\end{array}$ & $\begin{array}{l}\text { Reported stratigraphic top of geologic } \\
\text { unit }^{6}\end{array}$ \\
\hline 206 & $7 / 8-29 \mathrm{~J} 1$ & & & 236 & 246 & 71.9 & 75.0 & $\begin{array}{l}\text { clay with streaks of gravel } \\
\text { gravel, medium, loose; good }\end{array}$ & Clay and gravel & -- & -- & -- & \\
\hline 206 & $7 / 8-29 J 1$ & & & 246 & 252 & 75.0 & 76.8 & colors; good & Gravel & -- & -- & -- & \\
\hline 206 & $7 / 8-29 J 1$ & & & 252 & 256 & 76.8 & 78.0 & gravel, tight, and clay & Clay and gravel & -- & .- & -- & \\
\hline 206 & $7 / 8-29 \mathrm{~J} 1$ & & & 256 & 306 & 78.0 & 93.3 & clay, hard, sticky & Clay & hard & -- & -- & \\
\hline 206 & $7 / 8-29 J 1$ & & & 306 & 309 & 93.3 & 94.2 & gravel, small, and clay, soft & Clay and gravel & -- & -- & -- & \\
\hline 206 & $7 / 8-29 \mathrm{~J} 1$ & & & 309 & 344 & 94.2 & 104.9 & clay, hard, sticky & Clay & hard & -- & -- & \\
\hline 206 & $7 / 8-2931$ & & & 344 & 345 & 104.9 & 105.2 & gravel and clay; poor & Clay and gravel & - & -- & -- & \\
\hline 206 & $7 / 8-29 J 1$ & & & 345 & 355 & 105.2 & 108.2 & $\begin{array}{l}\text { clay } \\
\text { clituliay, pour }\end{array}$ & $\begin{array}{l}\text { Clay } \\
\text { Clut gravet }\end{array}$ & -- & -- & -- & \\
\hline 206 & $7 / 8-29 \mathrm{J1}$ & & & 355 & 357 & 108.2 & 108.8 & gravel and clay; poor & Clay and gravel & -- & -- & -- & \\
\hline 206 & $7 / 8-29 J 1$ & & & 357 & 360 & 108.8 & 109.7 & clay, soft & Clay & -- & -- & -- & \\
\hline 206 & $7 / 8-29 J 1$ & & & 360 & 366 & 109.7 & 111.6 & $\begin{array}{l}\text { gravel, medium; fair } \\
\text { clay, hard, with soft streaks of }\end{array}$ & Gravel & -- & -- & -- & \\
\hline 206 & $7 / 8-29 J 1$ & & & 366 & 408 & 111.6 & 124.4 & $\begin{array}{l}\text { white clay } \\
\text { clay, hard, sticky, with streaks of }\end{array}$ & Clay & hard & -- & -- & \\
\hline 206 & $7 / 8-29 J 1$ & & & 408 & 430 & 124.4 & 131.1 & gravel and soft clay & Clay and gravel & hard & .- & .- & \\
\hline 206 & $7 / 8-29 J 1$ & & & 430 & 432 & 131.1 & 131.7 & clay, soft & Clay & -- & -- & -- & \\
\hline 206 & $7 / 8-29 \mathrm{~J} 1$ & & & 432 & 434 & 131.7 & 132.3 & gravel and sand; fair & Sand (or sandstone) and gravel & .- & .- & -- & \\
\hline 206 & $7 / 8-29 J 1$ & & & 434 & 435 & 132.3 & 132.6 & $\begin{array}{l}\text { clay and gravel } \\
\text { clin }\end{array}$ & $\begin{array}{l}\text { Clay and gravel }\end{array}$ & -- & -- & -- & \\
\hline 206 & $7 / 8-29 J 1$ & & & 435 & 443 & 132.6 & 135.0 & sand and small gravel, fair & Sand (or sandstone) and gravel & -- & -- & -- & \\
\hline 206 & $7 / 8-29 J 1$ & & & 443 & 450 & 135.0 & 137.2 & $\begin{array}{l}\text { clay, hard, cemented } \\
\text { all }\end{array}$ & Clay & cemented & .- & .- & \\
\hline 206 & $7 / 8-29 J 1$ & & & 450 & 453 & 137.2 & 138.1 & clay, soft & Clay & -- & -- & -- & \\
\hline 206 & $7 / 8-29 \mathrm{~J} 1$ & & & 453 & 456 & 138.1 & 139.0 & sand and gravel; fair & Sand (or sandstone) and gravel & .- & .- & .- & \\
\hline 206 & $7 / 8-29 J 1$ & & & 456 & 472 & 139.0 & 143.9 & sandy clay and gravel; poor & $\begin{array}{l}\text { Clay, sand, and gravel } \\
\text {. }\end{array}$ & -- & -- & -- & \\
\hline 206 & $7 / 8-2931$ & & & 472 & 476 & 143.9 & 145.1 & sand and gravel; fair & Sand (or sandstone) and gravel & -- & .- & .- & \\
\hline 206 & $7 / 8-29 J 1$ & & & 476 & 515 & 145.1 & 157.0 & clay hard & Clay & hard & -- & -- & \\
\hline 206 & $7 / 8-29 J 1$ & & & 515 & 536 & 157.0 & 163.4 & clay, hard, with soft streaks & Clay & hard & -- & -- & \\
\hline 206 & $7 / 8-29 J 1$ & & & 536 & 546 & 163.4 & 166.4 & 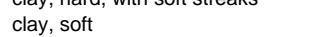 & Clay & -. & -- & -- & \\
\hline 206 & $7 / 8-29 \mathrm{~J} 1$ & & & 546 & 549 & 166.4 & 167.3 & gravel, small, and sand; poor & Sand (or sandstone) and gravel & -- & .- & -- & \\
\hline 206 & $7 / 8-29 J 1$ & & & 549 & 560 & 167.3 & 170.7 & clay & Clay & .- & .- & .- & \\
\hline 206 & $7 / 8-29 J 1$ & & & 560 & 563 & 170.7 & 171.6 & sand & Sand & -- & -- & -- & \\
\hline 206 & $7 / 8-29 J 1$ & & & 563 & 569 & 171.6 & 173.4 & gravel, medium; good & Gravel & -- & -- & -- & \\
\hline 206 & $7 / 8-29 J 1$ & & & 569 & 591 & 173.4 & 180.1 & $\begin{array}{l}\text { clay, hard, sticky } \\
\text { la }\end{array}$ & Clay & hard & -- & -- & \\
\hline 206 & $7 / 8-29 \mathrm{~J} 1$ & & & 591 & 592 & 180.1 & 180.4 & clay and gravel & Clay and gravel & - & -- & -- & \\
\hline 206 & $7 / 8-29 \mathrm{~J} 1$ & & & 592 & 622 & 180.4 & 189.6 & clay, hard sticky & Clay & hard & -- & -- & \\
\hline 206 & $7 / 8-29 J 1$ & & & 622 & 627 & 189.6 & 191.1 & gravel, light & Gravel & -- & -- & -- & \\
\hline 206 & $7 / 8-29 J 1$ & & & 627 & 632 & 191.1 & 192.6 & clay and gravel & Clay and gravel & -- & -- & -- & \\
\hline 206 & $7 / 8-29 \mathrm{J1}$ & & & 632 & 647 & 192.6 & 197.2 & clay & $\begin{array}{l}\text { Clay } \\
\text { Clut gravet }\end{array}$ & -- & -- & -- & \\
\hline 206 & $7 / 8-29 J 1$ & & & 647 & 661 & 197.2 & 201.5 & gravel, large, loose, good & Gravel & -- & -- & -- & \\
\hline 206 & $7 / 8-29 \mathrm{J1}$ & & & 661 & 700 & 201.5 & 213.4 & clay & Clay & -- & .- & -- & \\
\hline 207 & $7 / 8-25 R 1$ & 207 & 7/8-25R1 & 0 & 30 & 0.0 & 9.1 & no data & No data & -- & -- & -- & -- \\
\hline 207 & $7 / 8-25 R 1$ & & & 30 & 180 & 9.1 & 54.9 & perforated intervals & No data & .- & .- & -- & Tsv (?) \\
\hline 207 & $7 / 8-25 \mathrm{R} 1$ & & & 180 & 320 & $\begin{array}{l}.1 \\
54.9\end{array}$ & 97.5 & no data & No data & -- & -- & -- & $150(?)$ \\
\hline 208 & 7/9-33D2 & 208 & 7/9-33D2 & 0 & 300 & 0.0 & 91.4 & no data & No data & -- & -- & -- & QTm \\
\hline 209 & 7/9-33A1 & 209 & 7/9-33A1 & 0 & 245 & 0.0 & 74.7 & no data & No data & -- & -- & .- & QTm \\
\hline 210 & 7/9-31A1 & 210 & 7/9-31A1 & 0 & 240 & 0.0 & 73.2 & no data & No data & -- & -- & -- & QTm \\
\hline 211 & $7 / 8-36 \mathrm{D} 2$ & 211 & 7/8-36D2 & 0 & 8 & 0.0 & 2.4 & soil & Sand and clay & -- & -- & -- & Qyal and QTge \\
\hline 211 & $7 / 8-36 \mathrm{D} 2$ & & & 8 & 28 & 2.4 & 8.5 & $\begin{array}{l}\text { yellow clay } \\
\text { cemented gravel, first water at } 77\end{array}$ & Clay & -- & -- & -- & \\
\hline 211 & $7 / 8-36 \mathrm{D} 2$ & & & 28 & 77 & 8.5 & 23.5 & feet & Gravel & cemented & -- & -- & \\
\hline 211 & $7 / 8-36 \mathrm{D} 2$ & & & 77 & 92 & 23.5 & 28.0 & hard rock & Ash or tuff & hard & -- & -- & \\
\hline 211 & $7 / 8-36 \mathrm{D} 2$ & & & 92 & 114 & 28.0 & 34.7 & rock & Ash or tuff & hard & -- & -- & \\
\hline 211 & $7 / 8-36 \mathrm{D} 2$ & & & 114 & 185 & 34.7 & 56.4 & yellow clay & Clay & - & -- & -- & \\
\hline 211 & $7 / 8-36 \mathrm{D} 2$ & & & 185 & 225 & 56.4 & 68.6 & yellow sandy clay & Clay and sand (or sandstone) & .- & .- & .- & \\
\hline 211 & $7 / 8-36 \mathrm{D} 2$ & & & 225 & 241 & 68.6 & 73.5 & clay & Clay & -- & -- & -- & \\
\hline 211 & $7 / 8-36 \mathrm{D} 2$ & & & 241 & 249 & 73.5 & 75.9 & coarse sand, water & Sand & -- & -- & -- & \\
\hline 211 & $7 / 8-36 \mathrm{D} 2$ & & & 249 & 252 & 75.9 & 76.8 & yellow clay & Clay & -- & -- & -- & \\
\hline 212 & $7 / 8-30 \mathrm{P} 1$ & 212 & 7/8-30P1 & 0 & 2 & 0.0 & 0.6 & soil & Sand and clay & -- & -- & -- & QTge \\
\hline 212 & 7/8-30P1 & & & 2 & 12 & 0.6 & 3.7 & hardpan & Clay and gravel & hard & -- & -- & \\
\hline 212 & $7 / 8-30 \mathrm{P} 1$ & & & 12 & 15 & 3.7 & 4.6 & water sand & Sand & -- & -- & -- & \\
\hline 212 & 7/8-30P1 & & & 15 & 25 & 4.6 & 7.6 & clay, brown & Clay & -- & -- & -- & \\
\hline
\end{tabular}


Appendix 3. Lithologic data. - Continued

[Site IDs and well number correspond to locations in Appendix 1, a site ID and well number is listed for each drilled interval for a particular drill hole. For convenience, the site ID and well numbers are repeated at the shallowest drilled interval for each well. leaders $(-1$, n

\begin{tabular}{|c|c|c|c|c|c|c|c|c|c|c|c|c|c|}
\hline Site ID & Well number & $\begin{array}{l}\text { Site ID, } \\
\text { top of } \\
\text { well }\end{array}$ & $\begin{array}{l}\text { Well number, } \\
\text { top of well }\end{array}$ & $\begin{array}{l}\text { Top of } \\
\text { interval, in } \\
\text { feet }\end{array}$ & $\begin{array}{l}\text { Base of } \\
\text { interval, in } \\
\text { feet }\end{array}$ & $\begin{array}{c}\text { Top of } \\
\text { interval, in } \\
\mathrm{m}\end{array}$ & $\begin{array}{c}\text { Base of } \\
\text { interval, in } \\
\mathrm{m}\end{array}$ & Reported lithologic unit' & Interpreted lithologic class ${ }^{2}$ & $\begin{array}{c}\text { Reported } \\
\text { degree of } \\
\text { cementation or } \\
\text { induration }^{3} \\
\end{array}$ & $\begin{array}{c}\text { Fossils } \\
\text { reported in } \\
\text { interval }^{4}\end{array}$ & $\begin{array}{c}\text { Organic } \\
\text { matter } \\
\text { reported in } \\
\text { interval }^{5}\end{array}$ & $\begin{array}{l}\text { Reported stratigraphic top of geologic } \\
\text { unit }^{6}\end{array}$ \\
\hline 212 & 7/8-30P1 & & & 25 & 48 & 7.6 & 14.6 & clay, blue & Clay & -- & -- & -- & \\
\hline 212 & $7 / 8-30 \mathrm{P} 1$ & & & 48 & 54 & 14.6 & 16.5 & gravel & Gravel & -- & -- & -- & \\
\hline 212 & 7/8-30P1 & & & 54 & 63 & 16.5 & 19.2 & clay, blue & Clay & -- & -- & -- & \\
\hline 212 & 7/8-30P1 & & & 63 & 72 & 19.2 & 21.9 & clay, brown & Clay & -- & -- & -- & \\
\hline 212 & $7 / 8-30 \mathrm{P} 1$ & & & 72 & 76 & 21.9 & 23.2 & clay, blue & Clay & -- & -- & -- & \\
\hline 212 & 7/8-30P1 & & & 76 & 123 & 23.2 & 37.5 & clay, blue & Clay & -- & -- & -- & \\
\hline 212 & 7/8-30P1 & & & 123 & 142 & 37.5 & 43.3 & sand and clay & Sand and clay & -- & -- & -- & QTm \\
\hline 212 & 7/8-30P1 & & & 142 & 145 & 43.3 & 44.2 & clay, blue & Clay & -- & -- & -- & \\
\hline 212 & $7 / 8-30 \mathrm{P} 1$ & & & 145 & 164 & 44.2 & 50.0 & sandstone & Sandstone & cemented & -- & -- & \\
\hline 212 & 7/8-30P1 & & & 164 & 178 & 50.0 & 54.3 & clay and gravel & Clay and gravel & -- & -- & -- & QTge \\
\hline 212 & 7/8-30P1 & & & 178 & 183 & 54.3 & 55.8 & gravel, cement & Gravel & cemented & -- & -- & \\
\hline 212 & $7 / 8-30 \mathrm{P} 1$ & & & 183 & 205 & 55.8 & 62.5 & clay, blue & Clay & -- & -- & -- & \\
\hline 213 & $7 / 8-31 \mathrm{C} 1$ & 213 & 7/8-31C1 & 0 & 190 & 0.0 & 57.9 & old well (no record) & No data & -- & -- & -- & QTge \\
\hline 213 & $7 / 8-31 \mathrm{C} 1$ & & & 190 & 305 & 57.9 & 93.0 & yellow sandstone & Sandstone & cemented & -- & -- & QTm \\
\hline 213 & $7 / 8-31 \mathrm{C} 1$ & & & 305 & 320 & 93.0 & 97.5 & gravel & Gravel & -- & -- & -- & \\
\hline 214 & $7 / 8-35 \mathrm{H} 1$ & 214 & $7 / 8-35 \mathrm{H} 1$ & 0 & 270 & 0.0 & 82.3 & no data & No data & -- & -- & -- & QTge \\
\hline 215 & 7/9-34G1 & 215 & 7/9-34G1 & 0 & 349 & 0.0 & 106.4 & no data & No data & -- & -- & -- & -- \\
\hline 215 & 7/9-34G1 & & & 349 & 358 & 106.4 & 109.1 & coarse soft sandstone & Sandstone & cemented & -- & -- & QTm \\
\hline 216 & $7 / 9-32 F 2$ & 216 & $7 / 9-32 F 2$ & 0 & 365 & 0.0 & 111.3 & no data & No data & -- & -- & -- & QTm \\
\hline 217 & 7/9-33F1 & 217 & 7/9-33F1 & 0 & 200 & 0.0 & 61.0 & sand, sandstone & Sandstone & cemented & -- & -- & QTm \\
\hline 218 & 7/9-35E1 & 218 & 7/9-35E1 & 0 & 253 & 0.0 & 77.1 & no data & No data & -- & -- & -- & QTm \\
\hline 219 & 7/9-32E1 & 219 & 7/9-32E1 & 0 & 200 & 0.0 & 61.0 & (Uncased below $6 \mathrm{ft}$ ) & No data & -- & -- & -- & QTm \\
\hline 220 & $7 / 9-36 \mathrm{H} 1$ & 220 & 7/9-36H1 & 0 & 2 & 0.0 & 0.6 & topsoil & Sand and clay & -- & -- & -- & QTge \\
\hline 220 & $7 / 9-36 \mathrm{H} 1$ & & & 2 & 30 & 0.6 & 9.1 & yellow clay & Clay & -- & -- & -- & \\
\hline 220 & $7 / 9-36 \mathrm{H} 1$ & & & 30 & 70 & 9.1 & 21.3 & blue clay & Clay & -- & -- & -- & \\
\hline 220 & $7 / 9-36 \mathrm{H} 1$ & & & 70 & 102 & 21.3 & 31.1 & gravel & Gravel & -- & -- & -- & \\
\hline 220 & $7 / 9-36 \mathrm{H} 1$ & & & 102 & 280 & 31.1 & 85.3 & blue clay & Clay & -- & -- & -- & \\
\hline 220 & $7 / 9-36 \mathrm{H} 1$ & & & 280 & 350 & 85.3 & 106.7 & hard sandstone & Sandstone & hard & -- & -- & QTm \\
\hline 220 & $7 / 9-36 \mathrm{H} 1$ & & & 350 & 485 & 106.7 & 147.8 & sandstone & Sandstone & cemented & -- & -- & \\
\hline 220 & $7 / 9-36 \mathrm{H} 1$ & & & 485 & 503 & 147.8 & 153.3 & blue sand & Sand & - & -- & -- & \\
\hline 220 & $7 / 9-36 \mathrm{H} 1$ & & & 503 & 510 & 153.3 & 155.4 & sandstone & Sandstone & cemented & -- & -- & \\
\hline 220 & $7 / 9-36 \mathrm{H} 1$ & & & 510 & 560 & 155.4 & 170.7 & gravel and sand; fair & Sand (or sandstone) and gravel & -- & -- & -- & \\
\hline 220 & $7 / 9-36 \mathrm{H} 1$ & & & 560 & 568 & 170.7 & 173.1 & blue clay & Clay & -- & -- & -- & \\
\hline 221 & $7 / 6-32 \mathrm{~F} 1$ & 221 & 7/6-32F1 & 0 & 21 & 0.0 & 6.4 & tule mud & Clay and sand (or sandstone) & -- & -- & tule & Qyal and QTge \\
\hline 221 & $7 / 6-32 F 1$ & & & 21 & 29 & 6.4 & 8.8 & rock and gravel & Sand (or sandstone) and gravel & -- & -- & -- & \\
\hline 221 & $7 / 6-32 \mathrm{~F} 1$ & & & 29 & 45 & 8.8 & 13.7 & clay, yellow, gravel, water & Clay and gravel & -- & -- & -- & \\
\hline 221 & 7/6-32F1 & & & 45 & 70 & 13.7 & 21.3 & gravel, cement & Gravel & cemented & -- & -- & \\
\hline 221 & $7 / 6-32 \mathrm{~F} 1$ & & & 70 & 80 & 21.3 & 24.4 & shale, blue, clay & Clay and sand (or sandstone) & cemented & -- & -- & \\
\hline 221 & $7 / 6-32 \mathrm{~F} 1$ & & & 80 & 99 & 24.4 & 30.2 & clay, blue & Clay & - & -- & -- & \\
\hline 221 & $7 / 6-32 \mathrm{~F} 1$ & & & 99 & 103 & 30.2 & 31.4 & gravel, some water & Gravel & -- & -- & -- & \\
\hline 221 & $7 / 6-32 \mathrm{~F} 1$ & & & 103 & 120 & 31.4 & 36.6 & clay, blue & Clay & -- & -- & -- & \\
\hline 221 & 7/6-32F1 & & & 120 & 137 & 36.6 & 41.8 & shale rock & Clay and sand (or sandstone) & cemented & -- & -- & \\
\hline 221 & $7 / 6-32 \mathrm{~F} 1$ & & & 137 & 160 & 41.8 & 48.8 & clay, yellow, gravel, and rock & Clay and gravel & -- & -- & -- & \\
\hline 221 & $7 / 6-32 \mathrm{~F} 1$ & & & 160 & 175 & 48.8 & 53.3 & clay, rock, and gravel & Clay and gravel & -- & -- & -- & \\
\hline 221 & $7 / 6-32 F 1$ & & & 175 & 190 & 53.3 & 57.9 & gravel, cement & Gravel & cemented & -- & -- & \\
\hline 222 & 7/9-34L1 & 222 & 7/9-34L1 & 0 & 300 & 0.0 & 91.4 & no data & No data & -- & -- & -- & -- \\
\hline 222 & 7/9-34L1 & & & 300 & 315 & 91.4 & 96.0 & sand & Sand & -- & -- & -- & QTm \\
\hline 223 & 7/9-36F1 & 223 & 7/9-36F1 & 0 & 30 & 0.0 & 9.1 & gumbo & Clay and sand (or sandstone) & -- & -- & -- & QTge \\
\hline 223 & 7/9-36F1 & & & 30 & 40 & 9.1 & 12.2 & clay and mixed gravel & Clay and gravel & -- & -- & -- & \\
\hline 223 & $7 / 9-36 \mathrm{~F} 1$ & & & 40 & 45 & 12.2 & 13.7 & gravel, pea; some water & Gravel & -- & -- & -- & \\
\hline 223 & $7 / 9-36 \mathrm{~F} 1$ & & & 45 & 60 & 13.7 & 18.3 & clay & Clay & -- & -- & -- & \\
\hline 223 & 7/9-36F1 & & & 60 & 70 & 18.3 & 21.3 & gravel, fine & Gravel & -- & -- & -- & \\
\hline 223 & $7 / 9-36 \mathrm{~F} 1$ & & & 70 & 90 & 21.3 & 27.4 & gravel large water & Gravel & -- & -- & -- & \\
\hline 223 & $7 / 9-36 \mathrm{~F} 1$ & & & 90 & 122 & 27.4 & 37.2 & shale and small boulders & Clay, sand, and gravel & cemented & -- & -- & \\
\hline 223 & $7 / 9-36 \mathrm{~F} 1$ & & & 122 & 133 & 37.2 & 40.5 & pack sand, solid & Sand & -- & -- & -- & \\
\hline 223 & 7/9-36F1 & & & 133 & 155 & 40.5 & 47.2 & dirt, black & Sand and clay & -- & -- & -- & \\
\hline 223 & 7/9-36F1 & & & 155 & 160 & 47.2 & 48.8 & mud & Sand and clay & -- & -- & -- & \\
\hline 223 & $7 / 9-36 \mathrm{~F} 1$ & & & 160 & 188 & 48.8 & 57.3 & sand, river; water & Sand & -- & -- & -- & QTm \\
\hline 223 & $7 / 9-36 \mathrm{~F} 1$ & & & 188 & 200 & 57.3 & 61.0 & clay and mixed sand & Clay and gravel & -- & -- & -- & \\
\hline 223 & $7 / 9-36 \mathrm{~F} 1$ & & & 200 & 228 & 61.0 & 69.5 & sand, quick; water & Sand & -- & -- & -- & \\
\hline 223 & $7 / 9-36 \mathrm{~F} 1$ & & & 228 & 251 & 69.5 & 76.5 & gravel, fine; water & Gravel & -- & -- & -- & \\
\hline
\end{tabular}


Appendix 3. Lithologic data. - Continued

[Site IDs and well number correspond to locations in Appendix 1, a site ID and well number is listed for each drilled interval for a particular drill hole. For convenience, the site ID and well numbers are repeated at the shallowest drilled interval for each well. leaders

\begin{tabular}{|c|c|c|c|c|c|c|c|c|c|c|c|c|c|}
\hline Site ID & Well number & $\begin{array}{l}\text { Site ID, } \\
\text { top of } \\
\text { well }\end{array}$ & $\begin{array}{l}\text { Well number, } \\
\text { top of well }\end{array}$ & $\begin{array}{l}\text { Top of } \\
\text { interval, in } \\
\text { feet }\end{array}$ & $\begin{array}{l}\text { Base of } \\
\text { interval, in } \\
\text { feet }\end{array}$ & $\begin{array}{c}\text { Top of } \\
\text { interval, in } \\
m\end{array}$ & $\begin{array}{c}\text { Base of } \\
\text { interval, in } \\
m\end{array}$ & Reported lithologic unit' & Interpreted lithologic class ${ }^{2}$ & $\begin{array}{c}\text { Reported } \\
\text { degree of } \\
\text { cementation or } \\
\text { induration }^{3}\end{array}$ & $\begin{array}{c}\text { Fossils } \\
\text { reported in } \\
\text { interval }^{4}\end{array}$ & $\begin{array}{c}\text { Organic } \\
\text { matter } \\
\text { reported in } \\
\text { interval }\end{array}$ & $\begin{array}{l}\text { Reported stratigraphic top of geologic } \\
\text { unit }^{6}\end{array}$ \\
\hline 223 & $7 / 9-36 \mathrm{~F} 1$ & & & 251 & 270 & 76.5 & 82.3 & gravel, coarse; water & Gravel & -- & -- & -- & \\
\hline 224 & $7 / 9-31 \mathrm{J1}$ & 224 & 7/9-31J1 & 0 & 40 & 0.0 & 12.2 & no data & No data & -- & -- & -- & -- \\
\hline 224 & 7/9-31J1 & & & 40 & 62 & 12.2 & 18.9 & fine sand & Sand & -- & -- & -- & QTm \\
\hline 224 & $7 / 9-31 J 1$ & & & 62 & 185 & 18.9 & 56.4 & no data & No data & -- & -- & -- & \\
\hline 224 & 7/9-31J1 & & & 185 & 241 & 56.4 & 73.5 & sandstone with clam shells & Sandstone & cemented & shells & .- & \\
\hline 224 & $7 / 9-31 J 1$ & & & 241 & 296 & 73.5 & 90.2 & $\begin{array}{l}\text { no data } \\
\text { sandstone containing streak }\end{array}$ & No data & - & -- & -- & \\
\hline 224 & 7/9-31J1 & & & 296 & 352 & 90.2 & 107.3 & $\begin{array}{l}\text { gravel } \\
\text { gen }\end{array}$ & Sand (or sandstone) and gravel & cemented & -- & -- & \\
\hline 225 & 7/9-34J1 & 225 & 7/9-34J1 & 0 & 230 & 0.0 & 70.1 & (cased to $204 \mathrm{ft}$ ) & No data & -- & .- & -- & QTm \\
\hline 226 & 7/7-32G1 & 226 & 7/7-32G1 & 0 & 40 & 0.0 & 12.2 & gravel & Gravel & .- & -- & .- & Qyal \\
\hline 226 & $7 / 7-32 \mathrm{G} 1$ & & & 40 & 110 & 12.2 & 33.5 & clay and volcanic ash & Ash or tuff & -- & -- & -- & QTge \\
\hline 226 & $7 / 7-32 \mathrm{G} 1$ & & & 110 & 130 & 33.5 & 39.6 & clay and volcanic ash & Ash or tuff & -- & -- & -- & \\
\hline 226 & 7/7-32G1 & & & 130 & 210 & 39.6 & 64.0 & basalt & Basalt & .- & .- & -- & Tsv \\
\hline 226 & $7 / 7-32 \mathrm{G1}$ & & & 210 & 260 & 64.0 & 79.2 & ash, white, volcanic & Ash or tuff & -- & -- & -- & 150 \\
\hline 226 & 7/7-32G1 & & & 260 & 270 & 79.2 & 82.3 & basalt and white volcanic ash & Basalt & -- & -- & -- & \\
\hline 226 & $7 / 7-32 \mathrm{G} 1$ & & & 270 & 330 & 82.3 & 100.6 & $\begin{array}{l}\text { white volcanic ash } \\
\text { white volcanic ash and basalt } \\
\text { cuttings (probably basalt boulders }\end{array}$ & Ash or tuff & -- & - & -- & \\
\hline 226 & 7/7-32G1 & & & 330 & 340 & 100.6 & 103.6 & in basaltic tuff) & Ash or tuff & .- & .- & .- & \\
\hline 226 & $7 / 7-32 \mathrm{G} 1$ & & & 340 & 370 & 103.6 & 112.8 & dark gray volcanic ash & Ash or tuff & -- & -- & -- & \\
\hline & & & & & & & & $\begin{array}{l}\text { gray volcanic ash, and dark gray } \\
\text { clay (also woody material reported }\end{array}$ & & & & & \\
\hline 226 & 7/7-32G1 & & & 370 & 390 & 112.8 & 118.9 & by driller as redwood log) & Ash or tuff & .- & .- & wood & \\
\hline 226 & 7/7-32G1 & & & 390 & 402 & 118.9 & 122.5 & white volcanic ash & Ash or tuff & .- & .- & -- & \\
\hline 226 & $7 / 7-32 \mathrm{G1}$ & & & 402 & 403 & 122.5 & 122.8 & basalt & $\begin{array}{l}\text { Basalt } \\
\text { Bain } \\
\text { Bas }\end{array}$ & -- & -- & -- & \\
\hline 227 & 7/9-34J2 & 227 & $7 / 9-34 J 2$ & 0 & 310 & 0.0 & 94.5 & no data & No data & .- & .- & .- & .- \\
\hline 227 & $7 / 9-34 J 2$ & & & 310 & 330 & 94.5 & 100.6 & (perforated Intervals) & No data & .- & .- & .- & QTm \\
\hline 227 & 7/9-34J2 & & & 330 & 350 & 100.6 & 106.7 & no data & No data & .- & .- & .- & \\
\hline 228 & 7/8-33M1 & 228 & 7/8-33M1 & 0 & 40 & 0.0 & 12.2 & clay & Clay & -- & -- & -- & QTge \\
\hline 228 & 7/8-33M1 & & & 40 & 63 & 12.2 & 19.2 & gravel & Gravel & .- & .- & .- & \\
\hline 228 & 7/8-33M1 & & & 63 & 87 & 19.2 & 26.5 & clay & Clay & .- & .- & -- & \\
\hline 228 & 7/8-33M1 & & & 87 & 104 & 26.5 & 31.7 & sand and gravel & Sand (or sandstone) and gravel & .- & .- & .- & \\
\hline 228 & 7/8-33M1 & & & 104 & 146 & 31.7 & 44.5 & clay & Clay & .- & .- & .- & \\
\hline 228 & 7/8-33M1 & & & 146 & 153 & 44.5 & 46.6 & sand and gravel & Sand (or sandstone) and gravel & -- & -- & -- & \\
\hline 228 & 7/8-33M1 & & & 153 & 172 & 46.6 & 52.4 & clay & Clay & -- & -- & -- & \\
\hline 228 & 7/8-33M1 & & & 172 & 301 & 52.4 & 91.7 & gravel & Gravel & .- & .- & .- & \\
\hline 228 & 7/8-33M1 & & & 301 & 346 & 91.7 & 105.5 & blue clay & Clay & .- & .- & .- & \\
\hline 228 & 7/8-33M1 & & & 346 & 375 & 105.5 & 114.3 & sand and gravel & Sand (or sandstone) and gravel & -- & -- & -- & \\
\hline 228 & $7 / 8-33 \mathrm{M} 1$ & & & 375 & 422 & 114.3 & 128.6 & $\begin{array}{l}\text { blue clay } \\
\text { blaver }\end{array}$ & Clay & -- & -- & -- & \\
\hline 228 & 7/8-33M1 & & & 422 & 452 & 128.6 & 137.8 & sand and gravel & Sand (or sandstone) and gravel & -- & -- & -- & \\
\hline 229 & 7/9-31Q1 & 229 & 7/9-31Q1 & 0 & 90 & 0.0 & 27.4 & no data & No data & -- & -- & -- & -- \\
\hline 229 & 7/9-31Q1 & & & 90 & 235 & 27.4 & $\begin{array}{l}21.4 \\
71.6\end{array}$ & sandstone & Sandstone & cemented & -- & -- & QTm \\
\hline 230 & 7/9-36M1 & 230 & 7/9-36M1 & 0 & 4 & 0.0 & 1.2 & adobe & Sand and clay & -- & -- & -- & QTge \\
\hline 230 & 7/9-36M1 & & & 4 & 8 & 1.2 & 2.4 & sandstone, yellow & Sandstone & cemented & -- & .- & \\
\hline 230 & 7/9-36M1 & & & 8 & 38 & 2.4 & 11.6 & clay, blue & Clay & - & -- & -- & \\
\hline 230 & 7/9-36M1 & & & 38 & 46 & 11.6 & 14.0 & sandstone and gravel & Sand (or sandstone) and gravel & cemented & -- & -- & \\
\hline 230 & 7/9-36M1 & & & 46 & 72 & 14.0 & 21.9 & $\begin{array}{l}\text { clay, blue } \\
\text { sand, blue, and some gravel; }\end{array}$ & Clay & -- & -- & -- & \\
\hline 230 & 7/9-36M1 & & & 72 & 86 & 21.9 & 26.2 & 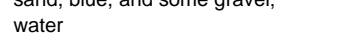 & Sand (or sandstone) and gravel & -- & .- & .- & \\
\hline 230 & 7/9-36M1 & & & 86 & 88 & 26.2 & 26.8 & sandstone, blue & Sandstone & cemented & -- & -- & QTm \\
\hline 231 & 7/9-31Q3 & 231 & 7/9-31Q3 & 0 & 250 & 0.0 & 76.2 & (Uncased below $8 \mathrm{ft.}$ ) & No data & - & -- & -- & QTm \\
\hline 232 & 7/9-35R1 & 232 & 7/9-35R1 & 0 & 4 & 0.0 & 1.2 & soil & Sand and clay & .- & .- & .- & QTm \\
\hline 232 & 7/9-35R1 & & & 4 & 28 & 1.2 & 8.5 & sand blue and gravel & Sand (or sandstone) and gravel & -- & -- & -- & \\
\hline 232 & 7/9-35R1 & & & 28 & 37 & 8.5 & 11.3 & sand, gray, and gravel & Sand (or sandstone) and gravel & .- & -- & .- & \\
\hline 232 & 7/9-35R1 & & & 37 & 42 & 11.3 & 12.8 & sand, yellow, dry, and clay & Sand & .- & -- & .- & \\
\hline 232 & 7/9-35R1 & & & 42 & 52 & 12.8 & 15.8 & $\begin{array}{l}\text { sand and gravel } \\
\text { savilu }\end{array}$ & Sand (or sandstone) and gravel & -- & -- & -- & \\
\hline 232 & 7/9-35R1 & & & 52 & 55 & 15.8 & 16.8 & sand, red, and gravel & Sand & .- & .- & .- & \\
\hline 232 & 7/9-35R1 & & & 55 & 61 & 16.8 & 18.6 & gravel and some sand & Sand (or sandstone) and gravel & .- & -- & -- & \\
\hline 232 & 7/9-35R1 & & & 61 & 63 & 18.6 & 19.2 & gravel & Gravel & -- & -- & -- & \\
\hline 232 & 7/9-35R1 & & & 63 & 70 & 19.2 & 21.3 & sand, clay, and gravel & Clay, sand, and gravel & -- & -- & -- & \\
\hline
\end{tabular}


Appendix 3. Lithologic data. - Continued

ISite IDs and well number correspond to locations in Appendix 1, a site ID and well number is listed for each drilled interval for a particular drill hole. For convenience, the site ID and well numbers are repeated at the shallowest drilled interval for eac $w$

\begin{tabular}{|c|c|c|c|c|c|c|c|c|c|c|c|c|c|}
\hline Site ID & Well number & $\begin{array}{l}\text { Site ID, } \\
\text { top of } \\
\text { well }\end{array}$ & $\begin{array}{l}\text { Well number, } \\
\text { top of well }\end{array}$ & $\begin{array}{l}\text { Top of } \\
\text { interval, in } \\
\text { feet }\end{array}$ & $\begin{array}{l}\text { Base of } \\
\text { interval, in } \\
\text { feet }\end{array}$ & $\begin{array}{c}\text { Top of } \\
\text { interval, in } \\
\mathrm{m}\end{array}$ & $\begin{array}{c}\text { Base of } \\
\text { interval, in } \\
\mathrm{m}\end{array}$ & Reported lithologic unit ${ }^{1}$ & Interpreted lithologic class ${ }^{2}$ & $\begin{array}{c}\text { Reported } \\
\text { degree of } \\
\text { cementation or } \\
\text { induration }^{3} \\
\end{array}$ & $\begin{array}{c}\text { Fossils } \\
\text { reported in } \\
\text { interval }^{4}\end{array}$ & $\begin{array}{c}\text { Organic } \\
\text { matter } \\
\text { reported in } \\
\text { interval }^{5} \\
\end{array}$ & $\begin{array}{l}\text { Reported stratigraphic top of geologic } \\
\text { unit }^{6}\end{array}$ \\
\hline 232 & $7 / 9-35 \mathrm{R} 1$ & & & 70 & 90 & 21.3 & 27.4 & clay, hard, and white sand & Clay and sand (or sandstone) & hard & -- & -- & \\
\hline 233 & 6/9-3A1 & 233 & 6/9-3A1 & 0 & 4 & 0.0 & 1.2 & soil & Sand and clay & -- & -- & -- & QTm \\
\hline 233 & 6/9-3A1 & & & 4 & 30 & 1.2 & 9.1 & sand, dry & Sand & -- & -- & -- & \\
\hline 233 & 6/9-3A1 & & & 30 & 155 & 9.1 & 47.2 & sand, yellow & Sand & -- & -- & -- & \\
\hline 233 & 6/9-3A1 & & & 155 & 250 & 47.2 & 76.2 & clay, blue, and sand & Clay and sand (or sandstone) & -- & -- & -- & \\
\hline 233 & 6/9-3A1 & & & 250 & 260 & 76.2 & 79.2 & sandstone, blue & Sandstone & cemented & -- & -- & \\
\hline 234 & $6 / 9-2 \mathrm{C} 1$ & 234 & $6 / 9-2 \mathrm{C} 1$ & 0 & 45 & 0.0 & 13.7 & topsoil, red & Sand and clay & -- & -- & -- & QTm \\
\hline 234 & $6 / 9-2 \mathrm{C} 1$ & & & 45 & 48 & 13.7 & 14.6 & sandstone & Sandstone & cemented & -- & -- & \\
\hline 234 & $6 / 9-2 \mathrm{C} 1$ & & & 48 & 52 & 14.6 & 15.8 & sand & Sand & - & -- & -- & \\
\hline 234 & $6 / 9-2 \mathrm{C} 1$ & & & 52 & 53 & 15.8 & 16.2 & sandstone & Sandstone & cemented & -- & -- & \\
\hline 234 & $6 / 9-2 \mathrm{C} 1$ & & & 53 & 59 & 16.2 & 18.0 & sand & Sand & -- & -- & -- & \\
\hline 234 & $6 / 9-2 \mathrm{C} 1$ & & & 59 & 61 & 18.0 & 18.6 & sandstone & Sandstone & cemented & -- & -- & \\
\hline 234 & $6 / 9-2 \mathrm{C} 1$ & & & 61 & 64 & 18.6 & 19.5 & sand & Sand & -- & -- & -- & \\
\hline 234 & $6 / 9-2 \mathrm{C} 1$ & & & 64 & 66 & 19.5 & 20.1 & sandstone & Sandstone & cemented & -- & -- & \\
\hline 234 & $6 / 9-2 \mathrm{C} 1$ & & & 66 & 70 & 20.1 & 21.3 & sand & Sand & -- & -- & -- & \\
\hline 234 & $6 / 9-2 \mathrm{C} 1$ & & & 70 & 71 & 21.3 & 21.6 & sandstone & Sandstone & cemented & -- & -- & \\
\hline 234 & $6 / 9-2 \mathrm{C} 1$ & & & 71 & 76 & 21.6 & 23.2 & sand & Sand & -- & -- & -- & \\
\hline 234 & $6 / 9-2 \mathrm{C} 1$ & & & 76 & 77 & 23.2 & 23.5 & sandstone & Sandstone & cemented & -- & -- & \\
\hline 234 & $6 / 9-2 \mathrm{C} 1$ & & & 77 & 80 & 23.5 & 24.4 & sand, yellow & Sand & -- & -- & -- & \\
\hline 234 & $6 / 9-2 \mathrm{C} 1$ & & & 80 & 82 & 24.4 & 25.0 & sandstone & Sandstone & cemented & -- & -- & \\
\hline 234 & $6 / 9-2 \mathrm{C} 1$ & & & 82 & 86 & 25.0 & 26.2 & sand & Sand & - & -- & -- & \\
\hline 234 & $6 / 9-2 \mathrm{C} 1$ & & & 86 & 87 & 26.2 & 26.5 & sandstone & Sandstone & cemented & -- & -- & \\
\hline 234 & $6 / 9-2 \mathrm{C} 1$ & & & 87 & 110 & 26.5 & 33.5 & sand & Sand & -- & -- & -- & \\
\hline 234 & $6 / 9-2 \mathrm{C} 1$ & & & 110 & 112 & 33.5 & 34.1 & sandstone & Sandstone & cemented & -- & -- & \\
\hline 234 & $6 / 9-2 \mathrm{C} 1$ & & & 112 & 172 & 34.1 & 52.4 & sand, yellow & Sand & -- & -- & -- & \\
\hline 234 & $6 / 9-2 \mathrm{C} 1$ & & & 172 & 175 & 52.4 & 53.3 & rock, lime & Sandstone & hard & -- & -- & \\
\hline 234 & $6 / 9-2 \mathrm{C} 1$ & & & 175 & 204 & 53.3 & 62.2 & shells and sand, blue & Sand & -- & shells & -- & \\
\hline 234 & $6 / 9-2 \mathrm{C} 1$ & & & 204 & 210 & 62.2 & 64.0 & $\begin{array}{l}\text { shells, petrified } \\
\text { late }\end{array}$ & Sandstone & cemented & shells & -- & \\
\hline 234 & $6 / 9-2 \mathrm{C} 1$ & & & 210 & 216 & 64.0 & 65.8 & sand, blue & Sand & -- & -- & -- & \\
\hline 234 & $6 / 9-2 \mathrm{C} 1$ & & & 216 & 218 & 65.8 & 66.4 & sand, rock & Sandstone & cemented & -- & -- & \\
\hline 234 & $6 / 9-2 \mathrm{C} 1$ & & & 218 & 222 & 66.4 & 67.7 & sand, blue & Sand & -- & -- & -- & \\
\hline 234 & $6 / 9-2 \mathrm{C} 1$ & & & 222 & 230 & 67.7 & 70.1 & sand, rock, blue & Sandstone & cemented & -- & -- & \\
\hline 234 & $6 / 9-2 \mathrm{C} 1$ & & & 230 & 235 & 70.1 & 71.6 & sand, blue and shells & Sand & -- & shells & -- & \\
\hline 234 & $6 / 9-2 \mathrm{C} 1$ & & & 235 & 236 & 71.6 & 71.9 & sand rock, blue & Sandstone & cemented & -- & -- & \\
\hline 234 & $6 / 9-2 \mathrm{C} 1$ & & & 236 & 243 & 71.9 & 74.1 & sand, blue & Sand & -- & -- & -- & \\
\hline 234 & $6 / 9-2 \mathrm{C} 1$ & & & 243 & 244 & 74.1 & 74.4 & sand rock, blue & Sandstone & cemented & -- & -- & \\
\hline 234 & $6 / 9-2 \mathrm{C} 1$ & & & 244 & 255 & 74.4 & 77.7 & sand, blue & Sand & -- & -- & -- & \\
\hline 234 & $6 / 9-2 \mathrm{C} 1$ & & & 255 & 258 & 77.7 & 78.6 & sand rock, blue & Sandstone & cemented & -- & -- & \\
\hline 234 & $6 / 9-2 \mathrm{C} 1$ & & & 258 & 268 & 78.6 & 81.7 & sand, blue and shells & Sand & -- & shells & -- & \\
\hline 234 & $6 / 9-2 \mathrm{C} 1$ & & & 268 & 273 & 81.7 & 83.2 & sand rock, gray & Sandstone & cemented & -- & -- & \\
\hline 234 & $6 / 9-2 \mathrm{C} 1$ & & & 273 & 302 & 83.2 & 92.0 & sand rock, blue, and shells & Sandstone & cemented & shells & -- & \\
\hline 234 & $6 / 9-2 \mathrm{C} 1$ & & & 302 & 304 & 92.0 & 92.7 & clam shells, fair & Sand & -- & shells & -- & \\
\hline 234 & $6 / 9-2 \mathrm{C} 1$ & & & 304 & 322 & 92.7 & 98.1 & sand, blue & Sand & -- & -- & -- & \\
\hline 234 & $6 / 9-2 \mathrm{C} 1$ & & & 322 & 323 & 98.1 & 98.5 & clam shells, fair & Sand & -- & shells & -- & \\
\hline 234 & $6 / 9-2 \mathrm{C} 1$ & & & 323 & 332 & 98.5 & 101.2 & $\begin{array}{l}\text { clay and sand, blue } \\
\text { and }\end{array}$ & Clay and sand (or sandstone) & -- & -- & -- & \\
\hline 234 & $6 / 9-2 \mathrm{C} 1$ & & & 332 & 335 & 101.2 & 102.1 & clam shells, good & Sand & -- & shells & -- & \\
\hline 234 & $6 / 9-2 \mathrm{C} 1$ & & & 335 & 337 & 102.1 & 102.7 & shells, petrified & Sandstone & cemented & shells & -- & \\
\hline 234 & $6 / 9-2 \mathrm{C} 1$ & & & 337 & 342 & 102.7 & 104.2 & clam shells and sand & Sand & -- & shells & -- & \\
\hline 234 & $6 / 9-2 \mathrm{C} 1$ & & & 342 & 345 & 104.2 & 105.2 & sand rock & Sandstone & cemented & -- & -- & \\
\hline 234 & $6 / 9-2 \mathrm{C} 1$ & & & 345 & 350 & 105.2 & 106.7 & clam shells, good & Sand & -- & shells & -- & \\
\hline 234 & $6 / 9-2 \mathrm{C} 1$ & & & 350 & 361 & 106.7 & 110.0 & sand, blue & Sand & -- & -- & -- & \\
\hline 234 & $6 / 9-2 \mathrm{C} 1$ & & & 361 & 362 & 110.0 & 110.3 & sand rock & Sandstone & cemented & -- & -- & \\
\hline 234 & $6 / 9-2 \mathrm{C} 1$ & & & 362 & 364 & 110.3 & 110.9 & clam shells and rock & Sandstone & -- & shells & -- & \\
\hline 234 & $6 / 9-2 \mathrm{C} 1$ & & & 364 & 374 & 110.9 & 114.0 & sand, blue & Sand & -- & -- & -- & \\
\hline 234 & $6 / 9-2 \mathrm{C} 1$ & & & 374 & 378 & 114.0 & 115.2 & rock and shells & Sandstone & -- & shells & -- & \\
\hline 234 & $6 / 9-2 \mathrm{C} 1$ & & & 378 & 399 & 115.2 & 121.6 & sand, blue & Sand & -- & -- & -- & \\
\hline 234 & $6 / 9-2 \mathrm{C} 1$ & & & 399 & 429 & 121.6 & 130.8 & clay, blue & Clay & -- & -- & -- & \\
\hline 234 & $6 / 9-2 \mathrm{C} 1$ & & & 429 & 444 & 130.8 & 135.3 & sand, blue & Sand & -- & -- & -- & \\
\hline 234 & $6 / 9-2 \mathrm{C} 1$ & & & 444 & 480 & 135.3 & 146.3 & sediments, blue & Sand & -- & -- & -- & \\
\hline 234 & $6 / 9-2 \mathrm{C} 1$ & & & 480 & 487 & 146.3 & 148.4 & sand, shells, and rock & Sand (or sandstone) and gravel & -- & shells & -- & \\
\hline 234 & $6 / 9-2 \mathrm{C} 1$ & & & 487 & 499 & 148.4 & 152.1 & shells and sand & Sand & -- & shells & -- & \\
\hline
\end{tabular}


Appendix 3. Lithologic data. - Continued

[Site IDs and well number correspond to locations in Appendix 1, a site ID and well number is listed for each drilled interval for a particular drill hole. For convenience, the site ID and well numbers are repeated at the shallowest drilled interval for each well. leaderst.

\begin{tabular}{|c|c|c|c|c|c|c|c|c|c|c|c|c|c|}
\hline Site ID & Well number & $\begin{array}{l}\text { Site ID, } \\
\text { top of } \\
\text { well }\end{array}$ & $\begin{array}{l}\text { Well number, } \\
\text { top of well }\end{array}$ & $\begin{array}{c}\text { Top of } \\
\text { interval, in } \\
\text { feet }\end{array}$ & $\begin{array}{l}\text { Base of } \\
\text { interval, in } \\
\text { feet }\end{array}$ & $\begin{array}{c}\text { Top of } \\
\text { interval, in } \\
\mathrm{m}\end{array}$ & $\begin{array}{c}\text { Base of } \\
\text { interval, in } \\
\mathrm{m}\end{array}$ & Reported lithologic unit' & Interpreted lithologic class ${ }^{2}$ & $\begin{array}{c}\text { Reported } \\
\text { degree of } \\
\text { cementation or } \\
\text { induration }^{3} \\
\end{array}$ & $\begin{array}{c}\text { Fossils } \\
\text { reported in } \\
\text { interval }^{4}\end{array}$ & $\begin{array}{c}\text { Organic } \\
\text { matter } \\
\text { reported in } \\
\text { interval }^{5}\end{array}$ & $\begin{array}{l}\text { Reported stratigraphic top of geologic } \\
\text { unit }^{6}\end{array}$ \\
\hline 234 & $6 / 9-2 \mathrm{C} 1$ & & & 499 & 505 & 152.1 & 153.9 & sand rock & Sandstone & cemented & -- & -- & \\
\hline 234 & $6 / 9-2 \mathrm{C} 1$ & & & 505 & 512 & 153.9 & 156.1 & sand, blue & Sand & -- & -- & -- & \\
\hline 234 & $6 / 9-2 \mathrm{C} 1$ & & & 512 & 514 & 156.1 & 156.7 & sand, grit, and shells & Sand & -- & shells & -- & \\
\hline 234 & $6 / 9-2 \mathrm{C} 1$ & & & 514 & 530 & 156.7 & 161.5 & sand, blue & Sand & -- & -- & -- & \\
\hline 234 & $6 / 9-2 \mathrm{C} 1$ & & & 530 & 533 & 161.5 & 162.5 & rock, sand, and shells & Sand (or sandstone) and gravel & hard & shells & -- & \\
\hline 234 & $6 / 9-2 \mathrm{C} 1$ & & & 533 & 539 & 162.5 & 164.3 & shells, sand, and grit & Sand & -- & shells & -- & \\
\hline 234 & $6 / 9-2 \mathrm{C} 1$ & & & 539 & 590 & 164.3 & 179.8 & sediment, blue, and blue sand & Sand & -- & -- & -- & \\
\hline 234 & $6 / 9-2 \mathrm{C} 1$ & & & 590 & 600 & 179.8 & 182.9 & clam shells and sand & Sand & -- & shells & -- & \\
\hline 235 & 6/8-5E1 & 235 & 6/8-5E1 & 0 & 10 & 0.0 & 3.0 & coarse, brown sand & Sand & -- & -- & -- & QTge \\
\hline 235 & 6/8-5E1 & & & 10 & 20 & 3.0 & 6.1 & brown sand and gravel & Sand (or sandstone) and gravel & -- & -- & -- & \\
\hline 235 & 6/8-5E1 & & & 20 & 30 & 6.1 & 9.1 & gray silt and gravel & Sand (or sandstone) and gravel & -- & -- & -- & \\
\hline 235 & 6/8-5E1 & & & 30 & 40 & 9.1 & 12.2 & gray sandy silt & Sand and clay & -- & -- & -- & \\
\hline 235 & 6/8-5E1 & & & 40 & 50 & 12.2 & 15.2 & gray silt and gravel & Sand (or sandstone) and gravel & -- & -- & -- & \\
\hline 235 & 6/8-5E1 & & & 50 & 60 & 15.2 & 18.3 & coarse dark-green sand & Sand & -- & -- & -- & \\
\hline 235 & 6/8-5E1 & & & 60 & 70 & 18.3 & 21.3 & silt and fine gravel & Sand (or sandstone) and gravel & -- & -- & -- & \\
\hline 235 & 6/8-5E1 & & & 70 & 90 & 21.3 & 27.4 & silt and gravel & Sand (or sandstone) and gravel & -- & -- & -- & \\
\hline 235 & 6/8-5E1 & & & 90 & 100 & 27.4 & 30.5 & sandy silt and gravel & Sand (or sandstone) and gravel & -- & -- & -- & \\
\hline 235 & 6/8-5E1 & & & 100 & 120 & 30.5 & 36.6 & blue clay & Clay & -- & -- & -- & \\
\hline 235 & 6/8-5E1 & & & 120 & 140 & 36.6 & 42.7 & blue clay and gravel & Clay and gravel & -- & -- & -- & \\
\hline 235 & 6/8-5E1 & & & 140 & 150 & 42.7 & 45.7 & blue clay & Clay & -- & -- & -- & \\
\hline 235 & 6/8-5E1 & & & 150 & 160 & 45.7 & 48.8 & blue clay and coarse gravel & Clay and gravel & -- & -- & -- & \\
\hline 235 & 6/8-5E1 & & & 160 & 170 & 48.8 & 51.8 & gravel, medium & Gravel & -- & -- & -- & \\
\hline 235 & 6/8-5E1 & & & 170 & 220 & 51.8 & 67.1 & gravel & Gravel & -- & -- & -- & \\
\hline 235 & 6/8-5E1 & & & 220 & 250 & 67.1 & 76.2 & sand and gravel & Sand (or sandstone) and gravel & -- & -- & -- & \\
\hline 235 & 6/8-5E1 & & & 250 & 260 & 76.2 & 79.2 & gray sand & Sand & -- & -- & -- & QTm \\
\hline 235 & 6/8-5E1 & & & 260 & 280 & 79.2 & 85.3 & clay and gravel & Clay and gravel & -- & -- & -- & \\
\hline 235 & 6/8-5E1 & & & 280 & 300 & 85.3 & 91.4 & gray sandy silt & Sand and clay & -- & -- & -- & \\
\hline 235 & 6/8-5E1 & & & 300 & 310 & 91.4 & 94.5 & blue clay & Clay & -- & -- & -- & \\
\hline 235 & 6/8-5E1 & & & 310 & 330 & 94.5 & 100.6 & sandy silt and gravel & Sand (or sandstone) and gravel & -- & -- & -- & \\
\hline 235 & 6/8-5E1 & & & 330 & 340 & 100.6 & 103.6 & gravel & Gravel & -- & -- & -- & \\
\hline 235 & 6/8-5E1 & & & 340 & 350 & 103.6 & 106.7 & blue sand and gravel & Sand (or sandstone) and gravel & -- & -- & -- & \\
\hline 235 & 6/8-5E1 & & & 350 & 370 & 106.7 & 112.8 & gray sand & Sand & -- & -- & -- & \\
\hline 235 & 6/8-5E1 & & & 370 & 380 & 112.8 & 115.8 & sandy silt and gravel & Sand (or sandstone) and gravel & -- & -- & -- & \\
\hline 235 & 6/8-5E1 & & & 380 & 390 & 115.8 & 118.9 & sand & Sand & -- & -- & -- & \\
\hline 235 & 6/8-5E1 & & & 390 & 400 & 118.9 & 121.9 & sand and gravel & Sand (or sandstone) and gravel & -- & -- & -- & \\
\hline 235 & 6/8-5E1 & & & 400 & 410 & 121.9 & 125.0 & blue clay & Clay & -- & -- & -- & \\
\hline 235 & 6/8-5E1 & & & 410 & 430 & 125.0 & 131.1 & sandy silt and gravel & Sand (or sandstone) and gravel & -- & -- & -- & \\
\hline 235 & 6/8-5E1 & & & 430 & 440 & 131.1 & 134.1 & sand and gravel & Sand (or sandstone) and gravel & -- & -- & -- & \\
\hline 235 & 6/8-5E1 & & & 440 & 460 & 134.1 & 140.2 & blue sand & Sand & -- & -- & -- & \\
\hline 235 & 6/8-5E1 & & & 460 & 480 & 140.2 & 146.3 & blue clay & Clay & -- & -- & -- & \\
\hline 235 & 6/8-5E1 & & & 480 & 490 & 146.3 & 149.4 & sand silt & Sand and clay & -- & -- & -- & \\
\hline 235 & 6/8-5E1 & & & 490 & 510 & 149.4 & 155.4 & sand & Sand & -- & -- & -- & \\
\hline 235 & 6/8-5E1 & & & 510 & 530 & 155.4 & 161.5 & sand and gravel & Sand (or sandstone) and gravel & -- & -- & -- & \\
\hline 235 & 6/8-5E1 & & & 530 & 638 & 161.5 & 194.5 & sand, medium coarse & Sand & -- & -- & -- & \\
\hline & & & & & & & & $\begin{array}{l}\text { volcanic conglomerate formation } \\
\text { (probably includes thin interval of }\end{array}$ & & & & & \\
\hline 236 & 6/6-4J1 & 236 & 6/6-4J1 & 0 & 80 & 0.0 & 24.4 & terrace or fan deposits) & Ash or tuff & -- & -- & -- & Tsv \\
\hline 236 & $6 / 6-4 J 1$ & & & 80 & 153 & 24.4 & 46.6 & rock, black volcanic & Ash or tuff & hard & -- & -- & \\
\hline 236 & $6 / 6-4 J 1$ & & & 153 & 190 & 46.6 & 57.9 & rock, red and black volcanic & Ash or tuff & hard & -- & -- & \\
\hline 237 & 6/7-3Q1 & 237 & 6/7-3Q1 & 0 & 27 & 0.0 & 8.2 & clay & Clay & -- & -- & -- & QTge \\
\hline 237 & 6/7-3Q1 & & & 27 & 48 & 8.2 & 14.6 & gravel & Gravel & -- & -- & -- & \\
\hline 237 & 6/7-3Q1 & & & 48 & 50 & 14.6 & 15.2 & gravel, brown, washed & Gravel & -- & -- & -- & \\
\hline 237 & 6/7-3Q1 & & & 50 & 98 & 15.2 & 29.9 & clay & Clay & -- & -- & -- & \\
\hline 237 & 6/7-3Q1 & & & 98 & 110 & 29.9 & 33.5 & gravel, wash & Gravel & -- & -- & -- & \\
\hline 237 & 6/7-3Q1 & & & 110 & 125 & 33.5 & 38.1 & basalt & Basalt & -- & -- & -- & Tsv \\
\hline 237 & 6/7-3Q1 & & & 125 & 417 & 38.1 & 127.1 & ash, white & Ash or tuff & -- & -- & -- & \\
\hline 238 & 6/8-7D1 & 238 & 6/8-7D1 & 0 & 4 & 0.0 & 1.2 & $\begin{array}{l}\text { topsoil } \\
\text { sand, blue, and gravel; good }\end{array}$ & Sand and clay & -- & -- & -- & Qyal and QTge (?) \\
\hline 238 & 6/8-7D1 & & & 0 & 271 & 0.0 & 82.6 & looking gravel & Clay and sand (or sandstone) & -- & -- & -- & \\
\hline 238 & 6/8-7D1 & & & 4 & 17 & 1.2 & 5.2 & clay sandy yellow & Clay and sand (or sandstone) & -- & -- & -- & \\
\hline
\end{tabular}


Appendix 3. Lithologic data. - Continued

[Site IDs and well number correspond to locations in Appendix 1, a site ID and well number is listed for each drilled interval for a particular drill hole. For convenience, the site ID and well numbers are repeated at the shallowest drilled interval for each well. leaders

\begin{tabular}{|c|c|c|c|c|c|c|c|c|c|c|c|c|c|}
\hline Site ID & Well number & $\begin{array}{l}\text { Site ID, } \\
\text { top of } \\
\text { well }\end{array}$ & $\begin{array}{l}\text { Well number, } \\
\text { top of well }\end{array}$ & $\begin{array}{c}\text { Top of } \\
\text { interval, in } \\
\text { feet }\end{array}$ & $\begin{array}{c}\text { Base of } \\
\text { interval, in } \\
\text { feet }\end{array}$ & $\begin{array}{c}\text { Top of } \\
\text { interval, in } \\
m\end{array}$ & $\begin{array}{c}\text { Base of } \\
\text { interval, in } \\
\mathrm{m}\end{array}$ & Reported lithologic unit' & Interpreted lithologic class ${ }^{2}$ & $\begin{array}{c}\text { Reported } \\
\text { degree of } \\
\text { cementation or } \\
\text { induration } \\
\end{array}$ & $\begin{array}{c}\text { Fossils } \\
\text { reported in } \\
\text { interval }^{4}\end{array}$ & $\begin{array}{c}\text { Organic } \\
\text { matter } \\
\text { reported in } \\
\text { interval }^{5}\end{array}$ & $\begin{array}{l}\text { Reported stratigraphic top of geologic } \\
\text { unit }^{6}\end{array}$ \\
\hline 238 & $6 / 8-7 D 1$ & & & 17 & 24 & 5.2 & 7.3 & sand, yellow, and gravel & Sand (or sandstone) and gravel & -- & -- & -- & \\
\hline 238 & 6/8-7D1 & & & 24 & 35 & 7.3 & 10.7 & $\begin{array}{l}\text { clay, blue } \\
\text { and gor }\end{array}$ & Clay & -- & -- & -- & \\
\hline 238 & 6/8-7D1 & & & 35 & 42 & 10.7 & 12.8 & clay, sandy, blue & Clay and sand (or sandstone) & -- & -- & -- & \\
\hline 238 & 6/8-7D1 & & & 42 & 130 & 12.8 & 39.6 & clay, sandy, yellow & Clay and sand (or sandstone) & -- & -- & -- & QTm \\
\hline 238 & 6/8-7D1 & & & 130 & 135 & 39.6 & 41.1 & clay, yellow & Clay & -- & -- & -- & \\
\hline 238 & 6/8-7D1 & & & 135 & 138 & 41.1 & 42.1 & sand, yellow, and some gravel & Sand (or sandstone) and gravel & -- & -.- & -- & \\
\hline 238 & 6/8-7D1 & & & 138 & 140 & 42.1 & 42.7 & sand, yellow, and little gravel & Sand (or sandstone) and gravel & - & - & -- & \\
\hline 238 & 6/8-7D1 & & & 140 & 225 & 42.7 & 68.6 & clay, sandy, yellow & Clay and sand (or sandstone) & -- & -- & -- & \\
\hline 238 & 6/8-7D1 & & & 225 & 236 & 68.6 & 71.9 & sand, yellow, fairly coarse & Sand & -- & -- & -- & \\
\hline 238 & 6/8-7D1 & & & 236 & 276 & 71.9 & 84.1 & sand, yellow & Sand (or sandstone) and gravel & -- & -- & -- & \\
\hline 238 & 6/8-7D1 & & & 271 & 410 & 82.6 & 125.0 & sand, blue, and gravel & Clay and gravel & -- & -- & -- & \\
\hline 238 & 6/8-7D1 & & & 276 & 315 & 84.1 & 96.0 & clay, blue, and streaks of black & Clay & -- & -- & -- & \\
\hline 238 & 6/8-7D1 & & & 315 & 325 & 96.0 & 99.1 & sandstone, yellow, soft & Sand (or sandstone) and gravel & cemented & -- & -- & \\
\hline 238 & 6/8-7D1 & & & 325 & 332 & 99.1 & 101.2 & clay, sandy, blue & Clay and gravel & -- & -- & -- & \\
\hline 238 & 6/8-7D1 & & & 332 & 360 & 101.2 & 109.7 & $\begin{array}{l}\text { clay, blue, and streaks of black } \\
\text { gravel }\end{array}$ & Clay and gravel & .. & .. & .. & \\
\hline 239 & $6 / 8-12 J 1$ & 239 & 6/8-12J1 & 0 & 7 & 0.0 & 2.1 .1 & soil, black & Sand and clay & -. & -. & -- & QTge \\
\hline 239 & $6 / 8-12 \mathrm{J1}$ & & & 7 & 27 & 2.1 & 8.2 & sandy, yellow clay & Clay and sand (or sandstone) & -- & -- & -- & \\
\hline 239 & $6 / 8-12 \mathrm{~J} 1$ & & & 27 & 28 & 8.2 & 8.5 & yellow clay and gravel & Clay and gravel & -- & -- & -- & \\
\hline 239 & $6 / 8-12 J 1$ & & & 28 & 39 & 8.5 & 11.9 & yellow clay and gravel & Clay and gravel & -- & -- & -- & \\
\hline 239 & $6 / 8-12 J 1$ & & & 39 & 50 & $\begin{array}{l}.0 \\
11.9\end{array}$ & 15.2 & $\begin{array}{l}\text { blue clay } \\
\text { all ave }\end{array}$ & $\begin{array}{l}\text { Clay } \\
\text { Clut }\end{array}$ & - &.- & - & \\
\hline 239 & $6 / 8-12 J 1$ & & & 50 & 72 & 15.2 & 21.9 & yellow clay & Clay & -- & -. & -.- & \\
\hline 239 & $6 / 8-12 J 1$ & & & 72 & 76 & 21.9 & 23.2 & yellow clay and gravel & Clay and gravel & -- & -- & -- & \\
\hline 239 & $6 / 8-12 J 1$ & & & 76 & 110 & 23.2 & 33.5 & yellow clay & Clay & -- & -- & -- & \\
\hline 239 & 6/8-12J1 & & & 110 & 135 & 33.5 & 41.1 & yellow clay and gravel & Clay and gravel & -- & -- & -- & \\
\hline 239 & $6 / 8-12 J 1$ & & & 135 & 205 & 41.1 & 62.5 & yellow clay & Clay & -- & -- & -- & \\
\hline 239 & $6 / 8-12 J 1$ & & & 205 & 223 & 62.5 & 68.0 & sandy yellow clay and gravel & Clay, sand, and gravel & -- & -- & -- & \\
\hline 240 & $6 / 6-8 \mathrm{~N} 1$ & 240 & $6 / 6-8 \mathrm{~N} 1$ & 0 & 16 & 0.0 & 4.9 & surface & Sand and clay & -- & -- & -. & QTge \\
\hline 240 & $6 / 6-8 \mathrm{~N} 1$ & & & 16 & 20 & 4.9 & 6.1 & gravel & Gravel & -- & -- & -- & \\
\hline 240 & $6 / 6-8 \mathrm{~N} 1$ & & & 20 & 65 & 6.1 & 19.8 & clay, sandy, blue & Clay and sand (or sandstone) & -- & -- & -.- & \\
\hline 240 & $6 / 6-8 \mathrm{~N} 1$ & & & 65 & 85 & 19.8 & 25.9 & gravel and boulders & Gravel & -- & -- & -- & \\
\hline 240 & $6 / 6-8 \mathrm{~N} 1$ & & & 85 & 100 & 25.9 & 30.5 & boulders & Gravel & -- & -- & -- & \\
\hline 240 & $6 / 6-8 \mathrm{~N} 1$ & & & 100 & 195 & 30.5 & 59.4 & clay, yellow & Clay & - & -- & - & \\
\hline 240 & $6 / 6-8 \mathrm{~N} 1$ & & & 195 & 205 & 59.4 & 62.5 & $\begin{array}{l}\text { boulders and gravel } \\
\text { volcanic rock, soft (probably }\end{array}$ & Gravel & -- & -- & -- & \\
\hline 240 & $6 / 6-8 \mathrm{~N} 1$ & & & 205 & 445 & 62.5 & 135.6 & tuffaceous Glen Ellen) & Ash or tuff & .- & -- & -. & \\
\hline 240 & $6 / 6-8 \mathrm{~N} 1$ & & & 445 & 490 & 135.6 & 149.4 & lava rock, blue & Basalt & hard & -- & -. & Tsv \\
\hline 241 & 6/6-17A1 & 241 & 6/6-17A1 & 0 & 33 & 0.0 & 10.1 & dirt and boulders & Sand (or sandstone) and gravel & -- & -- & -- & Qt \\
\hline 241 & 6/6-17A1 & & & 33 & 98 & 10.1 & 29.9 & clay, yellow, and boulders & Clay and gravel & -- & -- & -- & QTge \\
\hline 241 & 6/6-17A1 & & & 98 & 128 & 29.9 & 39.0 & clay and gravel & Clay and gravel & -- & -- & -- & \\
\hline 241 & 6/6-17A1 & & & 128 & 130 & 39.0 & 39.6 & gravel & Gravel & -- & -- & -- & \\
\hline 241 & 6/6-17A1 & & & 130 & 150 & 39.6 & 45.7 & clay and gravel & Clay and gravel & -- & -- & -- & \\
\hline 242 & $6 / 8-17 \mathrm{~K} 1$ & 242 & 6/8-17K1 & 0 & 10 & 0.0 & 3.0 & clay, yellow and gravel & Clay and gravel & -- & -- & -- & QTm \\
\hline 242 & 6/8-17K1 & & & 10 & 45 & 3.0 & 13.7 & clay, sandy, yellow, and gravel & Clay, sand, and gravel & -- & -- & -- & \\
\hline 242 & $6 / 8-17 \mathrm{~K} 1$ & & & 45 & 50 & 13.7 & 15.2 & sand, yellow, and gravel & Sand (or sandstone) and gravel & - & -- & -- & \\
\hline 242 & 6/8-17K1 & & & 50 & 55 & 15.2 & 16.8 & sand, blue; some gravel & Sand (or sandstone) and gravel & -- & -- & -- & \\
\hline 242 & 6/8-17K1 & & & 55 & 129 & 16.8 & 39.3 & sand, blue, and gravel & Sand (or sandstone) and gravel & -- & -- & -- & \\
\hline 242 & $6 / 8-17 \mathrm{~K} 1$ & & & 129 & 134 & 39.3 & 40.8 & clay, sandy, blue, and gravel & Clay, sand, and gravel & -- & -- & -- & \\
\hline 242 & 6/8-17K1 & & & 134 & 136 & 40.8 & 41.5 & sand, blue, and gravel & Sand (or sandstone) and gravel & -- & -- & -- & \\
\hline 242 & 6/8-17K1 & & & 136 & 145 & 41.5 & 44.2 & clay, blue, and gravel & Clay and gravel & -- & -- & -. & \\
\hline 242 & 6/8-17K1 & & & 145 & 156 & 44.2 & 47.5 & sand, blue, and gravel & Sand (or sandstone) and gravel & -- & -- & -- & \\
\hline 242 & 6/8-17K1 & & & 156 & 162 & 47.5 & 49.4 & $\begin{array}{l}\text { clay, sandy, blue } \\
\text { later }\end{array}$ & Clay and sand (or sandstone) & .- & -- & .- & \\
\hline 242 & 6/8-17K1 & & & 162 & 210 & 49.4 & 64.0 & sand, blue, and gravel & Sand (or sandstone) and gravel & -- & -- & -- & \\
\hline 243 & 6/8-16R1 & 243 & 6/8-16R1A & 0 & 15 & 0.0 & 4.6 & sandy, yellow clay & Clay and sand (or sandstone) & -- & -- & -- & QTge \\
\hline 243 & 6/8-16R1 & & 6/8-16R1B & 15 & 40 & 4.6 & 12.2 & blue clay & Clay & -- & -- & -- & \\
\hline 243 & 6/8-16R1 & & & 40 & 72 & 12.2 & 21.9 & yellow clay & Clay & -- & -- & -. & \\
\hline 243 & 6/8-16R1 & & & 72 & 84 & 21.9 & 25.6 & blue clay & Clay & -- & -- & -- & \\
\hline 243 & 6/8-16R1 & & & 84 & 100 & 25.6 & 30.5 & blue clay and gravel & Clay and gravel & -- & -- & - & \\
\hline 243 & 6/8-16R1 & & & 100 & 101 & 30.5 & 30.8 & sandy yellow clay & Clay and sand (or sandstone) & -- & -- & -. & \\
\hline 243 & 6/8-16R1 & & & 101 & 123 & 30.8 & 37.5 & clay and gravel & Clay and gravel & -- & -- & -. & \\
\hline 243 & 6/8-16R1 & & & 123 & 385 & 37.5 & 117.3 & sandy yellow clay & Clay and sand (or sandstone) & -- & -- & -- & QTm (?) \\
\hline
\end{tabular}


Appendix 3. Lithologic data. - Continued

[Site IDs and well number correspond to locations in Appendix 1, a site ID and well number is listed for each drilled interval for a particular drill hole. For convenience, the site ID and well numbers are repeated at the shallowest drilled interval for each well. leaders $1-$

\begin{tabular}{|c|c|c|c|c|c|c|c|c|c|c|c|c|c|}
\hline Site ID & Well number & $\begin{array}{l}\text { Site ID, } \\
\text { top of } \\
\text { well }\end{array}$ & $\begin{array}{l}\text { Well number, } \\
\text { top of well }\end{array}$ & $\begin{array}{c}\text { Top of } \\
\text { interval, in } \\
\text { feet }\end{array}$ & $\begin{array}{c}\text { Base of } \\
\text { interval, in } \\
\text { feet }\end{array}$ & $\begin{array}{c}\text { Top of } \\
\text { interval, in } \\
m\end{array}$ & $\begin{array}{c}\text { Base of } \\
\text { interval, in } \\
\mathrm{m}\end{array}$ & Reported lithologic unit ${ }^{1}$ & Interpreted lithologic class ${ }^{2}$ & $\begin{array}{c}\text { Reported } \\
\text { degree of } \\
\text { cementation or } \\
\text { induration } \\
\end{array}$ & $\begin{array}{c}\text { Fossils } \\
\text { reported in } \\
\text { interval }^{4}\end{array}$ & $\begin{array}{c}\text { Organic } \\
\text { matter } \\
\text { reported in } \\
\text { interval }^{5}\end{array}$ & $\begin{array}{l}\text { Reported stratigraphic top of geologic } \\
\text { unit }^{6}\end{array}$ \\
\hline 243 & 6/8-16R1 & & & 385 & 400 & 117.3 & 121.9 & blue clay & Clay & -- & -- & -- & \\
\hline 243 & 6/8-16R1 & & & 400 & 420 & 121.9 & 128.0 & sandy blue clay & Clay and sand (or sandstone) & -- & -- & -- & \\
\hline 243 & 6/8-16R1 & & & 420 & 512 & 128.0 & 156.1 & blue clay & Clay & -- & -- & -- & \\
\hline 243 & 6/8-16R1 & & & 512 & 526 & 156.1 & 160.3 & blue sand & Sand & -- & -- & -- & \\
\hline 243 & 6/8-16R1 & & & 526 & 794 & 160.3 & 242.0 & blue clay & Clay & -- & -- & -- & \\
\hline 243 & 6/8-16R1 & & & 794 & 805 & 242.0 & 245.4 & gravel and sand & Sand (or sandstone) and gravel & -- & -- & -- & \\
\hline 243 & 6/8-16R1 & & & 805 & 915 & 245.4 & 278.9 & blue clay and some gravel & Clay and gravel & -- & -- & -- & \\
\hline 243 & $6 / 8-16 \mathrm{R} 1$ & & & 915 & 930 & 278.9 & 283.5 & blue clay & Clay & -- & -- & -- & \\
\hline 243 & 6/8-16R1 & & & 930 & 937 & 283.5 & 285.6 & $\begin{array}{l}\text { gravel and sand } \\
\text { blue clay and streaks of yellow }\end{array}$ & Sand (or sandstone) and gravel & -- & -- & -- & \\
\hline 243 & 6/8-16R1 & & & 937 & 1050 & 285.6 & 320.0 & clay & Clay and gravel & -- & -- & -. & \\
\hline 243 & $6 / 8-16 \mathrm{R} 1$ & & & 1050 & 1065 & 320.0 & 324.6 & sandy blue cement gravel & Sand (or sandstone) and gravel & cemented & -- & -- & \\
\hline 243 & 6/8-16R1 & & & 1065 & 1085 & 324.6 & 330.7 & blue clay & Clay & -- & -- & -- & \\
\hline 243 & 6/8-16R1 & & & 1085 & 1115 & 330.7 & 339.9 & blue clay and gravel & Clay and gravel & -- & -- & -- & \\
\hline 243 & 6/8-16R1 & & & 1115 & 1150 & 339.9 & 350.5 & rock & Ash or tuff & hard & -- & -- & \\
\hline 243 & $6 / 8-16 \mathrm{R} 1$ & & & 1150 & 1198 & 350.5 & 365.2 & blue clay and gravel & Clay and gravel & -- & -- & -- & \\
\hline 243 & $6 / 8-16 \mathrm{R} 1$ & & & 1198 & 1204 & 365.2 & 367.0 & boulders & Gravel & -- & -- & -- & \\
\hline 244 & 6/9-22A1 & 244 & 6/9-22A1 & 0 & 8 & 0.0 & 2.4 & topsoil, sandy loam & Sand and clay & -- & -- & -- & QTm \\
\hline 244 & 6/9-22A1 & & & 8 & 20 & 2.4 & 6.1 & clay, hard sandy yellow & Clay & hard & -- & -- & \\
\hline 244 & 6/9-22A1 & & & 20 & 94 & 6.1 & 28.7 & blue sandstone, medium hard & Sandstone & hard & -- & -- & \\
\hline 244 & $6 / 9-22 A 1$ & & & 94 & 112 & 28.7 & 34.1 & blue sandstone, with shells & Sandstone & cemented & shells & - & \\
\hline 244 & 6/9-22A1 & & & 112 & 158 & 34.1 & 48.2 & blue sandstone, medium hard & Sandstone & hard & -- & -- & \\
\hline 244 & 6/9-22A1 & & & 158 & 180 & 48.2 & 54.9 & blue sandstone with shells & Sandstone & cemented & shells & -- & \\
\hline 244 & 6/9-22A1 & & & 180 & 226 & 54.9 & 68.9 & blue sandstone, medium hard & Sandstone & hard & -- & -.- & \\
\hline 244 & $6 / 9-22 A 1$ & & & 226 & 228 & 68.9 & 69.5 & hard gray rock & Sandstone & hard & -- & -- & \\
\hline 244 & $6 / 9-22 \mathrm{~A} 1$ & & & 228 & 247 & 69.5 & 75.3 & hard gray sandstone & Sandstone & hard & -- & -- & \\
\hline 244 & 6/9-22A1 & & & 247 & 288 & 75.3 & 87.8 & blue sandstone, medium hard & Sandstone & hard & -- & -- & \\
\hline 244 & 6/9-22A1 & & & 288 & 360 & 87.8 & $\begin{array}{l}109.7 \\
109\end{array}$ & $\begin{array}{l}\text { blue sandstone with shells } \\
\text { sal }\end{array}$ & Sandstone & cemented & shells & - & \\
\hline 245 & 6/7-19D1 & 245 & 6/7-19D1 & 0 & 8 & 0.0 & 2.4 & topsoil & Sand and clay & -- & -- & -- & Qyal and QTge \\
\hline 245 & 6/7-19D1 & & & 8 & 21 & 2.4 & 6.4 & clay, blue & Clay & -- & -- & -.- & \\
\hline 245 & 6/7-19D1 & & & 21 & 100 & 6.4 & 30.5 & clay, sandy, brown, gravelly & Clay, sand, and gravel & -- & -- & -- & \\
\hline 245 & 6/7-19D1 & & & 100 & 104 & 30.5 & 31.7 & clay and gravel & Clay and gravel & -- & -- & -- & \\
\hline 245 & 6/7-19D1 & & & 104 & 108 & 31.7 & 32.9 & clay, sandy, brown & Clay and sand (or sandstone) & - & -- & - & \\
\hline 246 & $6 / 8-23 C 1$ & 246 & $6 / 8-23 \mathrm{C} 1$ & 0 & 3 & 0.0 & 0.9 & adobe & Sand and clay & -- & -- & -- & Qyal and QTge (?) \\
\hline 246 & $6 / 8-23 C 1$ & & & 0 & 108 & 0.0 & 32.9 & clay, blue & Clay & -- & -- & -- & \\
\hline 246 & $6 / 8-23 \mathrm{C} 1$ & & & 3 & 15 & 0.9 & 4.6 & $\begin{array}{l}\text { clay, yellow } \\
\text { clay, yellow and gravel; some }\end{array}$ & Clay & -- & -- & -- & \\
\hline 246 & $6 / 8-23 \mathrm{C} 1$ & & & 15 & 25 & 4.6 & 7.6 & water & Clay and gravel & -- & -- & -- & \\
\hline 246 & $6 / 8-23 \mathrm{C} 1$ & & & 25 & 60 & 7.6 & 18.3 & clay, blue and yellow & Clay & -- & -- & -- & \\
\hline 246 & $6 / 8-23 \mathrm{C} 1$ & & & 60 & 65 & 18.3 & 19.8 & gravel & Gravel & .. & -. & .- & \\
\hline 246 & $6 / 8-23 \mathrm{C} 1$ & & & 65 & 130 & 19.8 & 39.6 & clay and gravel & Clay and gravel & -- & -- & -- & \\
\hline 246 & $6 / 8-23 \mathrm{C} 1$ & & & 108 & 260 & 32.9 & 79.2 & clay, yellow, sandy, and gravel & Clay and sand (or sandstone) & -- & -- & -- & \\
\hline 246 & $6 / 8-23 C 1$ & & & 130 & 172 & 39.6 & 52.4 & clay, yellow, and gravelly sand & Clay, sand, and gravel & -- & -- & -.- & QTm \\
\hline 246 & $6 / 8-23 \mathrm{C} 1$ & & & 172 & 194 & 52.4 & 59.1 & clay, sandy yellow & Clay, sand, and gravel & -- & -- & -- & \\
\hline 246 & 6/8-23C1 & & & 260 & 265 & 79.2 & 80.8 & very soft streak & Clay, sand, and gravel & - & -- & -- & \\
\hline 246 & $6 / 8-23 \mathrm{C1}$ & & & 265 & 336 & 80.8 & 102.4 & clay, yellow, sandy, and gravel & Sand & -- & -- & -- & \\
\hline 246 & $6 / 8-23 \mathrm{C} 1$ & & & 336 & 342 & 102.4 & 104.2 & sand, soft, wet & Clay and sand (or sandstone) & -- & -- & -- & \\
\hline 246 & $6 / 8-23 \mathrm{C} 1$ & & & 342 & 350 & 104.2 & 106.7 & $\begin{array}{l}\text { clay, yellow, and sand } \\
\text { clay, yellow and gravelly; sticky }\end{array}$ & Clay and gravel & -- & -- & -- & \\
\hline 246 & $6 / 8-23 \mathrm{C} 1$ & & & 350 & 411 & 106.7 & 125.3 & last $10 \mathrm{ft}$. & Clay & -- & -- & -. & \\
\hline 246 & $6 / 8-23 \mathrm{C} 1$ & & & 411 & 430 & 125.3 & 131.1 & clay, blue & Clay & -- & -- & -- & \\
\hline 246 & $6 / 8-23 \mathrm{C} 1$ & & & 430 & 438 & 131.1 & 133.5 & $\begin{array}{l}\text { clay, yellow } \\
\text { clats }\end{array}$ & Clay & -- & -- & -- & \\
\hline 247 & 6/8-22N1 & 247 & 6/8-22N1 & 0 & 3 & 0.0 & 0.9 & soil & Sand and clay & -- & -- & -- & QTm \\
\hline 247 & 6/8-22N1 & & & 3 & 15 & 0.9 & 4.6 & clay, pink, sandy & Clay and sand (or sandstone) & -- & -- & -- & \\
\hline 247 & $6 / 8-22 N 1$ & & & 15 & 21 & 4.6 & 6.4 & clay, sandy, white, and gravel & Clay, sand, and gravel & -- & -- & -- & \\
\hline 247 & $6 / 8-22 N 1$ & & & 21 & 28 & 6.4 & 8.5 & sand and gravel & Sand (or sandstone) and gravel & -- & -- & -. & \\
\hline 247 & 6/8-22N1 & & & 28 & 70 & 8.5 & 21.3 & clay, sandy, yellow & Clay and sand (or sandstone) & -. & -- & -- & \\
\hline 247 & 6/8-22N1 & & & 70 & 95 & 21.3 & 29.0 & clay, sandy, blue & Clay and sand (or sandstone) & -- & -- & - & \\
\hline 247 & $6 / 8-22 \mathrm{~N} 1$ & & & 95 & 147 & 29.0 & 44.8 & clay, blue & Clay & .- & -- & -. & \\
\hline 247 & 6/8-22N1 & & & 147 & 155 & 44.8 & 47.2 & clay, sandy, blue & Clay and sand (or sandstone) & -- & -- & -- & \\
\hline 247 & $6 / 8-22 N 1$ & & & 155 & 170 & 47.2 & 51.8 & clay, sandy, blue & Clay and sand (or sandstone) & .. & --. & -. & \\
\hline
\end{tabular}


Appendix 3. Lithologic data. - Continued

[Site IDs and well number correspond to locations in Appendix 1, a site ID and well number is listed for each drilled interval for a particular drill hole. For convenience, the site ID and well numbers are repeated at the shallowest drilled interval for each well. leaders

\begin{tabular}{|c|c|c|c|c|c|c|c|c|c|c|c|c|c|}
\hline Site ID & Well number & $\begin{array}{l}\text { Site ID, } \\
\text { top of } \\
\text { well }\end{array}$ & $\begin{array}{l}\text { Well number, } \\
\text { top of well }\end{array}$ & $\begin{array}{c}\text { Top of } \\
\text { interval, in } \\
\text { feet }\end{array}$ & $\begin{array}{c}\text { Base of } \\
\text { interval, in } \\
\text { feet }\end{array}$ & $\begin{array}{c}\text { Top of } \\
\text { interval, in } \\
m\end{array}$ & $\begin{array}{c}\text { Base of } \\
\text { interval, in } \\
\mathrm{m}\end{array}$ & Reported lithologic unit ${ }^{1}$ & Interpreted lithologic class ${ }^{2}$ & $\begin{array}{c}\text { Reported } \\
\text { degree of } \\
\text { cementation or } \\
\text { induration } \\
\end{array}$ & $\begin{array}{l}\text { Fossils } \\
\text { reported in } \\
\text { interval }^{4}\end{array}$ & $\begin{array}{c}\text { Organic } \\
\text { matter } \\
\text { reported in } \\
\text { interval }^{5}\end{array}$ & $\begin{array}{l}\text { Reported stratigraphic top of geologic } \\
\text { unit }^{6}\end{array}$ \\
\hline 247 & 6/8-22N1 & & & 170 & 185 & 51.8 & 56.4 & clay, yellow & Clay & -- & -- & -- & \\
\hline 247 & 6/8-22N1 & & & 185 & 190 & 56.4 & 57.9 & sand, and clay, red & Sand and clay & -- & -- & -- & \\
\hline 247 & $6 / 8-22 \mathrm{~N} 1$ & & & 190 & 195 & 57.9 & 59.4 & clay, sandy, yellow & Clay and sand (or sandstone) & -- & -- & -- & \\
\hline 247 & $6 / 8-22 N 1$ & & & 195 & 200 & 59.4 & 61.0 & 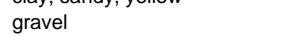 & Gravel & -- & -- & -- & \\
\hline 248 & 6/7-29P1 & 248 & 6/7-29P1 & 0 & 1 & 0.0 & 0.3 & "Hill formation" & Clay and gravel & -- & -- & -- & Tsv \\
\hline 248 & 6/7-29P1 & & & 1 & 78 & 0.3 & 23.8 & rock & Ash or tuff & hard & -- & -- & \\
\hline 248 & 6/7-29P1 & & & 78 & 104 & 23.8 & 31.7 & clay, blue and gravel & Clay and gravel & -- & -- & -- & \\
\hline 248 & 6/7-29P1 & & & 104 & 127 & 31.7 & 38.7 & blue clay & Clay & -- & -- & -- & \\
\hline 248 & 6/7-29P1 & & & 127 & 140 & 38.7 & 42.7 & decayed rock & Ash or tuff & -- & -- & -- & \\
\hline 248 & 6/7-29P1 & & & 140 & 168 & 42.7 & 51.2 & decayed rock, blue & Ash or tuff & -- & -- & -- & \\
\hline 248 & 6/7-29P1 & & & 168 & 185 & 51.2 & 56.4 & no data & Ash or tuff & -- & -- & -- & \\
\hline 249 & 6/8-35A2 & 249 & 6/8-35A2 & 0 & 3 & 0.0 & 0.9 & soil & Sand and clay & -- & -- & -- & Qyal \\
\hline 249 & $6 / 8-35 \mathrm{~A} 2$ & & & 3 & 28 & 0.9 & 8.5 & clay, yellow & Clay & -- & -- & -- & \\
\hline 249 & 6/8-35A2 & & & 28 & 38 & 8.5 & 11.6 & clay, blue & Clay & -- & -- & -- & \\
\hline 249 & 6/8-35A2 & & & 38 & 42 & 11.6 & 12.8 & clay, blue, and gravel & Clay and gravel & -- & -- & -- & \\
\hline 249 & $6 / 8-35 \mathrm{~A} 2$ & & & 42 & 80 & 12.8 & 24.4 & clay, yellow & Clay & -- & -- & -- & QTm \\
\hline 249 & 6/8-35A2 & & & 80 & 101 & 24.4 & 30.8 & clay, sandy, yellow, and gravel & Clay, sand, and gravel & -- & -- & -- & \\
\hline 249 & 6/8-35A2 & & & 101 & 125 & 30.8 & 38.1 & clay, sandy, yellow & Clay and sand (or sandstone) & -- & -- & -- & \\
\hline 249 & $6 / 8-35 \mathrm{~A} 2$ & & & 125 & 137 & 38.1 & 41.8 & clay, blue & Clay & -- & -- & -- & \\
\hline 249 & $6 / 8-35 \mathrm{~A} 2$ & & & 137 & 157 & 41.8 & 47.9 & clay, yellow & Clay & -- & -- & -- & \\
\hline 249 & $6 / 8-35 \mathrm{~A} 2$ & & & 157 & 161 & 47.9 & 49.1 & rock (probably cemented sand) & Sandstone & cemented & -- & -- & \\
\hline 249 & 6/8-35A2 & & & 161 & 235 & 49.1 & 71.6 & clay, yellow & Clay & -- & -- & -- & \\
\hline 249 & 6/8-35A2 & & & 235 & 238 & 71.6 & 72.5 & clay, yellow, sandy & Clay and sand (or sandstone) & -- & -- & -- & \\
\hline 249 & $6 / 8-35 \mathrm{~A} 2$ & & & 238 & 240 & 72.5 & 73.2 & clay, yellow, sandy; little gravel & Clay, sand, and trace gravel & -- & -- & -- & \\
\hline 249 & $6 / 8-35 \mathrm{~A} 2$ & & & 240 & 246 & 73.2 & 75.0 & $\begin{array}{l}\text { clay, blue } \\
\text {. }\end{array}$ & Clay & -. & -- & -- & \\
\hline 249 & $6 / 8-35 \mathrm{~A} 2$ & & & 246 & 298 & 75.0 & 90.8 & clay, yellow & Clay & -- & -- & -- & \\
\hline 249 & $6 / 8-35 \mathrm{~A} 2$ & & & 298 & 368 & 90.8 & 112.2 & $\begin{array}{l}\text { clay, blue } \\
\text { cla }\end{array}$ & Clay & -- & -- & -- & \\
\hline 249 & $6 / 8-35 \mathrm{~A} 2$ & & & 368 & 369 & 112.2 & 112.5 & redwood & No data & -- & -- & wood & \\
\hline 249 & $6 / 8-35 \mathrm{~A} 2$ & & & 369 & 400 & 112.5 & 121.9 & clay, blue, and gravel & Clay and gravel & -- & -- & -- & \\
\hline 249 & $6 / 8-35 \mathrm{~A} 2$ & & & 400 & 425 & 121.9 & 129.5 & clay, blue & Clay & -- & -- & -- & \\
\hline 249 & $6 / 8-35 \mathrm{~A} 2$ & & & 425 & 515 & 129.5 & 157.0 & clay, sandy, blue & Clay and sand (or sandstone) & -- & -- & -- & \\
\hline 249 & $6 / 8-35 \mathrm{~A} 2$ & & & 515 & 528 & 157.0 & 160.9 & sand, yellow & Sand & -- & -- & -- & \\
\hline 249 & $6 / 8-35 \mathrm{~A} 2$ & & & 528 & 548 & 160.9 & 167.0 & sand, yellow & Sand & -- & -- & -- & \\
\hline 249 & 6/8-35A2 & & & 548 & 554 & 167.0 & 168.9 & clay, brown, and redwood & Clay & -- & -- & wood & \\
\hline 249 & 6/8-35A2 & & & 554 & 564 & 168.9 & 171.9 & clay, blue & Clay & -- & -- & -- & \\
\hline 249 & $6 / 8-35 \mathrm{~A} 2$ & & & 564 & 567 & 171.9 & 172.8 & clay blue, and gravel & Clay and gravel & - & -- & -- & \\
\hline 249 & $6 / 8-35 \mathrm{~A} 2$ & & & 567 & 585 & 172.8 & 178.3 & clay and gravel & Clay and gravel & -- & -- & -- & \\
\hline 249 & $6 / 8-35 \mathrm{~A} 2$ & & & 585 & 605 & 178.3 & 184.4 & clay blue & Clay & -- & -- & -- & \\
\hline 249 & 6/8-35A2 & & & 605 & 615 & 184.4 & 187.5 & clay and gravel & Clay and gravel & -- & -- & -- & \\
\hline 249 & 6/8-35A2 & & & 615 & 623 & 187.5 & 189.9 & sand blue, and gravel & Sand (or sandstone) and gravel & -- & -- & -- & \\
\hline 249 & $6 / 8-35 \mathrm{~A} 2$ & & & 623 & 635 & 189.9 & 193.5 & clay, blue, tough & Clay & -- & -- & -- & \\
\hline 249 & $6 / 8-35 \mathrm{~A} 2$ & & & 635 & 647 & 193.5 & 197.2 & clay, blue tough; some gravel & Clay and gravel & -- & -- & -- & \\
\hline 249 & 6/8-35A2 & & & 647 & 650 & 197.2 & 198.1 & clay, blue & Clay & -- & -- & -- & \\
\hline 249 & 6/8-35A2 & & & 650 & 660 & 198.1 & 201.2 & shale & Clay and sand (or sandstone) & cemented & -- & -. & \\
\hline 250 & $6 / 8-36 \mathrm{~A} 1$ & 250 & 6/8-36A1 & 0 & 4 & 0.0 & 1.2 & soil & Sand and clay & -- & -- & -- & Qyal and QTm \\
\hline 250 & 6/8-36A1 & & & 4 & 7 & 1.2 & 2.1 & clay, yellow & Clay & -- & -- & -- & \\
\hline 250 & 6/8-36A1 & & & 7 & 31 & 2.1 & 9.4 & clay, yellow, and gravel; mixed & Clay and gravel & -- & -- & -- & \\
\hline 250 & 6/8-36A1 & & & 31 & 40 & 9.4 & 12.2 & clay, yellow & Clay & -- & -- & -- & \\
\hline 250 & 6/8-36A1 & & & 40 & 42 & 12.2 & 12.8 & clay yellow, and gravel & Clay and gravel & -- & -- & -. & \\
\hline 250 & $6 / 8-36 \mathrm{~A} 1$ & & & 42 & 60 & 12.8 & 18.3 & clay, yellow & Clay & -- & -- & -- & \\
\hline 250 & $6 / 8-36 \mathrm{~A} 1$ & & & 60 & 64 & 18.3 & 19.5 & clay, yellow, and gravel & Clay and gravel & -- & -- & -- & \\
\hline 250 & 6/8-36A1 & & & 64 & 80 & 19.5 & 24.4 & clay sandy yellow & Clay and sand (or sandstone) & -- & -- & -- & \\
\hline 250 & 6/8-36A1 & & & 80 & 85 & 24.4 & 25.9 & clay, sandy, yellow, and gravel & Clay, sand, and gravel & -- & -- & -- & \\
\hline 250 & 6/8-36A1 & & & 85 & 108 & 25.9 & 32.9 & clay, yellow & Clay & -- & -- & - & \\
\hline 250 & 6/8-36A1 & & & 108 & 128 & 32.9 & 39.0 & sand, yellow & Sand & -- & -- & -- & \\
\hline 250 & 6/8-36A1 & & & 128 & 130 & 39.0 & 39.6 & clay, yellow & Clay & -- & -- & -- & \\
\hline 250 & 6/8-36A1 & & & 130 & 136 & 39.6 & 41.5 & clay, sandy, yellow, and gravel & Clay, sand, and gravel & -- & -- & -- & \\
\hline 250 & 6/8-36A1 & & & 136 & 150 & 41.5 & 45.7 & clay, sandy, yellow & Clay and sand (or sandstone) & -- & -- & -. & \\
\hline 250 & 6/8-36A1 & & & 150 & 158 & 45.7 & 48.2 & sand, yellow, and sandy gravel & Sand (or sandstone) and gravel & -- & -- & -- & \\
\hline 250 & 6/8-36A1 & & & 158 & 178 & 48.2 & 54.3 & clay, yellow & Clay & -- & -- & -- & \\
\hline
\end{tabular}


Appendix 3. Lithologic data. - Continued

[Site IDs and well number correspond to locations in Appendix 1, a site ID and well number is listed for each drilled interval for a particular drill hole. For convenience, the site ID and well numbers are repeated at the shallowest drilled interval for each well. leaders (--), not reported]

\begin{tabular}{|c|c|c|c|c|c|c|c|c|c|c|c|c|c|}
\hline Site ID & Well number & $\begin{array}{l}\text { Site ID, } \\
\text { top of } \\
\text { well }\end{array}$ & $\begin{array}{l}\text { Well number, } \\
\text { top of well }\end{array}$ & $\begin{array}{l}\text { Top of } \\
\text { interval, in } \\
\text { feet }\end{array}$ & $\begin{array}{l}\text { Base of } \\
\text { interval, in } \\
\text { feet }\end{array}$ & $\begin{array}{c}\text { Top of } \\
\text { interval, in } \\
\mathrm{m}\end{array}$ & $\begin{array}{c}\text { Base of } \\
\text { interval, in } \\
m\end{array}$ & Reported lithologic unit' & Interpreted lithologic class ${ }^{2}$ & $\begin{array}{c}\text { Reported } \\
\text { degree of } \\
\text { cementation or } \\
\text { induration }^{3} \\
\end{array}$ & $\begin{array}{c}\text { Fossils } \\
\text { reported in } \\
\text { interval }^{4}\end{array}$ & $\begin{array}{c}\text { Organic } \\
\text { matter } \\
\text { reported in } \\
\text { interval }^{5}\end{array}$ & $\begin{array}{c}\text { Reported stratigraphic top of geologic } \\
\text { unit }^{6}\end{array}$ \\
\hline 250 & 6/8-36A1 & & & 178 & 185 & 54.3 & 56.4 & clay, yellow, and little fine gravel & Clay and gravel & -- & -- & -- & \\
\hline 250 & 6/8-36A1 & & & 185 & 240 & 56.4 & 73.2 & clay yellow & Clay & -- & -- & -- & \\
\hline 250 & 6/8-36A1 & & & 240 & 264 & 73.2 & 80.5 & clay, sandy, yellow & Clay and sand (or sandstone) & -- & -- & -- & \\
\hline 250 & 6/8-36A1 & & & 264 & 282 & 80.5 & 86.0 & sandstone, yellow & Sandstone & cemented & -- & -- & \\
\hline 250 & 6/8-36A1 & & & 282 & 284 & 86.0 & 86.6 & clay, yellow & Clay & -- & -- & -- & \\
\hline 250 & 6/8-36A1 & & & 284 & 287 & 86.6 & 87.5 & $\begin{array}{l}\text { sand, fine, yellow } \\
\text { clay, yellow, and gravel; small }\end{array}$ & Sand & -- & -- & -- & \\
\hline 250 & 6/8-36A1 & & & 287 & 290 & 87.5 & 88.4 & amount & Clay and gravel & -- & -- & -- & \\
\hline 250 & 6/8-36A1 & & & 290 & 324 & 88.4 & 98.8 & clay, sandy, yellow & Clay and sand (or sandstone) & -- & -- & -- & \\
\hline 250 & 6/8-36A1 & & & 324 & 338 & 98.8 & 103.0 & gravel, yellow, cemented & Gravel & cemented & -- & -- & \\
\hline 250 & 6/8-36A1 & & & 338 & 344 & 103.0 & 104.9 & clay, yellow & Clay & -- & -- & -- & \\
\hline 250 & 6/8-36A1 & & & 344 & 347 & 104.9 & 105.8 & clay, blue & Clay & -- & -- & -- & \\
\hline 250 & 6/8-36A1 & & & 347 & 350 & 105.8 & 106.7 & $\begin{array}{l}\text { clay, yellow } \\
\text { clay, yellow, and small amount }\end{array}$ & Clay & -- & -- & -- & \\
\hline 250 & 6/8-36A1 & & & 350 & 353 & 106.7 & 107.6 & gravel & Clay, sand, and trace gravel & -- & -- & -- & \\
\hline 250 & 6/8-36A1 & & & 353 & 360 & 107.6 & 109.7 & clay, yellow & Clay & -- & -- & -- & \\
\hline 250 & 6/8-36A1 & & & 360 & 376 & 109.7 & 114.6 & clay blue & Clay & -- & -- & -- & \\
\hline 250 & 6/8-36A1 & & & 376 & 391 & 114.6 & 119.2 & gravel, blue, cemented & Gravel & cemented & -- & -- & \\
\hline 250 & 6/8-36A1 & & & 391 & 392 & 119.2 & 119.5 & clay, blue & Clay & -- & -- & -- & \\
\hline 250 & $6 / 8-36 \mathrm{~A} 1$ & & & 392 & 402 & 119.5 & 122.5 & gravel, blue, cemented & Gravel & cemented & -- & -- & \\
\hline 250 & 6/8-36A1 & & & 402 & 424 & 122.5 & 129.2 & clay, sandy, yellow & Clay and sand (or sandstone) & -- & -- & -- & \\
\hline 250 & 6/8-36A1 & & & 424 & 433 & 129.2 & 132.0 & clay, yellow & Clay & -- & -- & -- & \\
\hline 250 & 6/8-36A1 & & & 433 & 443 & 132.0 & 135.0 & $\begin{array}{l}\text { clay, yellow } \\
\text { clay, sandy, blue; little gravel, } 471-\end{array}$ & Clay & -- & -- & -- & \\
\hline 250 & 6/8-36A1 & & & 443 & 476 & 135.0 & 145.1 & $476 \mathrm{ft}$. & Clay, sand, and trace gravel & -- & -- & -- & \\
\hline 250 & 6/8-36A1 & & & 476 & 490 & 145.1 & 149.4 & clay, sandy, green & Clay and sand (or sandstone) & -- & -- & -- & \\
\hline 250 & 6/8-36A1 & & & 490 & 500 & 149.4 & 152.4 & clay, sandy, blue & Clay and sand (or sandstone) & -- & -- & -- & \\
\hline 250 & 6/8-36A1 & & & 500 & 506 & 152.4 & 154.2 & clay, blue & Clay & -- & -- & -- & \\
\hline 250 & 6/8-36A1 & & & 506 & 512 & 154.2 & 156.1 & gravel, cemented & Gravel & cemented & -- & -- & \\
\hline 250 & 6/8-36A1 & & & 512 & 522 & 156.1 & 159.1 & clay, hard, blue & Clay & hard & -- & -- & \\
\hline 250 & 6/8-36A1 & & & 522 & 530 & 159.1 & 161.5 & clay, sandy, blue & Clay and sand (or sandstone) & -- & -- & -- & \\
\hline 250 & 6/8-36A1 & & & 530 & 547 & 161.5 & 166.7 & gravel, cemented & Gravel & cemented & -- & -- & \\
\hline 250 & 6/8-36A1 & & & 547 & 574 & 166.7 & 175.0 & clay, blue & Clay & -- & -- & -- & \\
\hline 250 & 6/8-36A1 & & & 574 & 603 & 175.0 & 183.8 & gravel, cemented & Gravel & cemented & -- & -- & \\
\hline 250 & 6/8-36A1 & & & 603 & 622 & 183.8 & 189.6 & clay, blue & Clay & -- & -- & -- & \\
\hline 250 & 6/8-36A1 & & & 622 & 638 & 189.6 & 194.5 & clay, blue, gravel, and clam shells & Clay and gravel & -- & shells & -- & \\
\hline 250 & 6/8-36A1 & & & 638 & 695 & 194.5 & 211.8 & clay, blue & Clay & -- & -- & -- & $T p(?)$ \\
\hline 251 & $6 / 7-31 \mathrm{H} 1$ & 251 & 6/7-31H1 & 0 & 5 & 0.0 & 1.5 & soil & Sand and clay & -- & -- & -- & Qyal and QTge \\
\hline 251 & $6 / 7-31 \mathrm{H} 1$ & & & 5 & 12 & 1.5 & 3.7 & clay yellow & Clay & -- & -- & -- & \\
\hline 251 & 6/7-31H1 & & & 12 & 18 & 3.7 & 5.5 & clay, yellow, and gravel & Clay and gravel & -- & -- & -- & \\
\hline 251 & 6/7-31H1 & & & 18 & 29 & 5.5 & 8.8 & clay yellow & Clay & -- & -- & -- & \\
\hline 251 & $6 / 7-31 \mathrm{H} 1$ & & & 29 & 44 & 8.8 & 13.4 & clay, yellow, and gravel & Clay and gravel & -- & -- & -- & \\
\hline 251 & 6/7-31H1 & & & 44 & 80 & 13.4 & 24.4 & clay, yellow & Clay & -- & -- & -- & \\
\hline 251 & $6 / 7-31 \mathrm{H} 1$ & & & 80 & 115 & 24.4 & 35.1 & clay, yellow, and gravel & Clay and gravel & -- & -- & -- & \\
\hline 251 & 6/7-31H1 & & & 115 & 175 & 35.1 & 53.3 & clay, yellow & Clay & -- & -- & -- & \\
\hline 251 & $6 / 7-31 \mathrm{H} 1$ & & & 175 & 252 & 53.3 & 76.8 & clay, yellow, and boulders & Clay and gravel & -- & -- & -- & \\
\hline 251 & 6/7-31H1 & & & 252 & 255 & 76.8 & 77.7 & clay, yellow & Clay & -- & -- & -- & \\
\hline 251 & $6 / 7-31 \mathrm{H} 1$ & & & 255 & 298 & 77.7 & 90.8 & clay, blue & Clay & -- & -- & -- & \\
\hline 252 & $6 / 7-31 \mathrm{G} 1$ & 252 & $6 / 7-31 \mathrm{G} 1$ & 0 & 6 & 0.0 & 1.8 & black soil & Sand and clay & -- & -- & -- & Qyal and QTge \\
\hline 252 & $6 / 7-31 \mathrm{G} 1$ & & & 6 & 40 & 1.8 & 12.2 & clay, yellow, and gravel & Clay and gravel & -- & -- & -- & \\
\hline 252 & $6 / 7-31 \mathrm{G} 1$ & & & 40 & 272 & 12.2 & 82.9 & clay, yellow & Clay & -- & -- & -- & \\
\hline 252 & 6/7-31G1 & & & 272 & 289 & 82.9 & 88.1 & volcanic rock & Ash or tuff & -- & -- & -- & Tsv (?) \\
\hline 252 & 6/7-31G1 & & & 289 & 324 & 88.1 & 98.8 & volcanic rock & Ash or tuff & -- & -- & -- & \\
\hline 252 & 6/7-31G1 & & & 324 & 445 & 98.8 & 135.6 & clay, blue & Clay & -- & -- & -- & QTm (?) \\
\hline 252 & 6/7-31G1 & & & 445 & 446 & 135.6 & 135.9 & $\begin{array}{l}\text { sand } \\
\text { hard shell (probably cemented }\end{array}$ & Sand & -- & -- & -- & \\
\hline 252 & 6/7-31G1 & & & 446 & 447 & 135.9 & 136.2 & sand) & Sandstone & hard & shells & -- & \\
\hline 252 & 6/7-31G1 & & & 447 & 468 & 136.2 & 142.6 & sand & Sand & -- & -- & -- & \\
\hline 252 & 6/7-31G1 & & & 468 & 470 & 142.6 & 143.3 & hard & Sandstone & hard & -- & -- & \\
\hline
\end{tabular}


Appendix 3. Lithologic data. - Continued

ISite IDs and well number correspond to locations in Appendix 1, a site ID and well number is listed for each drilled interval for a particular drill hole. For convenience, the site ID and well numbers are repeated at the shallowest drilled interval for each well. leaders

\begin{tabular}{|c|c|c|c|c|c|c|c|c|c|c|c|c|c|}
\hline Site ID & Well number & $\begin{array}{l}\text { Site ID, } \\
\text { top of } \\
\text { well }\end{array}$ & $\begin{array}{l}\text { Well number, } \\
\text { top of well }\end{array}$ & $\begin{array}{c}\text { Top of } \\
\text { interval, in } \\
\text { feet }\end{array}$ & $\begin{array}{c}\text { Base of } \\
\text { interval, in } \\
\text { feet }\end{array}$ & $\begin{array}{c}\text { Top of } \\
\text { interval, in } \\
m\end{array}$ & $\begin{array}{c}\text { Base of } \\
\text { interval, in } \\
\mathrm{m}\end{array}$ & Reported lithologic unit ${ }^{1}$ & Interpreted lithologic class ${ }^{2}$ & $\begin{array}{c}\text { Reported } \\
\text { degree of } \\
\text { cementation or } \\
\text { induration } \\
\end{array}$ & $\begin{array}{c}\text { Fossils } \\
\text { reported in } \\
\text { interval }^{4}\end{array}$ & $\begin{array}{c}\text { Organic } \\
\text { matter } \\
\text { reported in } \\
\text { interval }^{5}\end{array}$ & $\begin{array}{l}\text { Reported stratigraphic top of geologic } \\
\text { unit }^{6}\end{array}$ \\
\hline $\begin{array}{l}252 \\
252\end{array}$ & $\begin{array}{l}\text { 6/7-31G1 } \\
6 / 7-31 \mathrm{G} 1\end{array}$ & & & $\begin{array}{l}470 \\
480\end{array}$ & $\begin{array}{l}480 \\
490\end{array}$ & $\begin{array}{l}143.3 \\
146.3\end{array}$ & $\begin{array}{l}146.3 \\
149.4\end{array}$ & (no record) & $\begin{array}{l}\text { No data } \\
\text { Clay }\end{array}$ & $\begin{array}{l}-- \\
--\end{array}$ & $\overline{--}$ & -- & \\
\hline 252 & $\begin{array}{l}\text { 6/7-31G1 } \\
6 / 7-31 \mathrm{G} 1\end{array}$ & & & $\begin{array}{l}480 \\
490\end{array}$ & $\begin{array}{l}490 \\
508\end{array}$ & $\begin{array}{l}146.3 \\
149.4\end{array}$ & $\begin{array}{l}149.4 \\
154.8\end{array}$ & $\begin{array}{l}\text { clay, blue } \\
\text { shale, blue }\end{array}$ & $\begin{array}{l}\text { Clay and sand (or sandstone) } \\
\text { Clat }\end{array}$ & cemented & $\overline{-}$ & -- & $\operatorname{Tp}(?)$ \\
\hline 253 & 6/8-33E1 & 253 & 6/8-33E1 & 0 & 3 & 0.0 & 0.9 & topsoil & Sand and clay & - & - & -- & $\mathrm{Tp}$ \\
\hline 253 & 6/8-33E1 & & & 3 & 17 & 0.9 & 5.2 & clay, sandy, yellow & Clay and sand (or sandstone) & -- & -- & -- & \\
\hline 253 & 6/8-33E1 & & & 17 & 35 & 5.2 & 10.7 & clay, sandy, blue & Clay and sand (or sandstone) & -- & -- & -- & \\
\hline 253 & 6/8-33E1 & & & 35 & 80 & 10.7 & 24.4 & clay, blue & Clay & -- & -- & -- & \\
\hline 253 & 6/8-33E1 & & & 80 & 90 & 24.4 & 27.4 & gravel, first water & Gravel & -- & -- & -- & \\
\hline 253 & 6/8-33E1 & & & 90 & 97 & 27.4 & 29.6 & gravel cemented & Gravel & cemented & -- & -- & \\
\hline 253 & 6/8-33E1 & & & 97 & 117 & 29.6 & 35.7 & gravel, cemented, blue & Gravel & cemented & -. & -- & \\
\hline 253 & 6/8-33E1 & & & 117 & 125 & 35.7 & 38.1 & gravel, cemented & Gravel & cemented & -.- & -- & \\
\hline 253 & 6/8-33E1 & & & 125 & 146 & 38.1 & 44.5 & sand and clay, blue & Sand and clay & -- & -- & -- & \\
\hline 253 & 6/8-33E1 & & & 146 & 153 & 44.5 & 46.6 & gravel, free & Gravel & -- & -- & -- & \\
\hline 253 & 6/8-33E1 & & & 153 & 165 & 46.6 & 50.3 & gravel, cemented & Gravel & cemented & -- & -- & \\
\hline 253 & 6/8-33E1 & & & 165 & 175 & 50.3 & 53.3 & sand and clay, blue & Sand and clay & -- & -. & -- & \\
\hline 253 & 6/8-33E1 & & & 175 & 195 & 53.3 & 59.4 & clay, blue and yellow, sticky & Clay & -- & -- & -- & \\
\hline 253 & 6/8-33E1 & & & 195 & 215 & 59.4 & 65.5 & clay and rock, jointed clay & Clay and gravel & -- & -- & -- & \\
\hline 254 & 6/7-31L1 & 254 & 6/7-31L1 & 0 & 3 & 0.0 & 0.9 & loam black & Sand and clay & -- & -. & -- & Qyal and QTge \\
\hline 254 & 6/7-31L1 & & & 3 & 8 & 0.9 & 2.4 & sand, gray & Sand & -- & -- & -- & \\
\hline 254 & 6/7-31L1 & & & 8 & 18 & 2.4 & 5.5 & clay, yellow, and gravel & Clay and gravel & -- & -- & -- & \\
\hline 254 & 6/7-31L1 & & & 18 & 21 & 5.5 & 6.4 & $\begin{array}{l}\text { gravel } \\
\text { gave, and graver }\end{array}$ & Gravel & -. & -. & -- & \\
\hline 254 & 6/7-31L1 & & & 21 & 30 & 6.4 & 9.1 & clay, yellow, sandy & Clay and sand (or sandstone) & -- & -- & -- & \\
\hline 254 & 6/7-31L1 & & & 30 & 50 & 9.1 & 15.2 & clay, yellow & Clay & -- & -. & -- & \\
\hline 254 & 6/7-31L1 & & & 50 & 63 & 15.2 & 19.2 & sand, yellow & Sand & -- & -. & .- & \\
\hline 254 & 6/7-31L1 & & & 63 & 75 & 19.2 & 22.9 & gravel & Gravel & -. & -- & -- & \\
\hline 254 & 6/7-31L1 & & & 75 & 79 & 22.9 & 24.1 & clay, yellow & Clay & -- & -- & .- & \\
\hline 255 & 6/7-32M1 & 255 & 6/7-32M1 & 0 & 2 & 0.0 & 0.6 & soil, black & Sand and clay & -- & -- & -- & Qyal and QTge \\
\hline 255 & $6 / 7-32 \mathrm{M} 1$ & & & 2 & 3 & 0.6 & 0.9 & sand blue, and gravel & Sand (or sandstone) and gravel & - & - & - & \\
\hline 255 & 6/7-32M1 & & & 3 & 5 & 0.9 & 1.5 & soil, black & Sand and clay & -- & -- & -- & \\
\hline 255 & 6/7-32M1 & & & 5 & 39 & 1.5 & 11.9 & clay, yellow, and gravel & Clay and gravel & -- & -- & -- & \\
\hline 255 & 6/7-32M1 & & & 39 & 40 & 11.9 & 12.2 & gravel & Gravel & -- & -- & -- & \\
\hline 255 & 6/7-32M1 & & & 40 & 70 & 12.2 & 21.3 & boulders & Gravel & -- & -- & -- & \\
\hline 255 & 6/7-32M1 & & & 70 & 75 & 21.3 & 22.9 & clay and gravel & Clay and gravel & - & -- & - & \\
\hline 255 & 6/7-32M1 & & & 75 & 95 & 22.9 & 29.0 & clay, yellow, and gravel & Clay and gravel & -- & -- & -- & \\
\hline 255 & 6/7-32M1 & & & 95 & 108 & 29.0 & 32.9 & clay, blue & Clay & -- & -- & -- & QTm \\
\hline 255 & 6/7-32M1 & & & 108 & 111 & 32.9 & 33.8 & sand, yellow & Sand & - & -- & -- & \\
\hline 255 & 6/7-32M1 & & & 111 & 127 & 33.8 & 38.7 & sand, blue, and yellow clay & Sand and clay & -- & -- & .- & \\
\hline 255 & 6/7-32M1 & & & 127 & 131 & 38.7 & 39.9 & sand, blue; streak of blue clay & Sand and clay & -- & -- & -- & \\
\hline 255 & 6/7-32M1 & & & 131 & 209 & 39.9 & 63.7 & clay, blue & Clay & -- & -- & .. & \\
\hline 255 & 6/7-32M1 & & & 209 & 220 & 63.7 & 67.1 & clay, blue, and gravel & Clay and gravel & .- & -. & .- & \\
\hline 255 & 6/7-32M1 & & & 220 & 240 & 67.1 & 73.2 & clay, blue & Clay & -- & -- & -- & \\
\hline 255 & 6/7-32M1 & & & 240 & 260 & 73.2 & 79.2 & clay, blue, sandy & Clay and sand (or sandstone) & -- & -- & .- & \\
\hline 255 & 6/7-32M1 & & & 260 & 290 & 79.2 & 88.4 & $\begin{array}{l}\text { clay, blue } \\
\text { clay, sandy, blue and streaks of }\end{array}$ & Clay & - & -- & -- & \\
\hline 255 & 6/7-32M1 & & & 290 & 325 & 88.4 & 99.1 & sandstone & Clay and sand (or sandstone) & cemented & -- & -. & \\
\hline 255 & 6/7-32M1 & & & 325 & 332 & 99.1 & 101.2 & sandstone, blue, and fine gravel & Sandstone & cemented & -- & -- & \\
\hline 255 & 6/7-32M1 & & & 332 & 335 & 101.2 & 102.1 & $\begin{array}{l}\text { clay, sandy, blue } \\
\text { lantion }\end{array}$ & Clay and sand (or sandstone) & -- & -- & -. & \\
\hline 255 & 6/7-32M1 & & & 335 & 397 & 102.1 & 121.0 & clay, blue & Clay & -- & -- & -. & \\
\hline 255 & 6/7-32M1 & & & 397 & 408 & 121.0 & 124.4 & clay, yellow & Clay & -. & -- & -. & \\
\hline 255 & 6/7-32M1 & & & 408 & 425 & 124.4 & 129.5 & clay, blue & Clay & -- & -- & -- & \\
\hline 255 & 6/7-32M1 & & & 425 & 460 & 129.5 & 140.2 & clay, yellow & Clay & -- & -- & -- & \\
\hline 255 & 6/7-32M1 & & & 460 & 474 & 140.2 & 144.5 & $\begin{array}{l}\text { clay, blue } \\
\text { clus }\end{array}$ & Clay & -- & -- & -. & \\
\hline 255 & 6/7-32M1 & & & 474 & 475 & 144.5 & 144.8 & clay, blue, and gravel & Clay and gravel & -- & -- & -- & \\
\hline 255 & 6/7-32M1 & & & 475 & 497 & 144.8 & 151.5 & clay, blue & Clay & -- & -- & -- & \\
\hline 255 & 6/7-32M1 & & & 497 & 502 & 151.5 & 153.0 & clay, yellow & Clay & -- & -- & -- & \\
\hline 255 & 6/7-32M1 & & & 502 & 800 & 153.0 & 243.8 & clay & Clay & -- & -- & -. & \\
\hline 255 & 6/7-32M1 & & & 800 & 815 & 243.8 & 248.4 & gravel, hard, cemented & Gravel & cemented & -- & -- & \\
\hline 255 & 6/7-32M1 & & & 815 & 850 & 248.4 & 259.1 & soft & Clay & -. & -. & .. & \\
\hline 255 & 6/7-32M1 & & & 850 & 920 & 259.1 & 280.4 & clay and streaks of gravel & Clay and gravel & -. & -- & .. & \\
\hline 255 & 6/7-32M1 & & & 920 & 963 & 280.4 & 293.5 & clay & Clay & -- & -- & -- & \\
\hline 255 & 6/7-32M1 & & & 963 & 980 & 293.5 & 298.7 & rock & Ash or tuff & hard & -. & -- & Tsv \\
\hline
\end{tabular}


Appendix 3. Lithologic data. - Continued

[Site IDs and well number correspond to locations in Appendix 1, a site ID and well number is listed for each drilled interval for a particular drill hole. For convenience, the site ID and well numbers are repeated at the shallowest drilled

\begin{tabular}{|c|c|c|c|c|c|c|c|c|c|c|c|c|c|}
\hline Site ID & Well number & $\begin{array}{l}\text { Site ID, } \\
\text { top of } \\
\text { well }\end{array}$ & $\begin{array}{l}\text { Well number, } \\
\text { top of well }\end{array}$ & $\begin{array}{c}\text { Top of } \\
\text { interval, in } \\
\text { feet }\end{array}$ & $\begin{array}{c}\text { Base of } \\
\text { interval, in } \\
\text { feet }\end{array}$ & $\begin{array}{c}\text { Top of } \\
\text { interval, in } \\
m\end{array}$ & $\begin{array}{c}\text { Base of } \\
\text { interval, in } \\
\mathrm{m}\end{array}$ & Reported lithologic unit ${ }^{1}$ & Interpreted lithologic class ${ }^{2}$ & $\begin{array}{c}\text { Reported } \\
\text { degree of } \\
\text { cementation or } \\
\text { induration }^{3} \\
\end{array}$ & $\begin{array}{c}\text { Fossils } \\
\text { reported in } \\
\text { interval }^{4}\end{array}$ & $\begin{array}{c}\text { Organic } \\
\text { matter } \\
\text { reported in } \\
\text { interval }^{5}\end{array}$ & $\begin{array}{l}\text { Reported stratigraphic top of geologic } \\
\text { unit }^{6}\end{array}$ \\
\hline 255 & $6 / 7-32 \mathrm{M} 1$ & & & 980 & 1008 & 298.7 & 307.2 & clay and gravel & Clay and gravel & -- & -- & -- & \\
\hline 255 & $6 / 7-32 \mathrm{M} 1$ & & & 1008 & 1010 & 307.2 & 307.8 & rock & Ash or tuff & hard & -- & -- & \\
\hline 255 & 6/7-32M1 & & & 1010 & 1138 & 307.8 & 346.9 & $\begin{array}{l}\text { clay and gravel } \\
\text {. }\end{array}$ & Clay and gravel & -- & -- & -- & \\
\hline 255 & 6/7-32M1 & & & 1138 & 1147 & 346.9 & 349.6 & $\begin{array}{l}\text { rock } \\
\text { lativet }\end{array}$ & Ash or tuff & hard & -- & -- & \\
\hline 255 & 6/7-32M1 & & & 1147 & 1163 & 349.6 & 354.5 & clay, hard & Clay & hard & -- & -- & $T p$ \\
\hline 256 & $6 / 7-3131$ & 256 & 6/7-31J1 & 0 & 6 & 0.0 & 1.8 & adobe & Sand and clay & -- & -- & -- & Qyal and QTge \\
\hline 256 & $6 / 7-31 \mathrm{J1}$ & & & 6 & 19 & 1.8 & 5.8 & gray clay & Clay & -- & -- & -- & \\
\hline 256 & $6 / 7-31 \mathrm{J1}$ & & & 19 & 45 & 5.8 & 13.7 & $\begin{array}{l}\text { yellow clay } \\
\text { sandy vellow clay and gravel: }\end{array}$ & Clay & -- & -- & -- & \\
\hline 256 & $6 / 7-31 \mathrm{J1}$ & & & 45 & 94 & 13.7 & 28.7 & water at $80 \mathrm{ft}$. & Clay, sand, and gravel & -- & -- & -- & \\
\hline 256 & $6 / 7-31 \mathrm{J1}$ & & & 94 & 103 & 28.7 & 31.4 & yellow clay & Clay & -- & -- & -- & \\
\hline 256 & $6 / 7-31 \mathrm{J1}$ & & & 103 & 128 & 31.4 & 39.0 & yellow clay and gravel & Clay and gravel & -- & -- & -- & \\
\hline 256 & 6/7-31J1 & & & 128 & 140 & 39.0 & 42.7 & yellow cemented gravel & Clay, sand, and gravel & cemented & -- & -- & \\
\hline 256 & $6 / 7-3131$ & & & 140 & 170 & 42.7 & 51.8 & yellow clay & Clay & -- & -- & -- & \\
\hline 256 & $6 / 7-31 \mathrm{J1}$ & & & 170 & 183 & 51.8 & 55.8 & yellow clay and gravel & Clay and gravel & -- & -- & -- & \\
\hline 256 & $6 / 7-31 \mathrm{J1}$ & & & 183 & 198 & 55.8 & 60.4 & $\begin{array}{l}\text { boulders } \\
\text { and }\end{array}$ & Gravel & -- & -. & -. & \\
\hline 256 & $6 / 7-3131$ & & & 198 & 218 & 60.4 & 66.4 & blue clay & Clay & -- & -- & -- & QTm \\
\hline 256 & $6 / 7-3131$ & & & 218 & 220 & 66.4 & 67.1 & blue sand and gravel & Sand (or sandstone) and gravel & -- & -- & -- & \\
\hline 256 & $6 / 7-3131$ & & & 220 & 221 & 67.1 & 67.4 & sand and gravel & Sand (or sandstone) and gravel & -- & -- & -- & \\
\hline 256 & $6 / 7-3131$ & & & 221 & 241 & 67.4 & 73.5 & blue sand, packed & Sand & -- & -- & -- & \\
\hline 256 & 6/7-31J1 & & & 241 & 251 & 73.5 & 76.5 & blue sand & Sand & -- & -. & .- & \\
\hline 256 & $6 / 7-3131$ & & & 251 & 274 & 76.5 & 83.5 & blue sandstone & Sandstone & cemented & -- & -- & \\
\hline 256 & $6 / 7-3131$ & & & 274 & 280 & 83.5 & 85.3 & blue clay & Clay & -- & -- & -. & \\
\hline 257 & $5 / 7-6 \mathrm{E} 1$ & 257 & 5/7-6E1 & 0 & 3 & 0.0 & 0.9 & loam, black & Sand and clay & -- & -- & .- & Qyal and QTm \\
\hline 257 & $5 / 7-6 \mathrm{E} 1$ & & & 3 & 24 & 0.9 & 7.3 & clay, yellow & Clay & -- & -- & -- & \\
\hline 257 & $5 / 7-6 \mathrm{E} 1$ & & & 24 & 40 & 7.3 & 12.2 & sand, yellow; and gravel; water & Sand & -- & -- & -- & \\
\hline 257 & $5 / 7-6 \mathrm{E} 1$ & & & 40 & 45 & 12.2 & 13.7 & $\begin{array}{l}\text { clay blue } \\
\text { sand and clay, gray; water at } 85\end{array}$ & Clay & -- & -- & - & \\
\hline 257 & $5 / 7-6 \mathrm{E} 1$ & & & 45 & 92 & 13.7 & 28.0 & ft. & Sand and clay & -- & -- & -- & \\
\hline 257 & 5/7-6E1 & & & 92 & 104 & 28.0 & 31.7 & sand and gravel, gray & Sand (or sandstone) and gravel & -- & -- & -.- & \\
\hline 257 & $5 / 7-6 \mathrm{E} 1$ & & & 104 & 115 & 31.7 & 35.1 & clay, blue & Clay & -- & -- & -- & \\
\hline 258 & $5 / 8-1 \mathrm{H} 1$ & 258 & $5 / 8-1 \mathrm{H} 1$ & 0 & 2.5 & 0.0 & 0.8 & soil & Sand and clay & -- & -- & -- & Qyal and QTm \\
\hline 258 & $5 / 8-1 \mathrm{H} 1$ & & & 2.5 & 35 & 0.8 & $\begin{array}{l}10.7 \\
10.7\end{array}$ & clay, yellow & Clay & -- & - & - & \\
\hline 258 & $5 / 8-1 \mathrm{H} 1$ & & & 35 & 43 & 10.7 & 13.1 & $\begin{array}{l}\text { clay, blue } \\
\text { lat }\end{array}$ & Clay & -. & -- & -. & \\
\hline 258 & 5/8-1H1 & & & 43 & 50 & 13.1 & 15.2 & clay, yellow & Clay & -. & -- & -- & \\
\hline 258 & $5 / 8-1 \mathrm{H} 1$ & & & 50 & 62 & 15.2 & 18.9 & clay, blue & Clay & -- & -- & -- & \\
\hline 258 & $5 / 8-1 \mathrm{H} 1$ & & & 62 & 78 & 18.9 & 23.8 & $\begin{array}{l}\text { clay, yellow } \\
\text { cin }\end{array}$ & Clay & -. & -- & -- & \\
\hline 258 & $5 / 8-1 \mathrm{H} 1$ & & & 78 & 88 & 23.8 & 26.8 & clay, blue & Clay & -- & -- & -- & \\
\hline 258 & 5/8-1H1 & & & 88 & 98 & 26.8 & 29.9 & clay, yellow & Clay & -- & -- & .. & \\
\hline 258 & $5 / 8-1 \mathrm{H} 1$ & & & 98 & 110 & 29.9 & 33.5 & $\begin{array}{l}\text { clay, blue } \\
\text { lat }\end{array}$ & Clay & .. & -. & .. & \\
\hline 258 & $5 / 8-1 \mathrm{H} 1$ & & & 110 & 116 & 33.5 & 35.4 & clay, yellow & Clay & -- & -- & -- & \\
\hline 258 & 5/8-1H1 & & & 116 & 136 & 35.4 & 41.5 & $\begin{array}{l}\text { clay, blue } \\
\text { la }\end{array}$ & Clay & -. & -- & -- & \\
\hline 258 & 5/8-1H1 & & & 136 & 140 & 41.5 & 42.7 & clay, yellow & Clay & -- & -- & -- & \\
\hline 258 & 5/8-1H1 & & & 140 & 151 & 42.7 & 46.0 & $\begin{array}{l}\text { clay, blue } \\
\text { las }\end{array}$ & Clay & -- & -- & -- & \\
\hline 258 & $5 / 8-1 \mathrm{H} 1$ & & & 151 & 176 & 46.0 & 53.6 & clay, blue and gravel & Clay and gravel & -- & -- & -. & \\
\hline 258 & $5 / 8-1 \mathrm{H} 1$ & & & 176 & 186 & 53.6 & 56.7 & clay blue & Clay & -- & -- & -- & \\
\hline 258 & 5/8-1H1 & & & 186 & 190 & 56.7 & 57.9 & clay, yellow & Clay & -- & -- & -. & \\
\hline 258 & $5 / 8-1 \mathrm{H} 1$ & & & 190 & 219 & 57.9 & 66.8 & clay, sandy, blue & Clay and sand (or sandstone) & -- & -- & -- & \\
\hline 258 & 5/8-1H1 & & & 219 & 224 & 66.8 & 68.3 & $\begin{array}{l}\text { clay, blue } \\
\text { sand and gravel, slightly }\end{array}$ & Clay & -- & -- & - & \\
\hline 258 & $5 / 8-1 \mathrm{H} 1$ & & & 224 & 230 & 68.3 & 70.1 & cemented & Sand (or sandstone) and gravel & cemented & -- & -- & \\
\hline 258 & $5 / 8-1 \mathrm{H} 1$ & & & 230 & 232 & 70.1 & 70.7 & $\begin{array}{l}\text { clay, blue } \\
\text { clay, yellow, sandy; water at } 70\end{array}$ & Clay & -- & -- & -- & \\
\hline 259 & $5 / 7-5 \mathrm{~K} 1$ & 259 & $5 / 7-5 K 1$ & 0 & 74 & 0.0 & 22.6 & feet & Clay and sand (or sandstone) & -- & -- & -- & QTm and Tp \\
\hline 259 & $5 / 7-5 \mathrm{~K} 1$ & & & 74 & 131 & 22.6 & 39.9 & clay, blue & Clay & -- & -- & -- & \\
\hline 259 & 5/7-5K1 & & & 131 & 147 & 39.9 & 44.8 & clay, brown & Clay & -- & -- & -. & \\
\hline 259 & $5 / 7-5 \mathrm{~K} 1$ & & & 147 & 204 & 44.8 & 62.2 & clay, blue & Clay & -- & -- & -- & \\
\hline 259 & $5 / 7-5 K 1$ & & & 204 & 218 & 62.2 & 66.4 & clay, yellow & Clay & -- & -- & -- & \\
\hline 259 & 5/7-5K1 & & & 218 & 253 & 66.4 & 77.1 & clay, blue & Clay & -- & -- & -- & \\
\hline 259 & $5 / 7-5 \mathrm{~K} 1$ & & & 253 & 265 & 77.1 & 80.8 & clay, blue and gravel & Clay and gravel & -- & -- & -- & \\
\hline 259 & 5/7-5K1 & & & 265 & 317 & 80.8 & 96.6 & $\begin{array}{l}\text { clay, blue } \\
\text { last }\end{array}$ & Clay & -- & -- & -- & \\
\hline
\end{tabular}


Appendix 3. Lithologic data. - Continued

[Site IDs and well number correspond to locations in Appendix 1, a site ID and well number is listed for each drilled interval for a particular drill hole. For convenience, the site ID and well numbers are repeated at the shallowest drilled interval for each well. leader.

\begin{tabular}{|c|c|c|c|c|c|c|c|c|c|c|c|c|c|}
\hline Site ID & Well number & $\begin{array}{l}\text { Site ID, } \\
\text { top of } \\
\text { well }\end{array}$ & $\begin{array}{l}\text { Well number, } \\
\text { top of well }\end{array}$ & $\begin{array}{l}\text { Top of } \\
\text { interval, in } \\
\text { feet }\end{array}$ & $\begin{array}{l}\text { Base of } \\
\text { interval, in } \\
\text { feet }\end{array}$ & $\begin{array}{c}\text { Top of } \\
\text { interval, in } \\
\mathrm{m}\end{array}$ & $\begin{array}{c}\text { Base of } \\
\text { interval, in } \\
\mathrm{m}\end{array}$ & Reported lithologic unit' & Interpreted lithologic class ${ }^{2}$ & $\begin{array}{c}\text { Reported } \\
\text { degree of } \\
\text { cementation or } \\
\text { induration }^{3} \\
\end{array}$ & $\begin{array}{l}\text { Fossils } \\
\text { reported in } \\
\text { interval }^{4}\end{array}$ & $\begin{array}{c}\text { Organic } \\
\text { matter } \\
\text { reported in } \\
\text { interval }^{5}\end{array}$ & $\begin{array}{l}\text { Reported stratigraphic top of geologic } \\
\text { unit }^{6}\end{array}$ \\
\hline 259 & $5 / 7-5 K 1$ & & & 317 & 326 & 96.6 & 99.4 & clay, joint & Clay & -- & -- & -- & \\
\hline 259 & $5 / 7-5 K 1$ & & & 326 & 337 & 99.4 & 102.7 & gravel & Gravel & -- & -- & -- & \\
\hline 259 & $5 / 7-5 K 1$ & & & 337 & 339 & 102.7 & 103.3 & clay, blue and gravel & Clay and gravel & -- & -- & -- & \\
\hline 260 & $5 / 8-1 \mathrm{J1}$ & 260 & 5/8-1J1 & 0 & 27 & 0.0 & 8.2 & no record & No data & -- & -- & -- & QTm \\
\hline 260 & $5 / 8-1 \mathrm{J1}$ & & & 27 & 44 & 8.2 & 13.4 & clay, blue & Clay & -- & -- & -- & \\
\hline 260 & $5 / 8-1 \mathrm{J1}$ & & & 44 & 49 & $\begin{array}{l}13.4 \\
13.4\end{array}$ & $\begin{array}{l}10.4 \\
14.9\end{array}$ & gravel, cement & Gravel & cemented & -- & -- & \\
\hline 260 & $5 / 8-1 \mathrm{J1}$ & & & 49 & 170 & 14.9 & 51.8 & clay & Clay & -- & -- & -- & \\
\hline 260 & $5 / 8-1 \mathrm{J1}$ & & & 170 & 180 & 51.8 & 54.9 & gravel, cement & Gravel & cemented & -- & -- & \\
\hline 260 & $5 / 8-1 \mathrm{~J} 1$ & & & 180 & 280 & 54.9 & 85.3 & $\begin{array}{l}\text { clay, blue } \\
\text { ches }\end{array}$ & Clay & -- & -- & -- & \\
\hline 260 & $5 / 8-1 \mathrm{J1}$ & & & 280 & 290 & 85.3 & 88.4 & gravel, cement & Gravel & cemented & -- & -- & \\
\hline 261 & 5/8-1L1 & 261 & 5/8-1L1 & 0 & 3 & 0.0 & 0.9 & topsoil & Sand and clay & -- & .- & .- & OTm \\
\hline 261 & 5/8-1ட1 & & & 3 & 22 & 0.9 & 6.7 & clay, yellow, and rock & Clay and gravel & -- & -- & -- & \\
\hline 261 & $5 / 8-1\llcorner 1$ & & & 22 & 30 & 6.7 & 9.1 & 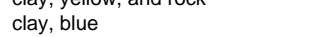 & $\begin{array}{l}\text { Clay } \\
\text { Cling }\end{array}$ & -- & -- & -- & \\
\hline 261 & 5/8-1L1 & & & 30 & 47 & 9.1 & 14.3 & $\begin{array}{l}\text { clay, yellow, and rock } \\
\text { gravel and clay , blue: } 1 \text { st water }\end{array}$ & Clay and gravel & -- & -- & -- & \\
\hline 261 & 5/8-1L1 & & & 47 & 52 & 14.3 & 15.8 & $52^{\prime}$ & Clay and gravel & .- & .- & -- & \\
\hline 261 & 5/8-1L1 & & & 52 & 82 & 15.8 & 25.0 & clay, blue & Clay & -- & -- & -- & \\
\hline 261 & 5/8-1L1 & & & 82 & 90 & 25.0 & 27.4 & gravel & Gravel & -- & -- & -- & \\
\hline 261 & 5/8-1ட1 & & & 90 & 105 & 27.4 & 32.0 & clay, blue, and gravel & Clay and gravel & -- & -- & -- & \\
\hline 261 & 5/8-1ட1 & & & 105 & 110 & 32.0 & 33.5 & clay, yellow, and gravel & Clay and gravel & -- & -- & -- & \\
\hline 261 & 5/8-1L1 & & & 110 & 155 & 33.5 & 47.2 & clay, blue, and gravel & Clay and gravel & .- & .- & .- & \\
\hline 261 & 5/8-1ட1 & & & 155 & 170 & 47.2 & 51.8 & $\begin{array}{l}\text { clay, sandy, blue } \\
\text { che }\end{array}$ & Clay and sand (or sandstone) & -- & -- & -- & \\
\hline 261 & 5/8-1L1 & & & 170 & 175 & 51.8 & 53.3 & clay, sandy, blue, and gravel & $\begin{array}{l}\text { Clay, sand, and gravel } \\
\text {. }\end{array}$ & -- & -- & -- & \\
\hline 261 & 5/8-1L1 & & & 175 & 202 & 53.3 & 61.6 & clay, blue & Clay & -- & -- & -- & \\
\hline 261 & 5/8-1L1 & & & 202 & 205 & 61.6 & 62.5 & clay, blue, sandy & Clay and sand (or sandstone) & -- & -- & -- & \\
\hline 261 & 5/8-1ட1 & & & 205 & 211 & 62.5 & 64.3 & gravel and clay, blue & Clay and gravel & -- & -- & -- & \\
\hline 261 & 5/8-1ட1 & & & 211 & 226 & 64.3 & 68.9 & $\begin{array}{l}\text { clay, blue, jointed } \\
\text { lated }\end{array}$ & Clay & -- & .- & .- & $\mathrm{Tp}(?)$ \\
\hline 261 & 5/8-1L1 & & & 226 & 253 & 68.9 & 77.1 & clay, blue & Clay & -- & -- & -- & \\
\hline 262 & 5/8-13B1 & 262 & 5/8-13B1 & 0 & 3 & 0.0 & 0.9 & abode soil & Sand and clay & -- & -- & -- & Qyal and QTm \\
\hline 262 & $5 / 8-13 \mathrm{~B} 1$ & & & 3 & 92 & 0.9 & 28.0 & sand; first water at 22 feet & Sand & -- & -- & -- & \\
\hline 262 & 5/8-13B1 & & & 92 & 172 & 28.0 & 52.4 & sand, blue and gravel & Sand (or sandstone) and gravel & -- & -- & -- & \\
\hline 263 & $5 / 7-15 \mathrm{~K} 1$ & 263 & 5/7-15K1 & 0 & 5 & 0.0 & 1.5 & 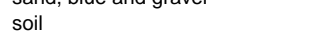 & Sand and clay & .- & .- & .- & Qyal and Qoal (?) \\
\hline 263 & $5 / 7-15 \mathrm{~K} 1$ & & & 5 & 15 & 1.5 & 4.6 & boulders & Gravel & -- & -- & -- & \\
\hline 263 & 5/7-15K1 & & & 15 & 42 & 4.6 & 12.8 & yellow clay & Clay & -- & -- & -- & $T p$ \\
\hline 263 & 5/7-15K1 & & & 42 & 60 & 12.8 & 18.3 & clay, blue, no water & Clay & -- & -- & -- & ק \\
\hline 263 & 5/7-15K1 & & & 60 & 95 & 18.3 & 29.0 & yellow sandstone & Sandstone & cemented & -- & -- & \\
\hline 263 & 5/7-15K1 & & & 95 & 106 & 29.0 & 32.3 & blue clay and gravel & Clay and gravel & - & -- & -- & \\
\hline 264 & $5 / 7-2001$ & 264 & $5 / 7-2001$ & 0 & 20 & 0.0 & 6.1 & clay & Clay & -- & -- & -- & Qyal and Qoal (?) \\
\hline 264 & $5 / 7-20 \mathrm{C} 1$ & & & 20 & 66 & 6.1 & 20.1 & sand and gravel & Sand (or sandstone) and gravel & -- & -- & -- & \\
\hline 264 & $5 / 7-2001$ & & & 66 & 96 & 20.1 & 29.3 & conglomerate & Clay, sand, and gravel & -- & -- & -- & \\
\hline 264 & $5 / 7-20 \mathrm{C} 1$ & & & 96 & 202 & 29.3 & 61.6 & $\begin{array}{l}\text { sand and gravel with streaks of } \\
\text { clay } \\
\text { sandy clay with streaks of sand }\end{array}$ & Sand (or sandstone) and gravel & -- & -- & -- & \\
\hline 264 & $5 / 7-20 \mathrm{C} 1$ & & & 202 & 290 & 61.6 & 88.4 & and gravel & Clay, sand, and trace gravel & .- & -- & -- & \\
\hline 264 & $5 / 7-20 C 1$ & & & 290 & 310 & 88.4 & 94.5 & conglomerate & Clay, sand, and gravel & .- & .- & .- & \\
\hline 264 & $5 / 7-20 \mathrm{C} 1$ & & & 310 & 315 & 94.5 & 96.0 & clay & Clay & -- & -- & -- & \\
\hline 264 & $5 / 7-20 C 1$ & & & 315 & 322 & 96.0 & 98.1 & rock (probably cemented sand) & Sandstone & cemented & .- & .- & QTm \\
\hline 264 & $5 / 7-2001$ & & & 322 & 355 & 98.1 & 108.2 & sandy clay & Clay and sand (or sandstone) & - & -- & -- & \\
\hline 264 & $5 / 7-20 C 1$ & & & 355 & 410 & 108.2 & 125.0 & clay, blue, sandy & Clay and sand (or sandstone) & -. & -- & - & \\
\hline 264 & $5 / 7-20 C 1$ & & & 410 & 430 & 125.0 & 131.1 & rock (probably cemented sand) & Sandstone & cemented & .- & .- & \\
\hline 264 & $5 / 7-20 \mathrm{C} 1$ & & & 430 & 435 & 131.1 & 132.6 & 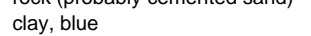 & Clay & - & -- & -- & \\
\hline 264 & $5 / 7-20 c_{1}$ & & & 435 & 506 & 132.6 & 154.2 & $\begin{array}{l}\text { clay, sandy, blue } \\
\text { cond }\end{array}$ & Clay and sand (or sandstone) &.-- & -- & -- & \\
\hline 264 & $5 / 7-20 \mathrm{C} 1$ & & & 506 & 513 & 154.2 & 156.4 & shelf & Sandstone & cemented & -- & -- & \\
\hline 264 & $5 / 7-20 \mathrm{Cl}$ & & & 513 & 548 & 156.4 & 167.0 & clay, hard; with gravel & Clay and gravel & hard & -- & -- & \\
\hline 264 & $5 / 7-20 \mathrm{C1}$ & & & 548 & 558 & 167.0 & 170.1 & clay, blue and gravel & Clay and gravel & -- & .- & .- & \\
\hline 264 & $5 / 7-20 \mathrm{C1}$ & & & 558 & 595 & 170.1 & 181.4 & $\begin{array}{l}\text { free gravel } \\
\text { fravel }\end{array}$ & Gravel & .- & .- & -- & \\
\hline 264 & $5 / 7-2001$ & & & 595 & 615 & 181.4 & 187.5 & clay, soft & Clay & -- & -- & -- & \\
\hline 264 & $5 / 7-20 \mathrm{C} 1$ & & & 615 & 617 & 187.5 & 188.1 & shelf (probably cemented sand) & Sandstone & cemented & -- & -- & \\
\hline 264 & $5 / 7-20 \mathrm{C} 1$ & & & 617 & 654 & 188.1 & 199.3 & gravel & Gravel & -. & .- & .- & \\
\hline 264 & $5 / 7-20 \mathrm{C} 1$ & & & 654 & 658 & 199.3 & 200.6 & free gravel & Gravel & -- & .- & -- & \\
\hline
\end{tabular}


Appendix 3. Lithologic data. - Continued

[Site IDs and well number correspond to locations in Appendix 1, a site ID and well number is listed for each drilled interval for a particular drill hole. For convenience, the site ID and well numbers are repeated at the shallowest drilled interval for each well. leaders (-

\begin{tabular}{|c|c|c|c|c|c|c|c|c|c|c|c|c|c|}
\hline Site ID & Well number & $\begin{array}{l}\text { Site ID, } \\
\text { top of } \\
\text { well }\end{array}$ & $\begin{array}{l}\text { Well number, } \\
\text { top of well }\end{array}$ & $\begin{array}{l}\text { Top of } \\
\text { interval, in } \\
\text { feet }\end{array}$ & $\begin{array}{l}\text { Base of } \\
\text { interval, in } \\
\text { feet }\end{array}$ & $\begin{array}{c}\text { Top of } \\
\text { interval, in } \\
m\end{array}$ & $\begin{array}{c}\text { Base of } \\
\text { interval, in } \\
\mathrm{m}\end{array}$ & Reported lithologic unit ${ }^{1}$ & Interpreted lithologic class ${ }^{2}$ & $\begin{array}{c}\text { Reported } \\
\text { degree of } \\
\text { cementation or } \\
\text { induration }^{3} \\
\end{array}$ & $\begin{array}{l}\text { Fossils } \\
\text { reported in }^{\text {interval }}{ }^{4}\end{array}$ & $\begin{array}{c}\text { Organic } \\
\text { matter } \\
\text { reported in } \\
\text { interval }^{5}\end{array}$ & $\begin{array}{l}\text { Reported stratigraphic top of geologic } \\
\text { unit }^{6}\end{array}$ \\
\hline 264 & $5 / 7-20 \mathrm{C} 1$ & & & 658 & 666 & 200.6 & 203.0 & $\begin{array}{l}\text { sand and shelf (probably } \\
\text { cemented layers) }\end{array}$ & Sandstone & cemented & .- & .- & \\
\hline 264 & $5 / 7-2001$ & & & 666 & 675 & 203.0 & 205.7 & gravel & Gravel & -- & -- & -- & \\
\hline 264 & $5 / 7-20 \mathrm{C} 1$ & & & 675 & 688 & 205.7 & 209.7 & gravel, hard & Gravel & hard & -- & -- & \\
\hline 265 & 5/7-19N1 & 265 & 5/7-19N1 & 0 & 6 & 0.0 & 1.8 & sand & Sand & -- & -- & -- & QTm \\
\hline 265 & 5/7-19N1 & & & 6 & 15 & 1.8 & 4.6 & clay, sandy, yellow & Clay and sand (or sandstone) & -- & -- & -- & \\
\hline 265 & $5 / 7-19 \mathrm{~N} 1$ & & & 15 & 25 & 4.6 & 7.6 & sand, yellow; and gravel; water & Sand (or sandstone) and gravel & -- & -- & -- & \\
\hline 265 & 5/7-19N1 & & & 25 & 30 & 7.6 & 9.1 & clay, blue & Clay & -- & -- & -- & \\
\hline 265 & 5/7-19N1 & & & 30 & 72 & 9.1 & 21.9 & clay, sandy yellow and gravel & Clay, sand, and gravel & .- & .- & -- & \\
\hline 265 & 5/7-19N1 & & & 72 & 75 & 21.9 & 22.9 & clay, sandy, yellow & Clay and sand (or sandstone) & -- & -- & -- & \\
\hline 265 & 5/7-19N1 & & & 75 & 90 & 22.9 & 27.4 & clay, blue & Clay & -- & -- & -- & \\
\hline 265 & 5/7-19N1 & & & 90 & 105 & 27.4 & 32.0 & $\begin{array}{l}\text { oyster, sandy, blue; and } \\
\text { sandstone }\end{array}$ & Sandstone & cemented & shells & -- & \\
\hline 265 & 5/7-19N1 & & & 105 & 140 & 32.0 & 42.7 & $\begin{array}{l}\text { clay, sandy, blue; and sandstone } \\
\text { clay, sandy, blue; and blue }\end{array}$ & Clay and sand (or sandstone) & cemented & -- & -- & \\
\hline 265 & 5/7-19N1 & & & 140 & 180 & 42.7 & 54.9 & sandstone & Clay and sand (or sandstone) & cemented & .- & .- & \\
\hline 266 & 5/7-25A1 & 266 & 5/7-25A1 & 0 & 136 & 0.0 & 41.5 & no data & No data & - & -- & -- & $T p$ \\
\hline 266 & 5/7-25A1 & & & 136 & 145 & 41.5 & 44.2 & clay, blue, sandy & Clay and sand (or sandstone) & .- & .- & -- & \\
\hline 266 & 5/7-25A1 & & & 145 & 149 & 44.2 & 45.4 & sand, blue & Sand & -- & -- & -- & \\
\hline 266 & $5 / 7-25 \mathrm{~A} 1$ & & & $\begin{array}{l}145 \\
149\end{array}$ & 182 & 45.4 & 55.5 & $\begin{array}{l}\text { clay, sandy, blue } \\
\text { clutur }\end{array}$ & Clay and sand (or sandstone) & -- & -- & -- & \\
\hline 266 & 5/7-25A1 & & & 182 & 205 & 55.5 & 62.5 & clay, blue & Clay & -- & -- & -- & \\
\hline 266 & 5/7-25A1 & & & 205 & 210 & 62.5 & 64.0 & clay, green & Clay & .- & .- & .- & \\
\hline 266 & 5/7-25A1 & & & 210 & 241 & 64.0 & 73.5 & clay, blue & Clay & -- & -- & -- & \\
\hline 266 & 5/7-25A1 & & & 241 & 265 & 73.5 & 80.8 & clay, green & Clay & -- & -- & -- & \\
\hline 267 & $5 / 7-25 \mathrm{C} 1$ & 267 & $5 / 7-25 \mathrm{C} 1$ & 0 & 4 & 0.0 & 1.2 & soil & Sand and clay & -- & -- & -- & Qoal \\
\hline 267 & $5 / 7-25 \mathrm{C} 1$ & & & 4 & 25 & 1.2 & 7.6 & clay, yellow & Clay & -- & -- & -- & \\
\hline 267 & $5 / 7-25 \mathrm{C} 1$ & & & 25 & 35 & 7.6 & 10.7 & boulders with water & Gravel & .- & .- & .- & \\
\hline 267 & $5 / 7-25 \mathrm{C1}$ & & & 35 & 65 & 10.7 & 19.8 & blue clay & Clay & .- & .- & .- & $\mathrm{Tp}$ \\
\hline 267 & $5 / 7-25 \mathrm{C1}$ & & & 65 & 75 & 19.8 & 22.9 & sand & Sand & .- & .- & .- & \\
\hline 267 & $5 / 7-25 \mathrm{Cl}$ & & & 75 & 200 & 22.9 & 61.0 & blue clay & Clay & -- & -- & -- & \\
\hline 267 & $5 / 7-25 \mathrm{C} 1$ & & & 200 & 225 & 61.0 & 68.6 & hard sandstone & Sandstone & cemented & -- & -- & \\
\hline 267 & $5 / 7-25 \mathrm{Cl}$ & & & 225 & 235 & $\begin{array}{l}68.6 \\
68.6\end{array}$ & 71.6 & sand, water & Sand & .- & -- & -- & \\
\hline 268 & 5/7-28A3 & 268 & $5 / 7-28 A 3$ & 0 & 7 & 0.0 & 2.1 & adobe & Sand and clay & -- & .- & .- & Qyal and Qoal \\
\hline 268 & $5 / 7-28 \mathrm{~A} 3$ & & & 7 & 75 & 2.1 & 22.9 & clay, yellow, and gravel & Clay and gravel & -- & -- & .- & \\
\hline 268 & $5 / 7-28 \mathrm{A3}$ & & & 75 & 80 & 22.9 & 24.4 & clay, yellow, sandy & Clay and sand (or sandstone) & -- & -- & -- & \\
\hline 268 & $5 / 7-28 \mathrm{A3}$ & & & 80 & 90 & 24.4 & 27.4 & clay, yellow, sandy and gravel & Clay, sand, and gravel & -- & -- & -- & \\
\hline 268 & 5/7-28A3 & & & 90 & 125 & 27.4 & 38.1 & $\begin{array}{l}\text { clay, blue } \\
\text { surus }\end{array}$ & 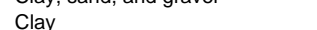 & .- & .- & .. & \\
\hline 268 & $5 / 7-28 \mathrm{~A} 3$ & & & 125 & 148 & 38.1 & 45.1 & sandstone & Sandstone & cemented & .- & .- & OTm \\
\hline 268 & $5 / 7-28 \mathrm{A3}$ & & & 148 & 186 & 45.1 & 56.7 & $\begin{array}{l}\text { clay, blue } \\
\text { che }\end{array}$ & $\begin{array}{l}\text { Clay } \\
\text { Clutic }\end{array}$ & - & -- & -- & \\
\hline 268 & $5 / 7-28 \mathrm{A3}$ & & & 186 & 220 & 56.7 & 67.1 & clay, blue, and fine gravel & Clay and gravel & -- & -- & -- & \\
\hline 268 & $5 / 7-28 \mathrm{A3}$ & & & 220 & 228 & 67.1 & 69.5 & sandstone & Sandstone & cemented & -- & -- & \\
\hline 268 & 5 & & & 228 & 231 & 69.5 & $\begin{array}{l}79.3 \\
70.4\end{array}$ & $\begin{array}{l}\text { gavel } \\
\text { gravite }\end{array}$ & $\begin{array}{l}\text { Gravel } \\
\text { Grite }\end{array}$ & chen & -- & -- & \\
\hline 268 & 5/7-28A3 & & & 231 & 238 & 70.4 & 72.5 & sandstone & Sandstone & cemented & .- & .- & \\
\hline 268 & $5 / 7-28 \mathrm{A3}$ & & & 238 & 239 & 72.5 & 72.8 & rock (probably cemented sand) & Sandstone & cemented & .- & .- & \\
\hline 268 & $5 / 7-28 \mathrm{A3}$ & & & 239 & 260 & 72.8 & 79.2 & clay, blue, and gravel & Clay and gravel & - & -- & -- & \\
\hline 268 & 5/7-28A3 & & & 260 & 280 & 79.2 & 85.3 & clay, blue & Clay & .- & .- & .- & \\
\hline 269 & $5 / 7-28 \mathrm{H} 5$ & 269 & $5 / 7-28 \mathrm{H} 5$ & 0 & 6 & 0.0 & 1.8 & adobe, black, and yellow clay & Sand and clay & .- & -- & .- & Oyal and Ooal \\
\hline 269 & $5 / 7-28 \mathrm{H} 5$ & & & 6 & 18 & 1.8 & 5.5 & gravel & Gravel & -- & -- & -- & \\
\hline 269 & 5/7-28H5 & & & 18 & 25 & 5.5 & 7.6 & clay, sandy, yellow & Clay and sand (or sandstone) & .- & -- & -- & \\
\hline 269 & $5 / 7-28 \mathrm{H} 5$ & & & 25 & 75 & 7.6 & 22.9 & clay, yellow & Clay & -- & -- & -- & \\
\hline 269 & $5 / 7-28 \mathrm{H} 5$ & & & 75 & 78 & 22.9 & 23.8 & $\begin{array}{l}\text { clay, sandy, yellow, and gravel } \\
\text { ay }\end{array}$ & Clay, sand, and gravel & -- & -- & -- & \\
\hline 269 & $5 / 7-28 \mathrm{H} 5$ & & & 78 & 95 & 23.8 & 29.0 & clay, sandy, yellow & Clay and sand (or sandstone) & .- & .- & .- & \\
\hline 269 & 5/7-28H5 & & & 95 & 130 & 29.0 & 39.6 & clay, sandy, blue, and gravel & $\begin{array}{l}\text { Clay, sand, and gravel } \\
\text { S }\end{array}$ & -- & .- & .- & \\
\hline 269 & 5/7-28H5 & & & 130 & 198 & 39.6 & 60.4 & $\begin{array}{l}\text { clay, blue } \\
\text { suc, ar }\end{array}$ & 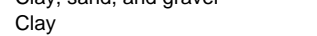 & -. & .- & .- & \\
\hline 269 & $5 / 7-28 \mathrm{H} 5$ & & & 198 & 208 & 60.4 & 63.4 & sandstone & Sandstone & cemented & -- & -- & \\
\hline 269 & $5 / 7-28 \mathrm{H} 5$ & & & 208 & 213 & 63.4 & $\begin{array}{l}6.4 \\
64.9\end{array}$ & $\begin{array}{l}\text { gravel } \\
\text { gravel }\end{array}$ & $\begin{array}{l}\text { Gravel } \\
\text { Grite }\end{array}$ & rentine & -- & -- & \\
\hline 269 & $5 / 7-28 \mathrm{H} 5$ & & & 213 & 217 & 64.9 & 66.1 & sandstone & Sandstone & cemented & .- & .- & \\
\hline 269 & $5 / 7-28 \mathrm{H} 5$ & & & 217 & 220 & 66.1 & 67.1 & $\begin{array}{l}\text { gravel } \\
\text { grite }\end{array}$ & $\begin{array}{l}\text { Gravel } \\
\text { Grel }\end{array}$ & - & .- & -. & \\
\hline 269 & $5 / 7-28 \mathrm{H} 5$ & & & 220 & 225 & 67.1 & 68.6 & sandstone & Sandstone & cemented & -- & -- & QTm \\
\hline 269 & $5 / 7-28 \mathrm{H} 5$ & & & 225 & 260 & 68.6 & 79.2 & $\begin{array}{l}\text { clay, blue } \\
\text { ches }\end{array}$ & Clay & -- & -- & -- & \\
\hline
\end{tabular}


Appendix 3. Lithologic data. - Continued

[Site IDs and well number correspond to locations in Appendix 1, a site ID and well number is listed for each drilled interval for a particular drill hole. For convenience, the site ID and well numbers are repeated at the shallowest drilled interval for each well. leaders $(-1$

\begin{tabular}{|c|c|c|c|c|c|c|c|c|c|c|c|c|c|}
\hline Site ID & Well number & $\begin{array}{l}\text { Site ID, } \\
\text { top of } \\
\text { well }\end{array}$ & $\begin{array}{c}\text { Well number, } \\
\text { top of well }\end{array}$ & $\begin{array}{c}\text { Top of } \\
\text { interval, in } \\
\text { feet }\end{array}$ & $\begin{array}{l}\text { Base of } \\
\text { interval, in } \\
\text { feet }\end{array}$ & $\begin{array}{c}\text { Top of } \\
\text { interval, in } \\
m\end{array}$ & $\begin{array}{c}\text { Base of } \\
\text { interval, in } \\
\mathrm{m}\end{array}$ & Reported lithologic unit ${ }^{1}$ & Interpreted lithologic class ${ }^{2}$ & $\begin{array}{c}\text { Reported } \\
\text { degree of } \\
\text { cementation or } \\
\text { induration }^{3}\end{array}$ & $\begin{array}{l}\text { Fossils } \\
\text { reported in } \\
\text { interval }^{4}\end{array}$ & $\begin{array}{c}\text { Organic } \\
\text { matter } \\
\text { reported in } \\
\text { interval }^{5}\end{array}$ & $\begin{array}{l}\text { Reported stratigraphic top of geologic } \\
\text { unit }^{6}\end{array}$ \\
\hline 269 & $5 / 7-28 \mathrm{H} 5$ & & & 260 & 265 & 79.2 & 80.8 & clay, blue, and shale & Clay and sand (or sandstone) & cemented & -- & -- & \\
\hline 269 & $5 / 7-28 \mathrm{H} 5$ & & & 265 & 276 & 80.8 & 84.1 & sandstone & Sandstone & cemented & -- & -- & \\
\hline 269 & $5 / 7-28 \mathrm{H} 5$ & & & 276 & 277 & 84.1 & 84.4 & rock & Sandstone & cemented & -- & -- & \\
\hline 269 & $5 / 7-28 \mathrm{H} 5$ & & & 277 & 283 & 84.4 & 86.3 & clay, blue & Clay & -. & -- & -- & \\
\hline 269 & $5 / 7-28 \mathrm{H} 5$ & & & 283 & 286 & 86.3 & 87.2 & clay, blue, and gravel & Clay and gravel & -- & -. & -- & \\
\hline 269 & $5 / 7-28 \mathrm{H} 5$ & & & 286 & 300 & 87.2 & 91.4 & clay, blue & Clay & -- & -- & -- & \\
\hline 269 & $5 / 7-28 \mathrm{H} 5$ & & & 300 & 304 & 91.4 & 92.7 & clay, blue, and gravel & Clay and gravel & -- & -- & -- & \\
\hline 269 & $5 / 7-28 \mathrm{H} 5$ & & & 304 & 317 & 92.7 & 96.6 & sandstone & Sandstone & cemented & -- & -- & \\
\hline 269 & $5 / 7-28 \mathrm{H} 5$ & & & 317 & 319 & 96.6 & 97.2 & clay, blue & Clay & -- & -- & -- & \\
\hline 269 & $5 / 7-28 \mathrm{H} 5$ & & & $\begin{array}{l}319 \\
319\end{array}$ & 320 & 97.2 & 97.5 & sandstone & Sandstone & cemented & -. & -- & \\
\hline 269 & 5/7-28H5 & & & 320 & 335 & 97.5 & 102.1 & clay, blue, and gravel & Clay and gravel & -- & .- & .- & \\
\hline 269 & $5 / 7-28 \mathrm{H} 5$ & & & 335 & 348 & 102.1 & 106.1 & $\begin{array}{l}\text { clay, blue } \\
\text {. }\end{array}$ & Clay & -- & -- & -- & \\
\hline 269 & $5 / 7-28 \mathrm{H} 5$ & & & 348 & 350 & 106.1 & 106.7 & sandstone & Sandstone & cemented & -- & -- & \\
\hline 269 & $5 / 7-28 \mathrm{H} 5$ & & & 350 & 360 & 106.7 & 109.7 & clay, sandy blue & Clay and sand (or sandstone) & -- & -- & -. & \\
\hline 269 & $5 / 7-28 \mathrm{H} 5$ & & & 360 & 385 & 109.7 & 117.3 & $\begin{array}{l}\text { clay, blue, gravel, and redwood } \\
\text { al }\end{array}$ & $\begin{array}{l}\text { Clay and gravel } \\
\text { Caliusion }\end{array}$ & -. & -. & wood & \\
\hline 269 & $5 / 7-28 \mathrm{H} 5$ & & & 385 & 390 & 117.3 & 118.9 & $\begin{array}{l}\text { clay, blue, and gravel } \\
\text { sandstone, (hard gravel) } \\
\text { (parentheses are driller's. }\end{array}$ & Clay and gravel & -- & -- & -- & \\
\hline 269 & $5 / 7-28 \mathrm{H} 5$ & & & 390 & 392 & 1189 & 1195 & Material is probably & Sand (or sandstone) and aravel & cemented & $\ldots$ & $\ldots$ & \\
\hline 269 & $5 / 7-28 \mathrm{H} 5$ & & & 392 & 398 & $\begin{array}{l}119.5 \\
119.5\end{array}$ & 121.3 & $\begin{array}{l}\text { Conglomerale) } \\
\text { gravel }\end{array}$ & $\begin{array}{l}\text { Sand (or sandstone) and gravel } \\
\text { Gravel }\end{array}$ & cemented & -- & -- & \\
\hline 269 & $5 / 7-28 \mathrm{H} 5$ & & & 398 & 400 & 121.3 & 121.9 & rock & Gravel & hard & -. & -. & \\
\hline 269 & $5 / 7-28 \mathrm{H} 5$ & & & 400 & 406 & 121.9 & 123.7 & gravel & Gravel & -- & -- & -- & \\
\hline 269 & $5 / 7-28 \mathrm{H} 5$ & & & 406 & 408 & 123.7 & 124.4 & rock & Gravel & hard & -- & -- & \\
\hline 269 & $5 / 7-28 \mathrm{H} 5$ & & & 408 & 435 & 124.4 & 132.6 & clay, blue, gravel, and rock & Clay and gravel & -. & .- & .- & \\
\hline 269 & $5 / 7-28 \mathrm{H} 5$ & & & 435 & 468 & 132.6 & 142.6 & clay, gray hard, compact & Clay and gravel & hard & -- & -- & $\mathrm{Tp}(?)$ \\
\hline 270 & $5 / 7-28 \mathrm{H} 1$ & 270 & $5 / 7-28 \mathrm{H} 1$ & 0 & 7 & 0.0 & 2.1 & topsoil, adobe & Sand and clay & -- & -- & .. & Qyal and Qoal \\
\hline 270 & $5 / 7-28 \mathrm{H} 1$ & & & 7 & 45 & 2.1 & 13.7 & $\begin{array}{l}\text { clay, sandy, yellow } \\
\text { con }\end{array}$ & Clay and sand (or sandstone) & -- & -- & -- & \\
\hline 270 & $5 / 7-28 \mathrm{H} 1$ & & & 45 & 66 & 13.7 & 20.1 & yellow clay & Clay & -- & -- & -- & \\
\hline 270 & $5 / 7-28 \mathrm{H} 1$ & & & 66 & 75 & 20.1 & 22.9 & blue clay & Clay & .- & -. & .. & \\
\hline 270 & $5 / 7-28 \mathrm{H} 1$ & & & 75 & 79 & 22.9 & 24.1 & yellow sand and gravel & Sand (or sandstone) and gravel & -. & -. & -. & \\
\hline 270 & $5 / 7-28 \mathrm{H} 1$ & & & 79 & 84 & 24.1 & 25.6 & $\begin{array}{l}\text { yellow sandy clay } \\
\text {. }\end{array}$ & Clay and sand (or sandstone) & -- & .- & .. & \\
\hline 270 & $5 / 7-28 \mathrm{H} 1$ & & & 84 & 112 & 25.6 & 34.1 & blue clay & Clay & -- & -- & -- & \\
\hline 270 & $5 / 7-28 \mathrm{H1}$ & & & 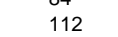 & 115 & 34.1 & 35.1 & yellow sand and fine gravel & Sand (or sandstone) and gravel & - &.- & - & \\
\hline 270 & $5 / 7-28 \mathrm{H} 1$ & & & 115 & 154 & 35.1 & 46.9 & blue clay & Clay & -- & -. & .. & \\
\hline 270 & $5 / 7-28 \mathrm{H} 1$ & & & 154 & 181 & 46.9 & 55.2 & blue cemented gravel & Gravel & cemented & .. & .. & $\mathrm{QTm}(?)$ \\
\hline 270 & $5 / 7-28 \mathrm{H} 1$ & & & 181 & 250 & 55.2 & 76.2 & blue clay & Clay & -- & -- & .. & \\
\hline 270 & $5 / 7-28 \mathrm{H} 1$ & & & 250 & 274 & 76.2 & 83.5 & blue clay and gravel & Clay and gravel & -- & .- & .- & \\
\hline 270 & $5 / 7-28 \mathrm{H1}$ & & & 274 & 460 & 83.5 & 140.2 & blue clay and boulders & Clay and gravel & - & -- &.- & \\
\hline 270 & $5 / 7-28 \mathrm{H} 1$ & & & 460 & 483 & 140.2 & 147.2 & blue clay & Clay & .- & -. & .- & \\
\hline 271 & $5 / 7-26 \mathrm{R} 1$ & 271 & 5/7-26R1 & 0 & 20 & 0.0 & 6.1 .2 & clay, adobe, yellow & Sand and clay & -- & .. & .. & Qyal and Qoal \\
\hline 271 & $5 / 7-26 \mathrm{R} 1$ & & & 20 & 75 & 6.1 & 22.9 & sandy, coarse, and gravel & Sand (or sandstone) and gravel & - & -- & -- & \\
\hline 271 & $5 / 7-26 \mathrm{R} 1$ & & & 75 & 110 & 22.9 & 33.5 & $\begin{array}{l}\text { clay, gray, sandy } \\
\text { and }\end{array}$ & Clay and sand (or sandstone) & -- & -- & -- & \\
\hline 271 & $5 / 7-26 \mathrm{R} 1$ & & & 110 & 120 & 33.5 & $\begin{array}{l}3.5 \\
36.6\end{array}$ & $\begin{array}{l}\text { sandy } \\
\text { san, saliuy }\end{array}$ & Clay & -. & -- & -- & \\
\hline 271 & $5 / 7-26 \mathrm{R} 1$ & & & 120 & 160 & 36.6 & 48.8 & $\begin{array}{l}\text { clay, yellow, sandy; and wood }\end{array}$ & Clay and sand (or sandstone) & -- & -- & wood & \\
\hline 271 & $5 / 7-26 \mathrm{R} 1$ & & & 160 & 180 & 48.8 & 54.9 & clay, yellow, sandy & Clay and sand (or sandstone) & - & -- & -. & \\
\hline 271 & $5 / 7-26 \mathrm{R} 1$ & & & 180 & 200 & 54.9 & 61.0 & clay, gray, sandy & Clay and sand (or sandstone) & - & -- & -- & \\
\hline 271 & $5 / 7-26 \mathrm{R} 1$ & & & 200 & 210 & 61.0 & 64.0 & wood & No data & -- & -- & wood & \\
\hline 271 & 5/7-26R1 & & & 210 & 220 & 64.0 & 67.1 & clay, rusty, sandy & Clay and sand (or sandstone) & .- & .- & -. & $\mathrm{QTm}(?)$ \\
\hline 271 & $5 / 7-26 \mathrm{R} 1$ & & & 220 & 230 & 67.1 & 70.1 & clay, gray, sandy & Clay and sand (or sandstone) & -- & -- & -. & \\
\hline 271 & $5 / 7-26 \mathrm{R} 1$ & & & 230 & 240 & 70.1 & 73.2 & $\begin{array}{l}\text { bentonite } \\
\text { belliuy }\end{array}$ & Clay & -- & -. & -. & \\
\hline 271 & $5 / 7-26 \mathrm{R} 1$ & & & 240 & 270 & 73.2 & 82.3 & shale, gray, sandy & Clay and sand (or sandstone) & cemented & -- & -- & \\
\hline 271 & $5 / 7-26 \mathrm{R} 1$ & & & 270 & 280 & 82.3 & 85.3 & clay, gray & Clay & -- & -- & -- & \\
\hline 271 & 5/7-26R1 & & & 280 & 300 & 85.3 & 91.4 & clay, gray, sandy & Clay and sand (or sandstone) & -- & -- & -- & \\
\hline 271 & $5 / 7-26 \mathrm{R} 1$ & & & 300 & 315 & 91.4 & 96.0 & $\begin{array}{l}\text { gravel } \\
\text { s. }\end{array}$ & Gravel & -. & -- & -- & \\
\hline 271 & $5 / 7-26 \mathrm{R} 1$ & & & 315 & 440 & $\begin{array}{l}91.4 \\
96.0\end{array}$ & 134.1 & clay, gray & Clay & -- & -. & -. & \\
\hline 271 & $5 / 7-26 \mathrm{R} 1$ & & & 440 & 450 & 134.1 & 137.2 & clay, gray, and hard gray rock & Clay and gravel & hard & -- & -- & \\
\hline 271 & $5 / 7-26 \mathrm{R} 1$ & & & 450 & 470 & 137.2 & 143.3 & $\begin{array}{l}\text { gravel, fine } \\
\text { ging }\end{array}$ & Gravel & -- & - & - & \\
\hline 271 & $5 / 7-26 \mathrm{R} 1$ & & & 470 & 490 & 143.3 & 149.4 & shale and rock, gray & Clay and sand (or sandstone) & cemented & -. & -- & $\operatorname{Tp}(?)$ \\
\hline 271 & $5 / 7-26 \mathrm{R} 1$ & & & 490 & 530 & 149.4 & 161.5 & shale, muddy & Clay and sand (or sandstone) & cemented & .- & -- & \\
\hline 271 & $5 / 7-26 \mathrm{R} 1$ & & & 530 & 600 & 161.5 & 182.9 & clay, gray & Clay & -- & -- & -- & \\
\hline
\end{tabular}


Appendix 3. Lithologic data. - Continued

[Site IDs and well number correspond to locations in Appendix 1, a site ID and well number is listed for each drilled interval for a particular drill hole. For convenience, the site ID and well numbers are repeated at the shallowest drilled

\begin{tabular}{|c|c|c|c|c|c|c|c|c|c|c|c|c|c|}
\hline Site ID & Well number & $\begin{array}{l}\text { Site ID, } \\
\text { top of } \\
\text { well }\end{array}$ & $\begin{array}{l}\text { Well number, } \\
\text { top of well }\end{array}$ & $\begin{array}{c}\text { Top of } \\
\text { interval, in } \\
\text { feet }\end{array}$ & $\begin{array}{c}\text { Base of } \\
\text { interval, in } \\
\text { feet }\end{array}$ & $\begin{array}{c}\text { Top of } \\
\text { interval, in } \\
m\end{array}$ & $\begin{array}{c}\text { Base of } \\
\text { interval, in } \\
\mathrm{m}\end{array}$ & Reported lithologic unit ${ }^{1}$ & Interpreted lithologic class ${ }^{2}$ & $\begin{array}{c}\text { Reported } \\
\text { degree of } \\
\text { cementation or } \\
\text { induration }^{3}\end{array}$ & $\begin{array}{c}\text { Fossils } \\
\text { reported in } \\
\text { interval }^{4}\end{array}$ & $\begin{array}{c}\text { Organic } \\
\text { matter } \\
\text { reported in } \\
\text { interval }^{5}\end{array}$ & $\begin{array}{l}\text { Reported stratigraphic top of geologic } \\
\text { unit }^{6}\end{array}$ \\
\hline 271 & $5 / 7-26 \mathrm{R} 1$ & & & 600 & 680 & 182.9 & 207.3 & clay mud, gray & Clay and sand (or sandstone) & -- & -- & -- & \\
\hline 271 & 5/7-26R1 & & & 680 & 800 & 207.3 & 243.8 & shale, gray & Clay and sand (or sandstone) & cemented & -- & -- & \\
\hline 271 & $5 / 7-26 \mathrm{R} 1$ & & & 800 & 835 & 243.8 & 254.5 & Franciscan & Undifferentiated basement & -- & -- & -- & $\mathrm{KJu}$ \\
\hline 272 & $5 / 7-35 \mathrm{H} 1$ & 272 & $5 / 7-35 \mathrm{H} 1$ & 0 & 5 & 0.0 & 1.5 & $\begin{array}{l}\text { adobe } \\
\text { sand, coarse, and small gravel; }\end{array}$ & Sand and clay & -- & -- & -- & Qyal and Qoal (?) \\
\hline 272 & $5 / 7-35 \mathrm{H} 1$ & & & 5 & 15 & 1.5 & 4.6 & bottomed in yellow clay & Sand (or sandstone) and gravel & -- & -. & -- & \\
\hline 272 & $5 / 7-35 \mathrm{H} 1$ & & & 15 & 30 & 4.6 & 9.1 & clay, yellow & Clay & -- & -- & -- & \\
\hline 272 & $5 / 7-35 \mathrm{H} 1$ & & & 30 & 35 & 9.1 & 10.7 & $\begin{array}{l}\text { gravel, small and coarse sand } \\
\text { sand, coarse; bottomed in yellow }\end{array}$ & Sand (or sandstone) and gravel & -- & -- & -- & \\
\hline 272 & $5 / 7-35 \mathrm{H} 1$ & & & 35 & 40 & 10.7 & 12.2 & 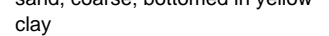 & Sand and clay & -- & -- & -- & \\
\hline 272 & 5/7-35H1 & & & 40 & 50 & 12.2 & 15.2 & clay, yellow & Clay & -- & -- & -- & \\
\hline 272 & $5 / 7-35 \mathrm{H} 1$ & & & 50 & 70 & 15.2 & 21.3 & $\begin{array}{l}\text { gravel streaks in yellow clay } \\
\text { sand, coarse streaks in yellow }\end{array}$ & Clay and gravel & -- & -- & -- & \\
\hline 272 & $5 / 7-35 \mathrm{H} 1$ & & & 70 & 80 & 21.3 & 24.4 & clay & Sand and clay & -- & -- & -- & \\
\hline 272 & $5 / 7-35 \mathrm{H} 1$ & & & 80 & 90 & 24.4 & 27.4 & clay, yellow, changing to blue & Clay & -- & -- & -- & \\
\hline 272 & 5/7-35H1 & & & 90 & 110 & 27.4 & 33.5 & clay, blue & Clay & -- & -- & -- & \\
\hline 272 & $5 / 7-35 \mathrm{H} 1$ & & & 110 & 120 & 33.5 & 36.6 & clay, blue changing to yellow clay & Clay & -- & .. & -. & \\
\hline 272 & $5 / 7-35 \mathrm{H} 1$ & & & 120 & 160 & 36.6 & 48.8 & $\begin{array}{l}\text { clay, yellow } \\
\text {. }\end{array}$ & Clay & -- & -- & -- & \\
\hline 272 & $5 / 7-35 \mathrm{H} 1$ & & & 160 & 170 & 48.8 & 51.8 & clay, blue & Clay & -- & -- & -- & \\
\hline 272 & $5 / 7-35 \mathrm{H} 1$ & & & 170 & 180 & 51.8 & 54.9 & $\begin{array}{l}\text { clay, blue, brown spots } \\
\text { sand, coarse, and fine; some }\end{array}$ & Clay & -- & -- & -- & \\
\hline 272 & $5 / 7-35 \mathrm{H} 1$ & & & 180 & 190 & 54.9 & 57.9 & shell fossils & Sand & -- & shells & -- & QTm (?) \\
\hline 272 & $5 / 7-35 \mathrm{H} 1$ & & & 190 & 200 & 57.9 & 61.0 & $\begin{array}{l}\text { gravel, small; shell fossils } \\
\text { gravel, small, and fine sand; firm. }\end{array}$ & Gravel & -- & shells & -- & \\
\hline 272 & $5 / 7-35 \mathrm{H} 1$ & & & 200 & 210 & 61.0 & 64.0 & but friable & Sand (or sandstone) and gravel & -- & -- & -.- & \\
\hline 272 & $5 / 7-35 \mathrm{H} 1$ & & & 210 & 290 & 64.0 & 88.4 & clay, blue tan spots & Clay & -- & .- & .. & \\
\hline 272 & $5 / 7-35 \mathrm{H} 1$ & & & 290 & 440 & 88.4 & 134.1 & clay, blue & Clay & -- & -- & -- & \\
\hline 272 & $5 / 7-35 \mathrm{H} 1$ & & & 440 & 460 & 134.1 & 140.2 & clay, blue some very small gravel & Clay and gravel & .- & .- & .. & \\
\hline 272 & $5 / 7-35 \mathrm{H} 1$ & & & 460 & 480 & 140.2 & 146.3 & $\begin{array}{l}\text { clay, blue some grit } \\
\text {. }\end{array}$ & Clay and sand (or sandstone) & -- & -- & -- & \\
\hline 272 & $5 / 7-35 \mathrm{H} 1$ & & & 480 & 487 & 146.3 & 148.4 & clay, blue & Clay & -- & -- & -- & \\
\hline 272 & $5 / 7-35 \mathrm{H} 1$ & & & 487 & 512 & 148.4 & 156.1 & gravel, small & Gravel & -- & -- & -- & \\
\hline 272 & $5 / 7-35 \mathrm{H1}$ & & & 512 & 529 & $\begin{array}{l}156.1 \\
156.1\end{array}$ & 161.2 & shell, very hard & Sand & hard & shells & - & $\mathrm{Tp}$ \\
\hline 272 & $5 / 7-35 \mathrm{H} 1$ & & & 529 & 540 & 161.2 & 164.6 & shale, blue & Clay and sand (or sandstone) & cemented & -- & .- & \\
\hline 272 & $5 / 7-35 \mathrm{H} 1$ & & & 540 & 542 & 164.6 & 165.2 & shale, blue gravel, small & Clay, sand, and trace gravel & cemented & .. & -- & \\
\hline 273 & 5/7-33M1 & 273 & 5/7-33м1 & 0 & 21 & 0.0 & 6.4 & no record & No data & -- & -- & -- & QTm \\
\hline 273 & 5/7-33M1 & & & 21 & 21 & 6.4 & 6.4 & gravel, water & Gravel & -- & -- & -- & \\
\hline 273 & $5 / 7-33 \mathrm{M} 1$ & & & 21 & 50 & $\begin{array}{l}0.4 \\
6.4\end{array}$ & $\begin{array}{l}15.2 \\
15.2\end{array}$ & $\begin{array}{l}\text { clay, blue, and gravel } \\
\text { clate }\end{array}$ & Clay and gravel & -- & -- & -. & \\
\hline 273 & 5/7-33M1 & & & 50 & 90 & 15.2 & 27.4 & $\begin{array}{l}\text { clay, blue } \\
\text {. }\end{array}$ & Clay & -- & -- & -- & \\
\hline 273 & 5/7-33M1 & & & 90 & 132 & 27.4 & 40.2 & rock & Gravel & hard & -. & .. & Tsv \\
\hline 274 & 5/7-33Q1 & 274 & 5/7-33Q1 & 0 & 30 & 0.0 & 9.1 & sand and clay, yellow & Clay & -- & -- & -- & Qyal \\
\hline 274 & $5 / 7-3301$ & & & 30 & 35 & 9.1 & 10.7 & sand and gravel (water) & Sand (or sandstone) and gravel & .- & .- & .. & \\
\hline 274 & 5/7-33Q1 & & & 35 & 65 & $\begin{array}{l}.1 \\
10.7\end{array}$ & 19.8 & $\begin{array}{l}\text { clay, blue } \\
\text { laver (fvaler) }\end{array}$ & 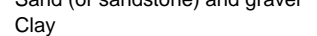 & -- & -- & -. & \\
\hline 274 & 5/7-33Q1 & & & 65 & 125 & 19.8 & 38.1 & sandstone, blue; water & Sandstone & cemented & -- & -- & QTm \\
\hline 274 & 5/7-33Q1 & & & 125 & 135 & 38.1 & 41.1 & $\begin{array}{l}\text { rock, sand } \\
\text { roue, waller }\end{array}$ & Sandstone & cemented & -- & .. & \\
\hline 274 & 5/7-33Q1 & & & 135 & 145 & 41.1 & 44.2 & rock, blue & Sandstone & hard & -- & -- & $\mathrm{KJu}$ \\
\hline 274 & $5 / 7-3301$ & & & 145 & 150 & 44.2 & 45.7 & rock, serpentine & Undifferentiated basement & hard & .- & .- & \\
\hline 274 & 5/7-33Q1 & & & $\begin{array}{l}145 \\
150\end{array}$ & 155 & 45.7 & $\begin{array}{l}47.1 \\
47.2\end{array}$ & rock, blue, and clay & Undifferentiated basement & hard & -- & -- & \\
\hline 274 & 5/7-33Q1 & & & 155 & 160 & 47.2 & 48.8 & gravel, cement; (water) & Undifferentiated basement & cemented & -- & -- & \\
\hline 274 & 5/7-33Q1 & & & 160 & 170 & 48.8 & 51.8 & rock, sand, and clay & Undifferentiated basement & hard & -. & -. & \\
\hline 274 & 5/7-33Q1 & & & 170 & 175 & 51.8 & 53.3 & clay, blue, and rock & Undifferentiated basement & -- & -- & -- & \\
\hline 274 & $5 / 7-33 Q 1$ & & & 175 & 185 & 53.3 & 56.4 & rock, blue, cracked & Undifferentiated basement & hard & -- & -- & \\
\hline 274 & 5/7-33Q1 & & & 185 & 205 & 56.4 & 62.5 & rock and clay & Undifferentiated basement & -- & .. & .. & \\
\hline 275 & 5/7-32R1 & 275 & 5/7-32R1 & 0 & 2 & 0.0 & 0.6 & adobe & Sand and clay & -- & -. & -- & Tsv \\
\hline 275 & 5/7-32R1 & & & 2 & 80 & 0.6 & 24.4 & rock, decomposed, yellow & Sandstone & hard & -- & .- & \\
\hline 275 & 5/7-32R1 & & & 80 & 107 & 24.4 & 32.6 & rock, decomposed, blue & Sandstone & hard & -- & -- & \\
\hline 275 & 5/7-32R1 & & & 107 & 111 & 32.6 & 33.8 & rock, decomposed, yellow & Sandstone & hard & - & - & \\
\hline 275 & 5/7-32R1 & & & 111 & 130 & 33.8 & 39.6 & rock, green & Undifferentiated basement & hard & -- & -- & $\mathrm{KJu}$ \\
\hline 275 & 5/7-32R1 & & & 130 & 200 & 39.6 & 61.0 & rock, blue & Undifferentiated basement & hard & -. & -- & \\
\hline 275 & $5 / 7-32 \mathrm{R} 1$ & & & 200 & 217 & 61.0 & 66.1 & rock, blue, with soft streaks & Undifferentiated basement & hard & -- & -- & \\
\hline
\end{tabular}


Appendix 3. Lithologic data. - Continued

[Site IDs and well number correspond to locations in Appendix 1, a site ID and well number is listed for each drilled interval for a particular drill hole. For convenience, the site ID and well numbers are repeated at the shallowest drilled interval for each well. leaders (--), not reported]

\begin{tabular}{|c|c|c|c|c|c|c|c|c|c|c|c|c|c|}
\hline Site ID & Well number & $\begin{array}{l}\text { Site ID, } \\
\text { top of } \\
\text { well }\end{array}$ & $\begin{array}{l}\text { Well number, } \\
\text { top of well }\end{array}$ & $\begin{array}{l}\text { Top of } \\
\text { interval, in } \\
\text { feet }\end{array}$ & $\begin{array}{l}\text { Base of } \\
\text { interval, in } \\
\text { feet }\end{array}$ & $\begin{array}{c}\text { Top of } \\
\text { interval, in } \\
m\end{array}$ & $\begin{array}{c}\text { Base of } \\
\text { interval, in } \\
\mathrm{m}\end{array}$ & Reported lithologic unit ${ }^{1}$ & Interpreted lithologic class ${ }^{2}$ & $\begin{array}{c}\text { Reported } \\
\text { degree of } \\
\text { cementation or } \\
\text { induration }^{3} \\
\end{array}$ & $\begin{array}{l}\text { Fossils } \\
\text { reported in }^{\text {interval }}{ }^{4}\end{array}$ & $\begin{array}{c}\text { Organic } \\
\text { matter } \\
\text { reported in } \\
\text { interval }^{5}\end{array}$ & $\begin{array}{l}\text { Reported stratigraphic top of geologic } \\
\text { unit }^{6}\end{array}$ \\
\hline 276 & 4/6-27L1 & 276 & 4/6-27L1 & 0 & 15 & 0.0 & 4.6 & no data & No data & -- & -- & -- & Qyal and Qoal (?) and QTge \\
\hline 276 & 4/6-27L1 & & & 15 & 98 & 4.6 & 29.9 & soil, clay, and gravel & Clay, sand, and gravel & -- & -- & -- & \\
\hline 276 & 4/6-27L1 & & & 98 & 102 & 29.9 & 31.1 & gravel & Gravel & -- & -- & -- & \\
\hline 276 & 4/6-27L1 & & & 102 & 158 & 31.1 & 48.2 & $\begin{array}{l}\text { clay, yellow; and gravel } \\
\text { clat }\end{array}$ & Clay and gravel & -- & -- & -- & \\
\hline 276 & 4/6-27L1 & & & 158 & 175 & 48.2 & 53.3 & sand, blue & Sand & -- & -- & -- & \\
\hline 276 & 4/6-27L1 & & & 175 & 235 & 53.3 & 71.6 & clay, blue & Clay & -- & -- & -- & \\
\hline 276 & 4/6-27L1 & & & 235 & 245 & 71.6 & 74.7 & clay, yellow & Clay & -- & -- & -- & \\
\hline 276 & 4/6-27L1 & & & 245 & 253 & 74.7 & 77.1 & $\begin{array}{l}\text { clay, blue } \\
\text { lat }\end{array}$ & Clay & -- & -- & -- & \\
\hline 276 & 4/6-27L1 & & & 253 & 295 & 77.1 & 89.9 & clay, yellow & Clay & -- & -- & -- & \\
\hline 276 & 4/6-27L1 & & & 295 & 320 & 89.9 & 97.5 & sand, yellow; (dry) & Sand & -- & -- & -- & \\
\hline 276 & 4/6-27L1 & & & 320 & 357 & 97.5 & 108.8 & clay, yellow & Clay & -- & -- & -- & \\
\hline 276 & 4/6-27L1 & & & 357 & 359 & 108.8 & 109.4 & gravel, pea & Gravel & -- & -- & -- & \\
\hline 276 & 4/6-27L1 & & & 359 & 450 & 109.4 & 137.2 & clay, hard, yellow & Clay & hard & -- & -- & \\
\hline 276 & 4/6-27L1 & & & 450 & 460 & 137.2 & 140.2 & conglomerate & Clay, sand, and gravel & -- & -- & -- & \\
\hline 276 & 4/6-27L1 & & & 460 & 485 & 140.2 & 147.8 & $\begin{array}{l}\text { clay, yellow } \\
\text { a }\end{array}$ & Clay & -- & -- & -- & \\
\hline 276 & 4/6-27L1 & & & 485 & 500 & 147.8 & 152.4 & gravel & Gravel & -. & .- & -- & \\
\hline 277 & 6/6-10F1 & 277 & 6/6-10F1 & 0 & 102 & 0.0 & 31.1 & hardpan and boulders & Clay and gravel & hard & -- & -- & QTge \\
\hline 277 & 6/6-10F1 & & & 102 & 350 & 31.1 & 106.7 & $\begin{array}{l}\text { rock, decomposed; not hard } \\
\text { dirt, gravel and clay, yellow- }\end{array}$ & Ash or tuff & hard & -- & -- & Tsv \\
\hline 278 & $6 / 6-16 \mathrm{~J} 2$ & 278 & 6/6-16J2 & 0 & 2 & 0.0 & 0.6 & mixture & Clay, sand, and gravel & -- & -- & -- & QTge \\
\hline 278 & $6 / 6-16 \mathrm{~J} 2$ & & & 2 & 39 & 0.6 & 11.9 & hardpan, boulders & Clay and gravel & hard & -- & -- & \\
\hline 278 & 6/6-16J2 & & & 39 & 51 & 11.9 & 15.5 & boulders and clay & Clay and gravel & - & -- & -- & \\
\hline 278 & 6/6-16J2 & & & 51 & 56 & 15.5 & 17.1 & boulders & Gravel & .- & .- & -- & \\
\hline 278 & $6 / 6-16 \mathrm{~J} 2$ & & & 56 & 93 & 17.1 & 28.3 & clay, yellow & Clay & -- & -- & .- & \\
\hline 278 & $6 / 6-16 \mathrm{~J} 2$ & & & 93 & 137 & 28.3 & 41.8 & $\begin{array}{l}\text { clay, blue } \\
\text { lat }\end{array}$ & Clay & -- & -- & -- & \\
\hline 278 & 6/6-16J2 & & & 137 & 160 & 41.8 & 48.8 & clay, yellow & Clay & -- & -- & -- & \\
\hline 278 & $6 / 6-16 \mathrm{~J} 2$ & & & 160 & 200 & 48.8 & 61.0 & shale, blue and gravel & Clay and sand (or sandstone) & cemented & -- & -- & \\
\hline 278 & 6/6-16J2 & & & 200 & 280 & $\begin{array}{l}61.0 \\
61.0\end{array}$ & 85.3 & $\begin{array}{l}\text { no record } \\
\text { nallation }\end{array}$ & $\begin{array}{l}\text { No data } \\
\text { datic }\end{array}$ & - & -- & -- & \\
\hline 279 & 6/6-22R3 & 279 & 6/6-22R3 & 0 & 90 & 0.0 & 27.4 & clay, yellow & Clay & -- & -- & .- & QTge \\
\hline 279 & 6/6-22R3 & & & 90 & 167 & 27.4 & 50.9 & $\begin{array}{l}\text { clay, blue } \\
\text { lat }\end{array}$ & Clay & -- & -- & -- & \\
\hline 279 & 6/6-22R3 & & & 167 & 215 & 50.9 & 65.5 & quicksand & Sand and clay & -- & -- & -- & \\
\hline 279 & 6/6-22R3 & & & 215 & 276 & 65.5 & 84.1 & $\begin{array}{l}\text { clay, yellow } \\
\text {. }\end{array}$ & Clay & -- & -- & -- & \\
\hline 279 & 6/6-22R3 & & & 276 & 316 & 84.1 & 96.3 & $\begin{array}{l}\text { clay, blue } \\
\text { al }\end{array}$ & Clay & .- & .- & .- & \\
\hline 279 & 6/6-22R3 & & & 316 & 418 & 96.3 & 127.4 & solid rock & Ash or tuff & -- & -- & -- & Tsv \\
\hline 280 & 6/6-26E1 & 280 & 6/6-26E1 & 0 & 4 & 0.0 & 1.2 & soil & Sand and clay & -- & -- & -- & Qoal and QTge \\
\hline 280 & 6/6-26E1 & & & 4 & 94 & 1.2 & 28.7 & "hardpan" & Clay and gravel & hard & -- & -- & \\
\hline 280 & 6/6-26E1 & & & 94 & 108 & 28.7 & 32.9 & gravel cement & Gravel & cemented & .- & .- & \\
\hline 280 & 6/6-26E1 & & & 108 & 181 & 32.9 & 55.2 & clay, yellow sandy & Clay and sand (or sandstone) & - & -- & -- & \\
\hline 280 & 6/6-26E1 & & & 181 & 204 & 55.2 & 62.2 & gravel and clay & Clay and gravel & -- & -- & -- & \\
\hline 280 & 6/6-26E1 & & & 204 & 241 & 62.2 & 73.5 & "shale" & Clay and sand (or sandstone) & cemented & .- & .- & Tsv \\
\hline 280 & 6/6-26E1 & & & 241 & 304 & 73.5 & 92.7 & rock drilling & Ash or tuff & -- & -- & -- & \\
\hline 281 & 6/6-34C1 & 281 & 6/6-34C1 & 0 & 23 & 0.0 & 7.0 & clay, yellow & Clay & .- & -- & -- & QTge \\
\hline 281 & 6/6-34C1 & & & 23 & 25 & 7.0 & 7.6 & gravel and sand & Sand (or sandstone) and gravel & .- & -- & .- & \\
\hline 281 & 6/6-34C1 & & & 25 & 229 & 7.6 & 69.8 & clay, yellow and gray & Clay & -- & -- & -- & \\
\hline 281 & 6/6-34C1 & & & 229 & 343 & 69.8 & 104.5 & $\begin{array}{l}\text { clay, yellow } \\
\text { clav yellown blue sliaht }\end{array}$ & Clay & -- & -- & -- & \\
\hline 281 & 6/6-34C1 & & & 343 & 500 & 104.5 & 152.4 & $\begin{array}{l}\text { clay, yellow and blue, slight } \\
\text { mixture sand }\end{array}$ & Clay and sand (or sandstone) & -- & - & .- & \\
\hline $\begin{array}{l}201 \\
282\end{array}$ & $5 / 5-8 \mathrm{~B} 1$ & 282 & $5 / 5-8 \mathrm{~B} 1$ & 0 & 43 & 0.0 & $\begin{array}{l}132.4 \\
13.1\end{array}$ & dirt and gravel & $\begin{array}{l}\text { Clay, sand, and gravel } \\
\text { late) }\end{array}$ & -- & -- & -- & Qoal \\
\hline 282 & $5 / 5-8 \mathrm{~B} 1$ & & & 43 & 63 & 13.1 & 19.2 & hardpan & Clay and gravel & hard & -- & -- & \\
\hline 282 & $5 / 5-8 \mathrm{~B} 1$ & & & 63 & 80 & 19.2 & 24.4 & gravel cement & Gravel & cemented & -- & -- & \\
\hline 283 & 5/5-8A1 & 283 & 5/5-8A1 & 0 & 2 & 0.0 & 0.6 & soil & Sand and clay & - & -- & -- & Qoal \\
\hline 283 & 5/5-8A1 & & & 2 & 19 & 0.6 & 5.8 & boulder and clay & Clay and gravel & -- & -- & -- & \\
\hline 283 & 5/5-8A1 & & & 19 & 54 & 5.8 & 16.5 & clay, yellow & Clay & -- & -- & -- & Qh \\
\hline 283 & $5 / 5-8 \mathrm{~A} 1$ & & & 54 & 94 & 16.5 & 28.7 & shale, blue & Clay and sand (or sandstone) & cemented & -- & -- & \\
\hline 283 & $5 / 5-8 A 1$ & & & 94 & 124 & 28.7 & 37.8 & rock, yellow, soft & Ash or tuff & hard & -- & -- & Tsv \\
\hline 283 & 5/5-8A1 & & & 124 & 136 & 37.8 & 41.5 & rock, red some water & Ash or tuff & hard & .- & -- & \\
\hline 283 & 5/5-8A1 & & & 136 & 157 & 41.5 & 47.9 & rock, black and red & Ash or tuff & hard & .- & -- & \\
\hline 283 & $5 / 5-8 \mathrm{~A} 1$ & & & 157 & 175 & 47.9 & 53.3 & rock, red & Ash or tuff & hard & -- & -- & \\
\hline 284 & 5/5-7A1 & 284 & 5/5-7A1 & 0 & 10 & 0.0 & 3.0 & "surface" boulders & Sand (or sandstone) and gravel & -- & .- & .- & Tsv \\
\hline 284 & 5/5-7A1 & & & 10 & 85 & 3.0 & 25.9 & clay, red and boulders & Clay and gravel & -- & -- & -- & \\
\hline 284 & 5/5-7A1 & & & 85 & 150 & 25.9 & 45.7 & volcanic ash, red & Ash or tuff & -- & -- & -- & \\
\hline
\end{tabular}


Appendix 3. Lithologic data. - Continued

ISite IDs and well number correspond to locations in Appendix 1, a site ID and well number is listed for each drilled interval for a particular drill hole. For convenience, the site ID and well numbers are repeated at the shallowest drilled interval for each well. leaders (--), not reported]

\begin{tabular}{|c|c|c|c|c|c|c|c|c|c|c|c|c|c|}
\hline Site ID & Well number & $\begin{array}{l}\text { Site ID, } \\
\text { top of } \\
\text { well }\end{array}$ & $\begin{array}{l}\text { Well number, } \\
\text { top of well }\end{array}$ & $\begin{array}{c}\text { Top of } \\
\text { interval, in } \\
\text { feet }\end{array}$ & $\begin{array}{c}\text { Base of } \\
\text { interval, in } \\
\text { feet }\end{array}$ & $\begin{array}{c}\text { Top of } \\
\text { interval, in } \\
m\end{array}$ & $\begin{array}{c}\text { Base of } \\
\text { interval, in } \\
\mathrm{m}\end{array}$ & Reported lithologic unit ${ }^{1}$ & Interpreted lithologic class ${ }^{2}$ & $\begin{array}{c}\text { Reported } \\
\text { degree of } \\
\text { cementation or } \\
\text { induration } \\
\end{array}$ & $\begin{array}{c}\text { Fossils } \\
\text { reported in } \\
\text { interval| }^{4}\end{array}$ & $\begin{array}{c}\text { Organic } \\
\text { matter } \\
\text { reported in }^{\text {in }} \text { interval }^{5}\end{array}$ & $\begin{array}{l}\text { Reported stratigraphic top of geologic } \\
\text { unit }^{6}\end{array}$ \\
\hline 284 & $5 / 5-7 \mathrm{~A} 1$ & & & 150 & 160 & 45.7 & 48.8 & lava boulders & Basalt & -- & -- & -- & \\
\hline 284 & 5/5-7A1 & & & 160 & 195 & 48.8 & 59.4 & volcanic ash, red & Ash or tuff & -- & -- & -- & \\
\hline 284 & 5/5-7A1 & & & 195 & 230 & 59.4 & 70.1 & clay, brown and cemented gravel & Clay and gravel & cemented & $\ldots$ & .. & \\
\hline 284 & 5/5-7A1 & & & 230 & 325 & 70.1 & 99.1 & tuff, white & Ash or tuff & -- & -- & -- & \\
\hline 284 & 5/5-7A1 & & & 325 & 340 & 99.1 & 103.6 & tuff white & Ash or tuff & .- & -- & -- & \\
\hline 284 & 5/5-7A1 & & & 340 & 475 & 103.6 & 144.8 & $\begin{array}{l}\text { lava rock } \\
\text { cemented sand with streaks of }\end{array}$ & Basalt & hard & -- & -- & \\
\hline 284 & 5/5-7A1 & & & 475 & 560 & 144.8 & 170.7 & clay and sandstone & Sandstone & cemented & -- & -- & \\
\hline 284 & 5/5-7A1 & & & 560 & 575 & 170.7 & 175.3 & clay, yellow, and sand & Clay and gravel & -- & -. & -- & \\
\hline 285 & 5/5-8E1 & 285 & 5/5-8E1 & 0 & 116 & 0.0 & 35.4 & "hardpan" & Clay and gravel & hard & -- & -- & Qoal and Qh \\
\hline 285 & $5 / 5-8 \mathrm{E} 1$ & & & 116 & 155 & 35.4 & 47.2 & rock, basalt & Basalt & -- & -- & -- & Tsv \\
\hline 285 & 5/5-8E1 & & & 155 & 170 & 47.2 & 51.8 & rock, red volcanic, water bearing' & Ash or tuff & hard & -- & -- & \\
\hline 285 & 5/5-8E1 & & & 170 & 212 & 51.8 & 64.6 & rock, soft, porous & Ash or tuff & hard & -- & -- & \\
\hline 285 & 5/5-8E1 & & & 212 & 235 & 64.6 & 71.6 & volcanic ash, red & Ash or tuff & -- & -- & -- & \\
\hline 286 & 5/5-9M1 & 286 & 5/5-9M1 & 0 & 2 & 0.0 & 0.6 & soil & Sand and clay & -- & -- & -- & Qoal \\
\hline 286 & 5/5-9M1 & & & 2 & 37 & 0.6 & 11.3 & "hardpan" & Clay and gravel & hard & -. & -- & \\
\hline 286 & 5/5-9M1 & & & 37 & 107 & 11.3 & 32.6 & clay, yellow & Clay & -- & -- & -- & Qh \\
\hline 286 & 5/5-9M1 & & & 107 & 149 & 32.6 & 45.4 & $\begin{array}{l}\text { clay, blue } \\
\text { cla }\end{array}$ & Clay & -- & -- & -- & \\
\hline 286 & 5/5-9M1 & & & 149 & 184 & 45.4 & 56.1 & $\begin{array}{l}\text { clay, yellow } \\
\text { clats }\end{array}$ & Clay & - & - & - & \\
\hline 286 & 5/5-9M1 & & & 184 & 215 & 56.1 & 65.5 & rock drilling & Ash or tuff & -- & -- & -- & Tsv \\
\hline 287 & 5/6-10K1 & 287 & 5/6-10K1 & 0 & 70 & 0.0 & 21.3 & "hardpan" and boulders & Clay and gravel & hard & -. & -- & Qoal \\
\hline 287 & $5 / 6-10 \mathrm{~K} 1$ & & & 70 & 155 & 21.3 & 47.2 & clay, yellow and boulders & Clay and gravel & -. & -. & .- & QTge \\
\hline 287 & $5 / 6-10 \mathrm{~K} 1$ & & & 155 & 237 & 47.2 & 72.2 & 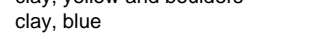 & Clay & -. & -- & -- & \\
\hline 287 & $5 / 6-10 K 1$ & & & 237 & 239 & 72.2 & 72.8 & sand, blue & Sand & -- & -- & .- & \\
\hline 288 & 5/5-9N2 & 288 & 5/5-9N2 & 0 & 2 & 0.0 & 0.6 & soil & Sand and clay & -- & -- & -- & Qoal and Qh \\
\hline 288 & 5/5-9N2 & & & 2 & 77 & 0.6 & 23.5 & clay, yellow & Clay & - & - & - & \\
\hline 288 & $5 / 5-9 \mathrm{~N} 2$ & & & 77 & 285 & 23.5 & 86.9 & clay, blue, and sand & Clay and sand (or sandstone) & -- & -- & -.- & \\
\hline 289 & 5/5-8P1 & 289 & 5/5-8P1 & 0 & 8 & 0.0 & 2.4 & soil & Sand and clay & -- & -. & -.- & Qoal \\
\hline 289 & $5 / 5-8 \mathrm{P} 1$ & & & 8 & 61 & 2.4 & 18.6 & clay, yellow, and gravel & Clay and gravel & -- & -- & -- & \\
\hline 289 & 5/5-8P1 & & & 61 & 234 & 18.6 & 71.3 & clay, yellow & Clay & -- & -- & -- & Qh \\
\hline 289 & $5 / 5-8 \mathrm{P} 1$ & & & 234 & 236 & 71.3 & 71.9 & sand brown, little water & Sand & - & -- & - & Qiा \\
\hline 289 & 5/5-8P1 & & & 236 & 333 & 71.9 & 101.5 & clay, blue & Clay & -- & -- & -- & \\
\hline 289 & 5/5-8P1 & & & 333 & 403 & 101.5 & 122.8 & shale, blue and sand & Clay and sand (or sandstone) & cemented & -- & -.- & \\
\hline 289 & 5/5-8P1 & & & 403 & 421 & 122.8 & 128.3 & rock formation, water bearing & Ash or tuff & -- & -- & -- & Tsv \\
\hline 290 & 5/5-17B1 & 290 & 5/5-17B1 & 0 & 2 & 0.0 & 0.6 & soil & Sand and clay & -- & -- & -. & Qoal \\
\hline 290 & 5/5-17B1 & & & 2 & 30 & 0.6 & 9.1 & hardpan and clay & Clay and gravel & hard & -- & -- & \\
\hline 290 & 5/5-17B1 & & & 30 & 180 & 9.1 & 54.9 & clay, yellow & Clay & -- & -- & -- & Qh \\
\hline 290 & 5/5-17B1 & & & 180 & 710 & 54.9 & 216.4 & $\begin{array}{l}\text { clay, blue } \\
\text { lat }\end{array}$ & Clay & -- & -- & -- & \\
\hline 290 & $5 / 5-17 \mathrm{~B} 1$ & & & 710 & 715 & 216.4 & 217.9 & clay, reddish and some scoria & Clay & -- & -- & -- & Tsv (?) \\
\hline 290 & 5/5-17B1 & & & 715 & 720 & 217.9 & 219.5 & $\begin{array}{l}\text { clay, blue } \\
\end{array}$ & Clay & -- & -- & -.- & \\
\hline 291 & 5/5-17C4 & 291 & 5/5-17C4 & 0 & 4 & 0.0 & 1.2 & soil & Sand and clay & -- & -- & -- & Qoal \\
\hline 291 & $5 / 5-17 \mathrm{C} 4$ & & & 4 & 22 & 1.2 & 6.7 & gravel and clay & Clay and gravel & - & -- & -- & \\
\hline 291 & $5 / 5-17 \mathrm{C} 4$ & & & 22 & 79 & 6.7 & 24.1 & clay, yellow & Clay & -- & -- & -. & Qh \\
\hline 291 & $5 / 5-17 \mathrm{C} 4$ & & & 79 & 149 & 24.1 & 45.4 & $\begin{array}{l}\text { clay, blue } \\
\text { s. }\end{array}$ & Clay & -- & -- & -- & \\
\hline 291 & $5 / 5-17 \mathrm{C} 4$ & & & 149 & 249 & 45.4 & 75.9 & clay, blue and yellow & Clay & -. & -- & .- & \\
\hline 291 & $5 / 5-17 \mathrm{C} 4$ & & & 249 & 265 & 75.9 & 80.8 & clay, blue & Clay & -- & -. & -. & \\
\hline 292 & $5 / 6-14 \mathrm{~B} 2$ & 292 & 5/6-14B2 & 0 & 1 & 0.0 & 0.3 & soil & Sand and clay & -. & -- & -. & Qyal \\
\hline 292 & $5 / 6-14 \mathrm{~B} 2$ & & & 1 & 5 & 0.3 & 1.5 & $\begin{array}{l}\text { topsoil and gravel } \\
\text { gravel and boulders, water }\end{array}$ & Sand and clay & -- & -- & -- & \\
\hline 292 & $5 / 6-14 \mathrm{~B} 2$ & & & 5 & 98 & 1.5 & 29.9 & bearing & Gravel & -- & -- & -. & \\
\hline 292 & $5 / 6-14 \mathrm{~B} 2$ & & & 98 & 147 & 29.9 & 44.8 & clay, yellow and boulders & Clay and gravel & -- & -- & -- & Qoal \\
\hline 292 & $5 / 6-14 \mathrm{~B} 2$ & & & 147 & 200 & 44.8 & 61.0 & clay, blue & Clay & -. & -.- & -. & QTge \\
\hline 293 & $5 / 5-16 \mathrm{E} 1$ & 293 & 5/5-16E1 & 0 & 3 & 0.0 & 0.9 & soil & Sand and clay & -- & -- & -- & Qoal and Qh \\
\hline 293 & 5/5-16E1 & & & 3 & 22 & 0.9 & 6.7 & rock & Gravel & hard & -- & -. & \\
\hline 293 & 5/5-16E1 & & & 22 & 100 & 6.7 & 30.5 & clay, yellow & Clay & -- & -. & -- & \\
\hline 293 & 5/5-16E1 & & & 100 & 101 & 30.5 & 30.8 & sand, little water & Sand & -- & -- & -- & \\
\hline 293 & 5/5-16E1 & & & 101 & 245 & 30.8 & 74.7 & clay, blue & Clay & -- & .. & -- & \\
\hline 293 & 5/5-16E1 & & & 245 & 322 & 74.7 & 98.1 & clay, yellow & Clay & -- & -- & -- & \\
\hline 293 & 5/5-16E1 & & & 322 & 336 & 98.1 & 102.4 & $\begin{array}{l}\text { clay, blue } \\
\text { al }\end{array}$ & Clay & -- & -- & -- & \\
\hline
\end{tabular}


Appendix 3. Lithologic data. - Continued

[Site IDs and well number correspond to locations in Appendix 1, a site ID and well number is listed for each drilled interval for a particular drill hole. For convenience, the site ID and well numbers are repeated at the shallowest drilled interval for each well. leaders

\begin{tabular}{|c|c|c|c|c|c|c|c|c|c|c|c|c|c|}
\hline Site ID & Well number & $\begin{array}{l}\text { Site ID, } \\
\text { top of } \\
\text { well }\end{array}$ & $\begin{array}{l}\text { Well number, } \\
\text { top of well }\end{array}$ & $\begin{array}{c}\text { Top of } \\
\text { interval, in } \\
\text { feet }\end{array}$ & $\begin{array}{c}\text { Base of } \\
\text { interval, in } \\
\text { feet }\end{array}$ & $\begin{array}{c}\text { Top of } \\
\text { interval, in } \\
m\end{array}$ & $\begin{array}{c}\text { Base of } \\
\text { interval, in } \\
\mathrm{m}\end{array}$ & Reported lithologic unit ${ }^{1}$ & Interpreted lithologic class ${ }^{2}$ & $\begin{array}{c}\text { Reported } \\
\text { degree of } \\
\text { cementation or } \\
\text { induration } \\
\end{array}$ & $\begin{array}{c}\text { Fossils } \\
\text { reported in } \\
\text { interval }^{4}\end{array}$ & $\begin{array}{c}\text { Organic } \\
\text { matter } \\
\text { reported in } \\
\text { interval }^{5}\end{array}$ & $\begin{array}{l}\text { Reported stratigraphic top of geologic } \\
\text { unit }^{6}\end{array}$ \\
\hline $\begin{array}{l}293 \\
293\end{array}$ & $\begin{array}{l}\text { 5/5-16E1 } \\
\text { 5/5-16E1 }\end{array}$ & & & $\begin{array}{l}336 \\
386\end{array}$ & $\begin{array}{l}386 \\
393\end{array}$ & $\begin{array}{l}102.4 \\
117.7\end{array}$ & $\begin{array}{l}117.7 \\
119.8\end{array}$ & $\begin{array}{l}\text { Shale, blue } \\
\text { shale and sand }\end{array}$ & Clay and sand (or sandstone) & $\begin{array}{c}\text { cemented } \\
\text { cemented }\end{array}$ & $\overline{-}$ & $\begin{array}{l}- \\
--\end{array}$ & \\
\hline $\begin{array}{l}293 \\
294\end{array}$ & $\begin{array}{l}\text { 5/5-16E1 } \\
\text { 5/6-13F1 }\end{array}$ & 294 & $5 / 6-13 F 1$ & $\begin{array}{l}386 \\
0\end{array}$ & $\begin{array}{l}393 \\
32\end{array}$ & $\begin{array}{l}11.1 \\
0.0\end{array}$ & $\begin{array}{l}111.8 \\
9.8\end{array}$ & $\begin{array}{l}\text { Snale and sand } \\
\text { clay }\end{array}$ & $\begin{array}{l}\text { Clay and sand (or sandistone) } \\
\text { Clay }\end{array}$ & cemented & -- & -- & Oyal and Qoal \\
\hline 294 & $5 / 6-13 F 1$ & & & 32 & 48 & 9.8 & 14.6 & gravel & Gravel & -- & -- & -- & \\
\hline 294 & $5 / 6-13 F 1$ & & & 48 & 68 & 14.6 & 20.7 & gravel and clay & Clay and gravel & -- & -- & -- & \\
\hline 294 & $5 / 6-13 \mathrm{~F} 1$ & & & 68 & 69 & 20.7 & 21.0 & gravel & Gravel & -- & -- & -- & \\
\hline 294 & $5 / 6-13 F 1$ & & & 69 & 80 & 21.0 & 24.4 & gravel and clay & Clay and gravel & -- & -- & -- & \\
\hline 294 & $5 / 6-13 F 1$ & & & 80 & 100 & 24.4 & 30.5 & gravel & Gravel & -- & -. & -- & \\
\hline 294 & $5 / 6-13 F 1$ & & & 100 & 108 & 30.5 & 32.9 & clay & Clay & -. & -- & -- & \\
\hline 294 & $5 / 6-13 F 1$ & & & 108 & 110 & 32.9 & 33.5 & clay & Clay & -. & -- & -- & \\
\hline 295 & $5 / 6-13 \mathrm{~F} 2$ & 295 & $5 / 6-13 F 2$ & 0 & 36 & 0.0 & 11.0 & clay & Clay & -- & -- & -- & Qyal and Qoal \\
\hline 295 & $5 / 6-13 F 2$ & & & 36 & 46 & 11.0 & 14.0 & clay, sand & Clay and sand (or sandstone) & .- & .- & .- & \\
\hline 295 & 5/6-13F2 & & & 46 & 62 & 14.0 & 18.9 & gravel & Gravel & -- & -- & -- & \\
\hline 295 & 5/6-13F2 & & & 62 & 65 & 18.9 & 19.8 & gravel and clay & Clay and gravel & .. & -- & -- & \\
\hline 295 & $5 / 6-13 F 2$ & & & 65 & 73 & $\begin{array}{l}19.9 \\
19.8-1\end{array}$ & $\begin{array}{l}19.0 \\
22.3\end{array}$ & $\begin{array}{l}\text { gravel } \\
\text { grich cia }\end{array}$ & Gravel & -. & -. & -. & \\
\hline 295 & 5/6-13F2 & & & 73 & 82 & 22.3 & 25.0 & gravel and clay & Clay and gravel & -- & - & -- & \\
\hline 295 & $5 / 6-13 F 2$ & & & 82 & 94 & 25.0 & 28.7 & $\begin{array}{l}\text { gravel } \\
\text { galial }\end{array}$ & Gravel & -. & -- & -- & \\
\hline 295 & $5 / 6-13 F 2$ & & & 94 & 98 & 28.7 & 29.9 & gravel and clay & Clay and gravel & .- & .- & -. & \\
\hline 295 & $5 / 6-13 F 2$ & & & 98 & 102 & 29.9 & 31.1 & gravel & Gravel & .. & -- & -. & \\
\hline 295 & $5 / 6-13 F 2$ & & & 102 & 105 & 31.1 & 32.0 & clay & Clay & -. & .- & .- & \\
\hline 296 & $5 / 5-17 \mathrm{J1}$ & 296 & 5/5-17J1 & 0 & 42 & 0.0 & 12.8 & clay, sandy & Clay and sand (or sandstone) & -- & .- & .- & Qoal \\
\hline 296 & $5 / 5-17 \mathrm{J1}$ & & & 42 & 60 & 12.8 & 18.3 & $\begin{array}{l}\text { clay, yellow } \\
\text { col y }\end{array}$ & Clay & -- & - & -- & Qh \\
\hline 296 & $5 / 5-17 \mathrm{~J} 1$ & & & 60 & 139 & 18.3 & 42.4 & clay, blue & Clay & .. & .- & .. & \\
\hline 296 & $5 / 5-17 J 1$ & & & 139 & 150 & 42.4 & 45.7 & $\begin{array}{l}\text { clay, yellow } \\
\text { cla }\end{array}$ & Clay & -- & -- & -- & \\
\hline 296 & 5/5-17J1 & & & 150 & 192 & 45.7 & 58.5 & clay, blue & Clay & -- & -- & -- & \\
\hline 296 & 5/5-17J1 & & & 192 & 229 & 58.5 & 69.8 & clay, yellow & Clay & -- & -- & -- & \\
\hline 296 & $5 / 5-17 \mathrm{J1}$ & & & 229 & 281 & 69.8 & 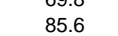 & $\begin{array}{l}\text { clay, blue } \\
\text { clas }\end{array}$ & Clay & -. & -- & -. & \\
\hline 296 & $5 / 5-17 J 1$ & & & 281 & 285 & 85.6 & 86.9 & $\begin{array}{l}\text { gravel } \\
\text { dirt, gravel and clay, yellow- }\end{array}$ & Gravel & -- & -- & - & \\
\hline 297 & 5/5-18K1 & 297 & 5/5-18K1 & 0 & 39 & 0.0 & 11.9 & mixture & Clay, sand, and gravel & -- & -- & -- & Qoal \\
\hline 297 & $5 / 5-18 \mathrm{~K} 1$ & & & 39 & 84 & 11.9 & 25.6 & clay, yellow and sand & Clay and sand (or sandstone) & -- & -- & -- & \\
\hline 297 & $5 / 5-18 \mathrm{~K} 1$ & & & 84 & 94 & 25.6 & 28.7 & clay, blue & Clay & -- & -- & -. & \\
\hline 297 & $5 / 5-18 \mathrm{~K} 1$ & & & 94 & 110 & 28.7 & 33.5 & clay, blue, sand and gravel & Clay, sand, and gravel & .. & -- & .. & \\
\hline 297 & 5/5-18K1 & & & 110 & 128 & 33.5 & 39.0 & sand, blue and gravel & Sand (or sandstone) and gravel & .. & .. & .. & \\
\hline 298 & 5/5-17N1 & 298 & 5/5-17N1 & 0 & 10 & 0.0 & 3.0 & surface & Sand and clay & -- & -- & -- & Qoal \\
\hline 298 & $5 / 5-17 \mathrm{~N} 1$ & & & 10 & 165 & 3.0 & 50.3 & clay, yellow, sand and gravel & Clay, sand, and gravel & .- & .- & .. & \\
\hline 298 & 5/5-17N1 & & & 165 & 290 & 50.3 & 88.4 & clay, yellow & Clay & -. & .- & .. & Qh \\
\hline 298 & 5/5-17N1 & & & 290 & 445 & 88.4 & 135.6 & $\begin{array}{l}\text { clay, blue } \\
\text { la }\end{array}$ & Clay & -. & -- & -- & \\
\hline 298 & 5/5-17N1 & & & 445 & 455 & 135.6 & 138.7 & volcanic rock & Ash or tuff & .- & -- & .. & \\
\hline 298 & 5/5-17N1 & & & 455 & 475 & 138.7 & 144.8 & sand fine & Sand & - & -- & -- & \\
\hline 299 & 5/5-17R2 & 299 & 5/5-17R2 & 0 & 1 & 0.0 & 0.3 & soil & Sand and clay & -- & -- & -- & Qoal and Qh \\
\hline 299 & 5/5-17R2 & & & 1 & 141 & 0.3 & 43.0 & clay, gray & Clay & -- & -- & -- & \\
\hline 299 & 5/5-17R2 & & & 70 & 104 & 21.3 & 31.7 & gravel & Gravel & .. & -. & .. & \\
\hline 299 & 5/5-17R2 & & & 84 & 94 & 25.6 & 28.7 & clay, blue & Clay & -- & -- & -- & \\
\hline 299 & 5/5-17R2 & & & 94 & 110 & 28.7 & 33.5 & clay, blue, sand and gravel & Clay, sand, and gravel & -- & -- & -- & \\
\hline 299 & 5/5-17R2 & & & 104 & 124 & 31.7 & 37.8 & 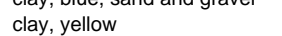 & Clay & - & - & -. & \\
\hline 299 & 5/5-17R2 & & & 110 & 128 & 33.5 & 39.0 & sand, blue and gravel & Sand (or sandstone) and gravel & -- & -- & -- & \\
\hline 299 & 5/5-17R2 & & & 124 & 150 & 37.8 & 45.7 & gravel & Gravel & -- & -- & -- & \\
\hline 299 & 5/5-17R2 & & & 128 & 70 & 39.0 & 21.3 & hardpan and clay & Clay and gravel & hard & -- & - & \\
\hline 299 & 5/5-17R2 & & & 141 & 151 & 43.0 & 46.0 & $\begin{array}{l}\text { clay, blue } \\
\text { latay }\end{array}$ & Clay & -- & -- & -. & \\
\hline 299 & $5 / 5-17 \mathrm{R} 2$ & & & 151 & 161 & 46.0 & 49.1 & $\begin{array}{l}\text { clay, brown } \\
\text { chent }\end{array}$ & Clay & -. & -- & -. & \\
\hline 299 & 5/5-17R2 & & & 161 & 191 & 49.1 & 58.2 & clay, gray & Clay & -- & -- & -- & \\
\hline 299 & 5/5-17R2 & & & 191 & 206 & 58.2 & 62.8 & clay, brown & Clay & .- & -- & .- & \\
\hline 299 & 5/5-17R2 & & & 206 & 230 & 62.8 & 70.1 & clay, blue & Clay & -- & -- & - & \\
\hline 299 & 5/5-17R2 & & & 230 & 246 & 70.1 & 75.0 & clay, brown & Clay & -. & -- & -. & \\
\hline 299 & 5/5-17R2 & & & 246 & 296 & 75.0 & 90.2 & clay, blue & Clay & -- & -- & -- & \\
\hline 299 & 5/5-17R2 & & & 296 & 300 & 90.2 & 91.4 & clay, brown & Clay & -. & -. & .- & \\
\hline 299 & 5/5-17R2 & & & 300 & 324 & 91.4 & 98.8 & clay, brown & Clay & -- & - & - & \\
\hline 299 & 5/5-17R2 & & & 324 & 341 & 98.8 & 103.9 & gravel, cemented & Gravel & cemented & -- & -- & \\
\hline 299 & 5/5-17R2 & & & 341 & 359 & 103.9 & 109.4 & $\begin{array}{l}\text { not described } \\
\text { not }\end{array}$ & No data & -- & -- & -- & \\
\hline 299 & 5/5-17R2 & & & 359 & 84 & 109.4 & 25.6 & clay, yellow and sand & Clay and sand (or sandstone) & -- & -- & -- & \\
\hline
\end{tabular}


Appendix 3. Lithologic data. - Continued [Site IDs and well number correspond to locations in Appendix 1, a site ID and well number is listed for each drilled interval for a particular drill hole. For convenience, the site ID and well numbers are repeated at the shallowest drilled
interval for each well. leaders (--) not reported]

\begin{tabular}{|c|c|c|c|c|c|c|c|c|c|c|c|c|c|}
\hline Site ID & Well number & $\begin{array}{l}\text { Site ID, } \\
\text { top of } \\
\text { well }\end{array}$ & $\begin{array}{c}\text { Well number, } \\
\text { top of well }\end{array}$ & $\begin{array}{c}\text { Top of } \\
\text { interval, in } \\
\text { feet }\end{array}$ & $\begin{array}{c}\text { Base of } \\
\text { interval, in } \\
\text { feet }\end{array}$ & $\begin{array}{c}\text { Top of } \\
\text { interval, in } \\
m\end{array}$ & $\begin{array}{c}\text { Base of } \\
\text { interval, in } \\
\mathrm{m}\end{array}$ & Reported lithologic unit ${ }^{1}$ & Interpreted lithologic class ${ }^{2}$ & $\begin{array}{c}\text { Reported } \\
\text { degree of } \\
\text { cementation or } \\
\text { induration }^{3}\end{array}$ & $\begin{array}{c}\text { Fossils } \\
\text { reported in } \\
\text { interval }^{4}\end{array}$ & $\begin{array}{c}\text { Organic } \\
\text { matter } \\
\text { reported in } \\
\text { interval }^{5}\end{array}$ & $\begin{array}{l}\text { Reported stratigraphic top of geologic } \\
\text { unit }^{6}\end{array}$ \\
\hline 300 & 5/5-21E1 & 300 & $5 / 5-21 \mathrm{E} 1$ & 0 & 215 & 0.0 & 65.5 & clay, blue & Clay & -- & -- & -- & $\mathrm{Qh}$ \\
\hline 300 & 5/5-21E1 & & & 215 & 221 & 65.5 & 67.4 & gravel free & Clay & -- & .- & -- & \\
\hline 300 & 5/5-21E1 & & & 221 & 250 & 67.4 & 76.2 & gravel, cemented and boulders & Gravel & cemented & -- & -- & \\
\hline 301 & $5 / 6-24 \mathrm{~K} 1$ & 301 & 5/6-24K1 & 0 & 3 & 0.0 & 0.9 & "Adobe" and sand & Sand and clay & -- & -- & -- & Qyal and Qoal \\
\hline 301 & $5 / 6-24 \mathrm{~K} 1$ & & & 3 & 22 & 0.9 & 6.7 & gravel. & Gravel & -- & -- & -- & \\
\hline 301 & 5/6-24K1 & & & 22 & 41 & 6.7 & 12.5 & gravel & Gravel & -- & -- & -- & \\
\hline 301 & 5/6-24K1 & & & 41 & 51 & 12.5 & 15.5 & gravel & Gravel & -- & -- & -- & \\
\hline 301 & $5 / 6-24 K 1$ & & & 51 & 92 & 15.5 & 28.0 & clay, yellow & Clay & -- & -- & -- & \\
\hline 301 & $5 / 6-24 \mathrm{~K} 1$ & & & 92 & 99 & 28.0 & 30.2 & gravel & Gravel & -- & -- & -- & \\
\hline 301 & $5 / 6-24 \mathrm{~K} 1$ & & & 99 & 133 & 30.2 & 40.5 & clay, yellow & Clay & -- & -. & -- & \\
\hline 301 & $5 / 6-24 K 1$ & & & 133 & 140 & 40.5 & 42.7 & gravel and clay & Clay and gravel & -- & -- & -- & \\
\hline 301 & $5 / 6-24 \mathrm{~K} 1$ & & & 140 & 151 & 42.7 & 46.0 & $\begin{array}{l}\text { clay, yellow } \\
\text { lat }\end{array}$ & 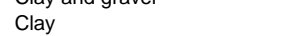 & -- & -- & -- & \\
\hline 302 & $5 / 5-22 L 1$ & 302 & 5/5-22L1 & 0 & 5 & 0.0 & 1.5 & soil & Sand and clay & -- & -- & -- & Qh \\
\hline 302 & $5 / 5-22 \mathrm{~L} 1$ & & & 5 & 150 & 1.5 & 45.7 & clay, yellow & Clay & -- & -- & -- & \\
\hline 302 & $5 / 5-22 L 1$ & & & 150 & 156 & 45.7 & 47.5 & gravel and sand & Sand (or sandstone) and gravel & -. & -. & -- & \\
\hline 302 & $5 / 5-22 \mathrm{L1}$ & & & 156 & 225 & 47.5 & 68.6 & clay, yellow & Clay & -- & - & -- & \\
\hline 302 & $5 / 5-22 L 1$ & & & 225 & 280 & 68.6 & 85.3 & $\begin{array}{l}\text { clay, blue } \\
\text { lats }\end{array}$ & Clay & -. & -- & -- & \\
\hline 302 & $5 / 5-22 \mathrm{L1}$ & & & 280 & 290 & 85.3 & 88.4 & sand, blue & Sand & -- & -- & -- & \\
\hline 302 & $5 / 5-22 \mathrm{~L} 1$ & & & 290 & 330 & 88.4 & 100.6 & lava rock, blue & Basalt & hard & -- & -- & Tsv \\
\hline 302 & $5 / 5-22 L 1$ & & & 330 & 400 & 100.6 & 121.9 & lava rock, red & Basalt & hard & -- & -.- & \\
\hline 302 & $5 / 5-22 L 1$ & & & 400 & 560 & 121.9 & 170.7 & not described & No data & -- & -- & -- & \\
\hline 303 & $5 / 5-19 L 5$ & 303 & 5/5-19L5 & 0 & 37 & 0.0 & 11.3 & hardpan and sand & Clay and gravel & hard & -- & -- & Qoal \\
\hline 303 & 5/5-19L5 & & & 37 & 60 & 11.3 & 18.3 & clay, yellow & Clay & -- & -- & -- & \\
\hline 303 & $5 / 5-19 L 5$ & & & 60 & 65 & 18.3 & 19.8 & gravel and sand & Sand (or sandstone) and gravel & .- & .- & .. & \\
\hline 304 & $5 / 5-22 \mathrm{~L} 2$ & 304 & 5/5-22L2 & 0 & 6 & $\begin{array}{l}1.0 \\
0.0\end{array}$ & $\begin{array}{l}1.8 \\
1.8\end{array}$ & soil & Sand and clay & -. & -- & -. & Qh \\
\hline 304 & $5 / 5-22 L 2$ & & & 6 & 70 & 1.8 & 21.3 & clay, yellow & Clay & -- & -- & -- & \\
\hline 304 & $5 / 5-22 L 2$ & & & 70 & 135 & 21.3 & 41.1 & lava rock, red & Basalt & hard & -- & -. & Tsv \\
\hline 304 & $5 / 5-22 L 2$ & & & 135 & 155 & 41.1 & 47.2 & volcanic ash, green & Ash or tuff & -- & - & -- & \\
\hline 304 & $5 / 5-22 \mathrm{~L} 2$ & & & 155 & 200 & 47.2 & 61.0 & lava rock, hard, black & Basalt & hard & .. & .. & \\
\hline 304 & $5 / 5-22 L 2$ & & & 200 & 212 & 61.0 & 64.6 & lava rock brown & Basalt & hard & -. & .. & \\
\hline 305 & 5/5-19L1 & 305 & 5/5-19L1 & 0 & 3 & 0.0 & 0.9 & soil & Sand and clay & -- & -- & -- & Qoal and Qh (?) \\
\hline 305 & 5/5-19L1 & & & 3 & 70 & 0.9 & 21.3 & hardpan and clay & Clay and gravel & hard & -- & -- & \\
\hline 305 & 5/5-19L1 & & & 70 & 104 & 21.3 & 31.7 & gravel & Gravel & -- & -- & -- & \\
\hline 305 & 5/5-19L1 & & & 104 & 124 & 31.7 & 37.8 & clay, yellow & Clay & - &.- & -. & \\
\hline 305 & 5/5-19L1 & & & 124 & 150 & 37.8 & 45.7 & gravel & Gravel & .- & -. & .. & \\
\hline 306 & $5 / 5-22 Q 1$ & 306 & 5/5-22Q1 & 0 & 10 & 0.0 & 3.0 & soil & Sand and clay & .- & .. & .. & Qh \\
\hline 306 & $5 / 5-22 \mathrm{Q} 1$ & & & 10 & 30 & 3.0 & 9.1 & clay, yellow & Clay & -- & -- & -- & \\
\hline 306 & $5 / 5-22 \mathrm{Q} 1$ & & & 30 & 45 & 9.1 & 13.7 & clay, blue and sand & Clay and sand (or sandstone) & -- & -- & -. & \\
\hline 306 & $5 / 5-22 \mathrm{Q1}$ & & & 45 & 75 & $\begin{array}{l}.1 \\
13.7\end{array}$ & 22.9 & $\begin{array}{l}\text { clay, yellow, boulders } \\
\text { clons }\end{array}$ & $\begin{array}{l}\text { Clay and gravel } \\
\text { Callusion }\end{array}$ & - & -- & - & \\
\hline 306 & $5 / 5-22 Q 1$ & & & 75 & 100 & 22.9 & 30.5 & $\begin{array}{l}\text { clay, blue and sand } \\
\text {. }\end{array}$ & Clay and sand (or sandstone) & .- & -. & .. & \\
\hline 306 & $5 / 5-22 Q 1$ & & & 100 & 130 & 30.5 & 39.6 & clay, yellow and sand & Clay and sand (or sandstone) & -- & .- & .. & \\
\hline 306 & $5 / 5-22 \mathrm{Q} 1$ & & & 130 & 215 & 39.6 & 65.5 & clay, yellow and boulders & Clay and gravel & - & -- & -- & \\
\hline 306 & $5 / 5-22 Q 1$ & & & 215 & 265 & 65.5 & 80.8 & clay, brown and boulders & Clay and gravel & -- & -- & -. & \\
\hline 306 & $5 / 5-22 Q 1$ & & & 265 & 300 & 80.8 & 91.4 & clay, blue and boulders & Clay and gravel & -. & -. & -. & \\
\hline 306 & 5/5-22Q1 & & & 300 & 315 & 91.4 & 96.0 & gravel and sand & Sand (or sandstone) and gravel & -- & -- & -- & \\
\hline 306 & 5/5-22Q1 & & & 315 & 320 & 96.0 & 97.5 & $\begin{array}{l}\text { clay, brown } \\
\text { chilu }\end{array}$ & Clay &.- & .- & .. & \\
\hline 306 & $5 / 5-22 Q 1$ & & & 320 & 500 & 97.5 & 152.4 & $\begin{array}{l}\text { clay, blue, sand, shattered rock } \\
\text { St. Helena rhyolite member. }\end{array}$ & Clay, sand, and gravel & -- & -- & -- & \\
\hline 306 & $5 / 5-22 \mathrm{Q} 1$ & & & 500 & 542 & 152.4 & 165.2 & basalt & Ash or tuff & -. & -- & -. & Tsv \\
\hline 307 & 5/5-20R1 & 307 & 5/5-20R1 & 0 & 10 & 0.0 & 3.0 & sand and dirt & Sand and clay & -- & .- & .. & Qoal \\
\hline 307 & $5 / 5-20 R 1$ & & & 10 & 100 & 3.0 & $\begin{array}{l}3.0 \\
30.5\end{array}$ & clay, yellow & Clay & -. & -. & .- & Qh \\
\hline 307 & $5 / 5-20 R 1$ & & & 100 & 125 & 30.5 & 38.1 & $\begin{array}{l}\text { clay, brown-yellow } \\
\text { cow }\end{array}$ & Clay & -- & -- & -- & \\
\hline 307 & 5/5-20R1 & & & 125 & 127 & 38.1 & 38.7 & water strata & Clay & -. & .- & -. & \\
\hline 307 & $5 / 5-20 R 1$ & & & 127 & 200 & 38.7 & 61.0 & clay, green colored & Clay & -- & -- & -- & \\
\hline 307 & $5 / 5-20 R 1$ & & & 200 & 250 & 61.0 & 76.2 & $\begin{array}{l}\text { clay, blue } \\
\text { laved }\end{array}$ & Clay & -. & -- & -- & \\
\hline 307 & $5 / 5-20 R 1$ & & & 250 & 275 & 76.2 & 83.8 & $\begin{array}{l}\text { clay, blue and sand } \\
\text { clat }\end{array}$ & Clay and sand (or sandstone) & -- & -. & -. & \\
\hline 307 & 5/5-20R1 & & & 275 & 300 & 83.8 & 91.4 & clay, green colored & Clay & -- & -- & -- & \\
\hline 307 & 5/5-20R1 & & & 300 & 350 & $\begin{array}{l}91.0 \\
91.4\end{array}$ & 106.7 & $\begin{array}{l}\text { clay, light blue } \\
\text { lal }\end{array}$ & Clay & - & - & - & \\
\hline 307 & 5/5-20R1 & & & 350 & 400 & 106.7 & 121.9 & clay, blue and gray colored & Clay & -- & -. & -- & \\
\hline 307 & 5/5-20R1 & & & 400 & 450 & 121.9 & 137.2 & $\begin{array}{l}\text { clay, green colored } \\
\text { lol d }\end{array}$ & Clay & -- & .- & -- & \\
\hline 307 & $5 / 5-20 R 1$ & & & 450 & 475 & 137.2 & 144.8 & $\begin{array}{l}\text { clay, blue } \\
\text { later }\end{array}$ & Clay & -- & -- & -- & \\
\hline
\end{tabular}


Appendix 3. Lithologic data. - Continued

[Site IDs and well number correspond to locations in Appendix 1, a site ID and well number is listed for each drilled interval for a particular drill hole. For convenience, the site ID and well numbers are repeated at the shallowest drilled interval for each well. leaders

\begin{tabular}{|c|c|c|c|c|c|c|c|c|c|c|c|c|c|}
\hline Site ID & Well number & $\begin{array}{l}\text { Site ID, } \\
\text { top of } \\
\text { well }\end{array}$ & $\begin{array}{l}\text { Well number, } \\
\text { top of well }\end{array}$ & $\begin{array}{c}\text { Top of } \\
\text { interval, in } \\
\text { feet }\end{array}$ & $\begin{array}{c}\text { Base of } \\
\text { interval, in } \\
\text { feet }\end{array}$ & $\begin{array}{c}\text { Top of } \\
\text { interval, in } \\
m\end{array}$ & $\begin{array}{c}\text { Base of } \\
\text { interval, in } \\
\mathrm{m}\end{array}$ & Reported lithologic unit ${ }^{1}$ & Interpreted lithologic class ${ }^{2}$ & $\begin{array}{c}\text { Reported } \\
\text { degree of } \\
\text { cementation or } \\
\text { induration } \\
\end{array}$ & $\begin{array}{l}\text { Fossils } \\
\text { reported in } \\
\text { interval }^{4}\end{array}$ & $\begin{array}{c}\text { Organic } \\
\text { matter } \\
\text { reported in } \\
\text { interval }^{5}\end{array}$ & $\begin{array}{l}\text { Reported stratigraphic top of geologic } \\
\text { unit }^{6}\end{array}$ \\
\hline $\begin{array}{l}307 \\
307\end{array}$ & $\begin{array}{l}\text { 5/5-20R1 } \\
\text { 5/5-20R1 }\end{array}$ & & & $\begin{array}{l}475 \\
500\end{array}$ & $\begin{array}{l}500 \\
504\end{array}$ & $\begin{array}{l}144.8 \\
152.4\end{array}$ & $\begin{array}{l}152.4 \\
153.6\end{array}$ & $\begin{array}{l}\text { clay, blue } \\
\text { clay, blue with sand }\end{array}$ & $\begin{array}{l}\text { Clay } \\
\text { Clay and sand (or sandstone) }\end{array}$ & $\begin{array}{l}-- \\
--\end{array}$ & $\begin{array}{l}-- \\
-\end{array}$ & -- & \\
\hline 308 & $5 / 5-21 \mathrm{R} 1$ & 308 & 5/5-21R1 & 0 & 7 & 0.0 & 2.1 & soil & $\begin{array}{l}\text { Sand and clay } \\
\text { sadiustie) }\end{array}$ & -. & -- & -- & Qoal \\
\hline 308 & $5 / 5-21 R 1$ & & & 7 & 46 & 2.1 & 14.0 & clay, sandy & Clay and sand (or sandstone) & -- & -. & -- & \\
\hline 308 & 5/5-21R1 & & & 46 & 116 & 14.0 & 35.4 & clay, yellow & Clay & -- & -- & -- & Qh \\
\hline 308 & 5/5-21R1 & & & 116 & 166 & 35.4 & 50.6 & $\begin{array}{l}\text { clay, blue } \\
\text { pla }\end{array}$ & Clay & -. & -- & -- & \\
\hline 308 & $5 / 5-21 \mathrm{R} 1$ & & & 166 & 236 & 50.6 & 71.9 & clay, yellow & Clay & -- & -- & -- & \\
\hline 308 & $5 / 5-21 \mathrm{R} 1$ & & & 236 & 242 & 71.9 & 73.8 & $\begin{array}{l}\text { clay, blue } \\
\text { cla }\end{array}$ & Clay & -- & -- & -- & \\
\hline 309 & 5/5-30E1 & 309 & 5/5-30E1 & 0 & 10 & 0.0 & $\begin{array}{l}3.0 \\
3.0\end{array}$ & soil wile & Sand and clay & - & - & - & \\
\hline 309 & 5/5-30E1 & & & 10 & 30 & 3.0 & 9.1 & clay, blue & Clay & -- & -- & -- & \\
\hline 309 & 5/5-30E1 & & & 30 & 50 & 9.1 & 15.2 & gravel, free & Gravel & -. & -- & -- & \\
\hline 309 & $5 / 5-30 E 1$ & & & 50 & 150 & 15.2 & 45.7 & clay, yellow, sand and gravel & Clay, sand, and gravel & -- & -- & -- & \\
\hline 309 & $5 / 5-30 E 1$ & & & 150 & 225 & 45.7 & 68.6 & clay, blue & Clay & -- & -- & -- & \\
\hline 309 & 5/5-30E1 & & & 225 & 350 & 68.6 & 106.7 & clay, yellow & Clay & -- & -- & -- & \\
\hline 309 & 5/5-30E1 & & & 350 & 360 & 106.7 & 109.7 & clay, yellow and sand & Clay and sand (or sandstone) & -- & -- & -- & \\
\hline 309 & 5/5-30E1 & & & 360 & 385 & 109.7 & 117.3 & $\begin{array}{l}\text { clay, yellow } \\
\text { s. }\end{array}$ & Clay & -- & -- & -- & \\
\hline 309 & $5 / 5-30 E 1$ & & & 385 & 395 & 117.3 & 120.4 & sand and yellow clay & Sand and clay & -- & -- & -- & \\
\hline 309 & 5/5-30E1 & & & 395 & 400 & 120.4 & 121.9 & sand and gravel & Sand (or sandstone) and gravel & -- & -- & -- & \\
\hline 309 & 5/5-30E1 & & & 400 & 450 & 121.9 & 137.2 & clay, blue and sand & Clay and sand (or sandstone) & -- & -- & -- & \\
\hline 309 & 5/5-30E1 & & & 450 & 460 & 137.2 & 140.2 & gravel & Gravel & -- & -- & -- & \\
\hline 309 & 5/5-30E1 & & & 460 & 475 & 140.2 & 144.8 & clay, blue, sand and gravel & Clay, sand, and gravel & -- & -- & .- & \\
\hline 309 & 5/5-30E1 & & & 475 & 488 & 144.8 & 148.7 & clay, yellow and sand & Clay and sand (or sandstone) & -- & -- & -- & \\
\hline 309 & 5/5-30E1 & & & 488 & 526 & 148.7 & 160.3 & clay, blue and sand & Clay and sand (or sandstone) & -- & -- & -- & \\
\hline 309 & 5/5-30E1 & & & 526 & 538 & 160.3 & 164.0 & gravel, free & Gravel & -- & -- & .- & \\
\hline 309 & 5/5-30E1 & & & 538 & 555 & 164.0 & 169.2 & sand & Sand & -. & -- & -- & \\
\hline 309 & $5 / 5-30 E 1$ & & & 555 & 560 & 169.2 & 170.7 & clay, blue and sand & Clay and sand (or sandstone) & -- & -- & -- & \\
\hline 309 & 5/5-30E1 & & & 560 & 577 & 170.7 & 175.9 & tuff, white & Ash or tuff & -- & -- & -- & \\
\hline 309 & 5/5-30E1 & & & 577 & 579 & 175.9 & 176.5 & gravel, free & Gravel & - & - & - & \\
\hline 309 & 5/5-30E1 & & & 579 & 590 & 176.5 & 179.8 & clay, blue, sand and gravel & Clay, sand, and gravel & -- & -- & -.- & \\
\hline 309 & 5/5-30E1 & & & 590 & 605 & 179.8 & 184.4 & clay, blue & Clay & -- & -- & -- & \\
\hline 309 & $5 / 5-30 E 1$ & & & 605 & 610 & 184.4 & 185.9 & sand & Sand & -- & -- & -- & \\
\hline 309 & 5/5-30E1 & & & 610 & 630 & 185.9 & 192.0 & gravel, free and sand & Sand (or sandstone) and gravel & -- & -- & -- & \\
\hline 309 & 5/5-30E1 & & & 630 & 636 & 192.0 & 193.9 & clay, blue and gravel & $\begin{array}{l}\text { Clay and gravel } \\
\text { (a) }\end{array}$ & - & -- & - & \\
\hline 309 & 5/5-30E1 & & & 636 & 642 & 193.9 & 195.7 & gravel, free and sand & Sand (or sandstone) and gravel & -- & -- & -- & \\
\hline 309 & 5/5-30E1 & & & 642 & 645 & 195.7 & 196.6 & $\begin{array}{l}\text { gravel, cemented } \\
\text { and }\end{array}$ & Gravel & cemented & -- & -.- & \\
\hline 309 & $5 / 5-30 E 1$ & & & 645 & 650 & 196.6 & 198.1 & sand & Sand & -- & -- & - & \\
\hline 309 & 5/5-30E1 & & & 650 & 655 & 198.1 & 199.6 & sand and gravel & Sand (or sandstone) and gravel & -- & -- & -. & \\
\hline 309 & 5/5-30E1 & & & 655 & 675 & 199.6 & 205.7 & clay, blue & Clay & -- & -- & -- & \\
\hline 309 & 5/5-30E1 & & & 675 & 685 & 205.7 & 208.8 & $\begin{array}{l}\text { clay, blue and sand } \\
\text {. blo }\end{array}$ & Clay and sand (or sandstone) & -- & -- & -- & \\
\hline 309 & 5/5-30E1 & & & 685 & 697 & 208.8 & 212.4 & gravel, free & Gravel & -- & -- & -. & \\
\hline 309 & $5 / 5-30 E 1$ & & & 697 & 705 & 212.4 & 214.9 & $\begin{array}{l}\text { clay, blue and sand } \\
\text { s. }\end{array}$ & Clay and sand (or sandstone) & -- & -- & - & \\
\hline 309 & 5/5-30E1 & & & 705 & 710 & 214.9 & 216.4 & 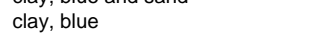 & Clay & -- & -- & -. & \\
\hline 309 & 5/5-30E1 & & & 710 & 725 & 216.4 & 221.0 & sand and gravel & Sand (or sandstone) and gravel & -- & -- & .- & \\
\hline 309 & 5/5-30E1 & & & 725 & 730 & 221.0 & 222.5 & clay, blue & Clay & -- & -- & -- & \\
\hline 309 & $5 / 5-30 E 1$ & & & 730 & 740 & 222.5 & 225.6 & clay, blue, sand and gravel & Clay, sand, and gravel & -- & -- & -. & \\
\hline 309 & 5/5-30E1 & & & 740 & 743 & 225.6 & 226.5 & clay, blue & Clay & -- & -- & -- & \\
\hline 309 & 5/5-30E1 & & & 743 & 755 & 226.5 & 230.1 & sand and free & Sand & -- & -- & -. & \\
\hline 310 & 5/5-27H1 & 310 & 5/5-27H1 & 0 & 20 & 0.0 & 6.1 & soil & Sand and clay & -- & -- & -- & Qh \\
\hline 310 & 5/5-27H1 & & & 20 & 50 & 6.1 & 15.2 & clay, blue, some fine sand & Clay and sand (or sandstone) & -- & -- & -- & \\
\hline 310 & $5 / 5-27 \mathrm{H} 1$ & & & 50 & 75 & 15.2 & 22.9 & clay, blue, sticky & Clay & -- & -- & -- & \\
\hline 310 & $5 / 5-27 \mathrm{H} 1$ & & & 75 & 110 & 22.9 & 33.5 & clay, blue, some fine sand & Clay and sand (or sandstone) & -- & -- & -- & \\
\hline 310 & $5 / 5-27 \mathrm{H} 1$ & & & 110 & 115 & 33.5 & 35.1 & clay, blue, sticky & Clay & -- & -- & -- & \\
\hline 310 & $5 / 5-27 \mathrm{H} 1$ & & & 115 & 168 & 35.1 & 51.2 & clay, blue, fine sand, some gravel & Clay and sand (or sandstone) & -- & -- & .- & \\
\hline 310 & 5/5-27H1 & & & 168 & 235 & 51.2 & 71.6 & clay, blue, sticky & Clay & -- & -- & -- & \\
\hline 310 & $5 / 5-27 \mathrm{H} 1$ & & & 235 & 300 & 71.6 & 91.4 & clay, yellow, sticky & Clay & -- & -- & -- & \\
\hline 310 & $5 / 5-27 \mathrm{H} 1$ & & & 300 & 310 & 91.4 & 94.5 & sand and gravel & Sand (or sandstone) and gravel & -- & -- & -- & \\
\hline 310 & 5/5-27H1 & & & 310 & 360 & 94.5 & 109.7 & clay, gray & Clay & -- & -- & -- & \\
\hline 310 & $5 / 5-27 \mathrm{H1}$ & & & 360 & 370 & 109.7 & 112.8 & clay, blue & Clay & -- & -- & -- & \\
\hline 310 & $5 / 5-27 \mathrm{H} 1$ & & & 370 & 380 & 112.8 & 115.8 & clay, gray & Clay & -- & -- & -- & \\
\hline 310 & $5 / 5-27 \mathrm{H} 1$ & & & 380 & 423 & 115.8 & 128.9 & "tule mud," blue & Clay and sand (or sandstone) & .- & -.- & tule & \\
\hline
\end{tabular}


Appendix 3. Lithologic data. - Continued

ISite IDs and well number correspond to locations in Appendix 1, a site ID and well number is listed for each drilled interval for a particular drill hole. For convenience, the site ID and well numbers are repeated at the shallowest drilled interval for each well. leaders

\begin{tabular}{|c|c|c|c|c|c|c|c|c|c|c|c|c|c|}
\hline Site ID & Well number & $\begin{array}{l}\text { Site ID, } \\
\text { top of } \\
\text { well }\end{array}$ & $\begin{array}{l}\text { Well number, } \\
\text { top of well }\end{array}$ & $\begin{array}{c}\text { Top of } \\
\text { interval, in } \\
\text { feet }\end{array}$ & $\begin{array}{c}\text { Base of } \\
\text { interval, in } \\
\text { feet }\end{array}$ & $\begin{array}{c}\text { Top of } \\
\text { interval, in } \\
m\end{array}$ & $\begin{array}{c}\text { Base of } \\
\text { interval, in } \\
\mathrm{m}\end{array}$ & Reported lithologic unit ${ }^{1}$ & Interpreted lithologic class ${ }^{2}$ & $\begin{array}{c}\text { Reported } \\
\text { degree of } \\
\text { cementation or } \\
\text { induration } \\
\end{array}$ & $\begin{array}{l}\text { Fossils } \\
\text { reported in } \\
\text { interval }^{4}\end{array}$ & $\begin{array}{c}\text { Organic } \\
\text { matter } \\
\text { reported in } \\
\text { interval }^{5}\end{array}$ & $\begin{array}{l}\text { Reported stratigraphic top of geologic } \\
\text { unit }^{6}\end{array}$ \\
\hline 310 & $5 / 5-27 \mathrm{H1}$ & & & 423 & 465 & 128.9 & 141.7 & sand, blue, and shattered rock & Sand (or sandstone) and gravel & -- & -- & -- & \\
\hline 310 & 5/5-27H1 & & & 465 & 470 & 141.7 & 143.3 & clay, red & Clay & -- & -- & -- & \\
\hline 310 & $5 / 5-27 \mathrm{H} 1$ & & & 470 & 482 & 143.3 & 146.9 & clay, blue & Clay & -- & -- & -- & \\
\hline 310 & $5 / 5-27 \mathrm{H} 1$ & & & 482 & 680 & 146.9 & 207.3 & sand, cemented and gravel & Sand (or sandstone) and gravel & cemented & -. & -- & \\
\hline 310 & 5/5-27H1 & & & 680 & 750 & 207.3 & 228.6 & basalt & Basalt & -- & -- & -- & Tsv \\
\hline 311 & $6 / 6-9 \mathrm{R} 1$ & 311 & 6/6-9R1 & 0 & 35 & 0.0 & 10.7 & $\begin{array}{l}\text { rock, brown } \\
\text { clay, yellow, and gravel; water at }\end{array}$ & Ash or tuff & hard & -- & -- & QTge \\
\hline 311 & 6/6-9R1 & & & 35 & 78 & 10.7 & 23.8 & 70 feet & Clay and gravel & .- & .. & .- & \\
\hline 311 & 6/6-9R1 & & & 78 & 172 & 23.8 & 52.4 & clay, blue, and gravel & Clay and gravel & -- &.- & - & \\
\hline 311 & 6/6-9R1 & & & 172 & 190 & 52.4 & 57.9 & gravel & Gravel & -- & -- & -- & \\
\hline 311 & 6/6-9R1 & & & 190 & 193 & 57.9 & 58.8 & clay, yellow, and gravel & Clay and gravel & -- & -- & -- & \\
\hline 312 & 6/6-16B1 & 312 & 6/6-16B1 & 0 & 87 & 0.0 & 26.5 & clay and rock & Clay and gravel & -- & -- & -- & QTge \\
\hline 312 & 6/6-16B1 & & & 87 & 128 & 26.5 & 39.0 & clay, yellow & Clay & -- & -- & -- & \\
\hline 312 & 6/6-16B1 & & & 128 & 150 & 39.0 & 45.7 & clay, blue & Clay & -- & -- & -- & \\
\hline 312 & 6/6-16B1 & & & 150 & 184 & 45.7 & 56.1 & clay, yellow & Clay & -- & -- & -- & \\
\hline 312 & 6/6-16B1 & & & 184 & 240 & 56.1 & 73.2 & $\begin{array}{l}\text { clay, blue } \\
\text { lat }\end{array}$ & Clay & -- & -- & -- & \\
\hline 312 & 6/6-16B1 & & & 240 & 317 & 73.2 & 96.6 & clay, yellow & Clay & -- & -- & -- & \\
\hline 312 & 6/6-16B1 & & & 317 & 352 & 96.6 & 107.3 & shale, blue & Clay and sand (or sandstone) & cemented & -- & -- & \\
\hline 312 & 6/6-16B1 & & & 352 & 426 & 107.3 & 129.8 & clay, volcanic red with gravel & Clay and gravel & -- & -- & -- & \\
\hline 313 & $5 / 6-25 \mathrm{P} 1$ & 313 & 5/6-25P1 & 0 & 3 & 0.0 & 0.9 & soil & Sand and clay & -- & -- & -. & Qoal \\
\hline 313 & $5 / 6-25 \mathrm{P} 1$ & & & 3 & 22 & 0.9 & 6.7 & hardpan & Clay and gravel & hard & -- & -- & \\
\hline 313 & 5/6-25P1 & & & 22 & 69 & 6.7 & 21.0 & clay, yellow & Clay & -- & -- & -- & \\
\hline 313 & 5/6-25P1 & & & 69 & 156 & 21.0 & 47.5 & $\begin{array}{l}\text { clay, blue } \\
\text { che }\end{array}$ & Clay & -- & -- & -- & \\
\hline 313 & $5 / 6-25 \mathrm{P} 1$ & & & 156 & 168 & 47.5 & 51.2 & clay, blue and sand & Clay and sand (or sandstone) & -- & -- & -- & \\
\hline 313 & $5 / 6-25 \mathrm{P} 1$ & & & 168 & 171 & 51.2 & 52.1 & sand & Sand & -- & -- & -. & \\
\hline 314 & $5 / 5-31 \mathrm{~A} 1$ & 314 & 5/5-31A1 & 0 & 15 & 0.0 & 4.6 & not described & No data & -- & -- & -- & Qyal (?) \\
\hline 314 & 5/5-31A1 & & & 15 & 17 & 4.6 & 5.2 & gravel, water & Gravel & -- & -- & -- & \\
\hline 314 & 5/5-31A1 & & & 17 & 42 & 5.2 & 12.8 & clay & Clay & - & - & - & \\
\hline 314 & 5/5-31A1 & & & 42 & 45 & 12.8 & 13.7 & gravel, water & Gravel & -- & -- & -. & \\
\hline 314 & 5/5-31A1 & & & 45 & 49 & 13.7 & 14.9 & rock and clay & Clay and gravel & -- & -- & -.- & \\
\hline 314 & $5 / 5-31 A 1$ & & & 49 & 60 & 14.9 & 18.3 & gravel, water & Gravel & -- & -- & -- & \\
\hline 314 & 5/5-31A1 & & & 60 & 160 & 18.3 & 48.8 & clay, yellow & Clay & -- & -- & -- & \\
\hline $\begin{array}{l}314 \\
314\end{array}$ & 5/5-31A1 & & & 160 & 167 & 48.8 & 50.9 & clay, some water & Clay & - & -- &.- & \\
\hline 314 & 5/5-31A1 & & & 167 & 195 & 50.9 & 59.4 & clay & Clay & - & -- & -- & \\
\hline 314 & 5/5-31A1 & & & 195 & 205 & 59.4 & 62.5 & quicksand & Sand and clay & -- & -- & .- & \\
\hline 314 & $5 / 5-31 A 1$ & & & 205 & 225 & 62.5 & 68.6 & clay & Clay & - & -- & - & \\
\hline 314 & 5/5-31A1 & & & 225 & 230 & 68.6 & 70.1 & clay, gravel, some water & Clay and gravel & -- & -- & -. & \\
\hline 314 & $5 / 5-31 \mathrm{~A} 1$ & & & 230 & 352 & 70.1 & 107.3 & quicksand & Sand and clay & -- & -- & -- & \\
\hline 314 & 5/5-31A1 & & & 352 & 380 & 107.3 & 115.8 & clay & Clay & -- & -- & -- & \\
\hline 314 & 5/5-31A1 & & & 380 & 381 & 115.8 & 116.1 & sand, flowing water & Sand & -- & -- & -. & \\
\hline 314 & $5 / 5-31 A 1$ & & & 381 & 393 & 116.1 & 119.8 & clay & Clay & -- & -- & - & \\
\hline 314 & 5/5-31A1 & & & 393 & 408 & 119.8 & 124.4 & sand, gravel, water & Sand (or sandstone) and gravel & -- & -- & -. & \\
\hline 315 & 5/5-31H1 & 315 & 5/5-31H1 & 0 & 7 & 0.0 & 2.1 & soil & Sand and clay & - & -- & -- & Qyal (?) \\
\hline 315 & 5/5-31H1 & & & 7 & 48 & 2.1 & 14.6 & "muck," blue & Clay and sand (or sandstone) & - & -- & -- & \\
\hline 315 & 5/5-31H1 & & & 48 & 51 & 14.6 & 15.5 & sand, salty water, cased off & Sand & -- & -- & -- & \\
\hline 315 & 5/5-31H1 & & & 51 & 88 & 15.5 & 26.8 & clay, blue & Clay & -- & -- & -- & \\
\hline 315 & 5/5-31H1 & & & 88 & 102 & 26.8 & 31.1 & clay, yellow & Clay & -- & -- & -. & \\
\hline 315 & 5/5-31H1 & & & 102 & 202 & 31.1 & 61.6 & clay, blue & Clay & -- & -- & -- & \\
\hline 315 & $5 / 5-31 \mathrm{H} 1$ & & & 202 & 203 & 61.6 & 61.9 & gravel & Gravel & .- & .- & .. & \\
\hline 316 & $4 / 5-3 \mathrm{Cl}$ & 316 & $4 / 5-3 \mathrm{C} 1$ & 0 & 5 & 0.0 & 1.5 & boulders & Gravel & -- & -- & -- & Qt \\
\hline 316 & 4/5-3C1 & & & 5 & 107 & 1.5 & 32.6 & $\begin{array}{l}\text { clay, yellow } \\
\text { y }\end{array}$ & Clay & -- & -- & -- & Qh \\
\hline 316 & $4 / 5-3 \mathrm{C} 1$ & & & 107 & 110 & 32.6 & 33.5 & gravel, tuff, water & Gravel & -- & -- & -- & \\
\hline 316 & $4 / 5-3 \mathrm{C} 1$ & & & 110 & 127 & 33.5 & 38.7 & clay, gray & Clay & -- & -- & -- & \\
\hline 316 & $4 / 5-3 \mathrm{C} 1$ & & & 127 & 142 & 38.7 & 43.3 & clay, blue & Clay & -- & -- & -- & \\
\hline 316 & $4 / 5-3 \mathrm{C} 1$ & & & 142 & 165 & 43.3 & 50.3 & clay, gray & Clay & -- & -- & -- & \\
\hline 316 & $4 / 5-3 \mathrm{C} 1$ & & & 165 & 174 & 50.3 & 53.0 & clay, glue & Clay & -- & -- & -- & \\
\hline 316 & $4 / 5-3 \mathrm{C} 1$ & & & 174 & 231 & 53.0 & 70.4 & clay, gray & Clay & -- & -- & -- & \\
\hline 316 & $4 / 5-3 \mathrm{C} 1$ & & & 231 & 258 & 70.4 & 78.6 & blue and gray clay, gravel, water & Clay and gravel & -- & -. & -- & \\
\hline 316 & $4 / 5-3 \mathrm{C} 1$ & & & 258 & 261 & 78.6 & 79.6 & clay, yellow & Clay & -- & -- & -- & \\
\hline 317 & 4/5-14D2 & 317 & $4 / 5-14 \mathrm{D} 2$ & 0 & 10 & 0.0 & 3.0 & peat and adobe & Clay and sand (or sandstone) & -- & -- & peat & \\
\hline
\end{tabular}


Appendix 3. Lithologic data. - Continued

ISite IDs and well number correspond to locations in Appendix 1, a site ID and well number is listed for each drilled interval for a particular drill hole. For convenience, the site ID and well numbers are repeated at the shallowest drilled interval for each well. leaders

\begin{tabular}{|c|c|c|c|c|c|c|c|c|c|c|c|c|c|}
\hline Site ID & Well number & $\begin{array}{l}\text { Site ID, } \\
\text { top of } \\
\text { well }\end{array}$ & $\begin{array}{l}\text { Well number, } \\
\text { top of well }\end{array}$ & $\begin{array}{c}\text { Top of } \\
\text { interval, in } \\
\text { feet }\end{array}$ & $\begin{array}{c}\text { Base of } \\
\text { interval, in } \\
\text { feet }\end{array}$ & $\begin{array}{c}\text { Top of } \\
\text { interval, in } \\
m\end{array}$ & $\begin{array}{c}\text { Base of } \\
\text { interval, in } \\
\mathrm{m}\end{array}$ & Reported lithologic unit ${ }^{1}$ & Interpreted lithologic class ${ }^{2}$ & $\begin{array}{c}\text { Reported } \\
\text { degree of } \\
\text { cementation or } \\
\text { induration } \\
\end{array}$ & $\begin{array}{c}\text { Fossils } \\
\text { reported in } \\
\text { interval| }^{4}\end{array}$ & $\begin{array}{c}\text { Organic } \\
\text { matter } \\
\text { reported in } \\
\text { interval }^{5}\end{array}$ & $\begin{array}{c}\text { Reported stratigraphic top of geologic } \\
\text { unit }^{6}\end{array}$ \\
\hline 317 & $4 / 5-14 \mathrm{D} 2$ & & & 10 & 48 & 3.0 & 14.6 & peat and silt & Clay and sand (or sandstone) & -- & -- & peat & \\
\hline 317 & $4 / 5-14 \mathrm{D} 2$ & & & 48 & 60 & 14.6 & 18.3 & silt sandy & Clay and sand (or sandstone) & -- & -- & -- & \\
\hline 317 & $4 / 5-14 \mathrm{D} 2$ & & & 60 & 75 & 18.3 & 22.9 & silt, coarse, sandy yellow & Clay and sand (or sandstone) & -- & -- & -- & \\
\hline 317 & $4 / 5-14 \mathrm{D} 2$ & & & 75 & 105 & 22.9 & 32.0 & silt & Clay and sand (or sandstone) & -- & -- & -- & \\
\hline 317 & $4 / 5-14 \mathrm{D} 2$ & & & 105 & 152 & 32.0 & 46.3 & sand, coarse and silt. yellow & Sand & -- & -- & -- & \\
\hline 317 & 4/5-14D2 & & & 152 & 156 & 46.3 & 47.5 & clay, blue & Clay & -- & -- & .- & \\
\hline 317 & $4 / 5-14 \mathrm{D} 2$ & & & 156 & 200 & 47.5 & 61.0 & clay, blue and peat in layers & Clay and sand (or sandstone) & -- & -- & peat & \\
\hline 317 & 4/5-14D2 & & & 200 & 250 & 61.0 & 76.2 & clay, blue & Clay & -- & -- & -- & \\
\hline 317 & 4/5-14D2 & & & 250 & 266 & 76.2 & 81.1 & silt, sandy & Clay and sand (or sandstone) & -- & -- & -- & \\
\hline 317 & $4 / 5-14 \mathrm{D} 2$ & & & 266 & 275 & 81.1 & 83.8 & clay, blue & Clay & -- & -- & -- & \\
\hline 317 & $4 / 5-14 \mathrm{D} 2$ & & & 275 & 320 & 83.8 & 97.5 & clay, blue and sand, coarse & Clay and sand (or sandstone) & -- & -- & -- & \\
\hline 317 & $4 / 5-14 \mathrm{D} 2$ & & & 320 & 330 & 97.5 & 100.6 & gravel, fine & Gravel & -- & -- & -- & \\
\hline 317 & $4 / 5-14 \mathrm{D} 2$ & & & 330 & 338 & 100.6 & 103.0 & sand and gravel & Sand (or sandstone) and gravel & -- & -- & -- & \\
\hline 317 & $4 / 5-14 \mathrm{D} 2$ & & & 338 & 365 & 103.0 & 111.3 & clay, sandy, yellow & Clay and sand (or sandstone) &.- & - & - & \\
\hline 317 & $4 / 5-14 \mathrm{D} 2$ & & & 365 & 370 & 111.3 & 112.8 & clay, sandy, yellow and gravel & Clay, sand, and gravel & -- & -- & -- & \\
\hline 317 & $4 / 5-14 \mathrm{D} 2$ & & & 370 & 386 & 112.8 & 117.7 & $\begin{array}{l}\text { clay, yellow and silt } \\
\text { lation }\end{array}$ & Clay and sand (or sandstone) & -- & -- & -- & \\
\hline 317 & $4 / 5-14 \mathrm{D} 2$ & & & 386 & 398 & 117.7 & 121.3 & clay, yellow and gravel & Clay and gravel & -- & -- & -- & \\
\hline 317 & 4/5-14D2 & & & 398 & 420 & 121.3 & 128.0 & silt, hard or clay, sandy, yellow & Clay and sand (or sandstone) & hard & -- & -. & \\
\hline 317 & 4/5-14D2 & & & 420 & 435 & 128.0 & 132.6 & silt, soft or clay, sandy, yellow & Clay and sand (or sandstone) & -- & -- & weed & \\
\hline 317 & $4 / 5-14 \mathrm{D} 2$ & & & 435 & 454 & 132.6 & 138.4 & silt, hard, yellow & Clay and sand (or sandstone) & hard & -- & -- & \\
\hline 317 & $4 / 5-14 \mathrm{D} 2$ & & & 454 & 464 & 138.4 & 141.4 & gravel and clay & $\begin{array}{l}\text { Clay and gravel } \\
\text { Sariusurie }\end{array}$ & -. & - & - & \\
\hline 317 & $4 / 5-14 \mathrm{D} 2$ & & & 464 & 474 & 141.4 & 144.5 & gravel, fine & Gravel & -- & -- & -- & \\
\hline 317 & $4 / 5-14 \mathrm{D} 2$ & & & 474 & 509 & 144.5 & 155.1 & clay and sand & Clay and sand (or sandstone) & -- & -. & -- & \\
\hline 317 & $4 / 5-14 \mathrm{D} 2$ & & & 509 & 520 & 155.1 & 158.5 & clay and sand & Clay and sand (or sandstone) & -- & -. & .- & \\
\hline 317 & $4 / 5-14 \mathrm{D} 2$ & & & 520 & 521 & 158.5 & 158.8 & gravel & Gravel & -- & -- & -- & \\
\hline 317 & $4 / 5-14 \mathrm{D} 2$ & & & 521 & 544 & 158.8 & 165.8 & sand and clay & Sand and clay & -- & -- & -- & \\
\hline 317 & $4 / 5-14 \mathrm{D} 2$ & & & 544 & 667 & 165.8 & 203.3 & $\begin{array}{l}\text { clay, yellow and tough, sticky } \\
\text { gravel, pea size, and sand, }\end{array}$ & Clay & -- & -- & - & \\
\hline 317 & $4 / 5-14 \mathrm{D} 2$ & & & 667 & 683 & 203.3 & 208.2 & $\begin{array}{l}\text { coarse } \\
\text { clay, blue, some fine gravel or }\end{array}$ & Sand (or sandstone) and gravel & -- & -- & -- & \\
\hline 317 & $4 / 5-14 \mathrm{D} 2$ & & & 683 & 710 & 208.2 & 216.4 & coarse sand & Clay and gravel & -- & -- & -- & \\
\hline 317 & $4 / 5-14 \mathrm{D} 2$ & & & 710 & 740 & 216.4 & 225.6 & $\begin{array}{l}\text { clay, blue, sticky } \\
\text { clay some sand and streaks of }\end{array}$ & Clay & -- & -- & -- & \\
\hline 317 & $4 / 5-14 \mathrm{D} 2$ & & & 740 & 782 & 225.6 & 238.4 & 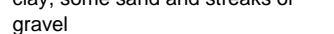 & Clay and gravel & -- & -- & .- & \\
\hline 317 & $4 / 5-14 \mathrm{D} 2$ & & & 782 & 785 & 238.4 & 239.3 & clay, hard & Clay & hard & -- & -- & \\
\hline 317 & $4 / 5-14 \mathrm{D} 2$ & & & 785 & 818 & 239.3 & 249.3 & clay, blue & Clay & -- & -- & -- & \\
\hline 317 & $4 / 5-14 \mathrm{D} 2$ & & & 818 & 819 & 249.3 & 249.6 & clay, hard & Clay & hard & -- & -- & \\
\hline 317 & 4/5-14D2 & & & 819 & 920 & 249.6 & 280.4 & clay, blue, some fine gravel & Clay and gravel & -- & -- & -- & \\
\hline 317 & 4/5-14D2 & & & 920 & 926 & 280.4 & 282.2 & clay, yellow, silty, some sand & Clay and sand (or sandstone) & -- & -- & -- & \\
\hline & & & & & & & & clay, alternating yellow and blue & & & & & \\
\hline 317 & 4/5-14D2 & & & 926 & 968 & 282.2 & 295.0 & with some angular rock fragments & Clay and gravel & -- & -- & -- & \\
\hline 317 & $4 / 5-14 \mathrm{D} 2$ & & & 968 & 1090 & 295.0 & 332.2 & $\begin{array}{l}\text { clay, yellow and blue } \\
\text { clay, gray, some small sized }\end{array}$ & Clay & - & -- & -- & \\
\hline 317 & $4 / 5-14 \mathrm{D} 2$ & & & 1090 & 1094 & 332.2 & 333.5 & gravel & Clay and gravel & -- & -- & -- & \\
\hline 317 & $4 / 5-14 \mathrm{D} 2$ & & & 1094 & 1221 & 333.5 & 372.2 & clay, alternating, blue, gray, yellow & Clay & -- & -- & -- & \\
\hline 317 & 4/5-14D2 & & & 1221 & 1222 & 372.2 & 372.5 & clay, hard & Clay & hard & -- & .- & \\
\hline 317 & 4/5-14D2 & & & 1222 & 1292 & 372.5 & 393.8 & clay, blue & Clay & -. & .- & .- & \\
\hline 317 & $4 / 5-14 \mathrm{D} 2$ & & & 1292 & 1299 & 393.8 & 395.9 & clay, gray-green, silty, soft & Clay and sand (or sandstone) & -- & -- & -- & \\
\hline 317 & $4 / 5-14 \mathrm{D} 2$ & & & 1299 & 1365 & 395.9 & 416.1 & clay, gray & Clay & -- & -- & -- & \\
\hline 317 & $4 / 5-14 \mathrm{D} 2$ & & & 1365 & 1370 & 416.1 & 417.6 & $\begin{array}{l}\text { clay, blue } \\
\text { hard zone. change to rock bit }\end{array}$ & Clay & -- & -- & -- & \\
\hline 317 & $4 / 5-14 \mathrm{D} 2$ & & & 1370 & 1371 & 417.6 & 417.9 & necessary to continue drilling & Clay & hard & -. & .- & \\
\hline 317 & $4 / 5-14 \mathrm{D} 2$ & & & 1371 & 1590 & 417.9 & 484.6 & gravel, cemented, and clay & Sand (or sandstone) and gravel & cemented & -- & -- & \\
\hline 317 & $4 / 5-14 \mathrm{D} 2$ & & & 1590 & 1620 & 484.6 & 493.8 & clay, mostly blue, no gravel & Clay & -- & -- & -. & \\
\hline 318 & 4/5-14D1 & 318 & 4/5-14D1 & 0 & 2 & 0.0 & 0.6 & soil & Sand and clay & -. & -- & -- & \\
\hline 318 & 4/5-14D1 & & & 2 & 12 & 0.6 & 3.7 & clay blue & Clay & -- & -- & - & \\
\hline 318 & $4 / 5-14 \mathrm{D} 1$ & & & 12 & 49 & 3.7 & 14.9 & clay, blue & Clay & -. & -- & -. & \\
\hline 318 & $4 / 5-14 \mathrm{D} 1$ & & & 49 & 55 & 14.9 & 16.8 & gravel & Gravel & -- & -- & -- & \\
\hline 318 & $4 / 5-14 \mathrm{D} 1$ & & & 55 & 60 & 16.8 & 18.3 & clay, blue & Clay & ..- & .- & -- & \\
\hline
\end{tabular}


Appendix 3. Lithologic data. - Continued

ISite IDs and well number correspond to locations in Appendix 1, a site ID and well number is listed for each drilled interval for a particular drill hole. For convenience, the site ID and well numbers are repeated at the shallowest drilled interval for each well. leaders

\begin{tabular}{|c|c|c|c|c|c|c|c|c|c|c|c|c|c|}
\hline Site ID & Well number & $\begin{array}{l}\text { Site ID, } \\
\text { top of } \\
\text { well }\end{array}$ & $\begin{array}{l}\text { Well number, } \\
\text { top of well }\end{array}$ & $\begin{array}{c}\text { Top of } \\
\text { interval, in } \\
\text { feet }\end{array}$ & $\begin{array}{c}\text { Base of } \\
\text { interval, in } \\
\text { feet }\end{array}$ & $\begin{array}{c}\text { Top of } \\
\text { interval, in } \\
m\end{array}$ & $\begin{array}{c}\text { Base of } \\
\text { interval, in } \\
\mathrm{m}\end{array}$ & Reported lithologic unit ${ }^{1}$ & Interpreted lithologic class ${ }^{2}$ & $\begin{array}{c}\text { Reported } \\
\text { degree of } \\
\text { cementation or } \\
\text { induration }^{3}\end{array}$ & $\begin{array}{c}\text { Fossils } \\
\text { reported in } \\
\text { interval }^{4}\end{array}$ & $\begin{array}{c}\text { Organic } \\
\text { matter } \\
\text { reported in } \\
\text { interval }^{5}\end{array}$ & $\begin{array}{l}\text { Reported stratigraphic top of geologic } \\
\text { unit }^{6}\end{array}$ \\
\hline 318 & $4 / 5-14 \mathrm{D} 1$ & & & 60 & 85 & 18.3 & 25.9 & clay, yellow & Clay & -- & -- & -- & \\
\hline 318 & 4/5-14D1 & & & 85 & 90 & 25.9 & 27.4 & clay, yellow, and gravel & Clay and gravel & -- & -. & -- & \\
\hline 318 & 4/5-14D1 & & & 90 & 106 & 27.4 & 32.3 & clay, blue & Clay & -- & -- & -- & \\
\hline 318 & $4 / 5-14 \mathrm{D} 1$ & & & 106 & 115 & 32.3 & 35.1 & clay, blue, and gravel & Clay and gravel & -- & -- & -- & \\
\hline 318 & 4/5-14D1 & & & 115 & 185 & 35.1 & 56.4 & $\begin{array}{l}\text { clay, blue } \\
\text {. }\end{array}$ & $\begin{array}{l}\text { Clay } \\
\text { Clution }\end{array}$ & -- & .. & -- & \\
\hline 318 & 4/5-14D1 & & & 185 & 208 & 56.4 & 63.4 & clay, yellow & Clay & -- & -- & -- & \\
\hline 318 & 4/5-14D1 & & & 208 & 220 & 63.4 & 67.1 & $\begin{array}{l}\text { clay, blue } \\
\text { lat }\end{array}$ & Clay & -. & -- & -- & \\
\hline 318 & 4/5-14D1 & & & 220 & 245 & 67.1 & 74.7 & clay, blue and gravel & Clay and gravel & -- & -- & -- & \\
\hline 318 & $4 / 5-14 \mathrm{D} 1$ & & & 245 & 271 & 74.7 & 82.6 & clay, blue, sandy & Clay and sand (or sandstone) & -- & -- & -- & \\
\hline 318 & 4/5-14D1 & & & 271 & 300 & 82.6 & 91.4 & $\begin{array}{l}\text { clay, blue } \\
\text { clumy }\end{array}$ & Clay & -- & -- & -- & \\
\hline 318 & 4/5-14D1 & & & 300 & 324 & 91.4 & 98.8 & clay, blue, and gravel & Clay and gravel & -- & -- & -- & \\
\hline 318 & 4/5-14D1 & & & 324 & 412 & 98.8 & 125.6 & $\begin{array}{l}\text { clay, yellow } \\
\text { latave }\end{array}$ & Clay & -- & -- & -- & \\
\hline 318 & $4 / 5-14 \mathrm{D} 1$ & & & 412 & 495 & 125.6 & 150.9 & clay, yellow, sandy & Clay and sand (or sandstone) & -- & -- & -- & \\
\hline 318 & 4/5-14D1 & & & 495 & 502 & 150.9 & 153.0 & clay, yellow, sandy, and gravel & Clay, sand, and gravel & -- & -- & -- & \\
\hline $\begin{array}{l}100 \\
318\end{array}$ & 4/5-14D1 & & & 502 & 512 & 153.0 & 156.1 & yellow sand and gravel & Sand (or sandstone) and gravel & -. & -. & -. & \\
\hline 318 & 4/5-14D1 & & & 512 & 535 & 156.1 & 163.1 & yellow sand and gravel & Sand (or sandstone) and gravel & -- & - & -- & \\
\hline 318 & 4/5-14D1 & & & 535 & 537 & 163.1 & 163.7 & yellow cement and gravel & Clay, sand, and gravel & cemented & -- & -- & \\
\hline 318 & $4 / 5-14 \mathrm{D} 1$ & & & 537 & 540 & 163.7 & 164.6 & clay, yellow, and gravel & Clay and gravel & -- & -- & -- & \\
\hline 319 & 4/5-14L1 & 319 & 4/5-14L1 & 0 & 58 & 0.0 & 17.7 & dark "surface" mud and peat & Clay and sand (or sandstone) & -- & -- & peat & \\
\hline $\begin{array}{l}199 \\
319\end{array}$ & $4 / 5-14 \mathrm{~L} 1$ & & & 58 & 66 & 17.7 & 20.1 & $\begin{array}{l}\text { clay, tough light blue } \\
\text { cod }\end{array}$ & Clay & -. & -- & peat & \\
\hline 319 & 4/5-14L1 & & & 66 & 86 & 20.1 & 26.2 & clay, tough brownish-yellow & Clay & -- & -- & -- & \\
\hline 319 & 4/5-14L1 & & & 86 & 100 & 26.2 & 30.5 & $\begin{array}{l}\text { clay, tough light blue } \\
\text { chent }\end{array}$ & Clay & -- & -- & -- & \\
\hline 319 & 4/5-14L1 & & & 100 & 135 & 30.5 & 41.1 & clay, soft dark blue & Clay & -- & -- & -- & \\
\hline 319 & 4/5-14L1 & & & 135 & 151 & 41.1 & 46.0 & blue "tule bed" & Clay and sand (or sandstone) & -. & -. & tule & \\
\hline 319 & 4/5-14L1 & & & 151 & 166 & 46.0 & 50.6 & clay blue & Clay & -- & .. & -. & \\
\hline 319 & 4/5-14L1 & & & 166 & 170 & 50.6 & 51.8 & black "tule bed" & Clay and sand (or sandstone) & -- & -- & tule & \\
\hline 319 & 4/5-14L1 & & & 170 & 175 & 51.8 & 53.3 & clay, clay & Clay & - & -- & -- & \\
\hline 319 & 4/5-14L1 & & & 175 & 179 & 53.3 & 54.6 & sandy blue clay with small gravel & Clay, sand, and gravel & .. & .. & .. & \\
\hline 319 & 4/5-14L1 & & & 179 & 202 & 54.6 & 61.6 & clay, blue & Clay & .- & -. & .. & \\
\hline 319 & 4/5-14L1 & & & 202 & 207 & 61.6 & 63.1 & blue clay with small gravel & Clay and gravel & -- & -- & -- & \\
\hline 319 & 4/5-14L1 & & & 207 & 221 & 63.1 & 67.4 & clay, brown & Clay & -- & -- & - & \\
\hline 319 & 4/5-14L1 & & & 221 & 244 & 67.4 & 74.4 & clay, blue & Clay & -- & -- & -- & \\
\hline 319 & 4/5-14L1 & & & 244 & 246 & 74.4 & 75.0 & black "tule bed" & Clay and sand (or sandstone) & - & -. & tule & \\
\hline 319 & 4/5-14L1 & & & 246 & 250 & 75.0 & 76.2 & gray and blue clay with gravel & Clay and gravel & .- & -. & -- & \\
\hline 319 & 4/5-14L1 & & & 250 & 252 & 76.2 & 76.8 & $\begin{array}{l}\text { sandy blue clay } \\
\text { coarse blue and gray gravel and }\end{array}$ & Clay and sand (or sandstone) & -. & -- & - & \\
\hline 319 & 4/5-14L1 & & & 252 & 255 & 76.8 & 77.7 & rocks & Gravel & -- & -- & -- & \\
\hline 319 & 4/5-14L1 & & & 255 & 285 & 77.7 & 86.9 & blue clay with streaks of sand & Clay and sand (or sandstone) & - & -- & -. & \\
\hline 319 & 4/5-14L1 & & & 285 & 298 & 86.9 & 90.8 & silty mud and "weed" & Clay and sand (or sandstone) & -- & -- & -- & \\
\hline 319 & 4/5-14L1 & & & 298 & 300 & 90.8 & 91.4 & dark sticky clay & Clay & -. & .. & .. & \\
\hline 319 & 4/5-14L1 & & & 300 & 316 & 91.4 & 96.3 & sand, silt, soft blue clay & Sand and clay & -- & -- & -- & \\
\hline 319 & 4/5-14L1 & & & 316 & 327 & 96.3 & 99.7 & black tough mud and tule & Clay and sand (or sandstone) & -- & -- & tule & \\
\hline 319 & 4/5-14L1 & & & 327 & 364 & 99.7 & 110.9 & $\begin{array}{l}\text { clay, blue, sand streaks } \\
\text { hard blue clay with embedded }\end{array}$ & Clay and sand (or sandstone) & -- & -- & -- & \\
\hline 319 & 4/5-14L1 & & & 364 & 371 & 110.9 & 113.1 & boulders & Clay and gravel & hard & .. & .. & \\
\hline 319 & 4/5-14L1 & & & 371 & 385 & 113.1 & 117.3 & clay, blue & Clay & -- & -- & -- & \\
\hline 319 & 4/5-14L1 & & & 385 & 451 & 117.3 & 137.5 & clay, brown, and rocks & Clay and gravel & -- & -- & -. & \\
\hline 319 & 4/5-14L1 & & & 451 & 466 & 137.5 & 142.0 & tough dark gray blue clay & Clay & hard & -- & - & \\
\hline 319 & 4/5-14L1 & & & 466 & 490 & 142.0 & 149.4 & clay, brownish-yellow & Clay & -- & -- & -. & \\
\hline 319 & $\begin{array}{l}4 / 3-141 \\
4 / 5-14 L 1\end{array}$ & & & $\begin{array}{l}400 \\
490\end{array}$ & 527 & 149.4 & $\begin{array}{l}14.4 \\
160.6\end{array}$ & $\begin{array}{l}\text { shale, gray } \\
\text { clay, brownish-yellow, slightly }\end{array}$ & Clay and sand (or sandstone) & cemented & -- & - & \\
\hline 319 & 4/5-14L1 & & & 527 & 565 & 160.6 & 172.2 & sandy & Clay and sand (or sandstone) & -. & .. & .. & \\
\hline 319 & 4/5-14L1 & & & 565 & 595 & 172.2 & 181.4 & gray sticky clay & Clay & -- & -- & - & \\
\hline 319 & 4/5-14L1 & & & 595 & 610 & 181.4 & 185.9 & gray sticky clay & Clay & -. & -- & -. & \\
\hline 319 & 4/5-14L1 & & & 610 & 645 & 185.9 & 196.6 & gravel and sand & Sand (or sandstone) and gravel & -. & -. & .. & \\
\hline 319 & 4/5-14L1 & & & 645 & 660 & 196.6 & 201.2 & clay, yellow & Clay & - & -- & -- & \\
\hline 320 & $9 / 7-26 \mathrm{C} 1$ & 320 & $9 / 7-26 \mathrm{C} 1$ & 0 & 5 & 0.0 & 1.5 & surface & Sand and clay & - & - & -. & \\
\hline 320 & $9 / 7-26 C 1$ & & & 5 & 60 & 1.5 & 18.3 & clay, yellow and boulders & Clay and gravel & .- & .. & .. & \\
\hline 320 & $9 / 7-26 \mathrm{C} 1$ & & & 60 & 85 & 18.3 & 25.9 & tuff & Ash or tuff & -- & .- & -- & Tsv \\
\hline 320 & $9 / 7-26 \mathrm{C} 1$ & & & 85 & 150 & 25.9 & 45.7 & lava ash & Basalt & -- & -- & -- & \\
\hline
\end{tabular}


Appendix 3. Lithologic data. - Continued

ISite IDs and well number correspond to locations in Appendix 1, a site ID and well number is listed for each drilled interval for a particular drill hole. For convenience, the site ID and well numbers are repeated at the shallowest drilled interval for each well. leaderst.

\begin{tabular}{|c|c|c|c|c|c|c|c|c|c|c|c|c|c|}
\hline Site ID & Well number & $\begin{array}{l}\text { Site ID, } \\
\text { top of } \\
\text { well }\end{array}$ & $\begin{array}{l}\text { Well number, } \\
\text { top of well }\end{array}$ & $\begin{array}{c}\text { Top of } \\
\text { interval, in } \\
\text { feet }\end{array}$ & $\begin{array}{c}\text { Base of } \\
\text { interval, in } \\
\text { feet }\end{array}$ & $\begin{array}{c}\text { Top of } \\
\text { interval, in } \\
m\end{array}$ & $\begin{array}{c}\text { Base of } \\
\text { interval, in } \\
\mathrm{m}\end{array}$ & Reported lithologic unit ${ }^{1}$ & Interpreted lithologic class ${ }^{2}$ & $\begin{array}{c}\text { Reported } \\
\text { degree of } \\
\text { cementation or } \\
\text { induration }^{3}\end{array}$ & $\begin{array}{c}\text { Fossils } \\
\text { reported in } \\
\text { interval }^{4}\end{array}$ & $\begin{array}{c}\text { Organic } \\
\text { matter } \\
\text { reported in } \\
\text { interval }^{5}\end{array}$ & $\begin{array}{l}\text { Reported stratigraphic top of geologic } \\
\text { unit }^{6}\end{array}$ \\
\hline 320 & $9 / 7-26 \mathrm{C} 1$ & & & 150 & 180 & 45.7 & 54.9 & lava rock, gray & Basalt & hard & -- & -- & \\
\hline 320 & $9 / 7-26 \mathrm{Cl}$ & & & 180 & 220 & 54.9 & 67.1 & basalt & Basalt & -- & -- & -- & \\
\hline 320 & $9 / 7-26 \mathrm{C} 1$ & & & 220 & 275 & 67.1 & 83.8 & tuff & Ash or tuff & -- & -- & -- & \\
\hline 320 & $9 / 7-26 \mathrm{Cl}$ & & & 275 & 310 & 83.8 & $\begin{array}{l}90.0 \\
94.5\end{array}$ & shale, hard, brown & Clay and sand (or sandstone) & hard & -. & -- & \\
\hline 320 & $9 / 7-26 \mathrm{C} 1$ & & & 310 & 340 & 94.5 & 103.6 & tuff & Ash or tuff & -- & -- & -- & \\
\hline 320 & $9 / 7-26 \mathrm{C} 1$ & & & 340 & 365 & 103.6 & 111.3 & basalt & Basalt & -- & -- & -- & \\
\hline 320 & $9 / 7-26 \mathrm{C} 1$ & & & 365 & 385 & 111.3 & 117.3 & lava ash & Basalt & -- & -- & -- & \\
\hline 320 & $9 / 7-26 \mathrm{C} 1$ & & & 385 & 450 & 117.3 & 137.2 & tuff & Ash or tuff & -- & -- & -- & \\
\hline 321 & $9 / 7-26 \mathrm{G} 1$ & 321 & 9/7-26G1 & 0 & 5 & 0.0 & 1.5 & surface & Sand and clay & -- & -- & -- & Slope wash \\
\hline 321 & $9 / 7-26 \mathrm{G} 1$ & & & 5 & 15 & 1.5 & 4.6 & sand and gravel & Sand (or sandstone) and gravel & -- & -- & -- & \\
\hline 321 & 9/7-26G1 & & & 15 & 30 & 4.6 & 9.1 & $\begin{array}{l}\text { boulders } \\
\text { bover }\end{array}$ & Gravel & -- & -- & -- & \\
\hline 321 & $9 / 7-26 \mathrm{G} 1$ & & & 30 & 40 & 9.1 & 12.2 & basalt, hard & Basalt & hard & .- & .- & Tsv \\
\hline 321 & $9 / 7-26 \mathrm{G} 1$ & & & 40 & 55 & 12.2 & 16.8 & basalt, medium soft & Basalt & -- & -- & -- & \\
\hline 321 & $9 / 7-26 \mathrm{G} 1$ & & & 55 & 305 & 16.8 & 93.0 & tuff & Ash or tuff & -- & -- & -. & \\
\hline 322 & 8/6-14R1 & 322 & 8/6-14R1 & 0 & 15 & 0.0 & 4.6 & soil & Sand and clay & -- & -- & -- & Qyal \\
\hline 322 & $8 / 6-14 \mathrm{R} 1$ & & & 15 & 25 & 4.6 & 7.6 & gravel and boulders & Gravel & -- & -- & -- & \\
\hline 322 & $8 / 6-14 \mathrm{R} 1$ & & & 25 & 73 & 7.6 & 22.3 & tuff, yellow & Ash or tuff & -- & -- & -- & Tsv \\
\hline 322 & $8 / 6-14 R 1$ & & & 73 & 97 & 22.3 & 29.6 & tuff, gray & Ash or tuff & -- & .- & .- & \\
\hline 322 & $8 / 6-14 R 1$ & & & 97 & 107 & 29.6 & 32.6 & rock, boulders, sand & Sand (or sandstone) and gravel & hard & -- & -- & \\
\hline 322 & $8 / 6-14 R 1$ & & & 107 & 123 & 32.6 & 37.5 & rock, boulders, sand, clay, yellow & Clay, sand, and gravel & hard & -- & -.- & \\
\hline 322 & 8/6-14R1 & & & 123 & 132 & 37.5 & 40.2 & gravel and sand, fine & Sand (or sandstone) and gravel & -- & - & -- & \\
\hline 323 & 8/6-23M1 & 323 & 8/6-23M1 & 0 & 4 & 0.0 & 1.2 & soil and boulders & Clay, sand, and gravel & -- & .. & -- & Qyal and Qoal \\
\hline 323 & 8/6-23M1 & & & 4 & 55 & 1.2 & 16.8 & "hardpan" and boulders & Clay and gravel & hard & ..- & .. & \\
\hline 323 & 8/6-23M1 & & & 55 & 57 & 16.8 & 17.4 & gravel, water & Gravel & -- & -- & -- & \\
\hline 323 & 8/6-23M1 & & & 57 & 97 & 17.4 & 29.6 & clay and boulders & Clay and gravel & -. & -- & -- & \\
\hline 323 & 8/6-23M1 & & & 97 & 100 & 29.6 & 30.5 & $\begin{array}{l}\text { gravel, water } \\
\text { nats }\end{array}$ & Gravel & -- & -- & -- & \\
\hline 323 & $8 / 6-23 \mathrm{M} 1$ & & & 100 & 129 & 30.5 & 39.3 & $\begin{array}{l}\text { clay and boulders, blue } \\
\text { chater }\end{array}$ & Clay and gravel & -- & -- &.- & \\
\hline 324 & 7/5-15A1 & 324 & 7/5-15A1 & 0 & 4 & 0.0 & 1.2 & soil & Sand and clay & -- & -- & -- & Qyal and Qoal \\
\hline 324 & 7/5-15A1 & & & 4 & 6 & 1.2 & 1.8 & & Clay and sand (or sandstone) & -- & -- & - & \\
\hline 324 & 7/5-15A1 & & & 6 & 32 & 1.8 & 9.8 & $\begin{array}{l}\text { clay, boulders; some water at } 15 \\
\text { ft. }\end{array}$ & Clay and gravel & .. & .. & .. & \\
\hline 324 & 7/5-15A1 & & & 32 & 40 & $\begin{array}{l}1.0 \\
9.8\end{array}$ & $\begin{array}{l}1.0 \\
12.2\end{array}$ & boulders and clay; some water & Clay and gravel & -. & -- & -. & \\
\hline 324 & 7/5-15A1 & & & 40 & 95 & 12.2 & 29.0 & clay and boulders & Clay and gravel & -. & .- & .. & \\
\hline 324 & $7 / 5-15 \mathrm{~A} 1$ & & & 95 & 105 & 29.0 & 32.0 & boulders; some water & Gravel & .. & -. & .- & \\
\hline 324 & 7/5-15A1 & & & 105 & 355 & 32.0 & 108.2 & boulders and clay & Clay and gravel & -- & -- & -- & \\
\hline 325 & $7 / 5-14 \mathrm{G} 1$ & 325 & 7/5-14G1 & 0 & 5 & 0.0 & 1.5 & surface & Sand and clay & -- & -- & -- & Qoal and Qyal \\
\hline 325 & $7 / 5-14 \mathrm{G} 1$ & & & 5 & 20 & 1.5 & 6.1 & clay, yellow & Clay & -. & -- & -. & Qual a diu Qyal \\
\hline 325 & $7 / 5-14 \mathrm{G} 1$ & & & 20 & 50 & 6.1 & 15.2 & $\begin{array}{l}\text { clay and some boulders, yellow } \\
\text {. }\end{array}$ & Clay and gravel & -- & -- & -- & \\
\hline 325 & $7 / 5-14 \mathrm{G} 1$ & & & 50 & 65 & $\begin{array}{l}.1 \\
15.2\end{array}$ & 19.8 & gravel, free & Gravel & .. & .. & .. & \\
\hline 325 & $7 / 5-14 \mathrm{G} 1$ & & & 65 & 227 & 19.8 & 69.2 & clay, sand, and gravel, yellow & Clay, sand, and gravel & -- & -- & -- & \\
\hline 325 & $7 / 5-14 \mathrm{G} 1$ & & & 227 & 232 & 69.2 & 70.7 & gravel, free & Gravel & -. & -- & -- & \\
\hline 325 & $7 / 5-14 \mathrm{G} 1$ & & & 232 & 255 & 70.7 & 77.7 & volcanic rock & Ash or tuff & .- & -. & .. & Tsv \\
\hline 325 & 7/5-14G1 & & & 255 & 265 & 77.7 & 80.8 & volcanic clay, red & Ash or tuff & -- & -- & -- & \\
\hline 326 & 7/5-16ட1 & 326 & 7/5-16L1 & 0 & 4 & 0.0 & 1.2 & soil & Sand and clay & -. & -- & - & Oyal and Qoal \\
\hline 326 & 7/5-16L1 & & & 4 & 20 & 1.2 & 6.1 & soil and boulders & Clay, sand, and gravel & -- & -- & -- & \\
\hline 326 & 7/5-16ட1 & & & 20 & 57 & 6.1 & 17.4 & clay, yellow; and boulders & Clay and gravel & .- & .- & .. & \\
\hline 326 & 7/5-16L1 & & & 57 & 70 & 17.4 & 21.3 & clay, yellow & Clay & -- & -- & -- & \\
\hline 326 & 7/5-16ட1 & & & 70 & 71 & 21.3 & 21.6 & gravel and small amount of water & Gravel & -. & -. & .. & \\
\hline 326 & 7/5-16L1 & & & 71 & 120 & 21.6 & 36.6 & $\begin{array}{l}\text { clay and boulders } \\
\text { nat }\end{array}$ & Clay and gravel & .- & -. & ..- & \\
\hline 326 & 7/5-16ட1 & & & 120 & 121 & 36.6 & 36.9 & gravel and water & Gravel & - & - &.- & \\
\hline 326 & 7/5-16ட1 & & & 121 & 218 & 36.9 & 66.4 & clay and boulders & Clay and gravel & .- & .. & .. & \\
\hline 326 & $7 / 5-16 \mathrm{~L} 1$ & & & 218 & 220 & 66.4 & 67.1 & gravel and water & Gravel & -- & -. & -- & \\
\hline 326 & 7/5-16L1 & & & 220 & 221 & 67.1 & 67.4 & blue cement and gravel & Clay and gravel & cemented & -. & .- & \\
\hline 327 & $7 / 5-17 \mathrm{J1}$ & 327 & 7/5-17J1 & 0 & 4 & 0.0 & 1.2 & soil & Sand and clay & - & -. & .. & Tsv \\
\hline 327 & $7 / 5-17 \mathrm{J1}$ & & & 4 & 55 & 1.2 & 16.8 & clay, yellow; and broken rock & Clay and gravel & -- & -- & - & \\
\hline 327 & $7 / 5-17 \mathrm{J1}$ & & & 55 & 70 & 16.8 & 21.3 & rock, broken, water & Ash or tuff & hard & .. & .. & \\
\hline 327 & $7 / 5-17 \mathrm{J1}$ & & & 70 & 86 & 21.3 & 26.2 & basalt & Basalt & -. & -. & -. & \\
\hline 327 & $7 / 5-17 J 1$ & & & 86 & 130 & 26.2 & 39.6 & basalt, broken; water & Basalt & -- & -- & -. & \\
\hline 327 & $7 / 5-17 \mathrm{J1}$ & & & 130 & 190 & 39.6 & 57.9 & sandstone & Sandstone & cemented & -. & -. & \\
\hline
\end{tabular}


Appendix 3. Lithologic data. - Continued

[Site IDs and well number correspond to locations in Appendix 1, a site ID and well number is listed for each drilled interval for a particular drill hole. For convenience, the site ID and well numbers are repeated at the shallowest drilled interval for each well. leaderst.

\begin{tabular}{|c|c|c|c|c|c|c|c|c|c|c|c|c|c|}
\hline Site ID & Well number & $\begin{array}{l}\text { Site ID, } \\
\text { top of } \\
\text { well }\end{array}$ & $\begin{array}{l}\text { Well number, } \\
\text { top of well }\end{array}$ & $\begin{array}{c}\text { Top of } \\
\text { interval, in } \\
\text { feet }\end{array}$ & $\begin{array}{c}\text { Base of } \\
\text { interval, in } \\
\text { feet }\end{array}$ & $\begin{array}{c}\text { Top of } \\
\text { interval, in } \\
m\end{array}$ & $\begin{array}{c}\text { Base of } \\
\text { interval, in } \\
\mathrm{m}\end{array}$ & Reported lithologic unit ${ }^{1}$ & Interpreted lithologic class ${ }^{2}$ & $\begin{array}{c}\text { Reported } \\
\text { degree of } \\
\text { cementation or } \\
\text { induration }^{3}\end{array}$ & $\begin{array}{c}\text { Fossils } \\
\text { reported in } \\
\text { interval }^{4}\end{array}$ & $\begin{array}{c}\text { Organic } \\
\text { matter } \\
\text { reported in } \\
\text { interval }^{5}\end{array}$ & $\begin{array}{l}\text { Reported stratigraphic top of geologic } \\
\text { unit }^{6}\end{array}$ \\
\hline 327 & $7 / 5-17 \mathrm{J1}$ & & & 190 & 250 & 57.9 & 76.2 & basalt & Basalt & -- & -- & -- & \\
\hline 327 & $7 / 5-17 \mathrm{J1}$ & & & 250 & 340 & 76.2 & 103.6 & sandstone, hard & Sandstone & cemented & -- & -- & \\
\hline 327 & 7/5-17J1 & & & 340 & 430 & 103.6 & 131.1 & basalt & Basalt & -- & -. & -- & \\
\hline 327 & $7 / 5-17 J 1$ & & & 430 & 470 & 131.1 & 143.3 & sandstone & Sandstone & cemented & -. & -- & \\
\hline 327 & $7 / 5-17 J 1$ & & & 470 & 480 & 143.3 & 146.3 & shale and broken rock & Clay, sand, and gravel & cemented & -- & -- & \\
\hline 327 & $7 / 5-17 \mathrm{J1}$ & & & 480 & 534 & 146.3 & 162.8 & sandstone & Sandstone & cemented & -- & -- & \\
\hline 327 & $7 / 5-17 \mathrm{J1}$ & & & 534 & 537 & 162.8 & 163.7 & sand, gravel and water & Sand (or sandstone) and gravel & -- & -- & -- & \\
\hline 327 & $7 / 5-17 \mathrm{J1}$ & & & 537 & 564 & 163.7 & 171.9 & sandstone & Sandstone & cemented & -. & -- & \\
\hline 328 & $7 / 5-16 \mathrm{~N} 1$ & 328 & $7 / 5-16 \mathrm{~N} 1$ & 0 & 6 & 0.0 & 1.8 & soil & Sand and clay & -- & -. & -- & Qyal and Qoal \\
\hline 328 & 7/5-16N1 & & & 6 & 35 & 1.8 & 10.7 & clay & Clay & -- & -- & -- & \\
\hline 328 & $7 / 5-16 \mathrm{~N} 1$ & & & 35 & 40 & 10.7 & 12.2 & clay and gravel, water & Clay and gravel & -- & -- & -- & \\
\hline 328 & $7 / 5-16 \mathrm{~N} 1$ & & & 40 & 140 & 12.2 & 42.7 & clay and boulders & Clay and gravel & -- & -- & -- & \\
\hline 328 & $7 / 5-16 \mathrm{~N} 1$ & & & 140 & 150 & 42.7 & 45.7 & clay and gravel, water & Clay and gravel & -- & -- & -- & \\
\hline 328 & $7 / 5-16 \mathrm{~N} 1$ & & & 150 & 213 & 45.7 & 64.9 & clay & Clay & -- & -- & -- & \\
\hline 328 & 7/5-16N1 & & & 213 & 218 & 64.9 & 66.4 & clay and gravel, blue, water & Clay and gravel & -- & -- & -- & \\
\hline 328 & $7 / 5-16 \mathrm{~N} 1$ & & & 218 & 225 & 66.4 & 68.6 & $\begin{array}{l}\text { clay, blue } \\
\text { clet, }\end{array}$ & Clay & - & -- & -- & \\
\hline 328 & $7 / 5-16 \mathrm{~N} 1$ & & & 225 & 252 & 68.6 & 76.8 & clay and gravel, blue, water & Clay and gravel & -- & -- & -- & \\
\hline 329 & 7/5-23L1 & 329 & 7/5-23L1 & 0 & 20 & 0.0 & 6.1 & soil & Sand and clay & ..- & .- & -. & Qyal and Qoal \\
\hline 329 & $7 / 5-23\llcorner 1$ & & & 20 & 35 & 6.1 & 10.7 & clay, yellow & Clay & .. & ..- & -. & \\
\hline 329 & 7/5-23L1 & & & 35 & 37 & 10.7 & 11.3 & clay, yellow; sand and gravel & Clay, sand, and gravel & -. & -- & -- & \\
\hline 329 & $7 / 5-23 L 1$ & & & 37 & 50 & 11.3 & 15.2 & clay, sandy, yellow; and gravel & Clay, sand, and gravel & -- & -. & .- & \\
\hline 329 & $7 / 5-23 L 1$ & & & 50 & 59 & 15.2 & 18.0 & sand and gravel, blue & Sand (or sandstone) and gravel & - & -- & -- & \\
\hline 329 & $7 / 5-23\llcorner 1$ & & & 59 & 77 & 18.0 & 23.5 & clay, sandy, yellow & Clay and sand (or sandstone) & .- & .. & .. & \\
\hline 329 & $7 / 5-23\llcorner 1$ & & & 77 & 83 & 23.5 & 25.3 & clay, sandy, blue and gravel & Clay, sand, and gravel & .- & ..- & .. & \\
\hline 329 & 7/5-23L1 & & & 83 & 89 & 25.3 & 27.1 & gravel & Gravel & -. & -- & -- & \\
\hline 329 & $7 / 5-23 L 1$ & & & 89 & 100 & 27.1 & 30.5 & clay, blue & Clay & -. & .- & .- & \\
\hline 329 & 7/5-23L1 & & & 100 & 127 & 30.5 & 38.7 & clay, yellow and gravel & Clay and gravel & -- & -- & -- & \\
\hline 329 & 7/5-23L1 & & & 127 & 132 & 38.7 & 40.2 & $\begin{array}{l}\text { clay, yellow } \\
\text { cin grave }\end{array}$ & $\begin{array}{l}\text { Clay } \\
\text { Clut giver }\end{array}$ & -- & -- &.- & \\
\hline 329 & 7/5-23L1 & & & 132 & 134 & 40.2 & 40.8 & $\begin{array}{l}\text { clay, blue } \\
\text { nats }\end{array}$ & Clay & .. & -. & .- & \\
\hline 329 & 7/5-23L1 & & & 134 & 150 & 40.8 & 45.7 & clay, yellow and gravel & Clay and gravel & .. & .. & .. & \\
\hline 330 & 7/5-27D1 & 330 & 7/5-27D1 & 0 & 3 & 0.0 & 0.9 & soil & Sand and clay & -- & -- & .- & Qyal and Qoal \\
\hline 330 & 7/5-27D1 & & & 3 & 30 & 0.9 & 9.1 & clay & Clay & -- & -- & -- & \\
\hline 330 & $7 / 5-27 \mathrm{D} 1$ & & & 30 & 40 & 9.1 & $\begin{array}{l}.1 \\
12.2\end{array}$ & gravel, water & Gravel & - & -- & -. & \\
\hline 330 & $7 / 5-27 \mathrm{D} 1$ & & & 40 & 100 & 12.2 & 30.5 & clay & Clay & .- & .- & .. & \\
\hline 330 & $7 / 5-27 \mathrm{D} 1$ & & & 100 & 190 & 30.5 & 57.9 & clay, gravel seams; some water & Clay and gravel & -- & -- & -- & \\
\hline 330 & $7 / 5-27 \mathrm{D} 1$ & & & 190 & 260 & 57.9 & 79.2 & clay, yellow & Clay & -- & -- & -- & \\
\hline 330 & $7 / 5-27 \mathrm{D} 1$ & & & 260 & 263 & 79.2 & 80.2 & mud, white; some water & Clay and sand (or sandstone) & -. & -- & -. & Tsv \\
\hline 330 & $7 / 5-27 \mathrm{D} 1$ & & & 263 & 285 & 80.2 & 86.9 & clay, white & Clay & -. & -- & -. & 150 \\
\hline 330 & $7 / 5-27 \mathrm{D} 1$ & & & 285 & 295 & 86.9 & 89.9 & clay, brownish-gray & Clay & .- & -- & -. & \\
\hline 330 & $7 / 5-27 \mathrm{D} 1$ & & & 295 & 321 & 89.9 & 97.8 & $\begin{array}{l}\text { clay, white } \\
\text { lation }\end{array}$ & Clay & .. & .. & .. & \\
\hline 330 & $7 / 5-27 \mathrm{D} 1$ & & & 321 & 335 & 97.8 & 102.1 & clay, brown & Clay & -- & -- & - & \\
\hline 330 & $7 / 5-27 \mathrm{D} 1$ & & & 335 & 345 & 102.1 & 105.2 & rock, red soft; some water & Ash or tuff & hard & -- & -. & \\
\hline 330 & $7 / 5-27 \mathrm{D} 1$ & & & 345 & 360 & 105.2 & 109.7 & rock, red, hard & Ash or tuff & hard & -. & .- & \\
\hline 330 & $7 / 5-27 \mathrm{D} 1$ & & & 360 & 410 & 109.7 & 125.0 & sandstone & Sandstone & cemented & -- & -- & \\
\hline 331 & 7/4-30L1 & 331 & 7/4-30L1 & 0 & 21 & 0.0 & 6.4 & soil and boulders & Clay, sand, and gravel & -- & -- & - & Qyal \\
\hline 331 & 7/4-30L1 & & & 21 & 22 & 6.4 & 6.7 & water & Clay, sand, and gravel & -- & -- & -- & \\
\hline 331 & $7 / 4-30\llcorner 1$ & & & 22 & 40 & 6.7 & 12.2 & cement and boulders & Clay, sand, and gravel & cemented & .- & .. & \\
\hline $\begin{array}{l}351 \\
331\end{array}$ & 7/4-30L1 & & & 40 & 60 & $\begin{array}{l}0.1 \\
12.2\end{array}$ & 18.3 & tuff, gray & Ash or tuff & - & -- & -- & Tsv \\
\hline 331 & 7/4-30L1 & & & 60 & 140 & 18.3 & 42.7 & tuff, brown & Ash or tuff & -- & -- & -- & \\
\hline 331 & 7/4-30L1 & & & 140 & 168 & 42.7 & 51.2 & tuff, green & Ash or tuff & -. & .. & -. & \\
\hline 331 & 7/4-30L1 & & & 168 & 171 & 51.2 & 52.1 & tuff, gray and water & Ash or tuff & -- & -- & -- & \\
\hline 332 & 7/4-31E1 & 332 & 7/4-31E1 & 0 & 7 & 0.0 & 2.1 & soil & Sand and clay & .. & .. & .. & Oyal and Qoal \\
\hline 332 & 7/4-31E1 & & & 7 & 40 & 2.1 & 12.2 & clay & Clay & -. & .- & -. & \\
\hline 332 & 7/4-31E1 & & & 40 & 65 & 12.2 & 19.8 & gravel & Gravel & -- & $\ldots$ & .. & \\
\hline 332 & 7/4-31E1 & & & 65 & 109 & 19.8 & 33.2 & clay & Clay & .- & .- & .. & \\
\hline 332 & 7/4-31E1 & & & 109 & 140 & 33.2 & 42.7 & clay and boulders & Clay and gravel & -- & -- & -- & \\
\hline 332 & 7/4-31E1 & & & 140 & 242 & 42.7 & 73.8 & clay & $\begin{array}{l}\text { Clay } \\
\text { Clin }\end{array}$ & - & - & - & \\
\hline 332 & 7/4-31E1 & & & 242 & 272 & 73.8 & 82.9 & gravel, small & Gravel & -- & -- & -- & \\
\hline 333 & 7/5-36N1 & 333 & $7 / 5-36 \mathrm{~N} 1$ & 0 & 3 & 0.0 & 0.9 & soil & Sand and clay &.- & $\ldots$ &.- & Qoal \\
\hline 333 & $7 / 5-36 \mathrm{~N} 1$ & & & 3 & 12 & 0.9 & 3.7 & clay, brown & Clay & -- & -- & -- & \\
\hline 333 & $7 / 5-36 \mathrm{~N} 1$ & & & 12 & 20 & 3.7 & 6.1 & clay, hard & Clay & hard & .- & -- & \\
\hline
\end{tabular}


Appendix 3. Lithologic data. - Continued

[Site IDs and well number correspond to locations in Appendix 1, a site ID and well number is listed for each drilled interval for a particular drill hole. For convenience, the site ID and well numbers are repeated at the shallowest drilled interval for each well. leaders

\begin{tabular}{|c|c|c|c|c|c|c|c|c|c|c|c|c|c|}
\hline Site ID & Well number & $\begin{array}{l}\text { Site ID, } \\
\text { top of } \\
\text { well }\end{array}$ & $\begin{array}{l}\text { Well number, } \\
\text { top of well }\end{array}$ & $\begin{array}{c}\text { Top of } \\
\text { interval, in } \\
\text { feet }\end{array}$ & $\begin{array}{c}\text { Base of } \\
\text { interval, in } \\
\text { feet }\end{array}$ & $\begin{array}{c}\text { Top of } \\
\text { interval, in } \\
m\end{array}$ & $\begin{array}{c}\text { Base of } \\
\text { interval, in } \\
\mathrm{m}\end{array}$ & Reported lithologic unit ${ }^{1}$ & Interpreted lithologic class ${ }^{2}$ & $\begin{array}{c}\text { Reported } \\
\text { degree of } \\
\text { cementation or } \\
\text { induration } \\
\end{array}$ & $\begin{array}{c}\text { Fossils } \\
\text { reported in } \\
\text { interval }^{4}\end{array}$ & $\begin{array}{c}\text { Organic } \\
\text { matter } \\
\text { reported in } \\
\text { interval }^{5}\end{array}$ & $\begin{array}{l}\text { Reported stratigraphic top of geologic } \\
\text { unit }^{6}\end{array}$ \\
\hline $\begin{array}{l}333 \\
333\end{array}$ & $\begin{array}{l}7 / 5-36 \mathrm{~N} 1 \\
7 / 5-36 \mathrm{~N} 1\end{array}$ & & & $\begin{array}{l}20 \\
26\end{array}$ & $\begin{array}{l}26 \\
34\end{array}$ & $\begin{array}{l}6.1 \\
7.9\end{array}$ & & $\begin{array}{l}\text { gravel, water } \\
\text { clay, hard }\end{array}$ & $\begin{array}{l}\text { Gravel } \\
\text { Clay }\end{array}$ & $\begin{array}{c}-- \\
\text { hard }\end{array}$ & $\overline{--}$ & $\begin{array}{l}-- \\
--\end{array}$ & \\
\hline 333 & $7 / 5-36 \mathrm{~N} 1$ & & & $\begin{array}{l}26 \\
34\end{array}$ & $\begin{array}{l}34 \\
48\end{array}$ & $\begin{array}{l}7.9 \\
10.4\end{array}$ & $\begin{array}{l}10.4 \\
14.6\end{array}$ & $\begin{array}{l}\text { clay, hard } \\
\text { clay, white }\end{array}$ & $\begin{array}{l}\text { Clay } \\
\text { Clay }\end{array}$ & hard & $\begin{array}{l}-- \\
-\end{array}$ & -- & Tsv \\
\hline 333 & $7 / 5-36 \mathrm{~N} 1$ & & & 48 & 97 & 14.6 & 29.6 & tuff, brown and gravel & Ash or tuff & -- & -. & -- & \\
\hline 333 & $7 / 5-36 \mathrm{~N} 1$ & & & 97 & 103 & 29.6 & 31.4 & gravel, water & Gravel & -- & -- & -- & \\
\hline 333 & $7 / 5-36 N 1$ & & & 103 & 104 & 31.4 & 31.7 & tuff & Ash or tuff & .- & -- & -- & \\
\hline 334 & $6 / 5-1 C 3$ & 334 & 6/5-1C3 & 0 & 3 & 0.0 & 0.9 & soil & Sand and clay & -- & -- & -- & Qoal \\
\hline 334 & $6 / 5-1 \mathrm{C3}$ & & & 3 & 42 & 0.9 & 12.8 & clay, boulders, yellow & Clay and gravel & -- & -- & -- & \\
\hline 334 & $6 / 5-1 \mathrm{C} 3$ & & & 42 & 45 & 12.8 & 13.7 & water & Clay and gravel & -- & -- & -- & \\
\hline 334 & $6 / 5-1 \mathrm{C} 3$ & & & 45 & 81 & 13.7 & 24.7 & clay, yellow & Clay & -- & -- & -- & \\
\hline 334 & $6 / 5-1 \mathrm{C} 3$ & & & 81 & 90 & 24.7 & 27.4 & blue & Clay & -. & -- & -- & \\
\hline 334 & 6/5-1C3 & & & 90 & 92 & 27.4 & 28.0 & water & Clay & -- & -- & -- & \\
\hline 334 & $6 / 5-1 C 3$ & & & 92 & 125 & 28.0 & 38.1 & clay, yellow & Clay & -- & -- & -- & \\
\hline 335 & $6 / 4-5 \mathrm{~A} 1$ & 335 & $6 / 4-5 \mathrm{~A} 1$ & 0 & 4 & 0.0 & 1.2 & soil & Sand and clay & .- & .- & .- & Oyal and Ooal \\
\hline 335 & 6/4-5A1 & & & 4 & 18 & 1.2 & 5.5 & $\begin{array}{l}\text { hardpan and clay } \\
\text { boulders, gravel, sand; and some }\end{array}$ & Clay and gravel & hard & -- & -- & \\
\hline 335 & 6/4-5A1 & & & 18 & 59 & 5.5 & 18.0 & clay & Clay, sand, and gravel & -- & -- & -- & \\
\hline 335 & 6/4-5A1 & & & 59 & 81 & 18.0 & 24.7 & clay & Clay & -- & -- & -- & \\
\hline 335 & 6/4-5A1 & & & 81 & 89 & 24.7 & 27.1 & gravel, boulders and clay & Sand (or sandstone) and gravel & -- & -- & -- & \\
\hline 335 & 6/4-5A1 & & & 89 & 99 & 27.1 & 30.2 & boulders and clay & Clay and gravel & -- & -- & -- & \\
\hline 335 & 6/4-5A1 & & & 99 & 125 & 30.2 & 38.1 & hard, sand and gravel & Sand (or sandstone) and gravel & hard & -. & -- & \\
\hline 335 & 6/4-5A1 & & & 125 & 138 & 38.1 & 42.1 & cemented conglomerate & Clay, sand, and gravel & -- & -- & -- & \\
\hline 335 & $6 / 4-5 \mathrm{~A} 1$ & & & 138 & 319 & 42.1 & 97.2 & rock & Undifferentiated basement & hard & -- & -. & KJu \\
\hline 336 & $6 / 4-5 B 1$ & 336 & 6/4-5B1 & 0 & 10 & 0.0 & 3.0 & soil & Sand and clay & -. & -- & .- & Qyal and Qoal \\
\hline 336 & $6 / 4-5 \mathrm{~B} 1$ & & & 10 & 13 & 3.0 & 4.0 & clay & Clay & -- & -- & -- & \\
\hline 336 & $6 / 4-5 B 1$ & & & 13 & 54 & 4.0 & 16.5 & hardpan and clay & Clay and gravel & hard & -- & .- & \\
\hline 336 & $6 / 4-5 B 1$ & & & 54 & 58 & 16.5 & 17.7 & rock & Undifferentiated basement & hard & -- & -. & KJu \\
\hline 337 & $6 / 4-5 B 2$ & 337 & $6 / 4-5 B 2$ & 0 & 12 & 0.0 & 3.7 & soil & Sand and clay & -. & .- & -. & Qyal \\
\hline 337 & $6 / 4-5 B 2$ & & & 12 & 18 & 3.7 & 5.5 & clay & Clay & -- & -- & -.- & \\
\hline 337 & $6 / 4-5 B 2$ & & & 18 & 28.4 & 5.5 & 8.7 & rock & Undifferentiated basement & hard & -- & -- & KJu \\
\hline 338 & 6/4-4E1 & 338 & 6/4-4E1 & 0 & 5 & 0.0 & 1.5 & surface & Sand and clay & -- & -- & -- & Qyal \\
\hline 338 & 6/4-4E1 & & & 5 & 65 & 1.5 & 19.8 & $\begin{array}{l}\text { clay, yellow and boulders }\end{array}$ & Clay and gravel & -- & -- & -- & \\
\hline $\begin{array}{l}300 \\
338\end{array}$ & 6/4-4E1 & & & 65 & 115 & 19.8 & 35.1 & granite gray & Gravel & - & -- & - & Tsv \\
\hline 338 & 6/4-4E1 & & & 115 & 155 & 35.1 & 47.2 & tuff & Ash or tuff & -- & -. & .- & \\
\hline 338 & 6/4-4E1 & & & 155 & 210 & 47.2 & 64.0 & granite, gray & Ash or tuff & -- & -- & -.- & \\
\hline 338 & 6/4-4E1 & & & 210 & 427 & 64.0 & 130.1 & tuff & Ash or tuff & -- & -- & -- & \\
\hline 338 & 6/4-4E1 & & & 427 & 450 & 130.1 & 137.2 & volcanic ash & Ash or tuff & -- & -- & -- & \\
\hline 338 & 6/4-4E1 & & & 450 & 455 & 137.2 & 138.7 & tuff & Ash or tuff & -- & -- & -- & \\
\hline 338 & 6/4-4E1 & & & 455 & 460 & 138.7 & 140.2 & volcanic & Ash or tuff & .- & -. & .. & \\
\hline 338 & 6/4-4E1 & & & 460 & 463 & 140.2 & 141.1 & volcanic rock, gray & Ash or tuff & .- & -. & .- & \\
\hline 338 & 6/4-4E1 & & & 463 & 475 & 141.1 & 144.8 & volcanic rock, black & Ash or tuff & -- & -- & -- & \\
\hline 338 & 6/4-4E1 & & & 475 & 478 & 144.8 & 145.7 & volcanic rock, gray & Ash or tuff & -- & -- & -- & \\
\hline 338 & 6/4-4E1 & & & 478 & 600 & 145.7 & 182.9 & shale rock, black & Clay and sand (or sandstone) & cemented & -- & -.- & \\
\hline 339 & $6 / 4-5 J 1$ & 339 & 6/4-5J1 & 0 & 25 & 0.0 & 7.6 & soil & Sand and clay & -- & -- & -- & Qyal and Qoal \\
\hline 339 & $6 / 4-5 J 1$ & & & 25 & 42 & 7.6 & 12.8 & gravel and clay & Clay and gravel & -- & -- & -- & \\
\hline 339 & $6 / 4-5 \mathrm{~J} 1$ & & & 42 & 64 & 12.8 & 19.5 & clay, gravel; and boulders & Clay and gravel & -- & -- & -- & \\
\hline 339 & $6 / 4-5 J 1$ & & & 64 & 76 & 19.5 & 23.2 & gravel, hard & Gravel & hard & -- & -- & \\
\hline 339 & $6 / 4-5 \mathrm{~J} 1$ & & & 76 & 78 & 23.2 & 23.8 & clay and boulders & Clay and gravel & -- & -- & -- & \\
\hline 339 & $6 / 4-5 \mathrm{~J} 1$ & & & 78 & 80 & 23.8 & 24.4 & clay & Clay & ..- & -- & ..- & \\
\hline 339 & 6/4-5J1 & & & 80 & 82 & 24.4 & 25.0 & clay and boulders & Clay and gravel & -- & -- & -- & \\
\hline 339 & 6/4-5J1 & & & 82 & 89 & 25.0 & 27.1 & gravel & Gravel & -- & -- & -- & \\
\hline 339 & $6 / 4-5 \mathrm{~J} 1$ & & & 89 & 94 & 27.1 & 28.7 & clay & Clay & -. & -- & -. & \\
\hline 339 & $6 / 4-5 J 1$ & & & 94 & 96 & 28.7 & 29.3 & clay and boulders & Clay and gravel & -- & -- & -- & \\
\hline 339 & $6 / 4-5 J 1$ & & & 96 & 120 & 29.3 & 36.6 & clay and gravel & Clay and gravel & -- & -- & -- & \\
\hline 339 & 6/4-5J1 & & & 120 & 122 & 36.6 & 37.2 & boulders & Gravel & -- & -- & -- & \\
\hline 339 & $6 / 4-5 J 1$ & & & 122 & 128 & 37.2 & 39.0 & clay, soft & Clay & -. & -- & -. & \\
\hline 339 & $6 / 4-5 \mathrm{~J} 1$ & & & 128 & 160 & 39.0 & 48.8 & clay, soft & Clay & -. & -- & -- & \\
\hline 339 & $6 / 4-5 J 1$ & & & 160 & 178 & 48.8 & 54.3 & clay, tough & Clay & -- & -- & -- & \\
\hline 339 & $6 / 4-5 \mathrm{~J} 1$ & & & 178 & 182 & 54.3 & 55.5 & gravel, good & Gravel & -. & -- & -. & \\
\hline 339 & $6 / 4-5 J 1$ & & & 182 & 186 & 55.5 & 56.7 & clay & Clay & -- & -- & -- & \\
\hline 339 & 6/4-5J1 & & & 186 & 192 & 56.7 & 58.5 & gravel, good & Gravel & -. & -- & -- & \\
\hline
\end{tabular}


Appendix 3. Lithologic data. - Continued

[Site IDs and well number correspond to locations in Appendix 1, a site ID and well number is listed for each drilled interval for a particular drill hole. For convenience, the site ID and well numbers are repeated at the shallowest drilled interval for each well. leaders

\begin{tabular}{|c|c|c|c|c|c|c|c|c|c|c|c|c|c|}
\hline Site ID & Well number & $\begin{array}{l}\text { Site ID, } \\
\text { top of } \\
\text { well }\end{array}$ & $\begin{array}{l}\text { Well number, } \\
\text { top of well }\end{array}$ & $\begin{array}{c}\text { Top of } \\
\text { interval, in } \\
\text { feet }\end{array}$ & $\begin{array}{c}\text { Base of } \\
\text { interval, in } \\
\text { feet }\end{array}$ & $\begin{array}{c}\text { Top of } \\
\text { interval, in } \\
m\end{array}$ & $\begin{array}{c}\text { Base of } \\
\text { interval, in } \\
\mathrm{m}\end{array}$ & Reported lithologic unit ${ }^{1}$ & Interpreted lithologic class ${ }^{2}$ & $\begin{array}{c}\text { Reported } \\
\text { degree of } \\
\text { cementation or } \\
\text { induration } \\
\end{array}$ & $\begin{array}{c}\text { Fossils } \\
\text { reported in } \\
\text { interval }^{4}\end{array}$ & $\begin{array}{c}\text { Organic } \\
\text { matter } \\
\text { reported in } \\
\text { interval }^{5}\end{array}$ & $\begin{array}{l}\text { Reported stratigraphic top of geologic } \\
\text { unit }^{6}\end{array}$ \\
\hline 339 & $6 / 4-5 J 1$ & & & 192 & 211 & 58.5 & 64.3 & clay and gravel & Clay and gravel & -- & -- & -- & \\
\hline 339 & $6 / 4-5 \mathrm{~J} 1$ & & & 211 & 292 & 64.3 & 89.0 & rock & Undifferentiated basement & hard & -- & -- & $\mathrm{KJu}$ \\
\hline 340 & $6 / 4-6 \mathrm{R} 1$ & 340 & 6/4-6R1 & 0 & 18 & 0.0 & 5.5 & adobe soil & Sand and clay & -- & -- & -- & Qyal \\
\hline 340 & 6/4-6R1 & & & 18 & 32 & 5.5 & 9.8 & clay, yellow & Clay & -- & - & -- & \\
\hline 340 & 6/4-6R1 & & & 32 & 36 & 9.8 & 11.0 & sand and clay & Sand and clay & -- & -- & -- & \\
\hline 340 & 6/4-6R1 & & & 36 & 43 & 11.0 & 13.1 & sand and gravel & Sand (or sandstone) and gravel & -- & -- & -- & \\
\hline 340 & $6 / 4-6 \mathrm{R} 1$ & & & 43 & 45 & 13.1 & 13.7 & clay, yellow & Clay & -- & -- & -- & \\
\hline 340 & $6 / 4-6 \mathrm{R} 1$ & & & 45 & 48 & 13.7 & 14.6 & sand rock & Sandstone & cemented & -- & -- & Qoal (?) \\
\hline 340 & 6/4-6R1 & & & 48 & 50 & 14.6 & 15.2 & yellow clay & Clay & -- & -- & -- & \\
\hline 340 & 6/4-6R1 & & & 50 & 51 & 15.2 & 15.5 & rock & Sandstone & hard & -- & -- & \\
\hline 340 & 6/4-6R1 & & & 51 & 75 & 15.5 & 22.9 & clay, blue & Clay & -- & -- & -- & \\
\hline 340 & $6 / 4-6 \mathrm{R} 1$ & & & 75 & 83 & 22.9 & 25.3 & $\begin{array}{l}\text { sand and gravel } \\
\text { sand, cemented; gravel and clay }\end{array}$ & Sand (or sandstone) and gravel & -- & -- & -- & \\
\hline 340 & 6/4-6R1 & & & 83 & 162 & 25.3 & 49.4 & mixture with soft streaks & Sand (or sandstone) and gravel & cemented & -- & -- & \\
\hline 340 & 6/4-6R1 & & & 162 & 181 & 49.4 & 55.2 & sand, cemented; and boulders & Sand (or sandstone) and gravel & cemented & -- & -- & \\
\hline 340 & $6 / 4-6 \mathrm{R} 1$ & & & 181 & 189 & 55.2 & 57.6 & clay, sandy & Clay and sand (or sandstone) & -- & - & -- & \\
\hline 340 & $6 / 4-6 \mathrm{R} 1$ & & & 189 & 233 & 57.6 & 71.0 & hardpan and boulders & Clay and gravel & hard & -- & -- & \\
\hline 340 & $6 / 4-6 \mathrm{R} 1$ & & & 233 & 242 & 71.0 & 73.8 & rocks & Gravel & hard & -. & -- & Tsv \\
\hline 341 & $6 / 4-6 \mathrm{R} 2$ & 341 & $6 / 4-6 \mathrm{R} 2$ & 0 & 15 & 0.0 & 4.6 & surface & Sand and clay & -- & -- & -- & Qyal and Qoal \\
\hline 341 & $6 / 4-6 \mathrm{R} 2$ & & & 15 & 38 & 4.6 & 11.6 & clay, yellow & Clay & -.- & -- & -- & \\
\hline 341 & $6 / 4-6 \mathrm{R} 2$ & & & 38 & 50 & 11.6 & 15.2 & gravel & Gravel & -- & -- & -- & \\
\hline 341 & $6 / 4-6 \mathrm{R} 2$ & & & 50 & 65 & 15.2 & 19.8 & clay, blue & Clay & -- & -- & -- & \\
\hline 341 & 6/4-6R2 & & & 65 & 80 & 19.8 & 24.4 & clay, blue and gravel & Clay and gravel & -- & -- & -- & \\
\hline 341 & $6 / 4-6 \mathrm{R} 2$ & & & 80 & 105 & 24.4 & 32.0 & clay, yellow and gravel & Clay and gravel & -- & -. & -- & \\
\hline 341 & 6/4-6R2 & & & 105 & 109 & 32.0 & 33.2 & gravel & Gravel & -- & -- & -- & \\
\hline 341 & $6 / 4-6 \mathrm{R} 2$ & & & 109 & 126 & 33.2 & 38.4 & clay, yellow and gravel & Clay and gravel & -- & -- & -- & \\
\hline 341 & $6 / 4-6 \mathrm{R} 2$ & & & 126 & 131 & 38.4 & 39.9 & gravel & Gravel & -- & -- & -- & \\
\hline 341 & $6 / 4-6 \mathrm{R} 2$ & & & 131 & 136 & $\begin{array}{l}39.4 \\
39.9\end{array}$ & 41.5 & clay, yellow and gravel & Clay and gravel & - &.- & - & \\
\hline 341 & $6 / 4-6 \mathrm{R} 2$ & & & 136 & 148 & 41.5 & 45.1 & clay, blue & Clay & -- & -- & -.- & \\
\hline 341 & $6 / 4-6 \mathrm{R} 2$ & & & 148 & 156 & 45.1 & 47.5 & clay, blue and gravel & Clay and gravel & -- & -- & -- & \\
\hline 341 & $6 / 4-6 \mathrm{R} 2$ & & & 156 & 165 & 47.5 & 50.3 & clay, yellow and gravel & Clay and gravel & -- & -- & -- & \\
\hline 341 & $6 / 4-6 \mathrm{R} 2$ & & & 165 & 205 & 50.3 & 62.5 & $\begin{array}{l}\text { clay, yellow and boulders }\end{array}$ & Clay and gravel & -- & -- & -- & \\
\hline 341 & $6 / 4-6 \mathrm{R} 2$ & & & 205 & 210 & 62.5 & 64.0 & gravel and boulders & Gravel & - & -- & - & \\
\hline 341 & 6/4-6R2 & & & 210 & 216 & 64.0 & 65.8 & boulders & Gravel & -- & -- & -- & \\
\hline 341 & 6/4-6R2 & & & 216 & 220 & 65.8 & 67.1 & rocks, solid white, very hard & Ash or tuff & hard & -- & -.- & Tsv \\
\hline 342 & $6 / 4-6 \mathrm{P} 1$ & 342 & 6/4-6P1 & 0 & 15 & 0.0 & 4.6 & topsoil & Sand and clay & -- & -- & -- & Qyal and Qoal \\
\hline 342 & 6/4-6P1 & & & 15 & 17 & 4.6 & 5.2 & sand & Sand & -. & -- & -- & \\
\hline 342 & $6 / 4-6 \mathrm{P} 1$ & & & 17 & 31 & 5.2 & 9.4 & gravel & Gravel & -- & -- & -- & \\
\hline 342 & 6/4-6P1 & & & 31 & 37 & 9.4 & 11.3 & clay, yellow & Clay & -- & -- & -- & \\
\hline 342 & 6/4-6P1 & & & 37 & 46 & 11.3 & 14.0 & gravel & Gravel & -- & -- & -- & \\
\hline 342 & $6 / 4-6 \mathrm{P} 1$ & & & 46 & 54 & 14.0 & 16.5 & sand & Sand & -- & -- & -- & \\
\hline 342 & 6/4-6P1 & & & 54 & 62 & 16.5 & 18.9 & clay, blue & Clay & -- & -- & -- & \\
\hline 342 & 6/4-6P1 & & & 62 & 72 & 18.9 & 21.9 & clay, sandy & Clay and sand (or sandstone) & -- & -- & -- & \\
\hline 342 & 6/4-6P1 & & & 72 & 78 & 21.9 & 23.8 & gravel, fine & Gravel & -. & -- & -. & \\
\hline 342 & 6/4-6P1 & & & 78 & 93 & 23.8 & 28.3 & clay blue & Clay & -- & -- & -- & \\
\hline 342 & 6/4-6P1 & & & 93 & 110 & 28.3 & 33.5 & gravel, fine & Gravel & -- & -- & -- & \\
\hline 342 & 6/4-6P1 & & & 110 & 120 & 33.5 & 36.6 & clay brown & Clay & -- & -- & -- & \\
\hline 343 & 6/4-5Q1 & 343 & 6/4-5Q1 & 0 & 12 & 0.0 & 3.7 & soil & Sand and clay & -- & -- & -- & Qyal and Qoal \\
\hline 343 & 6/4-5Q1 & & & 12 & 31 & 3.7 & 9.4 & clay, yellow & Clay & -- & -- & -- & \\
\hline 343 & $6 / 4-5 \mathrm{Q} 1$ & & & 31 & 49 & 9.4 & 14.9 & clay, sandy, yellow & Clay and sand (or sandstone) & -- & -- & -- & \\
\hline 343 & 6/4-5Q1 & & & 49 & 54 & 14.9 & 16.5 & clay, sandy, blue & Clay and sand (or sandstone) & -- & -- & -- & \\
\hline 343 & 6/4-5Q1 & & & 54 & 70 & 16.5 & 21.3 & clay, blue, tough & Clay & -- & -- & -- & \\
\hline 343 & 6/4-5Q1 & & & 70 & 73 & 21.3 & 22.3 & sand, blue; and clay & Sand and clay & -- & -- & -- & \\
\hline 343 & 6/4-5Q1 & & & 73 & 77 & 22.3 & 23.5 & $\begin{array}{l}\text { clay, yellow, tough } \\
\text { sand, gravel, and boulders, and }\end{array}$ & Clay & -- & -- & -- & \\
\hline 343 & 6/4-5Q1 & & & 77 & 107 & 23.5 & 32.6 & $\begin{array}{l}\text { clay mixtures } \\
\text { sand, gravel, and boulders, and }\end{array}$ & Sand (or sandstone) and gravel & -- & -- & -- & \\
\hline 343 & 6/4-5Q1 & & & 107 & 112 & 32.6 & 34.1 & clay mixtures & Sand (or sandstone) and gravel & -- & -- & -- & \\
\hline 343 & $6 / 4-5 \mathrm{Q} 1$ & & & 112 & 145 & 34.1 & 44.2 & sandy clay and gravels & Clay, sand, and gravel & -- & -- & .- & \\
\hline 343 & 6/4-5Q1 & & & 145 & 151 & 44.2 & 46.0 & sand and gravel & Sand (or sandstone) and gravel & - & -- & -- & \\
\hline 343 & $6 / 4-5 \mathrm{Q} 1$ & & & 151 & 154 & 46.0 & 46.9 & gravel, boulders, and sand & Gravel & -. & -- & -- & \\
\hline
\end{tabular}


Appendix 3. Lithologic data. - Continued

ISite IDs and well number correspond to locations in Appendix 1, a site ID and well number is listed for each drilled interval for a particular drill hole. For convenience, the site ID and well numbers are repeated at the shallowest drilled interval for each well. leaders $(-1, n$

\begin{tabular}{|c|c|c|c|c|c|c|c|c|c|c|c|c|c|}
\hline Site ID & Well number & $\begin{array}{l}\text { Site ID, } \\
\text { top of } \\
\text { well }\end{array}$ & $\begin{array}{l}\text { Well number, } \\
\text { top of well }\end{array}$ & $\begin{array}{c}\text { Top of } \\
\text { interval, in } \\
\text { feet }\end{array}$ & $\begin{array}{l}\text { Base of } \\
\text { interval, in } \\
\text { feet }\end{array}$ & $\begin{array}{c}\text { Top of } \\
\text { interval, in } \\
m\end{array}$ & $\begin{array}{c}\text { Base of } \\
\text { interval, in } \\
\mathrm{m}\end{array}$ & Reported lithologic unit ${ }^{1}$ & Interpreted lithologic class ${ }^{2}$ & $\begin{array}{c}\text { Reported } \\
\text { degree of } \\
\text { cementation or } \\
\text { induration } \\
\end{array}$ & $\begin{array}{c}\text { Fossils } \\
\text { reported in } \\
\text { interval }^{4}\end{array}$ & $\begin{array}{c}\text { Organic } \\
\text { matter } \\
\text { reported in } \\
\text { interval }^{5}\end{array}$ & $\begin{array}{l}\text { Reported stratigraphic top of geologic } \\
\text { unit }^{6}\end{array}$ \\
\hline 343 & $6 / 4-5 \mathrm{Q} 1$ & & & 154 & 166 & 46.9 & 50.6 & sand clay and gravel & Clay, sand, and gravel & -- & -- & -- & \\
\hline 343 & 6/4-5Q1 & & & 166 & 167 & 50.6 & 50.9 & rock & Undifferentiated basement & hard & -- & -- & KJu \\
\hline 344 & 6/4-17A1 & 344 & 6/4-17A1 & 0 & 10 & 0.0 & 3.0 & surface & Sand and clay & -- & -- & -- & Qyal and Qoal \\
\hline 344 & 6/4-17A1 & & & 10 & 70 & 3.0 & 21.3 & clay, yellow, and gravel & Clay and gravel & -- & - & -- & \\
\hline 344 & 6/4-17A1 & & & 70 & 120 & 21.3 & 36.6 & boulders and gravel & Gravel & -- & -. & -- & \\
\hline 344 & 6/4-17A1 & & & 120 & 180 & 36.6 & 54.9 & clay, yellow & Clay & -- & -- & -- & \\
\hline 344 & 6/4-17A1 & & & 180 & 200 & 54.9 & 61.0 & gravel & Gravel & -- & -- & -- & \\
\hline 344 & 6/4-17A1 & & & 200 & 225 & 61.0 & 68.6 & boulders and gravel & Gravel & -- & -- & -- & \\
\hline 344 & 6/4-17A1 & & & 225 & 250 & 68.6 & 76.2 & clay, yellow & Clay & -- & -- & -- & \\
\hline 345 & 6/4-18A3 & 345 & 6/4-18A3 & 0 & 3 & 0.0 & 0.9 & soil & Sand and clay & -- & -- & -- & Qyal \\
\hline 345 & 6/4-18A3 & & & 3 & 15 & 0.9 & 4.6 & clay & Clay & -. & -- & -- & \\
\hline 345 & 6/4-18A3 & & & 15 & 25 & 4.6 & 7.6 & gravel and sand & Sand (or sandstone) and gravel & -- & -- & -- & \\
\hline 345 & 6/4-18A3 & & & 25 & 40 & 7.6 & 12.2 & clay & Clay & -- & -- & -- & \\
\hline 345 & 6/4-18A3 & & & 40 & 43 & 12.2 & 13.1 & gravel & Gravel & -- & -- & -- & \\
\hline 345 & 6/4-18A3 & & & 43 & 82 & 13.1 & 25.0 & clay & Clay & -- & -- & -- & \\
\hline 345 & $6 / 4-18 \mathrm{~A} 3$ & & & 82 & 98 & 25.0 & 29.9 & gravel and boulders, loose & Gravel & .. & -- & -- & \\
\hline 345 & 6/4-18A3 & & & 98 & 140 & 29.9 & 42.7 & clay & Clay & -- & -- & -- & \\
\hline 345 & 6/4-18A3 & & & 140 & 158 & 42.7 & 48.2 & gravel and boulders, fairly loose & Gravel & -- & -- & -- & \\
\hline 345 & 6/4-18A3 & & & 158 & 172 & 48.2 & 52.4 & clay & Clay & -- & -- & -- & \\
\hline 345 & 6/4-18A3 & & & 172 & 204 & 52.4 & 62.2 & boulders and gravel & Gravel & -- & -- & -- & \\
\hline 345 & $6 / 4-18 A 3$ & & & 204 & 240 & 62.2 & 73.2 & clay and boulders & Clay and gravel & -. & -. & -. & \\
\hline 345 & 6/4-18A3 & & & 240 & 248 & 73.2 & 75.6 & gravel & Gravel & -- & -- & -- & \\
\hline 345 & 6/4-18A3 & & & 248 & 288 & 75.6 & 87.8 & clay and boulders, tight & Clay and gravel & .. & -. & -. & \\
\hline 345 & 6/4-18A3 & & & 288 & 304 & 87.8 & 92.7 & gravel, fairly loose & Gravel & -- & -. & .- & \\
\hline 345 & 6/4-18A3 & & & 304 & 320 & 92.7 & 97.5 & rock & Ash or tuff & hard & -- & -- & \\
\hline 345 & 6/4-18A3 & & & 320 & 328 & 97.5 & 100.0 & $\begin{array}{l}\text { clay, sticky and tight } \\
\text {. }\end{array}$ & Clay & -- & - & -- & \\
\hline 345 & 6/4-18A3 & & & 328 & 335 & 100.0 & 102.1 & rock & Ash or tuff & hard & -- & -- & \\
\hline 345 & 6/4-18A3 & & & 335 & 340 & 102.1 & 103.6 & mud, blue & Clay and sand (or sandstone) & -. & - & - & \\
\hline 345 & $6 / 4-18 A 3$ & & & 340 & 354 & 103.6 & 107.9 & gravel and boulders & Gravel & .. & -- & -.- & \\
\hline 345 & $6 / 4-18 \mathrm{~A} 3$ & & & 354 & 381 & 107.9 & 116.1 & clay and boulders & Clay and gravel & -- & .- & -.- & \\
\hline 345 & 6/4-18A3 & & & 381 & 395 & 116.1 & 120.4 & rock & Ash or tuff & hard & -- & -- & \\
\hline 345 & 6/4-18A3 & & & 395 & 408 & 120.4 & 124.4 & gravel & Gravel & -- & -- & -- & \\
\hline 345 & 6/4-18A3 & & & 408 & 414 & 124.4 & 126.2 & rock & Ash or tuff & hard & -- & - & \\
\hline 345 & 6/4-18A3 & & & 414 & 422 & 126.2 & 128.6 & gravel & Gravel & -- & .- & -. & \\
\hline 345 & 6/4-18A3 & & & 422 & 434 & 128.6 & 132.3 & rock & Ash or tuff & hard & -- & -- & \\
\hline 345 & 6/4-18A3 & & & 434 & 442 & 132.3 & 134.7 & clay & Clay & -- & -- & -- & \\
\hline 345 & 6/4-18A3 & & & 442 & 458 & 134.7 & 139.6 & rock & Ash or tuff & hard & -- & -- & \\
\hline 345 & 6/4-18A3 & & & 458 & 479 & 139.6 & 146.0 & gravel and boulders & Gravel & -- & - & - & \\
\hline 345 & 6/4-18A3 & & & 479 & 520 & 146.0 & 158.5 & clay and boulders & Clay and gravel & -- & -- & -- & \\
\hline 345 & 6/4-18A3 & & & 520 & 525 & 158.5 & 160.0 & gravel & Gravel & -- & -. & .- & \\
\hline 345 & 6/4-18A3 & & & 525 & 545 & 160.0 & 166.1 & rock & Ash or tuff & hard & -- & -- & \\
\hline 345 & 6/4-18A3 & & & 545 & 565 & 166.1 & 172.2 & clay & Clay & -- & -- & -- & \\
\hline 345 & 6/4-18A3 & & & 565 & 605 & 172.2 & 184.4 & boulders and clay & Clay and gravel & -- & -- & -.- & \\
\hline 345 & 6/4-18A3 & & & 605 & 620 & 184.4 & 189.0 & gravel and clay & Clay and gravel & -- & -- & -- & \\
\hline 345 & 6/4-18A3 & & & 620 & 630 & 189.0 & 192.0 & rock & Ash or tuff & hard & -- & -- & \\
\hline 345 & 6/4-18A3 & & & 630 & 650 & 192.0 & 198.1 & clay & Clay & -- & -- & -- & \\
\hline 345 & $6 / 4-18 \mathrm{~A} 3$ & & & 650 & 694 & 198.1 & 211.5 & boulders and clay & Clay and gravel & -- & -- & -- & \\
\hline 345 & $6 / 4-18 \mathrm{~A} 3$ & & & 694 & 702 & 211.5 & 214.0 & rock & Ash or tuff & hard & -- & .- & \\
\hline 345 & $6 / 4-18 \mathrm{~A} 3$ & & & 702 & 730 & 214.0 & 222.5 & clay and boulders & Clay and gravel & -. & .- & -. & \\
\hline 345 & 6/4-18A3 & & & 730 & 730 & 222.5 & 222.5 & rock & Ash or tuff & hard & -- & -- & \\
\hline 346 & 6/4-15Q1 & 346 & 6/4-15Q1 & 0 & 2 & 0.0 & 0.6 & soil & Sand and clay & - & -- & -- & Qoal \\
\hline 346 & 6/4-15Q1 & & & 2 & 48 & 0.6 & 14.6 & $\begin{array}{l}\text { clay and boulders } \\
\text {. }\end{array}$ & Clay and gravel & -. & -- & -. & \\
\hline 346 & 6/4-15Q1 & & & 48 & 75 & 14.6 & 22.9 & clay & Clay & -- & -- & -- & \\
\hline 346 & 6/4-15Q1 & & & 75 & 84 & 22.9 & 25.6 & rock, water & Ash or tuff & hard & -. & -. & \\
\hline 346 & 6/4-15Q1 & & & 84 & 105 & 25.6 & 32.0 & clay & Clay & -- & -- & -- & \\
\hline 346 & 6/4-15Q1 & & & 105 & 147 & 32.0 & 44.8 & clay and gravel & Clay and gravel & - & -- & -- & \\
\hline 346 & 6/4-15Q1 & & & 147 & 162 & 44.8 & 49.4 & gravel, dry & Gravel & -- & -- & -. & \\
\hline 346 & 6/4-15Q1 & & & 162 & 185 & 49.4 & 56.4 & tuff & Ash or tuff & -- & -- & -- & Qh (?) \\
\hline 346 & 6/4-15Q1 & & & 185 & 210 & 56.4 & 64.0 & gravel, some tuff; some water & Gravel & -. & -- & -. & \\
\hline 346 & 6/4-15Q1 & & & 210 & 245 & 64.0 & 74.7 & tuff, blue & Ash or tuff & -- & -- & -- & \\
\hline 346 & 6/4-15Q1 & & & 245 & 264 & 74.7 & 80.5 & tuff, gray; some water & Ash or tuff & -. & -- & -- & \\
\hline
\end{tabular}


Appendix 3. Lithologic data. - Continued

[Site IDs and well number correspond to locations in Appendix 1, a site ID and well number is listed for each drilled interval for a particular drill hole. For convenience, the site ID and well numbers are repeated at the shallowest drilled interval for each well. leaders

\begin{tabular}{|c|c|c|c|c|c|c|c|c|c|c|c|c|c|}
\hline Site ID & Well number & $\begin{array}{l}\text { Site ID, } \\
\text { top of } \\
\text { well }\end{array}$ & $\begin{array}{l}\text { Well number, } \\
\text { top of well }\end{array}$ & $\begin{array}{l}\text { Top of } \\
\text { interval, in } \\
\text { feet }\end{array}$ & $\begin{array}{l}\text { Base of } \\
\text { interval, in } \\
\text { feet }\end{array}$ & $\begin{array}{c}\text { Top of } \\
\text { interval, in } \\
m\end{array}$ & $\begin{array}{c}\text { Base of } \\
\text { interval, in } \\
\mathrm{m}\end{array}$ & Reported lithologic unit ${ }^{1}$ & Interpreted lithologic class ${ }^{2}$ & $\begin{array}{c}\text { Reported } \\
\text { degree of } \\
\text { cementation or } \\
\text { induration }^{3} \\
\end{array}$ & $\begin{array}{l}\text { Fossils } \\
\text { reported in } \\
\text { interval }^{4}\end{array}$ & $\begin{array}{c}\text { Organic } \\
\text { matter } \\
\text { reported in } \\
\text { interval }^{5}\end{array}$ & $\begin{array}{l}\text { Reported stratigraphic top of geologic } \\
\text { unit }^{6}\end{array}$ \\
\hline 346 & $6 / 4-15 Q 1$ & & & 264 & 273 & 80.5 & 83.2 & tuff, brown & Ash or tuff & -- & -- & -- & \\
\hline 346 & 6/4-15Q1 & & & 273 & 295 & 83.2 & 89.9 & lava and gravel, water & Basalt & -- & -- & -- & \\
\hline 346 & 6/4-15Q1 & & & 295 & 303 & 89.9 & 92.4 & tuff, gray & Ash or tuff & -- & -- & -- & \\
\hline 347 & 6/4-15Q3 & 347 & 6/4-15Q3 & 0 & 5 & 0.0 & 1.5 & soil & Sand and clay & -- & -. & -- & Qoal and Qh (?) \\
\hline 347 & 6/4-15Q3 & & & 5 & 27 & 1.5 & 8.2 & clay, and boulders & Clay and gravel & -- & -- & -- & \\
\hline 347 & 6/4-15Q3 & & & 27 & 30 & 8.2 & 9.1 & gravel, some water & Gravel & -- & -- & -- & \\
\hline 347 & 6/4-15Q3 & & & 30 & 54 & 9.1 & 16.5 & clay, and boulders & Clay and gravel & -- & -- & -- & \\
\hline 347 & 6/4-15Q3 & & & 54 & 57 & 16.5 & 17.4 & gravel, some water & Gravel & -- & -- & -- & \\
\hline 347 & 6/4-15Q3 & & & 57 & 132 & 17.4 & $\begin{array}{l}40.2 \\
40.2\end{array}$ & clay and boulders & Clay and gravel & -- & -- & -- & \\
\hline 347 & 6/4-15Q3 & & & 132 & 135 & 40.2 & 41.1 & gravel water & Gravel & -- & -. & .- & \\
\hline 347 & 6/4-15Q3 & & & 135 & 177 & 41.1 & 53.9 & boulders and clay & Clay and gravel & -- & -- & -- & \\
\hline 347 & 6/4-15Q3 & & & 177 & 180 & 53.9 & 54.9 & gravel, some water & Gravel & -- & -- & -- & \\
\hline 347 & 6/4-15Q3 & & & 180 & 309 & 54.9 & 94.2 & boulders and clay & Clay and gravel & -- & .- & -- & \\
\hline 347 & 6/4-15Q3 & & & 309 & 327 & 94.2 & 99.7 & gravel, water & Gravel & -- & -- & -- & \\
\hline 348 & 6/4-22B3 & 348 & 6/4-22B3 & 0 & 3 & 0.0 & 0.9 & soil and boulders & Clay, sand, and gravel & -- & -- & -- & Qoal \\
\hline 348 & 6/4-22B3 & & & 3 & 54 & 0.9 & 16.5 & hardpan and boulders & $\begin{array}{l}\text { Clay and gravel } \\
\text { Caver }\end{array}$ & hard & -- & -- & \\
\hline 348 & 6/4-22B3 & & & 54 & 55 & 16.5 & 16.8 & water, small amount & Clay and gravel & -- & -- & -- & \\
\hline 348 & 6/4-22B3 & & & 55 & 102 & 16.8 & 31.1 & clay and boulders & Clay and gravel & .- & -- & -- & \\
\hline 348 & 6/4-22B3 & & & 102 & 106 & $\begin{array}{l}31.1 \\
31.1\end{array}$ & 32.3 & boulders and water & Gravel & -- & -- & -- & \\
\hline 348 & 6/4-22B3 & & & 106 & 237 & 32.3 & 72.2 & $\begin{array}{l}\text { clay and boulders } \\
\text { a }\end{array}$ & Clay and gravel & -- & -- & -- & \\
\hline 349 & 6/4-22B2 & 349 & 6/4-22B2 & 0 & 50 & 0.0 & 15.2 & boulders and clay & Clay and gravel & .- & .- & .- & Qoal \\
\hline 349 & 6/4-22B2 & & & 50 & 53 & 15.2 & 16.2 & gravel, water & Gravel & -- & -- & -- & \\
\hline 349 & 6/4-22B2 & & & 53 & 110 & 16.2 & 33.5 & clay and boulders & Clay and gravel & .- & -- & .- & \\
\hline 349 & 6/4-22B2 & & & 110 & 113 & 33.5 & 34.4 & gravel, water & Gravel & -- & -- & -- & \\
\hline 349 & 6/4-22B2 & & & 113 & 117 & 34.4 & 35.7 & boulders and clay & Clay and gravel & -- & -- & -- & \\
\hline 350 & 6/4-19B1 & 350 & 6/4-19B1 & 0 & 80 & 0.0 & 24.4 & $\begin{array}{l}\text { clay and boulders } \\
\text { volcanic flow with pyrite }\end{array}$ & Clay and gravel & -- & -- & -- & Qyal and Qoal (?) \\
\hline 350 & 6/4-19B1 & & & 80 & 125 & 24.4 & 38.1 & mineralization & Ash or tuff & -- & -- & -- & Tsv \\
\hline 351 & 6/4-23F1 & 351 & 6/4-23F1 & 0 & 3 & 0.0 & 0.9 & soil & Sand and clay & .- & .- & -- & Qh \\
\hline 351 & $6 / 4-23 F 1$ & & & 3 & 18 & 0.9 & 5.5 & boulders & Gravel & -- & -- & -- & \\
\hline 351 & $6 / 4-23 F 1$ & & & 18 & 90 & 5.5 & 27.4 & rock, gray & Ash or tuff & hard & -- & -- & \\
\hline 351 & $6 / 4-23 F 1$ & & & 90 & 96 & 27.4 & 29.3 & rock, broken, and water & Ash or tuff & hard & -- & -- & \\
\hline 351 & $6 / 4-23 F 1$ & & & 96 & 160 & 29.3 & 48.8 & rock brown & Ash or tuff & -- & -- & -- & \\
\hline 351 & $6 / 4-23 F 1$ & & & 160 & 202 & 48.8 & 61.6 & rock, gray & Ash or tuff & hard & .- & .- & \\
\hline 351 & $6 / 4-23 F 1$ & & & 202 & 212 & 61.6 & 64.6 & rock, broken, water & Ash or tuff & hard & -- & -- & \\
\hline 351 & $6 / 4-23 F 1$ & & & 212 & 220 & 64.6 & 67.1 & rock, gray & Ash or tuff & hard & -- & -- & \\
\hline 352 & 6/3-19M1 & 352 & 6/3-19M1 & 0 & 60 & 0.0 & 18.3 & pumice & Ash or tuff & -. & .- & .- & Tsv \\
\hline 352 & 6/3-19M1 & & & 60 & 160 & 18.3 & 48.8 & volcanic rock, black & Ash or tuff & .- & .- & .- & \\
\hline 352 & 6/3-19M1 & & & 160 & 190 & 48.8 & 57.9 & rock, soft red, source of water & Ash or tuff & hard & -- & -- & \\
\hline 353 & $6 / 4-23 J 1$ & 353 & 6/4-23J1 & 0 & 4 & 0.0 & 1.2 & soil & Sand and clay & -. & -- & -- & Qh \\
\hline 353 & $6 / 4-23 J 1$ & & & 4 & 34 & 1.2 & 10.4 & clay, yellow & Clay & -- & -- & -- & \\
\hline 353 & $6 / 4-23 J 1$ & & & 34 & 99 & 10.4 & 30.2 & clay, blue & Clay & .- & -- & -- & \\
\hline 353 & $6 / 4-23 J 1$ & & & 99 & 123 & 30.2 & 37.5 & clay, tough & Clay & -.- & -- & -. & \\
\hline 353 & $6 / 4-23 J 1$ & & & 123 & 126 & 37.5 & 38.4 & rock & Ash or tuff & hard & -- & -- & \\
\hline 353 & $6 / 4-23 J 1$ & & & 126 & 147 & 38.4 & 44.8 & boulders, cemented & Gravel & cemented & .- & .- & \\
\hline 353 & $6 / 4-23 J 1$ & & & 147 & 288 & 44.8 & 87.8 & $\begin{array}{l}\text { tuff, brown } \\
\text { the }\end{array}$ & Ash or tuff & -- & -- & -- & \\
\hline 353 & $6 / 4-23 J 1$ & & & 288 & 333 & 87.8 & 101.5 & boulders and tuff & Ash or tuff & -. & .- & .- & \\
\hline 353 & $6 / 4-23 J 1$ & & & 333 & 437 & 101.5 & 133.2 & rock & Ash or tuff & hard & -- & -- & Tsv \\
\hline 353 & $6 / 4-23 J 1$ & & & 437 & 514 & 133.2 & 156.7 & diorite & Basalt & -- & .- & -- & \\
\hline 353 & $6 / 4-23 J 1$ & & & 514 & 545 & 156.7 & 166.1 & cement & Clay, sand, and gravel & cemented & -- & -- & \\
\hline 353 & $6 / 4-23 J 1$ & & & 545 & 592 & 166.1 & 180.4 & conglomerate rock & Clay, sand, and gravel & - & -- & -- & \\
\hline 353 & $6 / 4-23 J 1$ & & & 592 & 597 & 180.4 & 182.0 & lava, water, $75 \mathrm{gpm}$ or more & $\begin{array}{l}\text { Basalt } \\
\text {. }\end{array}$ & -- & -- & -- & \\
\hline 353 & $6 / 4-23 J 1$ & & & 597 & 654 & 182.0 & 199.3 & lava, cemented & Basalt & -. & -- & .- & \\
\hline 353 & $6 / 4-23 J 1$ & & & 654 & 675 & 199.3 & 205.7 & $\begin{array}{l}\text { lava, hard } \\
\text { las }\end{array}$ & Basalt & hard & .- & .- & \\
\hline 353 & 6/4-23J1 & & & 675 & 700 & 205.7 & 213.4 & lava & Basalt & -- & -- & -- & \\
\hline 354 & 6/4-21Q1 & 354 & 6/4-21Q1 & 0 & 3 & 0.0 & 0.9 & soil & Sand and clay & -- & -- & .- & Qyal \\
\hline 354 & 6/4-21Q1 & & & 3 & 30 & 0.9 & 9.1 & clay & Clay & -- & -- & -- & \\
\hline 354 & 6/4-21Q1 & & & 30 & 49 & 9.1 & 14.9 & water gravel & Gravel & -- & -- & -- & \\
\hline 354 & 6/4-21Q1 & & & 49 & 64 & 14.9 & 19.5 & clay & Clay & -- & .- & -- & Qoal \\
\hline 354 & 6/4-21Q1 & & & 64 & 75 & 19.5 & 22.9 & gravel, dry & Gravel & -- & -- & -- & \\
\hline 354 & 6/4-21Q1 & & & 75 & 89 & 22.9 & 27.1 & clay & Clay & .- & -- & -- & \\
\hline
\end{tabular}


Appendix 3. Lithologic data. - Continued

[Site IDs and well number correspond to locations in Appendix 1, a site ID and well number is listed for each drilled interval for a particular drill hole. For convenience, the site ID and well numbers are repeated at the shallowest drilled interval for each well. leaderst.

\begin{tabular}{|c|c|c|c|c|c|c|c|c|c|c|c|c|c|}
\hline Site ID & Well number & $\begin{array}{l}\text { Site ID, } \\
\text { top of } \\
\text { well }\end{array}$ & $\begin{array}{l}\text { Well number, } \\
\text { top of well }\end{array}$ & $\begin{array}{l}\text { Top of } \\
\text { interval, in } \\
\text { feet }\end{array}$ & $\begin{array}{l}\text { Base of } \\
\text { interval, in } \\
\text { feet }\end{array}$ & $\begin{array}{c}\text { Top of } \\
\text { interval, in } \\
m\end{array}$ & $\begin{array}{c}\text { Base of } \\
\text { interval, in } \\
\mathrm{m}\end{array}$ & Reported lithologic unit ${ }^{1}$ & Interpreted lithologic class ${ }^{2}$ & $\begin{array}{c}\text { Reported } \\
\text { degree of } \\
\text { cementation or } \\
\text { induration }^{3} \\
\end{array}$ & $\begin{array}{l}\text { Fossils } \\
\text { reported in }^{\text {interval }}{ }^{4}\end{array}$ & $\begin{array}{c}\text { Organic } \\
\text { matter } \\
\text { reported in } \\
\text { interval }^{5}\end{array}$ & $\begin{array}{l}\text { Reported stratigraphic top of geologic } \\
\text { unit }^{6}\end{array}$ \\
\hline 354 & 6/4-21Q1 & & & 89 & 94 & 27.1 & 28.7 & gravel & Gravel & -- & -- & -- & \\
\hline 354 & 6/4-21Q1 & & & 94 & 110 & 28.7 & 33.5 & clay & Clay & -- & -- & -- & \\
\hline 355 & 6/4-27C2 & 355 & 6/4-27C2 & 0 & 5 & 0.0 & 1.5 & soil & Sand and clay & -- & -- & -- & Qoal \\
\hline 355 & 6/4-27C2 & & & 5 & 20 & 1.5 & 6.1 & clay, yellow & Clay & -- & -- & -- & \\
\hline 355 & 6/4-27C2 & & & 20 & 27 & 6.1 & 8.2 & clay, blue & Clay & -- & -- & -- & \\
\hline 355 & 6/4-27C2 & & & 27 & 33 & 8.2 & 10.1 & gravel & Gravel & -- & -- & -- & \\
\hline 355 & $6 / 4-27 C 2$ & & & 33 & 93 & 10.1 & 28.3 & clay, yellow & Clay & -- & -- & -- & \\
\hline 355 & $6 / 4-27 \mathrm{C} 2$ & & & 93 & 98 & 28.3 & 29.9 & gravel & Gravel & -- & .- & -- & \\
\hline 355 & 6/4-27C2 & & & 98 & 110 & 29.9 & 33.5 & $\begin{array}{l}\text { clay } \\
\text { clater }\end{array}$ & Clay & -- & -- & -- & \\
\hline 355 & $6 / 4-27 \mathrm{C} 2$ & & & 110 & 115 & 33.5 & 35.1 & gravel & Gravel & -- & -. & .- & \\
\hline 355 & 6/4-27C2 & & & 115 & 132 & 35.1 & 40.2 & clay, blue & Clay & -- & -- & -- & \\
\hline 355 & $6 / 4-27 C^{2}$ & & & 132 & 145 & 40.2 & 44.2 & clay yellow & Clay & -- & -- & -- & \\
\hline 355 & $6 / 4-27 \mathrm{C} 2$ & & & 145 & 195 & 44.2 & 59.4 & gravel, cemented & Gravel & cemented & .- & -- & \\
\hline 355 & $6 / 4-27 \mathrm{C} 2$ & & & 195 & 210 & 59.4 & 64.0 & clay, yellow & Clay & -- & .- & .- & \\
\hline 355 & 6/4-27C2 & & & 210 & 240 & 64.0 & 73.2 & clay, yellow; and rock & Clay and gravel & -- & -- & -- & \\
\hline 356 & $6 / 4-30 \mathrm{C} 1$ & 356 & 6/4-30C1 & 0 & 20 & 0.0 & 6.1 & soil and clay & Sand and clay & -- & -- & -- & Qoal \\
\hline 356 & $6 / 4-30 C 1$ & & & 20 & 28 & 6.1 & 8.5 & gravel, water & Gravel & -- & -- & -- & \\
\hline 356 & $6 / 4-30 \mathrm{C1}$ & & & 28 & 35 & 8.5 & 10.7 & clay & Clay & .- & -- & -- & \\
\hline 356 & 6/4-30C1 & & & 35 & 50 & $\begin{array}{l}10.7 \\
10.7\end{array}$ & 15.2 & clay, gravel, water & Clay and gravel & -- & -- & -- & \\
\hline 356 & 6/4-30C1 & & & 50 & 90 & 15.2 & 27.4 & clay & Clay & -- & -- & -- & \\
\hline 356 & 6/4-30C1 & & & 90 & 95 & 27.4 & 29.0 & gravel, water & Gravel & -- & -- & -- & \\
\hline 356 & $6 / 4-3001$ & & & 95 & 105 & 29.0 & 32.0 & clay, boulders & Clay and gravel & -- & -- & -- & \\
\hline 356 & $6 / 4-30 \mathrm{C} 1$ & & & 105 & 114 & 32.0 & 34.7 & boulders, gravel & Gravel & .- & .- & .- & \\
\hline 357 & 6/4-25B1 & 357 & 6/4-25B1 & 0 & 18 & 0.0 & 5.5 & pumice & Ash or tuff & .- & .- & .- & Tsv \\
\hline 357 & 6/4-25B1 & & & 18 & 63 & 5.5 & 19.2 & tuff, blue & Ash or tuff & -- & -- & -. & \\
\hline 357 & 6/4-25B1 & & & 63 & 93 & 19.2 & 28.3 & tule mud, blue & Clay and sand (or sandstone) & -- & -- & tule & \\
\hline 357 & 6/4-25B1 & & & 93 & 105 & 28.3 & 32.0 & rock, hard & Ash or tuff & hard & -- & - & \\
\hline 357 & 6/4-25B1 & & & 105 & 123 & 32.0 & 37.5 & lava, gray; some water & Basalt & -- & -- & -- & \\
\hline 357 & 6/4-25B1 & & & 123 & 129 & 37.5 & 39.3 & lava, black broken; water & Basalt & -- & -- & -- & \\
\hline 358 & 6/4-25B2 & 358 & 6/4-25B2 & 0 & 5 & 0.0 & 1.5 & surface & Sand and clay & .- & .- & .- & Tsv \\
\hline 358 & 6/4-25B2 & & & 5 & 80 & 1.5 & 24.4 & pumice, gray & Ash or tuff & -- & -- & -- & \\
\hline 358 & 6/4-25B2 & & & 80 & 100 & 24.4 & 30.5 & pumice, gray and boulders & Ash or tuff & -. & -- & -- & \\
\hline 358 & 6/4-25B2 & & & 100 & 185 & 30.5 & 56.4 & $\begin{array}{l}\text { lava rock and boulders } \\
\text { rock }\end{array}$ & Basalt & hard & -- & -- & \\
\hline 359 & 6/4-25D1 & 359 & 6/4-25D1 & 0 & 32 & 0.0 & 9.8 & sandstone, yellow, soft & Sandstone & cemented & -- & -- & Qh \\
\hline 359 & 6/4-25D1 & & & 32 & 55 & 9.8 & 16.8 & gravelly sand, blue & Sand (or sandstone) and gravel & - & -- & -- & \\
\hline 359 & 6/4-25D1 & & & 55 & 85 & 16.8 & 25.9 & clay, greenish & Clay & -- & -- & -- & \\
\hline 359 & 6/4-25D1 & & & 85 & 140 & 25.9 & 42.7 & volcanic matter; water & Ash or tuff & -- & -- & -- & \\
\hline 359 & 6/4-25D1 & & & 140 & 255 & 42.7 & 77.7 & broken rock and sandstone; water & Ash or tuff & cemented & .- & .- & \\
\hline 359 & 6/4-25D1 & & & 255 & 305 & 77.7 & 93.0 & gravel and sand; water & Sand (or sandstone) and gravel & -- & -- & -- & \\
\hline 360 & $6 / 4-26 \mathrm{H} 1$ & 360 & 6/4-26H1 & 0 & 18 & 0.0 & 5.5 & soil & Sand and clay & -- & -- & -- & Qh \\
\hline 360 & 6/4-26H1 & & & 18 & 42 & 5.5 & 12.8 & hardpan & Clay and gravel & hard & -- & -- & \\
\hline 360 & $6 / 4-26 \mathrm{H} 1$ & & & 42 & 44 & 12.8 & 13.4 & gravel, water & Gravel & -- & -- & -- & \\
\hline 360 & 6/4-26H1 & & & 44 & 135 & 13.4 & 41.1 & muck, yellow and boulders; water & Clay and gravel & -- & .- & .- & \\
\hline 360 & $6 / 4-26 \mathrm{H} 1$ & & & 135 & 160 & 41.1 & 48.8 & boulders, cemented & Gravel & cemented & -- & -- & \\
\hline 360 & 6/4-26H1 & & & 160 & 243 & 48.8 & 74.1 & cement and cemented boulders & Gravel & cemented & -- & -- & \\
\hline 360 & $6 / 4-26 \mathrm{H} 1$ & & & 243 & 246 & 74.1 & 75.0 & gravel water & Gravel & -. & -- & -- & \\
\hline 360 & 6/4-26H1 & & & 246 & 258 & 75.0 & 78.6 & rock, broken, and cement water & Clay, sand, and gravel & cemented & -- & -- & \\
\hline 360 & $6 / 4-26 \mathrm{H} 1$ & & & 258 & 282 & 78.6 & 86.0 & sandy gravel and cement; water & Sand (or sandstone) and gravel & cemented & .- & -- & \\
\hline 360 & 6/4-26H1 & & & 282 & 327 & 86.0 & 99.7 & tule muck & Clay and sand (or sandstone) & - & -- & tule & \\
\hline 360 & 6/4-26H1 & & & 327 & 381 & 99.7 & 116.1 & gravel, sand, and rock; water & Sand (or sandstone) and gravel & -- & -- & -- & \\
\hline 360 & 6/4-26H1 & & & 381 & 418 & 116.1 & 127.4 & broken basalt; water & Basalt & -- & -- & -- & Tsv \\
\hline 360 & 6/4-26H1 & & & 418 & 432 & 127.4 & 131.7 & lava, red; rock & Basalt & hard & -- & -- & \\
\hline 360 & $6 / 4-26 \mathrm{H} 1$ & & & 432 & 477 & 131.7 & 145.4 & cement, red broken; water & Basalt & cemented & -- & -- & \\
\hline 360 & $6 / 4-26 \mathrm{H} 1$ & & & 477 & 477 & 145.4 & 145.4 & gravel, cemented & Gravel & cemented & .- & -- & \\
\hline 360 & 6/4-26H1 & & & 477 & 516 & 145.4 & 157.3 & gravel, cemented & Gravel & cemented & -- & -- & \\
\hline 360 & 6/4-26H1 & & & 516 & 540 & 157.3 & 164.6 & lava, hard, stratified; water & Basalt & hard & -- & -- & \\
\hline 360 & 6/4-26H1 & & & 540 & 595 & 164.6 & 181.4 & basalt, hard & Basalt & hard & .- & .- & \\
\hline 360 & $6 / 4-26 \mathrm{H} 1$ & & & 595 & 602 & 181.4 & 183.5 & lava, cemented & Basalt & -- & -- & -- & \\
\hline 360 & 6/4-26H1 & & & 602 & 643 & 183.5 & 196.0 & lava, broken, water & Basalt & -- & -- & -- & \\
\hline
\end{tabular}


Appendix 3. Lithologic data. - Continued

[Site IDs and well number correspond to locations in Appendix 1, a site ID and well number is listed for each drilled interval for a particular drill hole. For convenience, the site ID and well numbers are repeated at the shallowest drilled interval for each well. leaders (--), not reported]

\begin{tabular}{|c|c|c|c|c|c|c|c|c|c|c|c|c|c|}
\hline Site ID & Well number & $\begin{array}{c}\text { Site ID, } \\
\text { top of } \\
\text { well }\end{array}$ & $\begin{array}{l}\text { Well number, } \\
\text { top of well }\end{array}$ & $\begin{array}{c}\text { Top of } \\
\text { interval, in } \\
\text { feet }\end{array}$ & $\begin{array}{l}\text { Base of } \\
\text { interval, in } \\
\text { feet }\end{array}$ & $\begin{array}{c}\begin{array}{c}\text { Top of } \\
\text { interval, in } \\
\mathrm{m}\end{array} \\
\end{array}$ & $\begin{array}{c}\text { Base of } \\
\text { interval, in } \\
\mathrm{m}\end{array}$ & Reported lithologic unit' & Interpreted lithologic class ${ }^{2}$ & $\begin{array}{c}\text { Reported } \\
\text { degree of } \\
\text { cementation or } \\
\text { induration }^{3} \\
\end{array}$ & $\begin{array}{c}\text { Fossils } \\
\text { reported in } \\
\text { interval }^{4}\end{array}$ & $\begin{array}{c}\text { Organic } \\
\text { matter } \\
\text { reported in } \\
\text { interval }^{5}\end{array}$ & $\begin{array}{l}\text { Reported stratigraphic top of geologic } \\
\text { unit }^{6}\end{array}$ \\
\hline 361 & $6 / 4-28 F 1$ & 361 & $6 / 4-28 F 1$ & 0 & 3 & 0.0 & 0.9 & soil & Sand and clay & -- & -- & -- & Qoal \\
\hline 361 & $6 / 4-28 \mathrm{~F} 1$ & & & 3 & 15 & 0.9 & 4.6 & clay, yellow & Clay & -- & -- & -- & \\
\hline 361 & $6 / 4-28 F 1$ & & & 15 & 17 & 4.6 & 5.2 & gravel & Gravel & -- & -- & -- & \\
\hline 361 & $6 / 4-28 F 1$ & & & 17 & 25 & 5.2 & 7.6 & clay, yellow & Clay & -- & -- & -- & \\
\hline 361 & $6 / 4-28 \mathrm{~F} 1$ & & & 25 & 35 & 7.6 & 10.7 & gravel, water & Gravel & -- & -- & -- & \\
\hline 361 & $6 / 4-28 F 1$ & & & 35 & 50 & 10.7 & 15.2 & clay, yellow & Clay & -- & -- & -- & \\
\hline 361 & $6 / 4-28 F 1$ & & & 50 & 55 & 15.2 & 16.8 & gravel, water & Gravel & -- & -- & -- & \\
\hline 361 & 6/4-28F1 & & & 55 & 90 & 16.8 & 27.4 & clay, yellow & Clay & -- & -- & -- & \\
\hline 361 & $6 / 4-28 \mathrm{~F} 1$ & & & 90 & 95 & 27.4 & 29.0 & clay, yellow & Clay & -- & -- & -- & \\
\hline 362 & $6 / 4-26 \mathrm{~F} 1$ & 362 & 6/4-26F1 & 0 & 3 & 0.0 & 0.9 & soil & Sand and clay & -- & -- & -- & Qoal \\
\hline 362 & $6 / 4-26 F 1$ & & & 3 & 12 & 0.9 & 3.7 & clay & Clay & -- & -- & -- & \\
\hline 362 & 6/4-26F1 & & & 12 & 32 & 3.7 & 9.8 & clay and boulders & Clay and gravel & -- & -- & -- & \\
\hline 362 & 6/4-26F1 & & & 32 & 42 & 9.8 & 12.8 & clay, blue & Clay & -- & -- & -- & \\
\hline 362 & 6/4-26F1 & & & 42 & 45 & 12.8 & 13.7 & rocks, broken; water & Gravel & hard & -- & -- & \\
\hline 362 & 6/4-26F1 & & & 45 & 84 & 13.7 & 25.6 & $\begin{array}{l}\text { clay, blue } \\
\text { late }\end{array}$ & Clay & -- & -- & -- & \\
\hline 362 & $6 / 4-26 F 1$ & & & 84 & 101 & 25.6 & 30.8 & clay, gray & Clay & -- & -- & -- & \\
\hline 362 & 6/4-26F1 & & & 101 & 131 & 30.8 & 39.9 & clay, blue & Clay & -- & -- & -- & \\
\hline 362 & 6/4-26F1 & & & 131 & 167 & 39.9 & 50.9 & lava, mud & Basalt & -- & -- & -- & Qh \\
\hline 362 & $6 / 4-26 F 1$ & & & 167 & 171 & 50.9 & 52.1 & lava, water & Basalt & -- & -- & -- & \\
\hline 362 & 6/4-26F1 & & & 171 & 198 & 52.1 & 60.4 & mud, volcanic & Ash or tuff & -- & -- & -- & \\
\hline 362 & 6/4-26F1 & & & 198 & 204 & 60.4 & 62.2 & lava, water & Basalt & -- & -- & -- & \\
\hline 363 & 6/4-28K2 & 363 & 6/4-28K2 & 0 & 2 & 0.0 & 0.6 & soil & Sand and clay & -- & -- & -- & Qoal \\
\hline 363 & 6/4-28K2 & & & 2 & 35 & 0.6 & 10.7 & clay, yellow & Clay & -- & -- & -- & \\
\hline 363 & 6/4-28K2 & & & 35 & 55 & 10.7 & 16.8 & clay, yellow & Clay & -- & -- & -- & \\
\hline 363 & 6/4-28K2 & & & 55 & 57 & 16.8 & 17.4 & gravel, water & Gravel & -- & -- & -- & \\
\hline 363 & 6/4-28K2 & & & 57 & 82 & 17.4 & 25.0 & clay, yellow & Clay & -- & -- & -- & \\
\hline 363 & 6/4-28K2 & & & 82 & 87 & 25.0 & 26.5 & gravel, water & Gravel & -- & -- & -- & \\
\hline 363 & 6/4-28K2 & & & 87 & 93 & 26.5 & 28.3 & clay, sandy & Clay and sand (or sandstone) & -- & -- & -- & \\
\hline 363 & $6 / 4-28 \mathrm{~K} 2$ & & & 93 & 127 & 28.3 & 38.7 & clay, blue & Clay & -- & -- & -- & \\
\hline 363 & $6 / 4-28 K 2$ & & & 127 & 145 & 38.7 & 44.2 & gravel, water & Gravel & -- & -- & -- & \\
\hline 363 & 6/4-28K2 & & & 145 & 155 & 44.2 & 47.2 & clay, water & Clay & -- & -- & -- & \\
\hline 364 & 6/4-26L2 & 364 & 6/4-26L2 & 0 & 3 & 0.0 & 0.9 & soil & Sand and clay & -- & -- & -- & Qoal \\
\hline 364 & $6 / 4-26\llcorner 2$ & & & 3 & 20 & 0.9 & 6.1 & clay & Clay & -- & -- & -- & \\
\hline 364 & 6/4-26L2 & & & 20 & 21 & 6.1 & 6.4 & clay and gravel, some water & Clay and gravel & -- & -. & -- & \\
\hline 364 & 6/4-26ட2 & & & 21 & 36 & 6.4 & 11.0 & clay and boulders & Clay and gravel & -- & -- & -- & \\
\hline 364 & 6/4-26ட2 & & & 36 & 45 & 11.0 & 13.7 & clay, blue & Clay & -- & -- & -- & \\
\hline 364 & $6 / 4-26 \mathrm{~L} 2$ & & & 45 & 180 & 13.7 & 54.9 & tuff and gravel, water & Ash or tuff & -- & -- & -. & Qh (?) \\
\hline 365 & 6/4-26ட1 & 365 & 6/4-26ட1 & 0 & 174 & 0.0 & 53.0 & previously drilled & No data & -- & -- & -- & Qoal and Qh \\
\hline 365 & $6 / 4-26 \mathrm{~L} 1$ & & & 174 & 176 & 53.0 & 53.6 & clay, blue & Clay & -- & -- & -- & \\
\hline 365 & 6/4-26ட1 & & & 176 & 188 & 53.6 & 57.3 & rock, hard & Ash or tuff & hard & -- & -- & Tsv (?) \\
\hline 365 & 6/4-26ட1 & & & 188 & 194 & 57.3 & 59.1 & rock, broken; water & Ash or tuff & hard & -- & -- & \\
\hline 365 & 6/4-26ட1 & & & 194 & 225 & 59.1 & 68.6 & clay, gray & Clay & -- & -- & -- & \\
\hline 365 & 6/4-26L1 & & & 225 & 230 & 68.6 & 70.1 & rock, broken; water & Ash or tuff & hard & -- & -- & \\
\hline 365 & 6/4-26L1 & & & 230 & 249 & 70.1 & 75.9 & clay, gray & Clay & -- & -- & -- & \\
\hline 366 & 6/4-27M1 & 366 & 6/4-27M1 & 0 & 3 & 0.0 & 0.9 & soil & Sand and clay & -- & -- & -- & Qoal \\
\hline 366 & 6/4-27M1 & & & 3 & 24 & 0.9 & 7.3 & clay, yellow & Clay & -- & -- & -- & \\
\hline 366 & 6/4-27M1 & & & 24 & 26 & 7.3 & 7.9 & gravel, water & Gravel & -- & -- & -- & \\
\hline 366 & 6/4-27M1 & & & 26 & 90 & 7.9 & 27.4 & clay, yellow & Clay & -- & -- & -- & \\
\hline 366 & 6/4-27M1 & & & 90 & 100 & 27.4 & 30.5 & gravel, water & Gravel & -- & -- & -- & \\
\hline 366 & 6/4-27M1 & & & 100 & 108 & 30.5 & 32.9 & clay, yellow & Clay & -- & -- & -- & \\
\hline 367 & 6/4-26N1 & 367 & 6/4-26N1 & 0 & 12 & 0.0 & 3.7 & soil & Sand and clay & -- & -- & -- & Qyal and Qoal \\
\hline 367 & $6 / 4-26 \mathrm{~N} 1$ & & & 12 & 26 & 3.7 & 7.9 & clay & Clay & -- & -- & -- & \\
\hline 367 & $6 / 4-26 N 1$ & & & 26 & 39 & 7.9 & 11.9 & clay, gravel, water & Clay and gravel & -- & -- & -- & \\
\hline 367 & $6 / 4-26 \mathrm{~N} 1$ & & & 39 & 61 & 11.9 & 18.6 & clay & Clay & -- & -- & -- & \\
\hline 367 & $6 / 4-26 N 1$ & & & 61 & 63 & 18.6 & 19.2 & clay, gravel, water & Clay and gravel & -- & -- & -- & \\
\hline 367 & 6/4-26N1 & & & 63 & 96 & 19.2 & 29.3 & boulders and clay & Clay and gravel & - & -- & -- & \\
\hline 367 & $6 / 4-26 \mathrm{~N} 1$ & & & 96 & 102 & 29.3 & 31.1 & boulders, sand, gravel, water & Sand (or sandstone) and gravel & -- & -- & -- & \\
\hline 367 & $6 / 4-26 \mathrm{~N} 1$ & & & 102 & 123 & 31.1 & 37.5 & clay and gravel; some water & Clay and gravel & .- & -- & -. & \\
\hline 367 & $6 / 4-26 \mathrm{~N} 1$ & & & 123 & 144 & 37.5 & 43.9 & clay, blue some water & Clay & -- & -- & -- & \\
\hline 367 & 6/4-26N1 & & & 144 & 150 & 43.9 & 45.7 & boulders and clay & Clay and gravel & -- & -- & -- & \\
\hline 368 & 6/4-27N1 & 368 & 6/4-27N1 & 0 & 41 & 0.0 & 12.5 & not described & No data & -- & -- & -- & Qoal \\
\hline
\end{tabular}


Appendix 3. Lithologic data. - Continued

[Site IDs and well number correspond to locations in Appendix 1, a site ID and well number is listed for each drilled interval for a particular drill hole. For convenience, the site ID and well numbers are repeated at the shallowest drilled interval for each well. leaderst.

\begin{tabular}{|c|c|c|c|c|c|c|c|c|c|c|c|c|c|}
\hline Site ID & Well number & $\begin{array}{l}\text { Site ID, } \\
\text { top of } \\
\text { well }\end{array}$ & $\begin{array}{l}\text { Well number, } \\
\text { top of well }\end{array}$ & $\begin{array}{c}\text { Top of } \\
\text { interval, in } \\
\text { feet }\end{array}$ & $\begin{array}{c}\text { Base of } \\
\text { interval, in } \\
\text { feet }\end{array}$ & $\begin{array}{c}\text { Top of } \\
\text { interval, in } \\
m\end{array}$ & $\begin{array}{c}\text { Base of } \\
\text { interval, in } \\
\mathrm{m}\end{array}$ & Reported lithologic unit' & Interpreted lithologic class ${ }^{2}$ & $\begin{array}{c}\text { Reported } \\
\text { degree of } \\
\text { cementation or } \\
\text { induration } \\
\end{array}$ & $\begin{array}{l}\text { Fossils } \\
\text { reported in } \\
\text { interval }^{4}\end{array}$ & $\begin{array}{c}\text { Organic } \\
\text { matter } \\
\text { reported in } \\
\text { interval }^{5}\end{array}$ & $\begin{array}{l}\text { Reported stratigraphic top of geologic } \\
\text { unit }^{6}\end{array}$ \\
\hline 368 & $6 / 4-27 \mathrm{~N} 1$ & & & 41 & 54 & 12.5 & 16.5 & gravel, sand, water & Sand (or sandstone) and gravel & -- & -- & -- & \\
\hline 368 & $6 / 4-27 \mathrm{~N} 1$ & & & 54 & 58 & 16.5 & 17.7 & clay & Clay & -- & -- & -- & \\
\hline 368 & $6 / 4-27 \mathrm{~N} 1$ & & & 58 & 69 & 17.7 & 21.0 & gravel, water & Gravel & -- & -- & -- & \\
\hline 368 & 6/4-27N1 & & & 69 & 91 & 21.0 & 27.7 & gravel, boulders, water & Gravel & -- & -. & -- & \\
\hline 368 & $6 / 4-27 N 1$ & & & 91 & 99 & 27.7 & 30.2 & gravel, water & Gravel & -- & -- & -- & \\
\hline 368 & 6/4-27N1 & & & 99 & 102 & 30.2 & 31.1 & clay, soft & Clay & -. & -- & -- & \\
\hline 368 & $6 / 4-27 \mathrm{~N} 1$ & & & 102 & 115 & 31.1 & 35.1 & gravel water & Gravel & -- & -- & -- & \\
\hline 368 & $6 / 4-27 \mathrm{~N} 1$ & & & 115 & 141 & 35.1 & 43.0 & clay, gravel, water & Clay and gravel & -- & -- & -- & \\
\hline 369 & 6/4-28Q1 & 369 & 6/4-28Q1 & 0 & 30 & 0.0 & 9.1 & not described & No data & -- & -- & -- & Qoal \\
\hline 369 & 6/4-28Q1 & & & 30 & 40 & 9.1 & 12.2 & boulders and clay & Clay and gravel & -- & -- & -- & \\
\hline 369 & 6/4-28Q1 & & & 40 & 43 & 12.2 & 13.1 & gravel, water & Gravel & -- & -- & -- & \\
\hline 369 & 6/4-28Q1 & & & 43 & 50 & 13.1 & 15.2 & clay & Clay & -- & -- & -- & \\
\hline 369 & 6/4-28Q1 & & & 50 & 52 & 15.2 & 15.8 & gravel, water & Gravel & -- & -- & -- & \\
\hline 369 & 6/4-28Q1 & & & 52 & 69 & 15.8 & 21.0 & clay, yellow & Clay & -- & -- & -- & \\
\hline 369 & 6/4-28Q1 & & & 69 & 81 & 21.0 & 24.7 & clay, some water & Clay & -- & -- & -- & \\
\hline 370 & 6/4-34D2 & 370 & 6/4-34D2 & 0 & 10 & 0.0 & 3.0 & surface & Sand and clay & -- & -- & -- & Qoal \\
\hline 370 & 6/4-34D2 & & & 10 & 45 & 3.0 & 13.7 & clay, yellow & Clay & -- & -- & -- & \\
\hline 370 & 6/4-34D2 & & & 45 & 65 & 13.7 & 19.8 & gravel & Gravel & -- & -- & -.- & \\
\hline 370 & 6/4-34D2 & & & 65 & 85 & 19.8 & 25.9 & clay and gravel & Clay and gravel & -- & -- & -- & \\
\hline 370 & 6/4-34D2 & & & 85 & 105 & 25.9 & 32.0 & clay, yellow & Clay & -- & -- & -. & \\
\hline 370 & 6/4-34D2 & & & 105 & 110 & 32.0 & 33.5 & rock and clay, mixed & Clay and gravel & -- & -- & -- & \\
\hline 370 & 6/4-34D2 & & & 110 & 125 & 33.5 & 38.1 & clay, yellow & Clay & -- & -- & -- & \\
\hline 370 & 6/4-34D2 & & & 125 & 130 & 38.1 & 39.6 & boulders and clay & Clay and gravel & -- & -- & -- & \\
\hline 370 & 6/4-34D2 & & & 130 & 150 & 39.6 & 45.7 & clay, yellow & Clay & -- & -- & -.. & \\
\hline 370 & 6/4-34D2 & & & 150 & 155 & 45.7 & 47.2 & clay and gravel & Clay and gravel & -- & -- & -.- & \\
\hline 370 & 6/4-34D2 & & & 155 & 190 & 47.2 & 57.9 & clay, yellow & Clay & -- & -- & -- & \\
\hline 370 & 6/4-34D2 & & & 190 & 193 & 57.9 & 58.8 & gravel & Gravel & -- & -- & -- & \\
\hline 370 & 6/4-34D2 & & & 193 & 205 & 58.8 & 62.5 & clay, yellow & Clay & - & - & - & \\
\hline 370 & 6/4-34D2 & & & 205 & 235 & 62.5 & 71.6 & gravel and clay & Clay and gravel & -- & -- & -.- & \\
\hline 370 & 6/4-34D2 & & & 235 & 243 & 71.6 & 74.1 & $\begin{array}{l}\text { clay, yellow } \\
\text { lat }\end{array}$ & Clay & -. & -- & -. & \\
\hline 370 & 6/4-34D2 & & & 243 & 247 & 74.1 & 75.3 & gravel and clay & Clay and gravel & -- & -- & -- & \\
\hline 370 & 6/4-34D2 & & & 247 & 255 & 75.3 & 77.7 & $\begin{array}{l}\text { clay, yellow } \\
\text { col }\end{array}$ & Clay & -- & -- & -- & \\
\hline 370 & 6/4-34D2 & & & 255 & 265 & 77.7 & 80.8 & gravel and clay & Clay and gravel & - & -- &.- & \\
\hline 370 & 6/4-34D2 & & & 265 & 285 & 80.8 & 86.9 & clay, yellow & Clay & -- & -- & -. & \\
\hline 370 & 6/4-34D2 & & & 285 & 287 & 86.9 & 87.5 & gravel & Gravel & -- & -- & -- & \\
\hline 370 & 6/4-34D2 & & & 287 & 290 & 87.5 & 88.4 & clay, yellow & Clay & -- & -- & -- & \\
\hline 370 & 6/4-34D2 & & & 290 & 292 & 88.4 & 89.0 & gravel & Gravel & -- & -- & -. & \\
\hline 370 & 6/4-34D2 & & & 292 & 315 & 89.0 & 96.0 & clay, yellow & Clay & -- & -- & -- & \\
\hline 370 & 6/4-34D2 & & & 315 & 317 & 96.0 & 96.6 & boulders and gravel & Gravel & -- & -- & -- & \\
\hline 370 & 6/4-34D2 & & & 317 & 343 & 96.6 & 104.5 & clay, yellow & Clay & -. & -. & .. & \\
\hline 370 & 6/4-34D2 & & & 343 & 347 & 104.5 & 105.8 & gravel & Gravel & -- & -- & -- & \\
\hline 370 & 6/4-34D2 & & & 347 & 365 & 105.8 & 111.3 & gravel and clay & Clay and gravel & -- & -- & -- & \\
\hline 370 & 6/4-34D2 & & & 365 & 380 & 111.3 & 115.8 & clay, yellow & Clay & -. & -- & -- & \\
\hline 371 & 6/4-36C1 & 371 & 6/4-36C1 & 0 & 140 & 0.0 & 42.7 & not described & No data & -. & -- & -. & Qyal (?) \\
\hline 371 & $6 / 4-36 \mathrm{C} 1$ & STI & & 140 & 145 & 42.7 & 44.2 & rock, blue & Sandstone & hard & -- &.- & Syal (?) \\
\hline 371 & $6 / 4-36 \mathrm{C} 1$ & & & 145 & 162 & 44.2 & 49.4 & clay, blue & Clay & -- & -. & -- & \\
\hline 371 & $6 / 4-36 \mathrm{C} 1$ & & & 162 & 231 & 49.4 & 70.4 & gravel and rock, water & Gravel & -- & -- & -. & \\
\hline 371 & $6 / 4-36 \mathrm{C} 1$ & & & 231 & 240 & 70.4 & 73.2 & clay, blue & Clay & -- & -.- & -. & \\
\hline 371 & $6 / 4-36 \mathrm{C} 1$ & & & 240 & 387 & 73.2 & 118.0 & sandstone & Sandstone & cemented & .- & .- & \\
\hline 371 & $6 / 4-36 \mathrm{C} 1$ & & & 387 & 396 & 118.0 & 120.7 & gravel and water, good flow & Gravel & -- & -- & -- & \\
\hline 371 & $6 / 4-36 \mathrm{C} 1$ & & & 396 & 400 & 120.7 & 121.9 & rock, blue & Sandstone & hard & -- & -- & \\
\hline 371 & 6/4-36C1 & & & 400 & 405 & 121.9 & 123.4 & sand and gravel & Sand (or sandstone) and gravel & -- & -- & -. & \\
\hline 371 & $6 / 4-36 \mathrm{C} 1$ & & & 405 & 447 & 123.4 & 136.2 & clay & Clay & -- & -- & -- & \\
\hline 372 & 6/4-35G2 & 372 & $6 / 4-35 G 2$ & 0 & 4 & 0.0 & 1.2 & surface & Sand and clay & -- & -- & -- & Qoal \\
\hline 372 & $6 / 4-35 \mathrm{G} 2$ & & & 4 & 12 & 1.2 & 3.7 & clay and boulders & Clay and gravel & -- & -- & -- & \\
\hline 372 & 6/4-35G2 & & & 12 & 25 & 3.7 & 7.6 & clay and boulders, yellow & Clay and gravel & -- & -- & -- & \\
\hline 372 & 6/4-35G2 & & & 25 & 35 & 7.6 & 10.7 & clay and boulders, blue & Clay and gravel & -- & -- & -- & \\
\hline 372 & 6/4-35G2 & & & 35 & 85 & 10.7 & 25.9 & clay, yellow & Clay & -- & -- & -- & \\
\hline 372 & $6 / 4-35 \mathrm{G} 2$ & & & 85 & 97 & 25.9 & 29.6 & gravel and boulders, water & Gravel & -- & -- & -- & \\
\hline 372 & 6/4-35G2 & & & 97 & 140 & 29.6 & 42.7 & clay, yellow & Clay & -- & -- & -- & \\
\hline 372 & 6/4-35G2 & & & 140 & 190 & 42.7 & 57.9 & clay and gravel, yellow & Clay and gravel & -- & -- & -- & \\
\hline
\end{tabular}


Appendix 3. Lithologic data. - Continued

[Site IDs and well number correspond to locations in Appendix 1, a site ID and well number is listed for each drilled interval for a particular drill hole. For convenience, the site ID and well numbers are repeated at the shallowest drilled interval for each well.

\begin{tabular}{|c|c|c|c|c|c|c|c|c|c|c|c|c|c|}
\hline Site ID & Well number & $\begin{array}{l}\text { Site ID, } \\
\text { top of } \\
\text { well }\end{array}$ & $\begin{array}{l}\text { Well number, } \\
\text { top of well }\end{array}$ & $\begin{array}{c}\text { Top of } \\
\text { interval, in } \\
\text { feet }\end{array}$ & $\begin{array}{c}\text { Base of } \\
\text { interval, in } \\
\text { feet }\end{array}$ & $\begin{array}{c}\text { Top of } \\
\text { interval, in } \\
m\end{array}$ & $\begin{array}{c}\text { Base of } \\
\text { interval, in } \\
\mathrm{m}\end{array}$ & Reported lithologic unit ${ }^{1}$ & Interpreted lithologic class ${ }^{2}$ & $\begin{array}{c}\text { Reported } \\
\text { degree of } \\
\text { cementation or } \\
\text { induration }^{3} \\
\end{array}$ & $\begin{array}{c}\text { Fossils } \\
\text { reported in } \\
\text { interval }^{4}\end{array}$ & $\begin{array}{c}\text { Organic } \\
\text { matter } \\
\text { reported in } \\
\text { interval }^{5}\end{array}$ & $\begin{array}{l}\text { Reported stratigraphic top of geologic } \\
\text { unit }^{6}\end{array}$ \\
\hline 372 & $6 / 4-35 \mathrm{G} 2$ & & & 190 & 224 & 57.9 & 68.3 & clay, blue & Clay & -- & -- & -- & \\
\hline 372 & 6/4-35G2 & & & 224 & 250 & 68.3 & 76.2 & volcanic ash, blue, water & Ash or tuff & -- & -- & -- & Tsv \\
\hline 372 & $6 / 4-35 \mathrm{G} 2$ & & & 250 & 258 & 76.2 & 78.6 & clay, blue & Clay & -- & -- & -- & \\
\hline 373 & $6 / 4-35 \mathrm{G} 3$ & 373 & 6/4-35G3 & 0 & 10 & 0.0 & 3.0 & surface & Sand and clay & -- & -- & -- & Qoal \\
\hline 373 & 6/4-35G3 & & & 10 & 30 & 3.0 & 9.1 & clay, yellow; gravel, no water & Clay and gravel & -- & -- & -- & \\
\hline 373 & 6/4-35G3 & & & 30 & 39 & 9.1 & 11.9 & hard boulders & Gravel & hard & -- & -- & \\
\hline 373 & 6/4-35G3 & & & 39 & 80 & 11.9 & 24.4 & clay, yellow and boulders & Clay and gravel & -- & -- & -- & \\
\hline 373 & 6/4-35G3 & & & 80 & 85 & 24.4 & 25.9 & boulders, some water & Gravel & -- & -. & -- & \\
\hline 373 & 6/4-35G3 & & & 85 & 105 & 25.9 & 32.0 & boulders and clay & Clay and gravel & - & -. & - & \\
\hline 373 & 6/4-35G3 & & & 105 & 120 & 32.0 & 36.6 & clay, yellow & Clay & -- & -- & -- & \\
\hline 373 & 6/4-35G3 & & & 120 & 138 & 36.6 & 42.1 & sand and broken rock & Sand (or sandstone) and gravel & hard & -- & -- & \\
\hline 373 & $6 / 4-35 G 3$ & & & 138 & 147 & 42.1 & 44.8 & clay, yellow & Clay & -- & -- & -- & \\
\hline 373 & 6/4-35G3 & & & 147 & 160 & 44.8 & 48.8 & clay, yellow; and sand & Clay and gravel & -- & -- & -- & \\
\hline 373 & 6/4-35G3 & & & 160 & 220 & 48.8 & 67.1 & clay, blue sand and gravel & Clay, sand, and gravel & -- & -- & -- & \\
\hline 373 & 6/4-35G3 & & & 220 & 238 & 67.1 & 72.5 & clay, yellow, and sand and gravel & Clay and sand (or sandstone) & -- & -. & -- & \\
\hline 373 & 6/4-35G3 & & & 238 & 250 & 72.5 & 76.2 & volcanic ash, blue & Ash or tuff & -- & -- & -- & Tsv \\
\hline 373 & 6/4-35G3 & & & 250 & 260 & 76.2 & 79.2 & lava rock, black & Basalt & hard & -- & -- & \\
\hline 374 & 6/4-35G4 & 374 & 6/4-35G4 & 0 & 10 & 0.0 & 3.0 & surface & Sand and clay & -- & -- & -- & Qoal \\
\hline 374 & 6/4-35G4 & & & 10 & 40 & 3.0 & 12.2 & clay, yellow, and gravel & Clay and gravel & .. & -- & -. & \\
\hline 374 & 6/4-35G4 & & & 40 & 55 & 12.2 & 16.8 & $\begin{array}{l}\text { clay, yellow } \\
\text { lation }\end{array}$ & Clay & -- & -- & -- & \\
\hline 374 & $6 / 4-35 G 4$ & & & 55 & 120 & 16.8 & 36.6 & clay, blue, and boulders & Clay and gravel & -- & -- & -- & \\
\hline 374 & $6 / 4-35 \mathrm{G} 4$ & & & 120 & 135 & 36.6 & 41.1 & clay, yellow, sand and gravel & Clay, sand, and gravel & -- & -- & -- & \\
\hline 374 & $6 / 4-35 G 4$ & & & 135 & 145 & 41.1 & 44.2 & clay, yellow & Clay & -- & -- & -- & \\
\hline 374 & $6 / 4-35 \mathrm{G} 4$ & & & 145 & 200 & 44.2 & 61.0 & clay, blue and boulders & Clay and gravel & -- & -- & -- & \\
\hline 374 & 6/4-35G4 & & & 200 & 215 & 61.0 & 65.5 & clay, blue & Clay & -- & -- & -- & \\
\hline 374 & 6/4-35G4 & & & 215 & 245 & 65.5 & 74.7 & lava rock, soft, water & Basalt & hard & -- & -- & Tsv \\
\hline 374 & 6/4-35G4 & & & 245 & 250 & 74.7 & 76.2 & basalt, water & Basalt & -. & -- & -. & \\
\hline 375 & 6/4-33L2 & 375 & 6/4-33L2 & 0 & 6 & 0.0 & 1.8 & soil & Sand and clay & -- & -- & -- & Qyal and Qoal \\
\hline 375 & 6/4-33L2 & & & 6 & 21 & 1.8 & 6.4 & clay & Clay & -- & -- & -- & \\
\hline 375 & 6/4-33L2 & & & 21 & 40 & 6.4 & 12.2 & sand, boulders, water & Sand & -- & -- & -- & \\
\hline 375 & 6/4-33L2 & & & 40 & 56 & 12.2 & 17.1 & boulders and clay & Clay and gravel & -- & -- & -- & \\
\hline 375 & 6/4-33L2 & & & 56 & 65 & 17.1 & 19.8 & clay & Clay & -- & -- & -- & \\
\hline 375 & 6/4-33L2 & & & 65 & 140 & 19.8 & 42.7 & boulders and clay & Clay and gravel & -- & -- & -- & \\
\hline 375 & 6/4-33L2 & & & 140 & 155 & 42.7 & 47.2 & clay & Clay & -- & -- & -- & \\
\hline 375 & 6/4-33L2 & & & 155 & 170 & 47.2 & 51.8 & boulders and clay & Clay and gravel & -- & -- & -- & \\
\hline 375 & $6 / 4-33 L 2$ & & & 170 & 198 & 51.8 & 60.4 & clay & Clay & -- & -- & .- & \\
\hline 375 & 6/4-33L2 & & & 198 & 202 & 60.4 & 61.6 & gravel, sand, water & Sand (or sandstone) and gravel & -- & -- & -- & \\
\hline 376 & 6/4-35L2 & 376 & 6/4-35L2 & 0 & 6 & 0.0 & 1.8 & surface & Sand and clay & -- & -- & -- & Qyal \\
\hline 376 & $6 / 4-35 \mathrm{~L} 2$ & & & 6 & 15 & 1.8 & 4.6 & boulders & Gravel & -- & -- & -- & \\
\hline 376 & 6/4-35L2 & & & 15 & 68 & 4.6 & 20.7 & clay, yellow & Clay & -- & -- & -- & Qoal \\
\hline 376 & $6 / 4-35 L 2$ & & & 68 & 75 & 20.7 & 22.9 & boulders & Gravel & -- & -- & -.- & \\
\hline 376 & $6 / 4-35 L 2$ & & & 75 & 93 & 22.9 & 28.3 & clay, yellow & Clay & -- & -- & -- & \\
\hline 376 & $6 / 4-35 \mathrm{~L} 2$ & & & 93 & 102 & 28.3 & 31.1 & boulders & Gravel & - & -- & -- & \\
\hline 376 & 6/4-35L2 & & & 102 & 150 & 31.1 & 45.7 & clay, yellow & Clay & -- & -- & -. & \\
\hline 376 & 6/4-35L2 & & & 150 & 165 & 45.7 & 50.3 & clay and some gravel, yellow & Clay and gravel & -- & -- & -- & \\
\hline 376 & $6 / 4-35 \mathrm{~L} 2$ & & & 165 & 175 & 50.3 & 53.3 & clay and some gravel, blue & Clay and gravel & -. & -- & .- & \\
\hline 376 & $6 / 4-35 L 2$ & & & 175 & 250 & 53.3 & 76.2 & volcanic ash, black & Ash or tuff & -- & -- & -- & Tsv \\
\hline 376 & $6 / 4-35 \mathrm{~L} 2$ & & & 250 & 260 & 76.2 & 79.2 & hard lava or soft basalt & Basalt & hard & .- & -- & \\
\hline 377 & $6 / 4-36 \mathrm{J1}$ & 377 & 6/4-36J1 & 0 & 60 & 0.0 & 18.3 & boulders and conglomerate & Clay, sand, and gravel & -- & -- & -- & \\
\hline 377 & $6 / 4-36 J 1$ & & & 60 & 325 & 18.3 & 99.1 & tule mud & Clay and sand (or sandstone) & -- & -- & tule & \\
\hline 377 & $6 / 4-36 \mathrm{J1}$ & & & 325 & 328 & 99.1 & 100.0 & $\begin{array}{l}\text { gravel } \\
\text { gave }\end{array}$ & Gravel & -- & -- & -- & \\
\hline 377 & 6/4-36J1 & & & 328 & 432 & 100.0 & 131.7 & shale, blue, with gravel & Clay and sand (or sandstone) & cemented & -- & -- & \\
\hline 378 & 6/4-36L1 & 378 & 6/4-36L1 & 0 & 3 & 0.0 & 0.9 & soil & Sand and clay & -- & -- & -- & Qyal \\
\hline 378 & 6/4-36L1 & & & 3 & 25 & 0.9 & 7.6 & boulders and sand & Sand (or sandstone) and gravel & .. & -- & -.. & \\
\hline 378 & 6/4-36L1 & & & 25 & 115 & 7.6 & 35.1 & tuff and boulders; some water & Ash or tuff & -- & -. & -. & Tsv \\
\hline 378 & 6/4-36L1 & & & 115 & 228 & 35.1 & 69.5 & rock, hard & Ash or tuff & hard & -- & -- & \\
\hline 378 & 6/4-36L1 & & & 228 & 243 & 69.5 & 74.1 & lava, broken; some water & Basalt & -- & -- & - & \\
\hline 379 & $6 / 4-32 J 1$ & 379 & 6/4-32J1 & 0 & 10 & 0.0 & 3.0 & surface & Sand and clay & -. & -- & -. & Qyal and Qoal \\
\hline 379 & $6 / 4-32 \mathrm{~s} 1$ & & & 10 & 170 & 3.0 & 51.8 & clay, yellow, sand and gravel & Clay, sand, and gravel & -- & -- & -- & \\
\hline 379 & $6 / 4-32 \mathrm{J1}$ & & & 170 & 445 & 51.8 & 135.6 & clay, yellow & Clay & -- & -- & -- & \\
\hline
\end{tabular}


Appendix 3. Lithologic data. - Continued

ISite IDs and well number correspond to locations in Appendix 1, a site ID and well number is listed for each drilled interval for a particular drill hole. For convenience, the site ID and well numbers are repeated at the shallowest drilled interval for each well. leaders $1-$

\begin{tabular}{|c|c|c|c|c|c|c|c|c|c|c|c|c|c|}
\hline Site ID & Well number & $\begin{array}{l}\text { Site ID, } \\
\text { top of } \\
\text { well }\end{array}$ & $\begin{array}{l}\text { Well number, } \\
\text { top of well }\end{array}$ & $\begin{array}{l}\text { Top of } \\
\text { interval, in } \\
\text { feet }\end{array}$ & $\begin{array}{l}\text { Base of } \\
\text { interval, in } \\
\text { feet }\end{array}$ & $\begin{array}{c}\text { Top of } \\
\text { interval, in } \\
\mathrm{m}\end{array}$ & $\begin{array}{c}\text { Base of } \\
\text { interval, in } \\
\mathrm{m}\end{array}$ & Reported lithologic unit' & Interpreted lithologic class ${ }^{2}$ & $\begin{array}{c}\text { Reported } \\
\text { degree of } \\
\text { cementation or } \\
\text { induration }^{3} \\
\end{array}$ & $\begin{array}{c}\text { Fossils } \\
\text { reported in } \\
\text { interval }^{4}\end{array}$ & $\begin{array}{c}\text { Organic } \\
\text { matter } \\
\text { reported in } \\
\text { interval }^{5}\end{array}$ & $\begin{array}{l}\text { Reported stratigraphic top of geologic } \\
\text { unit }^{6}\end{array}$ \\
\hline 379 & $6 / 4-32 \mathrm{J1}$ & & & 445 & 462 & 135.6 & 140.8 & clay, yellow, sand and gravel & Clay, sand, and gravel & -- & -- & -- & \\
\hline 379 & 6/4-32J1 & & & 462 & 475 & 140.8 & 144.8 & clay, yellow & Clay & -- & -- & -- & \\
\hline 380 & 6/4-33M1 & 380 & 6/4-33M1 & 0 & 2 & 0.0 & 0.6 & topsoil & Sand and clay & -- & -- & -- & Qyal and Qoal \\
\hline 380 & 6/4-33M1 & & & 2 & 15 & 0.6 & 4.6 & clay, yellow & Clay & -- & -- & -- & \\
\hline 380 & 6/4-33M1 & & & 15 & 22 & 4.6 & 6.7 & clay and boulders, yellow & Clay and gravel & -- & -- & -- & \\
\hline 380 & 6/4-33M1 & & & 22 & 62 & 6.7 & 18.9 & clay, gravel, yellow & Clay and gravel & -- & -- & -- & \\
\hline 380 & 6/4-33M1 & & & 62 & 110 & 18.9 & 33.5 & clay, yellow & Clay & -- & -- & -- & \\
\hline 380 & 6/4-33M1 & & & 110 & 133 & 33.5 & 40.5 & gravel & Gravel & -- & -- & -- & \\
\hline 380 & 6/4-33M1 & & & 133 & 167 & 40.5 & 50.9 & clay and gravel, yellow & Clay and gravel & -- & -- & -- & \\
\hline 380 & 6/4-33M1 & & & 167 & 176 & 50.9 & 53.6 & clay, yellow & Clay & -- & -- & -- & \\
\hline 381 & 6/4-35P2 & 381 & 6/4-35P2 & 0 & 5 & 0.0 & 1.5 & soil & Sand and clay & -- & -- & -- & Qyal and Qoal \\
\hline 381 & 6/4-35P2 & & & 5 & 26 & 1.5 & 7.9 & sand loam & Sand and clay & -- & -- & -- & \\
\hline 381 & 6/4-35P2 & & & 26 & 48 & 7.9 & 14.6 & gravel and water & Gravel & -- & -- & -- & \\
\hline 381 & $6 / 4-35 P 2$ & & & 48 & 76 & 14.6 & 23.2 & lava mud, white & Sand and clay & -- & -- & -- & \\
\hline 381 & 6/4-35P2 & & & 76 & 77 & 23.2 & 23.5 & gravel and water & Gravel & -- & -- & -- & \\
\hline 381 & $6 / 4-35 P 2$ & & & 77 & 84 & 23.5 & 25.6 & cemented gravel and lava & Gravel & cemented & -- & -- & \\
\hline 381 & 6/4-35P2 & & & 84 & 85 & 25.6 & 25.9 & gravel and water & Gravel & -- & -- & -- & \\
\hline 381 & 6/4-35P2 & & & 85 & 97 & 25.9 & 29.6 & lava gray & Basalt & -- & -- & -- & Tsv (?) \\
\hline 381 & 6/4-35P2 & & & 97 & 100 & 29.6 & 30.5 & gravel and water & Gravel & -- & -- & -- & \\
\hline 381 & 6/4-35P2 & & & 100 & 105 & 30.5 & 32.0 & pumice & Ash or tuff & -- & -- & -- & \\
\hline 382 & $6 / 4-32 \mathrm{~N} 1$ & 382 & 6/4-32N1 & 0 & 30 & 0.0 & 9.1 & soil and clay & Sand and clay & -- & -- & -- & Knoxville and Horsetown Formations \\
\hline 382 & 6/4-32N1 & & & 30 & 165 & 9.1 & 50.3 & shale, blue & Clay and sand (or sandstone) & cemented & -- & -- & \\
\hline 382 & 6/4-32N1 & & & 165 & 246 & 50.3 & 75.0 & clay, yellow and boulders & Clay and gravel & -- & -- & -- & \\
\hline 382 & $6 / 4-32 \mathrm{~N} 1$ & & & 246 & 247 & 75.0 & 75.3 & gravel, some water & Gravel & -- & -- & -- & \\
\hline 382 & 6/4-32N1 & & & 247 & 398 & 75.3 & 121.3 & clay, yellow, and some boulders & Clay and gravel & -- & -- & -- & \\
\hline 382 & 6/4-32N1 & & & 398 & 502 & 121.3 & 153.0 & 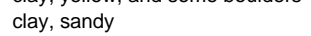 & Clay and sand (or sandstone) & -- & -- & -- & \\
\hline 382 & 6/4-32N1 & & & 502 & 519 & 153.0 & 158.2 & sand, gravel, some water & Sand (or sandstone) and gravel & -- & -- & -- & \\
\hline 383 & 6/4-35Q1 & 383 & 6/4-35Q1 & 0 & 60 & 0.0 & 18.3 & tuff and rock & Ash or tuff & -- & -- & -- & Tsv \\
\hline 383 & 6/4-35Q1 & & & 60 & 81 & 18.3 & 24.7 & rock, broken & Ash or tuff & hard & -- & -- & \\
\hline 383 & 6/4-35Q1 & & & 81 & 86 & 24.7 & 26.2 & not described & No data & - & -- & -- & \\
\hline 383 & 6/4-35Q1 & & & 86 & 117 & 26.2 & 35.7 & rock, red & Ash or tuff & hard & -- & -- & \\
\hline 383 & 6/4-35Q1 & & & 117 & 135 & 35.7 & 41.1 & tuff and boulders, yellow & Ash or tuff & -- & -- & -- & \\
\hline 383 & 6/4-35Q1 & & & 135 & 142 & 41.1 & 43.3 & lava, water & Basalt & -- & -- & -- & \\
\hline 383 & 6/4-35Q1 & & & 142 & 162 & 43.3 & 49.4 & rock and tuff, white & Ash or tuff & -- & -- & -- & \\
\hline 383 & 6/4-35Q1 & & & 162 & 173 & 49.4 & 52.7 & lava, water & Basalt & -- & -- & -- & \\
\hline 383 & 6/4-35Q1 & & & 165 & 192 & 50.3 & 58.5 & lava, water & Basalt & -. & -- & -- & \\
\hline 383 & 6/4-35Q1 & & & 173 & 165 & 52.7 & 50.3 & rock & Ash or tuff & hard & -- & -- & \\
\hline 384 & $6 / 4-35 R 1$ & 384 & 6/4-35R1 & 0 & 15 & 0.0 & 4.6 & surface boulders & Sand and clay & -- & -- & -- & Tsv \\
\hline 384 & $6 / 4-35 R 1$ & & & 15 & 170 & 4.6 & 51.8 & volcanic rock, hard, red & Ash or tuff & hard & -- & -- & \\
\hline 384 & $6 / 4-35 R 1$ & & & 170 & 190 & 51.8 & 57.9 & boulders & Gravel & -- & -- & -- & \\
\hline 384 & 6/4-35R1 & & & 190 & 240 & 57.9 & 73.2 & lava rock, red & Basalt & hard & -- & -- & \\
\hline 384 & $6 / 4-35 R 1$ & & & 240 & 265 & 73.2 & 80.8 & lava rock, gray & Basalt & hard & -- & -- & \\
\hline 384 & $6 / 4-35 R 1$ & & & 265 & 280 & 80.8 & 85.3 & tuff, gray & Ash or tuff & -- & -- & -- & \\
\hline 384 & $6 / 4-35 \mathrm{R} 1$ & & & 280 & 310 & 85.3 & 94.5 & basalt & Basalt & -- & -- & -- & \\
\hline 384 & 6/4-35R1 & & & 310 & 335 & 94.5 & 102.1 & basaltic conglomerate & Basalt & -- & -- & -- & \\
\hline 384 & $6 / 4-35 R 1$ & & & 335 & 360 & 102.1 & 109.7 & basalt & Basalt & -- & -- & -- & \\
\hline 384 & $6 / 4-35 R 1$ & & & 360 & 382 & 109.7 & 116.4 & tuff, hard gray & Ash or tuff & hard & -- & -- & \\
\hline 385 & $5 / 4-2 B 1$ & 385 & $5 / 4-2 \mathrm{~B} 1$ & 0 & 3 & 0.0 & 0.9 & soil & Sand and clay & -- & -- & -- & Qoal \\
\hline 385 & $5 / 4-2 \mathrm{~B} 1$ & & & 3 & 30 & 0.9 & 9.1 & hardpan & Clay and gravel & hard & -- & -- & \\
\hline 385 & $5 / 4-2 \mathrm{~B} 1$ & & & 30 & 56 & 9.1 & 17.1 & rock, yellow & Ash or tuff & hard & -- & -- & \\
\hline 385 & $5 / 4-2 B 1$ & & & 56 & 57 & 17.1 & 17.4 & water, little & Ash or tuff & -- & -- & -- & Tsv \\
\hline 385 & $5 / 4-2 \mathrm{~B} 1$ & & & 57 & 80 & 17.4 & 24.4 & rock, yellow & Ash or tuff & hard & -- & -- & \\
\hline 385 & $5 / 4-2 \mathrm{~B} 1$ & & & 80 & 130 & 24.4 & 39.6 & rock, brown & Ash or tuff & hard & -- & -- & \\
\hline 385 & $5 / 4-2 B 1$ & & & 130 & 160 & 39.6 & 48.8 & rock, red & Ash or tuff & hard & -- & -- & \\
\hline 385 & $5 / 4-2 B 1$ & & & 160 & 202 & 48.8 & 61.6 & rock, brown; water & Ash or tuff & hard & -- & -- & \\
\hline 386 & $5 / 4-1 \mathrm{G} 1$ & 386 & 5/4-1G1 & 0 & 3 & 0.0 & 0.9 & soil & Sand and clay & -- & -- & -- & Tsv \\
\hline 386 & $5 / 4-1 \mathrm{G} 1$ & & & 3 & 28 & 0.9 & 8.5 & tuff & Ash or tuff & -- & -- & -- & \\
\hline 386 & $5 / 4-1 \mathrm{G} 1$ & & & 28 & 49 & 8.5 & 14.9 & clay & Clay & -- & -- & .- & \\
\hline 386 & $5 / 4-1 \mathrm{G} 1$ & & & 49 & 58 & 14.9 & 17.7 & rocks, water at $57 \mathrm{ft}$. & Ash or tuff & hard & -- & -- & \\
\hline 386 & $5 / 4-1 \mathrm{G} 1$ & & & 58 & 61 & 17.7 & 18.6 & tuff & Ash or tuff & -- & -- & -- & \\
\hline
\end{tabular}


Appendix 3. Lithologic data. - Continued

ISite IDs and well number correspond to locations in Appendix 1, a site ID and well number is listed for each drilled interval for a particular drill hole. For convenience, the site ID and well numbers are repeated at the shallowest drilled interval for eachw

\begin{tabular}{|c|c|c|c|c|c|c|c|c|c|c|c|c|c|}
\hline Site ID & Well number & $\begin{array}{l}\text { Site ID, } \\
\text { top of } \\
\text { well }\end{array}$ & $\begin{array}{l}\text { Well number, } \\
\text { top of well }\end{array}$ & $\begin{array}{c}\text { Top of } \\
\text { interval, in } \\
\text { feet }\end{array}$ & $\begin{array}{c}\text { Base of } \\
\text { interval, in } \\
\text { feet }\end{array}$ & $\begin{array}{c}\text { Top of } \\
\text { interval, in } \\
m\end{array}$ & $\begin{array}{c}\text { Base of } \\
\text { interval, in } \\
\mathrm{m}\end{array}$ & Reported lithologic unit ${ }^{1}$ & Interpreted lithologic class ${ }^{2}$ & $\begin{array}{c}\text { Reported } \\
\text { degree of } \\
\text { cementation or } \\
\text { induration }^{3} \\
\end{array}$ & $\begin{array}{c}\text { Fossils } \\
\text { reported in } \\
\text { interval }^{4}\end{array}$ & $\begin{array}{c}\text { Organic } \\
\text { matter } \\
\text { reported in } \\
\text { interval }^{5}\end{array}$ & $\begin{array}{l}\text { Reported stratigraphic top of geologic } \\
\text { unit }^{6}\end{array}$ \\
\hline 386 & $5 / 4-1 \mathrm{G1}$ & & & 61 & 78 & 18.6 & 23.8 & rocks and water & Ash or tuff & hard & -- & -- & \\
\hline 387 & $5 / 4-5 \mathrm{H} 4$ & 387 & $5 / 4-5 \mathrm{H} 4$ & 0 & 8 & 0.0 & 2.4 & soil & Sand and clay & -- & -- & -- & Qyal and Qoal \\
\hline 387 & $5 / 4-5 \mathrm{H} 4$ & & & 8 & 17 & 2.4 & 5.2 & clay, yellow & Clay & -- & -- & -- & \\
\hline 387 & $5 / 4-5 \mathrm{H} 4$ & & & 17 & 26 & 5.2 & 7.9 & clay, yellow; and boulders & Clay and gravel & -- & -. & -- & \\
\hline 387 & $5 / 4-5 \mathrm{H} 4$ & & & 26 & 36 & 7.9 & 11.0 & clay, yellow & Clay & -- & -- & -- & \\
\hline 387 & $5 / 4-5 \mathrm{H} 4$ & & & 36 & 37 & 11.0 & 11.3 & gravel, water & Gravel & -- & -- & -- & \\
\hline 387 & $5 / 4-5 \mathrm{H} 4$ & & & 37 & 66 & 11.3 & 20.1 & clay, yellow & Clay & -- & -- & -- & \\
\hline 387 & $5 / 4-5 \mathrm{H} 4$ & & & 66 & 68 & 20.1 & 20.7 & gravel, water & Gravel & -- & -- & -- & \\
\hline 387 & $5 / 4-5 \mathrm{H} 4$ & & & 68 & 75 & 20.7 & 22.9 & boulders and clay & Clay and gravel & -- & -. & -- & \\
\hline 388 & 5/3-6M2 & 388 & $5 / 3-6 \mathrm{M} 2$ & 0 & 4 & 0.0 & 1.2 & $\begin{array}{l}\text { soil } \\
\text { chalk and rock (diatomite and }\end{array}$ & Sand and clay & -- & -- & -- & Tsv \\
\hline 388 & $5 / 3-6 \mathrm{M} 2$ & & & 4 & 24 & 1.2 & 7.3 & rock) & Clay and sand (or sandstone) & -- & -- & -- & \\
\hline 388 & 5/3-6M2 & & & 24 & 35 & 7.3 & 10.7 & boulders and clay & Clay and gravel & -- & -- & -- & \\
\hline 388 & $5 / 3-6 \mathrm{M} 2$ & & & 35 & 38 & 10.7 & 11.6 & gravel, water & Gravel & -- & -- & -- & \\
\hline 388 & 5/3-6M2 & & & 38 & 60 & 11.6 & 18.3 & rock, blue & Ash or tuff & hard & -- & -- & \\
\hline 388 & $5 / 3-6 \mathrm{M} 2$ & & & 60 & 65 & 18.3 & 19.8 & rock, water & Ash or tuff & hard & .- & -. & \\
\hline 389 & 5/3-6M1 & 389 & 5/3-6M1 & 0 & 5 & 0.0 & 1.5 & soil & Sand and clay & -- & -- & -- & Tsv \\
\hline 389 & $5 / 3-6 \mathrm{M} 1$ & & & 5 & 45 & 1.5 & 13.7 & clay & Clay & .- & .. & -. & \\
\hline 389 & $5 / 3-6 \mathrm{M} 1$ & & & 45 & 55 & 13.7 & 16.8 & clay, boulders; water & Clay and gravel & -- & ..- & -- & \\
\hline 389 & 5/3-6M1 & & & 55 & 147 & 16.8 & 44.8 & clay and boulders & Clay and gravel & -- & -- & -- & \\
\hline 389 & $5 / 3-6 \mathrm{M} 1$ & & & 147 & 163 & 44.8 & 49.7 & lava, blue; water & Basalt & -- & -- & -. & \\
\hline 389 & 5/3-6M1 & & & 163 & 170 & 49.7 & 51.8 & lava, hard & Basalt & hard & -- & -- & \\
\hline 389 & $5 / 3-6 \mathrm{M} 1$ & & & 170 & 190 & 51.8 & 57.9 & conglomerate, brown & Clay, sand, and gravel & -- & .. & .- & \\
\hline 389 & $5 / 3-6 \mathrm{M} 1$ & & & 190 & 197 & 57.9 & 60.0 & lava, red; water & Basalt & ..- & ..- & .- & \\
\hline 389 & 5/3-6M1 & & & 197 & 205 & 60.0 & 62.5 & conglomerate, gray & Clay, sand, and gravel & -- & -- & -- & \\
\hline 389 & 5/3-6M1 & & & 205 & 250 & 62.5 & 76.2 & not described & No data & -- & -. & -. & \\
\hline 389 & 5/3-6M1 & & & 250 & 295 & 76.2 & 89.9 & lava, water & Basalt & -- & -- & -- & \\
\hline $\begin{array}{l}509 \\
390\end{array}$ & 5/4-3M1 & 390 & 5/4-3M1 & 0 & 9 & 0.0 & $\begin{array}{l}2.7 \\
2.7\end{array}$ & soil & Sand and clay & -- &.- & -- & Qyal and Qoal \\
\hline 390 & 5/4-3M1 & & & 9 & 40 & 2.7 & 12.2 & clay & Clay & .- & ..- & -. & \\
\hline 390 & 5/4-3M1 & & & 40 & 44 & 12.2 & 13.4 & gravel and water & Gravel & -- & .. & -. & \\
\hline 390 & 5/4-3M1 & & & 44 & 80 & 13.4 & 24.4 & clay & Clay & -- & -- & -- & \\
\hline 390 & 5/4-3M1 & & & 80 & 85 & 24.4 & 25.9 & gravel & Gravel & -- & -. & -- & \\
\hline 390 & 5/4-3M1 & & & 85 & 107 & $\begin{array}{l}25.4 \\
25.9\end{array}$ & 32.6 & $\begin{array}{l}\text { clay } \\
\text { clave }\end{array}$ & Clay & - & -. & - & \\
\hline 390 & 5/4-3M1 & & & 107 & 110 & 32.6 & 33.5 & gravel & Gravel & -- & -- & -- & \\
\hline 390 & 5/4-3M1 & & & 110 & 168 & 33.5 & 51.2 & clay & Clay & -- & .. & .. & \\
\hline 390 & $5 / 4-3 \mathrm{M} 1$ & & & 168 & 174 & 51.2 & 53.0 & gravel, water & Gravel & -- & -- & -- & \\
\hline 390 & 5/4-3M1 & & & 174 & 200 & 53.0 & 61.0 & clay & Clay & -- & -. & -- & \\
\hline 390 & 5/4-3M1 & & & 200 & 204 & 61.0 & 62.2 & gravel & Gravel & -- & -. & -. & \\
\hline 390 & 5/4-3M1 & & & 204 & 278 & 62.2 & 84.7 & clay & Clay & - & -- & -- & \\
\hline 390 & $5 / 4-3 \mathrm{M} 1$ & & & 278 & 280 & 84.7 & 85.3 & gravel & Gravel & .. & ..- & .. & \\
\hline 390 & $5 / 4-3 \mathrm{M} 1$ & & & 280 & 290 & 85.3 & 88.4 & clay & Clay & -- & -- & -- & \\
\hline 390 & 5/4-3M1 & & & 290 & 304 & 88.4 & 92.7 & gravel, water & Gravel & -- & -- & -- & \\
\hline $\begin{array}{l}390 \\
390\end{array}$ & $\begin{array}{l}5 / 4-3 \mathrm{M} 1 \\
5 / 4\end{array}$ & & & 304 & $\begin{array}{l}304 \\
375\end{array}$ & $\begin{array}{l}80.4 \\
92.7\end{array}$ & $\begin{array}{l}9.1 \\
114.3\end{array}$ & $\begin{array}{l}\text { gravel, waler } \\
\text { clay }\end{array}$ & Clay & -- & -- & -- & \\
\hline 390 & 5/4-3M1 & & & 375 & 380 & 114.3 & 115.8 & water-sand & Sand & -- & -- & -- & \\
\hline 390 & $5 / 4-3 M 1$ & & & 380 & 665 & $\begin{array}{l}11.3 \\
115.8\end{array}$ & 202.7 & clay and gravel, mixed & Clay and gravel & .. & -- & .. & \\
\hline 390 & 5/4-3M1 & & & 665 & 675 & 202.7 & 205.7 & $\begin{array}{l}\text { gravel, no water } \\
\text { lats }\end{array}$ & Gravel & -- & -- & -- & \\
\hline 390 & 5/4-3M1 & & & 675 & 780 & 205.7 & 237.7 & clay & Clay & -- & -. & -- & \\
\hline 390 & 5/4-3M1 & & & 780 & 805 & 237.7 & 245.4 & gravel, water & Gravel & -. & -. & .- & \\
\hline 390 & 5/4-3M1 & & & 805 & 865 & 245.4 & 263.7 & clay & Clay & -- & -- & -- & \\
\hline 391 & $5 / 3-5 \mathrm{M} 2$ & 391 & $5 / 3-5 \mathrm{M} 2$ & 0 & $\begin{array}{l}005 \\
3\end{array}$ & $\begin{array}{l}24.4 \\
0.0\end{array}$ & $\begin{array}{l}20.9 \\
0.9\end{array}$ & soil & Sand and clay & -. & -- & -. & Tsv \\
\hline 391 & 5/3-5M2 & & & 3 & 66 & 0.9 & 20.1 & tuff & Ash or tuff & -- & -- & -- & \\
\hline 391 & $5 / 3-5 \mathrm{M} 2$ & & & 66 & 69 & 20.1 & 21.0 & rock, broken; water & Ash or tuff & hard & -- & - & \\
\hline 391 & $5 / 3-5 \mathrm{M} 2$ & & & 69 & 128 & 21.0 & 39.0 & tuff & Ash or tuff & -- & .. & .- & \\
\hline 391 & $5 / 3-5 \mathrm{M} 2$ & & & 128 & 132 & 39.0 & 40.2 & rock, broken; water & Ash or tuff & hard & -. & -- & \\
\hline 391 & 5/3-5M2 & & & 132 & 135 & 40.2 & 41.1 & tuff & Ash or tuff & -- & -. & .. & \\
\hline 392 & $5 / 3-6 \mathrm{J1}$ & 392 & 5/3-6J1 & 0 & 3 & 0.0 & 0.9 & soil & Sand and clay & -- & -. & -- & Tsv \\
\hline 392 & $5 / 3-6 \mathrm{~J} 1$ & & & 3 & 70 & 0.9 & 21.3 & diatomite & Clay and sand (or sandstone) & - & -- & -- & \\
\hline 392 & $5 / 3-6 \mathrm{J1}$ & & & 70 & 85 & 21.3 & 25.9 & tule mud & Clay and sand (or sandstone) & -- & -- & tule & \\
\hline 392 & $5 / 3-6 \mathrm{J1}$ & & & 85 & 150 & 25.9 & 45.7 & not described & No data & -- & -. & -- & \\
\hline 392 & $5 / 3-6 \mathrm{~J} 1$ & & & 150 & 176 & 45.7 & 53.6 & rock, hard & Ash or tuff & hard & -- & .- & \\
\hline 392 & $5 / 3-6 \mathrm{~J} 1$ & & & 176 & 255 & 53.6 & 77.7 & lava, vesicular & Basalt & -- & -. & -- & \\
\hline
\end{tabular}


Appendix 3. Lithologic data. - Continued

[Site IDs and well number correspond to locations in Appendix 1, a site ID and well number is listed for each drilled interval for a particular drill hole. For convenience, the site ID and well numbers are repeated at the shallowest drilled interval for each well. leaders $1-$

\begin{tabular}{|c|c|c|c|c|c|c|c|c|c|c|c|c|c|}
\hline Site ID & Well number & $\begin{array}{l}\text { Site ID, } \\
\text { top of } \\
\text { well }\end{array}$ & $\begin{array}{l}\text { Well number, } \\
\text { top of well }\end{array}$ & $\begin{array}{c}\text { Top of } \\
\text { interval, in } \\
\text { feet }\end{array}$ & $\begin{array}{l}\text { Base of } \\
\text { interval, in } \\
\text { feet }\end{array}$ & $\begin{array}{c}\text { Top of } \\
\text { interval, in } \\
\mathrm{m}\end{array}$ & $\begin{array}{c}\text { Base of } \\
\text { interval, in } \\
\mathrm{m}\end{array}$ & Reported lithologic unit' & Interpreted lithologic class ${ }^{2}$ & $\begin{array}{c}\text { Reported } \\
\text { degree of } \\
\text { cementation or } \\
\text { induration } \\
\end{array}$ & $\begin{array}{c}\text { Fossils } \\
\text { reported in } \\
\text { interval }^{4}\end{array}$ & $\begin{array}{c}\text { Organic } \\
\text { matter } \\
\text { reported in } \\
\text { interval }^{5}\end{array}$ & $\begin{array}{l}\text { Reported stratigraphic top of geologic } \\
\text { unit }^{6}\end{array}$ \\
\hline 392 & $5 / 3-6 J 1$ & & & 255 & 265 & 77.7 & 80.8 & $\begin{array}{l}\text { lava, brown } \\
\text { basalt, (some quartz grains in }\end{array}$ & Basalt & -- & -- & -- & \\
\hline 392 & $5 / 3-6 J 1$ & & & 265 & 290 & 80.8 & 88.4 & washed cuttings) & Basalt & -- & -- & -- & \\
\hline 393 & $5 / 4-4 K 2$ & 393 & $5 / 4-4 K 2$ & 0 & 3 & 0.0 & 0.9 & soil & Sand and clay & -- & -- & -- & Qyal and Qoal \\
\hline 393 & $5 / 4-4 K 2$ & & & 3 & 25 & 0.9 & 7.6 & clay, yellow & Clay & -- & -- & -- & \\
\hline 393 & $5 / 4-4 K 2$ & & & 25 & 27 & 7.6 & 8.2 & gravel, water & Gravel & -- & -- & -- & \\
\hline 393 & $5 / 4-4 K 2$ & & & 27 & 36 & 8.2 & 11.0 & clay, yellow & Clay & -- & -- & -- & \\
\hline 393 & $5 / 4-4 K 2$ & & & 36 & 38 & 11.0 & 11.6 & clay, blue & Clay & -- & -- & -- & \\
\hline 393 & $5 / 4-4 K 2$ & & & 38 & 51 & 11.6 & 15.5 & clay, yellow & Clay & -- & -- & -- & \\
\hline 393 & $5 / 4-4 K 2$ & & & 51 & 65 & 15.5 & 19.8 & gravel, water & Gravel & -- & -- & -- & \\
\hline 393 & $5 / 4-4 K 2$ & & & 65 & 87 & 19.8 & 26.5 & clay, yellow & Clay & -- & -- & -- & \\
\hline 393 & $5 / 4-4 K 2$ & & & 87 & 92 & 26.5 & 28.0 & gravel, water & Gravel & -- & -- & -- & \\
\hline 393 & $5 / 4-4 K 2$ & & & 92 & 100 & 28.0 & 30.5 & clay, yellow & Clay & -- & -- & -- & \\
\hline 394 & 5/3-5N3 & 394 & 5/3-5N3 & 0 & 22 & 0.0 & 6.7 & rock and clay & Clay and gravel & -- & -- & -- & Tsv \\
\hline 394 & $5 / 3-5 N 3$ & tot & & 22 & 32 & 6.7 & 9.8 & tule mud, brown & Clay and sand (or sandstone) & -- & -- & tule & rov \\
\hline 394 & $5 / 3-5 N 3$ & & & 32 & 51 & 9.8 & 15.5 & tule mud, black & Clay and sand (or sandstone) & -- & -- & tule & \\
\hline 394 & 5/3-5N3 & & & 51 & 124 & 15.5 & 37.8 & rock and lava & Ash or tuff & -- & -- & -- & \\
\hline 394 & 5/3-5N3 & & & 124 & 128 & 37.8 & 39.0 & basalt rock, hard & Basalt & hard & -- & -- & \\
\hline 394 & $5 / 3-5 N 3$ & & & 128 & 151 & 39.0 & 46.0 & lava, gray & Basalt & -- & -- & -- & \\
\hline 394 & $5 / 3-5 N 3$ & & & 151 & 182 & 46.0 & 55.5 & lava, blue; and water & Basalt & -- & -- & -- & \\
\hline 394 & 5/3-5N3 & & & 182 & 192 & 55.5 & 58.5 & basalt rock, hard & Basalt & hard & -- & -- & \\
\hline 395 & 5/3-5P1 & 395 & 5/3-5P1 & 0 & 30 & 0.0 & 9.1 & tuff & Ash or tuff & -- & -- & -- & Tsv \\
\hline 395 & 5/3-5P1 & & & 30 & 39 & 9.1 & 11.9 & chalk (diatomite) & Clay and sand (or sandstone) & -- & -- & -- & \\
\hline 395 & $5 / 3-5 \mathrm{P} 1$ & & & 39 & 84 & 11.9 & 25.6 & clay & Clay & -- & -- & -- & \\
\hline 395 & 5/3-5P1 & & & 84 & 183 & 25.6 & 55.8 & tuff & Ash or tuff & -- & -- & -- & \\
\hline 395 & 5/3-5P1 & & & 183 & 195 & 55.8 & 59.4 & gravel, water & Gravel & -- & -- & -- & \\
\hline 395 & 5/3-5P1 & & & 195 & 224 & 59.4 & 68.3 & rocks, hard & Ash or tuff & hard & -- & -- & \\
\hline 395 & $5 / 3-5 \mathrm{P} 1$ & & & 224 & 234 & 68.3 & 71.3 & gravel, water & Gravel & -- & -- & -- & \\
\hline 395 & 5/3-5P1 & & & 234 & 263 & 71.3 & 80.2 & $\begin{array}{l}\text { cement } \\
\text { cols }\end{array}$ & Clay, sand, and gravel & cemented & -- & -- & \\
\hline 395 & 5/3-5P1 & & & 263 & 278 & 80.2 & 84.7 & rocks & Ash or tuff & hard & -- & -- & \\
\hline 395 & 5/3-5P1 & & & 278 & 368 & 84.7 & 112.2 & cement & Clay, sand, and gravel & cemented & -- & -- & \\
\hline 395 & 5/3-5P1 & & & 368 & 391 & 112.2 & 119.2 & gravel, cemented & Gravel & cemented & -- & -- & \\
\hline 395 & 5/3-5P1 & & & 391 & 411 & 119.2 & 125.3 & gravel, water & Gravel & -- & -- & -- & \\
\hline 395 & 5/3-5P1 & & & 411 & 420 & 125.3 & 128.0 & cement & Clay, sand, and gravel & cemented & -- & -- & \\
\hline 395 & $5 / 3-5 P 1$ & & & 420 & 450 & 128.0 & 137.2 & diorite & Basalt & -- & -- & -- & \\
\hline 396 & $5 / 4-5 R 1$ & 396 & $5 / 4-5 R 1$ & 0 & 4 & 0.0 & 1.2 & soil, black & Sand and clay & -- & -- & -- & Qyal and Qoal \\
\hline 396 & $5 / 4-5 R 1$ & & & 4 & 25 & 1.2 & 7.6 & clay, yellow & Clay & -- & -- & -- & \\
\hline 396 & $5 / 4-5 R 1$ & & & 25 & 26 & 7.6 & 7.9 & gravel and water & Gravel & -- & -- & -- & \\
\hline 396 & $5 / 4-5 R 1$ & & & 26 & 40 & 7.9 & 12.2 & clay, blue & Clay & -- & -- & -- & \\
\hline 396 & $5 / 4-5 R 1$ & & & 40 & 41 & 12.2 & 12.5 & water, small amount & Clay & -- & -- & -- & \\
\hline 396 & $5 / 4-5 R 1$ & & & 41 & 46 & 12.5 & 14.0 & lava mud, green & Basalt & -- & -- & -- & Tsv \\
\hline 396 & 5/4-5R1 & & & 46 & 67 & 14.0 & 20.4 & lava, broken; water-bearing & Basalt & -- & -- & -- & 100 \\
\hline 396 & $5 / 4-5 R 1$ & & & 67 & 70 & 20.4 & 21.3 & shale, blue & Clay and sand (or sandstone) & cemented & -- & -- & $\mathrm{KJu}$ \\
\hline 397 & 5/3-6N2 & 397 & $5 / 3-6 N 2$ & 0 & 8 & 0.0 & 2.4 & soil whe & Sand and clay & -- & -- & -- & Tsv \\
\hline 397 & $5 / 3-6 N 2$ & & & 8 & 48 & 2.4 & 14.6 & chalk rock (diatomite) & Clay and sand (or sandstone) & -- & -- & -- & \\
\hline 397 & $5 / 3-6 N 2$ & & & 48 & 130 & 14.6 & 39.6 & rock, white (tuff) & Ash or tuff & hard & -- & -- & \\
\hline 397 & $5 / 3-6 \mathrm{~N} 2$ & & & 130 & 150 & 39.6 & 45.7 & rock, broken; water & Ash or tuff & hard & -- & -- & \\
\hline 397 & $5 / 3-6 N 2$ & & & 150 & 205 & 45.7 & 62.5 & rock, white & Ash or tuff & hard & -- & -- & \\
\hline 398 & $5 / 4-5 Q 2$ & 398 & 5/4-5Q2 & 0 & 50 & 0.0 & 15.2 & clay, blue, and silt & Clay and sand (or sandstone) & -- & -- & -- & Qyal \\
\hline 398 & $5 / 4-5 \mathrm{Q} 2$ & & & 50 & 150 & 15.2 & 45.7 & sandstone, yellow & Sandstone & cemented & -- & -. & \\
\hline 398 & $5 / 4-5 Q 2$ & & & 150 & 150 & 45.7 & 45.7 & shale, blue & Clay and sand (or sandstone) & cemented & -- & -- & \\
\hline 399 & 5/4-1R1 & 399 & $5 / 4-1 R 1$ & 0 & 2 & 0.0 & 0.6 & soil & Sand and clay & - & -- & -. & Tsv \\
\hline 399 & 5/4-1R1 & & & 2 & 20 & 0.6 & 6.1 & tuff, white & Ash or tuff & -- & -- & -- & \\
\hline 399 & $5 / 4-1 R 1$ & & & 20 & 41 & 6.1 & 12.5 & clay, yellow & Clay & -- & -- & -- & \\
\hline 399 & $5 / 4-1 R 1$ & & & 41 & 50 & 12.5 & 15.2 & rock & Ash or tuff & hard & -- & -- & \\
\hline 399 & 5/4-1R1 & & & 50 & 60 & 15.2 & 18.3 & tuff, white & Ash or tuff & -- & -- & -. & \\
\hline 399 & $5 / 4-1 \mathrm{R} 1$ & & & 60 & 71 & 18.3 & 21.6 & rock, brown & Ash or tuff & hard & -- & -- & \\
\hline 399 & 5/4-1R1 & & & 71 & 96 & 21.6 & 29.3 & tuff, gray; and boulders & Ash or tuff & -- & -- & -- & \\
\hline 399 & $5 / 4-1 R 1$ & & & 96 & 151 & 29.3 & 46.0 & $\begin{array}{l}\text { rock, broken } \\
\text { la }\end{array}$ & Ash or tuff & hard & -- & -. & \\
\hline 399 & 5/4-1R1 & & & 151 & 192 & 46.0 & 58.5 & rock, clay; some water & Clay and gravel & hard & -- & -- & \\
\hline 399 & $5 / 4-1 R 1$ & & & 192 & 199 & 58.5 & 60.7 & rock, hard & Ash or tuff & hard & -- & -- & \\
\hline
\end{tabular}


Appendix 3. Lithologic data. - Continued

[Site IDs and well number correspond to locations in Appendix 1, a site ID and well number is listed for each drilled interval for a particular drill hole. For convenience, the site ID and well numbers are repeated at the shallowest drilled interval for each well. leaders

\begin{tabular}{|c|c|c|c|c|c|c|c|c|c|c|c|c|c|}
\hline Site ID & Well number & $\begin{array}{l}\text { Site ID, } \\
\text { top of } \\
\text { well }\end{array}$ & $\begin{array}{l}\text { Well number, } \\
\text { top of well }\end{array}$ & $\begin{array}{c}\text { Top of } \\
\text { interval, in } \\
\text { feet }\end{array}$ & $\begin{array}{c}\text { Base of } \\
\text { interval, in } \\
\text { feet }\end{array}$ & $\begin{array}{c}\text { Top of } \\
\text { interval, in } \\
m\end{array}$ & $\begin{array}{c}\text { Base of } \\
\text { interval, in } \\
\mathrm{m}\end{array}$ & Reported lithologic unit ${ }^{1}$ & Interpreted lithologic class ${ }^{2}$ & $\begin{array}{c}\text { Reported } \\
\text { degree of } \\
\text { cementation or } \\
\text { induration } \\
\end{array}$ & $\begin{array}{c}\text { Fossils } \\
\text { reported in } \\
\text { interval }^{4}\end{array}$ & $\begin{array}{c}\text { Organic } \\
\text { matter } \\
\text { reported in } \\
\text { interval }^{5}\end{array}$ & $\begin{array}{l}\text { Reported stratigraphic top of geologic } \\
\text { unit }^{6}\end{array}$ \\
\hline 399 & 5/4-1R1 & & & 199 & 214 & 60.7 & 65.2 & clay & Clay & -- & -- & -- & \\
\hline 399 & $5 / 4-1 \mathrm{R} 1$ & & & 214 & 224 & 65.2 & 68.3 & rock, hard & Ash or tuff & hard & -- & -- & \\
\hline 399 & 5/4-1R1 & & & 224 & 228 & 68.3 & 69.5 & rock, brown & Ash or tuff & hard & -- & -- & \\
\hline 399 & 5/4-1R1 & & & 228 & 232 & 69.5 & 70.7 & rock, brown & Ash or tuff & hard & -. & -- & \\
\hline 399 & $5 / 4-1 R 1$ & & & 232 & 235 & 70.7 & 71.6 & rock, hard & Ash or tuff & hard & -- & -- & \\
\hline 399 & 5/4-1R1 & & & 235 & 283 & 71.6 & 86.3 & rock, hard & Ash or tuff & hard & -- & -- & \\
\hline 399 & $5 / 4-1 \mathrm{R} 1$ & & & 283 & 333 & 86.3 & 101.5 & lava; some water & Basalt & -- & -- & -- & \\
\hline 399 & $5 / 4-1 R 1$ & & & 333 & 339 & 101.5 & 103.3 & tuff & Ash or tuff & -- & -- & -- & \\
\hline 399 & 5/4-1R1 & & & 339 & 349 & 103.3 & 106.4 & $\begin{array}{l}\text { rock, white } \\
\text { guartz aravel cemented. some }\end{array}$ & Ash or tuff & hard & -- & -- & \\
\hline 399 & $5 / 4-1 \mathrm{R} 1$ & & & 349 & 369 & 106.4 & 112.5 & water & Clay, sand, and gravel & cemented & -.- & -- & \\
\hline 400 & $5 / 3-6 Q 1$ & 400 & 5/3-6Q1 & 0 & 3 & 0.0 & 0.9 & soil & Sand and clay & -- & -- & -- & Tsv \\
\hline 400 & 5/3-6Q1 & & & 3 & 10 & 0.9 & 3.0 & chalk rock (diatomite) & Clay and sand (or sandstone) & -- & -- & -- & \\
\hline 400 & 5/3-6Q1 & & & 10 & 35 & 3.0 & 10.7 & tule mud & Clay and sand (or sandstone) & -- & -- & tule & \\
\hline 400 & 5/3-6Q1 & & & 35 & 45 & 10.7 & 13.7 & lava, blue; water & Basalt & -- & -- & -- & \\
\hline 400 & $5 / 3-6 Q 1$ & & & 45 & 75 & 13.7 & 22.9 & lava, cemented, blue & Basalt & -- & -. & -- & \\
\hline 400 & $5 / 3-6 Q 1$ & & & 75 & 82 & 22.9 & 25.0 & lava, black; water & Basalt & -- & -- & -- & \\
\hline 400 & 5/3-6Q1 & & & 82 & 218 & 25.0 & 66.4 & lava, cemented, blue & Basalt & -- & -. & -- & \\
\hline 400 & 5/3-6Q1 & & & 218 & 225 & 66.4 & 68.6 & lava, water & Basalt & -- & -. & -- & \\
\hline 400 & 5/3-6Q1 & & & 225 & 228 & 68.6 & 69.5 & rock, blue, hard & Ash or tuff & hard & -- & -- & \\
\hline 401 & $5 / 3-5 \mathrm{~N} 1$ & 401 & $5 / 3-5 \mathrm{~N} 1$ & 0 & 8 & 0.0 & 2.4 & soil & Sand and clay & -. & - & - & Tsv \\
\hline 401 & $5 / 3-5 \mathrm{~N} 1$ & & & 8 & 36 & 2.4 & 11.0 & ash & Ash or tuff & -- & -- & -- & \\
\hline 401 & $5 / 3-5 N 1$ & & & 36 & 59 & 11.0 & 18.0 & tuff & Ash or tuff & -- & -. & -. & \\
\hline 401 & $5 / 3-5 N 1$ & & & 59 & 62 & 18.0 & 18.9 & rock, broken; water & Ash or tuff & hard & -. & -.- & \\
\hline 401 & 5/3-5N1 & & & 62 & 120 & 18.9 & 36.6 & lava, cemented & Basalt & -- & -- & -- & \\
\hline 401 & 5/3-5N1 & & & 120 & 130 & 36.6 & 39.6 & lava, broken; water & Basalt & -- & -- & -- & \\
\hline 401 & 5/3-5N1 & & & 130 & 135 & 39.6 & 41.1 & lava, cemented & Basalt & -- & -- & -- & \\
\hline 402 & $5 / 3-6 \mathrm{~N} 1$ & 402 & $5 / 3-6 \mathrm{~N} 1$ & 0 & 1 & 0.0 & 0.3 & soil & Sand and clay & -- & - & - & Tsv \\
\hline 402 & $5 / 3-6 N 1$ & & & 1 & 30 & 0.3 & 9.1 & hardpan and boulders & Clay and gravel & hard & -- & -.- & \\
\hline 402 & $5 / 3-6 \mathrm{~N} 1$ & & & 30 & 45 & 9.1 & 13.7 & rock, blue & Ash or tuff & hard & .- & .. & \\
\hline 402 & $5 / 3-6 \mathrm{~N} 1$ & & & 45 & 46 & 13.7 & 14.0 & water & Ash or tuff & -- & -- & -- & \\
\hline 402 & $5 / 3-6 \mathrm{~N} 1$ & & & 46 & 110 & 14.0 & 33.5 & rock, blue & Ash or tuff & hard & -- & -- & \\
\hline 402 & $5 / 3-6 \mathrm{~N} 1$ & & & 110 & 115 & 33.5 & 35.1 & rock, water & Ash or tuff & hard & -- & - & \\
\hline 402 & $5 / 3-6 N 1$ & & & 115 & 120 & 35.1 & 36.6 & rock & Ash or tuff & hard & -- & -- & \\
\hline 403 & $5 / 3-6 N 3$ & 403 & $5 / 3-6 N 3$ & 0 & 18 & 0.0 & 5.5 & rock and clay & Clay and gravel & -- & -- & -- & Tsv \\
\hline 403 & $5 / 3-6 N 3$ & & & 18 & 55 & 5.5 & 16.8 & clay & Clay & -- & -- & -. & \\
\hline 403 & $5 / 3-6 N 3$ & & & 55 & 63 & 16.8 & 19.2 & gravel and sand; water & Sand (or sandstone) and gravel & -- & -- & -.- & \\
\hline 403 & $5 / 3-6 \mathrm{~N} 3$ & & & 63 & 86 & 19.2 & 26.2 & rock and clay & Clay and gravel & - & - & - & \\
\hline 403 & $5 / 3-6 N 3$ & & & 86 & 87 & 26.2 & 26.5 & gravel, water & Gravel & -. & -- & .. & \\
\hline 403 & $5 / 3-6 N 3$ & & & 87 & 120 & 26.5 & 36.6 & rock & Ash or tuff & hard & -- & -- & \\
\hline 403 & $5 / 3-6 N 3$ & & & 120 & 146 & 36.6 & 44.5 & rocks, water & Ash or tuff & hard & -- & -- & \\
\hline 404 & $5 / 4-3 Q 1$ & 404 & 5/4-3Q1 & 0 & 40 & 0.0 & 12.2 & dug well not described & No data & -- & -- & -- & Qyal \\
\hline 404 & 5/4-3Q1 & & & 40 & 78 & 12.2 & 23.8 & clay, yellow & Clay & -- & -- & -.- & \\
\hline 404 & 5/4-3Q1 & & & 78 & 97 & 23.8 & 29.6 & boulders and clay; water & Clay and gravel & -- & -- & -- & Qoal (?) \\
\hline 404 & 5/4-3Q1 & & & 97 & 112 & 29.6 & 34.1 & $\begin{array}{l}\text { clay, yellow } \\
\text { lag, wote }\end{array}$ & 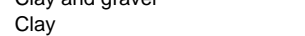 & -- & -- & -- & \\
\hline 404 & 5/4-3Q1 & & & 112 & 123 & 34.1 & 37.5 & clay, white & Clay & -- & -- & -- & \\
\hline 404 & $5 / 4-3 Q 1$ & & & 123 & 138 & 37.5 & 42.1 & clay & Clay & -- & -. & -. & \\
\hline 404 & $5 / 4-3 Q 1$ & & & 138 & 177 & 42.1 & 53.9 & clay and boulders & Clay and gravel & -- & -- & -- & \\
\hline 404 & 5/4-3Q1 & & & 177 & 178 & 53.9 & 54.3 & gravel, water & Gravel & -- & .. & .- & \\
\hline 404 & $5 / 4-3 Q 1$ & & & 178 & 196 & 54.3 & 59.7 & clay & Clay & -- & -- & -- & \\
\hline 404 & 5/4-3Q1 & & & 196 & 227 & 59.7 & 69.2 & $\begin{array}{l}\text { clay and boulders } \\
\text {. }\end{array}$ & Clay and gravel & -- & -- & -- & \\
\hline 404 & 5/4-3Q1 & & & 227 & 234 & 69.2 & 71.3 & $\begin{array}{l}\text { clay, yellow } \\
\text { lo s }\end{array}$ & $\begin{array}{l}\text { Clay } \\
\text { Clution }\end{array}$ & -. & -- & -- & \\
\hline 404 & 5/4-3Q1 & & & 234 & 239 & 71.3 & 72.8 & boulders and clay & Clay and gravel & -- & -- & -- & \\
\hline 404 & $5 / 4-3 Q 1$ & & & 239 & 241 & 72.8 & 73.5 & gravel, water & Gravel & -- & -- & -- & \\
\hline 405 & $5 / 4-12 B 1$ & 405 & $5 / 4-12 \mathrm{~B} 1$ & 0 & 2 & 0.0 & 0.6 & topsoil & Sand and clay & -- & -- & -- & Tsv \\
\hline 405 & $5 / 4-12 \mathrm{~B} 1$ & & & 2 & 3 & 0.6 & 0.9 & clay, gravel & Clay and gravel & -- & -- & -- & \\
\hline 405 & $5 / 4-12 \mathrm{~B} 1$ & & & 3 & 17 & 0.9 & 5.2 & rock, porous & Ash or tuff & hard &.- & -- & \\
\hline 405 & $5 / 4-12 \mathrm{~B} 1$ & & & 17 & 18 & 5.2 & 5.5 & clay, gravel & Clay and gravel & -- & -- & -- & \\
\hline 405 & $5 / 4-12 \mathrm{~B} 1$ & & & 18 & 26 & 5.5 & 7.9 & rock & Ash or tuff & hard & -. & .- & \\
\hline 405 & $5 / 4-12 B 1$ & & & 26 & 27 & 7.9 & 8.2 & sand rock & Sandstone & cemented & -- & -- & \\
\hline 405 & $5 / 4-12 \mathrm{~B} 1$ & & & 27 & 31 & 8.2 & 9.4 & rock, hard & Ash or tuff & hard & -- & -- & \\
\hline
\end{tabular}


Appendix 3. Lithologic data. - Continued

[Site IDs and well number correspond to locations in Appendix 1, a site ID and well number is listed for each drilled interval for a particular drill hole. For convenience, the site ID and well numbers are repeated at the shallowest drilled interval for each well. leaders

\begin{tabular}{|c|c|c|c|c|c|c|c|c|c|c|c|c|c|}
\hline Site ID & Well number & $\begin{array}{l}\text { Site ID, } \\
\text { top of } \\
\text { well }\end{array}$ & $\begin{array}{l}\text { Well number, } \\
\text { top of well }\end{array}$ & $\begin{array}{c}\text { Top of } \\
\text { interval, in } \\
\text { feet }\end{array}$ & $\begin{array}{c}\text { Base of } \\
\text { interval, in } \\
\text { feet }\end{array}$ & $\begin{array}{c}\text { Top of } \\
\text { interval, in } \\
\mathrm{m}\end{array}$ & $\begin{array}{c}\text { Base of } \\
\text { interval, in } \\
\mathrm{m}\end{array}$ & Reported lithologic unit ${ }^{1}$ & Interpreted lithologic class ${ }^{2}$ & $\begin{array}{c}\text { Reported } \\
\text { degree of } \\
\text { cementation or } \\
\text { induration } \\
\end{array}$ & $\begin{array}{c}\text { Fossils } \\
\text { reported in } \\
\text { interval }^{4}\end{array}$ & $\begin{array}{c}\text { Organic } \\
\text { matter } \\
\text { reported in } \\
\text { interval }^{5}\end{array}$ & $\begin{array}{l}\text { Reported stratigraphic top of geologic } \\
\text { unit }^{6}\end{array}$ \\
\hline 405 & $5 / 4-12 \mathrm{~B} 1$ & & & 31 & 38 & 9.4 & 11.6 & boulders & Gravel & -- & -- & -- & \\
\hline 405 & 5/4-12B1 & & & 38 & 46 & 11.6 & 14.0 & rock, coarse gravel & Gravel & hard & -.- & -- & \\
\hline 405 & $5 / 4-12 \mathrm{~B} 1$ & & & 46 & 49 & 14.0 & 14.9 & rock & Ash or tuff & hard & -. & -- & \\
\hline 405 & $5 / 4-12 B 1$ & & & 49 & 55 & 14.9 & 16.8 & rock, white & Ash or tuff & hard & -- & -- & \\
\hline 405 & 5/4-12B1 & & & 55 & 76 & 16.8 & 23.2 & rock, porous & Ash or tuff & hard & -- & -- & \\
\hline 405 & $5 / 4-12 \mathrm{~B} 1$ & & & 76 & 108 & 23.2 & 32.9 & rock, red, porous & Ash or tuff & hard & .. & -- & \\
\hline 405 & $5 / 4-12 \mathrm{~B} 1$ & & & 108 & 126 & 32.9 & 38.4 & shale & Clay and sand (or sandstone) & cemented & -- & -- & \\
\hline 405 & $5 / 4-12 \mathrm{~B} 1$ & & & 126 & 141 & 38.4 & 43.0 & rock & Ash or tuff & hard & -. & -- & \\
\hline 405 & $5 / 4-12 \mathrm{~B} 1$ & & & 141 & 150 & 43.0 & 45.7 & gravel, sandy & Sand (or sandstone) and gravel & -- & -- & -- & \\
\hline 406 & $5 / 3-8 C 1$ & 406 & $5 / 3-8 \mathrm{C} 1$ & 0 & 1 & 0.0 & 0.3 & soil & Sand and clay & -- & -- & -- & Tsv \\
\hline 406 & $5 / 3-8 \mathrm{Cl} 1$ & & & 1 & 20 & 0.3 & 6.1 & tuff, brown & Ash or tuff & - & - &.- & \\
\hline 406 & $5 / 3-8 \mathrm{C} 1$ & & & 20 & 54 & 6.1 & 16.5 & tuff, green & Ash or tuff & .- & .- & .- & \\
\hline 406 & $5 / 3-8 \mathrm{C} 1$ & & & 54 & 56 & 16.5 & 17.1 & lava and water & Basalt & -- & -- & -- & \\
\hline 406 & $5 / 3-8 \mathrm{C} 1$ & & & 56 & 80 & 17.1 & 24.4 & tuff, gray & Ash or tuff & -- & -- & -- & \\
\hline 406 & $5 / 3-8 \mathrm{C} 1$ & & & 80 & 90 & 24.4 & 27.4 & lava and water, flowing & Basalt & -- & -.. & .- & \\
\hline 406 & $5 / 3-8 \mathrm{C} 1$ & & & 90 & 95 & 27.4 & 29.0 & lava & Basalt & -- & -- & -- & \\
\hline 407 & 5/3-7D1 & 407 & 5/3-7D1 & 0 & 4 & 0.0 & 1.2 & soil & Sand and clay & -. & -. & -- & Tsv \\
\hline 407 & $5 / 3-7 \mathrm{D} 1$ & & & 4 & 8 & 1.2 & 2.4 & chalk rock (diatomite) & Clay and sand (or sandstone) & - & - & -- & \\
\hline 407 & 5/3-7D1 & & & 8 & 65 & 2.4 & 19.8 & tule mud & Clay and sand (or sandstone) & -- & -- & tule & \\
\hline 407 & 5/3-7D1 & & & 65 & 70 & 19.8 & 21.3 & lava, cemented, blue & Basalt & -. & -- & -- & \\
\hline 407 & 5/3-7D1 & & & 70 & 120 & 21.3 & 36.6 & lava, blue & Basalt & -- & .. & .. & \\
\hline 407 & 5/3-7D1 & & & 120 & 130 & 36.6 & 39.6 & rock, blue, hard & Ash or tuff & hard & -- & -- & \\
\hline 407 & $5 / 3-7 \mathrm{D} 1$ & & & 130 & 145 & 39.6 & 44.2 & broken lava; water & Ash or tuff & -. & -- & .- & \\
\hline 408 & 5/4-9D1 & 408 & 5/4-9D1 & 0 & 3 & 0.0 & 0.9 & soil & Sand and clay & -- & -- & -- & Tsv \\
\hline 408 & 5/4-9D1 & & & 3 & 63 & 0.9 & 19.2 & tuff & Ash or tuff & .- & .- & .- & \\
\hline 408 & 5/4-9D1 & & & 63 & 70 & 19.2 & 21.3 & boulders and sand; water & Sand (or sandstone) and gravel & -- & .- & .- & \\
\hline 408 & 5/4-9D1 & & & 70 & 93 & 21.3 & 28.3 & boulders, cemented & Gravel & cemented & -- & -- & \\
\hline 408 & 5/4-9D1 & & & 93 & 98 & 28.3 & 29.9 & lava, water & Basalt & -. & -- & .- & \\
\hline 408 & 5/4-9D1 & & & 98 & 127 & 29.9 & 38.7 & lava and cemented & Basalt & -- & -- & -- & \\
\hline 408 & $5 / 4-9 \mathrm{D} 1$ & & & 127 & 135 & 38.7 & 41.1 & $\begin{array}{l}\text { lava, water } \\
\text { lated }\end{array}$ & Basalt & -- &.- & - & \\
\hline 408 & 5/4-9D1 & & & 135 & 135 & 41.1 & 41.1 & cement & Clay, sand, and gravel & cemented & .- & .- & \\
\hline 409 & $5 / 4-12 \mathrm{E} 1$ & 409 & 5/4-12E1 & 0 & 4 & 0.0 & 1.2 & soil and boulders & Clay, sand, and gravel & -- & .- & .. & Tsv \\
\hline 409 & 5/4-12E1 & & & 4 & 80 & 1.2 & 24.4 & tuff, brown & Ash or tuff & -- & -- & -- & \\
\hline 409 & 5/4-12E1 & & & 80 & 82 & 24.4 & 25.0 & rock, broken, and water & Ash or tuff & hard & -- & -- & \\
\hline 409 & $5 / 4-12 \mathrm{E} 1$ & & & 82 & 167 & 25.0 & 50.9 & rock & Ash or tuff & hard & -- & -- & \\
\hline 409 & $5 / 4-12 \mathrm{E} 1$ & & & 167 & 180 & 50.9 & 54.9 & rock, hard & Ash or tuff & hard & .. & .. & \\
\hline 409 & $5 / 4-12 \mathrm{E} 1$ & & & 180 & 189 & 54.9 & 57.6 & rock, broken; and water & Ash or tuff & hard & -- & .. & \\
\hline 409 & 5/4-12E1 & & & 189 & 255 & 57.6 & 77.7 & tuff, blue & Ash or tuff & -- & -- & -- & \\
\hline 410 & 5/3-7E3 & 410 & 5/3-7E3 & 0 & 186 & 0.0 & 56.7 & no record & No data & -- & -- & -- & Tsv \\
\hline 410 & $5 / 3-7 \mathrm{E} 3$ & & & 186 & 224 & 56.7 & 68.3 & rocks & Ash or tuff & hard & -- & -. & \\
\hline 410 & 5/3-7E3 & & & 224 & 242 & 68.3 & 73.8 & rocks, soft; water & Ash or tuff & hard & -- & -- & \\
\hline 410 & 5/3-7E3 & & & 242 & 270 & 73.8 & 82.3 & clay, yellow & Clay & -- & .- & .. & \\
\hline 410 & 5/3-7E3 & & & 270 & 278 & 82.3 & 84.7 & rocks, blue soft; water & Sandstone & hard & -- & -- & \\
\hline 410 & 5/3-7E3 & & & 278 & 310 & 84.7 & 94.5 & rocks, yellow & Ash or tuff & hard & -- & -- & \\
\hline 410 & 5/3-7E3 & & & 310 & 402 & $\begin{array}{l}84.1 \\
94.5\end{array}$ & $\begin{array}{l}94.5 \\
122.5\end{array}$ & $\begin{array}{l}\text { rocks, blue soft; water } \\
\text { rocks }\end{array}$ & Sandstone & hard & -- & -- & \\
\hline 410 & 5/3-7E3 & & & 402 & 429 & 122.5 & 130.8 & cement & Clay, sand, and gravel & cemented & -- & -- & \\
\hline 410 & $5 / 3-7 \mathrm{E} 3$ & & & 429 & 431 & 130.8 & 131.4 & conglomerate, soft; water & Clay, sand, and gravel & - & -- & - & \\
\hline 410 & 5/3-7E3 & & & 431 & 444 & 131.4 & 135.3 & lava, hard & Basalt & hard & -- & -- & \\
\hline 410 & $5 / 3-7 \mathrm{E} 3$ & & & 444 & 448 & 135.3 & 136.6 & lava, brown, water & Basalt & -. & - & - & \\
\hline 410 & 5/3-7E3 & & & 448 & 478 & 136.6 & 145.7 & cement & Clay, sand, and gravel & cemented & .- & .. & \\
\hline 411 & 5/4-11E1 & 411 & 5/4-11E1 & 0 & 60 & 0.0 & 18.3 & clay & Clay & - & -- & -- & Qyal and Qoal \\
\hline 411 & 5/4-11E1 & & & 60 & 160 & $\begin{array}{l}18.3 \\
\end{array}$ & $\begin{array}{l}10.3 \\
48.8\end{array}$ & boulders & Gravel & -- & -- & .. & \\
\hline 411 & 5/4-11E1 & & & 160 & 170 & 48.8 & 51.8 & shale & Clay and sand (or sandstone) & cemented & -- & -- & \\
\hline 411 & 5/4-11E1 & & & 170 & 210 & 51.8 & 64.0 & sand & Sand & -- & -- & -- & \\
\hline 411 & 5/4-11E1 & & & 210 & 360 & 64.0 & 109.7 & $\begin{array}{l}\text { Clay, yellow; flow of water at } 360 \\
\text { ft. }\end{array}$ & Clay & .- & .- & ..- & \\
\hline 411 & 5/4-11E1 & & & 360 & 450 & 109.7 & 137.2 & shale, more water & Clay and sand (or sandstone) & cemented & -- & -. & Tsv (?) \\
\hline 411 & 5/4-11E1 & & & 450 & 500 & 137.2 & 152.4 & shale & Clay and sand (or sandstone) & cemented & -- & -- & \\
\hline 411 & 5/4-11E1 & & & 500 & 700 & 152.4 & 213.4 & shale, blue; warm water & Clay and sand (or sandstone) & cemented & - & - & \\
\hline 412 & 5/4-9E1 & 412 & 5/4-9E1 & 0 & 298 & 0.0 & 90.8 & lava & Basalt & -. & -- & .- & Tsv \\
\hline
\end{tabular}


Appendix 3. Lithologic data. - Continued

[Site IDs and well number correspond to locations in Appendix 1, a site ID and well number is listed for each drilled interval for a particular drill hole. For convenience, the site ID and well numbers are repeated at the shallowest drilled interval for each well. leaders

\begin{tabular}{|c|c|c|c|c|c|c|c|c|c|c|c|c|c|}
\hline Site ID & Well number & $\begin{array}{l}\text { Site ID, } \\
\text { top of } \\
\text { well }\end{array}$ & $\begin{array}{l}\text { Well number, } \\
\text { top of well }\end{array}$ & $\begin{array}{l}\text { Top of } \\
\text { interval, in } \\
\text { feet }\end{array}$ & $\begin{array}{l}\text { Base of } \\
\text { interval, in } \\
\text { feet }\end{array}$ & $\begin{array}{c}\text { Top of } \\
\text { interval, in } \\
\mathrm{m}\end{array}$ & $\begin{array}{c}\text { Base of } \\
\text { interval, in } \\
\mathrm{m}\end{array}$ & Reported lithologic unit ${ }^{1}$ & Interpreted lithologic class ${ }^{2}$ & $\begin{array}{c}\text { Reported } \\
\text { degree of } \\
\text { cementation or } \\
\text { induration }^{3} \\
\end{array}$ & $\begin{array}{c}\text { Fossils } \\
\text { reported in } \\
\text { interval }^{4}\end{array}$ & $\begin{array}{c}\text { Organic } \\
\text { matter } \\
\text { reported in } \\
\text { interval }^{5}\end{array}$ & $\begin{array}{c}\text { Reported stratigraphic top of geologic } \\
\text { unit }^{6}\end{array}$ \\
\hline & & & & & & & & $\begin{array}{l}\text { open hole, water reached } \\
\text { (probably tuffaceous quicksand) }\end{array}$ & & & & & \\
\hline 412 & 5/4-9E1 & & & 298 & 310 & 90.8 & 94.5 & sand, white, bailed $30-50$ yds. & No data & -- & -- & -- & \\
\hline 412 & 5/4-9E1 & & & 310 & 380 & 94.5 & 115.8 & $\begin{array}{l}\text { clay, blue, water } \\
\text { cos. }\end{array}$ & Clay & -- & -- & -- & \\
\hline 413 & $5 / 4-12 J 1$ & 413 & $5 / 4-12 \mathrm{~J} 1$ & 0 & 7 & 0.0 & 2.1 & soil & Sand and clay & -- & -- & -- & Qt \\
\hline 413 & $5 / 4-12 \mathrm{J1}$ & & & 7 & 12 & 2.1 & 3.7 & gravel, and clay, water & Clay and gravel & -- & -- & -- & \\
\hline 413 & $5 / 4-12 \mathrm{~J} 1$ & & & 12 & 82 & 3.7 & 25.0 & clay & Clay & -- & -- & -- & Tsv \\
\hline 413 & $5 / 4-12 \mathrm{J1}$ & & & 82 & 111 & 25.0 & 33.8 & tule clay & Clay and sand (or sandstone) & -- & -- & tule & \\
\hline 413 & $5 / 4-12 \mathrm{J1}$ & & & 111 & 170 & 33.8 & 51.8 & boulders, cemented & Gravel & cemented & -- & -- & \\
\hline 413 & $5 / 4-12 \mathrm{~J} 1$ & & & 170 & 209 & 51.8 & 63.7 & lava, cemented & Basalt & -- & -. & -- & \\
\hline 413 & $5 / 4-12 \mathrm{J1}$ & & & 209 & 258 & 63.7 & 78.6 & $\begin{array}{l}\text { lava, water } \\
\text { la }\end{array}$ & Basalt & -- & -- & -- & \\
\hline 413 & $5 / 4-12 \mathrm{~J} 1$ & & & 258 & 271 & 78.6 & 82.6 & lava, cemented; some water & Basalt & -- & -- & -- & \\
\hline 413 & $5 / 4-12 \mathrm{~J} 1$ & & & 271 & 293 & 82.6 & 89.3 & lava, water & Basalt & -- & -- & -- & \\
\hline 413 & $5 / 4-12 \mathrm{J1}$ & & & 293 & 303 & 89.3 & 92.4 & lava, cemented & Basalt & -- & -- & -- & \\
\hline 414 & $5 / 4-9 \mathrm{~K} 2$ & 414 & $5 / 4-9 \times 2$ & 0 & 7 & 0.0 & 2.1 & surface & Sand and clay & -- & -- & -- & Qyal and Qoal \\
\hline 414 & $5 / 4-9 \mathrm{~K} 2$ & & & 7 & 20 & 2.1 & 6.1 & $\begin{array}{l}\text { clay, yellow } \\
\text { clats }\end{array}$ & Clay & -- & -- & -- & Qy ar arlu Luar \\
\hline 414 & $5 / 4-9 k^{2}$ & & & 20 & 25 & 6.1 & 7.6 & clay, yellow; and gravel water & Clay and gravel & -- & -- & -- & \\
\hline 414 & $5 / 4-9 \mathrm{~K} 2$ & & & 25 & 47 & 7.6 & 14.3 & clay, yellow & Clay & -- & -- & -- & \\
\hline 414 & $5 / 4-9 K 2$ & & & 47 & 80 & 14.3 & 24.4 & $\begin{array}{l}\text { clay, yellow; good gravel water } \\
\text { caw }\end{array}$ & Clay & -- & -- & -- & \\
\hline 414 & $5 / 4-9 \mathrm{~K} 2$ & & & 80 & 108 & 24.4 & 32.9 & clay, yellow; some gravel & Clay and gravel & -- & -- & -- & \\
\hline 415 & 5/4-11L3 & 415 & 5/4-11L3 & 0 & 2 & 0.0 & 0.6 & soil & Sand and clay & -- & -. & -- & Qoal \\
\hline 415 & $5 / 4-11\llcorner 3$ & & & 2 & 10 & 0.6 & 3.0 & clay, boulders & Clay and gravel & -- & -- & -- & \\
\hline 415 & 5/4-11L3 & & & 10 & 28 & 3.0 & 8.5 & sand and clay & Sand and clay & -- & -- & -- & \\
\hline 415 & $5 / 4-11\llcorner 3$ & & & 28 & 31 & 8.5 & $\begin{array}{l}.5 \\
9.4\end{array}$ & clay, sandy; and boulders & Clay, sand, and gravel & -- & -- & -- & \\
\hline 415 & 5/4-11L3 & & & 31 & 50 & 9.4 & 15.2 & dry sand and gravel & Sand (or sandstone) and gravel & -- & -- & -- & \\
\hline 415 & 5/4-11L3 & & & 50 & 67 & 15.2 & 20.4 & gravel and water & Gravel & -- & -- & -- & \\
\hline 415 & 5/4-11L3 & & & 67 & 69 & 20.4 & 21.0 & lava rock and mud & Basalt & hard & -- & -- & Tsv \\
\hline 416 & 5/4-9L1 & 416 & 5/4-9L1 & 0 & 64 & 0.0 & 19.5 & soil and clay & Sand and clay & -. & -- & -- & Tsv \\
\hline 416 & 5/4-9L1 & & & 64 & 66 & 19.5 & 20.1 & gravel, some water & Gravel & -- & -- & -- & \\
\hline 416 & 5/4-9L1 & & & 66 & 105 & 20.1 & 32.0 & clay and boulders & Clay and gravel & -- & -- & -- & \\
\hline 416 & 5/4-9L1 & & & 105 & 108 & 32.0 & 32.9 & gravel, no water, lost some water & Gravel & .- & .- & .- & \\
\hline 416 & 5/4-9L1 & & & 108 & 175 & 32.9 & 53.3 & clay & Clay & -- & -- & -- & \\
\hline 416 & 5/4-9L1 & & & 175 & 200 & 53.3 & 61.0 & clay, gravel & Clay and gravel & -. & -- & .- & \\
\hline 416 & 5/4-9L1 & & & 200 & 220 & 61.0 & 67.1 & clay & Clay & -- & -- & -- & \\
\hline 416 & 5/4-9L1 & & & 220 & 240 & 67.1 & 73.2 & clay, gravel, some water & Clay and gravel & -- & -- & -- & \\
\hline 416 & 5/4-9L1 & & & 240 & 258 & 73.2 & 78.6 & clay & Clay & -- & -- & -. & \\
\hline 417 & $5 / 4-9 \mathrm{Q} 1$ & 417 & 5/4-9Q1 & 0 & 2 & 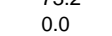 & 0.6 & soil & Sand and clay & -- & -- & -- & Qoal \\
\hline 417 & 5/4-9Q1 & & & 2 & 65 & 0.6 & 19.8 & clay, yellow & Clay & .- & -- & .- & \\
\hline 417 & $5 / 4-9 Q 1$ & & & 65 & 66 & 19.8 & 20.1 & gravel, water & Gravel & .- & -. & -- & \\
\hline 417 & $5 / 4-9 Q 1$ & & & 66 & 170 & 20.1 & 51.8 & clay, yellow & Clay & -- & -- & -- & \\
\hline 417 & 5/4-9Q1 & & & 170 & 172 & 51.8 & 52.4 & gravel, water & Gravel & -. & -- & -- & \\
\hline 417 & 5/4-9Q1 & & & 172 & 178 & $\begin{array}{l}52.4 \\
52.4\end{array}$ & 54.3 & clay, yellow & Clay & -- & -- & -- & \\
\hline 418 & 5/4-10P4 & 418 & 5/4-10P4 & 0 & 37 & 0.0 & 11.3 & clay & Clay & -- & -- & -- & Qyal and Qoal \\
\hline 418 & 5/4-10P4 & & & 37 & 75 & 11.3 & 22.9 & clay, blue & Clay & -- & -- & -- & \\
\hline 418 & 5/4-10P4 & & & 75 & 86 & 22.9 & 26.2 & gravel & Gravel & -- & -- & -- & \\
\hline 418 & 5/4-10P4 & & & 86 & 96 & 26.2 & 29.3 & clay and gravel & Clay and gravel & -. & -- & -- & \\
\hline 418 & 5/4-10P4 & & & 96 & 106 & 29.3 & 32.3 & clay & Clay & -.- & -- & -- & \\
\hline 418 & $5 / 4-10 \mathrm{P} 4$ & & & 106 & 116 & 32.3 & 35.4 & clay and gravel & Clay and gravel & .- & .- & -- & \\
\hline 418 & $5 / 4-10 \mathrm{P} 4$ & & & 116 & 162 & 35.4 & 49.4 & clay & Clay & -- & -- & -- & \\
\hline 418 & 5/4-10P4 & & & 162 & 194 & 49.4 & 59.1 & gravel & Gravel & -- & -- & -- & \\
\hline 418 & 5/4-10P4 & & & 194 & 228 & 59.1 & 69.5 & $\begin{array}{l}\text { clay } \\
\text { lat }\end{array}$ & Clay & -- & -- & -- & \\
\hline 418 & 5/4-10P4 & & & 228 & 241 & 69.5 & 73.5 & gravel & Gravel & -- & -- & -- & \\
\hline 418 & 5/4-10P4 & & & 241 & 247 & 73.5 & 75.3 & clay and gravel & Clay and gravel & -.- & -- & -- & \\
\hline 418 & $5 / 4-10 \mathrm{P} 4$ & & & 247 & 254 & 75.3 & 77.4 & gravel & Gravel & -- & -- & -- & \\
\hline 418 & 5/4-10P4 & & & 254 & 300 & 77.4 & 91.4 & clay & Clay & -- & -- & -- & \\
\hline 419 & $5 / 4-14 \mathrm{C} 1$ & 419 & $5 / 4-14 \mathrm{C} 1$ & 0 & 6 & 0.0 & $\begin{array}{l}1.4 \\
1.8\end{array}$ & surface & Sand and clay & - & -- & -- & Qoal \\
\hline 419 & $5 / 4-14 \mathrm{Cl}$ & & & 6 & 55 & 1.8 & 16.8 & clay, yellow & Clay & -- & -- & -- & \\
\hline 419 & $5 / 4-14 \mathrm{Cl} 1$ & & & 55 & 70 & 16.8 & 21.3 & gravel and boulders; water & Gravel & -- & -- & -- & \\
\hline 419 & $5 / 4-14 \mathrm{Cl}$ & & & 70 & 180 & 21.3 & 54.9 & clay, yellow & Clay & -- & -- & -- & \\
\hline 419 & $5 / 4-14 \mathrm{C} 1$ & & & 180 & 190 & 54.9 & 57.9 & gravel and boulders; water & Gravel & -- & -- & -- & \\
\hline
\end{tabular}


Appendix 3. Lithologic data. - Continued

[Site IDs and well number correspond to locations in Appendix 1, a site ID and well number is listed for each drilled interval for a particular drill hole. For convenience, the site ID and well numbers are repeated at the shallowest drilled interval for each well. leaders $(-1,0$

\begin{tabular}{|c|c|c|c|c|c|c|c|c|c|c|c|c|c|}
\hline Site ID & Well number & $\begin{array}{l}\text { Site ID, } \\
\text { top of } \\
\text { well }\end{array}$ & $\begin{array}{l}\text { Well number, } \\
\text { top of well }\end{array}$ & $\begin{array}{c}\text { Top of } \\
\text { interval, in } \\
\text { feet }\end{array}$ & $\begin{array}{l}\text { Base of } \\
\text { interval, in } \\
\text { feet }\end{array}$ & $\begin{array}{c}\text { Top of } \\
\text { interval, in } \\
\mathrm{m}\end{array}$ & $\begin{array}{c}\text { Base of } \\
\text { interval, in } \\
\mathrm{m}\end{array}$ & Reported lithologic unit' ${ }^{1}$ & Interpreted lithologic class ${ }^{2}$ & $\begin{array}{c}\text { Reported } \\
\text { degree of } \\
\text { cementation or } \\
\text { induration }^{3} \\
\end{array}$ & $\begin{array}{c}\text { Fossils } \\
\text { reported in } \\
\text { interval }^{4}\end{array}$ & $\begin{array}{c}\text { Organic } \\
\text { matter } \\
\text { reported in } \\
\text { interval }^{5}\end{array}$ & $\begin{array}{l}\text { Reported stratigraphic top of geologic } \\
\text { unit }^{6}\end{array}$ \\
\hline 419 & $5 / 4-14 \mathrm{C} 1$ & & & 190 & 220 & 57.9 & 67.1 & $\begin{array}{l}\text { clay and boulders, yellow; some } \\
\text { water }\end{array}$ & Clay and gravel & 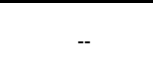 & -- & -- & \\
\hline 420 & $5 / 4-15 C 2$ & 420 & $5 / 4-15 C 2$ & 0 & 60 & 0.0 & 18.3 & old well, material not described & No data & -- & -- & -- & -- \\
\hline 420 & $5 / 4-15 C 2$ & & & 60 & 82 & 18.3 & 25.0 & $\begin{array}{l}\text { clay and some gravel } \\
\text { clay, blue, gravel at bottom; salty }\end{array}$ & Clay and gravel & -- & -- & -- & Qoal \\
\hline 420 & $5 / 4-15 \mathrm{C} 2$ & & & 82 & 86.6 & 25.0 & 26.4 & water & Clay & -- & -- & -- & \\
\hline 421 & $5 / 3-17 \mathrm{C} 1$ & 421 & 5/3-17C1 & 0 & 84 & 0.0 & 25.6 & tuff & Ash or tuff & -- & -- & -- & Tsv \\
\hline 421 & $5 / 3-17 C 1$ & & & 84 & 105 & 25.6 & 32.0 & rock, hard & Ash or tuff & hard & -- & -- & \\
\hline 421 & $5 / 3-17 \mathrm{C} 1$ & & & 105 & 185 & 32.0 & 56.4 & tuff & Ash or tuff & -- & -- & -- & \\
\hline 421 & $5 / 3-17 \mathrm{C} 1$ & & & 185 & 210 & 56.4 & 64.0 & lava, brown; some water & Basalt & -- & -- & -- & \\
\hline 421 & $5 / 3-17 C 1$ & & & 210 & 237 & 64.0 & 72.2 & tuff, blue & Ash or tuff & -- & -- & -- & \\
\hline 421 & $5 / 3-17 \mathrm{C} 1$ & & & 237 & 261 & 72.2 & 79.6 & lava, broken; water & Basalt & -- & -- & -- & \\
\hline 422 & 5/4-13E1 & 422 & 5/4-13E1 & 0 & 9 & 0.0 & 2.7 & soil & Sand and clay & -- & -- & -- & Qoal \\
\hline 422 & 5/4-13E1 & & & 9 & 30 & 2.7 & 9.1 & hardpan & Clay and gravel & hard & -- & -- & \\
\hline 422 & 5/4-13E1 & & & 30 & 35 & 9.1 & 10.7 & gravel water & Gravel & -- & -- & -- & \\
\hline 422 & 5/4-13E1 & & & 35 & 48 & 10.7 & 14.6 & hardpan & Clay and gravel & hard & -- & -- & \\
\hline 422 & 5/4-13E1 & & & 48 & 65 & 14.6 & 19.8 & clay, blue & Clay & -- & -- & -- & \\
\hline 422 & 5/4-13E1 & & & 65 & 140 & 19.8 & 42.7 & clay, blue shale & Clay and sand (or sandstone) & cemented & -- & -- & \\
\hline 422 & 5/4-13E1 & & & 140 & 148 & 42.7 & 45.1 & gravel, some water & Gravel & -- & -- & -- & \\
\hline 422 & 5/4-13E1 & & & 148 & 153 & 45.1 & 46.6 & shale & Clay and sand (or sandstone) & cemented & -- & -- & Tsv \\
\hline 423 & $5 / 4-14 \mathrm{H} 3$ & 423 & $5 / 4-14 \mathrm{H} 3$ & 0 & 10 & 0.0 & 3.0 & soil & Sand and clay & -- & -- & -- & Qoal \\
\hline 423 & $5 / 4-14 \mathrm{H} 3$ & & & 10 & 54 & 3.0 & 16.5 & clay, yellow & Clay & -- & -- & -- & \\
\hline 423 & $5 / 4-14 \mathrm{H} 3$ & & & 54 & 55 & 16.5 & 16.8 & gravel, water & Gravel & -- & -- & -- & \\
\hline 423 & $5 / 4-14 \mathrm{H} 3$ & & & 55 & 81 & 16.8 & 24.7 & clay & Clay & -- & -- & -- & \\
\hline 423 & $5 / 4-14 \mathrm{H} 3$ & & & 81 & 87 & 24.7 & 26.5 & gravel, water & Gravel & -- & -- & -- & \\
\hline 423 & $5 / 4-14 \mathrm{H} 3$ & & & 87 & 102 & 26.5 & 31.1 & clay and boulders & Clay and gravel & -- & -- & -- & \\
\hline 423 & $5 / 4-14 \mathrm{H} 3$ & & & 102 & 109 & 31.1 & 33.2 & boulders, clay and shale & Clay, sand, and gravel & cemented & -- & -- & \\
\hline 423 & $5 / 4-14 \mathrm{H} 3$ & & & 109 & 240 & 33.2 & 73.2 & gravel, sand, water & Sand (or sandstone) and gravel & -- & -- & -- & \\
\hline 424 & $5 / 4-17 F 1$ & 424 & $5 / 4-17 F 1$ & 0 & 160 & 0.0 & 48.8 & $\begin{array}{l}\text { shale, blue, salty water } \\
\text { washed clay, yellow, sandy, }\end{array}$ & Clay and sand (or sandstone) & cemented & -- & -- & Capay shale \\
\hline 425 & 5/4-13L1 & 425 & $5 / 4-13\llcorner 1$ & 0 & 49 & 0.0 & 14.9 & containing gravel & Clay, sand, and gravel & -- & -- & -- & Qoal \\
\hline 425 & $5 / 4-13\llcorner 1$ & & & 49 & 83 & 14.9 & 25.3 & $\begin{array}{l}\text { clay, blue, sandy } \\
\text { clay, black, slightly sand and } \\
\text { containing a large nercent of }\end{array}$ & Clay and sand (or sandstone) & -- & -- & -- & \\
\hline 425 & 5/4-13ட1 & & & 83 & 100 & 25.3 & 30.5 & vegetable matter & Clay and sand (or sandstone) & -- & -- & plants & \\
\hline 425 & $5 / 4-13\llcorner 1$ & & & 100 & 125 & 30.5 & 38.1 & clay, blue, sandy & Clay and sand (or sandstone) & -- & -- & -- & \\
\hline 425 & $5 / 4-13 L 1$ & & & 125 & 215 & 38.1 & 65.5 & tuff & Ash or tuff & -- & -- & .- & Tsv \\
\hline 425 & 5/4-13L1 & & & 215 & 260 & 65.5 & 79.2 & $\begin{array}{l}\text { tuff, chiefly fine white ash } \\
\text { tuff, chiefly fragments of pumice }\end{array}$ & Ash or tuff & -- & -- & -- & \\
\hline 425 & $5 / 4-13\llcorner 1$ & & & 260 & 265 & 79.2 & 80.8 & and basalt & Ash or tuff & -- & -- & -- & \\
\hline 425 & $5 / 4-13\llcorner 1$ & & & 265 & 270 & 80.8 & 82.3 & tuff & Ash or tuff & -- & -- & - & \\
\hline 425 & $5 / 4-13\llcorner 1$ & & & 270 & 370 & 82.3 & 112.8 & tuff, chiefly pumice & Ash or tuff & -- & -- & -- & \\
\hline 425 & 5/4-13L1 & & & 370 & 375 & 112.8 & 114.3 & tule & Clay and sand (or sandstone) & - & -- & tule & \\
\hline 425 & 5/4-13ட1 & & & 375 & 390 & 114.3 & 118.9 & tuff & Ash or tuff & -- & -- & -- & \\
\hline 425 & $5 / 4-13\llcorner 1$ & & & 390 & 401 & 118.9 & 122.2 & basalt & Basalt & -- & -- & -- & \\
\hline 425 & $5 / 4-13\llcorner 1$ & & & 401 & 406 & 122.2 & 123.7 & clay, blue & Clay & -- & -- & -- & \\
\hline 425 & 5/4-13L1 & & & 406 & 420 & 123.7 & 128.0 & tuff, brown & Ash or tuff & -- & -- & -- & \\
\hline 425 & $5 / 4-13\llcorner 1$ & & & 420 & 425 & 128.0 & 129.5 & clay, blue, sticky & Clay & -- & -- & -- & \\
\hline 425 & $5 / 4-13\llcorner 1$ & & & 425 & 430 & 129.5 & 131.1 & tuff, brown & Ash or tuff & -. & -- & -. & \\
\hline 425 & $5 / 4-13\llcorner 1$ & & & 430 & 432 & 131.1 & 131.7 & basalt & Basalt & -- & -- & -- & \\
\hline 425 & $5 / 4-13\llcorner 1$ & & & 432 & 438 & 131.7 & 133.5 & clay, brown, soft & Clay & -- & -- & -- & \\
\hline 425 & 5/4-13L1 & & & 438 & 440 & 133.5 & 134.1 & basalt, very hard & Basalt & hard & -- & -- & \\
\hline 425 & $5 / 4-13\llcorner 1$ & & & 440 & 445 & 134.1 & 135.6 & ash & Ash or tuff & -- & -- & -- & \\
\hline 425 & $5 / 4-13 L 1$ & & & 445 & 450 & 135.6 & 137.2 & clay, blue, sticky & Clay & -- & -- & -- & \\
\hline 425 & $5 / 4-13\llcorner 1$ & & & 450 & 455 & 137.2 & 138.7 & tuff, brown & Ash or tuff & -- & -- & -- & \\
\hline 425 & 5/4-13L1 & & & 455 & 472 & 138.7 & 143.9 & clay and rock & Clay and gravel & -- & -- & -. & \\
\hline 425 & $5 / 4-13\llcorner 1$ & & & 472 & 475 & 143.9 & 144.8 & basalt & Basalt & -- & -- & -- & \\
\hline 425 & $5 / 4-13\llcorner 1$ & & & 475 & 485 & 144.8 & 147.8 & basalt, porous & Basalt & -- & -- & -- & \\
\hline 425 & 5/4-13L1 & & & 485 & 500 & 147.8 & 152.4 & clay, blue, sickly & Clay & -- & -- & -- & \\
\hline 425 & $5 / 4-13\llcorner 1$ & & & 500 & 510 & 152.4 & 155.4 & basalt and lava & Basalt & -- & -- & -- & \\
\hline 425 & $5 / 4-13\llcorner 1$ & & & 510 & 518 & 155.4 & 157.9 & clay, blue, sticky & Clay & -- & -- & -- & \\
\hline
\end{tabular}


Appendix 3. Lithologic data. - Continued

[Site IDs and well number correspond to locations in Appendix 1, a site ID and well number is listed for each drilled interval for a particular drill hole. For convenience, the site ID and well numbers are repeated at the shallowest drilled interval for each well. leaders

\begin{tabular}{|c|c|c|c|c|c|c|c|c|c|c|c|c|c|}
\hline Site ID & Well number & $\begin{array}{l}\text { Site ID, } \\
\text { top of } \\
\text { well }\end{array}$ & $\begin{array}{l}\text { Well number, } \\
\text { top of well }\end{array}$ & $\begin{array}{c}\text { Top of } \\
\text { interval, in } \\
\text { feet }\end{array}$ & $\begin{array}{c}\text { Base of } \\
\text { interval, in } \\
\text { feet }\end{array}$ & $\begin{array}{c}\text { Top of } \\
\text { interval, in } \\
m\end{array}$ & $\begin{array}{c}\text { Base of } \\
\text { interval, in } \\
\mathrm{m}\end{array}$ & Reported lithologic unit ${ }^{1}$ & Interpreted lithologic class ${ }^{2}$ & $\begin{array}{c}\text { Reported } \\
\text { degree of } \\
\text { cementation or } \\
\text { induration } \\
\end{array}$ & $\begin{array}{l}\text { Fossils } \\
\text { reported in } \\
\text { interval }^{4}\end{array}$ & $\begin{array}{c}\text { Organic } \\
\text { matter } \\
\text { reported in } \\
\text { interval }^{5}\end{array}$ & $\begin{array}{l}\text { Reported stratigraphic top of geologic } \\
\text { unit }\end{array}$ \\
\hline 425 & 5/4-13L1 & & & 518 & 520 & 157.9 & 158.5 & basalt & Basalt & -- & -- & -- & \\
\hline 425 & $5 / 4-13\llcorner 1$ & & & 520 & 522 & 158.5 & 159.1 & clay, sticky & Clay & -- & -- & -- & \\
\hline 425 & $5 / 4-13\llcorner 1$ & & & 522 & 525 & 159.1 & 160.0 & basalt and lava & Basalt & -- & -- & -- & \\
\hline 425 & $5 / 4-13\llcorner 1$ & & & 525 & 530 & 160.0 & 161.5 & basalt and pink lava & Basalt & -- & -- & -- & \\
\hline 425 & $5 / 4-13 L 1$ & & & 530 & 535 & 161.5 & 163.1 & clay, sticky & Clay & -- & -- & -- & \\
\hline 425 & $5 / 4-13\llcorner 1$ & & & 535 & 537 & 163.1 & 163.7 & basalt and lava & Basalt & -- & -- & -- & \\
\hline 425 & 5/4-13L1 & & & 537 & 539 & 163.7 & 164.3 & $\begin{array}{l}\text { clay, sticky } \\
\text { lat }\end{array}$ & Clay & -- & -- & -- & \\
\hline 425 & $5 / 4-13\llcorner 1$ & & & 539 & 545 & 164.3 & 166.1 & basalt and lava & Basalt & -- & -- & -- & \\
\hline 425 & $5 / 4-13 L 1$ & & & 545 & 555 & 166.1 & 169.2 & basalt and lava, pink & Basalt & -- & -- & -- & \\
\hline 425 & 5/4-13L1 & & & 555 & 568 & 169.2 & 173.1 & basalt and lava, black & Basalt & -- & -- & - & \\
\hline 425 & $5 / 4-13\llcorner 1$ & & & 568 & 570 & 173.1 & 173.7 & sand and lava & Sand (or sandstone) and gravel & -- & -- & -- & \\
\hline 425 & $5 / 4-13 L 1$ & & & 570 & 580 & 173.7 & 176.8 & tuff and lava & Ash or tuff & -- & -- & -- & \\
\hline 425 & $5 / 4-13\llcorner 1$ & & & 580 & 585 & 176.8 & 178.3 & tuff and lava, red & Ash or tuff & -- & -- & -- & \\
\hline 425 & 5/4-13L1 & & & 585 & 610 & 178.3 & 185.9 & tuff and lava, blue & Ash or tuff & -- & -- & -- & \\
\hline 425 & $5 / 4-13\llcorner 1$ & & & 610 & 615 & 185.9 & 187.5 & lava and sandy & Basalt & -- & -- & -. & \\
\hline 425 & 5/4-13L1 & & & 615 & 620 & 187.5 & 189.0 & tuff, red and yellow & Ash or tuff & -- & -- & -- & \\
\hline 425 & $5 / 4-13\llcorner 1$ & & & 620 & 640 & 189.0 & 195.1 & basalt and lava, black & Basalt & -- & -- & -- & \\
\hline 425 & $5 / 4-13\llcorner 1$ & & & 640 & 650 & 195.1 & 198.1 & tuff, black & Ash or tuff & -- & -- & -- & \\
\hline 425 & 5/4-13L1 & & & 650 & 670 & 198.1 & 204.2 & tuff, and black sand & Ash or tuff & -- & -- & -- & \\
\hline 425 & $5 / 4-13\llcorner 1$ & & & 670 & 675 & 204.2 & 205.7 & clay, sticky, some rock & Clay and gravel & -- & -- & -- & \\
\hline 425 & $5 / 4-13\llcorner 1$ & & & 675 & 680 & 205.7 & 207.3 & tuff and sand & Ash or tuff & -- & -- & -- & \\
\hline 425 & $5 / 4-13\llcorner 1$ & & & 680 & 690 & 207.3 & 210.3 & sand, black & Sand & -- & -- & -- & \\
\hline 425 & 5/4-13L1 & & & 690 & 695 & 210.3 & 211.8 & tuff, and gray sand & Ash or tuff & -- & -- & -- & \\
\hline 425 & 5/4-13ட1 & & & 695 & 700 & 211.8 & 213.4 & tuff, various colors & Ash or tuff & -- & -- & -. & \\
\hline 425 & 5/4-13L1 & & & 700 & 705 & 213.4 & 214.9 & tuff and lava & Ash or tuff & -- & -- & -- & \\
\hline 425 & $5 / 4-13\llcorner 1$ & & & 705 & 720 & 214.9 & 219.5 & tuff and sand & Ash or tuff & -- & -- & -. & \\
\hline 425 & $5 / 4-13\llcorner 1$ & & & 720 & 733 & 219.5 & 223.4 & basalt & Basalt & -- & -- & -- & \\
\hline 425 & 5/4-13L1 & & & 733 & 736 & 223.4 & 224.3 & lava & Basalt & -- & -- & -- & \\
\hline 425 & 5/4-13L1 & & & 736 & 742 & 224.3 & 226.2 & basalt & Basalt & -- & -- & -- & \\
\hline 425 & $5 / 4-13\llcorner 1$ & & & 742 & 746 & 226.2 & 227.4 & lava and sandy & Basalt & -- & -- & -- & \\
\hline 425 & $5 / 4-13\llcorner 1$ & & & 746 & 750 & 227.4 & 228.6 & basalt & Basalt & -- & -- & -.- & \\
\hline 425 & $5 / 4-13\llcorner 1$ & & & 750 & 765 & 228.6 & 233.2 & tuff, blue & Ash or tuff & -- & -- & -- & \\
\hline 425 & 5/4-13L1 & & & 765 & 770 & 233.2 & 234.7 & sand, gray & Sand & -- & -- & -- & \\
\hline 425 & 5/4-13L1 & & & 770 & 779 & 234.7 & 237.4 & sand and lava, various colors & Sand (or sandstone) and gravel & -- & -- & -. & \\
\hline 425 & $5 / 4-13\llcorner 1$ & & & 779 & 785 & 237.4 & 239.3 & tuff and lava & Ash or tuff & -- & -- & -- & \\
\hline 425 & $5 / 4-13\llcorner 1$ & & & 785 & 792 & 239.3 & 241.4 & tuff and basalt & Ash or tuff & -- & -- & -- & \\
\hline 425 & $5 / 4-13\llcorner 1$ & & & 792 & 796 & 241.4 & 242.6 & basalt & Basalt & -- & -- & -- & \\
\hline 425 & $5 / 4-13\llcorner 1$ & & & 796 & 816 & 242.6 & 248.7 & basalt, very hard & Basalt & hard & -- & -- & \\
\hline 425 & $5 / 4-13\llcorner 1$ & & & 816 & 821 & 248.7 & 250.2 & basalt & Basalt & -- & -- & -- & \\
\hline 425 & 5/4-13L1 & & & 821 & 822 & 250.2 & 250.5 & basalt, very hard & Basalt & hard & -- & -- & \\
\hline 425 & 5/4-13L1 & & & 822 & 824 & 250.5 & 251.2 & basalt & Basalt & -- & -- & -- & \\
\hline 425 & 5/4-13L1 & & & 824 & 826 & 251.2 & 251.8 & basalt, very hard & Basalt & hard & -- & -- & \\
\hline 425 & $5 / 4-13\llcorner 1$ & & & 826 & 835 & 251.8 & 254.5 & basalt & Basalt & -- & -- & -- & \\
\hline 425 & 5/4-13ட1 & & & 835 & 860 & 254.5 & 262.1 & basalt, very hard & Basalt & hard & -- & -- & \\
\hline 425 & 5/4-13L1 & & & 860 & 865 & 262.1 & 263.7 & basalt, soft & Basalt & -- & -- & -- & \\
\hline 425 & 5/4-13L1 & & & 865 & 869 & 263.7 & 264.9 & basalt, black and hard & Basalt & hard & -- & -- & \\
\hline 425 & 5/4-13L1 & & & 869 & 875 & 264.9 & 266.7 & basalt, soft and pink & Basalt & -- & -- & -- & \\
\hline 425 & 5/4-13ட1 & & & 875 & 878 & 266.7 & 267.6 & basalt, hard & Basalt & hard & -- & -- & \\
\hline 425 & 5/4-13L1 & & & 878 & 886 & 267.6 & 270.1 & basalt, soft and pink & Basalt & -- & - & -- & \\
\hline 425 & 5/4-13ட1 & & & 886 & 895 & 270.1 & 272.8 & basalt, very hard & Basalt & hard & -- & -- & \\
\hline 425 & 5/4-13L1 & & & 895 & 904 & 272.8 & 275.5 & basalt, hard & Basalt & hard & -- & -- & \\
\hline 426 & 5/4-14L2 & 426 & $5 / 4-14 \mathrm{~L} 2$ & 0 & 56 & 0.0 & 17.1 & gravel and clay & Clay and gravel & -- & -- & -- & Qoal \\
\hline 426 & $5 / 4-14 \mathrm{~L} 2$ & & & 56 & 80 & 17.1 & 24.4 & clay, yellow & Clay & -- & -- & -- & \\
\hline 426 & $5 / 4-14 L 2$ & & & 80 & 85 & 24.4 & 25.9 & gravel, flowing water & Gravel & -- & -- & -- & \\
\hline 426 & $5 / 4-14 \mathrm{~L} 2$ & & & 85 & 105 & 25.9 & 32.0 & $\begin{array}{l}\text { tule mud } \\
\text { Sult }\end{array}$ & Clay and sand (or sandstone) & -- & -. & tule & \\
\hline 426 & 5/4-14L2 & & & 105 & 255 & 32.0 & 77.7 & $\begin{array}{l}\text { volcanic ash compact } \\
\text { volcanic rock and sands gradual } \\
\text { increase in water from } 225 \text { to } 323\end{array}$ & Ash or tuff & -- & -- & -- & Tsv \\
\hline 426 & $5 / 4-14 L 2$ & & & 255 & 323 & 77.7 & 98.5 & $\mathrm{ft.}$ & Ash or tuff & -. & .- & .- & \\
\hline 426 & $5 / 4-14 L 2$ & & & 323 & 325 & 98.5 & 99.1 & basalt rock & Basalt & hard & -. & -- & \\
\hline 427 & 5/4-14L1 & 427 & 5/4-14L1 & 0 & 5 & 0.0 & 1.5 & surface & Sand and clay & -- & -- & -- & Qoal \\
\hline
\end{tabular}


Appendix 3. Lithologic data. - Continued

[Site IDs and well number correspond to locations in Appendix 1, a site ID and well number is listed for each drilled interval for a particular drill hole. For convenience, the site ID and well numbers are repeated at the shallowest drilled interval for each well. leaders

\begin{tabular}{|c|c|c|c|c|c|c|c|c|c|c|c|c|c|}
\hline Site ID & Well number & $\begin{array}{l}\text { Site ID, } \\
\text { top of } \\
\text { well }\end{array}$ & $\begin{array}{l}\text { Well number, } \\
\text { top of well }\end{array}$ & $\begin{array}{c}\text { Top of } \\
\text { interval, in } \\
\text { feet }\end{array}$ & $\begin{array}{c}\text { Base of } \\
\text { interval, in } \\
\text { feet }\end{array}$ & $\begin{array}{c}\text { Top of } \\
\text { interval, in } \\
\mathrm{m}\end{array}$ & $\begin{array}{c}\text { Base of } \\
\text { interval, in } \\
\mathrm{m}\end{array}$ & Reported lithologic unit ${ }^{1}$ & Interpreted lithologic class ${ }^{2}$ & $\begin{array}{c}\text { Reported } \\
\text { degree of } \\
\text { cementation or } \\
\text { induration } \\
\end{array}$ & $\begin{array}{c}\text { Fossils } \\
\text { reported in } \\
\text { interval }^{4}\end{array}$ & $\begin{array}{c}\text { Organic } \\
\text { matter } \\
\text { reported in } \\
\text { interval }^{5}\end{array}$ & $\begin{array}{l}\text { Reported stratigraphic top of geologic } \\
\text { unit }^{6}\end{array}$ \\
\hline 427 & $5 / 4-14 \mathrm{~L} 1$ & & & 5 & 30 & 1.5 & 9.1 & clay, yellow & Clay & -- & -- & -- & \\
\hline 427 & 5/4-14L1 & & & 30 & 43 & 9.1 & 13.1 & gravel & Gravel & -- & -- & -- & \\
\hline 427 & $5 / 4-14 L 1$ & & & 43 & 50 & 13.1 & 15.2 & clay, yellow & Clay & -- & -. & -- & \\
\hline 427 & $5 / 4-14 L 1$ & & & 50 & 97 & 15.2 & 29.6 & clay, blue, gravel; and boulders & Clay and gravel & -- & -- & -- & \\
\hline 427 & 5/4-14L1 & & & 97 & 130 & 29.6 & 39.6 & tule mud & Clay and sand (or sandstone) & -- & -- & tule & Tsv \\
\hline 427 & $5 / 4-14 L 1$ & & & 130 & 175 & 39.6 & 53.3 & clay, blue & Clay & -- & -- & -- & \\
\hline 427 & 5/4-14L1 & & & 175 & 180 & 53.3 & 54.9 & tuff, brown, soft & Ash or tuff & -- & - & -- & \\
\hline 427 & $5 / 4-14 L 1$ & & & 180 & 200 & 54.9 & 61.0 & $\begin{array}{l}\text { tuff, hard, gray } \\
\text { nat }\end{array}$ & Ash or tuff & hard & -- & -- & \\
\hline 427 & 5/4-14L1 & & & 200 & 215 & 61.0 & 65.5 & tuff & Ash or tuff & -- & -- & -- & \\
\hline 427 & 5/4-14L1 & & & 215 & 263 & 65.5 & 80.2 & volcanic ash & Ash or tuff & -- & -- & -- & \\
\hline 427 & $5 / 4-14 L 1$ & & & 263 & 325 & 80.2 & $\begin{array}{l}90.2 \\
99.1\end{array}$ & conglomerate, basaltic & Basalt &.- & - & - & \\
\hline 427 & 5/4-14L1 & & & 325 & 350 & 99.1 & 106.7 & basalt, hard & Basalt & hard & -- & -- & \\
\hline 427 & $5 / 4-14 L 1$ & & & 350 & 354 & 106.7 & 107.9 & clay, blue rock & Clay and gravel & -- & -- & -- & \\
\hline 427 & 5/4-14L1 & & & 354 & 369 & 107.9 & 112.5 & basalt and clay & Basalt & -- & -- & -- & \\
\hline 427 & 5/4-14L1 & & & 369 & 382 & 112.5 & 116.4 & $\begin{array}{l}\text { clay blue } \\
\text { las }\end{array}$ & Clay & -- & -- & -- & \\
\hline 427 & 5/4-14L1 & & & 382 & 433 & 116.4 & 132.0 & basalt and clay & Basalt & -- & -- & -- & \\
\hline 427 & 5/4-14L1 & & & 433 & 438 & 132.0 & 133.5 & basalt, hard & Basalt & hard & -- & -- & \\
\hline 427 & $5 / 4-14 L 1$ & & & 438 & 440 & 133.5 & 134.1 & clay & Clay & -. & - & - & \\
\hline 427 & 5/4-14L1 & & & 440 & 465 & 134.1 & 141.7 & basalt, hard & Basalt & hard & -- & -- & \\
\hline 427 & $5 / 4-14 L 1$ & & & 465 & 488 & 141.7 & 148.7 & clay, hard, and rock & Clay and gravel & hard & -- & -- & \\
\hline 427 & 5/4-14L1 & & & 488 & 492 & 148.7 & 150.0 & clay & Clay & -. & -. & .. & \\
\hline 427 & $5 / 4-14 L 1$ & & & 492 & 502 & 150.0 & 153.0 & lava, red; water & Basalt & -- & -- & -- & \\
\hline 427 & $5 / 4-14 L 1$ & & & 502 & 520 & 153.0 & 158.5 & clay and rock & Clay and gravel & -- & - & - & \\
\hline 427 & 5/4-14L1 & & & 520 & 525 & 158.5 & 160.0 & basalt, hard; and clay & Basalt & hard & -- & -- & \\
\hline 428 & 5/4-15K1 & 428 & 5/4-15K1 & 0 & 3 & 0.0 & 0.9 & soil & Sand and clay & -- & .- & .- & Qyal and Qoal \\
\hline 428 & $5 / 4-15 K 1$ & & & 3 & 12 & 0.9 & 3.7 & clay, yellow & Clay & -- & -- & -- & \\
\hline 428 & $5 / 4-15 \mathrm{~K} 1$ & & & 12 & 13 & 3.7 & 4.0 & sand, water & Sand & .- & .- & .. & \\
\hline 428 & 5/4-15K1 & & & 13 & 20 & 4.0 & 6.1 & clay, yellow & Clay & -- & -- & -- & \\
\hline 428 & 5/4-15K1 & & & 20 & 28 & 6.1 & 8.5 & sand and gravel & Sand (or sandstone) and gravel & -- & -- & -- & \\
\hline 428 & $5 / 4-15 \mathrm{~K} 1$ & & & 28 & 35 & 8.5 & 10.7 & gravel and clay & $\begin{array}{l}\text { Clay and gravel } \\
\text {. }\end{array}$ & - & - &.- & \\
\hline 428 & $5 / 4-15 \mathrm{~K} 1$ & & & 35 & 41 & 10.7 & 12.5 & sand, blue; and clay & Sand and clay & - & -- & -- & \\
\hline 428 & $5 / 4-15 \mathrm{~K} 1$ & & & 41 & 70 & 12.5 & 21.3 & $\begin{array}{l}\text { clay, blue } \\
\text { land }\end{array}$ & Clay & -- & -- & -. & \\
\hline 428 & 5/4-15K1 & & & 70 & 101 & 21.3 & 30.8 & clay, yellow & Clay & - & -- & -- & \\
\hline 428 & 5/4-15K1 & & & 101 & 105 & 30.8 & 32.0 & clay and sand, yellow & Clay and sand (or sandstone) & -- & -- & -- & \\
\hline 428 & $5 / 4-15 \mathrm{~K} 1$ & & & 105 & 118 & 32.0 & 36.0 & $\begin{array}{l}\text { clay and sand } \\
\text { sation }\end{array}$ & Clay and sand (or sandstone) & - & -- & - & \\
\hline 428 & $5 / 4-15 \mathrm{~K} 1$ & & & 118 & 191 & 36.0 & 58.2 & clay, yellow & Clay & -- & -- & -- & \\
\hline 428 & 5/4-15K1 & & & 191 & 195 & 58.2 & 59.4 & gravel & Gravel & - & -- & -. & \\
\hline 428 & 5/4-15K1 & & & 195 & 205 & 59.4 & 62.5 & clay, yellow & Clay & -- & -- & -- & \\
\hline 428 & $5 / 4-15 \mathrm{~K} 1$ & & & 205 & 227 & 62.5 & 69.2 & clay, white & Clay & - & -- & -- & \\
\hline 428 & $5 / 4-15 K 1$ & & & 227 & 238 & 69.2 & 72.5 & gravel strips & Gravel & -- & -- & -. & \\
\hline 428 & 5/4-15K1 & & & 238 & 252 & 72.5 & 76.8 & clay, yellow & Clay & -- & -. & -. & \\
\hline 428 & $5 / 4-15 \mathrm{~K} 1$ & & & 252 & 276 & 76.8 & 84.1 & sand and gravel, blue & Sand (or sandstone) and gravel & - & -- & - & \\
\hline 428 & 5/4-15K1 & & & 276 & 282 & 84.1 & 86.0 & clay, blue & Clay & -- & -- & -- & \\
\hline 428 & $5 / 4-15 \mathrm{~K} 1$ & & & 282 & 298 & 86.0 & 90.8 & boulders & Gravel & -- & -- & -- & \\
\hline 428 & 5/4-15K1 & & & 298 & 327 & 90.8 & 99.7 & clay, blue & Clay & -- & -. & ..- & \\
\hline 428 & 5/4-15K1 & & & 327 & 328 & 99.7 & 100.0 & boulders & Gravel & -- & -- & -. & \\
\hline 428 & $5 / 4-15 \mathrm{~K} 1$ & & & 328 & 340 & 100.0 & 103.6 & clay, blue & Clay & - & - & -- & \\
\hline 428 & 5/4-15K1 & & & 340 & 510 & 103.6 & 155.4 & tule & Clay and sand (or sandstone) & -- & -- & tule & \\
\hline 428 & 5/4-15K1 & & & 510 & 580 & 155.4 & $\begin{array}{l}176.8 \\
176.8-5\end{array}$ & tule mud & Clay and sand (or sandstone) & - & - & $\begin{array}{l}\text { tule } \\
\text { tula }\end{array}$ & \\
\hline 428 & 5/4-15K1 & & & 580 & 658 & 176.8 & 200.6 & sand, blue & Sand & -- & .. & -- & \\
\hline 428 & 5/4-15K1 & & & 658 & 677 & 200.6 & 206.3 & rock, soft, blue & Sandstone & hard & -- & -- & \\
\hline 428 & 5/4-15K1 & & & $\begin{array}{l}500 \\
677\end{array}$ & 791 & 206.3 & 241.1 & $\begin{array}{l}\text { clay, blue } \\
\text { clue }\end{array}$ & Clay & Mal- & -. & -- & \\
\hline 428 & 5/4-15K1 & & & 791 & 802 & 241.1 & 244.4 & shale, possibly andesite & Ash or tuff & cemented & -- & -- & Tsv (?) \\
\hline 428 & $5 / 4-15 \mathrm{~K} 1$ & & & 802 & 869 & 244.4 & 264.9 & shale, brown & Ash or tuff & cemented & -- & -- & \\
\hline 428 & 5/4-15K1 & & & 869 & 891 & 264.9 & 271.6 & tule mud & Ash or tuff & -- & -- & tule & \\
\hline 428 & 5/4-15K1 & & & 891 & 892 & 271.6 & 271.9 & serpentine & Basalt & -- & -. & -- & \\
\hline 428 & 5/4-15K1 & & & 892 & 903 & 271.9 & 275.2 & tule mud & Ash or tuff & -- & -.- & tule & \\
\hline 428 & 5/4-15K1 & & & 903 & 910 & 275.2 & 277.4 & boulders & Ash or tuff & -- & -- & -- & \\
\hline 428 & 5/4-15K1 & & & 910 & 918 & 277.4 & 279.8 & shale brown & Ash or tuff & cemented & -.- & -- & \\
\hline 428 & 5/4-15K1 & & & 918 & 940 & 279.8 & 286.5 & clay, blue & Ash or tuff & -- & -. & -- & \\
\hline 428 & $5 / 4-15 \mathrm{~K} 1$ & & & 940 & 950 & 286.5 & 289.6 & $\begin{array}{l}\text { tule mud } \\
\text { taly }\end{array}$ & Ash or tuff &.- & - & tule & \\
\hline
\end{tabular}


Appendix 3. Lithologic data. - Continued

[Site IDs and well number correspond to locations in Appendix 1, a site ID and well number is listed for each drilled interval for a particular drill hole. For convenience, the site ID and well numbers are repeated at the shallowest drilled interval for each well. leaders

\begin{tabular}{|c|c|c|c|c|c|c|c|c|c|c|c|c|c|}
\hline Site ID & Well number & $\begin{array}{l}\text { Site ID, } \\
\text { top of } \\
\text { well }\end{array}$ & $\begin{array}{l}\text { Well number, } \\
\text { top of well }\end{array}$ & $\begin{array}{c}\text { Top of } \\
\text { interval, in } \\
\text { feet }\end{array}$ & $\begin{array}{c}\text { Base of } \\
\text { interval, in } \\
\text { feet }\end{array}$ & $\begin{array}{c}\text { Top of } \\
\text { interval, in } \\
m\end{array}$ & $\begin{array}{c}\text { Base of } \\
\text { interval, in } \\
\mathrm{m}\end{array}$ & Reported lithologic unit ${ }^{1}$ & Interpreted lithologic class ${ }^{2}$ & $\begin{array}{c}\text { Reported } \\
\text { degree of } \\
\text { cementation or } \\
\text { induration } \\
\end{array}$ & $\begin{array}{c}\text { Fossils } \\
\text { reported in } \\
\text { interval }^{4}\end{array}$ & $\begin{array}{c}\text { Organic } \\
\text { matter } \\
\text { reported in } \\
\text { interval }^{5}\end{array}$ & $\begin{array}{l}\text { Reported stratigraphic top of geologic } \\
\text { unit }^{6}\end{array}$ \\
\hline 428 & $5 / 4-15 K 1$ & & & 950 & 957 & 289.6 & 291.7 & rock & Ash or tuff & hard & -- & -- & \\
\hline 428 & $5 / 4-15 K 1$ & & & 957 & 980 & 291.7 & 298.7 & rock and clay & Ash or tuff & -- & -- & -- & \\
\hline 428 & 5/4-15K1 & & & 980 & 984 & 298.7 & 299.9 & rock, black & Basalt & hard & -- & -- & \\
\hline 428 & $5 / 4-15 \mathrm{~K} 1$ & & & 984 & 1030 & 299.9 & 313.9 & conglomerate, grayish blue & Basalt & -. & - & -- & \\
\hline 428 & 5/4-15K1 & & & 1030 & 1036 & 313.9 & 315.8 & rock, hard & Basalt & hard & -- & -- & \\
\hline 428 & 5/4-15K1 & & & 1036 & 1045 & 315.8 & 318.5 & sand and gravel & Basalt & -- & -- & -- & \\
\hline 428 & $5 / 4-15 K 1$ & & & 1045 & 1070 & 318.5 & 326.1 & $\begin{array}{l}\text { rock, loose } \\
\text { sandstone, gray and boulders, }\end{array}$ & Basalt & -- & -- & -- & \\
\hline 428 & 5/4-15K1 & & & 1070 & 1090 & 326.1 & 332.2 & black & Basalt & cemented & -- & -- & \\
\hline 428 & 5/4-15K1 & & & 1090 & 1152 & 332.2 & 351.1 & rock, volcanic gray & Basalt & hard & -. & -- & \\
\hline 428 & 5/4-15K1 & & & 1152 & 1168 & 351.1 & 356.0 & rock, black & Basalt & hard & -.- & -- & \\
\hline 429 & $5 / 4-14 \mathrm{P} 1$ & 429 & $5 / 4-14 \mathrm{P} 1$ & 0 & 5 & 0.0 & 1.5 & surface & Sand and clay & -- & -- & -- & Qyal \\
\hline 429 & $5 / 4-14 P 1$ & & & 5 & 40 & 1.5 & 12.2 & sand and gravel water & Sand (or sandstone) and gravel & -- & -- & -- & \\
\hline 429 & 5/4-14P1 & & & 40 & 60 & 12.2 & 18.3 & clay, blue and sand & Clay and sand (or sandstone) & -- & -- & -- & Qoal \\
\hline 429 & 5/4-14P1 & & & 60 & 75 & 18.3 & 22.9 & boulders & Gravel & -- & -- & -- & \\
\hline 429 & $5 / 4-14 \mathrm{P} 1$ & & & 75 & 100 & 22.9 & 30.5 & $\begin{array}{l}\text { clay blue } \\
\text { ches }\end{array}$ & Clay & -- & -- & -- & \\
\hline 429 & $5 / 4-14 \mathrm{P} 1$ & & & 100 & 108 & 30.5 & 32.9 & boulders & Gravel & -- & -- & -- & \\
\hline 429 & 5/4-14P1 & & & 108 & 130 & 32.9 & 39.6 & clay, gray & Clay & -- & -- & -- & \\
\hline 429 & 5/4-14P1 & & & 130 & 160 & 39.6 & 48.8 & clay, blue, with boulders & Clay and gravel & -. & -- & -- & \\
\hline 429 & $5 / 4-14 \mathrm{P} 1$ & & & 160 & 165 & 48.8 & 50.3 & clay, hard, gray & Clay & hard & -- & -- & \\
\hline 429 & $5 / 4-14 \mathrm{P} 1$ & & & 165 & 268 & 50.3 & 81.7 & clay, blue, with boulders & Clay and gravel & -- & -. & -- & \\
\hline 429 & 5/4-14P1 & & & 268 & 274 & 81.7 & 83.5 & boulders & Gravel & -- & -- & -- & \\
\hline 429 & 5/4-14P1 & & & 274 & 290 & 83.5 & 88.4 & clay, blue, with boulders & Clay and gravel & -- & -. & -- & \\
\hline 429 & 5/4-14P1 & & & 290 & 310 & 88.4 & 94.5 & conglomerate, basaltic & Basalt & -- & -. & -.- & \\
\hline 429 & $5 / 4-14 \mathrm{P} 1$ & & & 310 & 365 & 94.5 & 111.3 & clay, blue with boulders & Clay and gravel & -- & -- & -- & \\
\hline 429 & $5 / 4-14 \mathrm{P} 1$ & & & 365 & 385 & 111.3 & 117.3 & boulders & Gravel & -- & -- & -- & \\
\hline 429 & $5 / 4-14 \mathrm{P} 1$ & & & 385 & 500 & 117.3 & 152.4 & lava rock & Basalt & hard & -- & -- & Tsv \\
\hline 429 & $5 / 4-14 \mathrm{P} 1$ & & & 500 & 600 & 152.4 & 182.9 & basalt rock & Basalt & hard & - & - & \\
\hline 430 & $5 / 4-15 \mathrm{P} 2$ & 430 & 5/4-15P2 & 0 & 25 & 0.0 & 7.6 & clay, yellow & Clay & -. & -- & -.- & Qyal and Qoal \\
\hline 430 & $5 / 4-15 \mathrm{P} 2$ & & & 25 & 90 & 7.6 & 27.4 & clay, boulders & Clay and gravel & -- & -- & -.- & \\
\hline 430 & $5 / 4-15 \mathrm{P} 2$ & & & 90 & 105 & 27.4 & 32.0 & gravel, salty water & Gravel & -- & -- & -- & \\
\hline 430 & $5 / 4-15 \mathrm{P} 2$ & & & 105 & 295 & 32.0 & 89.9 & clay, yellow; boulders & Clay and gravel & -- & -- & -- & \\
\hline 430 & $5 / 4-15 \mathrm{P} 2$ & & & 295 & 350 & 89.9 & 106.7 & $\begin{array}{l}\text { tule mud, driftwood } \\
\text { the }\end{array}$ & Clay and sand (or sandstone) & - & -- & tule, wood & \\
\hline 430 & $5 / 4-15 \mathrm{P} 2$ & & & 350 & 370 & 106.7 & 112.8 & clay, blue & Clay & -- & -. & -. & \\
\hline 430 & $5 / 4-15 \mathrm{P} 2$ & & & 370 & 600 & 112.8 & 182.9 & clay and boulders, yellow & Clay and gravel & -- & -- & -.- & \\
\hline 430 & $5 / 4-15 \mathrm{P} 2$ & & & 600 & 750 & 182.9 & 228.6 & rock & Ash or tuff & hard & -- & -- & Tsv \\
\hline 430 & $5 / 4-15 \mathrm{P} 2$ & & & 750 & 860 & 228.6 & 262.1 & clay and boulders, yellow & Clay and gravel & -- & -- & -- & \\
\hline 430 & $5 / 4-15 \mathrm{P} 2$ & & & 860 & 990 & 262.1 & 301.8 & volcanic ash & Ash or tuff & -- & -- & -- & \\
\hline 430 & 5/4-15P2 & & & 990 & 1010 & 301.8 & 307.8 & gravel, cement, some water & Gravel & cemented & -- & .. & \\
\hline 430 & 5/4-15P2 & & & 1010 & 1195 & 307.8 & 364.2 & ash, brown & Ash or tuff & - & -. & .. & \\
\hline 430 & $5 / 4-15 \mathrm{P} 2$ & & & 1195 & 1214 & 364.2 & 370.0 & rock, broken; water & Ash or tuff & hard & -- & -- & \\
\hline 431 & $5 / 4-21 \mathrm{~A} 1$ & 431 & 5/4-21A1 & 0 & 4 & 0.0 & 1.2 & surface & Sand and clay & -- & -- & -.- & Qoal \\
\hline 431 & $5 / 4-21 \mathrm{~A} 1$ & & & 4 & 30 & 1.2 & 9.1 & boulders & Gravel & -- & -- & -.- & \\
\hline 431 & 5/4-21A1 & & & 30 & 57 & 9.1 & 17.4 & clay, red & Clay & -- & -- & -- & Tsv \\
\hline 431 & $5 / 4-21 A 1$ & & & 57 & 75 & 17.4 & 22.9 & clay, red; some sand and gravel & Clay, sand, and gravel & -- & -- & -- & 100 \\
\hline 431 & 5/4-21A1 & & & 75 & 95 & 22.9 & 29.0 & boulders & Gravel & -- & -- & -- & \\
\hline 431 & $5 / 4-21 \mathrm{~A} 1$ & & & 95 & 130 & 29.0 & 39.6 & $\begin{array}{l}\text { clay and sand, gray } \\
\text {. }\end{array}$ & Clay and sand (or sandstone) & -- & -- & -. & \\
\hline 431 & $5 / 4-21 \mathrm{~A} 1$ & & & 130 & 207 & 39.6 & 63.1 & clay, blue some sand & Clay and sand (or sandstone) & -- & -. & .- & \\
\hline 431 & $5 / 4-21 A 1$ & & & 207 & 225 & 63.1 & 68.6 & boulders & Gravel & -- & .. & .. & \\
\hline 431 & $5 / 4-21 A 1$ & & & 225 & 240 & 68.6 & 73.2 & clay, sand; some sand & Clay and sand (or sandstone) & -- & -- & .- & \\
\hline 431 & $5 / 4-21 A 1$ & & & 240 & 300 & 73.2 & 91.4 & clay and boulders, blue & Clay and gravel & -- & -- & -- & \\
\hline 431 & $5 / 4-21 \mathrm{~A} 1$ & & & 300 & 330 & 91.4 & 100.6 & $\begin{array}{l}\text { clay, sandy blue } \\
\text { latic }\end{array}$ & Clay and sand (or sandstone) & -- & -- & -. & \\
\hline 431 & $5 / 4-21 A 1$ & & & 330 & 390 & 100.6 & 118.9 & basalt, shattered & Basalt & -- & -- & -- & \\
\hline 431 & $5 / 4-21 A 1$ & & & 390 & 450 & 118.9 & 137.2 & lava ash, brown and basalt & Basalt & -- & -. & .. & \\
\hline 431 & $5 / 4-21 A 1$ & & & 450 & 545 & 137.2 & 166.1 & basalt & Basalt & -- & -- & -- & \\
\hline 432 & $5 / 4-19 \mathrm{~J} 1$ & 432 & 5/4-19J1 & 0 & 12 & 0.0 & 3.7 & soil & Sand and clay & -- & -- & -. & Qt \\
\hline 432 & $5 / 4-19 j 1$ & & & 12 & 100 & 3.7 & 30.5 & Markley sandstone, water & Sandstone & cemented & -- & -- & Markley sandstone \\
\hline 433 & 5/4-19j2 & 433 & 5/4-19J2 & 0 & 18 & 0.0 & 5.5 & clay, blue & Clay & -- & -- & -- & Qt \\
\hline 433 & $5 / 4-1932$ & & & 18 & 59 & 5.5 & 18.0 & Markley sandstone, blue & Sandstone & cemented & -. & .. & Markley sandstone \\
\hline 433 & $5 / 4-1932$ & & & 59 & 63 & 18.0 & 19.2 & rock, broken; water & Sandstone & hard & -- & -- & \\
\hline 433 & $5 / 4-19 \mathrm{~J} 2$ & & & 63 & 90 & 19.2 & 27.4 & sandstone, blue & Sandstone & cemented & -- & -- & \\
\hline
\end{tabular}


Appendix 3. Lithologic data. - Continued

ISite IDs and well number correspond to locations in Appendix 1, a site ID and well number is listed for each drilled interval for a particular drill hole. For convenience, the site ID and well numbers are repeated at the shallowest drilled interval for each well. leaders

\begin{tabular}{|c|c|c|c|c|c|c|c|c|c|c|c|c|c|}
\hline Site ID & Well number & $\begin{array}{l}\text { Site ID, } \\
\text { top of } \\
\text { well }\end{array}$ & $\begin{array}{l}\text { Well number, } \\
\text { top of well }\end{array}$ & $\begin{array}{c}\text { Top of } \\
\text { interval, in } \\
\text { feet }\end{array}$ & $\begin{array}{l}\text { Base of } \\
\text { interval, in } \\
\text { feet }\end{array}$ & $\begin{array}{c}\text { Top of } \\
\text { interval, in } \\
\mathrm{m}\end{array}$ & $\begin{array}{c}\text { Base of } \\
\text { interval, in } \\
\mathrm{m}\end{array}$ & Reported lithologic unit' & Interpreted lithologic class ${ }^{2}$ & $\begin{array}{c}\text { Reported } \\
\text { degree of } \\
\text { cementation or } \\
\text { induration }^{3} \\
\end{array}$ & $\begin{array}{c}\text { Fossils } \\
\text { reported in } \\
\text { interval }^{4}\end{array}$ & $\begin{array}{c}\text { Organic } \\
\text { matter } \\
\text { reported in } \\
\text { interval }^{5}\end{array}$ & $\begin{array}{l}\text { Reported stratigraphic top of geologic } \\
\text { unit }^{6}\end{array}$ \\
\hline 433 & $5 / 4-19 \mathrm{~J} 2$ & & & 90 & 93 & 27.4 & 28.3 & sandstone, broken; water & Sandstone & cemented & -- & -- & \\
\hline 433 & $5 / 4-19 \mathrm{~J} 2$ & & & 93 & 108 & 28.3 & 32.9 & sandstone & Sandstone & cemented & -- & -- & \\
\hline 434 & $5 / 4-21 P 2$ & 434 & 5/4-21P2 & 0 & 235 & 0.0 & 71.6 & shale, black & Clay and sand (or sandstone) & cemented & -- & -- & Qt \\
\hline 435 & $5 / 4-19 \mathrm{R} 1$ & 435 & $5 / 4-19 \mathrm{R} 1$ & 0 & 18 & 0.0 & 5.5 & $\begin{array}{l}\text { soil } \\
\text { Monterey shale, black, thin- }\end{array}$ & Sand and clay & -- & -- & -- & Qt \\
\hline 435 & $5 / 4-19 R 1$ & & & 18 & 135 & 5.5 & 41.1 & bedded & Clay and sand (or sandstone) & cemented & -- & -- & Monterey shale (?) \\
\hline 436 & $5 / 4-19 Q 1$ & 436 & 5/4-19Q1 & 0 & 45 & 0.0 & 13.7 & silt black & Clay and sand (or sandstone) & -- & -- & -- & Qyal \\
\hline 436 & 5/4-19Q1 & & & 45 & 175 & 13.7 & 53.3 & clay, yellow & Clay & -- & -- & -- & Qh \\
\hline 437 & $5 / 4-27 A 1$ & 437 & 5/4-27A1 & 0 & 10 & 0.0 & 3.0 & soil and gravel & Sand (or sandstone) and gravel & -- & -- & -- & Qoal \\
\hline 437 & $5 / 4-27 \mathrm{~A} 1$ & & & 10 & 12 & 3.0 & 3.7 & $\begin{array}{l}\text { gravel in yellow clay } \\
\text { soft sandstone, yellow, changing }\end{array}$ & Clay and gravel & -- & -- & -- & \\
\hline 437 & $5 / 4-27 A 1$ & & & 12 & 30 & 3.7 & 9.1 & to sandy yellow clay & Sandstone & cemented & -- & -- & \\
\hline 437 & 5/4-27A1 & & & 30 & 53 & 9.1 & 16.2 & stiff clay, yellow with gravel & Clay and gravel & -- & -- & -- & \\
\hline 437 & $5 / 4-27 A 1$ & & & 53 & 59 & 16.2 & 18.0 & sandy clay, blue with gravel & Clay, sand, and gravel & -- & -- & -- & \\
\hline 437 & 5/4-27A1 & & & 59 & 87 & 18.0 & 26.5 & dark stiff clay & Clay & -- & -- & -- & \\
\hline 437 & $5 / 4-27 \mathrm{~A} 1$ & & & 87 & 95 & 26.5 & 29.0 & blue sand with clay & Sand and clay & -- & -- & -- & \\
\hline 437 & $5 / 4-27 \mathrm{~A} 1$ & & & 95 & 102 & 29.0 & 31.1 & gravel, blue, water-worn & Gravel & -- & -- & -- & \\
\hline 437 & 5/4-27A1 & & & 102 & 130 & 31.1 & 39.6 & sand, blue, with clay & Sand and clay & -- & -- & -- & \\
\hline 437 & $5 / 4-27 \mathrm{~A} 1$ & & & 130 & 188 & 39.6 & 57.3 & volcanic tuff & Ash or tuff & -- & -- & -- & Tsv \\
\hline 437 & 5/4-27A1 & & & 188 & 194 & 57.3 & 59.1 & $\begin{array}{l}\text { very hard tuff or shale } \\
\text { blue gray clay with sand and }\end{array}$ & Clay and sand (or sandstone) & hard & -- & -- & rov \\
\hline 437 & $5 / 4-27 \mathrm{~A} 1$ & & & 194 & 204 & 59.1 & 62.2 & sharp gravel & Clay, sand, and gravel & -- & -- & -- & \\
\hline 437 & 5/4-27A1 & & & 204 & 242 & 62.2 & 73.8 & dark clay & Clay & -- & -- & -- & \\
\hline 437 & 5/4-27A1 & & & 242 & 252 & 73.8 & 76.8 & brown sandy clay & Clay and sand (or sandstone) & -- & -- & -- & \\
\hline 437 & $5 / 4-27 A 1$ & & & 252 & 255 & 76.8 & 77.7 & blue clay & Clay & -- & -- & -- & \\
\hline 437 & $5 / 4-27 \mathrm{~A} 1$ & & & 255 & 323 & 77.7 & 98.5 & brown clay & Clay & -- & -- & -- & \\
\hline 437 & 5/4-27A1 & & & 323 & 332 & 98.5 & 101.2 & gravel and sand & Sand (or sandstone) and gravel & -- & -- & -- & \\
\hline 437 & $5 / 4-27 \mathrm{~A} 1$ & & & 332 & 335 & 101.2 & 102.1 & $\begin{array}{l}\text { volcanic tuff } \\
\text { gravel, blue' sand and clay with } \\
\text { light green lava. Water seeping }\end{array}$ & Ash or tuff & -- & -- & -- & \\
\hline 437 & $5 / 4-27 \mathrm{~A} 1$ & & & 335 & 435 & 102.1 & 132.6 & over top & Clay, sand, and gravel & -- & -- & -- & \\
\hline 437 & $5 / 4-27 \mathrm{~A} 1$ & & & 435 & 442 & 132.6 & 134.7 & $\begin{array}{l}\text { clay, blue and sand } \\
\text { mud, brown: and sand Flow of }\end{array}$ & Clay and sand (or sandstone) & -- & -- & -- & \\
\hline 437 & $5 / 4-27 \mathrm{~A} 1$ & & & 442 & 475 & 134.7 & 144.8 & $\begin{array}{l}\text { water } 5 \mathrm{gpm} \\
\text { sand and gravel. Flow of water }\end{array}$ & Clay and sand (or sandstone) & -- & -- & -- & \\
\hline 437 & 5/4-27A1 & & & 475 & 492 & 144.8 & 150.0 & $12 \mathrm{gpm}$ & Sand (or sandstone) and gravel & -- & -- & -- & \\
\hline 437 & 5/4-27A1 & & & 492 & 504 & 150.0 & 153.6 & volcanic tuff & Ash or tuff & -- & -- & -- & \\
\hline 437 & $5 / 4-27 \mathrm{~A} 1$ & & & 504 & 522 & 153.6 & 159.1 & brown ash and cinders & Ash or tuff & -- & -- & -- & \\
\hline 437 & $5 / 4-27 \mathrm{~A} 1$ & & & 522 & 532 & 159.1 & 162.2 & $\begin{array}{l}\text { porous dark cinders } \\
\text { cinders, brick red. Flow of water }\end{array}$ & Basalt & -- & -- & -- & \\
\hline 437 & $5 / 4-27 \mathrm{~A} 1$ & & & 532 & 542 & 162.2 & 165.2 & $50 \mathrm{gpm}$ & Basalt & -- & -- & -- & \\
\hline 437 & 5/4-27A1 & & & 542 & 570 & 165.2 & 173.7 & tuff and volcanic cinders & Ash or tuff & -- & -- & -- & \\
\hline 437 & $5 / 4-27 \mathrm{~A} 1$ & & & 570 & 630 & 173.7 & 192.0 & hard lava & Basalt & hard & -- & -- & \\
\hline 437 & $5 / 4-27 A 1$ & & & 630 & 772 & 192.0 & 235.3 & tuff and lava rock & Ash or tuff & -- & -- & -- & \\
\hline 437 & $5 / 4-27 \mathrm{~A} 1$ & & & 772 & 795 & 235.3 & 242.3 & hard lava & Basalt & hard & -- & -- & \\
\hline 438 & 5/4-30B2 & 438 & 5/4-30B2 & 0 & 42 & 0.0 & 12.8 & silt, black & Clay and sand (or sandstone) & -- & -- & -- & Qyal \\
\hline 438 & 5/4-30B2 & & & 42 & 196 & 12.8 & 59.7 & clay, yellow & Clay & -- & -- & -- & Qh \\
\hline 438 & $5 / 4-30 \mathrm{~B} 2$ & & & 196 & 530 & 59.7 & 161.5 & rock, grayish-yellow & Ash or tuff & hard & -- & -- & Tsv (?) \\
\hline 438 & $5 / 4-30 B 2$ & & & 530 & 570 & 161.5 & 173.7 & Neroly sandstone, flow of water & Sandstone & cemented & -- & -- & Neroly sandstone \\
\hline 439 & $5 / 4-26 B 1$ & 439 & $5 / 4-26 B 1$ & 0 & 380 & 0.0 & 115.8 & no record & No data & -- & -- & -- & -- \\
\hline & 5/4-26B1 & & & 380 & 380 & 115.8 & 115.8 & $\begin{array}{l}\text { basalt, gray, slightly vesicular and } \\
\text { amygdaloidal, finely porphyritic }\end{array}$ & Basalt & $\ldots$ & $\ldots$ & $\ldots$ & Tsv \\
\hline $\begin{array}{l}439 \\
439\end{array}$ & $\begin{array}{l}5 / 4-26 \mathrm{~B} 1 \\
5 / 4-26 \mathrm{~B} 1\end{array}$ & & & $\begin{array}{l}880 \\
380\end{array}$ & $\begin{array}{l}380 \\
385\end{array}$ & $\begin{array}{l}115.8 \\
115.8\end{array}$ & $\begin{array}{l}115.8 \\
117.3\end{array}$ & $\begin{array}{l}\text { amygaatoliaal, rine-gy porphyyticic } \\
\text { basalt, gray; fine-grained }\end{array}$ & $\begin{array}{l}\text { Basalt } \\
\text { Basalt }\end{array}$ & -- & $\begin{array}{l}-- \\
--\end{array}$ & $\begin{array}{l}-- \\
--\end{array}$ & $15 \mathrm{~V}$ \\
\hline 439 & $5 / 4-26 \mathrm{~B} 1$ & & & 385 & 390 & 117.3 & 118.9 & basalt, gray, some red & Basalt & -- & -- & -- & \\
\hline 439 & $5 / 4-26 B 1$ & & & 390 & 393 & 118.9 & 119.8 & basalt, slightly oxidized, vesicular & Basalt & .- & -- & -- & \\
\hline 439 & $5 / 4-26 \mathrm{~B} 1$ & & & 393 & 395 & 119.8 & 120.4 & $\begin{array}{l}\text { basalt, gray, vesicular } \\
\text { basalt, red, vesicular; some gray }\end{array}$ & Basalt & -- & -- & -- & \\
\hline 439 & $5 / 4-26 B 1$ & & & 395 & 405 & 120.4 & 123.4 & vesicular basalt & Basalt & -- & -- & -- & \\
\hline
\end{tabular}


Appendix 3. Lithologic data. - Continued

[Site IDs and well number correspond to locations in Appendix 1, a site ID and well number is listed for each drilled interval for a particular drill hole. For convenience, the site ID and well numbers are repeated at the shallowest drilled interval for each well. leaders (--), not reported]

\begin{tabular}{|c|c|c|c|c|c|c|c|c|c|c|c|c|c|}
\hline Site ID & Well number & $\begin{array}{l}\text { Site ID, } \\
\text { top of } \\
\text { well }\end{array}$ & $\begin{array}{l}\text { Well number, } \\
\text { top of well }\end{array}$ & $\begin{array}{l}\text { Top of } \\
\text { interval, in } \\
\text { feet }\end{array}$ & $\begin{array}{l}\text { Base of } \\
\text { interval, in } \\
\text { feet }\end{array}$ & $\begin{array}{c}\text { Top of } \\
\text { interval, in } \\
\mathrm{m}\end{array}$ & $\begin{array}{c}\text { Base of } \\
\text { interval, in } \\
\mathrm{m}\end{array}$ & Reported lithologic unit' & Interpreted lithologic class ${ }^{2}$ & $\begin{array}{c}\text { Reported } \\
\text { degree of } \\
\text { cementation or } \\
\text { induration }^{3} \\
\end{array}$ & $\begin{array}{c}\text { Fossils } \\
\text { reported in } \\
\text { interval }^{4}\end{array}$ & $\begin{array}{c}\text { Organic } \\
\text { matter } \\
\text { reported in } \\
\text { interval }^{5} \\
\end{array}$ & $\begin{array}{l}\text { Reported stratigraphic top of geologic } \\
\text { unit }^{6}\end{array}$ \\
\hline 439 & $5 / 4-26 B 1$ & & & 405 & 420 & 123.4 & 128.0 & $\begin{array}{l}\text { basalt, mixed red and gray } \\
\text { vesicular }\end{array}$ & Basalt & 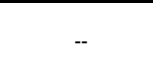 & -- & -- & \\
\hline 439 & $5 / 4-26 \mathrm{~B} 1$ & & & 420 & 435 & 128.0 & 132.6 & $\begin{array}{l}\text { basalt, light gray, fine-grained } \\
\text { fragments of vesicular basalt; }\end{array}$ & Basalt & -- & -- & -- & \\
\hline 439 & $5 / 4-26 B 1$ & & & 435 & 438 & 132.6 & 133.5 & some oxidized & Basalt & -- & -- & -- & \\
\hline 439 & $5 / 4-26 B 1$ & & & 438 & 485 & 133.5 & 147.8 & basalt, gray, fine-grained & Basalt & -- & -- & -- & \\
\hline 439 & $5 / 4-26 B 1$ & & & 485 & 492 & 147.8 & 150.0 & basalt, slightly oxidized, vesicular & Basalt & -- & -- & -- & \\
\hline 439 & $5 / 4-26 \mathrm{~B} 1$ & & & 492 & 495 & 150.0 & 150.9 & $\begin{array}{l}\text { basalt, red, vesicular } \\
\text { basalt, reddish-brown, vesicular }\end{array}$ & Basalt & -- & -- & -- & \\
\hline 439 & $5 / 4-26 B 1$ & & & 495 & 505 & 150.9 & 153.9 & $\begin{array}{l}\text { and amygdaloidal; some pyrite } \\
\text { basalt, dark gray, vesicular; }\end{array}$ & Basalt & -- & -- & -- & \\
\hline 439 & $5 / 4-26 \mathrm{~B} 1$ & & & 505 & 515 & 153.9 & 157.0 & $\begin{array}{l}\text { slightly amygdaloidal } \\
\text { basalt, dark gray, fine-grained, }\end{array}$ & Basalt & -- & -- & -- & \\
\hline 439 & $5 / 4-26 B 1$ & & & 515 & 525 & 157.0 & 160.0 & $\begin{array}{l}\text { fairly porphyritic } \\
\text { basalt, mixed oxidized and } \\
\text { unoxidized, vesicular and }\end{array}$ & Basalt & -- & -- & -- & \\
\hline 439 & $5 / 4-26 \mathrm{~B} 1$ & & & 525 & 530 & 160.0 & 161.5 & $\begin{array}{l}\text { amygdoloidal } \\
\text { basalt, slightly vesicular and }\end{array}$ & Basalt & -- & -- & -- & \\
\hline 439 & $5 / 4-26 B 1$ & & & 530 & 540 & 161.5 & 164.6 & $\begin{array}{l}\text { amygdaloidal } \\
\text { basalt, dark gray, slightly vesicular }\end{array}$ & Basalt & -- & -- & -- & \\
\hline 439 & $5 / 4-26 B 1$ & & & 540 & 555 & 164.6 & 169.2 & $\begin{array}{l}\text { and amygdaloidal } \\
\text { finely porphyritic with glassy } \\
\text { texture. Basalt, dark gray, some }\end{array}$ & Basalt & -- & -- & -- & \\
\hline 439 & $5 / 4-26 B 1$ & & & 555 & 565 & 169.2 & 172.2 & $\begin{array}{l}\text { red which might be cave } \\
\text { basalt, dark gray, slightly }\end{array}$ & Basalt & -- & -- & -- & \\
\hline 439 & $5 / 4-26 B 1$ & & & 565 & 570 & 172.2 & 173.7 & $\begin{array}{l}\text { porphyritic and amygdaloidal } \\
\text { basalt, mixed gray and brown }\end{array}$ & Basalt & -- & -- & -- & \\
\hline 439 & $5 / 4-26 B 1$ & & & 570 & 580 & 173.7 & 176.8 & $\begin{array}{l}\text { vesicular basalt } \\
\text { basalt, mixed gray and reddish- }\end{array}$ & Basalt & -- & -- & -- & \\
\hline 439 & $5 / 4-26 B 1$ & & & 580 & 585 & 176.8 & 178.3 & $\begin{array}{l}\text { brown, vesicular } \\
\text { basalt, mixed gray and brown }\end{array}$ & Basalt & -- & -- & -- & \\
\hline 439 & $5 / 4-26 B 1$ & & & 585 & 590 & 178.3 & 179.8 & $\begin{array}{l}\text { vesicular } \\
\text { basalt, dark gray, slightly vesicular }\end{array}$ & Basalt & -- & -- & -- & \\
\hline 439 & $5 / 4-26 \mathrm{~B} 1$ & & & 590 & 595 & 179.8 & 181.4 & and amygdaloidal & Basalt & -- & -- & -- & \\
\hline 439 & $5 / 4-26 B 1$ & & & 595 & 597 & 181.4 & 182.0 & $\begin{array}{l}\text { basalt, gray, slightly vesicular and } \\
\text { amygdaloidal, finely porphyritic }\end{array}$ & Basalt & -- & -- & -- & \\
\hline 439 & $5 / 4-26 \mathrm{~B} 1$ & & & 597 & 600 & 182.0 & 182.9 & $\begin{array}{l}\text { basaltic agglomerate vesicular } \\
\text { basalt, slightly oxidized, vesicular; }\end{array}$ & Basalt & -- & -- & -- & \\
\hline 439 & $5 / 4-26 \mathrm{~B} 1$ & & & 600 & 605 & 182.9 & 184.4 & $\begin{array}{l}\text { some pyrite } \\
\text { basalt, gray, slightly amygdoloidal }\end{array}$ & Basalt & -- & -- & -- & \\
\hline 439 & $5 / 4-26 B 1$ & & & 605 & 615 & 184.4 & 187.5 & and vesicular & Basalt & -- & -- & -- & \\
\hline 439 & $5 / 4-26 B 1$ & & & 615 & 620 & 187.5 & 189.0 & $\begin{array}{l}\text { basalt, fine-grained gray } \\
\text { basalt, gray, vesicular; some }\end{array}$ & Basalt & -- & -- & -- & \\
\hline 439 & $5 / 4-26 B 1$ & & & 620 & 625 & 189.0 & 190.5 & $\begin{array}{l}\text { pumice } \\
\text { tuff, consists of fragments of red } \\
\text { and gray basalt, pumice, obsidian, }\end{array}$ & Basalt & -- & -- & -- & \\
\hline 439 & $5 / 4-26 \mathrm{~B} 1$ & & & 625 & 728 & 190.5 & 221.9 & $\begin{array}{l}\text { and pyrite } \\
\text { basalt, red, sample contains cave }\end{array}$ & Ash or tuff & -- & -- & -- & \\
\hline 439 & $5 / 4-26 B 1$ & & & 728 & 735 & 221.9 & 224.0 & of tuff, vesicular & Basalt & -- & -- & -- & \\
\hline 439 & $5 / 4-26 \mathrm{~B} 1$ & & & 735 & 740 & 224.0 & 225.6 & $\begin{array}{l}\text { vesicular basalt, reddish-brown } \\
\text { vesicular basalt, mixed brown and }\end{array}$ & Basalt & -- & -- & -- & \\
\hline 439 & $5 / 4-26 B 1$ & & & 740 & 750 & 225.6 & 228.6 & gray & Basalt & -- & -- & -- & \\
\hline 439 & $5 / 4-26 \mathrm{~B} 1$ & & & 750 & 755 & 228.6 & 230.1 & $\begin{array}{l}\text { basalt, gray, fine-grained, sample } \\
\text { contains large percentage of cave } \\
\text { basalt, gray, fine-grained, slightly } \\
\text { glassy texture, slightly porphyritic } \\
\text { some cave of red vesicular basalt } \\
\end{array}$ & Basalt & -- & -- & -- & \\
\hline
\end{tabular}


Appendix 3. Lithologic data. - Continued

ISite IDs and well number correspond to locations in Appendix 1, a site ID and well number is listed for each drilled interval for a particular drill hole. For convenience, the site ID and well numbers are repeated at the shallowest drilled interval for each well. leaders (--), not reported]

\begin{tabular}{|c|c|c|c|c|c|c|c|c|c|c|c|c|c|}
\hline Site ID & Well number & $\begin{array}{l}\text { Site ID, } \\
\text { top of } \\
\text { well }\end{array}$ & $\begin{array}{l}\text { Well number, } \\
\text { top of well }\end{array}$ & $\begin{array}{l}\text { Top of } \\
\text { interval, in } \\
\text { feet }\end{array}$ & $\begin{array}{l}\text { Base of } \\
\text { interval, in } \\
\text { feet }\end{array}$ & $\begin{array}{l}\text { Top of } \\
\text { interval, in } \\
\mathrm{m}\end{array}$ & $\begin{array}{l}\text { Base of } \\
\text { interval, in } \\
\mathrm{m}\end{array}$ & Reported lithologic unit' & Interpreted lithologic class ${ }^{2}$ & $\begin{array}{c}\text { Reported } \\
\text { degree of } \\
\text { cementation or } \\
\text { induration } \\
\end{array}$ & $\begin{array}{l}\text { Fossils } \\
\text { reported in } \\
\text { interval }^{4} \\
\end{array}$ & $\begin{array}{c}\text { Organic } \\
\text { matter } \\
\text { reported in } \\
\text { interval }^{5}\end{array}$ & $\begin{array}{l}\text { Reported stratigraphic top of geologic } \\
\text { unit }^{6}\end{array}$ \\
\hline 439 & $5 / 4-26 \mathrm{~B} 1$ & & & 760 & 765 & 231.6 & 233.2 & $\begin{array}{l}\text { basalt, gray, fine-grained samples } \\
\text { contains considerable cave } \\
\text { basalt, gray, fine-grained slightly } \\
\text { glassy sample contains much }\end{array}$ & Basalt & -- & -- & -- & \\
\hline 439 & $5 / 4-26 \mathrm{~B} 1$ & & & 765 & 770 & 233.2 & 234.7 & cave & Basalt & -- & -- & -- & \\
\hline 439 & $5 / 4-26 \mathrm{~B} 1$ & & & 770 & 799 & 234.7 & 243.5 & $\begin{array}{l}\text { basalt, gray, fine-grained } \\
\text { agglomerate mixture of red and } \\
\text { black vesicular basalt and a little }\end{array}$ & Basalt & -- & -- & -- & \\
\hline 439 & $5 / 4-26 \mathrm{~B} 1$ & & & 799 & 805 & 243.5 & 245.4 & pumice & Basalt & -- & -- & -- & \\
\hline 439 & $5 / 4-26 \mathrm{~B} 1$ & & & 805 & 821 & 245.4 & 250.2 & agglomerate & Clay, sand, and gravel & -- & -- & -- & \\
\hline 439 & $5 / 4-26 \mathrm{~B} 1$ & & & 821 & 830 & 250.2 & 253.0 & $\begin{array}{l}\text { basalt, gray, fine-grained } \\
\text { basalt, dark gray, slightly vesicular }\end{array}$ & Basalt & -- & -- & -- & \\
\hline 439 & $5 / 4-26 \mathrm{~B} 1$ & & & 830 & 835 & 253.0 & 254.5 & $\begin{array}{l}\text { and amygdaloidal } \\
\text { basalt, vesicular and } \\
\text { amygdaloidal, some oxidized }\end{array}$ & Basalt & -- & -- & -- & \\
\hline 439 & $5 / 4-26 \mathrm{~B} 1$ & & & 835 & 850 & 254.5 & 259.1 & basalt in sample & Basalt & -- & -- & -- & \\
\hline 439 & $5 / 4-26 B 1$ & & & 850 & 855 & 259.1 & 260.6 & $\begin{array}{l}\text { basalt, slightly oxidized } \\
\text { agglomerate, basaltic, red and }\end{array}$ & Basalt & -- & -- & -- & \\
\hline 439 & $5 / 4-26 \mathrm{~B} 1$ & & & 855 & 860 & 260.6 & 262.1 & gray, vesicular & Basalt & -- & -- & -- & \\
\hline 439 & $5 / 4-26 \mathrm{~B} 1$ & & & 860 & 905 & 262.1 & 275.8 & agglomerate & Clay, sand, and gravel & -- & -- & -- & \\
\hline 439 & $5 / 4-26 \mathrm{~B} 1$ & & & 905 & 910 & 275.8 & 277.4 & agglomerate, slightly tuffaceous & Ash or tuff & -- & -- & -- & \\
\hline 439 & $5 / 4-26 \mathrm{~B} 1$ & & & 910 & 953 & 277.4 & 290.5 & agglomerate & Clay, sand, and gravel & -- & -- & -- & \\
\hline 439 & $5 / 4-26 \mathrm{~B} 1$ & & & 953 & 965 & 290.5 & 294.1 & $\begin{array}{l}\text { agglomerate, soft grayish brown } \\
\text { agglomerate, basaltic, chiefly } \\
\text { black, some red vesicular and }\end{array}$ & Ash or tuff & -- & -- & -- & \\
\hline 439 & $5 / 4-26 \mathrm{~B} 1$ & & & 965 & 980 & 294.1 & 298.7 & $\begin{array}{l}\text { amygdaloidal } \\
\text { agglomerate, basaltic, chiefly red }\end{array}$ & Basalt & -- & -- & -- & \\
\hline 439 & $5 / 4-26 \mathrm{~B} 1$ & & & 980 & 1015 & 298.7 & 309.4 & $\begin{array}{l}\text { vesicular basalt } \\
\text { agglomerate, basaltic, chiefly } \\
\text { brownish-gray vesicular and }\end{array}$ & Basalt & -- & -- & -- & \\
\hline 439 & $5 / 4-26 \mathrm{~B} 1$ & & & 1015 & 1025 & 309.4 & 312.4 & $\begin{array}{l}\text { amygdaloidal basalt } \\
\text { agglomerate, basaltic, chiefly }\end{array}$ & Basalt & -- & -- & -- & \\
\hline 439 & $5 / 4-26 \mathrm{~B} 1$ & & & 1025 & 1065 & 312.4 & 324.6 & $\begin{array}{l}\text { brownish-red vesicular basalt } \\
\text { basaltic, gray porphyritic, slightly }\end{array}$ & Basalt & -- & -- & -- & \\
\hline 439 & $5 / 4-26 \mathrm{~B} 1$ & & & 1065 & 1090 & 324.6 & 332.2 & $\begin{array}{l}\text { vesicular } \\
\text { tuffaceous agglomerate, chiefly }\end{array}$ & Basalt & -- & -- & -- & \\
\hline 439 & $5 / 4-26 \mathrm{~B} 1$ & & & 1090 & 1140 & 332.2 & 347.5 & $\begin{array}{l}\text { basalt, some pumice } \\
\text { agglomerate, basaltic, mixed } \\
\text { oxidized and unoxidized basalt, }\end{array}$ & Ash or tuff & -- & -- & -- & \\
\hline 439 & $5 / 4-26 \mathrm{~B} 1$ & & & 1140 & 1182 & 347.5 & 360.3 & $\begin{array}{l}\text { some pumice } \\
\text { agglomerate, basaltic red }\end{array}$ & Basalt & -- & -- & -- & \\
\hline 439 & $5 / 4-26 \mathrm{~B} 1$ & & & 1182 & 1187 & 360.3 & 361.8 & $\begin{array}{l}\text { vesicular } \\
\text { agglomerate, basaltic, mixed } \\
\text { oxidized and unoxidized basalt, }\end{array}$ & Basalt & -- & -- & -- & \\
\hline 439 & $5 / 4-26 \mathrm{~B} 1$ & & & 1187 & 1236 & 361.8 & 376.7 & some pumice & Basalt & -- & -- & -- & \\
\hline 439 & $5 / 4-26 \mathrm{~B} 1$ & & & 1236 & 1335 & 376.7 & 406.9 & tuff, oxidized & Ash or tuff & -- & -- & -- & \\
\hline 439 & $5 / 4-26 \mathrm{~B} 1$ & & & 1335 & 1390 & 406.9 & 423.7 & tuffaceous agglomerate, basaltic & Ash or tuff & -- & -- & -- & \\
\hline 439 & $5 / 4-26 \mathrm{~B} 1$ & & & 1390 & 1440 & 423.7 & 438.9 & tuff, top of tuff oxidized & Ash or tuff & -- & -- & -- & \\
\hline 440 & $5 / 4-26 E 1$ & 440 & 5/4-26E1 & 0 & 4 & 0.0 & 1.2 & soil & Sand and clay & -- & -- & -- & Qoal \\
\hline 440 & 5/4-26E1 & & & 4 & 10 & 1.2 & 3.0 & gravel, red & Gravel & -- & -- & -- & \\
\hline 440 & $5 / 4-26 E 1$ & & & 10 & 18 & 3.0 & 5.5 & $\begin{array}{l}\text { gravel, hard mixed } \\
\text { sandy yellow clay and gravel. }\end{array}$ & Gravel & hard & -- & -- & \\
\hline 440 & 5/4-26E1 & & & 18 & 62 & 5.5 & 18.9 & Fresh water (5 pts.) at $49^{\prime}$ & Clay, sand, and gravel & -- & -- & -- & \\
\hline 440 & 5/4-26E1 & & & 62 & 125 & 18.9 & 38.1 & $\begin{array}{l}\text { clay, blue, and gravel } \\
\text { agglomerate, volcanic with blue- } \\
\text { black clay. piece of redwood at }\end{array}$ & Clay and gravel & -- & -- & -- & \\
\hline 440 & 5/4-26E1 & & & 125 & 260 & 38.1 & 79.2 & $\begin{array}{l}250 \mathrm{ft} . \\
\text { tuff, contains pumice, sticky like }\end{array}$ & Ash or tuff & -- & -- & wood & Tsv \\
\hline 440 & 5/4-26E1 & & & 260 & 315 & 79.2 & 96.0 & clay & Ash or tuff & -- & -- & -- & \\
\hline
\end{tabular}


Appendix 3. Lithologic data. - Continued

ISite IDs and well number correspond to locations in Appendix 1, a site ID and well number is listed for each drilled interval for a particular drill hole. For convenience, the site ID and well numbers are repeated at the shallowest drilled interval for each well. leaders (--), not reported]

\begin{tabular}{|c|c|c|c|c|c|c|c|c|c|c|c|c|c|}
\hline Site ID & Well number & $\begin{array}{l}\text { Site ID, } \\
\text { top of } \\
\text { well }\end{array}$ & $\begin{array}{l}\text { Well number, } \\
\text { top of well }\end{array}$ & $\begin{array}{l}\text { Top of } \\
\text { interval, in } \\
\text { feet }\end{array}$ & $\begin{array}{l}\text { Base of } \\
\text { interval, in } \\
\text { feet }\end{array}$ & $\begin{array}{c}\text { Top of } \\
\text { interval, in } \\
\text { m }\end{array}$ & $\begin{array}{c}\text { Base of } \\
\text { interval, in } \\
\mathrm{m}\end{array}$ & Reported lithologic unit' & Interpreted lithologic class ${ }^{2}$ & $\begin{array}{c}\text { Reported } \\
\text { degree of } \\
\text { cementation or } \\
\text { induration }^{3} \\
\end{array}$ & $\begin{array}{l}\text { Fossils } \\
\text { reported in } \\
\text { interval }^{4}\end{array}$ & $\begin{array}{l}\text { Organic } \\
\text { matter } \\
\text { reported in } \\
\text { interval }^{5}\end{array}$ & $\begin{array}{c}\text { Reported stratigraphic top of geologic } \\
\text { unit }^{6}\end{array}$ \\
\hline & & & & & & & & $\begin{array}{l}\text { agglomerate, red to black, } \\
\text { basaltic, red at } 365 \mathrm{ft} \text {, vesicular }\end{array}$ & & & & & \\
\hline 440 & 5/4-26E1 & & & 315 & 375 & 96.0 & 114.3 & to nonvesicular, no pumice & Basalt & -- & -- & -- & \\
\hline 440 & 5/4-26E1 & & & 375 & 437 & 114.3 & 133.2 & $\begin{array}{l}\text { basalt, black, hard } \\
\text { agglomerate, red, basaltic, }\end{array}$ & Basalt & hard & -- & -- & \\
\hline 440 & $5 / 4-26 \mathrm{E} 1$ & & & 437 & 454 & 133.2 & 138.4 & $\begin{array}{l}\text { vesicular; some black } \\
\text { basalt, gray, fine-grained, shows }\end{array}$ & Basalt & -- & -- & -- & \\
\hline 440 & 5/4-26E1 & & & 454 & 465 & 138.4 & 141.7 & $\begin{array}{l}\text { some oxidization } \\
\text { agglomerate, red to brown, }\end{array}$ & Basalt & -- & -- & -- & \\
\hline 440 & 5/4-26E1 & & & 465 & 475 & 141.7 & 144.8 & $\begin{array}{l}\text { vesicular } \\
\text { basalt, gray, fine-grained, }\end{array}$ & Ash or tuff & -- & -- & -- & \\
\hline 440 & $5 / 4-26 \mathrm{E} 1$ & & & 475 & 480 & 144.8 & 146.3 & $\begin{array}{l}\text { vesicular to nonvesicular } \\
\text { agglomerate, gray, basaltic; }\end{array}$ & Basalt & -- & -- & -- & \\
\hline 440 & $5 / 4-26 \mathrm{E} 1$ & & & 480 & 495 & 146.3 & 150.9 & $\begin{array}{l}\text { pumice present } \\
\text { tuff, coarse-grained, pumice, }\end{array}$ & Basalt & -- & -- & -- & \\
\hline 440 & $5 / 4-26 \mathrm{E} 1$ & & & 495 & 502 & 150.9 & 153.0 & $\begin{array}{l}\text { some pyrite } \\
\text { agglomerate, grayish green, } \\
\text { vesicular; some pumice and }\end{array}$ & Ash or tuff & -- & -- & -- & \\
\hline 440 & $5 / 4-26 \mathrm{E} 1$ & & & 502 & 560 & 153.0 & 170.7 & $\begin{array}{l}\text { obsidian } \\
\text { red vesicular agglomerate, some }\end{array}$ & Ash or tuff & -- & -- & -- & \\
\hline 440 & $5 / 4-26 \mathrm{E} 1$ & & & 560 & 570 & 170.7 & 173.7 & $\begin{array}{l}\text { unoxidized basalt } \\
\text { basalt, light gray, some red } \\
\text { vesicular basalt near bottom of }\end{array}$ & Basalt & -- & -- & -- & \\
\hline 440 & $5 / 4-26 \mathrm{E} 1$ & & & 570 & 620 & 173.7 & 189.0 & $\begin{array}{l}\text { strata } \\
\text { agglomerate, basaltic, consists of } \\
\text { red and gray vesicular and }\end{array}$ & Basalt & -- & -- & -- & \\
\hline 440 & 5/4-26E1 & & & 620 & 630 & 189.0 & 192.0 & $\begin{array}{l}\text { nonvesicular basalt } \\
\text { tuff, agglomerate, contains } \\
\text { pumice, obsidian, red and gray } \\
\text { vesicular basalt, and nonvesicular }\end{array}$ & Basalt & -- & -- & -- & \\
\hline 440 & $5 / 4-26 \mathrm{E} 1$ & & & 630 & 638 & 192.0 & 194.5 & gray basalt & Ash or tuff & -- & -- & -- & \\
\hline & & & & & & & & $\begin{array}{l}\text { basalt, gray, fine-grained, finely } \\
\text { porphyritic, finely vesicular near } \\
\text { bottom, slightly oxidized at bottom }\end{array}$ & & & & & \\
\hline 440 & 5/4-26E1 & & & 638 & 698 & 194.5 & 212.8 & $\begin{array}{l}\text { of strata, jointed throughout } \\
\text { Tuff, agglomerate, vesicular and } \\
\text { nonvesicular gray basalt, and red }\end{array}$ & Basalt & -- & -- & -- & \\
\hline 440 & 5/4-26E1 & & & 698 & 713 & 212.8 & 217.3 & vesicular basalt & Ash or tuff & -- & -- & -- & \\
\hline & & & & & & & & $\begin{array}{l}\text { agglomerate, basaltic, consists } \\
\text { chiefly of dark gray, very vesicular }\end{array}$ & & & & & \\
\hline 440 & 5/4-26E1 & & & 713 & 742 & 217.3 & 226.2 & $\begin{array}{l}\text { basalt, some oxidized basalt } \\
\text { basalt, light gray, slightly } \\
\text { vesicular, very hard and jointed. } \\
\text { strata still persists at } 806 \mathrm{ft} \text {. } \\
\text { although much softer and more }\end{array}$ & Basalt & -- & -- & -- & \\
\hline 440 & $5 / 4-26 \mathrm{E} 1$ & & & 742 & 806 & 226.2 & 245.7 & vesicular than higher strata & Basalt & hard & -- & -- & \\
\hline 441 & $5 / 4-27 \mathrm{H} 2$ & 441 & $5 / 4-27 \mathrm{H} 2$ & 0 & 3 & 0.0 & 0.9 & soil & Sand and clay & -- & -- & -- & Qyal \\
\hline 441 & $5 / 4-27 \mathrm{H}^{2}$ & & & 3 & 65 & 0.9 & 19.8 & clay, yellow; and gravel & Clay and gravel & -- & -- & -- & \\
\hline 441 & $5 / 4-27 \mathrm{H} 2$ & & & 65 & 225 & 19.8 & 68.6 & clay, blue and gravel & Clay and gravel & -- & -- & -- & Qoal (?) \\
\hline 441 & $5 / 4-27 \mathrm{H} 2$ & & & 225 & 303 & 68.6 & 92.4 & mud, black & Clay and sand (or sandstone) & -- & -- & -- & \\
\hline 441 & $5 / 4-27 \mathrm{H} 2$ & & & 303 & 320 & 92.4 & 97.5 & clay, blue & Clay & -- & -- & -- & \\
\hline 441 & $5 / 4-27 \mathrm{H} 2$ & & & 320 & 354 & 97.5 & 107.9 & $\begin{array}{l}\text { clay, blue and black mud } \\
\text { clay, gravel blue; blue rock and }\end{array}$ & Clay and sand (or sandstone) & -- & -- & -- & \\
\hline 441 & $5 / 4-27 \mathrm{H} 2$ & & & 354 & 460 & 107.9 & 140.2 & boulders & Clay and gravel & -- & -- & -- & Tsv \\
\hline 441 & $5 / 4-27 \mathrm{H} 2$ & & & 460 & 470 & 140.2 & 143.3 & lava, hard & Basalt & hard & -- & -- & \\
\hline 441 & $5 / 4-27 \mathrm{H} 2$ & & & 470 & 492 & 143.3 & 150.0 & lava, very hard & Basalt & hard & -- & -- & \\
\hline 441 & $5 / 4-27 \mathrm{H} 2$ & & & 492 & 495 & 150.0 & 150.9 & lava, soft & Basalt & -- & -- & -- & \\
\hline 441 & $5 / 4-27 \mathrm{H} 2$ & & & 495 & 509 & 150.9 & 155.1 & clay, blue & Clay & -- & -- & -- & \\
\hline 441 & $5 / 4-27 \mathrm{H} 2$ & & & 509 & 518 & 155.1 & 157.9 & clay and lava rock & Clay and gravel & -- & -- & -- & \\
\hline
\end{tabular}


Appendix 3. Lithologic data. - Continued

ISite IDs and well number correspond to locations in Appendix 1, a site ID and well number is listed for each drilled interval for a particular drill hole. For convenience, the site ID and well numbers are repeated at the shallowest drilled interval for each well. leaders (--),

\begin{tabular}{|c|c|c|c|c|c|c|c|c|c|c|c|c|c|}
\hline Site ID & Well number & $\begin{array}{l}\text { Site ID, } \\
\text { top of } \\
\text { well }\end{array}$ & $\begin{array}{l}\text { Well number, } \\
\text { top of well }\end{array}$ & $\begin{array}{l}\text { Top of } \\
\text { interval, in } \\
\text { feet }\end{array}$ & $\begin{array}{l}\text { Base of } \\
\text { interval, in } \\
\text { feet }\end{array}$ & $\begin{array}{c}\text { Top of } \\
\text { interval, in } \\
m\end{array}$ & $\begin{array}{c}\text { Base of } \\
\text { interval, in } \\
\mathrm{m}\end{array}$ & Reported lithologic unit' & Interpreted lithologic class ${ }^{2}$ & $\begin{array}{c}\text { Reported } \\
\text { degree of } \\
\text { cementation or } \\
\text { induration } \\
\end{array}$ & $\begin{array}{c}\text { Fossils } \\
\text { reported in } \\
\text { interval }^{4}\end{array}$ & $\begin{array}{c}\text { Organic } \\
\text { matter } \\
\text { reported in } \\
\text { interval }^{5}\end{array}$ & $\begin{array}{l}\text { Reported stratigraphic top of geologic } \\
\text { unit }\end{array}$ \\
\hline 441 & $5 / 4-27 \mathrm{H} 2$ & & & 518 & 527 & 157.9 & 160.6 & lava, hard & Basalt & hard & -- & -- & \\
\hline 441 & $5 / 4-27 \mathrm{H} 2$ & & & 527 & 555 & 160.6 & 169.2 & rock and brown clay & Clay and gravel & -- & -- & -- & \\
\hline 441 & $5 / 4-27 \mathrm{H} 2$ & & & 555 & 562 & 169.2 & 171.3 & clay and hard rock & Clay and gravel & -- & -- & -- & \\
\hline 441 & $5 / 4-27 \mathrm{H} 2$ & & & 562 & 570 & 171.3 & 173.7 & lava rock & Basalt & hard & -- & -- & \\
\hline 441 & $5 / 4-27 \mathrm{H}^{2}$ & & & 570 & 580 & 173.7 & 176.8 & lava, hard & Basalt & hard & -- & -- & \\
\hline 441 & $5 / 4-27 \mathrm{H} 2$ & & & 580 & 608 & 176.8 & 185.3 & lava, black & Basalt & -- & -- & -- & \\
\hline 441 & $5 / 4-27 \mathrm{H} 2$ & & & 608 & 624 & 185.3 & 190.2 & lava, hard; boulder & Basalt & hard & -- & -- & \\
\hline 441 & $5 / 4-27 \mathrm{H} 2$ & & & 624 & 630 & 190.2 & 192.0 & lava, hard & Basalt & hard & -. & -- & \\
\hline 441 & $5 / 4-27 \mathrm{H} 2$ & & & 630 & 657 & 192.0 & 200.3 & clay, blue & Clay & -- & -- & -- & \\
\hline 441 & $5 / 4-27 \mathrm{H}^{2}$ & & & 657 & 664 & 200.3 & 202.4 & lava, hard & Basalt & hard & -- & -- & \\
\hline 441 & $5 / 4-27 \mathrm{H} 2$ & & & 664 & 668 & 202.4 & 203.6 & rock, soft & Ash or tuff & hard & -- & -- & \\
\hline 441 & $5 / 4-27 \mathrm{H} 2$ & & & 668 & 673 & 203.6 & 205.1 & cinders, red & Basalt & -- & -- & -- & \\
\hline 441 & $5 / 4-27 \mathrm{H} 2$ & & & 673 & 705 & 205.1 & 214.9 & lava, hard & Basalt & hard & -- & -- & \\
\hline 441 & $5 / 4-27 \mathrm{H} 2$ & & & 705 & 720 & 214.9 & 219.5 & lava, hard & Basalt & hard & -- & -- & \\
\hline 441 & $5 / 4-27 \mathrm{H} 2$ & & & 720 & 725 & 219.5 & 221.0 & cinders & Basalt & -- & .- & -- & \\
\hline 441 & $5 / 4-27 \mathrm{H} 2$ & & & 725 & 740 & 221.0 & 225.6 & hard lava & Basalt & hard & -- & -- & \\
\hline 441 & $5 / 4-27 \mathrm{H} 2$ & & & 740 & 745 & 225.6 & 227.1 & gray sandstone & Sandstone & cemented & -- & -- & \\
\hline 441 & $5 / 4-27 \mathrm{H} 2$ & & & 745 & 750 & 227.1 & 228.6 & lava, soft & Basalt & -- & -- & -- & \\
\hline 441 & $5 / 4-27 \mathrm{H}^{2}$ & & & 750 & 758 & 228.6 & 231.0 & hard lava & Basalt & hard & -- & -- & \\
\hline 441 & $5 / 4-27 \mathrm{H} 2$ & & & 758 & 802 & 231.0 & 244.4 & clay, green, sticky and sand & Clay and sand (or sandstone) & -- & -- & -- & \\
\hline 441 & $5 / 4-27 \mathrm{H} 2$ & & & 802 & 807 & 244.4 & 246.0 & boulders and clay & Clay and gravel & -- & -- & -- & \\
\hline 441 & $5 / 4-27 \mathrm{H} 2$ & & & 807 & 815 & 246.0 & 248.4 & $\begin{array}{l}\text { clay and sand } \\
\text {. }\end{array}$ & Clay and sand (or sandstone) & -- & -- & - & \\
\hline 441 & $5 / 4-27 \mathrm{H} 2$ & & & 815 & 822 & 248.4 & 250.5 & lava rock & Basalt & hard & -- & -- & \\
\hline 441 & $5 / 4-27 \mathrm{H}^{2}$ & & & 822 & 830 & 250.5 & 253.0 & clay and lava sand & Clay and gravel & -- & -- & -- & \\
\hline 441 & $5 / 4-27 \mathrm{H} 2$ & & & 830 & 860 & 253.0 & 262.1 & lava, black & Basalt & -- & -- & -- & \\
\hline 442 & $5 / 5-26 \mathrm{G} 1$ & 442 & 5/5-26G1 & 0 & 3 & 0.0 & 0.9 & surface & Sand and clay & -- & -- & -- & Tsv \\
\hline 442 & $5 / 5-26 \mathrm{G} 1$ & & & 3 & 45 & 0.9 & 13.7 & rock, gray tuff & Ash or tuff & hard & -- & -- & 100 \\
\hline 442 & $5 / 5-26 \mathrm{G} 1$ & & & 45 & 240 & 13.7 & 73.2 & rock, blue tuff, water & Sandstone & hard & -- & -- & \\
\hline 442 & 5/5-26G1 & & & 240 & 260 & 73.2 & 79.2 & basalt boulders, water & Basalt & -- & -- & -- & \\
\hline 442 & $5 / 5-26 \mathrm{G} 1$ & & & 260 & 290 & 79.2 & 88.4 & rock, blue tuff & Sandstone & hard & -- & -- & \\
\hline 442 & $5 / 5-26 \mathrm{G} 1$ & & & 290 & 300 & 88.4 & 91.4 & lava rock, red & Basalt & hard & -- & -- & \\
\hline 442 & $5 / 5-26 \mathrm{G} 1$ & & & 300 & 355 & 91.4 & 108.2 & volcanic ash, white & Ash or tuff & -- & -- & -- & \\
\hline 442 & $5 / 5-26 \mathrm{G} 1$ & & & 355 & 410 & 108.2 & 125.0 & rock, blue tuff & Ash or tuff & hard & -- & -- & \\
\hline 443 & $5 / 4-27 \mathrm{H} 1$ & 443 & 5/4-27H1 & 0 & 7 & 0.0 & 2.1 & soil & Sand and clay & -- & -- & -- & Qyal and Qoal \\
\hline 443 & $5 / 4-27 \mathrm{H} 1$ & & & 7 & 20 & 2.1 & 6.1 & clay, blue & Clay & -- & -- & -- & \\
\hline 443 & $5 / 4-27 \mathrm{H} 1$ & & & 20 & 55 & 6.1 & 16.8 & clay, yellow; and gravel & Clay and gravel & -- & -- & -- & \\
\hline 443 & $5 / 4-27 \mathrm{H} 1$ & & & 55 & 64 & 16.8 & 19.5 & clay, yellow & Clay & -- & -- & -- & \\
\hline 443 & $5 / 4-27 \mathrm{H} 1$ & & & 64 & 75 & 19.5 & 22.9 & clay and gravel & Clay and gravel & -- & -- & -- & \\
\hline 443 & $5 / 4-27 \mathrm{H} 1$ & & & 75 & 84 & 22.9 & 25.6 & $\begin{array}{l}\text { muck, blue } \\
\text { mats }\end{array}$ & Clay and sand (or sandstone) & -- & -- & -- & \\
\hline 443 & $5 / 4-27 \mathrm{H} 1$ & & & 84 & 120 & 25.6 & 36.6 & mud and gravel & Clay, sand, and gravel & -- & -- & -- & \\
\hline 443 & $5 / 4-27 \mathrm{H} 1$ & & & 120 & 130 & 36.6 & 39.6 & clay, blue and gravel & Clay and gravel & -- & -- & -- & \\
\hline 443 & $5 / 4-27 \mathrm{H} 1$ & & & 130 & 193 & 39.6 & 58.8 & clay, blue & Clay & -- & -- & -- & \\
\hline 443 & $5 / 4-27 \mathrm{H} 1$ & & & 193 & 245 & 58.8 & 74.7 & clay, blue and gravel & Clay and gravel & -- & -- & -- & \\
\hline 443 & $5 / 4-27 \mathrm{H} 1$ & & & 245 & 325 & 74.7 & 99.1 & clay, blue, black mud, and gravel & Clay and sand (or sandstone) & -- & -- & -- & \\
\hline 443 & $5 / 4-27 \mathrm{H} 1$ & & & 325 & 358 & 99.1 & 109.1 & $\begin{array}{l}\text { lava, gravel, water worn } \\
\text { latur }\end{array}$ & Basalt & -- & -- & -- & \\
\hline 443 & $5 / 4-27 \mathrm{H} 1$ & & & 358 & 362 & 109.1 & 110.3 & cinders, red & Basalt & -- & -- & -- & Tsv \\
\hline 443 & $5 / 4-27 \mathrm{H} 1$ & & & 362 & 387 & 110.3 & 118.0 & clay, blue, and gravel & Clay and gravel & -- & -- & -- & \\
\hline 443 & $5 / 4-27 \mathrm{H} 1$ & & & 387 & 395 & 118.0 & 120.4 & $\begin{array}{l}\text { mud and gravel, black } \\
\text { clay, blue, and boulders; smooth }\end{array}$ & Clay, sand, and gravel & -- & -- & -- & \\
\hline 443 & $5 / 4-27 \mathrm{H} 1$ & & & 395 & 412 & 120.4 & 125.6 & $\begin{array}{l}\text { lava boulders } \\
\text { lations }\end{array}$ & Clay and gravel & -- & -- & -- & \\
\hline 443 & $5 / 4-27 \mathrm{H} 1$ & & & 412 & 472 & 125.6 & 143.9 & cinders, red and black & Basalt & -- & -- & -- & \\
\hline 443 & $5 / 4-27 \mathrm{H} 1$ & & & 472 & 505 & 143.9 & 153.9 & lava, blue-black & Basalt & -- & -- & -- & \\
\hline 443 & $5 / 4-27 \mathrm{H} 1$ & & & 505 & 509 & 153.9 & 155.1 & lava ash, red & Basalt & -- & -- & -- & \\
\hline 443 & $5 / 4-27 \mathrm{H} 1$ & & & 509 & 523 & 155.1 & 159.4 & lava, blue, hard & Basalt & hard & -- & -- & \\
\hline 443 & $5 / 4-27 \mathrm{H} 1$ & & & 523 & 539 & 159.4 & 164.3 & lava, black & Basalt & -- & -- & -. & \\
\hline 443 & $5 / 4-27 \mathrm{H} 1$ & & & 539 & 575 & 164.3 & 175.3 & lava rock & Basalt & hard & -- & -- & \\
\hline 443 & $5 / 4-27 \mathrm{H} 1$ & & & 575 & 672 & 175.3 & 204.8 & lava, blue, soft & Basalt & -. & -- & -- & \\
\hline 443 & $5 / 4-27 \mathrm{H} 1$ & & & 672 & 677 & 204.8 & 206.3 & hard lava & Basalt & hard & -- & -- & \\
\hline 443 & $5 / 4-27 \mathrm{H} 1$ & & & 677 & 695 & 206.3 & 211.8 & soft lava & Basalt & - & -- & -- & \\
\hline 443 & $5 / 4-27 \mathrm{H} 1$ & & & 695 & 720 & 211.8 & 219.5 & hard lava & Basalt & hard & -- & -- & \\
\hline
\end{tabular}


Appendix 3. Lithologic data. - Continued

ISite IDs and well number correspond to locations in Appendix 1, a site ID and well number is listed for each drilled interval for a particular drill hole. For convenience, the site ID and well numbers are repeated at the shallowest drilled interval for each well. leaderst.

\begin{tabular}{|c|c|c|c|c|c|c|c|c|c|c|c|c|c|}
\hline Site ID & Well number & $\begin{array}{l}\text { Site ID, } \\
\text { top of } \\
\text { well }\end{array}$ & $\begin{array}{l}\text { Well number, } \\
\text { top of well }\end{array}$ & $\begin{array}{c}\text { Top of } \\
\text { interval, in } \\
\text { feet }\end{array}$ & $\begin{array}{c}\text { Base of } \\
\text { interval, in } \\
\text { feet }\end{array}$ & $\begin{array}{c}\text { Top of } \\
\text { interval, in } \\
m\end{array}$ & $\begin{array}{c}\text { Base of } \\
\text { interval, in } \\
\mathrm{m}\end{array}$ & Reported lithologic unit ${ }^{1}$ & Interpreted lithologic class ${ }^{2}$ & $\begin{array}{c}\text { Reported } \\
\text { degree of } \\
\text { cementation or } \\
\text { induration } \\
\end{array}$ & $\begin{array}{c}\text { Fossils } \\
\text { reported in } \\
\text { interval| }^{4}\end{array}$ & $\begin{array}{c}\text { Organic } \\
\text { matter } \\
\text { reported in }^{\text {in }} \text { interval }^{5}\end{array}$ & $\begin{array}{l}\text { Reported stratigraphic top of geologic } \\
\text { unit }^{6}\end{array}$ \\
\hline 443 & $5 / 4-27 \mathrm{H} 1$ & & & 720 & 820 & 219.5 & 249.9 & lava, blue, soft and sticky & Basalt & -- & -- & -- & \\
\hline 443 & $5 / 4-27 \mathrm{H} 1$ & & & 820 & 830 & 249.9 & 253.0 & loose lava, light blue & Basalt & -- & -- & -- & \\
\hline 443 & $5 / 4-27 \mathrm{H} 1$ & & & 830 & 842 & 253.0 & 256.6 & $\begin{array}{l}\text { hard dark lava } \\
\text { volcanic ash and lava particles, }\end{array}$ & Basalt & hard & -- & -- & \\
\hline 443 & $5 / 4-27 \mathrm{H} 1$ & & & 842 & 960 & 256.6 & 292.6 & sticky like clay & Ash or tuff & -- & -- & -- & \\
\hline 443 & $5 / 4-27 \mathrm{H} 1$ & & & 960 & 980 & 292.6 & 298.7 & hard black lava & Basalt & hard & -- & -- & \\
\hline 443 & $5 / 4-27 \mathrm{H} 1$ & & & 980 & 990 & 298.7 & 301.8 & lava rock and red cinders & Basalt & hard & -- & -- & \\
\hline 443 & $5 / 4-27 \mathrm{H} 1$ & & & 990 & 1005 & 301.8 & 306.3 & lava, gray & Basalt & -- & -- & -- & \\
\hline 443 & $5 / 4-27 \mathrm{H} 1$ & & & 1005 & 1015 & 306.3 & 309.4 & tuff, gray & Ash or tuff & -- & -- & -- & \\
\hline 443 & $5 / 4-27 \mathrm{H} 1$ & & & 1015 & 1070 & 309.4 & 326.1 & lava, blue-gray & Basalt & -- & -- & -- & \\
\hline 443 & $5 / 4-27 \mathrm{H} 1$ & & & 1070 & 1085 & 326.1 & 330.7 & tuff and pumice, gray & Ash or tuff & -- & -- & .- & \\
\hline 443 & $5 / 4-27 \mathrm{H} 1$ & & & 1085 & 1110 & 330.7 & 338.3 & tuff, gray; hard lava & Ash or tuff & hard & -- & -- & \\
\hline 443 & $5 / 4-27 \mathrm{H} 1$ & & & 1110 & 1226 & 338.3 & 373.7 & tuff, gray or sandy clay & Ash or tuff & -- & -- & -- & \\
\hline 444 & $5 / 4-29 F 1$ & 444 & 5/4-29F1 & 0 & 3 & 0.0 & 0.9 & soil & Sand and clay &.- & - & - & Qh \\
\hline 444 & 5/4-29F1 & & & 3 & 38 & 0.9 & 11.6 & clay, yellow & Clay & -- & -- & -- & \\
\hline 444 & $5 / 4-29 F 1$ & & & 38 & 57 & 11.6 & 17.4 & $\begin{array}{l}\text { clay, blue } \\
\text { lat }\end{array}$ & Clay & -- & -- & -.- & \\
\hline 444 & $5 / 4-29 \mathrm{~F} 1$ & & & 57 & 58 & 17.4 & 17.7 & water & Clay & -- & -- & -- & \\
\hline 444 & 5/4-29F1 & & & 58 & 84 & 17.7 & 25.6 & clay, blue & Clay & -- & -- & -.- & \\
\hline 444 & 5/4-29F1 & & & 84 & 90 & 25.6 & 27.4 & sandstone & Sandstone & cemented & -- & -- & Knoxville and Horsetown Formations \\
\hline 444 & $5 / 4-29 \mathrm{~F} 1$ & & & 90 & 106 & 27.4 & 32.3 & shale & Clay and sand (or sandstone) & cemented & -- & -- & \\
\hline 445 & $5 / 4-27 \mathrm{~K} 2$ & 445 & 5/4-27K2 & 0 & 3 & 0.0 & 0.9 & soil & 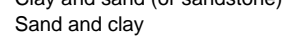 & - & - & -- & Qyal and Qoal \\
\hline 445 & $5 / 4-27 \mathrm{~K} 2$ & & & 3 & 10 & 0.9 & 3.0 & tule mud & Clay and sand (or sandstone) & -- & -- & tule & \\
\hline 445 & $5 / 4-27 \mathrm{~K} 2$ & & & 10 & 42 & 3.0 & 12.8 & $\begin{array}{l}\text { clay or mud, blue } \\
\text { clay, sandy, yellow; gravel with }\end{array}$ & Clay and sand (or sandstone) & -- & -- & -- & \\
\hline 445 & $5 / 4-27 \mathrm{~K} 2$ & & & 42 & 51 & 12.8 & 15.5 & clay & Clay, sand, and gravel & -- & -- & -. & \\
\hline 445 & 5/4-27K2 & & & 51 & 60 & 15.5 & 18.3 & stiff clay, sandy, blue & Clay and sand (or sandstone) & -- & -- & .- & \\
\hline 445 & $5 / 4-27 \mathrm{~K} 2$ & & & 60 & 77 & 18.3 & 23.5 & gravel blue with clay & Clay and gravel & -- & -- & -- & \\
\hline 445 & $5 / 4-27 \mathrm{~K} 2$ & & & 77 & 83 & 23.5 & 25.3 & $\begin{array}{l}\text { clay, sandy, blue } \\
\text { can }\end{array}$ & Clay and sand (or sandstone) & - & - & - & \\
\hline 445 & $5 / 4-27 \mathrm{~K} 2$ & & & 83 & 92 & 25.3 & 28.0 & sand, blue with clay & Sand and clay & -- & -- & -- & \\
\hline 445 & $5 / 4-27 \mathrm{~K} 2$ & & & 92 & 105 & 28.0 & 32.0 & $\begin{array}{l}\text { tule mud } \\
\text { thing }\end{array}$ & Clay and sand (or sandstone) & -- & -- & tule & \\
\hline 445 & $5 / 4-27 K 2$ & & & 105 & 114 & 32.0 & 34.7 & stiff clay, sandy, blue & Clay and sand (or sandstone) & -- & -- & -- & \\
\hline 445 & $5 / 4-27 \mathrm{~K} 2$ & & & 114 & 118 & 34.7 & 36.0 & sand, blue with clay & Sand and clay & -- & -- & -- & \\
\hline 445 & $5 / 4-27 \mathrm{~K} 2$ & & & 118 & 125 & 36.0 & 38.1 & stiff clay, blue & Clay & -- & -- & -- & \\
\hline 445 & $5 / 4-27 \mathrm{~K} 2$ & & & 125 & 135 & 38.1 & 41.1 & sand, blue, and gravel with clay & Clay, sand, and gravel & -- & -- & -- & \\
\hline 445 & $5 / 4-27 \mathrm{~K} 2$ & & & 135 & 148 & 41.1 & 45.1 & $\begin{array}{l}\text { stiff, clay, blue } \\
\text { clay, sandy, blue with gravel }\end{array}$ & Clay & -- & -- & - & \\
\hline 445 & $5 / 4-27 \mathrm{~K} 2$ & & & 148 & 170 & 45.1 & 51.8 & streaks & Clay and gravel & -- & -- & -- & \\
\hline 445 & $5 / 4-27 \mathrm{~K} 2$ & & & 170 & 230 & 51.8 & 70.1 & $\begin{array}{l}\text { stiff clay, blue with gravel streaks } \\
\text { volcanic tuff, slight increase in }\end{array}$ & Clay and gravel & -- & -- & -- & \\
\hline 445 & $5 / 4-27 \mathrm{~K} 2$ & & & 230 & 259 & 70.1 & 78.9 & $\begin{array}{l}\text { water } \\
\text { mud or clay, brownish with fine }\end{array}$ & Ash or tuff & -- & -- & -- & Tsv (?) \\
\hline 445 & $5 / 4-27 \mathrm{~K} 2$ & & & 259 & 280 & 78.9 & 85.3 & sand and gravel & Clay and sand (or sandstone) & -- & -- & -- & \\
\hline 445 & $5 / 4-27 \mathrm{~K} 2$ & & & 280 & 292 & 85.3 & 89.0 & $\begin{array}{l}\text { fine sand and gravel with clay } \\
\text { struck a redwood log. fine sand }\end{array}$ & Clay, sand, and gravel & - & -- & -- & \\
\hline 445 & $5 / 4-27 \mathrm{~K} 2$ & & & 292 & 310 & 89.0 & 94.5 & $\begin{array}{l}\text { and clay } \\
\text { gravel, blue water-worn with } \\
\text { green lava rock. Increase in flow }\end{array}$ & Sand and clay & -- & -- & wood & \\
\hline 445 & $5 / 4-27 \mathrm{~K} 2$ & & & 310 & 329 & 94.5 & 100.3 & of water & Clay, sand, and gravel & -- & -- & -- & \\
\hline 445 & $5 / 4-27 \mathrm{~K} 2$ & & & 329 & 343 & 100.3 & 104.5 & gravel with more clay & Clay and gravel & - & - & -- & \\
\hline 445 & $5 / 4-27 \mathrm{~K} 2$ & & & 343 & 355 & 104.5 & 108.2 & mud or ash, brown & Clay and sand (or sandstone) & -- & -- & -- & \\
\hline 445 & $5 / 4-27 \mathrm{~K} 2$ & & & 355 & 358 & 108.2 & 109.1 & gravel, blue, water-worn & Gravel & -- & -- & -. & \\
\hline 445 & $5 / 4-27 \mathrm{~K} 2$ & & & 358 & 382 & 109.1 & 116.4 & ash brown, with gravel & Ash or tuff & -- & -- & -- & \\
\hline 445 & $5 / 4-27 \mathrm{~K} 2$ & & & 382 & 393 & 116.4 & 119.8 & gravel, blue, water-worn & Gravel & -- & -- & -. & \\
\hline 445 & $5 / 4-27 \mathrm{~K} 2$ & & & 393 & 400 & 119.8 & 121.9 & fine gray sand and clay & Sand and clay & -- & -- & -- & \\
\hline 445 & $5 / 4-27 \mathrm{~K} 2$ & & & 400 & 407 & 121.9 & 124.1 & $\begin{array}{l}\text { clay, sandy, blue } \\
\text { conglomerate of burnt rock, }\end{array}$ & Clay and sand (or sandstone) & - & -- & - & \\
\hline 445 & $5 / 4-27 \mathrm{~K} 2$ & & & 407 & 450 & 124.1 & 137.2 & cinders & Clay, sand, and gravel & .- & -. & .. & \\
\hline 445 & $5 / 4-27 \mathrm{~K} 2$ & & & 450 & 456 & 137.2 & 139.0 & rock, dark, close-grained & Clay, sand, and gravel & hard & -- & -. & \\
\hline 446 & 5/4-26M1 & 446 & $5 / 4-26 \mathrm{M} 1$ & 0 & 4 & 0.0 & 1.2 & soil & Sand and clay & -- & -- & -- & Qyal and Qoal \\
\hline 446 & $5 / 4-26 \mathrm{M} 1$ & & & 4 & 44 & 1.2 & 13.4 & clay, yellow, and gravel & Clay and gravel & -. & -- & -- & \\
\hline
\end{tabular}


Appendix 3. Lithologic data. - Continued

[Site IDs and well number correspond to locations in Appendix 1, a site ID and well number is listed for each drilled interval for a particular drill hole. For convenience, the site ID and well numbers are repeated at the shallowest drilled interval for each well. leaders (- -

\begin{tabular}{|c|c|c|c|c|c|c|c|c|c|c|c|c|c|}
\hline Site ID & Well number & $\begin{array}{l}\text { Site ID, } \\
\text { top of } \\
\text { well }\end{array}$ & $\begin{array}{l}\text { Well number, } \\
\text { top of well }\end{array}$ & $\begin{array}{l}\text { Top of } \\
\text { interval, in } \\
\text { feet }\end{array}$ & $\begin{array}{l}\text { Base of } \\
\text { interval, in } \\
\text { feet }\end{array}$ & $\begin{array}{c}\text { Top of } \\
\text { interval, in } \\
m\end{array}$ & $\begin{array}{c}\text { Base of } \\
\text { interval, in } \\
\mathrm{m}\end{array}$ & Reported lithologic unit' & Interpreted lithologic class ${ }^{2}$ & $\begin{array}{c}\text { Reported } \\
\text { degree of } \\
\text { cementation or } \\
\text { induration }^{3} \\
\end{array}$ & $\begin{array}{l}\text { Fossils } \\
\text { reported in } \\
\text { interval }^{4}\end{array}$ & $\begin{array}{c}\text { Organic } \\
\text { matter } \\
\text { reported in } \\
\text { interval }^{5}\end{array}$ & $\begin{array}{l}\text { Reported stratigraphic top of geologic } \\
\text { unit }^{6}\end{array}$ \\
\hline 446 & $5 / 4-26 \mathrm{M} 1$ & & & 44 & 95 & 13.4 & 29.0 & clay, blue, and gravel & Clay and gravel & -- & -- & -- & \\
\hline 446 & 5/4-26M1 & & & 95 & 135 & 29.0 & 41.1 & lava, black, soft vesicular & Basalt & -- & -- & -- & \\
\hline 446 & $5 / 4-26 \mathrm{M} 1$ & & & 135 & 280 & 41.1 & 85.3 & tuff, dark color, very little pumice & Ash or tuff & -- & -- & -- & \\
\hline 446 & 5/4-26M1 & & & 280 & 340 & 85.3 & 103.6 & $\begin{array}{l}\text { basalt, gray } \\
\text { basast, mixed gray, vesicular and }\end{array}$ & Basalt & -- & -- & -- & \\
\hline 446 & 5/4-26M1 & & & 340 & 370 & 103.6 & 112.8 & red oxidized basalt & Basalt & -- & -- & -- & \\
\hline 446 & 5/4-26M1 & & & 370 & 405 & 112.8 & 123.4 & basalt, gray & Basalt & -- & -- & -- & \\
\hline 446 & 5/4-26M1 & & & 405 & 410 & 123.4 & 125.0 & mixed tuff and basalt & Ash or tuff & -- & -- & -- & \\
\hline 446 & $5 / 4-26 \mathrm{M} 1$ & & & 410 & 416 & 125.0 & 126.8 & basalt, grayish-black & Basalt & -- & -- & -- & \\
\hline 446 & 5/4-26M1 & & & 416 & 430 & 126.8 & 131.1 & tuff & Ash or tuff & -- & -- & -- & \\
\hline 446 & $5 / 4-26 \mathrm{M} 1$ & & & 430 & 435 & 131.1 & 132.6 & $\begin{array}{l}\text { basalt, gray } \\
\text { basalt, reddish-brown vesicular }\end{array}$ & Basalt & -- & -- & -- & \\
\hline 446 & 5/4-26M1 & & & 435 & 440 & 132.6 & 134.1 & oxidized & Basalt & -- & -- & -- & \\
\hline 446 & $5 / 4-26 \mathrm{M} 1$ & & & 440 & 536 & 134.1 & 163.4 & $\begin{array}{l}\text { basalt, gray, glassy to vesicular } \\
\text { tuff, sticky like clay, contains }\end{array}$ & Basalt & -- & -- & -- & \\
\hline 446 & 5/4-26M1 & & & 536 & 592 & 163.4 & 180.4 & pumice & Ash or tuff & -- & -- & -- & \\
\hline 446 & 5/4-26M1 & & & 592 & 630 & 180.4 & 192.0 & $\begin{array}{l}\text { basalt, gray, glassy to vesicular } \\
\text { basalt, red oxidized, glassy to }\end{array}$ & Basalt & -- & -- & -- & \\
\hline 446 & 5/4-26M1 & & & 630 & 655 & 192.0 & 199.6 & vesicular & Basalt & -- & -- & -- & \\
\hline 446 & 5/4-26M1 & & & 655 & 675 & 199.6 & 205.7 & basalt, blue-black & Basalt & -- & -- & -- & \\
\hline 446 & 5/4-26M1 & & & 675 & 692 & 205.7 & 210.9 & basalt, blue-black, vesicular & Basalt & -- & -- & -- & \\
\hline 446 & $5 / 4-26 \mathrm{M} 1$ & & & 692 & 835 & 210.9 & 254.5 & tuff, sticky, contains pumice & Ash or tuff & -- & -- & -- & \\
\hline 446 & 5/4-26M1 & & & 835 & 840 & 254.5 & 256.0 & mixed tuff and glassy basalt & Ash or tuff & -- & .- & .- & \\
\hline 446 & $5 / 4-26 \mathrm{M} 1$ & & & 840 & 875 & 256.0 & 266.7 & tuff & Ash or tuff & -- & -- & -- & \\
\hline 446 & 5/4-26M1 & & & 875 & 890 & 266.7 & 271.3 & mixed tuff and basalt & Ash or tuff & -- & -- & -- & \\
\hline 446 & $5 / 4-26 \mathrm{M} 1$ & & & 890 & 900 & 271.3 & 274.3 & basalt, light-gray, porphyritic & Basalt & -- & -- & -- & \\
\hline 446 & $5 / 4-26 \mathrm{M} 1$ & & & 900 & 939 & 274.3 & 286.2 & mixed tuff and basalt, very hard & Ash or tuff & hard & -- & -- & \\
\hline 447 & 5/4-27K1 & 447 & 5/4-27K1 & 0 & 6 & 0.0 & 1.8 & adobe, black & Sand and clay & -- & -- & -- & Qyal and Qoal \\
\hline 447 & $5 / 4-27 \mathrm{~K} 1$ & & & 6 & 13 & 1.8 & 4.0 & fine gravel in clay & Clay and gravel & -- & -- & -- & \\
\hline 447 & $5 / 4-27 \mathrm{~K} 1$ & & & 13 & 24 & 4.0 & 7.3 & $\begin{array}{l}\text { stiff clay, yellow } \\
\text { s. }\end{array}$ & Clay & -- & -- & -- & \\
\hline 447 & $5 / 4-27 K 1$ & & & 24 & 40 & 7.3 & 12.2 & gravel with yellow clay & Clay and gravel & -- & -- & -- & \\
\hline 447 & $5 / 4-27 \mathrm{~K} 1$ & & & 40 & 43 & 12.2 & 13.1 & $\begin{array}{l}\text { stiff clay, yellow } \\
\text { sum }\end{array}$ & Clay & -- & -- & -- & \\
\hline 447 & $5 / 4-27 K 1$ & & & 43 & 68 & 13.1 & 20.7 & stuff clay, blue & Clay & -- & -- & -- & \\
\hline 447 & $5 / 4-27 K 1$ & & & 68 & 75 & 20.7 & 22.9 & $\begin{array}{l}\text { sand, blue with clay } \\
\text { gravel, blue, water-worn. Water }\end{array}$ & Sand and clay & -- & -- & -- & \\
\hline 447 & $5 / 4-27 \mathrm{~K} 1$ & & & 75 & 105 & 22.9 & 32.0 & rose to $12 \mathrm{ft}$ ' below surface & Gravel & -- & -- & -- & \\
\hline 447 & $5 / 4-27 \mathrm{~K} 1$ & & & 105 & 110 & 32.0 & 33.5 & gravel in clay & Clay and gravel & -. & -- & -- & \\
\hline 447 & $5 / 4-27 \mathrm{~K} 1$ & & & 110 & 117 & 33.5 & 35.7 & boulder, hard lava (?) & Gravel & hard & -- & -- & Tsv \\
\hline 447 & $5 / 4-27 \mathrm{~K} 1$ & & & 117 & 135 & 35.7 & 41.1 & lava rock, porous & Basalt & hard & -- & -- & \\
\hline 447 & $5 / 4-27 K 1$ & & & 135 & 190 & 41.1 & 57.9 & $\begin{array}{l}\text { lava rock, greenish color } \\
\text { clinker and gravel. Increase in }\end{array}$ & Basalt & hard & -- & -- & \\
\hline 447 & $5 / 4-27 \mathrm{~K} 1$ & & & 190 & 204 & 57.9 & 62.2 & $\begin{array}{l}\text { water } \\
\text { cinders, reddish-brown, slight flow }\end{array}$ & Basalt & -- & -- & -- & \\
\hline 447 & $5 / 4-27 \mathrm{~K} 1$ & & & 204 & 235 & 62.2 & 71.6 & of water & Basalt & -- & -- & -- & \\
\hline 447 & $5 / 4-27 K 1$ & & & 235 & 243 & 71.6 & 74.1 & ash or mud, dark brown & Ash or tuff & -. & -- & -- & \\
\hline 447 & $5 / 4-27 \mathrm{~K} 1$ & & & 243 & 267 & 74.1 & 81.4 & lava rock, green & Basalt & hard & -- & -- & \\
\hline 447 & $5 / 4-27 K 1$ & & & 267 & 273 & 81.4 & 83.2 & $\begin{array}{l}\text { cinders. Increase in flow of water } \\
\text { rock, very hard close-grained dark. }\end{array}$ & Basalt & -- & -- & -- & \\
\hline 447 & $5 / 4-27 K 1$ & & & 273 & 276 & 83.2 & 84.1 & colored & Ash or tuff & hard & -- & -- & \\
\hline 448 & $5 / 4-26 \mathrm{M} 2$ & 448 & 5/4-26M2 & 0 & 3 & 0.0 & 0.9 & soil & Sand and clay & -- & -- & -- & Qyal \\
\hline 448 & $5 / 4-26 \mathrm{M} 2$ & & & 3 & 30 & 0.9 & 9.1 & sandy clay, yellow & Clay and sand (or sandstone) & -- & -- & -- & \\
\hline 448 & $5 / 4-26 \mathrm{M} 2$ & & & 30 & 50 & 9.1 & 15.2 & clay, blue, hard & Clay & hard & -- & -- & \\
\hline 448 & 5/4-26M2 & & & 50 & 93 & 15.2 & 28.3 & sandy clay, blue & Clay and sand (or sandstone) & -- & -- & -- & \\
\hline 448 & $5 / 4-26 \mathrm{M} 2$ & & & 93 & 150 & 28.3 & 45.7 & agglomerate, red, vesicular & Ash or tuff & -- & -- & -- & \\
\hline 448 & 5/4-26M2 & & & 150 & 325 & 45.7 & 99.1 & $\begin{array}{l}\text { basalt, blue-gray } \\
\text { agglomerate, mixed red and }\end{array}$ & Basalt & -- & -- & -- & \\
\hline 448 & 5/4-26M2 & & & 325 & 340 & 99.1 & 103.6 & basalt & Basalt & -- & -- & -- & \\
\hline 448 & 5/4-26M2 & & & 340 & 375 & 103.6 & 114.3 & basalt, gray & Basalt & -- & -- & -- & \\
\hline 448 & 5/4-26M2 & & & 375 & 408 & 114.3 & 124.4 & basalt, blue-gray & Basalt & -- & -- & -- & \\
\hline
\end{tabular}


Appendix 3. Lithologic data. - Continued

[Site IDs and well number correspond to locations in Appendix 1, a site ID and well number is listed for each drilled interval for a particular drill hole. For convenience, the site ID and well numbers are repeated at the shallowest drilled

\begin{tabular}{|c|c|c|c|c|c|c|c|c|c|c|c|c|c|}
\hline Site ID & Well number & $\begin{array}{l}\text { Site ID, } \\
\text { top of } \\
\text { well }\end{array}$ & $\begin{array}{l}\text { Well number, } \\
\text { top of well }\end{array}$ & $\begin{array}{l}\text { Top of } \\
\text { interval, in } \\
\text { feet }\end{array}$ & $\begin{array}{l}\text { Base of } \\
\text { interval, in } \\
\text { feet }\end{array}$ & $\begin{array}{c}\begin{array}{c}\text { Top of } \\
\text { interval, in } \\
\mathrm{m}\end{array} \\
\end{array}$ & $\begin{array}{c}\text { Base of } \\
\text { interval, in } \\
\mathrm{m}\end{array}$ & Reported lithologic unit' & Interpreted lithologic class ${ }^{2}$ & $\begin{array}{c}\text { Reported } \\
\text { degree of } \\
\text { cementation or } \\
\text { induration }^{3} \\
\end{array}$ & $\begin{array}{l}\text { Fossils } \\
\text { reported in } \\
\text { interval }^{4}\end{array}$ & $\begin{array}{c}\text { Organic } \\
\text { matter } \\
\text { reported in } \\
\text { interval }^{5}\end{array}$ & $\begin{array}{l}\text { Reported stratigraphic top of geologic } \\
\text { unit }^{6}\end{array}$ \\
\hline 448 & $5 / 4-26 \mathrm{M} 2$ & & & 408 & 432 & 124.4 & 131.7 & agglomerate, red, vesicular & Ash or tuff & -- & -- & -- & \\
\hline 448 & 5/4-26M2 & & & 432 & 442 & 131.7 & 134.7 & basalt & Basalt & -- & -- & -- & \\
\hline 448 & $5 / 4-26 \mathrm{M} 2$ & & & 442 & 483 & 134.7 & 147.2 & agglomerate, red, vesicular & Ash or tuff & -- & -- & -- & \\
\hline 448 & $5 / 4-26 \mathrm{M} 2$ & & & 483 & 590 & 147.2 & 179.8 & basalt, brownish-black, vesicular & Basalt & -- & -- & -- & \\
\hline 448 & $5 / 4-26 \mathrm{M} 2$ & & & 590 & 605 & 179.8 & 184.4 & basalt, light-gray & Basalt & -- & -- & -- & \\
\hline 448 & $5 / 4-26 \mathrm{M} 2$ & & & 605 & 620 & 184.4 & 189.0 & $\begin{array}{l}\text { basalt, dark-gray } \\
\text { basalt, mixed gray, vesicular and }\end{array}$ & Basalt & -- & -- & -- & \\
\hline 448 & 5/4-26M2 & & & 620 & 682 & 189.0 & 207.9 & red vesicular agglomerate & Basalt & -- & -- & -- & \\
\hline 449 & $5 / 4-29 K 1$ & 449 & $5 / 4-29 K 1$ & 0 & 2 & 0.0 & 0.6 & soil & Sand and clay & -- & -- & -- & Qh \\
\hline 449 & $5 / 4-29 K 1$ & & & 2 & 25 & 0.6 & 7.6 & clay, yellow & Clay & -- & -- & -- & \\
\hline 449 & $5 / 4-29 K 1$ & & & 25 & 26 & 7.6 & 7.9 & boulders and clay & Clay and gravel & -- & -- & -- & \\
\hline 449 & $5 / 4-29 k 1$ & & & 26 & 33 & 7.9 & 10.1 & $\begin{array}{l}\text { clay, yellow } \\
\text { lath }\end{array}$ & Clay & -- & -- & -- & \\
\hline 449 & $5 / 4-29 \mathrm{~K} 1$ & & & 33 & 42 & 10.1 & 12.8 & boulders and clay & Clay and gravel & -- & -- & -- & \\
\hline 449 & $5 / 4-29 K 1$ & & & 42 & 105 & 12.8 & 32.0 & clay, yellow & Clay & -- & -- & -- & \\
\hline 450 & $5 / 4-26 N 1$ & 450 & 5/4-26N1 & 0 & 2 & 0.0 & 0.6 & soil & Sand and clay & -. & -- & -- & Qoal \\
\hline 450 & $5 / 4-26 \mathrm{~N} 1$ & & & 2 & 6 & 0.6 & 1.8 & hardpan & Clay and gravel & hard & -- & -- & \\
\hline 450 & $5 / 4-26 \mathrm{~N} 1$ & & & 6 & 12 & 1.8 & 3.7 & clay, sandy & Clay and sand (or sandstone) & - & -- & -- & \\
\hline 450 & $5 / 4-26 \mathrm{~N} 1$ & & & 12 & 45 & 3.7 & 13.7 & clay and gravel & Clay and gravel & -- & -- & -- & \\
\hline 450 & $5 / 4-26 \mathrm{~N} 1$ & & & 45 & 50 & 13.7 & 15.2 & clay, yellow & Clay & -- & -- & -- & \\
\hline 450 & $5 / 4-26 \mathrm{~N} 1$ & & & 50 & 385 & 15.2 & 117.3 & $\begin{array}{l}\text { agglomerate, red, vesicular } \\
\text { basalt, blue, platy to blocky, some }\end{array}$ & Ash or tuff & -- & -- & -- & Tsv \\
\hline 450 & $5 / 4-26 \mathrm{~N} 1$ & & & 385 & 450 & 117.3 & 137.2 & $\begin{array}{l}\text { blue clay } \\
\text { clay, brown (Not enough water }\end{array}$ & Basalt & -- & -- & -- & \\
\hline 451 & $5 / 4-29 N 2$ & 451 & 5/4-29N2 & 0 & 310 & 0.0 & 94.5 & for domestic use) & Clay & -- & -- & -- & Qh \\
\hline 451 & 5/4-29N2 & & & 105 & 310 & 32.0 & 94.5 & no data & No data & -- & -- & -- & \\
\hline 452 & $5 / 4-30 \mathrm{R} 1$ & 452 & 5/4-30R1 & 0 & 900 & 0.0 & 274.3 & clay, yellow & Clay & -- & -- & -- & Qal and Qh \\
\hline 452 & 5/4-30R1 & & & 900 & 1400 & 274.3 & 426.7 & $\begin{array}{l}\text { shale, gray or Neroly sandstone, } \\
\text { flow of brackish water }\end{array}$ & Clay and sand (or sandstone) & cemented & .- & .- & Neroly sandstone \\
\hline 453 & $5 / 4-29 \mathrm{~N} 1$ & 453 & 5/4-29N1 & 0 & 33 & 0.0 & 10.1 & $\begin{array}{l}\text { flow of brackisn water } \\
\text { no data }\end{array}$ & No data & cemented & -- & -- & Neroly sandstone \\
\hline 454 & $5 / 4-29 Q 1$ & 454 & 5/4-29Q1 & 0 & 170 & 0.0 & 51.8 & clay, yellow & Clay & -- & -- & -- & Qh \\
\hline 455 & 5/4-29Q2 & 455 & 5/4-29Q2 & 0 & 8 & 0.0 & 2.4 & soil & Sand and clay & -- & -- & -- & Qh \\
\hline 455 & $5 / 4-29 Q 2$ & & & 8 & 45 & 2.4 & 13.7 & clay, yellow & Clay & -- & -- & -- & \\
\hline 455 & $5 / 4-29 Q 2$ & & & 45 & 70 & 13.7 & 21.3 & sand, yellow and water & Sand & -- & -- & -- & \\
\hline 455 & $5 / 4-29 Q 2$ & & & 70 & 139 & 21.3 & 42.4 & $\begin{array}{l}\text { clay, blue } \\
\text { clution }\end{array}$ & Clay & -- & -- & -- & \\
\hline 455 & $5 / 4-2902$ & & & 139 & 143 & 42.4 & 43.6 & gravel and water & Gravel & .- & .- & .- & \\
\hline 455 & 5/4-29Q2 & & & 143 & 149 & 43.6 & 45.4 & clay, yellow and boulders & Clay and gravel & -- & -- & -- & \\
\hline 456 & $5 / 4-27 \mathrm{R} 1$ & 456 & 5/4-27R1 & 0 & 42 & 0.0 & 12.8 & mud blue & Clay and sand (or sandstone) & -- & -- & -- & Qyal \\
\hline 456 & $5 / 4-27 \mathrm{R} 1$ & & & 42 & 82 & 12.8 & 25.0 & gravel & Gravel & -- & -- & -- & \\
\hline 456 & $5 / 4-27 \mathrm{R} 1$ & & & 82 & 107 & 25.0 & 32.6 & clay, blue and gravel & Clay and gravel & -- & -- & -- & \\
\hline 456 & $5 / 4-27 \mathrm{R} 1$ & & & 107 & 125 & 32.6 & 38.1 & clay green impervious & Clay & -- & -- & -- & Qoal \\
\hline 456 & $5 / 4-27 R 1$ & & & 125 & 128 & 38.1 & 39.0 & 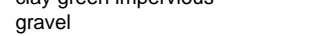 & Gravel & -- & -- & -- & \\
\hline 456 & $5 / 4-27 \mathrm{R} 1$ & & & 128 & 168 & 39.0 & 51.2 & clay, blue and gravel & Clay and gravel & -- & -- & -- & \\
\hline 456 & $5 / 4-27 R 1$ & & & 168 & 171 & 51.2 & 52.1 & dark-lava gravel & Gravel & -- & -- & -- & \\
\hline 456 & $5 / 4-27 R 1$ & & & 171 & 175 & 52.1 & 53.3 & lava, blue-gray & Basalt & -- & -- & -- & Tsv \\
\hline 456 & $5 / 4-27 \mathrm{R} 1$ & & & 175 & 178 & 53.3 & 54.3 & tuff, maroon, with lava & Ash or tuff & .- & .- & .- & \\
\hline 456 & $5 / 4-27 R 1$ & & & 178 & 180 & 54.3 & 54.9 & lava with maroon tuff & Basalt & -- & -- & -- & \\
\hline 456 & $5 / 4-27 \mathrm{R} 1$ & & & 180 & 267 & 54.9 & 81.4 & tuff, maroon & Ash or tuff & -- & -- & -- & \\
\hline 456 & $5 / 4-27 \mathrm{R} 1$ & & & 267 & 270 & 81.4 & 82.3 & tuff with lava & Ash or tuff & -- & -- & -- & \\
\hline 456 & $5 / 4-27 R 1$ & & & 270 & 275 & 82.3 & 83.8 & tuff maroon & Ash or tuff & -- & -- & -- & \\
\hline 456 & $5 / 4-27 \mathrm{R} 1$ & & & 275 & 317 & 83.8 & 96.6 & lava, dark, porous & Basalt & -- & -- & -- & \\
\hline 456 & $5 / 4-27 \mathrm{R} 1$ & & & 317 & 322 & 96.6 & 98.1 & $\begin{array}{l}\text { lava, dark, with tuff } \\
\text { lava, dark; some blue clay with }\end{array}$ & Basalt & -- & -- & -- & \\
\hline 456 & $5 / 4-27 \mathrm{R} 1$ & & & 322 & 357 & 98.1 & 108.8 & sand & Basalt & -- & .- & .- & \\
\hline 456 & $5 / 4-27 \mathrm{R} 1$ & & & 357 & 368 & 108.8 & 112.2 & sand, dark colored with clay & Sand and clay & -- & -- & -- & \\
\hline 456 & $5 / 4-27 \mathrm{R} 1$ & & & 368 & 485 & 112.2 & 147.8 & lava, dark, fine-grained & Basalt & -- & -- & -- & \\
\hline 457 & $5 / 4-30 \mathrm{P} 1$ & 457 & 5/4-30P1 & 0 & 15 & 0.0 & 4.6 & surface & Sand and clay & -- & -- & -- & Qh \\
\hline 457 & 5/4-30P1 & & & 15 & 200 & 4.6 & 61.0 & clay, yellow & Clay & -- & -- & -- & \\
\hline 457 & 5/4-30P1 & & & 200 & 253 & 61.0 & 77.1 & clay, blue & Clay & -- & -- & -- & \\
\hline 457 & 5/4-30P1 & & & 253 & 257 & 77.1 & 78.3 & clay and gravel; some water & Clay and gravel & -- & -- & -- & \\
\hline
\end{tabular}


Appendix 3. Lithologic data. - Continued

ISite IDs and well number correspond to locations in Appendix 1, a site ID and well number is listed for each drilled interval for a particular drill hole. For convenience, the site ID and well numbers are repeated at the shallowest drilled interval for each well. leaders 1.

\begin{tabular}{|c|c|c|c|c|c|c|c|c|c|c|c|c|c|}
\hline Site ID & Well number & $\begin{array}{l}\text { Site ID, } \\
\text { top of } \\
\text { well }\end{array}$ & $\begin{array}{l}\text { Well number, } \\
\text { top of well }\end{array}$ & $\begin{array}{c}\text { Top of } \\
\text { interval, in } \\
\text { feet }\end{array}$ & $\begin{array}{c}\text { Base of } \\
\text { interval, in } \\
\text { feet }\end{array}$ & $\begin{array}{c}\text { Top of } \\
\text { interval, in } \\
m\end{array}$ & $\begin{array}{c}\text { Base of } \\
\text { interval, in } \\
\mathrm{m}\end{array}$ & Reported lithologic unit ${ }^{1}$ & Interpreted lithologic class ${ }^{2}$ & $\begin{array}{c}\text { Reported } \\
\text { degree of } \\
\text { cementation or } \\
\text { induration } \\
\end{array}$ & $\begin{array}{c}\text { Fossils } \\
\text { reported in } \\
\text { interval }^{4}\end{array}$ & $\begin{array}{c}\text { Organic } \\
\text { matter } \\
\text { reported in } \\
\text { interval }^{5}\end{array}$ & $\begin{array}{l}\text { Reported stratigraphic top of geologic } \\
\text { unit }^{6}\end{array}$ \\
\hline 457 & $5 / 4-30 \mathrm{P} 1$ & & & 257 & 347 & 78.3 & 105.8 & clay, blue & Clay & -- & -- & -- & \\
\hline 458 & 5/4-27Q1 & 458 & 5/4-27Q1 & 0 & 6 & 0.0 & 1.8 & soil & Sand and clay & -- & -- & -- & Qyal \\
\hline 458 & 5/4-27Q1 & & & 6 & 34 & 1.8 & 10.4 & hard gravel, salt water & Gravel & hard & -- & -- & \\
\hline 458 & 5/4-27Q1 & & & 34 & 115 & 10.4 & 35.1 & loose gravel, salty water & Gravel & -. & -- & -- & \\
\hline 458 & $5 / 4-27 Q 1$ & & & 115 & 120 & 35.1 & 36.6 & clay, blue & Clay & -- & -- & -- & Qoal \\
\hline 458 & 5/4-27Q1 & & & 120 & 252 & 36.6 & 76.8 & sand, blue, and clay & Sand and clay & -- & -- & -- & \\
\hline 458 & 5/4-27Q1 & & & 252 & 287 & 76.8 & 87.5 & shale, brown & Undifferentiated basement & cemented & -- & -- & Knoxville and Horsetown Formations \\
\hline 458 & $5 / 4-27 Q 1$ & & & 287 & 315 & 87.5 & 96.0 & $\begin{array}{l}\text { shale, brown; and blue sand } \\
\text { shale, brown, blue clay, yellow }\end{array}$ & Undifferentiated basement & cemented & -- & -- & \\
\hline 458 & 5/4-27Q1 & & & 315 & 382 & 96.0 & 116.4 & clay, blue shale & Undifferentiated basement & cemented & -. & -- & \\
\hline 458 & 5/4-27Q1 & & & 382 & 490 & 116.4 & 149.4 & $\begin{array}{l}\text { clay, yellow; and gravel } \\
\text { clay, blue and yellow; volcanic }\end{array}$ & Undifferentiated basement & -- & -- & - & \\
\hline 458 & 5/4-27Q1 & & & 490 & 555 & 149.4 & 169.2 & rock gravel & Undifferentiated basement & -- & -- & -- & \\
\hline 458 & 5/4-27Q1 & & & 555 & 570 & 169.2 & 173.7 & sand, blue, close & Undifferentiated basement & -- & -- & -- & \\
\hline 458 & 5/4-27Q1 & & & 570 & 575 & 173.7 & 175.3 & sand, blue, compact & Undifferentiated basement & & -- & -- & \\
\hline 458 & $5 / 4-27 \mathrm{Q} 1$ & & & 575 & 712 & 175.3 & 217.0 & not described & No data & - & -. & -- & \\
\hline 459 & $5 / 4-27 \mathrm{R} 2$ & 459 & 5/4-27R2 & 0 & 4 & 0.0 & 1.2 & tule mud & Clay and sand (or sandstone) & -- & -- & tule & Qyal \\
\hline 459 & 5/4-27R2 & & & 4 & 25 & 1.2 & 7.6 & clay, blue & Clay & -- & -- & -- & \\
\hline 459 & 5/4-27R2 & & & 25 & 45 & 7.6 & 13.7 & clay, sandy, blue & Clay and sand (or sandstone) & -- & -- & -- & \\
\hline 459 & $5 / 4-27 \mathrm{R} 2$ & & & 45 & 73 & 13.7 & 22.3 & loose gravel & Gravel & -- & -- & -- & \\
\hline 459 & $5 / 4-27 \mathrm{R} 2$ & & & 73 & 94 & 22.3 & 28.7 & boulders and gravel & Gravel & - & - & - & \\
\hline 459 & $5 / 4-27 R_{2} 2$ & & & 94 & 105 & 28.7 & 32.0 & light gravel & Gravel & -- & -- & -- & \\
\hline 459 & 5/4-27R2 & & & 105 & 107 & 32.0 & 32.6 & clay and gravel & Clay and gravel & -- & -- & -- & \\
\hline 459 & 5/4-27R2 & & & 107 & 150 & 32.6 & 45.7 & volcanic clay, green & Ash or tuff & -- & -- & .- & Qoal \\
\hline 459 & $5 / 4-27 \mathrm{R} 2$ & & & 150 & 160 & 45.7 & 48.8 & rock, green & Ash or tuff & hard & -- & -- & \\
\hline 459 & $5 / 4-27 \mathrm{R} 2$ & & & 160 & 250 & 48.8 & 76.2 & lava, red; rock & Basalt & hard & -- & -- & Tsv \\
\hline 459 & 5/4-27R2 & & & 250 & 266 & 76.2 & 81.1 & rock, red, harder & Basalt & hard & -- & -- & \\
\hline 459 & 5/4-27R2 & & & 266 & 269 & 81.1 & 82.0 & rock, blue hard & Basalt & hard & -. & - & \\
\hline 459 & $5 / 4-27 R 2$ & & & 269 & 272 & 82.0 & 82.9 & rock, red, hard & Basalt & hard & -. & -.- & \\
\hline 459 & $5 / 4-27 \mathrm{R} 2$ & & & 272 & 302 & 82.9 & 92.0 & rock, red, softer & Basalt & hard & -- & -- & \\
\hline 459 & $5 / 4-27 R 2$ & & & 302 & 330 & 92.0 & 100.6 & rock, very hard & Basalt & hard & -- & -- & \\
\hline 460 & $5 / 4-35 \mathrm{R} 4$ & 460 & 5/4-35R4 & 0 & 5 & 0.0 & 1.5 & topsoil & Sand and clay & -- & -- & -- & Qyal and Qoal (?) \\
\hline 460 & $5 / 4-35 R 4$ & & & 5 & 30 & 1.5 & 9.1 & gravel in clay & Clay and gravel & -. & -- & - & \\
\hline 460 & $5 / 4-35 R 4$ & & & 30 & 68 & 9.1 & 20.7 & $\begin{array}{l}\text { clay yellow } \\
\text { San Pablo group undifferentiated, }\end{array}$ & Clay & -- & -- & -- & \\
\hline 460 & $5 / 4-35 R 4$ & & & 68 & 217 & 20.7 & 66.1 & shale, gray & Undifferentiated basement & cemented & -- & -. & San Pablo Group \\
\hline 460 & $5 / 4-35 R 4$ & & & 217 & 429 & 66.1 & 130.8 & shale, black & Undifferentiated basement & cemented & -- & -- & \\
\hline 460 & $5 / 4-35 R 4$ & & & 429 & 439 & 130.8 & 133.8 & gum, black, asphalt like & Undifferentiated basement & -- & -- & black gum & \\
\hline 460 & $5 / 4-35 R 4$ & & & 439 & 453 & 133.8 & 138.1 & rock & Undifferentiated basement & hard & -- & -- & \\
\hline 460 & $5 / 4-35 R 4$ & & & 453 & 1082 & 138.1 & 329.8 & shale, gray & Undifferentiated basement & cemented & -. & .. & \\
\hline 460 & $5 / 4-35 R 4$ & & & 1082 & 1090 & 329.8 & 332.2 & rock & Undifferentiated basement & hard & -- & -- & \\
\hline 460 & $5 / 4-35 R 4$ & & & 1090 & 1105 & 332.2 & 336.8 & shale, hard & Undifferentiated basement & hard & -- & -- & \\
\hline 460 & $5 / 4-35 R 4$ & & & 1105 & 1115 & 336.8 & 339.9 & boulders & Undifferentiated basement & -. & -- & -.- & \\
\hline 460 & 5/4-35R4 & & & 1115 & 1202 & 339.9 & 366.4 & shale, black & Undifferentiated basement & cemented & -- & -- & \\
\hline 460 & 5/4-35R4 & & & 1202 & 1205 & 366.4 & 367.3 & sand, black & Undifferentiated basement & - & -- & -- & \\
\hline 460 & $5 / 4-35 R 4$ & & & 1205 & 1208 & 367.3 & 368.2 & shale, light & Undifferentiated basement & cemented & -- & -- & \\
\hline 461 & $4 / 4-4 C 1$ & 461 & 4/4-4C1 & 0 & 10 & 0.0 & 3.0 & surface & Sand and clay & -- & -- & .- & Qyal \\
\hline 461 & $4 / 4-4 \mathrm{C} 1$ & & & 10 & 25 & 3.0 & 7.6 & clay, yellow & Clay & -- & -- & -- & $\mathrm{Qh}(?)$ \\
\hline 461 & $4 / 4-4 \mathrm{C} 1$ & & & 25 & 60 & 7.6 & 18.3 & $\begin{array}{l}\text { clay, yellow; sand and some } \\
\text { gravel }\end{array}$ & Clay, sand, and gravel & -- & -- & -- & \\
\hline 461 & $4 / 4-4 \mathrm{C} 1$ & & & 60 & 80 & 18.3 & 24.4 & gravel and sand; some yellow clay & Sand (or sandstone) and gravel & -- & -- & .- & \\
\hline 462 & $4 / 4-5 \mathrm{C} 1$ & 462 & $4 / 4-5 \mathrm{C} 1$ & 0 & 92 & 0.0 & 28.0 & clay, red & Clay & -- & -- & -- & Qh \\
\hline 462 & $4 / 4-5 \mathrm{C} 1$ & & & 92 & 98 & 28.0 & 29.9 & gravel, water & Gravel & -- & -- & -- & \\
\hline 462 & $4 / 4-5 C 1$ & & & 98 & 155 & 29.9 & 47.2 & clay, red & Clay & -- & -- & -- & \\
\hline 463 & $4 / 4-16 \mathrm{D} 1$ & 463 & 4/4-16D1 & 0 & 70 & 0.0 & 21.3 & tule mud & Clay and sand (or sandstone) & -- & -- & tule & Qyal and Qoal \\
\hline 463 & $4 / 4-16 \mathrm{D} 1$ & & & 70 & 108 & 21.3 & 32.9 & gravel and water & Gravel & -. & -- & -- & \\
\hline 463 & $4 / 4-16 \mathrm{D} 1$ & & & 108 & 118 & 32.9 & 36.0 & clay, gray & Clay & -- & -- & -- & \\
\hline 463 & $4 / 4-16 \mathrm{D} 1$ & & & 118 & 130 & 36.0 & 39.6 & clay, yellow & Clay & -. & -- & -. & \\
\hline 463 & $4 / 4-16 \mathrm{D} 1$ & & & 130 & 141 & 39.6 & 43.0 & clay, blue & Clay & -- & -- & -- & \\
\hline 463 & $4 / 4-16 \mathrm{D} 1$ & & & 141 & 149 & 43.0 & 45.4 & gravel and water & Gravel & -. & -- & -- & \\
\hline
\end{tabular}


Appendix 3. Lithologic data. - Continued

ISite IDs and well number correspond to locations in Appendix 1, a site ID and well number is listed for each drilled interval for a particular drill hole. For convenience, the site ID and well numbers are repeated at the shallowest drilled interval for each well. leaders (--), not reported]

\begin{tabular}{|c|c|c|c|c|c|c|c|c|c|c|c|c|c|}
\hline Site ID & Well number & $\begin{array}{l}\text { Site ID, } \\
\text { top of } \\
\text { well }\end{array}$ & $\begin{array}{l}\text { Well number, } \\
\text { top of well }\end{array}$ & $\begin{array}{l}\text { Top of } \\
\text { interval, in } \\
\text { feet }\end{array}$ & $\begin{array}{c}\text { Base of } \\
\text { interval, in } \\
\text { feet }\end{array}$ & $\begin{array}{l}\text { Top of } \\
\text { interval, in } \\
\mathrm{m}\end{array}$ & $\begin{array}{l}\text { Base of } \\
\text { interval, in } \\
\mathrm{m}\end{array}$ & Reported lithologic unit' & Interpreted lithologic class ${ }^{2}$ & $\begin{array}{c}\text { Reported } \\
\text { degree of } \\
\text { cementation or } \\
\text { induration }^{3} \\
\end{array}$ & $\begin{array}{l}\text { Fossils } \\
\text { reported in } \\
\text { interval }^{4}\end{array}$ & $\begin{array}{c}\text { Organic } \\
\text { matter } \\
\text { reported in } \\
\text { interval }^{5}\end{array}$ & $\begin{array}{l}\text { Reported stratigraphic top of geologic } \\
\text { unit }^{6}\end{array}$ \\
\hline 463 & $4 / 4-16 \mathrm{D} 1$ & & & 149 & 178 & 45.4 & 54.3 & clay, gray & Clay & -- & -- & -- & \\
\hline 463 & $4 / 4-16 \mathrm{D} 1$ & & & 178 & 182 & 54.3 & 55.5 & gravel and water & Gravel & -- & -- & -- & \\
\hline 463 & 4/4-16D1 & & & 182 & 216 & 55.5 & 65.8 & $\begin{array}{l}\text { clay blue } \\
\text { chat }\end{array}$ & Clay & -- & -- & -- & \\
\hline 463 & $4 / 4-16 \mathrm{D} 1$ & & & 216 & 219 & 65.8 & $\begin{array}{l}66.8 \\
66.8\end{array}$ & gravel and water & Gravel & -- & - & -- & \\
\hline 463 & 4/4-16D1 & & & 219 & 228 & 66.8 & 69.5 & cement, gray & Clay and gravel & cemented & -- & -- & \\
\hline 463 & $4 / 4-16 \mathrm{D} 1$ & & & 228 & 231 & 69.5 & 70.4 & clay, yellow; and boulders & Clay and gravel & -- & -- & -- & Qh (?) \\
\hline 464 & 4/4-16E1 & 464 & 4/4-16E1 & 0 & 52 & 0.0 & 15.8 & tule mud blue & Clay and sand (or sandstone) & -- & -- & tule & Qyal and Qoal \\
\hline 464 & 4/4-16E1 & & & 52 & 56 & 15.8 & 17.1 & gravel, salty water & Gravel & -- & -- & -- & \\
\hline 464 & 4/4-16E1 & & & 56 & 93 & $\begin{array}{l}17.1 \\
17.1\end{array}$ & 28.3 & tule mud, blue & Clay and sand (or sandstone) & - & -- & tule & \\
\hline 464 & 4/4-16E1 & & & 93 & 99 & 28.3 & 30.2 & gravel, salty water & Gravel & -- & -- & - & \\
\hline 464 & 4/4-16E1 & & & 99 & 138 & 30.2 & 42.1 & tule mud, blue & Clay and sand (or sandstone) & -- & -- & tule & \\
\hline 464 & 4/4-16E1 & & & 138 & 143 & 42.1 & 43.6 & sand & Sand & -- & -- & - & \\
\hline 464 & 4/4-16E1 & & & 143 & 164 & 43.6 & 50.0 & gravel & Gravel & -- & - & -- & \\
\hline 464 & 4/4-16E1 & & & 164 & 239 & 50.0 & 72.8 & tule mud, blue & Clay and sand (or sandstone) & -- & - & tule & \\
\hline 464 & 4/4-16E1 & & & 239 & 241 & 72.8 & 73.5 & sand & Sand & -- & -- & - & \\
\hline 464 & 4/4-16E1 & & & 241 & 279 & 73.5 & $\begin{array}{l}85.0 \\
85.0\end{array}$ & clay, sandy, yellow & Clay and sand (or sandstone) & - & - & -- & \\
\hline 464 & 4/4-16E1 & & & 279 & 280 & 85.0 & 85.3 & gravel, coarse, fresh water & Gravel & -- & -- & .- & \\
\hline 464 & 4/4-16E1 & & & 280 & 301 & 85.3 & 91.7 & sand, coarse, fresh water & Sand (or sandstone) and gravel & -- & -- & _- & \\
\hline 464 & $4 / 4-16 \mathrm{E} 1$ & & & 301 & 306 & 91.7 & 93.3 & clay & Clay & -- & -- & -- & \\
\hline
\end{tabular}

Lithology transcribed exactly from driller's descriptions, as published in the three Water-Supply Papers.

${ }^{2}$ Assignment of interpreted lithologic class explained in table 2.

${ }^{3}$ Degree of cementation transcribed exactly from driller's descriptions, as published in the three Water-Supply Papers.

4Description of fossils transcribed exactly from driller's descriptions, as published in the three Water-Supply Papers.

${ }^{5}$ Presence of organic matter transcribed exactly from driller's descriptions, as published in the three Water-Supply Papers.

${ }^{6}$ Top of stratigraphic units listed as published in the three Water-Supply Papers; units queried as in original publications. Abbreviations of stratigraphic units and deposits: KJu, Franciscan and Knoxville Formations,

undifferentiated; Qal, Alluvium; Qh, Huichica Formation; Qoal, Older alluvium; Qt, Terrace deposits; QTc, Continental deposits; QTge, Glen Ellen Formation; QTm, Merced Formation; Qyal, Younger alluvium; Tp, Petalum;

Formation; Tsv, Sonoma volcanics, undifferentiated. Blank cells imply the same stratigraphic unit unit as the overlying formation top. 